\title{
A quantitative analysis of the molecular organization of dendritic spines from hippocampal neurons
}

\author{
Dissertation \\ for the award of the degree \\ Doctor rerum naturalium
}

by the Georg-August-Universität Göttingen

within the doctoral program IMPRS for Molecular Biology

of the Göttingen Graduate School for Neurosciences, Biophysics, and Molecular Biosciences

submitted by

Martin Helm

from Stuttgart, Germany.

Göttingen, January 2019 


\section{Thesis Committee and Examination Board}

Prof. Dr. Silvio O. Rizzoli (first referee)

Department of Neuro- and Sensory Physiology

University Medical Center Göttingen, Göttingen, Germany

Prof. Dr. Nils Brose (second referee)

Department of Molecular Neurobiology

Max-Planck-Institute for Experimental Medicine, Göttingen, Germany

Prof. Dr. Bert de Groot

Research Group Computational Biomolecular dynamics

Max-Planck-Institute for Biophysical Chemistry

\section{Further members of the Examination Board}

Dr. Camin Dean

Group of Trans-synaptic Signaling

European Neuroscience Institute, Göttingen, Germany

Dr. Dieter Klopfenstein

Department of Biophysics

University Göttingen, Göttingen, Germany

Prof. Dr. Ralf Heinrich

Department for Cellular Neurobiology

University Göttingen, Göttingen, Germany

Date of oral examination: 26.03.2019 


\section{Affidavit}

I hereby declare that I prepared this dissertation independently, and with no other sources and aids than quoted.

Martin Helm 
Dedication

Für Tabea 


\section{Table of Contents}

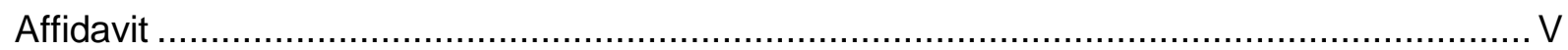

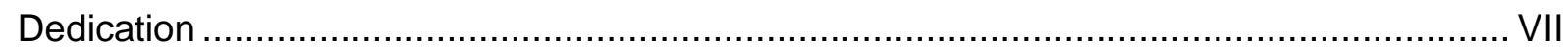

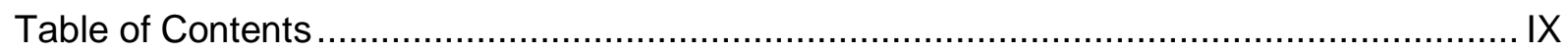

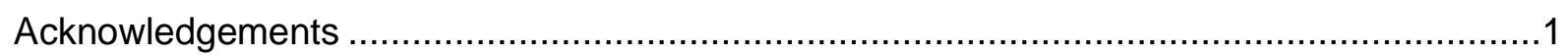

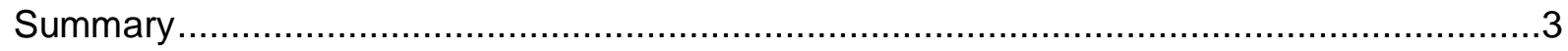

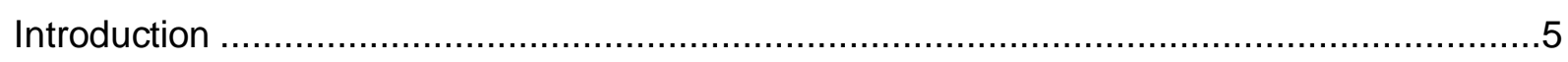

The postsynapse - In need of a quantitative understanding ..................................... 5

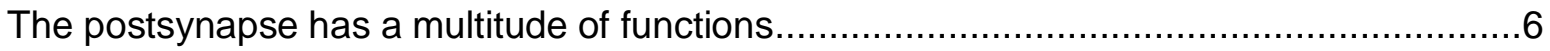

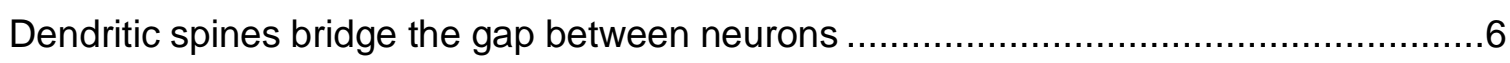

The PSD is the central active zone on dendritic spines .........................................6

Dendritic spines differentiate between inputs from many sources - Electrochemical

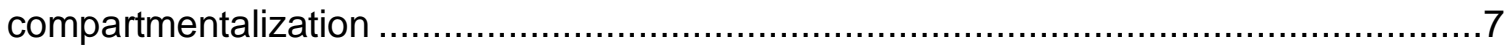

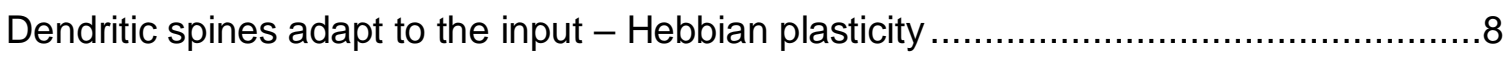

Dendritic spines are controlled by the neuron as a whole - Homeostatic plasticity ........11

Dendritic spines respond to and modulate the input - Retrograde signaling.................12

Dendritic spines sustain themselves - Local translation and organelles ......................12

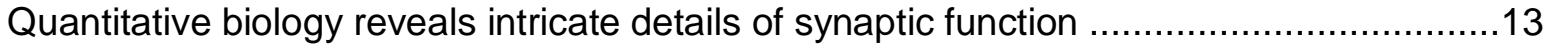

The current understanding of dendritic spines is insufficient ....................................14

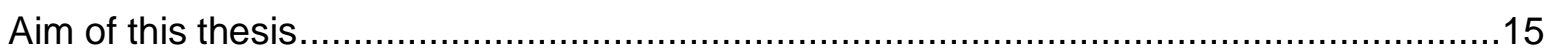

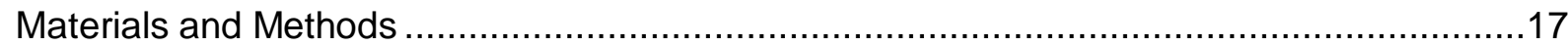

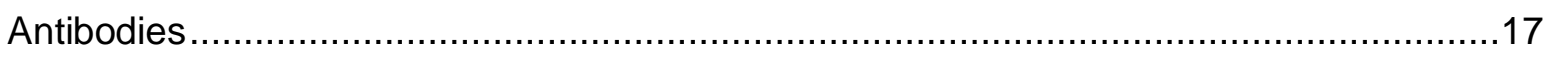

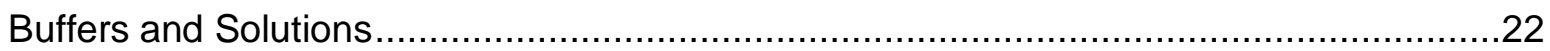

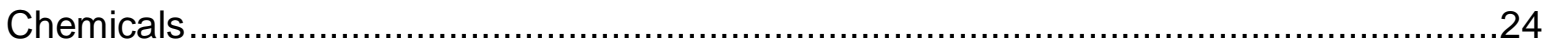

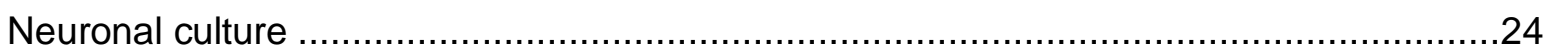

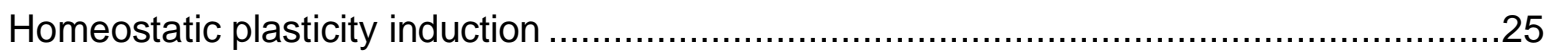

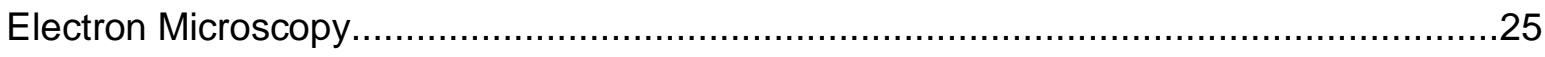

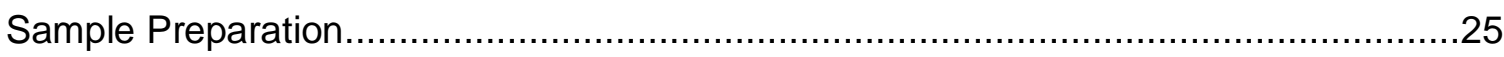

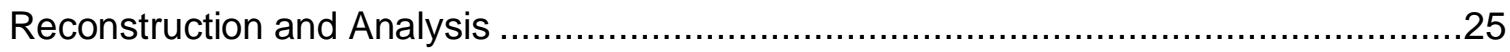

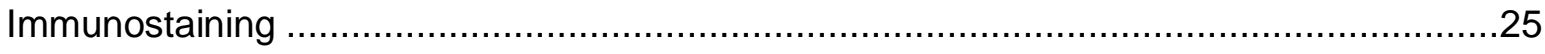




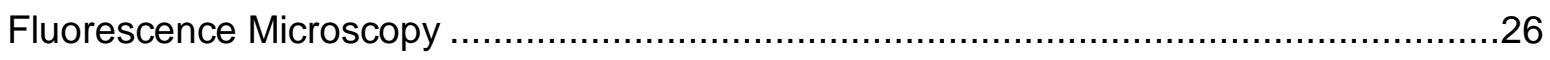

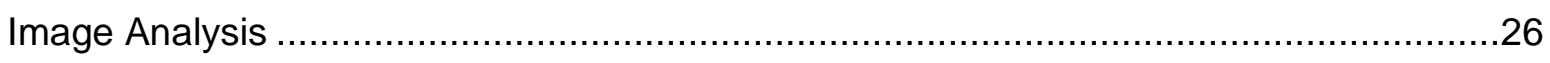

Calculating the average protein distributions in dendritic spines ................................27

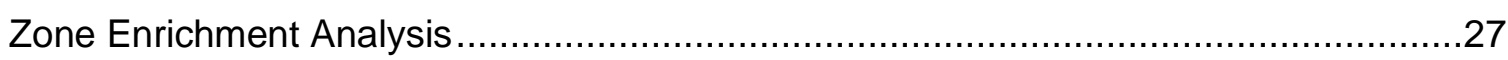

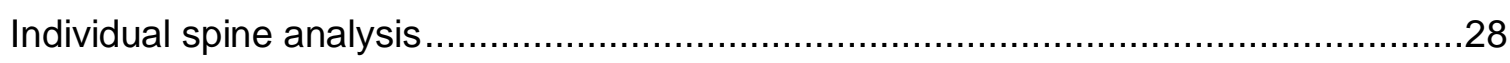

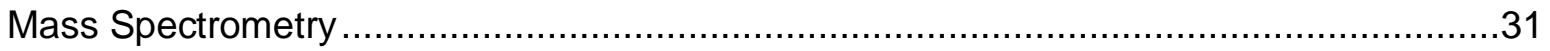

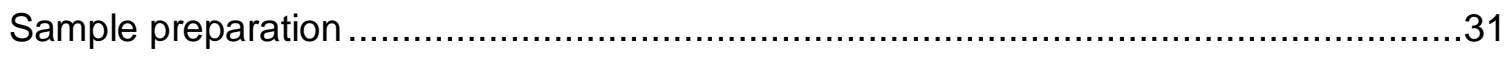

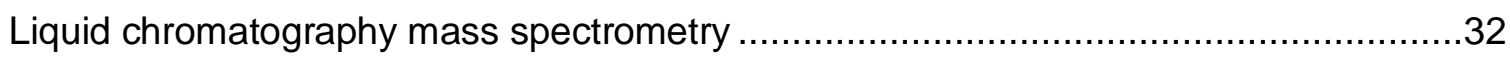

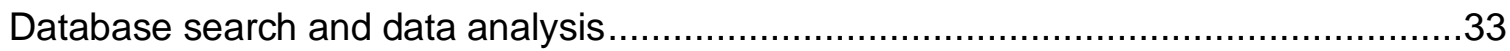

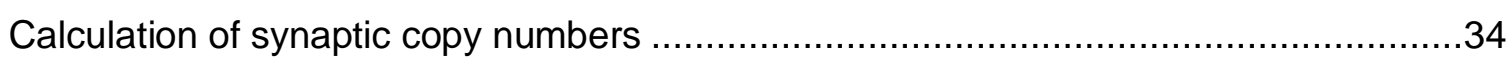

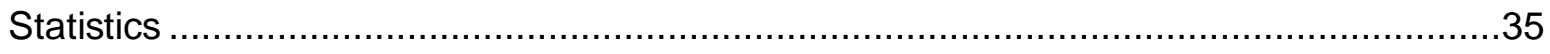

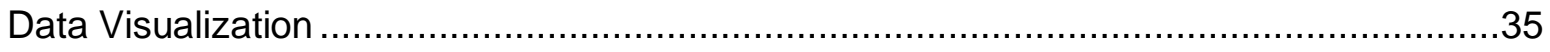

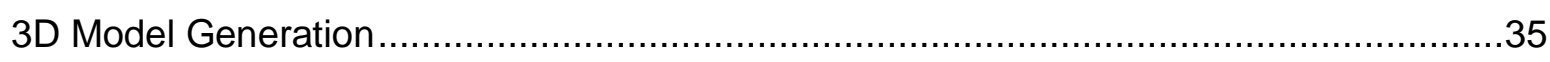

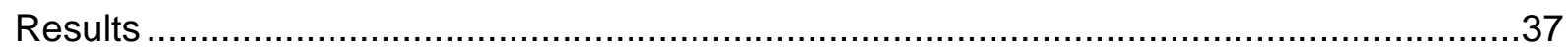

The Banker culture is a well-defined model to study dendritic spines ...............................37

The ultrastructure of dendritic spines and its organelles in Banker cultures .....................40

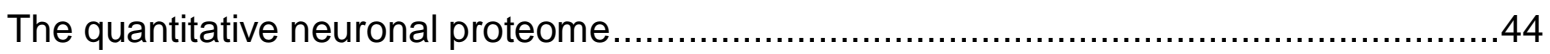

Label-free mass spectrometry reveals the quantitative neuronal proteome …................44

Subcellular copy numbers can be derived using imaging methods ................................47

The quantitative average distribution and abundance of 105 postsynaptic proteins ..........51

Cytoskeleton and associated proteins ........................................................................5

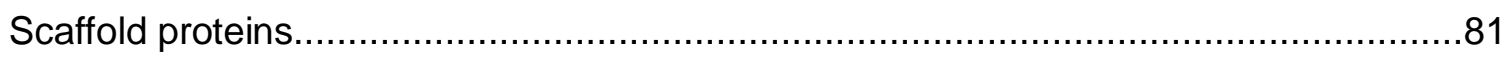

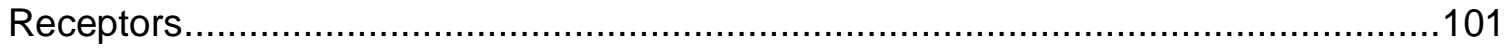

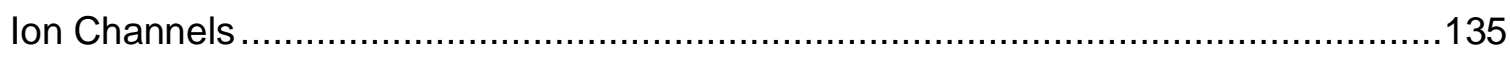

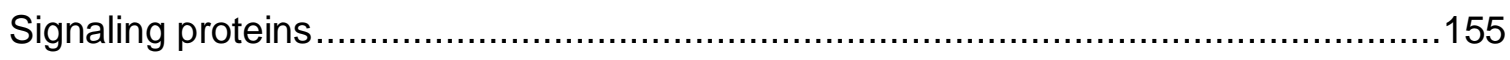

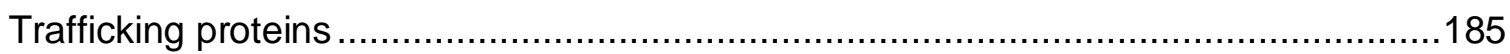

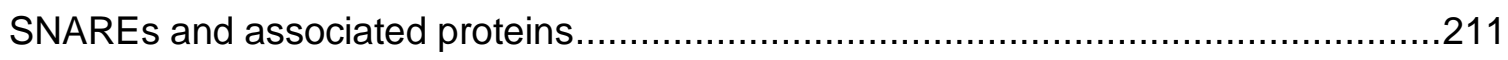

Organelle proteins

Synaptic scaling changes the nanoscale localization and abundance of key proteins .....275

Actin 


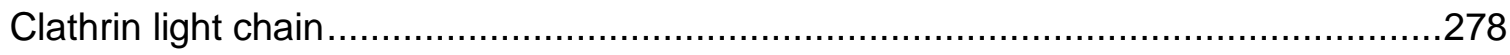

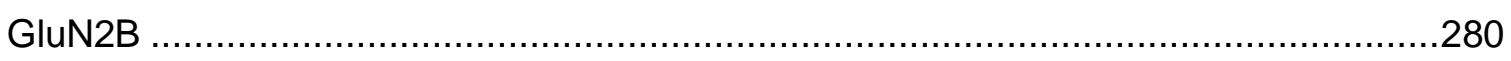

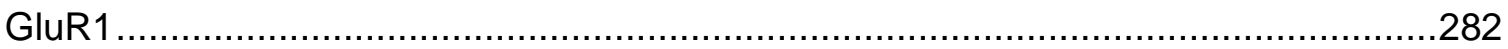

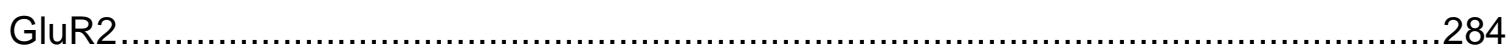

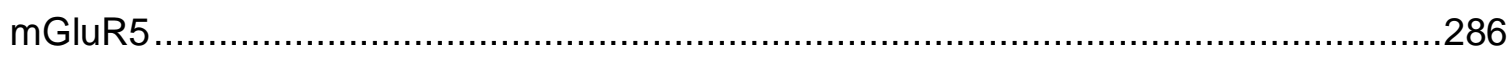

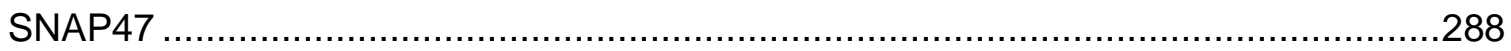

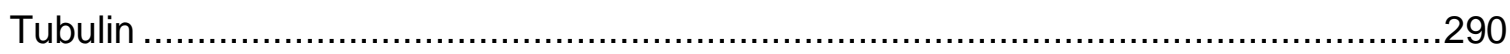

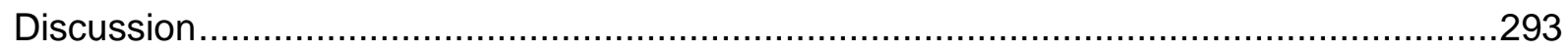

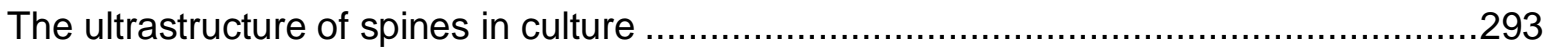

Mushroom and stubby spines have similar morphology ......................................293

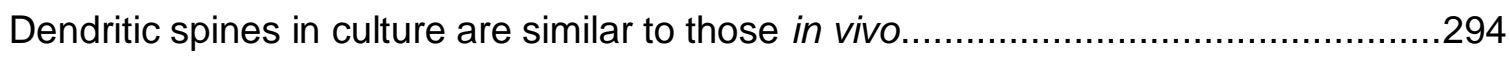

The quantitative neuronal proteome reveals general regulatory principles ....................296

Subcellular copy numbers show a highly skewed distribution ..................................296

The copy number decreases with protein size ....................................................297

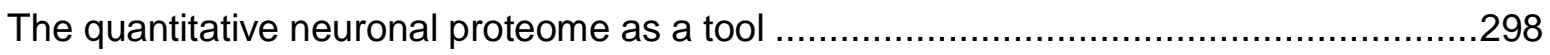

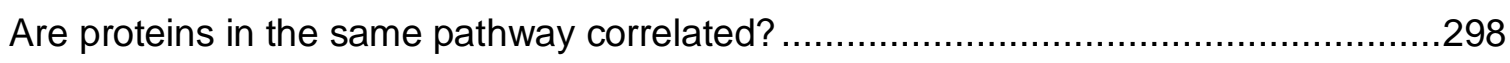

What are neuronal copy numbers in other compartments? ...................................298

How strong is the molecular crowding effect in neurons? ......................................298

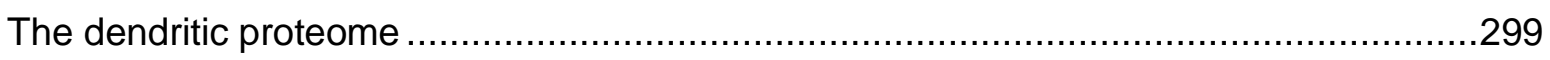

The Nanomap is a window to dendritic spines at unprecedented detail.........................301

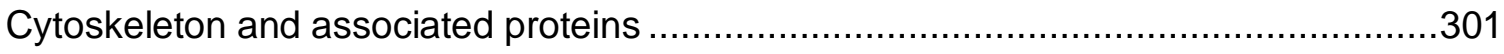

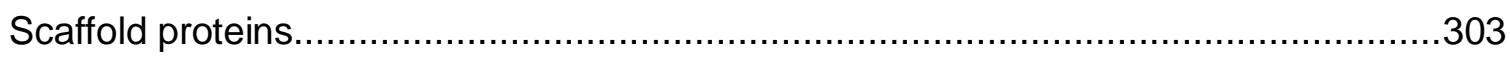

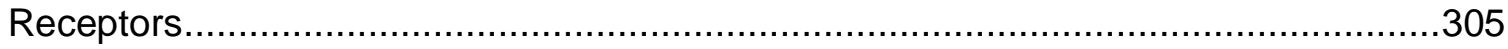

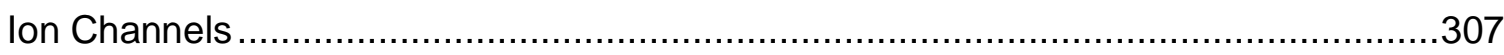

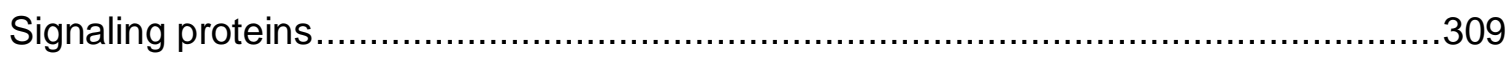

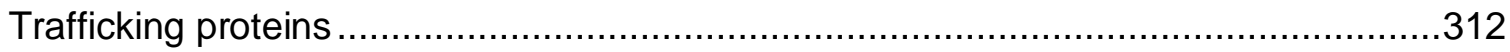

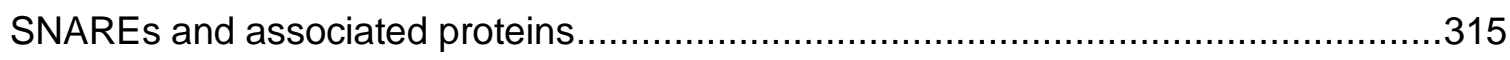

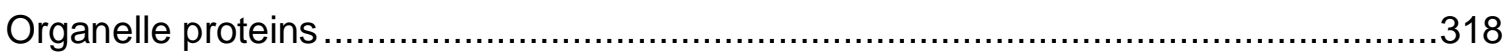

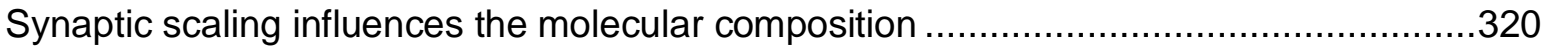

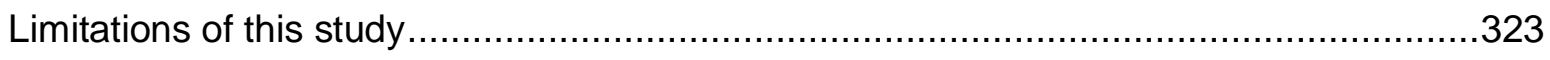


The detection of Homer1 and DiO was limited to confocal resolution 323

The in vitro culture misses some elements present in the brain ...............................324

The manual morphology assessment is limiting protein throughput ...........................324

The Nanomap as a quantitative foundation for further studies .....................................325

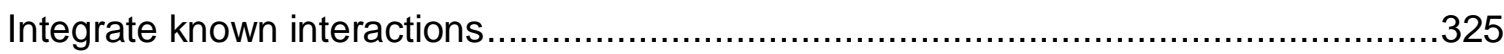

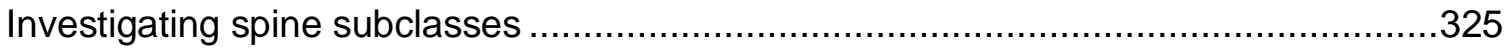

What is the function and composition of synapses at the base of mushroom spines? .325

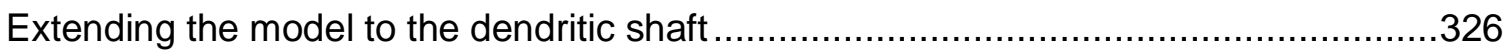

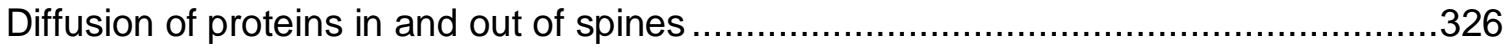

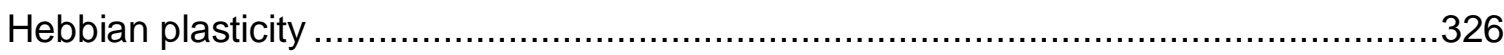

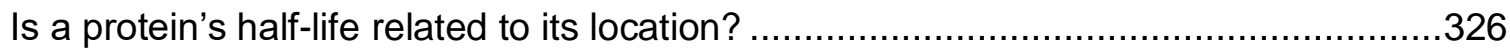

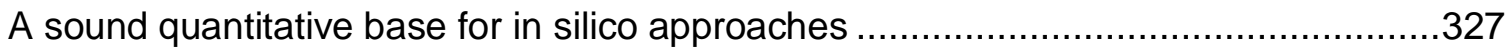

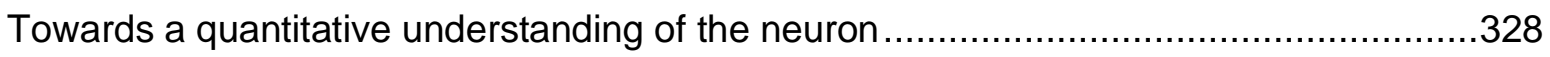

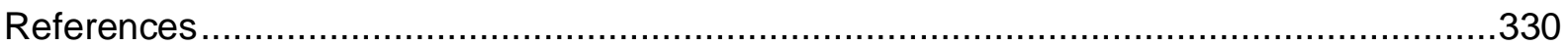

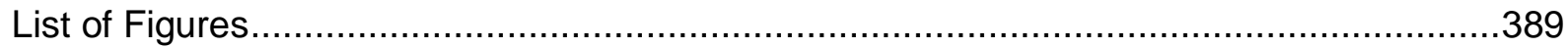

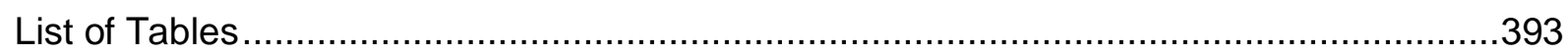

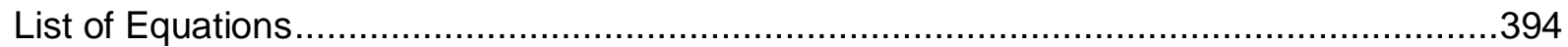

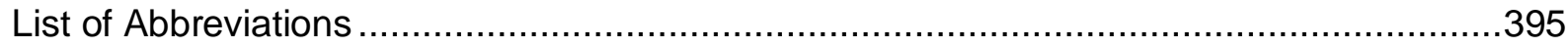

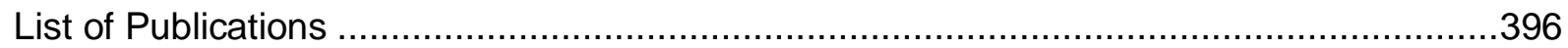

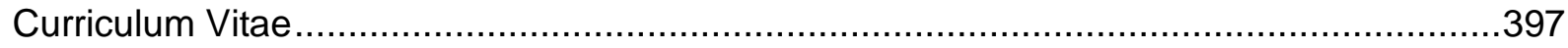




\section{Acknowledgements}

First of all, I am highly indebted to Silvio Rizzoli for letting me pursue my passion for quantitative biology in this work. You have been supportive in every way during this project, way beyond what a PhD supervisor is required to do. I highly benefitted from the many insightful advice on the project and beyond, while also giving me freedom to develop my own ideas. The time in your lab let me expand my skills in all directions and provided me with a great foundation. But most of all, you have made the time fun with your humor. I could not have wished for more.

I would like to thank the members of my thesis committee, Nils Brose and Bert de Groot for their advice and guidance during this project.

I am indebted to the IMPRS Molecular Biology office, for putting in all the extra work required to run such a well-organized graduate program. This program challenged and motivated to go the extra mile to reach my goals and has certainly sharpened my focus. Thank you as well for all the amazing extracurricular opportunities, especially giving me the chance to organize Horizons, for all the insight I gained during my time as a $\mathrm{PhD}$ representative as well as being available at any time for any question.

I would like to thank Sven for convincing me to join this lab. It is amazing how many interests we share. I will always remember all the fun we had during D\&D, our trips to the Spiel', and for answering countless questions and discussions. You always put in the extra mile, regardless whether it was regarding work or hobby. You are a big inspiration to me.

I am indebted to Thomas Schikorski for providing electron microscopy data and to Christina Koerbs and Vanessa Salimi for the data on dendritic spine morphology they obtained during their medical dissertations here. I would also like to thank Tal Dankovich for the amazing amount of work she performed in during her lab rotation and master's thesis on the plasticity changes of dendritic spines. Thank you as well for all our great discussions about programming, everything geek and being part of the nPreSC. I am also indebted to Malena for her work as a research assistant.

I want to thank Kathi and Sebastian for all the input you gave into this project and for the tools you developed, and I could use. Thank you for all great times we had on conferences and in the lab. I want to thank Nicole and Christina for countless hours spent reliably preparing neuronal cultures. I am indebted to Eugenio, for knowing seemingly every aspect of neuroscience and being able to answer so many of my questions, especially on mass 
spectrometry. I want to thank Sunit for doing mass spectrometric analysis of my samples, and Burkhard for providing the 3D protein images. Thank you, Ina, the Adobe Wizard, for helping me with all my questions about Illustrator and InDesign. I also want to thank all the member of the Rizzoli lab. It was a pleasure working here, thank you creating a great atmosphere of teamwork, creativity and fun!

Thank you to all my friends in Witzenhausen. Since we have been here, we have been close like family and I treasure all the times we had here.

Am meisten möchte ich meiner Familie danken. Ihr habt mein Leben mit Freude und Spaß gefüllt und mir das Leben aus immer neuen Blickwinkeln gezeigt. Danke, dass ich mit euch die Welt entdecken darf. Tabea, danke für deine unendliche Unterstützung. Du bist und warst immer da für mich, und du bringst das Beste in mir hervor. Ohne dich wäre diese Arbeit nie möglich gewesen und ich wäre heute nicht da wo ich bin. 


\section{Summary}

Dendritic spines are the major excitatory postsynaptic compartment in the brain. Despite its essential function in synaptic transmission and plasticity, we are still lacking a comprehensive and quantitative understanding of its molecular composition. However, this knowledge is crucial to appreciate the minute regulatory details that affect learning and memory. Here, I investigated the molecular architecture of dendritic spines in absolute quantitative terms and on the nanoscale. I report the localization and abundance of 105 postsynaptic proteins, differentiating between spine classes. Surprisingly, the investigated spine types show highly similar morphology as well as molecular composition. I found that proteins belonging of the same pathway do localize with each other and that their abundance is equally correlated. Using pharmacological treatment, I also show that the organization of dendritic spines is relatively stable during homeostatic plasticity, with only minor changes. Interestingly, the copy number of a given protein on the postsynapse correlated with its copy number in the presynapse, corroborating a tight link between both compartments. In addition, I report the neuronal proteome of pure hippocampal excitatory neurons in absolute values. This revealed that the abundance of a protein decreases with its size, probably because larger proteins do attract damage faster, or because it is energetically costly to produce large proteins. Also, the copy number distribution of mitochondria is highly different to all other investigated compartments, most likely due to its highly specialized function and prokaryotic origin. In total, the database I provide is the most extensive quantitative description of dendritic spines to date. It provides the foundation for highly detailed in silico modelling approaches and will be an important reference tool for synaptic function. 


\section{Introduction}

\section{The postsynapse - In need of a quantitative understanding}

Understanding the brain is one of the fundamental goals of our century. It is currently tackled on many levels, ranging from whole-brain perspectives such as connectomics or transcriptomics, to minute details of individual pathways and proteins. Many of these efforts are quantifying the brain on the systems level, for example by elucidating the ultrastructure of brain regions or even whole brains (Bock et al., 2011; Oh et al., 2014; Zheng et al., 2018) , measuring protein expression on the cell level (Hawrylycz et al., 2012; Kang et al., 2011; Lein et al., 2007) or following its activity in real time (Dombeck et al., 2007; Glasser et al., 2016). However, we are still lacking a quantitative understanding of the individual neuron and of the location where it receives input, its dendrites and the postsynapses located thereon. This is especially aggravating, as it has been shown that even tiny perturbations to the protein composition of its corresponding presynaptic side or their localization can affect its function. For example, we know that changes as small as the addition of a handful of SNAP25 copies to the synaptic vesicle (SV) will inactivate it (Truckenbrodt et al., 2018), that the positioning of SVs in relation to calcium channels is crucial for efficient fusion (Jahn and Fasshauer, 2012), and that presynaptic release zones need to be precisely placed opposite to postsynaptic receptors, to ensure accurate synaptic function (Tang et al., 2016).

Accordingly, the neuron tightly regulates probably many quantitative aspects of presynaptic function, such as molecule and organelle positions or copy numbers, to ensure proper neurotransmitter release. It is reasonable to assume that it is similarly controlling postsynaptic function just as strictly, and in as much detail. This regulation, however, has been more difficult to investigate on the postsynaptic side, whose composition is far less understood than that of the presynaptic bouton. Therefore, it is essential that we gain a quantitative understanding of the postsynaptic compartment, especially since many aspects of learning and plasticity are thought to be primarily realized there (Herring and Nicoll, 2016a; Turrigiano, 2008). For this reason, I set out to chart a comprehensive, quantitative description of the postsynapse, with a special focus on the main excitatory compartment, the dendritic spine. I determined the nanoscale architecture of dendritic spines by measuring the location and copy number of 105 proteins and analyzed the changes of key proteins during homeostatic plasticity. In addition, I report an extensive proteome of pure hippocampal neurons with absolute quantification, revealing general regulatory principles of protein homeostasis. Finally, this database is the most extensive quantification of a subcellular compartment to date and will serve as the basis for in silico studies of dendritic spine function. 


\section{The postsynapse has a multitude of functions}

\section{Dendritic spines bridge the gap between neurons}

In the late 1800s, Ramon y Cajal showed that the brain is made up of individual neurons, which exhibited many spiny protrusions (Ramón y Cajal, 1888). He also already argued that these dendritic spines are the location where the individual neurons receive their input from other neurons (Cajal, 1894). It took over 60 years until synapses at the central nervous system could be observed in detail for the first time (Gray, 1959), and to discover that neurotransmitters released by SVs are the medium by which neurons send signals (Heuser and Reese, 1973). The opposed postsynaptic membrane showed a protein dense area, which was further on termed the postsynaptic density (PSD).

\section{The PSD is the central active zone on dendritic spines}

The most important function of a synapse is the transformation of the presynaptic electrical signal to a chemical signal, the release of neurotransmitter. This chemical signal then needs to be recognized by the receiving neuron, via receptors, and transformed back to an electrical signal. The receptors that perform this function on the postsynapse are located mostly within the PSD. The signaling can either be excitatory or inhibitory, I will focus here exclusively on the excitatory synapse, as this is the topic of my presented work.

When glutamate is released into the synaptic cleft, it is recognized by a-amino-3-hydroxy-5methyl-4-isoxazolepropionic acid (AMPA) type glutamate receptors. These are heterotetrameric ionotropic receptors, which conduct mostly potassium and sodium ions, thereby depolarizing the postsynapse upon activation. The second important glutamate receptors class, N-methyl-D-aspartic acid (NMDA) type glutamate receptors are blocked by magnesium at resting potential (Mayer et al., 1984). Only when the postsynaptic compartment is sufficiently depolarized is this block released and NMDA receptors (NMDAR) open. They are therefore often referred to as coincidence detectors and usually only open upon the synchronous release of multiple SV, whereas asynchronous release does not activate NMDAR (Citri and Malenka, 2008). In addition to potassium and sodium, they are also permeable for calcium. This influx of calcium depolarizes it further, but also triggers many signaling cascades within the postsynapse. The depolarization of the postsynapse then propagates mostly electrotonically, although dendritic spikes have also been demonstrated (Jarsky et al., 2005; Sabatini et al., 2001).

The positioning of these receptors is crucial for synaptic function and the localization of the receptors in relation to the presynaptic release site heavily influences the strength of the synapse (MacGillavry et al., 2013; Raghavachari and Lisman, 2004). It is therefore controlled by many different scaffolding proteins that bind AMPA receptors (AMPAR) and NMDAR 
directly or indirectly. The best studied one is PSD95, which binds NMDAR, among others (Sheng and Kim, 2011). AMPAR do not directly interact with PSD95, but they have associated proteins, the transmembrane AMPA receptor regulating proteins (TARP), which in turn can bind to PSD95 (Schnell et al., 2002; Schwenk et al., 2012). The alignment of the presynaptic release site and the postsynaptic PSD is brought about by a transsynaptic nanocolumn, involving PSD95 and RIM1 (Tang et al., 2016). Other scaffolds, such as PSD93, Homer and Shank proteins connect the receptor complexes to the cytoskeleton, signaling molecules or kinases (for review see Foa and Gasperini, 2009; Frank and Grant, 2017; Monteiro and Feng, 2017). This complex network of interactions causes the typical electron dense appearance in electron microscopy (EM).

\section{Dendritic spines differentiate between inputs from many sources - Electrochemical compartmentalization}

Using EM, it quickly became clear that many excitatory presynaptic terminals signal onto these spiny protrusions, called dendritic spines. They can have vastly different morphologies and the exact implications of this variability is still not completely understood. Most of the times, they are made up of a spherical head that is connected to the dendritic shaft by a thin neck. In the hippocampus, the volume of the head varies between $0.003-0.55 \mu \mathrm{m}^{3}$, whereas the neck diameter is between 0.038 and $0.46 \mu \mathrm{m}$ (Harris and Stevens, 1989). The length of the neck is equally variably, typically between 0.160 and $2.13 \mu \mathrm{m}$. They can be classified, based mostly on the relation between the head and neck of the spine, most often dividing them into stubby, thin, mushroom and branched spines (Harris et al., 1992), others also include filopodia as a separate group (Figure 1A; Berry and Nedivi, 2017). This classification remains controversial though. Several studies have shown that spine morphology is stable, especially in vivo (Grutzendler et al., 2002; Zuo et al., 2005). This is supported by the observation that PSD area is correlated to presynaptic number of SVs, the spine head volume and the amplitude of excitatory postsynaptic currents (Chicurel and Harris, 1992; Harris and Stevens, 1989; Matsuzaki et al., 2001; Noguchi et al., 2011; Spacek and Harris, 1997). Live-imaging studies have shown that a strong stimulation often leads to an increase of the spine head and it assumes a more rounded shape (Korkotian and Segal, 2001; Lendvai et al., 2000; Matsuzaki et al., 2004a; Schiffelholz and Aldenhoff, 2002). Similarly, the neck often increases its diameter upon strong stimulation (Tønnesen et al., 2014). Therefore, it is widely believed that mushroom type spines are a morphological analogue to learning. Conversely, other live-imaging experiments revealed that spines can change their morphology continuously and show a wide variety of shapes (Dunaevsky et al., 1999; Fischer et al., 2000; Parnass et al., 2000; Tønnesen et al., 2014). Also, the large variability in spine morphology lead to classes that in themselves still have large variability. All dendritic spines though, fulfill two basic functions: 
First, they dramatically increase the possible complexity of neural networks (Sorra and Harris, 2000). By virtue of having a longer connection to the dendritic shaft, dendritic spines enable the postsynaptic neuron to receive input also from axons not directly adjacent to the shaft (Figure 1B). This is important in the brain, as space is highly limited.

Second, the morphology of dendritic spines electrochemically isolates the postsynapse from the shaft (Tønnesen and Nägerl, 2016). Therefore, the depolarization generated at the PSD is effectively amplified locally, allowing strong depolarizations to occur with only few released SVs. Also, the diffusion of proteins in and out of the spine is limited (Guthrie et al., 1991; Müller and Connor, 1991), generating a confined space where signaling can take place without affecting other postsynapses (Figure 1C). This input-specificity is important, because the neuron does need to differentiate between the different inputs it receives (Citri and Malenka, 2008). Only then it can adjust the strength of synapses independently, a process essential to learning.

\section{Dendritic spines adapt to the input - Hebbian plasticity}

For learning to occur, synapses need to have a molecular counterpart to information storage. To do so, synapses can change their efficiency in signal transduction, often also referred to as a synapses' strength. This change in strength can be on short or long timescale, and can either be brought about by presynaptic changes, i.e. a higher probability of SV release per action potential, or a stronger depolarization in the postsynapse per SV released (Malenka and Bear, 2004). I will only cover the postsynaptic mechanisms here:

The classical mechanism for learning requires long term changes in a synapses' characteristic. Depending on the frequency and amplitude of the signal a dendritic spine receives, it either increases its strength upon high frequency stimulation, a mechanism known as long-term potentiation (LTP), or it decreases its strength if it receives low frequency stimulation, long-term depression (LTD). Sometimes, multiple spines work together to reach the depolarization necessary for LTP. This mechanism is known as cooperativity, when multiple synapses are receiving weak stimuli at the same time, or associativity, when one synapses receives weak, another one strong input (Citri and Malenka, 2008). The input specificity is still maintained, as only the involved dendritic spines are potentiated.

Several different forms of LTP exist, the prototypical one being NMDAR-dependent LTP. Here, the influx of calcium through NMDAR activates several calcium dependent proteins and kinases, which induce a significant remodeling of the dendritic spine. One of the major players is calmodulin dependent kinase II (CaMKII; Hell, 2014). The changes in synaptic strength can be caused by different adjustments: 
A

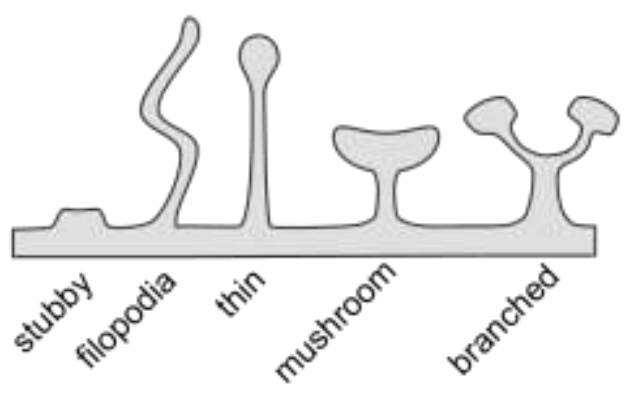

B

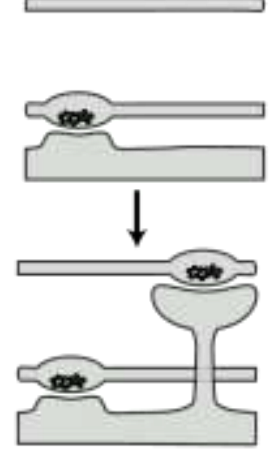

D
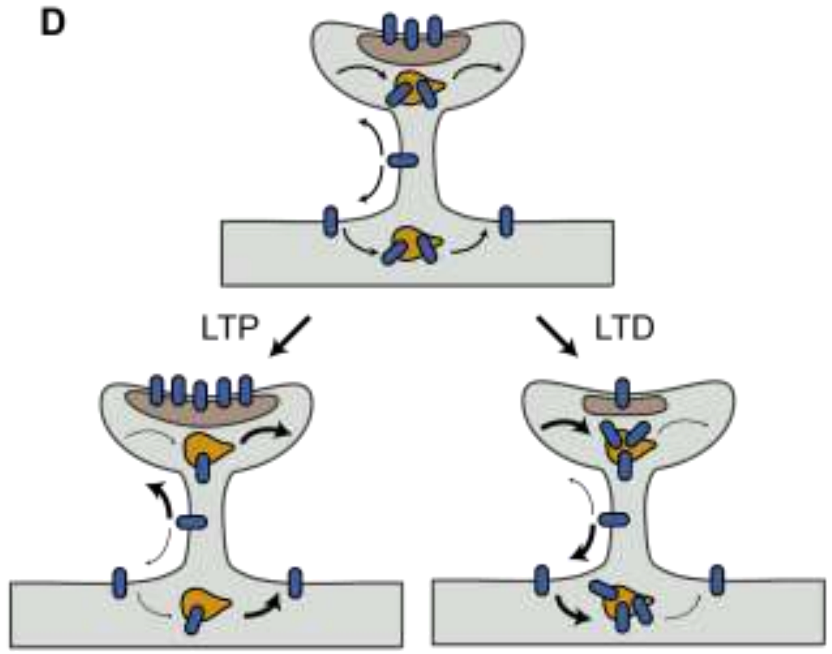

$\mathbf{F}$

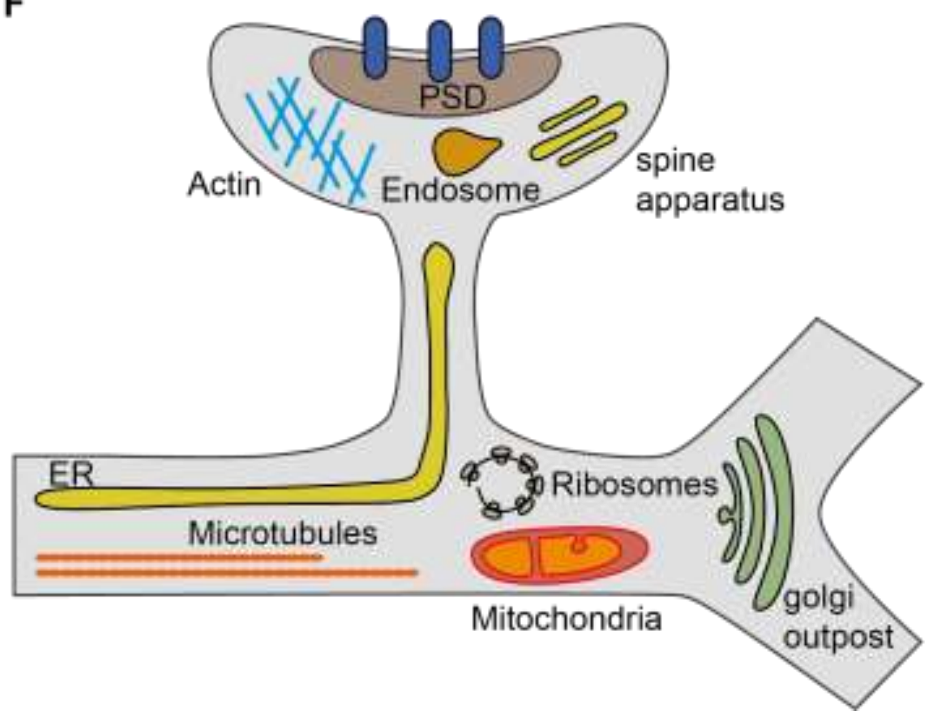

C

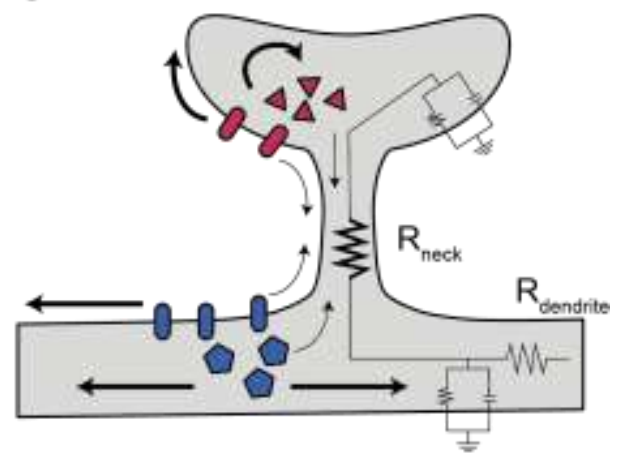

E
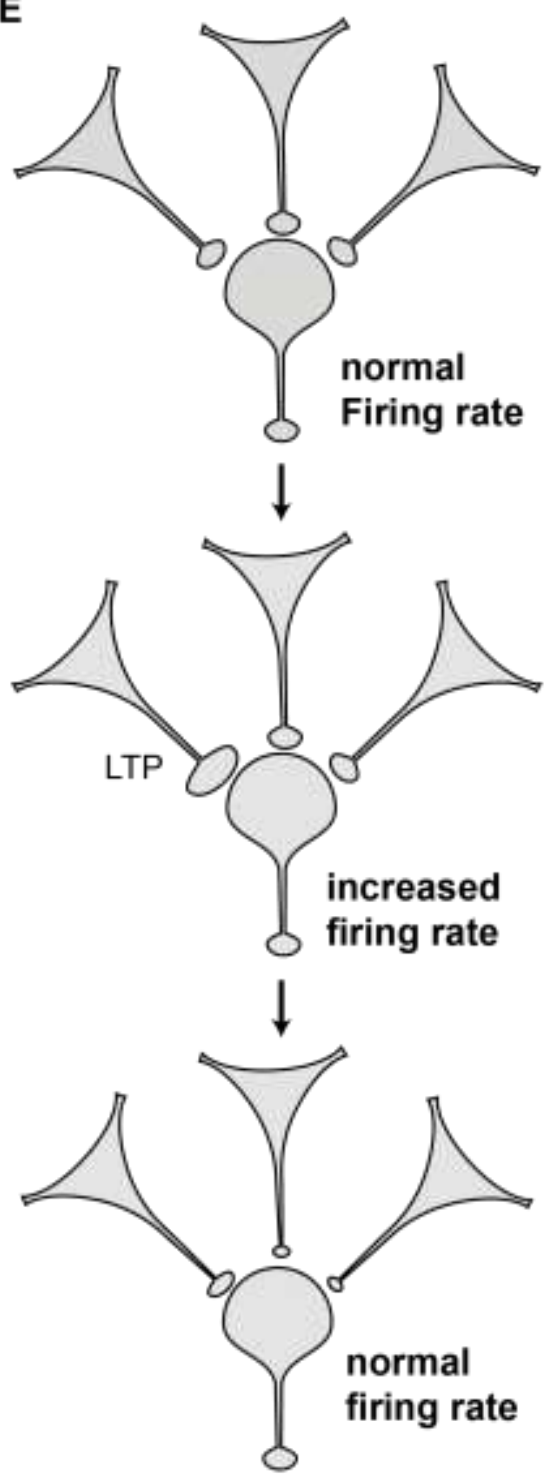

Figure 1: Dendritic spines fulfill a multitude of functions. A) Dendritic spines have many different morphologies that can be divided into 5 classes. B) Dendritic spines enable the dendrite to form synapses with more axons, increasing the complexity of the neural network. C) Dendritic spines compartmentalize the cytosol. Especially the thin neck limits diffusion of proteins in and out of the spine head, but it also has a high resistance, creating an electrically isolated compartment as well. D) The composition of dendritic spines changes during Hebbian plasticity. At resting conditions, AMPAR are present in equilibrium between the PSD, intracellular stores and extrasynaptic membranes (upper panel). Upon LTP induction, AMPAR are transported from endosomes to the membrane and 
the diffusion into the head is increased (left panel). Conversely, during LTD AMPAR are internalized and released from the PSD, resulting in their diffusion away from it. E) Homeostatic plasticity prevents excitotoxicity in neurons. When enough synapses undergo LTP, the firing of the postsynaptic neuron can increase significantly. To reset it back to its set point, the neuron globally scales down all input it receives, retaining relative synaptic strength. F) Dendritic spines house many organelles. The PSD is filled with glutamate receptors that receive the incoming signal (blue rods). The spine head is mostly shaped by actin filaments, whereas the dendritic shaft contains microtubules. Within the head, endosomes are present, as well as the spine apparatus. The ER tubules traverse the dendritic shaft but can also invade into the neck. Ribosomes are mostly present at the base of the dendritic neck, Mitochondria are in the dendritic shaft as well. Golgi outposts often are present at dendritic arbors.

First, the dendritic spine can change the number of receptors at its PSD (Figure 1D). During LTP, it increases the number of AMPAR receptors at its PSD (Lin et al., 2009; Park et al., 2004; Shi et al., 2001; Yudowski et al., 2007), therefore increasing its ability to depolarize, creating stronger excitatory postsynaptic potentials (EPSP). This increase in AMPAR is thought to mainly occur by exocytosis of GluR1/GluR2 AMPAR receptor at perisynaptic sites, which are subsequently diffuse into the PSD and are trapped there by scaffolding proteins such as PSD95. How fusion is initiated is not yet understood, although myosin motors are involved in the delivery of AMPAR containing endosomes (Wang et al., 2008). It is likely that SNARE proteins are involved as well, as infusion of dendritic spines with botulinum neurotoxin blocks LTP (Lledo et al., 1998). Which SNARE proteins exactly are involved in AMPAR exocytosis is not completely known, but several have been suggested (Jurado et al., 2013; Kennedy et al., 2010; Suh et al., 2010). Especially, no Synaptotagmin-like calcium sensor has yet been identified for this process.

In addition, the diffusion of AMPAR already on the postsynaptic membrane can be changed by the phosphorylation of their TARPS through CaMKII, to facilitate their binding to PSD95 (Bats et al., 2007; Opazo et al., 2010; Schnell et al., 2002).

Similarly, during LTD the number of AMPAR receptors is decreased (Figure 1D; Carroll et al., 1999; Ribrault et al., 2011). This endocytosis is even less understood, only very recently a synaptotagmin isoform responsible for GluR2 internalization has been reported (Awasthi et al., 2018).

Second, dendritic spines can change the conductance of the AMPAR it already has. This is either brought about by phosphorylation of the AMPAR subunits, also mediated by CamKII (Benke et al., 1998; Lee et al., 2000), or by the interaction of AMPAR with TARPs that modulate its characteristics (Shaikh et al., 2016; Tomita et al., 2005).

Third, the spine can change its morphology. During LTP, dendritic spines have been shown to increase their size and PSD, widen their neck transiently and the growth of new spines occurs (Bosch et al., 2014; Kopec et al., 2006; Okamoto et al., 2004; Tønnesen et al., 2014). The cytoskeleton of dendritic spines is mainly composed of actin, which is highly dynamic. This 
actin cytoskeleton is regulated by several Rho type GTPases, the main mediators being Rac1 and CDC42 for spine enlargement, and RhoA for spine shrinkage Their respective guanine nucleotide exchange factors (GEF) are equally controlled by phosphorylation through CaMKII. (Herring and Nicoll, 2016b; Jaudon et al., 2015; Murakoshi et al., 2011; Nakayama et al., 2000).

Fourth, to ensure that the changes in synaptic strength are stable, protein translation is necessary. Although how translation is regulated in the spine is not fully understood and the translated proteins are largely unknown (Citri and Malenka, 2008), it has been shown that blocking translation does occlude the late phase of LTP (Krug et al., 1984).

Recently, also short-term changes in synaptic strength have been attributed to postsynaptic mechanisms. Up until then, it has been thought that short-term changes in synaptic strength are exclusively mediated by changes in the presynaptic release probability, mainly by an accumulation of calcium. Now, elegant experiments tracking AMPAR and manipulating their diffusion revealed that also the postynapse is involved in these processes (Constals et al., 2015; Heine et al., 2008). AMPAR undergo desensitization after they have been activated by glutamate. It has been proposed that these desensitized receptors are released from the PSD and replaced by other AMPAR receptors, that have not been stimulated yet. Therefore, when another SV is released within a short time frame, they can now respond to the released glutamate, whereas desensitized AMPAR could not. When this exchange is blocked or slowed, short term plasticity is equally affected.

\section{Dendritic spines are controlled by the neuron as a whole - Homeostatic plasticity}

Once LTP is triggered in a synapse, it is easy to imagine that this would cause a positive feedback loop: Since the synaptic strength is increased, the same stimulation more easily would trigger a further increase in synaptic strength, ultimately leading to hyperexcitation of the postsynaptic neuron. This hyperexcitation would eventually lead to the death of the neuron (Turrigiano, 2008). The question therefore arises how neurons escape this loop. The discovery of homeostatic plasticity answered parts of this question, although it is still incompletely understood (Lissin et al., 1998; O'Brien et al., 1998; Turrigiano et al., 1998). In homeostatic plasticity, it is hypothesized that a neuron can sense its own activation pattern. At the same time, it has a set frequency of activation that it wants to maintain (Turrigiano and Nelson, 2004). Once LTP is induced on a sufficient amount of postsynapses, the neuron's firing rat increases (Figure 1E). To reverse to its target activity, one mechanism is to decrease the synaptic strength of all excitatory input it receives. This is known as synaptic scaling and can happen on a global (Turrigiano et al., 1998), as well as local levels (Ju et al., 2004; Sutton et al., 2006; Thiagarajan et al., 2005). This elegant solution resets its firing rate back to the set point, while also maintaining the relative synaptic strength of the input it receives. 
Synaptic scaling often involves changes in the AMPAR and NMDAR content in the PSD (Lissin et al., 1998; O'Brien et al., 1998; Stellwagen and Malenka, 2006; Turrigiano et al., 1998; Watt et al., 2000). When a neuron is excited too much, it retrieves these receptors from its membranes, when it is stimulated too little, it adds more to the PSD. How the neuron senses its own activation pattern, and how the signal to scale up or down is transmitted to the synapses is poorly understood though. One prime candidate is the somatic calcium level, that could be recognized by calcium binding proteins, such as CaMKIV. But also other molecules have been shown to be involved in synaptic scaling, for example Arc or BDNF, or also TNFa released from glia (Turrigiano, 2008).

\section{Dendritic spines respond to and modulate the input - Retrograde signaling}

Upon stimulation, the postsynapse also communicates back to the presynapse, a process termed retrograde signaling. This signaling is mostly brought about by the release of chemical factors, for example brain derived neurotrophic factor (BDNF), endocannabinoids or nitric oxide (Bon and Garthwaite, 2003; Korte et al., 1995; Minichiello et al., 2002). These are then recognized by presynaptic receptors and likely induce the concerted growth or shrinkage of the presynaptic active zone with the postsynaptic PSD. This is further supported by the finding that both structures often correlate in their size (Harris and Stevens, 1989; Harris et al., 1992).

\section{Dendritic spines sustain themselves - Local translation and organelles}

All above mentioned processes together put high demands on the dendritic spine. It is therefore not surprising that dendritic spines have an equally complex organelle composition (Figure 1F). First, dendrites have the ability to locally translate proteins (Hanus and Schuman, 2013). Ribosomes and mRNA have been found throughout the dendrite (Cajigas et al., 2012), where ribosomes usually are at the base of the dendritic neck (Ostroff et al., 2002). Upon stimulation of the dendrite, and often a concomitant increase of translation, ribosomes can move into the head. Also, local regulation of translation on the mRNA level has been demonstrated, for example Dicer, a protein involved in miRNA production, is also present in the dendrite (Lugli et al., 2005; Sambandan et al., 2017).

Since many of the crucial postsynaptic proteins are inserted in the membrane, the biosynthetic pathway for transmembrane proteins is also present in dendrites. The endoplasmic reticulum $(E R)$ is found in tubules stretching along the dendritic shaft, where it sometimes also invaginates into dendritic spines (Spacek and Harris, 1997). There, it also forms stacked tubules, the spine apparatus. The function of this peculiar structure is still not fully understood, although it has been implicated in the calcium regulation and protein biosynthesis (Bourne and Harris, 2008; Holbro et al., 2009; Jedlicka et al., 2008; Pierce et al., 2000). Additionally, organelles similar to the Golgi apparatus have been found in dendrites, preferentially at 
dendrite branching points. These compartments have therefore been termed Golgi outposts. Beyond their function in posttranslational protein modification, they also regulate dendrite morphology and arborization by acting as microtubule nucleation sites (Gardiol et al., 1999; Horton and Ehlers, 2003, 2004; Jan and Jan, 2010).

The trafficking between these organelles and the plasma membrane occurs via endosomes. All regular endosomal compartments, early, late, sorting and recycling endosomes have been reported in the dendrite as well as the dendritic spine (Spacek and Harris, 1997). In addition, multivesicular bodies, lysosomes and the proteasome have also been described, complementing the translation machinery with the degradation pathways (von Figura and Hasilik, 1986; Hamilton and Zito, 2013; Spacek and Harris, 1997).

To provide the energy necessary for this multitude of functions, mitochondria are found throughout the dendritic shaft. They are only rarely present in dendritic spines themselves, this mostly occurs at large mushroom or branched spines (Chicurel and Harris, 1992). An interesting observation is that glycolytic enzymes are present in the PSD directly, which suggests local ATP production in this compartment (Wu et al., 1997). The majority of ATP is probably still produced by the mitochondria in the shaft and diffuses into the dendritic spine.

\section{Quantitative biology reveals intricate details of synaptic function}

Tiny changes, that are difficult to pick up during qualitative work may heavily influence synaptic function. For example, it has been shown that the position of AMPAR strongly determines synaptic strength (MacGillavry et al., 2013; Raghavachari and Lisman, 2004). This is largely due to the low affinity of AMPAR to glutamate, which causes them to only get activated when they are close to the presynaptic release site. Once AMPAR receptors are over $100 \mathrm{~nm}$ away from the release site, their open probability starts to drop. Because this difference is beyond the regular diffraction limit of light microscopy, it has been difficult to assess without modelling. Using super resolution microscopy, it has been shown that AMPAR do indeed form $80 \mathrm{~nm}$ large clusters, meaning that most of these receptors will get activated if the release site is aligned with it (MacGillavry et al., 2013; Nair et al., 2013). Consistently, it has recently been found that the presynaptic release site is aligned with these postsynaptic receptor clusters, forming a 'transsynaptic nanocolumn' (Tang et al., 2016). This discovery has only been able through the quantitative analysis of PSD95 and RIM1 distribution in super resolution.

Besides analyzing the nanoscale distribution using super resolution, the absolute number of proteins present is of equal interest. However, many biochemical or mass spectrometric studies have been limited to report relative amounts(Cheng et al., 2006; Collins et al., 2006; 
Lowenthal et al., 2015; Peng et al., 2004a). Also, an inherent problem to western blotting or quantitative imaging is the nature of antibody binding (Ehlen et al., 2014). For the vast majority of antibodies, their affinity is not known, making the comparison of signal between proteins virtually impossible. This restricts many works to comparisons of a protein to itself, for example during different treatments. A comparison between proteins is only seldomly done.

Two major studies have attempted to quantify protein distribution and number of organelles in absolute terms. In 2006, Takamori and colleagues published the molecular anatomy of SV, using quantitative western blotting (Takamori et al., 2006). They found that exocytotic soluble NSF attachment protein receptors (SNAREs) are present in excess on the average vesicle, most likely to ensure fast release upon stimulation. On the other hand, some proteins were present in very low numbers, for example CSP $\alpha$ was found to be present in only 2.8 copies per vesicle. Later, this was found to have important implications for SV aging, as they pick up SNAP25 from the plasma membrane during recycling, which sequesters CSPa in a cis complex, preventing fusion (Truckenbrodt et al., 2018). Understanding this process was only possible through the quantitative knowledge of protein abundance on SVs.

Taking this one step further, Wilhelm and colleagues studied the quantitative composition of the presynapse with quantitative western blotting and mass spectrometry, but also determined the position of the counted molecules using stimulated emission depletion (STED) microscopy (Wilhelm et al., 2014). Interestingly, they also found exocytotic SNAREs to be much more abundant than needed for efficient SV fusion, similar to the situation on SV themselves, whereas endocytosis is limited by the number of clathrin molecules present (4000 molecules accounting for a maximum of $\sim 20$ simultaneous endocytosis events). In addition, they also revealed the fine architecture of the presynaptic active zone.

\section{The current understanding of dendritic spines is insufficient}

Because of the intricacy of dendritic spines, its different functions are often analyzed independently from another. For example, some groups focus on AMPAR trafficking, others on structural plasticity or calcium dynamics in spines. Even for individual proteins, such as CamKII with its many functions, only parts of their signaling cascades are considered by individual groups. This is understandable to reduce the complexity, but it leads to isolated viewpoints of spines. On the other hand, many of the proteins are involved in various pathways, information which is rarely considered at the moment. Also, apart from electrophysiology, most research done on dendritic spines is of qualitative nature, which is at odds with the observation that especially in small and highly specialized compartments, such as the synapse, quantitative 
changes on the nanoscale can result in large functional changes. Therefore, it is essential that we arrive at an integrated and quantitative understanding of the synapse.

In addition to that, the large variability in morphology of dendritic spines have so far made it difficult to grasp the functional implications of changes in protein abundance and localization, because it is virtually impossible to control for all the different conditions. This can only be achieved in modelling approaches, where one is in full control over all parameters. However, modelling dendritic spines has been challenging because of the lack of quantitative information. Because of the complexity of dendritic spines, it is not possible to simulate long time steps and the models rely on good initial estimates for their parameters, but we are lacking these. Because of these problems, in silico approaches tackling major function in spines, such as plasticity are rare.

On example is the work by Gallimore and colleagues, studying the regulation of LTP and LTD in the cerebellum (Gallimore et al., 2018). They studied the regulation of AMPAR at the postsynaptic membrane by phosphatases and kinases, constructing a molecular model that comprised both the post- as well as parts of the presynapse. For the initialization of their model, they had to set the concentration of almost 100 proteins and molecules, many of which they needed to estimate $a b$ initiio, because no measurements where available. Also, they had to assume a well-mixed system, as the nanoscale localization of many of the proteins where not known. On the other hand, this assumption does not resemble in vivo spines, which do show strong compartmentalization (Tønnesen and Nägerl, 2016). If better data on protein numbers and localization were available, this would first, create models that do represent real spine much closer, and second, free up computing time that could be used to take additional molecules or parameters, such as compartmentalization and diffusion, into account. One could then also start to vary the conditions the spine is faced, for example changing the morphology, ion concentration, stimulation frequency and amplitude to really understand their influence on the function of dendritic spines.

\section{Aim of this thesis}

Therefore, it is clear that we need to know the quantitative architecture of the whole synapse to understand its function in all aspects. Especially dendritic spines are not equally well understood as their presynaptic counterpart. This is in part due to its inaccessibility to subcellular fractionation techniques; so far, no pure dendritic spine compartment could be isolated in large quantities. In this study, I set out to determine the absolute quantity and nanoscale distribution of 105 proteins in dendritic spines. To circumvent the lack of subcellular fractionation, I developed new methods, enabling me to study the quantify the synaptic 
proteome without the need to isolate this compartment. This is the first comprehensive description of not only the PSD but also the cytosol in dendritic spines and also extends into the dendritic shaft. Because the PSD has repeatedly been shown to exhibit a modular organization, elucidating it molecular architecture will prove to be widely applicable, both in vitro as well as in vivo.

Second, I investigated how the composition of dendritic spines changes during plasticity and determine whether its organization is stable or fragile.

Third, I addressed whether spine classes do indeed show different molecular composition and I will study if there are distinct, yet unknown spine subtypes with unique molecular compositions.

Finally, this exploratory study generates a database available to the neuroscientific community in general, which can be used and extended easily by other researchers according to their own needs. With the molecular anatomy of dendritic spines revealed, synaptic neuroscience is ready for the transition to a quantitative age. 


\section{Materials and Methods}

\section{Antibodies}

Table 1: Primary antibodies, Nanobodies and small molecules used to detect proteins.

\begin{tabular}{|c|c|c|c|c|}
\hline Target protein & Species & Dilution & Manufacturer & $\begin{array}{l}\text { Catalogue } \\
\text { Number }\end{array}$ \\
\hline ADAM22 & mouse & 500 & Novus Biologicals & NBP2-22425 \\
\hline Akt (pan) & rabbit & 400 & Cell Signaling & 4691 \\
\hline$\alpha / \beta$ SNAP & mouse & 100 & Jahn Laboratory & 77.2 \\
\hline$\alpha$-internexin & rabbit & 500 & LSBio & LS-B10413 \\
\hline AP 180 & rabbit & 100 & Synaptic Systems & 155003 \\
\hline APP & mouse & 100 & Millipore & MAB-348 \\
\hline Arc & rabbit & 1000 & Synaptic Systems & 156003 \\
\hline BDNF & rabbit & 100 & Biorbyt & orb38809 \\
\hline$\beta 2$ spectrin & mouse & 100 & BDBiosciences & 612562 \\
\hline B3-Tubulin & rabbit & 500 & Cell Signaling & 5568 \\
\hline$\beta$-Actin & mouse & 100 & Sigma-Aldrich & A1978 \\
\hline Calbindin-D28K & rabbit & 500 & Synaptic Systems & 214002 \\
\hline Calcineurin A & rabbit & 1000 & Synaptic Systems & 387002 \\
\hline Calmodulin & rabbit & 100 & Abcam & ab45689 \\
\hline Calreticulin & rabbit & 200 & Cell Signaling & 12238 \\
\hline Calretinin & rabbit & 250 & Novus Biologicals & NBP1-88220 \\
\hline CaMKII & mouse & 500 & Abnova & MAB6627 \\
\hline CAPS1 & rabbit & 500 & Abcam & $a b 69797$ \\
\hline Cav1.3 & rabbit & 50 & Alomone Labs & ACC-311 \\
\hline Cav2.1 & rabbit & 500 & Synaptic Systems & 152203 \\
\hline CDC42 & rabbit & 100 & Thermo Scientific & PA1-092 \\
\hline Chromogranin A & rabbit & 500 & Synaptic Systems & 259003 \\
\hline Chromogranin B & rabbit & 500 & Synaptic Systems & 259103 \\
\hline Chromogranin C & rabbit & 250 & Abcam & $a b 12241$ \\
\hline Clathrin heavy chain & mouse & 100 & BD Biosciences & 610499 \\
\hline Clathrin light chain & mouse & 1000 & Synaptic Systems & 113011 \\
\hline Cortactin & mouse & 500 & Synaptic Systems & 313111 \\
\hline DLGAP1 & rabbit & 50 & Novus Biologicals & NBP1-76911 \\
\hline Dopamine receptor 1 & rabbit & 1000 & Abcam & $a b 40653$ \\
\hline
\end{tabular}




\begin{tabular}{|c|c|c|c|c|}
\hline Dopamine receptor 2 & rabbit & 500 & Merck & AB5084P \\
\hline Drebrin1 & mouse & 100 & Novus Biologicals & NB100-1951 \\
\hline Dynamin 1/2/3 & mouse & 100 & BDBiosciences & 610245 \\
\hline ERp72 & rabbit & 100 & Cell Signaling & 5033 \\
\hline GluK1 & rabbit & 100 & Alomone & AGC-008 \\
\hline GluN1 & mouse & 1000 & Synaptic Systems & 114011 \\
\hline GluN2A & mouse & 100 & NeuroMab & $75-288$ \\
\hline GluN2B & mouse & 100 & NeuroMab & $75-101$ \\
\hline GluR1 & rabbit & 500 & Synaptic Systems & 182003 \\
\hline GluR2 & rabbit & 100 & Alomone Labs & AGC-005 \\
\hline GluR3 & mouse & 100 & Invitrogen & $32-0400$ \\
\hline GRIP1/2 & rabbit & 100 & Synaptic Systems & 151003 \\
\hline Homer 1 & mouse & 500 & Synaptic Systems & 160011 \\
\hline Homer 1 & rabbit & 500 & Synaptic Systems & 160003 \\
\hline Homer 2 & rabbit & 500 & Synaptic Systems & 160203 \\
\hline Homer 3 & rabbit & 250 & Synaptic Systems & 160303 \\
\hline HSC70 & mouse & 100 & Santa Cruz & sc-7298 \\
\hline IGF-1 Receptor & rabbit & 300 & Cell Signaling & 3027 \\
\hline KCNJ2 & rabbit & 100 & Novus Biologicals & NBP1-95482 \\
\hline Kv1.1 & rabbit & 100 & Thermo Scientific & PA5-19593 \\
\hline Kv2.1 & rabbit & 500 & Synaptic Systems & 231002 \\
\hline LNGFR & rabbit & 1000 & Cell Signaling & 8238 \\
\hline m-AChR-1 & rabbit & 100 & Novus Biologicals & NBP1-87466 \\
\hline MAP2 & rabbit & 1000 & Synaptic Systems & 188002 \\
\hline mGluR1 $\alpha$ & rabbit & 250 & Abcam & ab51314 \\
\hline mGluR2 & rabbit & 100 & Abcam & ab150387 \\
\hline mGluR5 & rabbit & 100 & Abcam & $a b 76316$ \\
\hline myosin 5a & rabbit & 200 & Sigma-Aldrich & M5062 \\
\hline $\mathrm{Na} \beta 1$ & rabbit & 50 & Alomone Labs & ASC-041 \\
\hline $\mathrm{Na}^{+} / \mathrm{K}^{+}$ATPase & mouse & 1000 & Thermo Scientific & MA3-915 \\
\hline Nav1.1 & rabbit & 100 & Merck & $06-811$ \\
\hline Nav1.3 & rabbit & 250 & Alomone Labs & ASC-004 \\
\hline Neurofilament H & rabbit & 1000 & LSBio & LS-C143052 \\
\hline Neurofilament L & rabbit & 500 & Synaptic Systems & 171002 \\
\hline Neurogranin & rabbit & 1000 & Synaptic Systems & 357003 \\
\hline nicotinic AChR $\beta 2$ & rabbit & 100 & Alomone Labs & ANC-012 \\
\hline
\end{tabular}




\begin{tabular}{|c|c|c|c|c|}
\hline $\mathrm{nNOS}$ & rabbit & 100 & Thermo Scientific & PA1-033 \\
\hline NSF & rabbit & 500 & Synaptic Systems & 123002 \\
\hline Parvalbumin & rabbit & 500 & Swant & PV25 \\
\hline Phalloidin-ATTO647N & $\begin{array}{l}\text { Small } \\
\text { molecule }\end{array}$ & 1000 & Sigma-Aldrich & 65906-10NMOL \\
\hline Phosphodiesterase & rabbit & 100 & Cell Signaling & 3501 \\
\hline PSD93 & rabbit & 300 & Invitrogen & $34-4700$ \\
\hline PSD95 & rabbit & 100 & Cell Signaling & 3450 \\
\hline PSD95 & mouse & 200 & Thermo Scientific & MA1-046 \\
\hline Rab11 & rabbit & 100 & Cell Signaling & 3539 \\
\hline Rab3 & mouse & 100 & BD Biosciences & 610379 \\
\hline Rab4 & mouse & 100 & BD Biosciences & 610888 \\
\hline Rab5 & mouse & 100 & Jahn Laboratory & cl. 621.3 \\
\hline Rab7 & rabbit & 100 & Cell Signaling & 9367 \\
\hline Rab9 & rabbit & 100 & Cell Signaling & 5118 \\
\hline Rapsyn & rabbit & 100 & Atlas Antibodies & HPA039475 \\
\hline $\begin{array}{l}\text { Ribosomal protein } \\
\text { L7a }\end{array}$ & rabbit & 100 & Cell Signaling & 2403 \\
\hline Ribosomal protein S3 & rabbit & 50 & Cell Signaling & 9538 \\
\hline Ribosomal protein S6 & rabbit & 100 & Cell Signaling & 2217 \\
\hline Sec22b & rabbit & 100 & Synaptic Systems & 186003 \\
\hline Septin7 & rabbit & 50 & Atlas Antibodies & HPA029524 \\
\hline Shank1 & rabbit & 500 & Synaptic Systems & 162013 \\
\hline Shank2 & rabbit & 500 & Synaptic Systems & 162202 \\
\hline Shank3 & rabbit & 500 & Synaptic Systems & 162302 \\
\hline SNAP23 & rabbit & 100 & Synaptic Systems & 111202 \\
\hline SNAP25 & mouse & 100 & Synaptic Systems & 111011 \\
\hline SNAP 29 & rabbit & 500 & Synaptic Systems & 111302 \\
\hline SNAP 47 & rabbit & 200 & Synaptic Systems & 111403 \\
\hline$\beta$ tubulin nanobody & Ilama & 100 & Self-made & na \\
\hline Synaptophysin & guinea pig & 1000 & Synaptic Systems & 101004 \\
\hline Synaptotagmin 4 & rabbit & 1000 & Synaptic Systems & 105143 \\
\hline Synaptotagmin 5/9 & rabbit & 100 & Synaptic Systems & 105053 \\
\hline Synaptotagmin 7 & rabbit & 250 & Synaptic Systems & 105173 \\
\hline SynGAP1 & rabbit & 1000 & Thermo Scientific & PA1-046 \\
\hline Syntaxin1 & mouse & 200 & Synaptic Systems & 110011 \\
\hline
\end{tabular}




\begin{tabular}{|l|l|l|l|l|}
\hline Syntaxin13 & mouse & 100 & Jahn Laboratory & cl. 151.1 \\
\hline Syntaxin16 & rabbit & 100 & Synaptic Systems & 110162 \\
\hline Syntaxin2 & rabbit & 100 & Synaptic Systems & 110022 \\
\hline Syntaxin3 & rabbit & 100 & Synaptic Systems & 110033 \\
\hline Syntaxin4 & rabbit & 100 & Synaptic Systems & 110042 \\
\hline Syntaxin5 & rabbit & 100 & Synaptic Systems & 110053 \\
\hline Syntaxin6 & rabbit & 100 & Cell Signaling & 2869 \\
\hline Syntaxin8 & rabbit & 100 & Synaptic Systems & 110083 \\
\hline TGN38 & rabbit & 100 & Sigma-Aldrich & T9826 \\
\hline TOM20 & mouse & 200 & Sigma-Aldrich & WH0009804M1 \\
\hline Transferrin Receptor & rabbit & 100 & Abcam & ab84036 \\
\hline TrkB & rabbit & 500 & Abcam & ab33655 \\
\hline vAChT & rabbit & 100 & Synaptic Systems & 139103 \\
\hline Vamp1 & rabbit & 500 & Synaptic Systems & 104002 \\
\hline Vamp2 & mouse & 1000 & Synaptic Systems & 104211 \\
\hline VAMP7 & rabbit & 100 & Abcam & ab68776 \\
\hline Vti1a & mouse & 100 & BDBiosciences & 611220 \\
\hline
\end{tabular}


Table 2: Antibodies used as cell-type markers

\begin{tabular}{|l|l|l|l|l|}
\hline Target protein & Species & Dilution & Manufacturer & $\begin{array}{l}\text { Catalogue } \\
\text { Number }\end{array}$ \\
\hline GAD65 & mouse & 500 & Synaptic Systems & 198111 \\
\hline GFAP & mouse & 500 & Synaptic Systems & 173011 \\
\hline Iba1 & guinea pig & 500 & Synaptic Systems & 234004 \\
\hline Olig2 & rabbit & 500 & Synaptic Systems & 292003 \\
\hline SMI310 & mouse & 200 & Abcam & 24570 \\
\hline
\end{tabular}

Table 3: Secondary antibodies and labels

\begin{tabular}{|l|l|l|l|l|}
\hline Target protein & Species & Dilution & Manufacturer & $\begin{array}{l}\text { Catalogue } \\
\text { Number }\end{array}$ \\
\hline ATTO647N anti mouse & goat & 500 & Rockland & $610-156-121$ \\
\hline ATTO647N anti rabbit & goat & 500 & Rockland & $611-156-122$ \\
\hline Alexa488 anti guinea pig & goat & 100 & Dianova & \\
\hline Cy3 Fab anti mouse & goat & 100 & Dianova & $715-166-150$ \\
\hline Cy3 Fab anti rabbit & goat & 100 & Dianova & $711-166-152$ \\
\hline $\begin{array}{l}\text { STAR635P nanobody } \\
\text { anti guinea pig }\end{array}$ & lama & 1000 & Nanotag & N0602 \\
\hline Hoechst & NA & $1: 2000$ & Thermo Scientific & 62249 \\
\hline
\end{tabular}




\section{Buffers and Solutions}

Table 4: Buffers and Solutions used in this study

\begin{tabular}{|c|c|c|c|}
\hline Name & Used for & Composition & Manufacturer \\
\hline \multirow{4}{*}{$\begin{array}{l}\text { PBS pH 7.4 (in } \\
\mathrm{dd}_{2} \mathrm{O} \text { ) }\end{array}$} & \multirow{4}{*}{$\begin{array}{l}\text { Cell culture, } \\
\text { Immunostaining }\end{array}$} & $137 \mathrm{mM} \mathrm{NaCl}$ & Merck, 1.06404 .1000 \\
\hline & & $2.7 \mathrm{mM} \mathrm{KCl}$ & Merck, 1.04936 .0250 \\
\hline & & $10 \mathrm{mM} \mathrm{Na}_{2} \mathrm{HPO}_{4}$ & Merck, 1.06580 .1000 \\
\hline & & $1.8 \mathrm{mM} \mathrm{KH}_{2} \mathrm{PO}_{4}$ & $\begin{array}{l}\text { Merck, } \\
1.04873 .10000\end{array}$ \\
\hline \multirow[t]{6}{*}{ Glia Medium } & \multirow[t]{6}{*}{ Cell culture } & MEM & $\begin{array}{l}\text { Life Technologies, } \\
51200-046\end{array}$ \\
\hline & & $0.6 \%$ (wt/vol) D-glucose & Sigma, G8769 \\
\hline & & 1\% (vol/vol) L-glutamine & Biozym, 882027 \\
\hline & & $100 \mathrm{U} / \mathrm{ml}$ penicillin & Biozym, 882082 \\
\hline & & $100 \mu \mathrm{g} / \mathrm{ml}$ Streptomycin & Biozym, 882082 \\
\hline & & $10 \%$ (vol/vol) horse serum & Biochrom, S9135 \\
\hline \multirow[t]{4}{*}{$\begin{array}{l}\text { Neuronal } \\
\text { Medium }\end{array}$} & \multirow[t]{4}{*}{ Cell culture } & MEM & $\begin{array}{l}\text { Life Technologies, } \\
51200-046\end{array}$ \\
\hline & & $0.6 \%$ (wt/vol) D-glucose & Sigma, G8769 \\
\hline & & 1\% (vol/vol) L-glutamine & Biozym, 882027 \\
\hline & & $10 \%$ (vol/vol) horse serum & Biochrom, S9135 \\
\hline \multirow{3}{*}{$\begin{array}{l}\text { Neuronal } \\
\text { Maintenance } \\
\text { Medium }\end{array}$} & \multirow[t]{3}{*}{ Cell culture } & MEM & $\begin{array}{l}\text { Life Technologies, } \\
51200-046\end{array}$ \\
\hline & & $0.6 \%$ (wt/vol) D-glucose & Sigma, G8769 \\
\hline & & 1\% N2 supplement & $\begin{array}{l}\text { Life Technologies } \\
17502-048\end{array}$ \\
\hline \multirow[t]{2}{*}{ CMF-HBSS } & \multirow[t]{2}{*}{ Cell culture } & CMF-HBSS & Invitrogen, 14175-095 \\
\hline & & 1\% 1M HEPES & Invitrogen, 15630-056 \\
\hline \multirow[t]{5}{*}{ Neurobasal-A } & \multirow[t]{5}{*}{ Cell culture } & Neurobasal-A & Invitrogen, 10888-022 \\
\hline & & $\begin{array}{ll}2 \% \quad(\mathrm{vol} / \mathrm{vol}) & \mathrm{B} 27 \\
\text { Supplement } & \end{array}$ & $\begin{array}{l}\text { Life Technologies, } \\
\text { 17504-044 }\end{array}$ \\
\hline & & $20 \mathrm{U} / \mathrm{ml}$ Penicillin & Biozym, 882082 \\
\hline & & $\begin{array}{l}0.02 \% \\
\text { Streptomycin }\end{array}$ & Biozym, 882082 \\
\hline & & $\begin{array}{l}1 \% \quad(\text { vol } / \text { vol }) \text { Glutamax-I } \\
\text { Supplement }\end{array}$ & $\begin{array}{l}\text { Life Technologies, } \\
\text { 35050-038 }\end{array}$ \\
\hline
\end{tabular}




\begin{tabular}{|c|c|c|c|}
\hline \multirow[t]{4}{*}{ Plating Medium } & \multirow[t]{4}{*}{ Cell culture } & MEM & $\begin{array}{l}\text { Life Technologies, } \\
51200-046\end{array}$ \\
\hline & & 3.3 mM D-Glucose & Sigma, G8769 \\
\hline & & 2 mM L-Glutamine & Biozym 882027 \\
\hline & & $10 \%$ (vol/vol) Horse serum & Biochrom, S9135 \\
\hline \multirow[t]{2}{*}{ Borate buffer } & \multirow[t]{2}{*}{ Cell culture } & Boric acid & Sigma, B0252 \\
\hline & & $\begin{array}{ll}\text { Sodium } & \text { tetraborate } \\
\text { decahydrate } & \end{array}$ & Sigma, S9640 \\
\hline \multirow[t]{5}{*}{ Enzyme solution } & \multirow[t]{5}{*}{ Cell culture } & DMEM & Biozym, 880026-12 \\
\hline & & $0.2 \%$ (wt/vol) Cysteine & Sigma, 30090 \\
\hline & & $1 \mathrm{mM} \mathrm{CaCl} 2$ & Merck, 1.02382 .1000 \\
\hline & & $0.5 \mathrm{mM}$ EDTA & Merck, 1.08418 .1000 \\
\hline & & 20-25 U/mI Papain $(100 \mu \mathrm{l})$ & $\begin{array}{ll}\text { Cell } & \text { Systems, } \\
\text { LS003124 } & \end{array}$ \\
\hline \multirow[t]{7}{*}{ Inactivating solution } & \multirow[t]{7}{*}{ Cell culture } & DMEM & Biozym, 880026-12 \\
\hline & & $10 \%$ (vol/vol) FCS & Biochrom, S0415 \\
\hline & & $2 \%$ (vol/vol) Glutamine & Biozym 882027 \\
\hline & & $20 \mathrm{U} / \mathrm{ml}$ Penicillin & Biozym, 882082 \\
\hline & & $\begin{array}{l}0.02 \% \text { (wt/vol) } \\
\text { Streptomycin }\end{array}$ & Biozym, 882082 \\
\hline & & $\begin{array}{l}2.5 \% \text { (wt/vol) Bovine serum } \\
\text { albumin }\end{array}$ & $\begin{array}{l}\text { Applichem, } \\
\text { A1391,0250 }\end{array}$ \\
\hline & & $\begin{array}{l}2.5 \% \text { (wt/vol) Trypsin- } \\
\text { Inhibitor }\end{array}$ & Sigma, T9253 \\
\hline \multirow{4}{*}{$\begin{array}{l}\text { Glyoxal solution } \mathrm{pH} \\
4\end{array}$} & \multirow[t]{4}{*}{ Immunostaining } & $7.15 \mathrm{ml} \mathrm{ddH} \mathrm{H}_{2} \mathrm{O}$ & - \\
\hline & & $1.99 \mathrm{ml} \mathrm{100 \%} \mathrm{Ethanol}$ & Merck, 1.00983.1011 \\
\hline & & $0.79 \mathrm{ml} 40 \%$ Glyoxal & Sigma, 128465 \\
\hline & & $0.08 \mathrm{ml} \mathrm{100 \%} \mathrm{Acetic} \mathrm{acid}$ & Roth, 3738.2 \\
\hline \multirow[t]{4}{*}{ Mowiol solution } & \multirow[t]{4}{*}{ Immunostaining } & $68 \%$ (wt/wt) dd $\mathrm{H}_{2} \mathrm{O}$ & AriumPro, Sartorius \\
\hline & & 100 mM TRIS (pH 8.5) & Sigma, 252859 \\
\hline & & $23 \%$ (wt/wt) glycerol & Sigma, G5516 \\
\hline & & 9\% (wt/wt) Mowiol 4-88 & Merck, 475904 \\
\hline \multirow[t]{3}{*}{ Floxuridine stock } & \multirow[t]{3}{*}{ Cell culture } & DMEM & Biozym, 880026-12 \\
\hline & & Uridine & Sigma, U-3003 \\
\hline & & 5-Fluoro-2'deoxyuridine & Sigma, F-0503 \\
\hline
\end{tabular}




\section{Chemicals}

Table 5: Chemicals used in this study

\begin{tabular}{|c|c|c|}
\hline Name & Manufacturer & Used for \\
\hline Nitric acid & Sigma, 695033-2.5L & Cell culture \\
\hline Poly-L-Lysine hydrochloride & Sigma, P2658 & Cell culture \\
\hline $2.5 \%$ (wt/vol) Trypsin & Invitrogen, 15090-046 & Cell culture \\
\hline DNase & Roche, 10104159001 & Cell culture \\
\hline Paraffin & Merck, 1.07158 .1000 & Cell culture \\
\hline Bovine serum albumin & Applichem, A1391,0250 & Immunostaining \\
\hline $\mathrm{NH}_{4} \mathrm{Cl}$ & Merck, 1.01145 .1000 & Immunostaining \\
\hline Paraformaldehyde (PFA) & Sigma, P6148 & Immunostaining \\
\hline Bicuculline & Sigma, 14340 & Plasticity Induction \\
\hline Tetrodotoxin (TTX) & Tocris, 1069 & Plasticity Induction \\
\hline $\begin{array}{l}\text { 6-cyano-7-nitroquinoxaline-2,3- } \\
\text { dione (CNQX) }\end{array}$ & Sigma, C127 & Plasticity Induction \\
\hline $\begin{array}{l}\text { (2R)-amino-5-phosphonovaleric } \\
\text { acid (AP5) }\end{array}$ & Abcam, ab144498 & Plasticity Induction \\
\hline LiChrosolv grade Water & Merck, 115333 & Mass spectrometry \\
\hline $\begin{array}{l}\text { LiChrosolv grade Acetonitrile } \\
(\mathrm{ACN})\end{array}$ & Merck, 100030 & Mass spectrometry \\
\hline Ammonium bicarbonate (ABC) & Sigma Aldrich, 09830 & Mass spectrometry \\
\hline $\begin{array}{l}\text { Sequencing Grade Modified } \\
\text { Trypsin }\end{array}$ & Promega, V5111 & Mass spectrometry \\
\hline Trifluoroacetic acid (TFA) & Fluka, 302031 & Mass spectrometry \\
\hline Pierce BCA Protein Assay kit & $\begin{array}{l}\text { Thermo Fisher Scientific, } \\
23225\end{array}$ & Mass spectrometry \\
\hline UPS2 protein standard & Sigma Aldrich, UPS2 & Mass spectrometry \\
\hline
\end{tabular}

\section{Neuronal culture}

Hippocampal neuron culture were prepared from E18 Spragley-Dawley rats and grown in a sandwich configuration as described before using an N2 supplemented medium (Kaech and Banker, 2006). The following modifications were made: Glia were seeded at 10000 cells per well in 12 well plates, 3 days before dissection day. Neurons were seeded at 30000 cells on $18 \mathrm{~mm}$ coverslips with paraffin dots. The culture was fed by exchanging $500 \mu \mathrm{L}$ of medium twice a week. 
For mass spectrometry analysis I did not use paraffin dots as spacers, as they impair mass spectrometric analysis. Instead, I punched C-shaped Teflon rings with a $14 \mathrm{~mm}$ inner diameter, $20 \mathrm{~mm}$ outer diameter and $1 \mathrm{~mm}$ thickness from Teflon sheets (Alt Industriebedarf, Neresheim, Germany). They were rinsed thoroughly in $70 \%$ ethanol and autoclaved before use.

\section{Homeostatic plasticity induction}

The hippocampal cultures were treated for $72 \mathrm{~h}$, using either $20 \mu \mathrm{M}$ Bicuculline to increase network activity and induce synaptic upscaling, $1 \mu \mathrm{M}$ TTX to block synchronous release and induce downscaling or $10 \mu \mathrm{M}$ CNQX + $50 \mu \mathrm{M}$ AP5 to block AMPA and NMDAR, also inducing downscaling but to a higher extent (Turrigiano, 2008). Cells were then processed for immunostaining.

\section{Electron Microscopy}

\section{Sample Preparation}

Samples and imaging for electron microscopy was performed by Thomas Schikorski (Universidad Central Del Caribe, Bayamon, PR, USA). Briefly, standard hippocampal neuronal cultures were grown and embedded in Epon according to published protocols (Schikorski and Stevens, 1997). Then, the samples were cut into $70 \mathrm{~nm}$ consecutive sections and analyzed using a Zeiss EM902 (Zeiss, Jena, Germany) equipped with a 1024×1024 CCD-detector (Proscan CCD HSS 512/1024; Proscan Electronics, Schering, Germany).

\section{Reconstruction and Analysis}

Excitatory synapses were identified in the images by the presence of a PSD and a region around the synapse was selected. The consecutive images were aligned using Photoshop (Adobe Systems, San Jose, CA, USA), the membrane and organelles were traced and analyzed using custom written functions in Matlab (MathWorks, Natick, MA, USA), written by Silvio Rizzoli. Image alignment and tracing were performed by Christina Koerbs and Vanessa Salimi during their medical dissertations (Koerbs, 2017; Salimi, 2017), which I supervised (with Silvio Rizzoli as official supervisor, or Erstbetreuer, according to the medical dissertation system).

\section{Immunostaining}

Neurons were fixed at DIV21-23 with glyoxal as described (Richter et al., 2017), using pH 4 for the glyoxal solution, or PFA. Briefly, the fixed cells were quenched for 30 min using PBS containing $100 \mathrm{mM} \mathrm{NH} 4 \mathrm{Cl}$ and then permeabilized for $3 \times 5 \mathrm{~min}$ in PBS with $0.3 \%$ Tween and $2.5 \%$ BSA. Antibodies were diluted in the same solution and incubated for $1 \mathrm{~h}$ each in a humid chamber. The cells were washed $3 x$ with high-salt PBS (containing $500 \mathrm{mM} \mathrm{NaCl}$ ) and 2x in 
normal PBS. Meanwhile, DiO was diluted in PBS to a concentration of $20 \mu \mathrm{g} / \mathrm{ml}$ and the DiO crystals sonicated for $30 \mathrm{~min}$ in a water bath sonicator. Afterwards, $\mathrm{DiO}$ was diluted further to $2 \mu \mathrm{g} / \mathrm{ml}$ in PBS and $1 \mathrm{ml}$ of the solution was added per well. The cells were incubated at $37^{\circ} \mathrm{C}$ for $20 \mathrm{~min}$, washed once and incubated overnight. The following day, they were washed $2 x$ with PBS, embedded in Mowiol and dried for $1 \mathrm{~h}$ at $37^{\circ} \mathrm{C}$.

\section{Fluorescence Microscopy}

In all images, DiO was used as a membrane marker, while the PSD was identified using Homer1 antibodies, recognized by Cy3-labeled secondary antibodies. The protein of interest was identified with ATTO647N labeled secondary antibodies.

Epifluorescence images were acquired with a Nikon Eclipse Ti-E (Nikon Instruments, Tokyo, Japan), using a 100x 1.4 NA UPlanSApo oil immersion objective. The signal was detected with a DS-Qi 2 CMOS camera (Nikon Instruments, Tokyo, Japan).

Confocal and STED images were acquired with a Leica TCS STED system (Leica Microsystems $\mathrm{GmbH}$, Mannheim, Germany) equipped with a 100x objective (1.4 numerical aperture, NA, 100x HCX PL APO CS oil; Leica Microsystems). For confocal imaging of DiO and Homer1, a helium-neon laser was used to excite DiO (using $488 \mathrm{~nm}$ excitation wavelength), Alexa 488 (488 nm) or Cy3 (543 nm or $561 \mathrm{~nm}$ ) while using acusto-optic tunable filters to select appropriate emission wavelengths. For STED imaging of the protein of interest, ATTO647N was excited with a $635 \mathrm{~nm}$ diode laser and a Spectra-Physics MaiTai multiphoton laser (Newport Spectra-Physics, Santa Clara, CA, USA) at $750 \mathrm{~nm}$ was used as depletion beam. Confocal images were acquired using photomultiplier tubes or Hybrid detectors, whereas ATTO $647 \mathrm{~N}$ in STED mode was detected with an avalanche photodiode (Leica Microsystems). For analyzing the nanoscale localization of proteins, a pixel size of $20.21 \mathrm{~nm}$ was chosen. For each protein, two neuronal cultures were analyzed, with at least 10 images taken per replicate. The number of spines used for an average image was between 72 and 276 for mushroom spines (Mean= 175 images) and 44 to 248 for stubby spines (Mean= 134 images).

\section{Image Analysis}

All image analysis was done using custom written functions in Matlab 2017b (MathWorks, Natick, MA, USA). All functions are written by me, except the initial spot selection function and EM analysis functions, which were written by Silvio Rizzoli. The initial synapse alignment tool was written by Tal Dankovich. 


\section{Calculating the average protein distributions in dendritic spines}

To calculate the average distribution of a protein in dendritic spines, several steps needed to be performed: Identification and classification of synapses, alignment of synapses and removal of signal outside of the dendritic spine:

After aligning the STED image to the confocal image stack (using the confocal ATTO647N image) the spine was selected using DiO as a mask and all Homer spots within this mask were selected by manual thresholding. For each selected putative synapse, a $6 \times 6 \mu \mathrm{m}$ sub-image, centered on the synapse was saved. All synapses were then manually curated and assigned a spine class based on their morphology and the presence of strong Homer1 staining (Mushroom, Stubby, Other). Then, several morphological landmarks were selected: Top, bottom, left edge and right edge of the head, position of the neck/shaft junction. Additionally, a rectangle on the shaft was selected to later calculate enrichment of proteins over the shaft, and the neck was traced. Using these landmarks, the spines for each protein were aligned to each other. Taking advantage of the single cell labeling with DiO, I removed fluorescence outside the spine using $\mathrm{DiO}$ as a mask. To combine the two replicates, I normalized all spines belonging to the same replicate by the maximum intensity observed in this replicate, to remove any bias due to different staining conditions between replicates. Finally, the signal was filtered and averaged over both replicates.

\section{Zone Enrichment Analysis}

To quantify the location and intensity of the staining, the dendritic spine was segmented into zones (Figure $2 \mathrm{~A}$ ). For each protein, the fraction of signal within a given zone was calculated and normalized by the size of the zone (Figure 2B). Then, the fold difference to the fraction of signal within the same zone from an average protein distribution was calculated (1)). The latter average distribution was determined by averaging across all investigated proteins.

$$
\text { Fold difference }=\frac{\% \text { of signal in zone }- \text { Average } \% \text { of signal in zone }}{\text { Average } \% \text { of signal in zone }}
$$

This results in an enrichment score I used for assessing where a protein is predominantly localized. 
A Mushroom zones

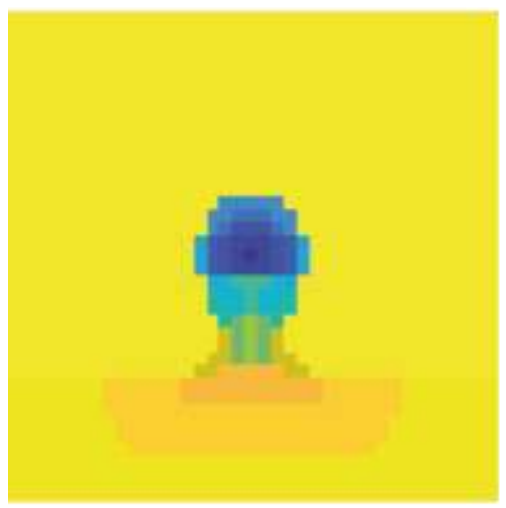

B

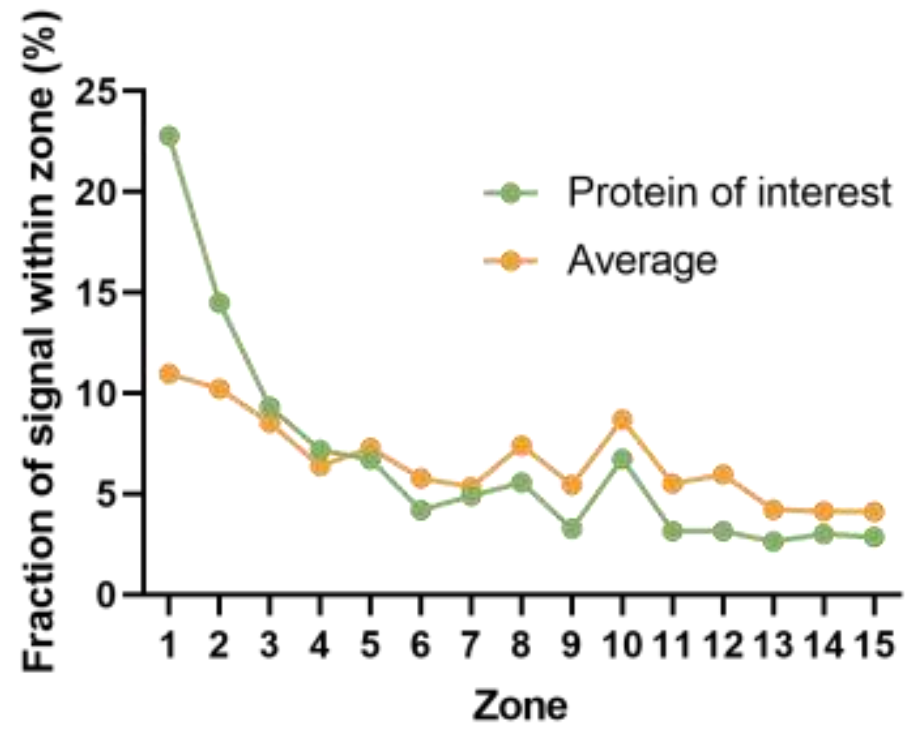

Figure 2 Zone Analysis explanation. A) Definitions for Mushroom and Stubby synapses. Darker colors represent zones closer to the PSD. B) For each protein the fraction of signal in a given zone was calculated. All proteins were also averaged and the average fraction of signal within a given zone was determined. The fold difference between the two was then calculated according to (1).

\section{Individual spine analysis}

In addition to the average analysis, I aimed to look at the individual spines and extract their morphological parameters and information on the protein signal within them. Also, these measurements served as the basis for morphologically clustering the spines to detect additional subclasses in the population.

Using the landmarks selected above, I could automatically reconstruct the different compartments of the spine, head, neck and shaft region, from the DiO signal (Figure 3). 
Head landmarks

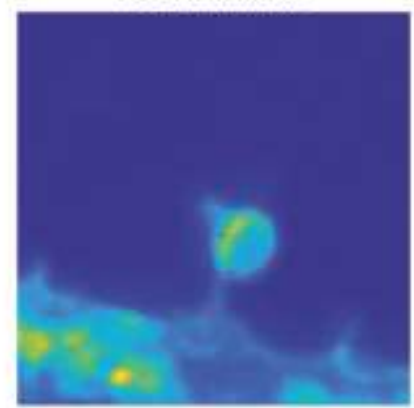

Head outline

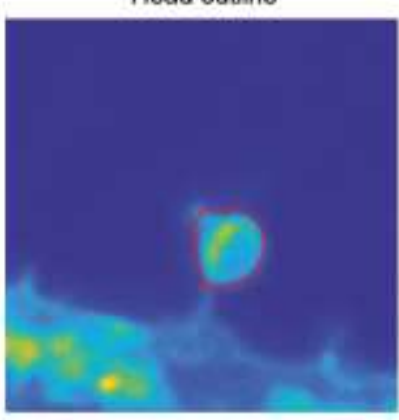

Compartment definitions

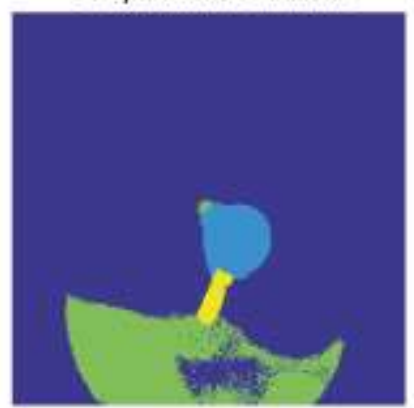

Figure 3: Automatic morphology detection of dendritic spines. In the compartment definitions, light blue corresponds to head, yellow to neck and green to shaft.

Individual STED spots were detected using wavelet transformation with the Spot Detector plugin for icy (De Chaumont et al., 2012; Olivo-Marin, 2002) using scale 2 with a threshold of $80 \%$. For Homer I mostly used the FWHM of the signal, which is a good estimate of its localization in super-resolution ( $\mathrm{Li}$ and Blanpied, 2016). I then extracted the following parameters:

Table 6: Individual spine parameters measured.

\begin{tabular}{|l|l|}
\hline \multicolumn{2}{|c|}{ Parameter name } \\
\hline Classification & Neck Area \\
\hline Head Area & Neck Length \\
\hline Head Center Column & Root Area \\
\hline Head Center Row & Spot Compartment \\
\hline Head Eccentricity & Spot File \\
\hline Head Height & STED Area \\
\hline Head Major Axis Length & STED Background Intensity \\
\hline Head Major Minor Axis Orientation & STED Centroid X \\
\hline Head Minor Axis Length & STED Centroid Y \\
\hline Head Width & STED DiO Distance \\
\hline Homer Area & STED Distribution \\
\hline Homer Center Angle & STED Eccentricity \\
\hline Homer Center Distance & STED Head Bottom Distance \\
\hline Homer Centroid X & STED Head Center Distance \\
\hline Homer Centroid Y & STED Head Enrichment \\
\hline Homer DiO Distance & STED Head Intensity \\
\hline Homer Eccentricity & STED Head Top Distance \\
\hline Homer Major Axis Length & STED Homer Enrichment \\
\hline Homer Major Minor Axis Orientation & STED Homer Intensity \\
\hline Homer Max Intensity & STED HomerFWHM Distance \\
\hline Homer Mean Intensity & STED HomerFWHM Enrichment \\
\hline Homer Minor Axis Length & STED HomerFWHM Intensity \\
\hline Homer Number & STED Laterality \\
\hline HomerFWHM Center Angle & STED Major Axis Length \\
\hline
\end{tabular}




\begin{tabular}{|l|l|}
\hline HomerFWHM Center Distance & STED Max Intensity \\
\hline HomerFWHM Centroid X & STED Mean Intensity \\
\hline HomerFWHM Centroid Y & STED Minor Axis Length \\
\hline HomerFWHM DiO Distance & STED Neck Bottom Distance \\
\hline HomerFWHM Eccentricity & STED Shaft Intensity \\
\hline HomerFWHM Major Axis Length & STED Spot Number \\
\hline HomerFWHM Major Minor Axis Orientation & STED Total Intensity \\
\hline HomerFWHM Max Intensity & \\
\hline HomerFWHM Mean Intensity & \\
\hline HomerFWHM Minor Axis Length & \\
\hline
\end{tabular}

In the presented analysis, I used the following parameters, which I explain in more detail here:

- Distance to membrane: The distance of every spot to its closest membrane is measured.

- Distance to PSD: The distance of spots to the PSD is measured. Only spots within the head/synaptic regions for stubby, are considered here.

- Enrichment in head: The fluorescence signal within the head is averaged and compared to the average fluorescence signal in the shaft. For each spine an individual rectangle was drawn on the shaft, which is used here.

- Enrichment in PSD: Same as enrichment in PSD, but the signal in the PSD, determined by Homer1 FWHM signal, is calculated.

- Eccentricity: The position of all spots in the head on the top-bottom axis is determined. This is normalized, 1 is the top of the head, -1 the bottom of the head ((2)

$$
\text { Eccentricity }=\frac{D_{\text {Bottom }}-D_{T o p}}{D_{\text {Bottom }}+D_{\text {Top }}}
$$

With $D_{\text {Bottom }}=$ the distance of the spot to the bottom of the head and $D_{\text {Top }}=$ the distance to the top of the head.

- Laterality: The position of all spots in the head on the left-right axis is determined. Because of the assumption of rotational symmetry, left and right are treated equally, reducing the dimension to central - left/right. The position is normalized, where 0 is the center of the spine, 1 is the most left/right position (3).

$$
\text { Laterality }=\frac{\left|D_{\text {Left }}-D_{\text {Right }}\right|}{D_{\text {Left }}-D_{\text {Right }}}
$$

With $D_{\text {Left }}=$ the distance of the spot to the left extreme of the head and $D_{\text {Right }}=$ the distance of the spot to the right extreme of the head

- Diameter: To each spot in the head an ellipse is fitted, the length of the major axis is shown.

- Distribution: For every spot in the head, the distance of this spot to all other spots in the head is calculated. 


\section{Mass Spectrometry}

\section{Sample preparation}

All samples were subjected to protein estimation using standard BCA protocol (Smith et al. 1985) as provided by Thermo Scientific online. First, $10 \mu \mathrm{l}$ of $1 \%$ RapiGest was added to 20 ug of protein sample (dendritic or UPS2 standard protein) and heated to $95^{\circ} \mathrm{C}$ for $5 \mathrm{~min}$. All subsequent steps were performed at $300 \mathrm{rpm}$ on a thermomixer at room temperature. Then, $10 \mu \mathrm{l}$ of $100 \mathrm{mM}$ ammonium bicarbonate solution was added to the sample and incubated for $5 \mathrm{~min}$. To reduce the cysteines, $10 \mu \mathrm{l}$ of $10 \mathrm{mM}$ dithiothreitol in $100 \mathrm{mM}$ ammonium bicarbonate was added and incubated for 1 hour. Reduced cysteines were alkylated by adding $10 \mu \mathrm{l}$ of $100 \mathrm{mM}$ iodoacetamide in $100 \mathrm{mM}$ ammonium bicarbonate and incubated for $20 \mathrm{~min}$ in the dark. To lower the detergent percentage to $0.1 \%, 180 \mathrm{ml}$ of $100 \mathrm{mM}$ ammonium bicarbonate was added. Finally, trypsin (1:50, ProMega) was added to the sample for digestion. For the long gradient, protein samples were digested for $12,14,16$ and 18 hours, while for high $\mathrm{pH}$ fractionation, $30 \mathrm{ug}$ of protein sample was digested using Trypsin for 16 hours. The trypsination was stopped by adding $20 \mathrm{ml}$ of $5 \%$ formic acid solution. The samples were incubated in $20 \mu \mathrm{l}$ of $5 \%$ trifluoro-acetic acid for 2 hours to deteriorate the RapiGest molecules. The samples were further desalted using StageTips. Briefly, at least four $\mathrm{C}_{18}$ plugs were filled in a micropipette tip to make one column. Prior to use, the column was washed twice with $50 \mu \mathrm{l}$ of methanol. The column was equilibrated by passing $50 \mu$ of $0.1 \%$ formic acid solution twice. The supernatant containing the peptides was loaded on the pre-equilibrated column, where the peptides bound to the $\mathrm{C}_{18}$ matrix. The column was washed four times with $50 \mu \mathrm{l}$ of $0.1 \%$ formic acid solution to remove ions. Finally, the bound peptides were eluted with $50 \mu \mathrm{l}$ of $80 \%$ acetonitrile, $0.1 \%$ formic acid solution twice. The eluted peptide solution was dried using a SpeedVac concentrator.

The samples were either directly processed for LC-MS on long gradient of 4 hours on Orbitrap Fusion Lumos Tribrid Mass Spectrometer or fractionated using high $\mathrm{pH}$ fractionation and subsequently processed for LC-MS for a short gradient of 1 hour on a Q-Exactive HF Mass Spectrometer.

For high $\mathrm{pH}$ fractionation, $40 \mu \mathrm{g}$ of protein digest was injected on a reversed phase column (XBridge Waters C18 column of dimension 3.5 $\mathrm{m}, 1.0 \times 150 \mathrm{~mm}$ ) connected to an Agilent 1200 high performance liquid chromatography (HPLC) system for $60 \mathrm{~min}$. A linear gradient was generated using dual highly basic buffer system (Buffer A: 100\% 10 mM Ammonia, Buffer B: $90 \%$ acetonitrile containing $10 \mathrm{mM}$ ammonia). The peptides were loaded on the column using buffer $A$ for $5 \mathrm{~min}$, followed by linear gradient of 0 to $50 \%$ of buffer $B$ for $45 \mathrm{~min}$. Finally, the column was washed using $95 \%$ of buffer $B$ and switched back to $100 \%$ of buffer $A$ for 5 min 
each. The flow rate was set to $50 \mu \mathrm{l} / \mathrm{min}$. A total of 60 fractions were collected and pooled to 12 fractions in a staggered manner (Table 7). The pooled fractions were dried using SpeedVac. Prior to MS, each fraction was resuspended in $25 \mu \mathrm{l}$ of sample loading buffer and $8 \mu \mathrm{l}$ injected thrice. $1 \mu \mathrm{g}$ of digested UPS2 standard protein mixture was analyzed between the samples to estimate the abundance of protein in the sample by label-free iBAQ approach.

Table 7: High pH fractionation pools

\begin{tabular}{|l|l|}
\hline Fraction number after pooling & High $\mathbf{p H}$ fractionation fractions (used for pooling) \\
\hline 1 & $1,13,25,37,49$ \\
\hline 2 & $2,14,26,38,50$ \\
\hline 3 & $3,15,27,39,51$ \\
\hline 4 & $4,16,28,40,52$ \\
\hline 5 & $5,17,29,41,53$ \\
\hline 6 & $6,18,30,42,54$ \\
\hline 7 & $7,19,31,43,55$ \\
\hline 8 & $8,20,32,44,56$ \\
\hline 9 & $9,21,33,45,57$ \\
\hline 10 & $10,22,34,46,58$ \\
\hline 11 & $11,23,35,47,59$ \\
\hline 12 & $12,24,36,48,60$ \\
\hline
\end{tabular}

\section{Liquid chromatography mass spectrometry}

The resuspended peptides in sample loading buffer ( $5 \%$ acetonitrile and $0.1 \%$ trifluoroacetic acid) were fractionated and analyzed by an online UltiMate 3000 RSLCnano HPLC system (Thermo Fisher Scientific) coupled online to the Q-Exactive HF or Orbitrap Fusion Lumos Tribrid Mass Spectrometer (Thermo Fisher). Firstly, the peptides were desalted on a reverse phase C18 pre-column ( $3 \mathrm{~cm}$ long, 100 $\mathrm{mm}$ inner diameter $360 \mathrm{~mm}$ outer diameter) for 3 minutes. After 3 minutes the pre-column was switched online with the analytical column (30 cm long, $75 \mu \mathrm{m}$ inner diameter) prepared in-house using ReproSil-Pur C18 AQ $1.9 \mu \mathrm{m}$ reversed phase resin (Dr. Maisch $\mathrm{GmbH}$ ). The peptides separated with a linear gradient of $5-30 \%$ buffer $\mathrm{B}(80 \%$ acetonitrile and $0.1 \%$ TFA acid) at flow rate of $10 \mathrm{nl} / \mathrm{min}$ either on a long gradient of 238 min on Lumos or a short gradient of 58 min on Q-Exactive HF. The pre-column and the column temperature were set to $50^{\circ} \mathrm{C}$ during the chromatography.

For long gradients on the Lumos spectrometer, the scan-range of precursors was set from $350-1500 \mathrm{~m} / \mathrm{z}$ at resolution of 120,000 in Top Speed mode. The top intense precursors were 
fragmented in HCD cell and analyzed at resolution 30,000. The AGC target and the isolation window was set at $5 \mathrm{e} 4$ and isolation window of $1.6 \mathrm{~m} / \mathrm{z}$.

For the short gradient on Q-Exactive HF, the precursors were scanned in the mass range from 350 to $1600 \mathrm{Da}$ at a resolution of 60,000 at $\mathrm{m} / \mathrm{z} 200$. Top 30 precursor ion were chosen for MS1 by using data-dependent acquisition (DDA) mode at a resolution of 15,000 at $\mathrm{m} / \mathrm{z} 200$ with maximum IT of $50 \mathrm{~ms}$. For MS2, HCD fragmentation was performed with the AGC target fill value of $1 \mathrm{e} 5$ ions. The precursors were isolated with a window of $1.4 \mathrm{Da}$. The lock mass option (m/z 445.1200; Olsen et al., 2005)) was used for internal recalibration.

\section{Database search and data analysis}

Proteins were identified using MaxQuant software (Cox and Mann, 2008) version 1.5.3.8 or 1.6.0.16 using the Andromeda search engine (Cox et al., 2011) with rat SwissProt (December 2016; containing 29795 entries) and Human Universal Proteome Standard (UPS2, SigmaAldrich) protein databases. For the database search, tolerance of $6 \mathrm{ppm}$ (for MS) and $10 \mathrm{ppm}$ (for MS/MS) were set. Oxidation of methionine and carbamidomethylation of cysteines were set as variable and fixed modifications respectively. Tryptic specificity with no proline restriction and up to 2 missed cleavages was used. False discovery rate (FDR) was set at $1 \%$. Additionally, the $\mathrm{iBAQ}$ option was enabled for quantification (using the $\log _{10}$ fit).

Absolute quantification can be acquired accurately 1) when a protein is fully digested and 2) no post-digestion modification has taken place to the peptides (Shuford et al., 2012). This is the reason why I chose to digest the four biological replicates for 8, 12, 16, 24 and 48 hours. In addition, the high $\mathrm{pH}$ fractionation enables efficient fragmentation due to a simplification of the MS spectrum, as less proteins are injected into the spectrometer per time. Thus, the maximum $\mathrm{iBAQ}$ value (from long and short gradient) was chosen to determine the absolute abundance of a protein.

The amount of a protein (in moles) is directly proportional to its iBAQ value (Schwanhäusser et al., 2011). Therefore, the logarithms of known amounts of UPS2 proteins were plotted against the logarithm of $\mathrm{BAQ}$ values. For determination of accurate absolute abundance, the slopes and intercepts of UPS2 proteins was calculated. Later, the slope value was used to determine absolute value of protein in the sample by linear regression. Next, the number of cells present in the sample was determined. To do so, for each biological replicate used, I stained coverslips from this replicate for their nuclei using Hoechst. I then counted the number of nuclei in random areas of the coverslips and extrapolated the number of neurons in the sample (Table 8). 
Table 8: Number of neurons for each biological replicate.

\begin{tabular}{|l|l|}
\hline Replicate & Number of neurons \\
\hline A & 6226 \\
\hline B & 2392 \\
\hline C & 14850 \\
\hline D & 24987 \\
\hline
\end{tabular}

The copy numbers of proteins per cell were calculated by dividing the number of proteins in the sample by the number of cells present in the used samples. The contaminants were removed from the protein list. To combine the regular and high-pH fractionation dataset, I took the maximum observed copy number of each protein for separate replicate, a principle that is also within the $\mathrm{iBAQ}$ algorithm. The data were then median-normalized and the mean across all replicates was calculated. Gene ontology analysis was done using the DAVID Functional Annotation Bioinformatics Microarray Analysis (Huang et al., 2007).

\section{Calculation of synaptic copy numbers}

To calculate synaptic copy numbers, I employed two different imaging steps. First, I took large epifluorescence images of the protein counterstained with Homer1 as a postsynaptic marker. For each protein, two independent cultures were analyzed and 20 images per replicate were acquired. The Homer images were thresholded and the percentage of signal within this homer mask to total signal was calculated for the protein of interest. Additionally, I calculated the $\mathrm{R}^{2}$ of the protein of interest to Homer from the central synaptic region of the STED images already acquired. Because the increased resolution of STED inherently causes the correlation to drop, I normalized the $\mathrm{R}^{2}$ by the $\mathrm{R}^{2}$ of Homer1 STED to Homer1 confocal. This step is intended to remove presynaptic contamination, as they correlate very weakly, or anticorrelate with Homer. For example, Synaptophysin shows a weak anticorrelation of normalized $R^{2}=-0.005 \pm 0.024$ whereas PSD95 strongly correlates with Homer1 $\left(R^{2}=0.408 \pm 0.030\right)$. The final synaptic copy number was then calculated as follows:

$$
N_{\text {synapse }}=\frac{N_{\text {wholecell }} * \text { Percentage in Homer mask } * \frac{R_{P O I}^{2}}{R_{\text {Homer } 1}^{2}}}{\text { Number of synapses per neuron }}
$$

with the number of synapses per neuron = 299 (Sebastian Jähne, Institute for Neuro- and Sensory Physiology, University Medical Center Göttingen, personal communication). 


\section{Statistics}

Statistic were done in Matlab 2017b (The MathWorks, Natick, MA, USA) or GraphPad Prism 8 (GraphPad, San Diego, CA, USA). All data shown is mean \pm SEM, if not otherwise noted. If the data was normal distributed, significance was assessed using a two-tailed t-test for single comparisons or one-way ANOVA with Bonferroni correction for multiple testing. If the data was not normal distributed, single comparisons were done using Whitney rank-sum test or KruskalWallis with Dunn's correction for multiple comparisons. Whether data was normal distributed was assessed with Kolmogorov-Smirnov test.

\section{Data Visualization}

All data was visualized using Matlab 2017b (The MathWorks, Natick, MA, USA), GraphPad Prism 8 (GraphPad, San Diego, CA, USA), Adobe Illustrator or InDesign (Adobe, San José, CA, USA), or Excel (Microsoft, Redmond, WA, USA). The contrast of images was adjusted using ImageJ (Schneider et al., 2012).

\section{D Model Generation}

To generate a representative 3D model, I needed to combine the super-resolution images with EM and mass spectrometry data. I chose one representative mushroom and stubby spine, which optimally matched the average parameters calculated for their class. Using the zones defined for the super-resolution data (Figure 2) I defined the same zones in EM data of these spines and assigned the organelle identities (Figure 4).

Via this method, I could link the super-resolution data to the EM images and also take the known localization of proteins into account, e.g. transmembrane proteins will only be placed on the membrane, ER resident proteins only on the ER. To generate the model, I used my calculated synaptic copy number and placed them in the spine volume according to the distribution measured from STED imaging. The reconstructed spines and the molecular images of the single proteins were rendered by Burkhard Rammner with Autodesk Maya (Autodesk Inc., Mill Valley, CA, USA) and I-Tasser (Roy et al., 2010; Zhang, 2008). Using these images, I made preliminary 3D models in Matlab. In brief, the models represent a maximum projection of the shown volume, but without the size of the protein adjusted according to its z-axis position. 


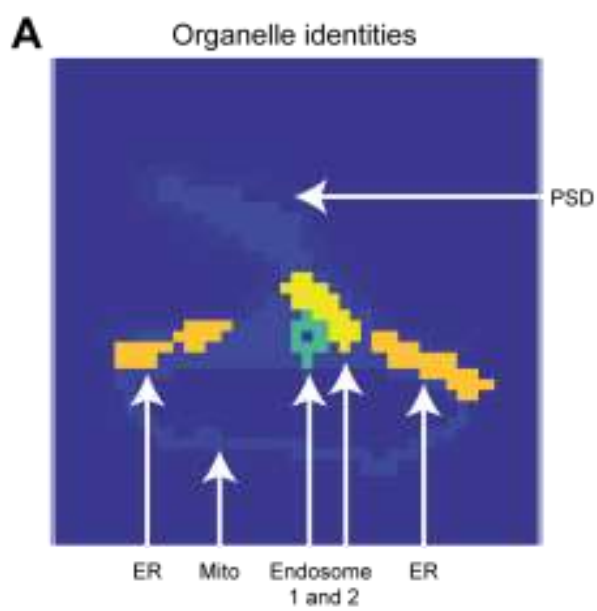

B

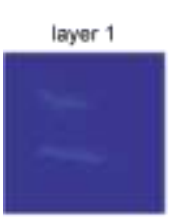

Mushroom organelle identities
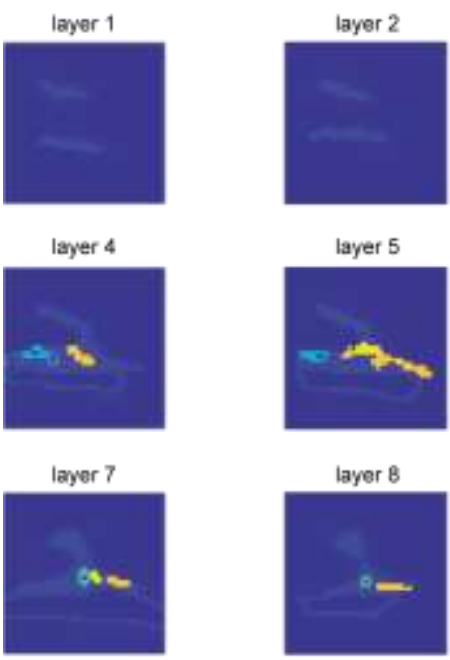

Stubby organelle identities
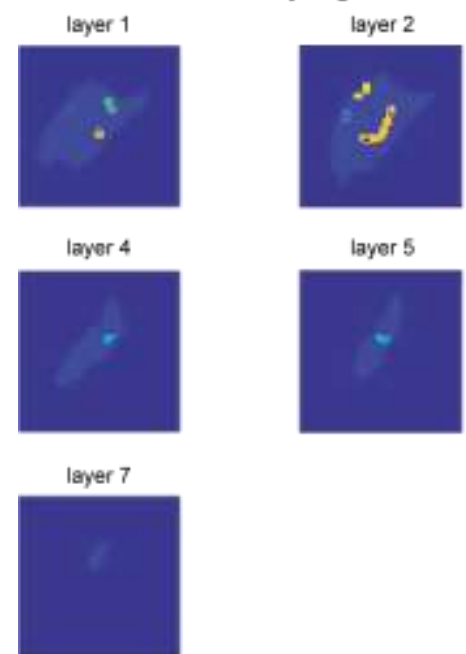

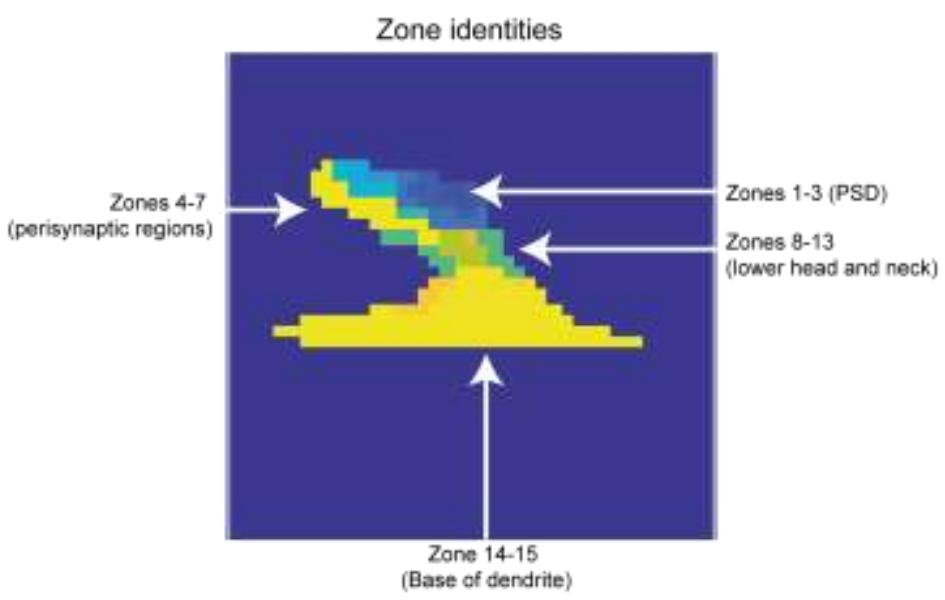

Mushroom zone identities
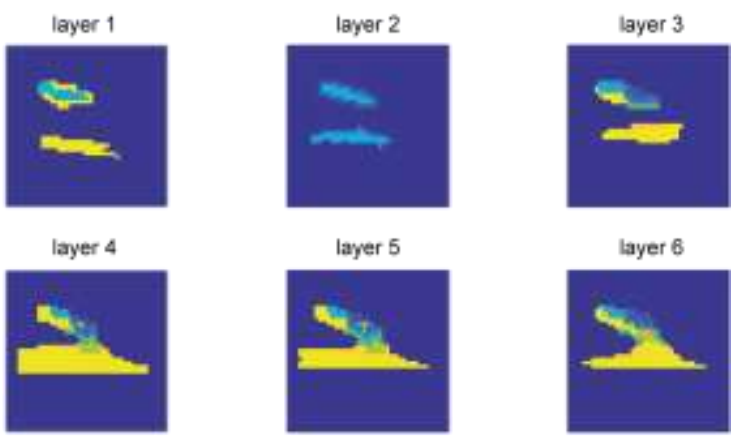

layer 7
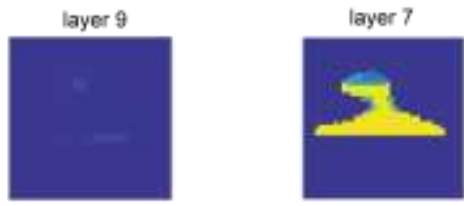

layer 8
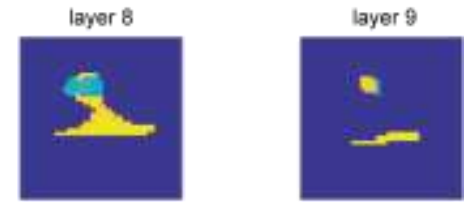

Stubby zone identities
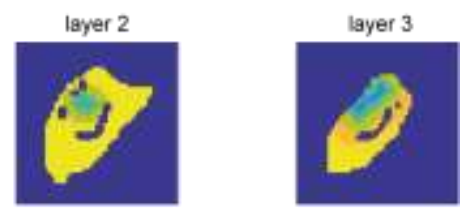

layer 6
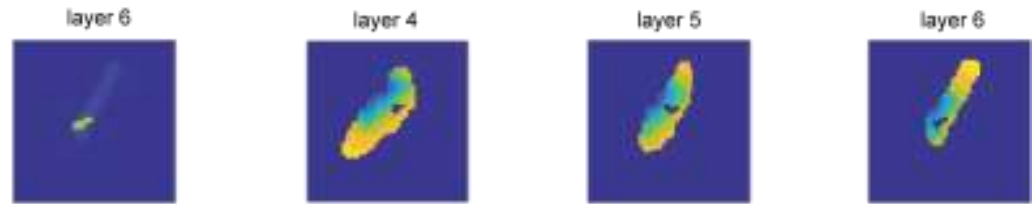

layer 7

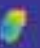

Figure 4: Assignment of organelle and zone identities for the construction of the 3D model. A) To apply the super resolution data on the EM ultrastructure, I needed to define the organelles and zones in the EM data. This way, membrane proteins could be mapped on the membrane, or organelle specific proteins only on their organelle. The EM data was binned and identities were assigned. The cytosol was also defined, depicted by the light blue color (left panel). Identical to the zone definitions of the super resolution data (Figure 2) the same zones were also assigned in the EM volume. B) This assignment was done for every layer of the EM reconstructions. 


\section{Results}

\section{The Banker culture is a well-defined model to study dendritic spines}

Deriving the quantitative information on protein copy numbers and localization requires an easy and well reproducible system. I could not use in vivo brain samples, as they are composed of many different neuron and glia types, making the biochemical analysis virtually impossible. Brain slices are also difficult to stain and image. I therefore opted to primary hippocampal neuron cultures, prepared in a Banker culture system (Kaech and Banker, 2006). It has several advantages:

- The neurons can easily be separated from the supporting glia, resulting in a pure neuronal sample. This way, I can eliminate glia contamination from the mass spectrometric analysis, which would otherwise confound our results.

- The majority of neurons in this type of culture are excitatory glutamatergic neurons, with only few inhibitory neurons present (Benson et al., 1994), yielding a well-defined neuronal population for the model. Additionally, the neurons can be grown in large quantities for biochemical analysis, single cells can be labeled with DiO (Figure 5A), and the preparation is easy to access for microscopy.

To confirm the purity of the culture I stained it for biomarkers of different neuronal populations or glia and compared it to the common coculture of neurons with astrocytes (Figure 5B). Both cultures showed little GABAergic neurons but large populations of glutamatergic neurons. I did also not detect appreciable populations of microglia or oligodendrocytes in either culture type. The most remarkable difference is in the content of astrocytes, marked by GFAP, which were present in large numbers in the coculture, whereas the Banker-type culture was almost completely devoid of them. 

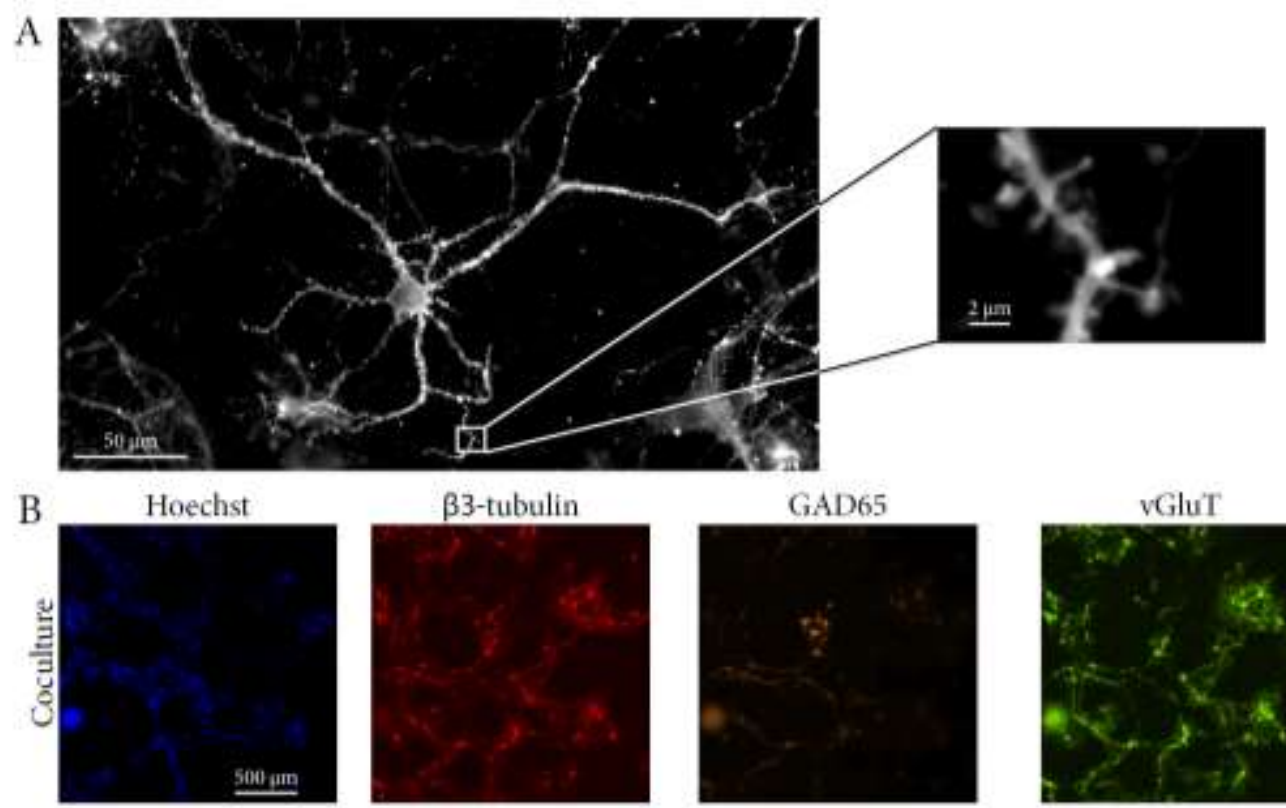

GAD65
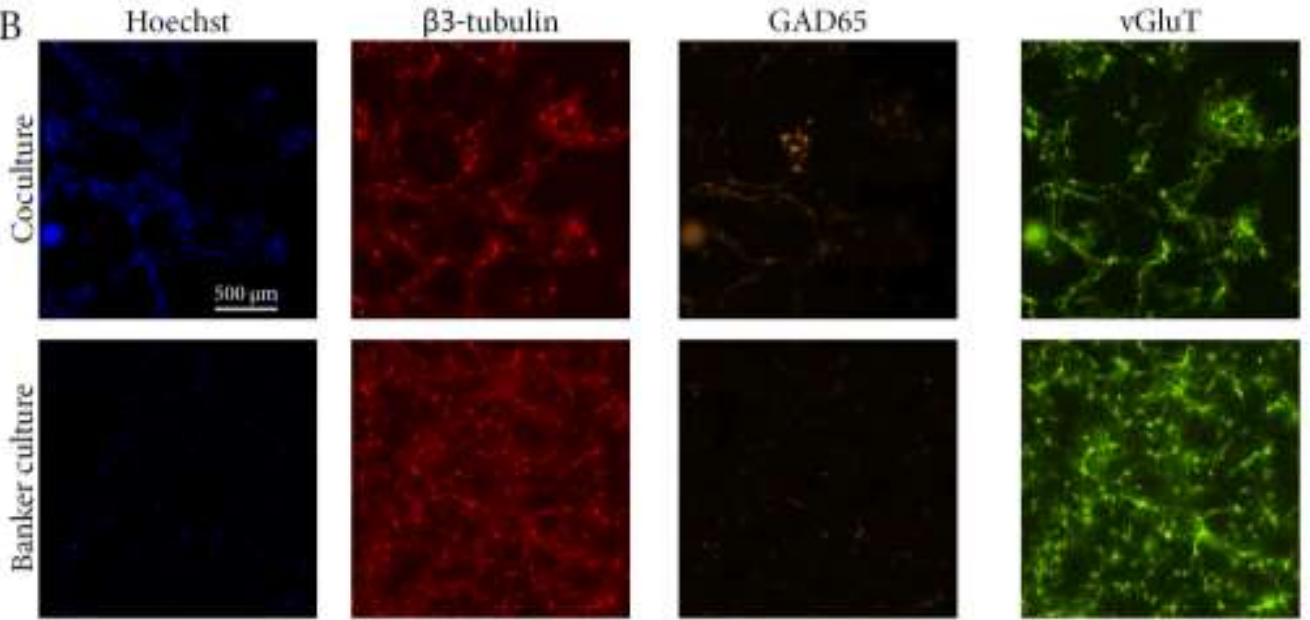

Hoechst

B3-tubulin
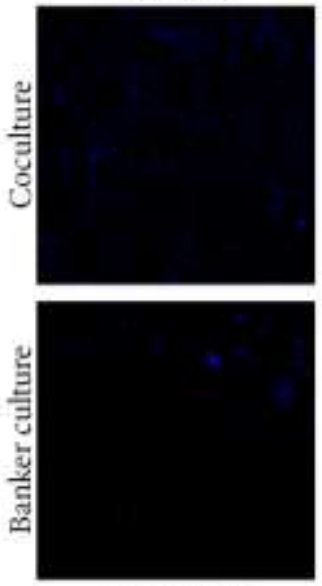

NF-H \& M

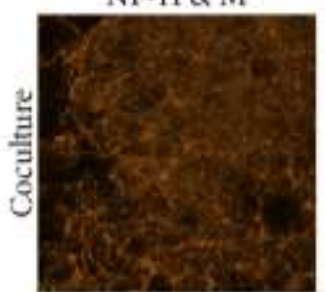

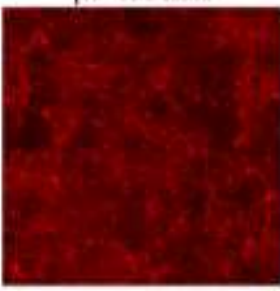

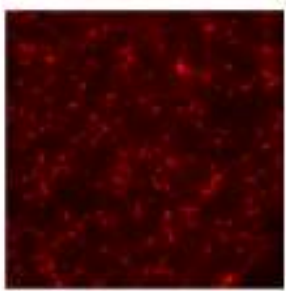

Olig2

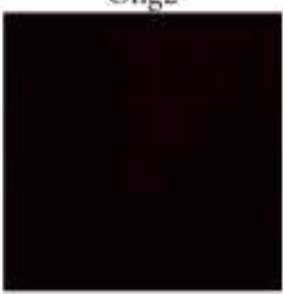

GFAP
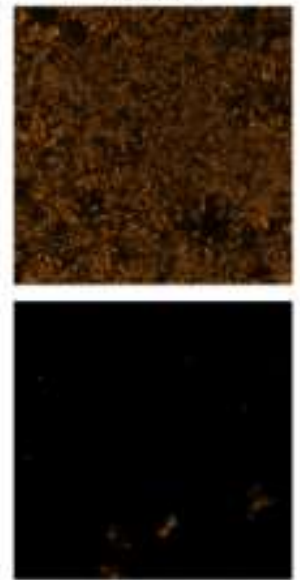

NF-H \& M

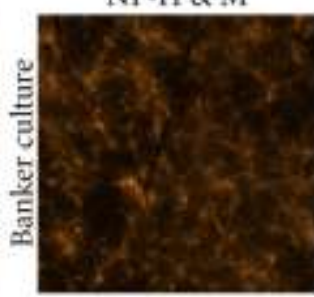

Iba]
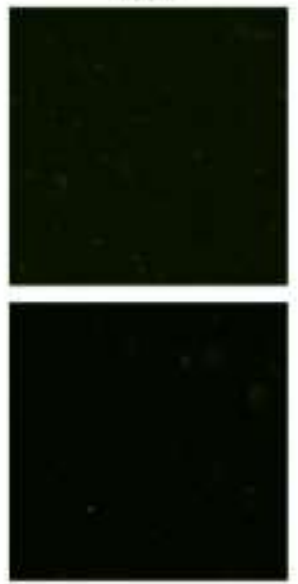

Olig2

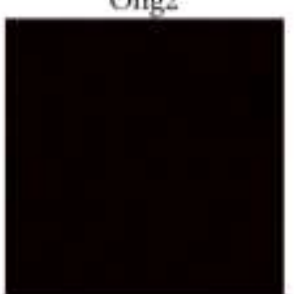

Figure 5: The Banker culture is a well-defined model. A) Using DiO labeling, I could label individual neurons, enabling me to visualize their spines in clear detail. B) Regular rat hippocampal neurons cocultured with astrocytes and the Banker type culture were stained for biomarkers of different neuron and glia populations. All neurons were either stained with $\beta 3$-tubulin, the antibody SMI310, labeling NF-H and NF-M. Cells were stained against GAD65 for GABAergic neurons, vGluT for glutamatergic neurons, GFAP for astrocytes, Iba1 for microglia and Olig2 for oligodendrocytes. 
In addition, I checked for the presence of glia biomarkers in our mass spectrometry dataset and compared it to numbers derived from whole-brain lysates (Figure 6). I did not detect most of the biomarkers in our culture, only GFAP was present in relatively larger amounts but markedly decreased compared to whole-brain lysates (reduced to 16.6\%). As GFAP is the major intermediate filament in astrocytes and abundantly expressed there, a few astrocytic cells can lead to a large number of proteins identified (Eng, 1985). In summary, the Banker type rat hippocampal cultures are a pure system with only few non-glutamatergic cells.

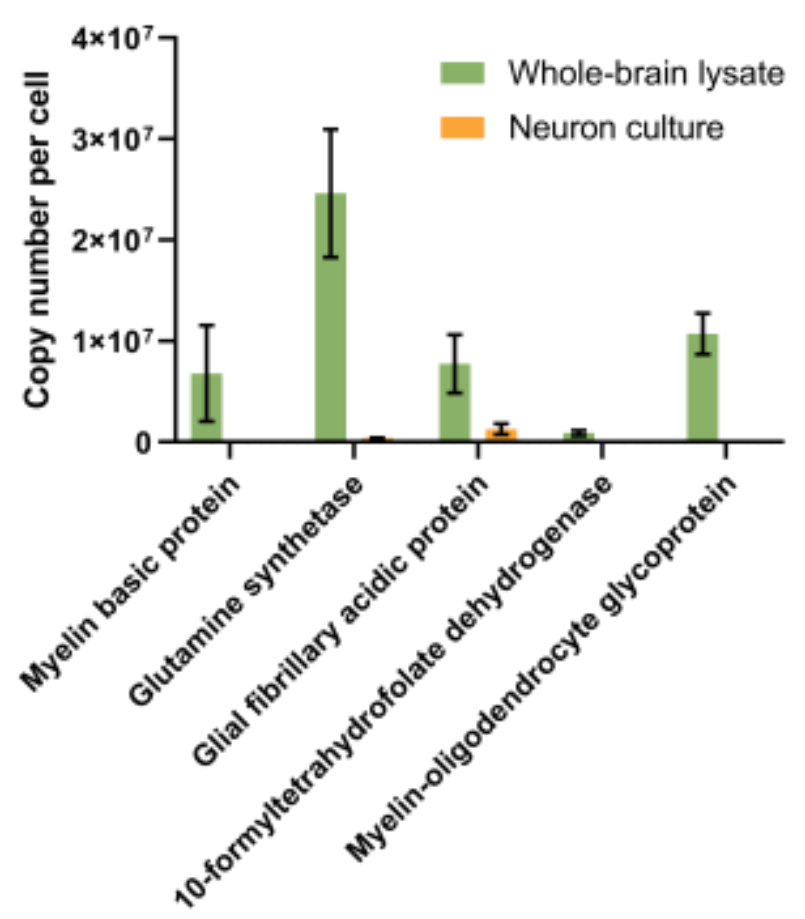

Figure 6: Biomarker analysis of neuronal cultures. In rat brain homogenate I could detect GFAP, glutamine synthetase, MBP and MOG in large quantities, but these were drastically reduced in neuron cultures. No statistical analysis was performed as only 1 experiment was done. 


\section{The ultrastructure of dendritic spines and its organelles in Banker cultures}

Next, I set out to measure the basic morphology of dendritic spines in the culture. Note that the presented analysis here is a recapitulation of the work done by Christina Koerbs and Vanessa Salimi during their medical dissertations under my supervision in our lab (Koerbs, 2017; Salimi, 2017). Mushroom class spines were traced from the head until the junction of the neck to the dendritic shaft. Stubby synapses usually showed a triangular protrusion from the shaft and were traced up to the point where this protrusion blended into the shaft (Figure 7). In total, I analyzed 30 mushroom and 34 stubby class synapses (Figure 8). 
A
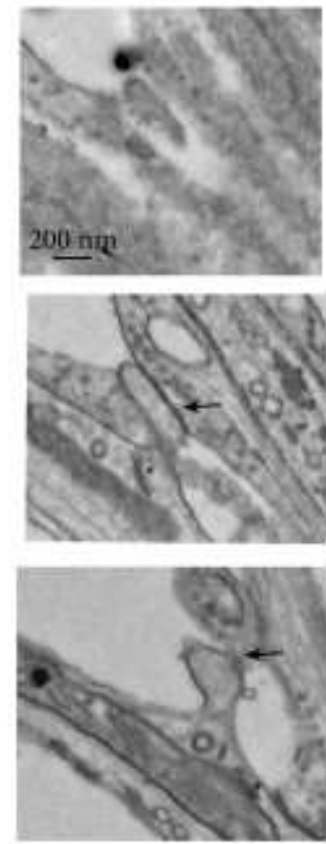

B
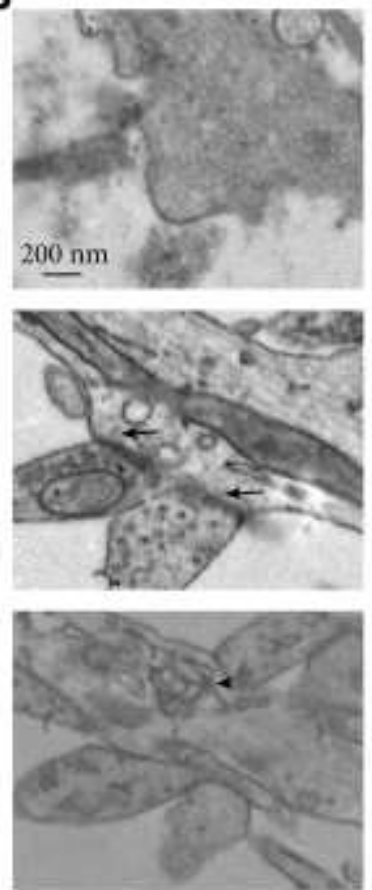

Mushroom
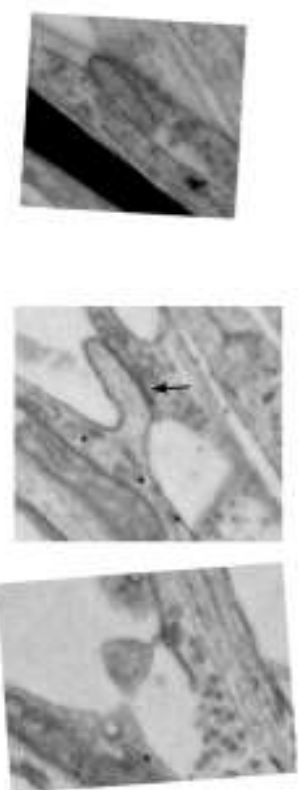

Stumpy
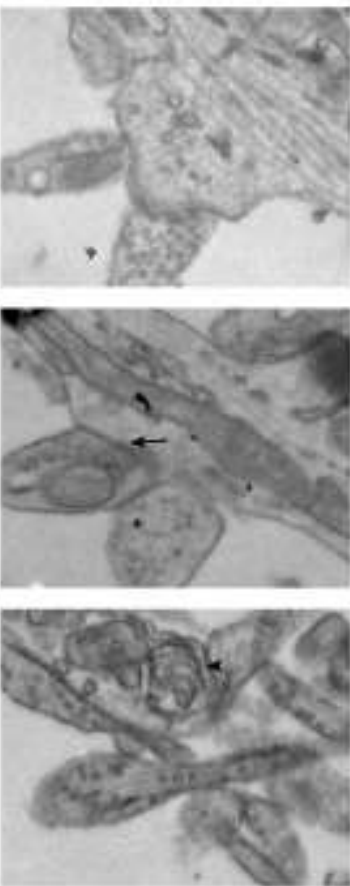
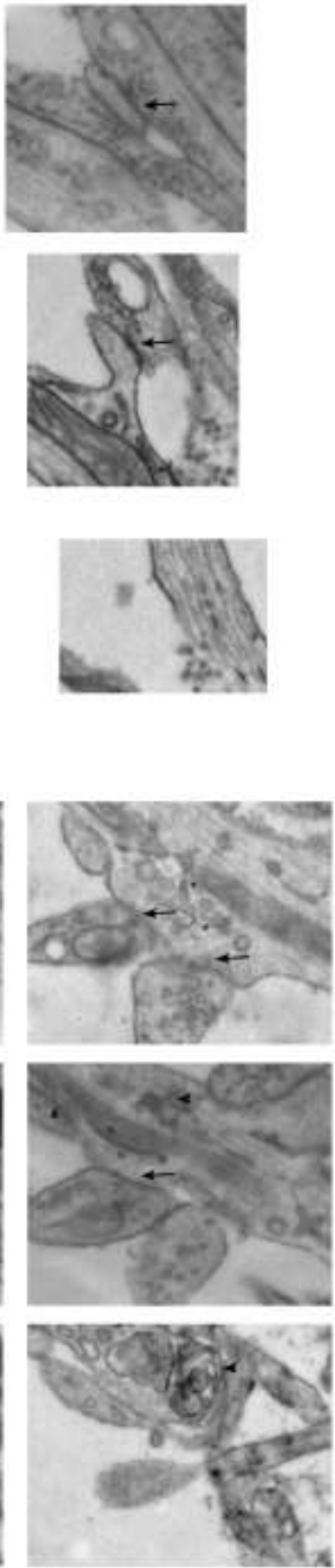

Figure 7: Representative serial sections. A) A mushroom class spine. The PSD is marked with an arrow. A continuous ER tubule is running along the dendrite, marked with an asterisk. Mitochondria are clearly identified by their dark stain and were often seen below the neck of the spine, but rarely invaginated into the neck or head. B) A stubby class spine with two presynapses and two corresponding PSDs side by side. The postsynapses are located on a bump-like protrusion from the dendritic shaft. The PSDs are marked with arrows, also here an ER tubule running along the shaft can be seen (marked with asterisks). In the upper portion of the shaft is a region with membrane ruffles, marked by arrowheads, of unknown identity. 
A
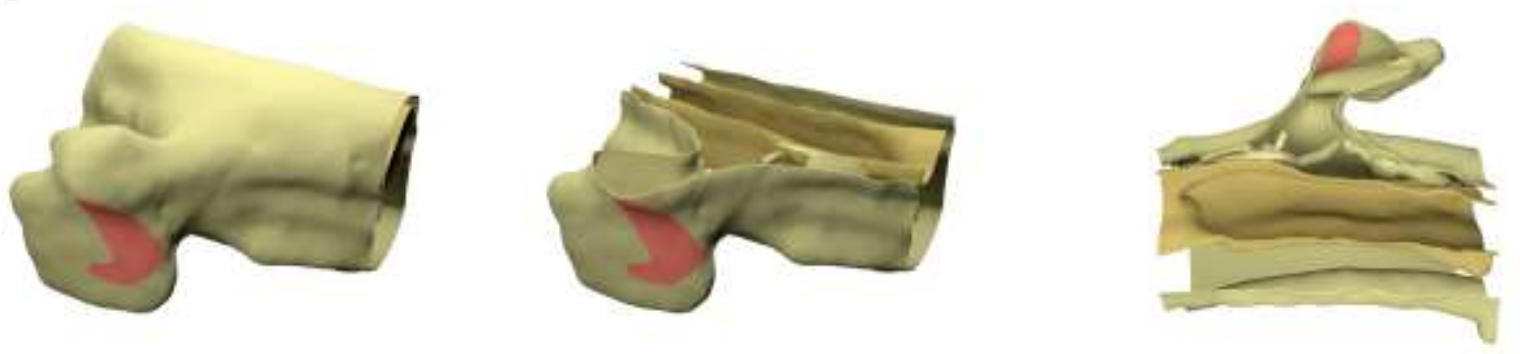

B
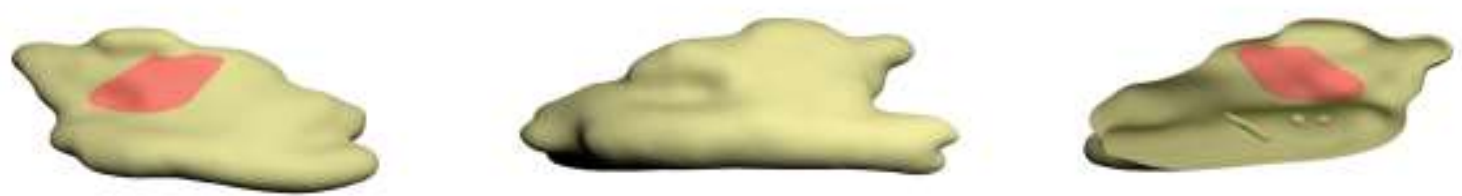

Figure 8: Representative 3D Reconstructions of dendritic spines. A) A Mushroom class spine is shown, with the PSD highlighted in red (left panel). The inside contains various organelles, in the dendritic shaft a large mitochondrion can be seen (middle panel). Many more organelles reside in the shaft and also in the spine itself, including ER, endosomes and vesicles (left panel) B) A stubby class spine is shown, looking at the face of the PSD. The dendritic shaft is not visualized, but it runs from left to right on the bottom of the spine (left panel). The back of the stubby spine does not have a PSD (middle panel). The cytosol of the stubby spine does show a few postsynaptic vesicles, as well as an ER tubule.

Interestingly, the morphology of the two classes were very similar in all the analyzed parameters, with no significant differences. Still, some variations could be observed: The PSD area of stubby synapses was on average $50 \%$ larger than those of mushroom synapses $\left(0.106 \pm 0.066 \mu \mathrm{m}^{2}\right.$ vs $\left.0.14 \pm 0.090 \mu \mathrm{m}^{2}\right)$. The PSD area of stubby postsynapses also showed a higher variability than those for mushroom class synapses, and it made up a larger fraction of the total surface of the synapse $(9.2 \pm 3.2 \%$ vs $6.6 \pm 1.4 \%)$. Also, the size of the PSD correlated with the postsynaptic volume for stubby synapses but did not for mushroom class synapses $(r=0.5016, p=0.0025$ and $r=0.3, p=0.173$ respectively). When I grouped the synapses by volume, I observed that there was a separate population of mushroom synapses with large volumes of $>0.4 \mu \mathrm{m}^{3}$, which is not present for stubby synapses. Interestingly, the number of vesicles per postsynapse was very variable: Many did not contain any vesicles, but some did contain up to 60 . The nature of these vesicles is unclear, they could be AMPAR containing postsynaptic vesicles, which have also been reported by others (Hussain and Davanger, 2015). 

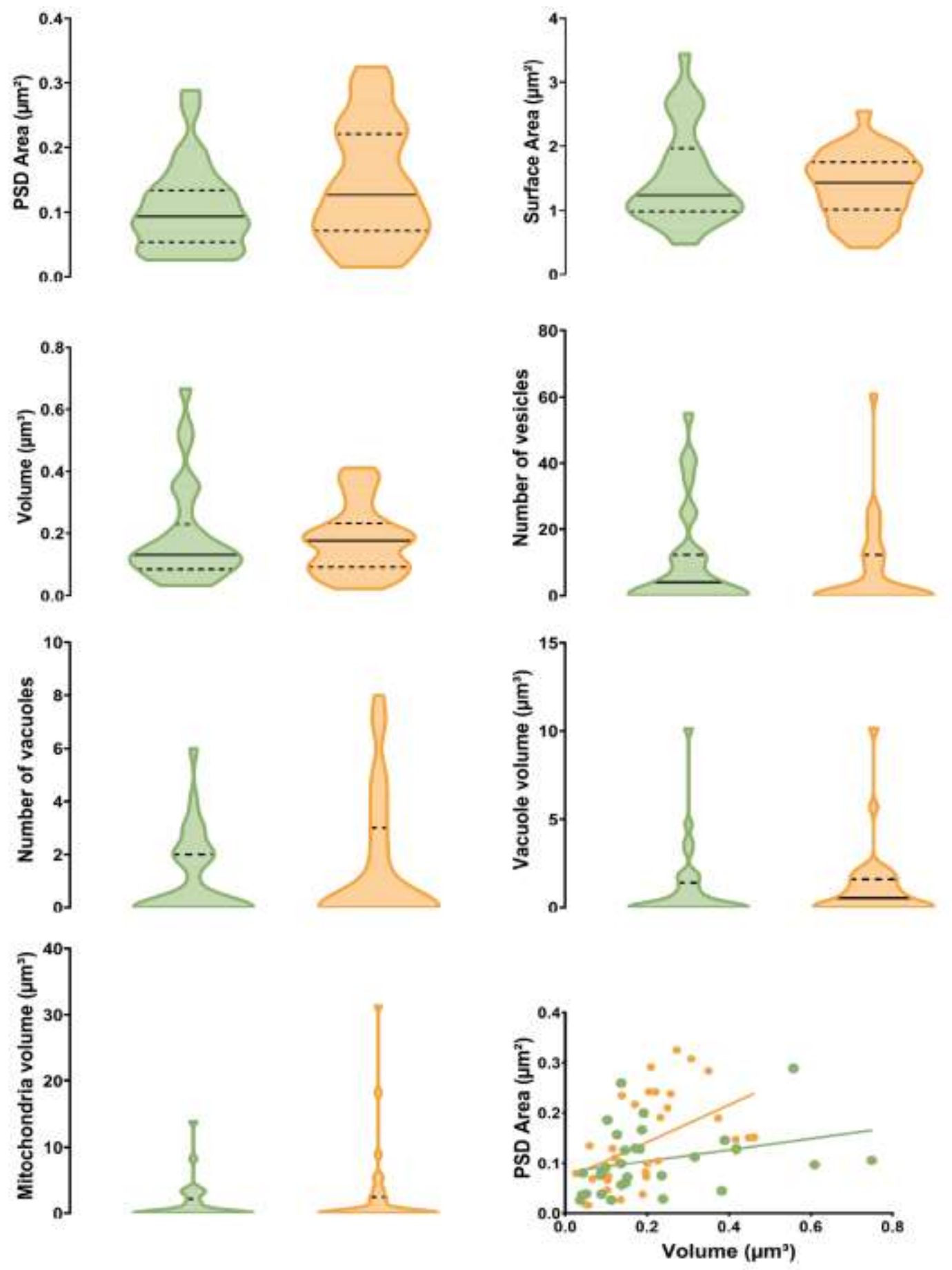

\begin{tabular}{|l|l|l|l|l|l|l|l|l|}
\hline Parameter & $\begin{array}{l}\text { PSD Area } \\
\left(\mu^{2}\right)\end{array}$ & $\begin{array}{l}\text { Surface Area } \\
\left(\mu \mathrm{m}^{2}\right)\end{array}$ & $\begin{array}{l}\text { Fraction PSD } \\
\text { of Surface } \\
\text { Area }(\%)\end{array}$ & $\begin{array}{l}\text { Volume } \\
\left(\mu \mathrm{m}^{7}\right)\end{array}$ & $\begin{array}{l}\text { Number of } \\
\text { vesicles }\end{array}$ & $\begin{array}{l}\text { Number of } \\
\text { vacuoles }\end{array}$ & $\begin{array}{l}\text { Vacuole } \\
\text { volume } \\
\left(\mu \mathrm{m}^{2}\right)\end{array}$ & $\begin{array}{l}\text { Mitochon- } \\
\text { dria volume } \\
\left(\mu \mathrm{m}^{2}\right)\end{array}$ \\
\hline Mushroom & $0.106 \pm 0.066$ & $1.513 \pm 0.743$ & $6.6 \pm 1.4$ & $0.189 \pm 0.158$ & $10.167 \pm 15.013$ & $1.067 \pm 1.552$ & $1.088 \pm 2.102$ & $1.502 \pm 2.985$ \\
\hline Stumpy & $0.142 \pm 0.090$ & $1.387 \pm 0.509$ & $9.2 \pm 3.2$ & $0.179 \pm 0.109$ & $6.882 \pm 12.526$ & $1.559 \pm 2.402$ & $1.139 \pm 1.959$ & $2.575 \pm 6.221$ \\
\hline
\end{tabular}

Figure 9: The quantitative morphological parameters of mushroom and stubby spines in culture are similar.

The distribution of the different measured parameters is shown, green is mushroom, orange are stubby spines. The PSD area follows a normal distribution, Surface and Volume are lognormal distributed (Kolmogorov-Smirnov test). None of the shown parameters were significantly different between the two classes. The continuous line shows the median, whereas the dotted lines are the quartiles. Stubby spines showed a correlation of their PSD area to their volume, which was not the case for mushroom spines $(r=0.5016, p=0.0025$ and $r=0.3, p=0.173$ respectively). The table shows the mean \pm SEM of the measured parameters. 


\section{The quantitative neuronal proteome}

Synaptic copy numbers for the dendrite are not easily obtainable, because there is no subcellular fractionation for dendritic spines available. It is possible to purify the PSD itself or synaptoneurosomes, but these contain the presynapse as well (Carlin et al., 1980; Hollingsworth et al., 1985), which will contaminate the analysis. I therefore devised an alternative strategy, where I first determined the average quantitative proteome of a complete hippocampal neuron using the label-free mass spectrometry technique iBAQ (Schwanhäusser et al., 2011). I then calculated the copy number per dendritic spine using imaging techniques. So far, most mass spectrometric analysis has focused on individual sub compartments (Cheng et al., 2006; Lowenthal et al., 2015; Peng et al., 2004a; Takamori et al., 2006; Wilhelm et al., 2014), whereas the whole neuron is seldomly analyzed. This is also the first quantitative description of the neuronal proteome in absolute terms, as so far only relative datasets are available for the neuron, which usually focus on the effect(s) of a specific treatment on the neuronal proteome (Schanzenbächer et al., 2016).

\section{Label-free mass spectrometry reveals the quantitative neuronal proteome}

I initially set out to determine the neuronal proteome using regular iBAQ mass spectrometry. Here, a standard protein mixture is analyzed before and after the sample in the mass spectrometer. The intensity for each peak is calculated and normalized by the number of theoretically observable peptides, derived from in silico digestion. Using the known protein numbers in the standard sample, one can then calculate a standard curve that relates peak intensity to copy number. This can then be used to calculate the copy number for each identified protein in the sample.

After optimizing the digestion and run times, I realized that several key proteins where not detected in the mass spectrometer, probably because the complexity of the sample was too high and because several important neuronal targets are proteins with extensive transmembrane domains, which are particularly difficult to tackle with mass spectrometry (Barrera and Robinson, 2011). I therefore introduced a high $\mathrm{pH}$ fractionation step right before the sample was injected into the mass spectrometer, to decrease sample complexity and increase the coverage of the neuronal proteome. To this purpose, I analyzed 4 biological replicates that showed a high correlation of their copy numbers (Figure 10A). The copy number per neuron showed a typical sigmoidal distribution (although not being normal or lognormal distributed, $p<0.0001$ for both tests), with many cytoskeleton or glycolytic proteins among the most abundant proteins (Figure 10B). 
A
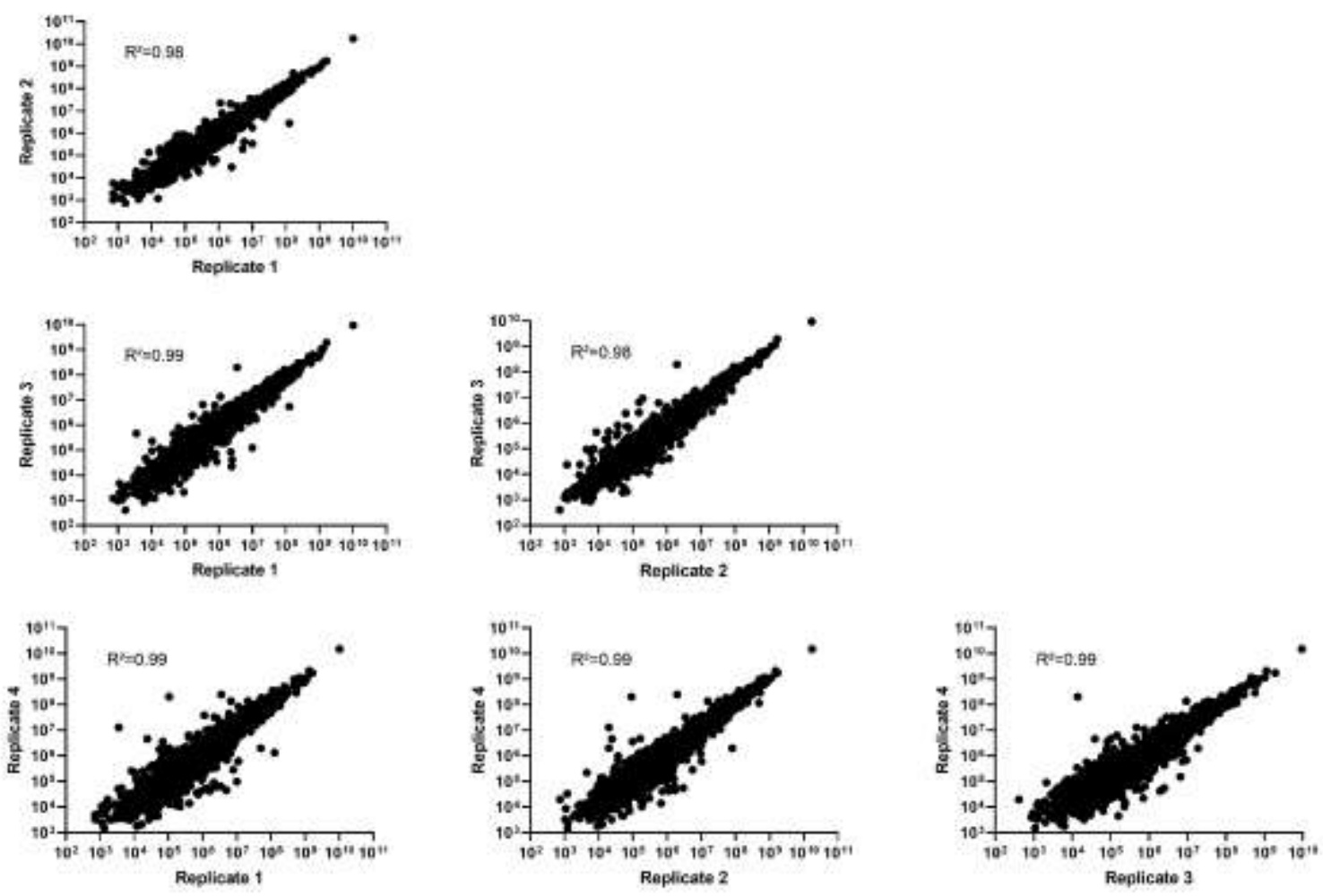

B

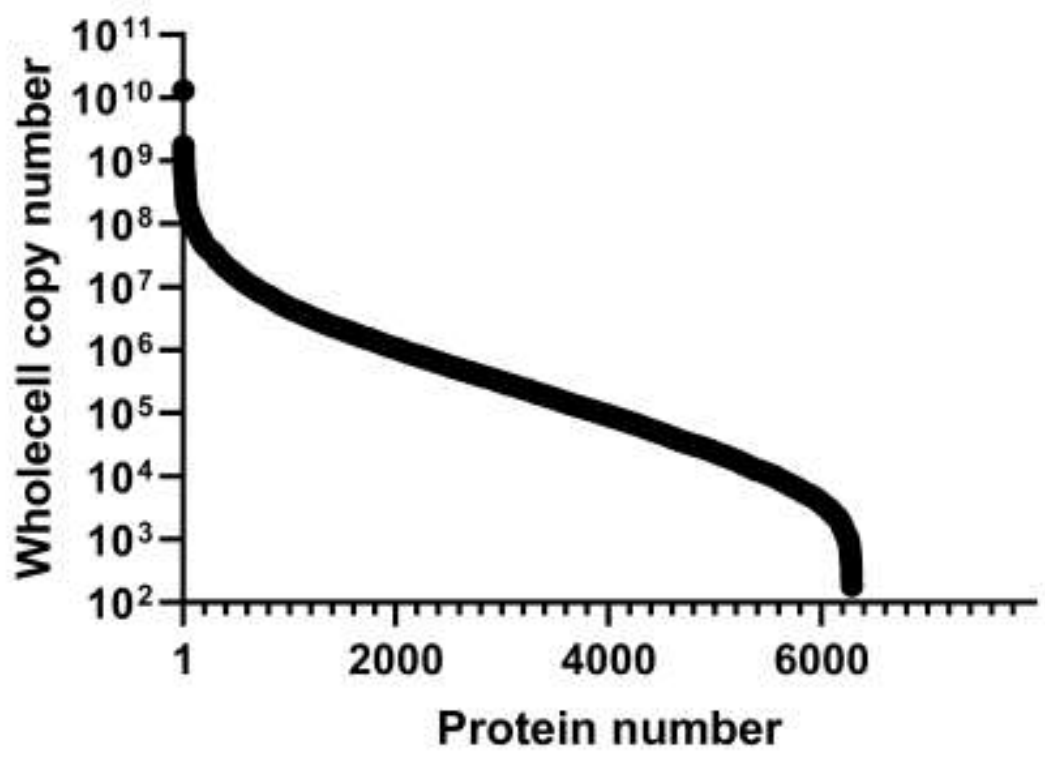

Figure 10: A general description of the quantitative neuronal proteome. A) I analyzed 4 biological replicates, which all showed a good correlation to each other. B) The distribution of the copy numbers showed few high or low abundant proteins, with most proteins in the range of $10^{4}$ to $10^{7}$ copies

In total, I was able to identify 6194 genes, which represents a very high coverage of pure hippocampal neuronal cultures. Because the rat proteome is not as highly curated as mouse or human, almost $2 / 3$ of the identifications were unreviewed entries (Figure 11A). Notably, VAMP2 was among the most abundant proteins with $7.5 \times 10^{8} \pm 9.4 \times 10^{7}$ copies. 
A

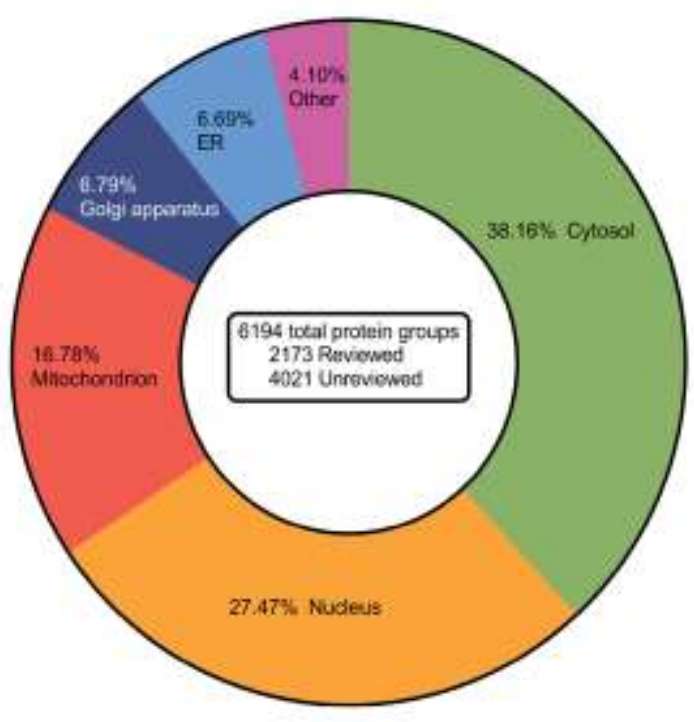

C

\begin{tabular}{|l|l|}
\hline Total protein number & $6.33 \times 10^{10}$ \\
\hline Total Molarity & $20.35 \mathrm{mM}$ \\
\hline Total protein & $2.2 \mathrm{ng}$ \\
\hline Total protein concentration & $4.36 \times 10^{5} \mathrm{mg} / \mathrm{ml}$ \\
\hline
\end{tabular}

D

\begin{tabular}{|l|l|l|}
\hline Protein & $\begin{array}{l}\text { Expected } \\
\text { stochiometry }\end{array}$ & $\begin{array}{l}\text { Measured } \\
\text { stochiometry }\end{array}$ \\
\hline $\begin{array}{l}\text { Rab geranylgeranyl } \\
\text { transferase }\end{array}$ & 1 & 1.28 \\
\hline vATPase subunit A & 3 & 2.89 \\
\hline vATPase subunit B & 3 & 3.64 \\
\hline
\end{tabular}

B

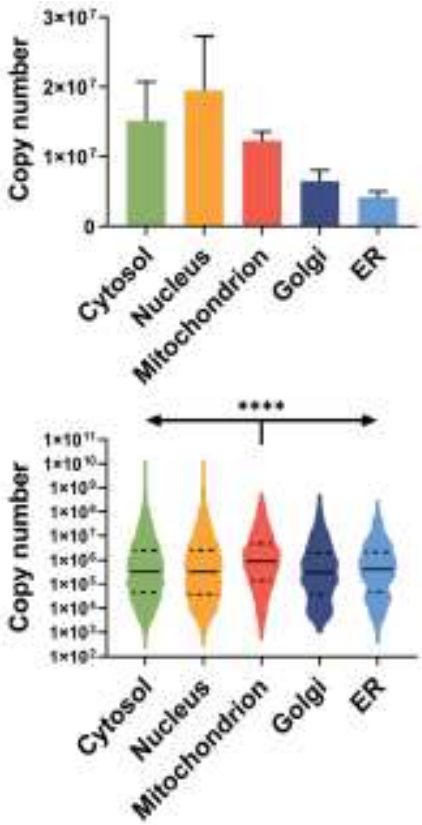

$\mathrm{E}$

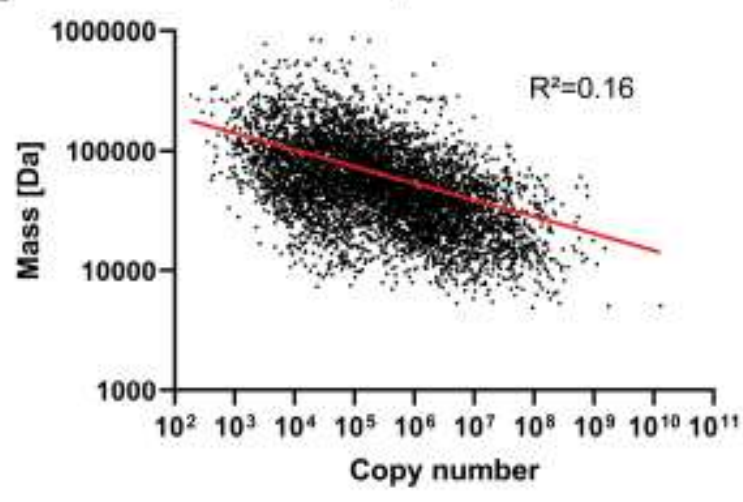

Figure 11: Quantitative mass spectrometry of neuronal cultures. A) In total I was able to identify 6194 protein groups. Using GO analysis, I assigned the proteins to the major subcellular compartments. B) The copy number distribution within each compartment was investigated. The mean copy number per subcellular compartment differs from the median (Mean \pm SEM, Median \pm quartiles are shown; Note the logarithmic scale of the violin plot). C) Summing up all protein numbers, the total molarity and protein content per neuron was calculated. The volume of the neuron was estimated to be $5000 \mu^{3}$. D) Two obligate protein interactions were checked for their occurrence in the data. E) Protein size shows a significant negative correlation with abundance (Spearman rho $=-0,51$, $p<0.001)$, that can also be fitted with a linear relationship $\left(R^{2}=0.16\right)$.

I also calculated the total protein number and amount per neuron and estimated the molarity and protein concentration (Figure 11C). Because there is no information in the literature on neuronal volumes in cultures, I estimated the volume to be $5000 \mu \mathrm{m}^{3}$, a value derived from mammalian cell culture volumes (Bohil et al., 2006; Cohen and Studzinski, 1967; Krombach et al., 1997; Luby-Phelps, 2000; PUCK et al., 1956; Zhao et al., 2008). I did so because neurons have many processes, that will increase the total volume. Neurons in vivo though can have much larger volumes, for example Purkinje cells have a volume of about $13700 \mu \mathrm{m}^{3}$ (Andersen et al., 2003). 
Using gene ontology analysis, I assigned the proteins to a subcellular compartment. The protein fraction within the compartment is similar to other studies (Schanzenbächer et al., 2016). Interestingly, the mean copy number between subcellular compartments was not significantly different, whereas the median copy number mitochondria was significantly different to those of the other compartments (Figure 11B). The lower median than mean indicates, that the data is skewed to higher values. This is more prominent for the non-mitochondrial compartments, which can also be seen from the violin plot. I checked the quality of the quantification by comparing two known obligate protein complexes with my measured data, the vATPase subunits and the rab geranylgeranyl transferase (Kitagawa et al., 2008; Zhu et al., 2006). These proteins are highly unstable when they are not bound by their interaction partner. Therefore, the whole cell copy numbers should represent the stochiometric ratio of the complex, which it did (Figure 11D). Intriguingly, the protein copy number showed highly significant anti-correlation with the mass of a protein (Figure 11E), a phenomenon that has also been observed in vivo (Mandad et al., 2018).

\section{Subcellular copy numbers can be derived using imaging methods}

As the goal of this study is to analyze the quantitative composition of dendritic spines, I devised a two-step method to calculate the copy number for a protein of interest in a dendritic spine. First, I needed to calculate the fraction of the protein of interest that is inside synapses. To do so, I used Homer1 as a postsynaptic marker, and coimmunostained against the protein of interest. Using Homer1 as a mask I calculated how much of the protein is within dendritic spines. I used epifluorescence imaging to cover large areas of the culture, but this has a lower resolution. Because of this, also some presynaptic regions are picked up within the homer mask. To correct for this, I used the super-resolution images already acquired. Here, I calculated the correlation coefficient of the protein of interest to Homer1. Together with the number of spines per neuron I can calculate the synaptic copy number for each protein analyzed this way (4).

The copy number within a dendritic spine followed a similar distribution as the neuronal copy numbers (Figure 12A). There were a few proteins that were only present in very few copies, suggesting that these are present only in a minor subset of dendritic spines. Interestingly, the postsynaptic copy number correlated with their presynaptic counterpart (Spearman rho $=0.68$, $p<0.0001$; Figure 12B), showing also that postsynaptic proteins are on average less abundant than in the presynapse (Presynaptic data from Wilhelm et al., 2014). In addition to the average postsynaptic copy number, I was interested whether different spine classes have different protein composition. I therefore calculated the synaptic copy number for mushroom and stubby synapses by comparing the immunofluorescence signal from the STED images between the two classes. Surprisingly, stubby synapses had more protein copy numbers on average (1.44 
\pm 0.34 ; Mean \pm SD) and several proteins showed a preferential enrichment in one class (Figure 12C). The proteins enriched in stubby synapses were mainly trafficking associated proteins, but also Homer1 showed an increased abundance in stubby synapses. Mushroom spines had, among others, more Actin and Drebrin, as well as PSD95. 
A

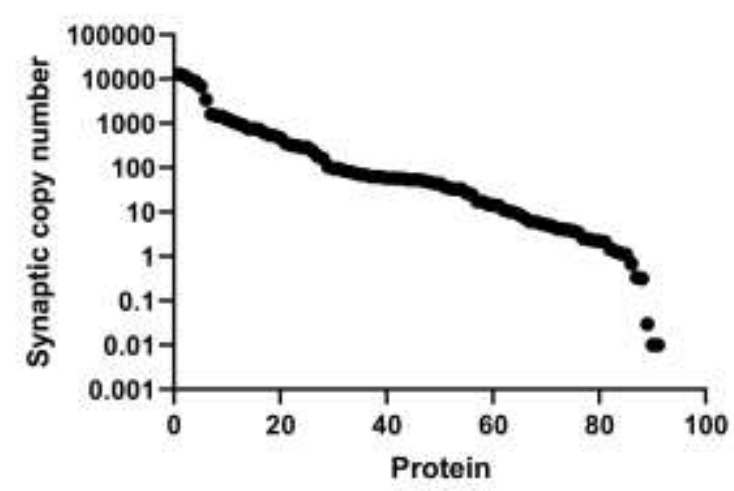

B

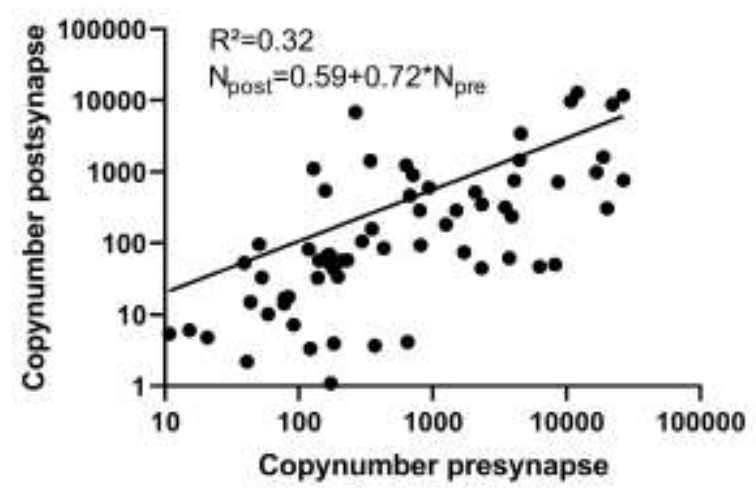

C

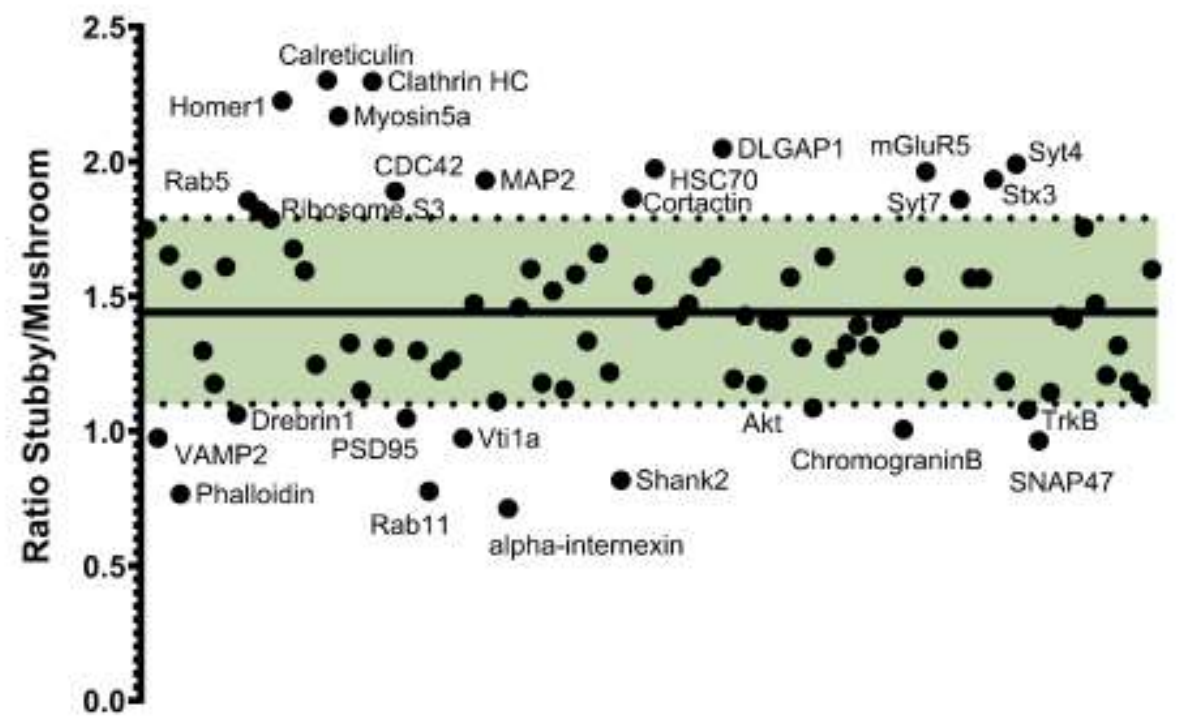

D

\begin{tabular}{|c|c|c|}
\hline $\begin{array}{c}\text { Total protein } \\
\text { number in spine }\end{array}$ & \multicolumn{2}{|c|}{$439565.05 \pm 147074.98$} \\
\hline $\begin{array}{c}\text { Total protein } \\
\text { number in PSD }\end{array}$ & Mushroom & Stubby \\
\hline $\begin{array}{c}\text { Total spine } \\
\text { protein number } \\
\text { Total PSD } \\
\text { protein number }\end{array}$ & $375772.51 \pm 123018.72$ & $516663.00 \pm 176305.10$ \\
\hline $\begin{array}{c}\text { Total molarity in } \\
\text { spine }[\mu M]\end{array}$ & $3149.45 \pm 1031.05$ & $2769.78 \pm 945.15$ \\
\hline $\begin{array}{c}\text { Total protein con- } \\
\text { tent in spine }[\mathrm{fg}]\end{array}$ & $71.88 \pm 26.43$ & $112.14 \pm 43.54$ \\
\hline
\end{tabular}

Figure 12: Dendritic spine copy numbers. A) The distribution of postsynaptic copy numbers. B) Pre- and postsynaptic copy numbers of the same protein are correlated (Spearman rho $=0.68, p<0.0001$; presynaptic data from Wilhelm et al., 2014). The postsynaptic protein is present in lower copies on average. Please note that the results are shown on logarithmic scales. C) Several proteins show a preference to either Stubby or Mushroom class spines. The line indicates the mean ratio, the dotted lines are mean \pm 1 SD. D) General parameters of the dendritic proteome. Spine means the whole dendritic spine, values are Mean \pm SEM. 


\section{The quantitative average distribution and abundance of 105 postsynaptic proteins}

After determining the synaptic copy number of the proteins of interest, I determined their nanoscale localization with dendritic spines. To do so, I used STED super-resolution microscopy. In total, I analyzed 40604 spines, of which 18429 were classified as mushroom, 14093 were stubby (Table 9). An additional class, termed 'Other', was also classified, that contains synapses that do not fit the two categories but show strong Homer1 signal. This class had 8082 entries but was not considered in the presented analysis.

Table 9: Distribution of the spine classes over the whole dataset.

\begin{tabular}{|l|l|l|}
\hline Spine class & Number & Percentage of total spines \\
\hline Mushroom & 18429 & $45.39 \%$ \\
\hline Stubby & 14093 & $34.71 \%$ \\
\hline Other & 8082 & $19.90 \%$ \\
\hline Total & 40604 & \multicolumn{1}{|l|}{} \\
\cline { 1 - 2 } & &
\end{tabular}

As this is a large dataset, and to make the comparison between proteins easier, I present it in a standardized form (Figure 13). For each protein, I briefly describe its known function, organization and interaction partners within this dataset, followed by representative microscopy images, together with the average distribution of the protein. Please note that the average image does only show the protein of interest. The violet color is not coming from mixing the homer signal in blue and the STED signal in red but is part of the used colormap. The color map extends from black over violet to red-white (Hunter, 2007).

Because the interpretation of the average images per se is difficult, and to extract more quantitative information about the localization of the protein, I performed an enrichment analysis. By analyzing each spine individually, I was also able to extract more detailed information on the protein localization and enrichment. This is presented as violin plots, which fits a frequency distribution to the data. The bold line indicates the median, the thin lines the lower and upper quartile respectively. The green plots correspond to Mushroom, the orange plots to Stubby class synapses.

The second page shows the copy number in the whole cell, the copy number in the whole spine or the PSD for both classes. I also calculated \%of total protein in the whole that a given protein constitutes and calculated the molarity. Finally, where available I show the Molecular model of a mushroom spine. 


\section{Proteinname (Synonyms, Gene name, Uniprot ID)}

Known functions within the postsynapse

Known organization, for example membrane-associated or not, multimerization if known

Known Interactions: Only interactions within this dataset are considered.

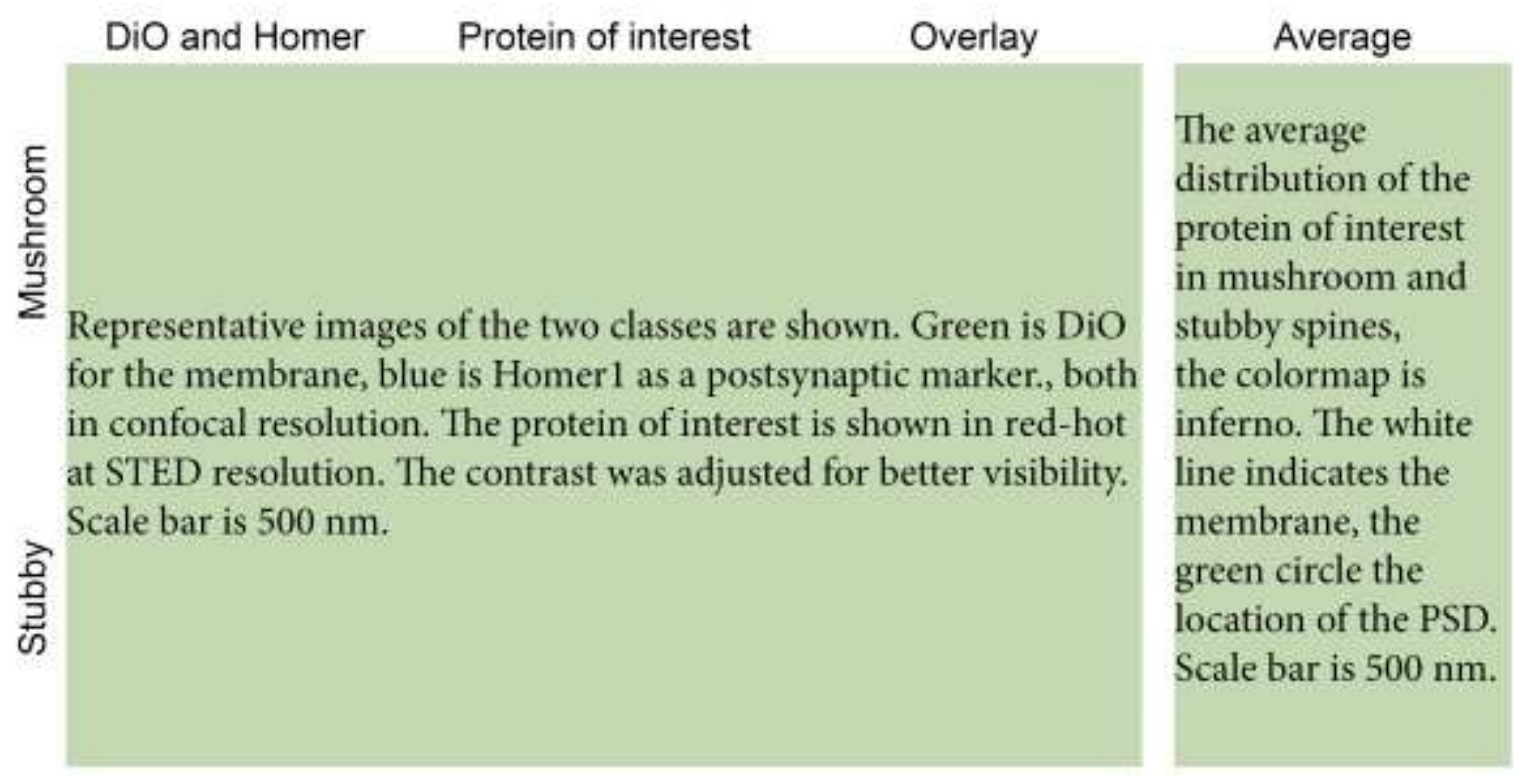

\section{Upper panel:}

The Enrichment of the protein across the different zones is depicted. It is calculated by dividing the fraction of signal from the protein of interest that falls into a given zone by the fraction of signal from the average of all investigated proteins that falls into the same zone. The zones corresponding to the PSD are highlighted in green, perisynaptic regions are yellow and extrasynaptic regions are red.

\section{Lower panel:}

Several parameteres were measured for each individual spine. The distribution was fitted with a kernel and is presented in a violin plot. The thick line indicates the median, the dotted lines the upper and lower quartiles. The number above the plot shows the median. Distance to membrane: The distance of every spot to its closest membrane is measured. Distance to PSD: The distance of spots to the PSD is measured. Only spots within the head/synaptic regions for stubby, are considered here.

Enrichment in head: The fluorescence signal within the head is averaged and compared to the average fluorescence signal in the shaft. For each spine an individual rectangle was drawn on the shaft, which is used here.

Enrichment in PSD: Same as enrichment in PSD, but the signal in the PSD, determined by Homerl FWHM signal, is calculated.

Eccentricity: The position of all spots in the head on the top-bottom axis is determined. This is normalized, where 1 is the top of the head, -1 the bottom of the head.

Laterality: The position of all spots in the head on the left-right axis is determined. The position is normalized, where 0 is the center of the spine, 1 is the most left/right position. Diameter: To each spot in the head an ellipse is fitted, the length of the major axis is shown. Distribution: For every spot in the head, the distance of this spot to all other spots in the head is calculated. 
A representative epifluorescence image of the protein is shown. Blue is Homer 1, red-hot is the protein of interest. For better visibility the contrast was adjusted.

Scale bar is $5 \mu \mathrm{m}$.

\begin{tabular}{|c|c|}
\hline $\begin{array}{c}\text { Whole cell copy } \\
\text { number }\end{array}$ & Mean \pm SEM \\
\hline $\begin{array}{c}\text { Spine copy } \\
\text { number }\end{array}$ & Mean \pm SEM \\
\hline$\%$ in PSD & Mean \\
\hline PSD copy number & Mean \pm SEM \\
\hline
\end{tabular}

\begin{tabular}{|c|c|c|}
\hline $\begin{array}{c}\text { Spine copy } \\
\text { number }\end{array}$ & Mushroom & Stubby \\
\hline PSD copy number & Mean \pm SEM & Mean \pm SEM \\
\hline$\%$ synaptic protein & Mean \pm SEM & Mean \pm SEM \\
\hline Molarity $[\mu \mathrm{M}]$ & Mean \pm SEM & Mean \pm SEM \\
\hline
\end{tabular}

Mushroom 3D model

The atomic

structure of the protein.

Where available, a preliminary 3D Model of mushroom spines is shown. Proteins were placed according to the calculated spine copy number and their average distribution.

\section{References}

Antibody: Manufacturer and Catalogue number

PDB Identifier: PDB entries used for the construction of the atomic model 


\section{Cytoskeleton and associated proteins}

The cytoskeleton fulfills many functions in the dendrite and spines. In the following section, I analyze the main components of the dendritic spine and shaft cytoskeleton.

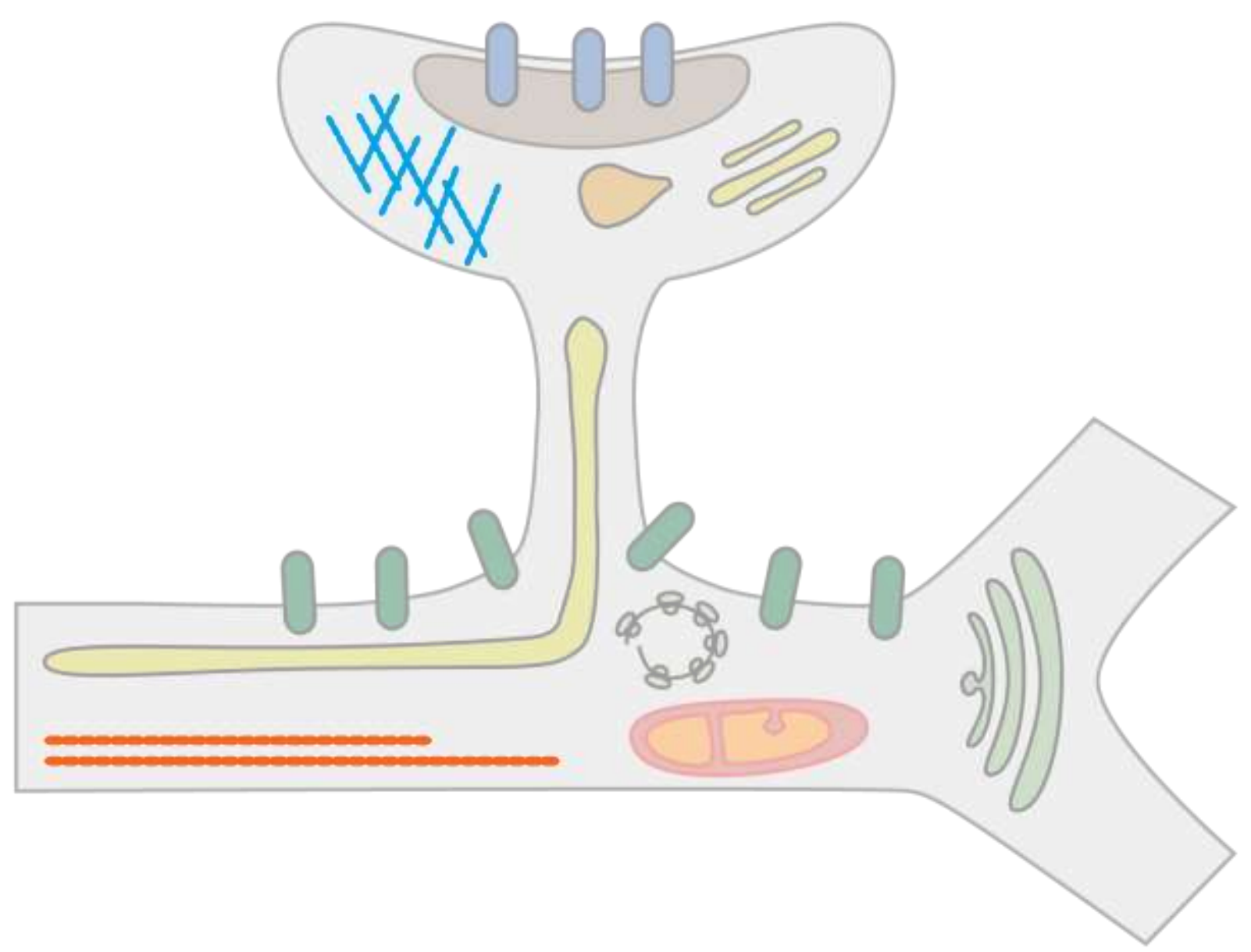

Figure 14: Depiction of the cytoskeleton in dendritic spines. 
Actin (Genes: Actg1, Actb, Actc1, Uniprot ID: P63259, P60711, P68035)

Known function: Shapes spine morphology, serves as scaffold for transport

Known organization: Cytosolic, forms filaments, rings in neck and shaft

Known Interactions: $\beta$-2-spectrin
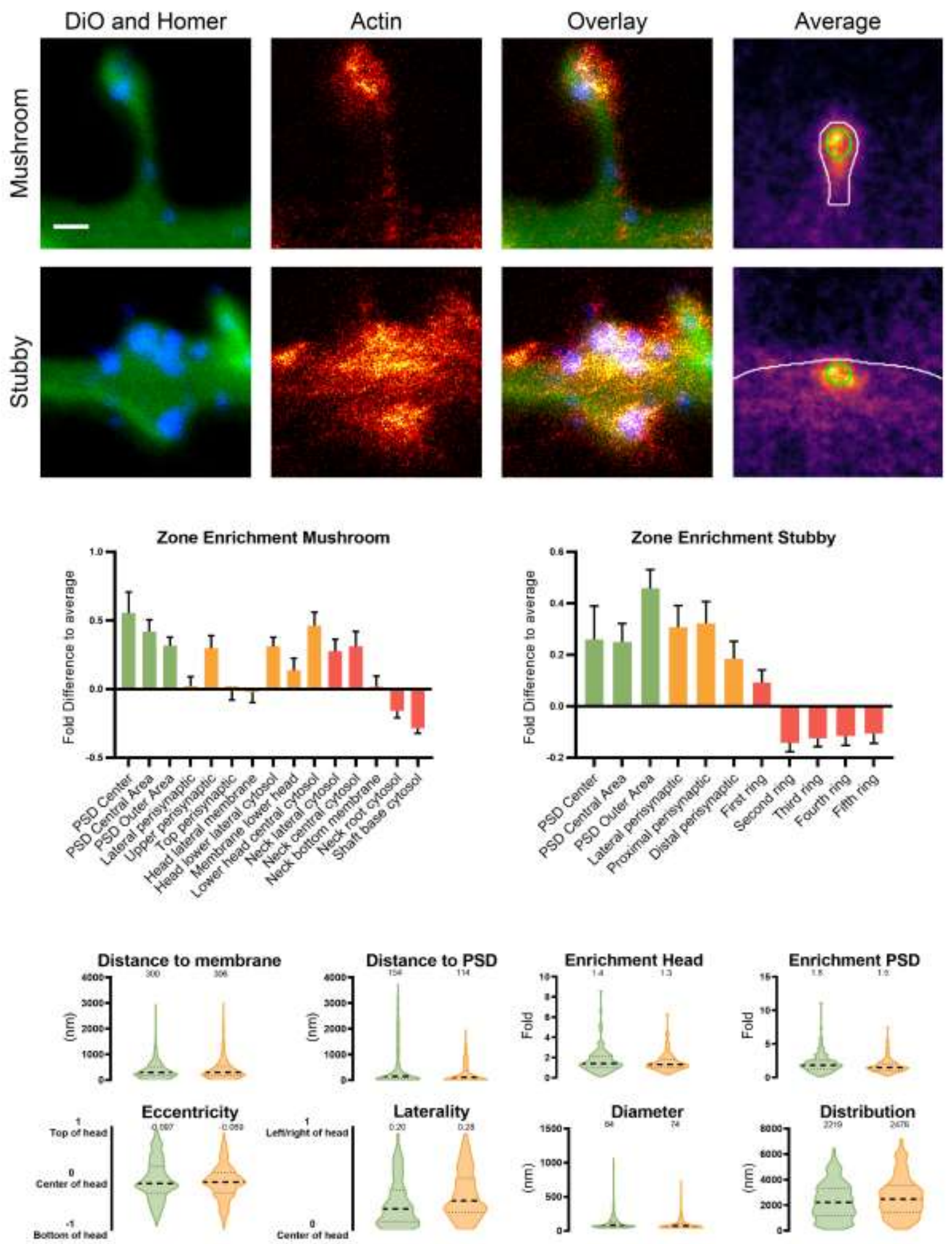

Figure 15: Actin nanoscale localization and abundance. Continued on next page. 


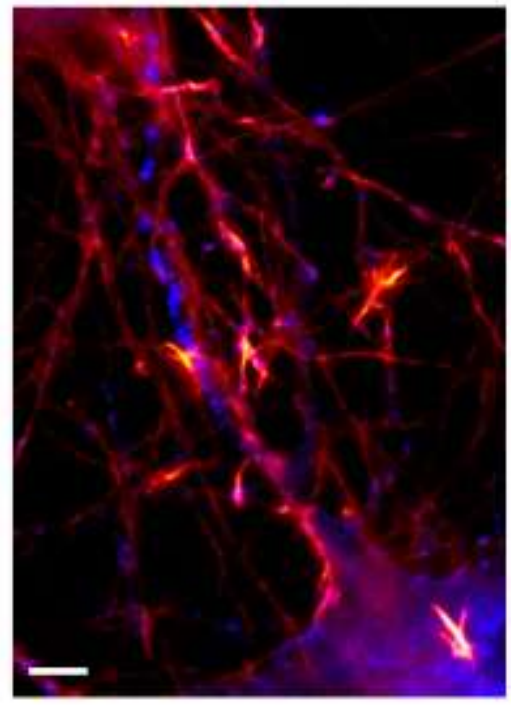

\begin{tabular}{|c|c|c|}
\hline $\begin{array}{c}\text { Whole cell copy } \\
\text { number }\end{array}$ & \multicolumn{2}{|c|}{$85789276.02 \pm 10990929.13$} \\
\hline $\begin{array}{c}\text { Spine copy } \\
\text { number }\end{array}$ & \multicolumn{2}{|c|}{$38100.16 \pm 6546.86$} \\
\hline$\%$ in PSD & \multicolumn{2}{|c|}{29.90} \\
\hline PSD copy number & \multicolumn{2}{|c|}{$8769.50 \pm 1506.89$} \\
\hline & Mushroom & Stubby \\
\hline \begin{tabular}{c|c|} 
Spine copy \\
number
\end{tabular} & $\begin{array}{c}42259.40 \\
\pm 7261.55\end{array}$ & $\begin{array}{c}32375.14 \\
\pm 5563.11\end{array}$ \\
\hline PSD copy number & $\begin{array}{c}9726.83 \\
\pm 1671.39\end{array}$ & \begin{tabular}{c}
7451.77 \\
\hline 1280.46
\end{tabular} \\
\hline$\%$ of protein & $13.03 \pm 2.24 \%$ & $6.40 \pm 1.10 \%$ \\
\hline Molarity $[\mu \mathrm{M}]$ & $354.19 \pm 60.86$ & $173.56 \pm 29.82$ \\
\hline
\end{tabular}

\section{References}

Phalloidin Sigma Aldrich 65906

PDB Identifier: $1 j 6 z$

\section{Literature:}

Bär et al., 2016; Chazeau and Giannone, 2016; D’Este et al., 2015, 2016; Sidenstein et al., 2016; Xu et al., 2013 


\section{a-Internexin (Gene: Ina , Uniprot ID: P23565)}

Known function: Part of the neurofilament filament

Known organization: Cytosolic

Known Interactions: Neurofilaments, Tubulins
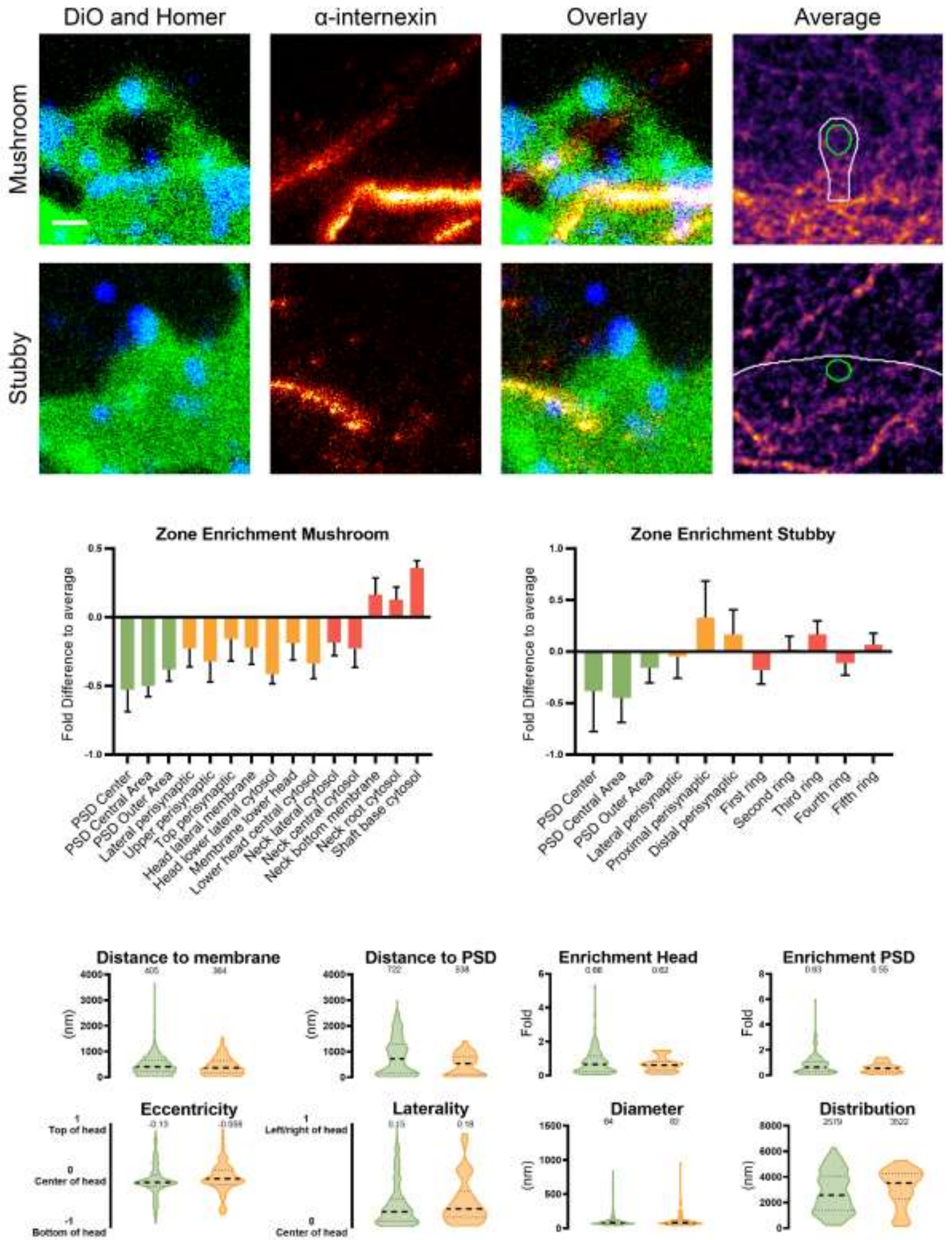

Figure 16: $\alpha$-Internexin nanoscale localization and abundance. Continued on next page. 


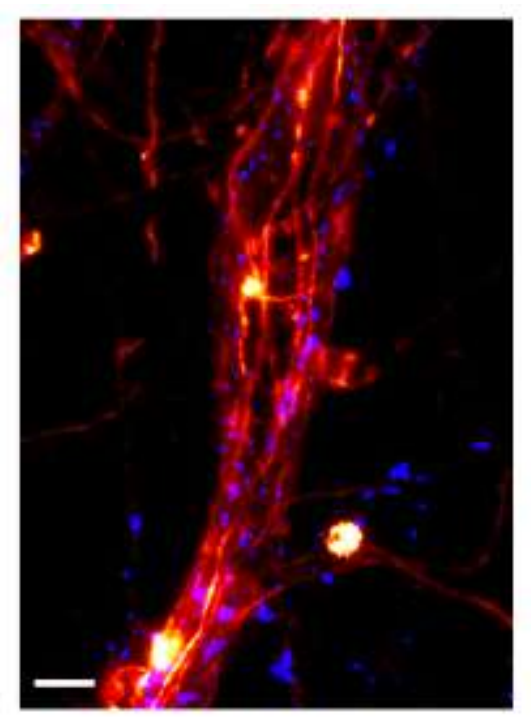

\begin{tabular}{|c|c|c|}
\hline $\begin{array}{c}\text { Whole cell copy } \\
\text { number }\end{array}$ & \multicolumn{2}{|c|}{$20247463.61 \pm 2146256.32$} \\
\hline $\begin{array}{c}\text { Spine copy } \\
\text { number }\end{array}$ & \multicolumn{2}{|c|}{$1045.72 \pm 1166.78$} \\
\hline$\%$ in PSD & \multicolumn{2}{|c|}{7.72} \\
\hline PSD copy number & \multicolumn{2}{|c|}{$74.98 \pm 83.66$} \\
\hline & Mushroom & Stubby \\
\hline \begin{tabular}{c|c|} 
Spine copy \\
number
\end{tabular} & $\begin{array}{c}1090.05 \\
71216.24\end{array}$ & \pm 867.77 \\
\hline PSD copy number & $78.16 \pm 87.21$ & $55.76 \pm 62.22$ \\
\hline$\%$ of protein & $0.14 \pm 0.16 \%$ & $0.06 \pm 0.07 \%$ \\
\hline Molarity $[\mu \mathrm{M}]$ & $9.14 \pm 10.19$ & $4.17 \pm 4.65$ \\
\hline
\end{tabular}

\section{References}

Antibody: LS Bio LS-B10413

PDB Identifier: modified NF-H

\section{Literature:}

Suzuki et al., 1997, 2018, Yuan et al., 2006, 2015b, 2015a 


\section{$\beta$-2-spectrin ( $\beta$-3-spectrin, Gene: Sptnb2, Uniprot ID: Q9QWN8)}

Known function: Acts as spacer for actin rings

Known organization: Cytosolic

Known Interactions: Actin
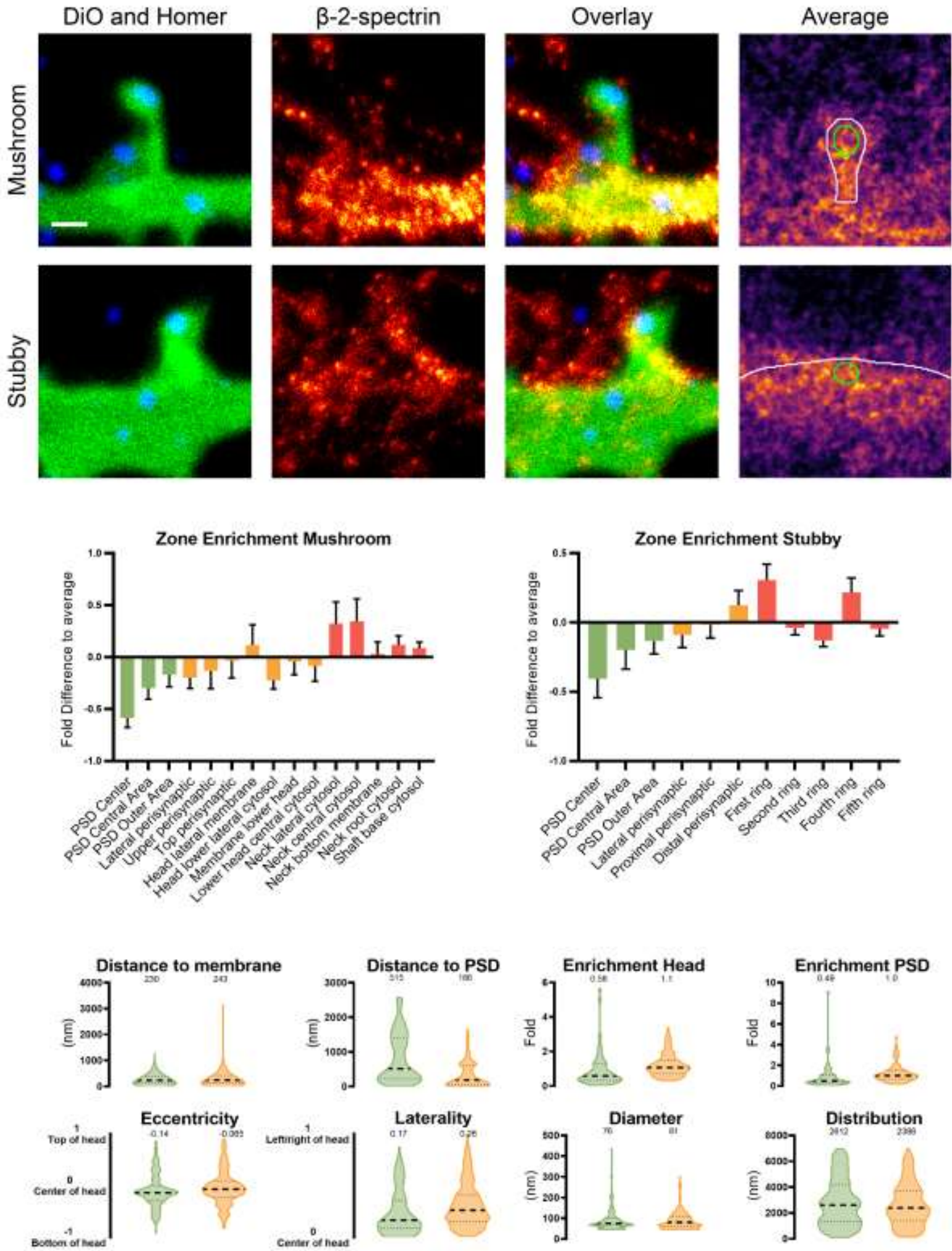

Figure 17: $\beta-2-s p e c t r i n$ nanoscale localization and abundance. Continued on next page. 


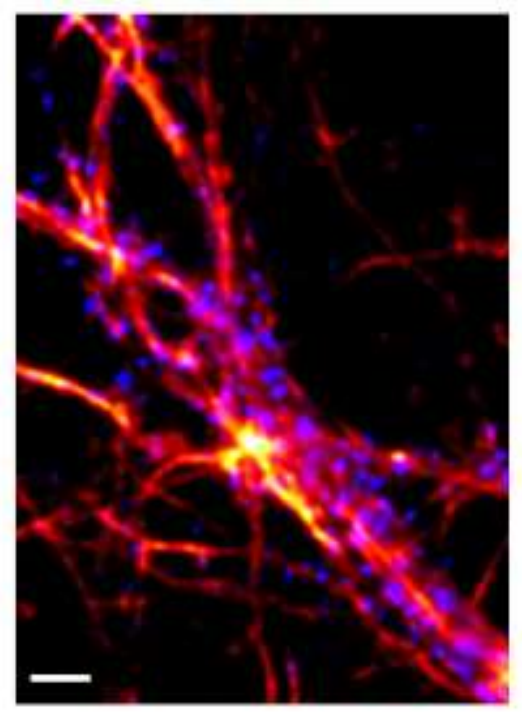

\begin{tabular}{|c|c|c|}
\hline $\begin{array}{c}\text { Whole cell copy } \\
\text { number }\end{array}$ & \multicolumn{2}{|c|}{$1169335.35 \pm 234131.07$} \\
\hline $\begin{array}{c}\text { Spine copy } \\
\text { number }\end{array}$ & \multicolumn{2}{|c|}{$32.79 \pm 43.22$} \\
\hline$\%$ in PSD & \multicolumn{2}{|c|}{14.56} \\
\hline PSD copy number & \multicolumn{2}{|c|}{$4.17 \pm 5.49$} \\
\hline & Mushroom & Stubby \\
\hline $\begin{array}{c}\text { Spine copy } \\
\text { number }\end{array}$ & $30.41 \pm 40.08$ & $36.11 \pm 47.58$ \\
\hline PSD copy number & $3.87 \pm 5.09$ & $4.59 \pm 6.05$ \\
\hline$\%$ of protein & $0.02 \pm 0.03 \%$ & $0.01 \pm 0.02 \%$ \\
\hline Molarity $[\mu \mathrm{M}]$ & $0.25 \pm 0.34$ & $0.19 \pm 0.26$ \\
\hline
\end{tabular}

\section{References}

Antibody: BD Biosciences 612562

PDB Identifier: 1s35

\section{Literature:}

Bär et al., 2016; Sidenstein et al., 2016; Xu et al., 2013 


\section{$\beta$-3-tubulin (Gene: Tubb3, Uniprot ID: Q4QRB4)}

Known function: Forms microtubules

Known organization: Cytosolic, Forms hollow microtubule, Neuron specific

Known Interactions: $\alpha$-tubulins, $\alpha$-internexin
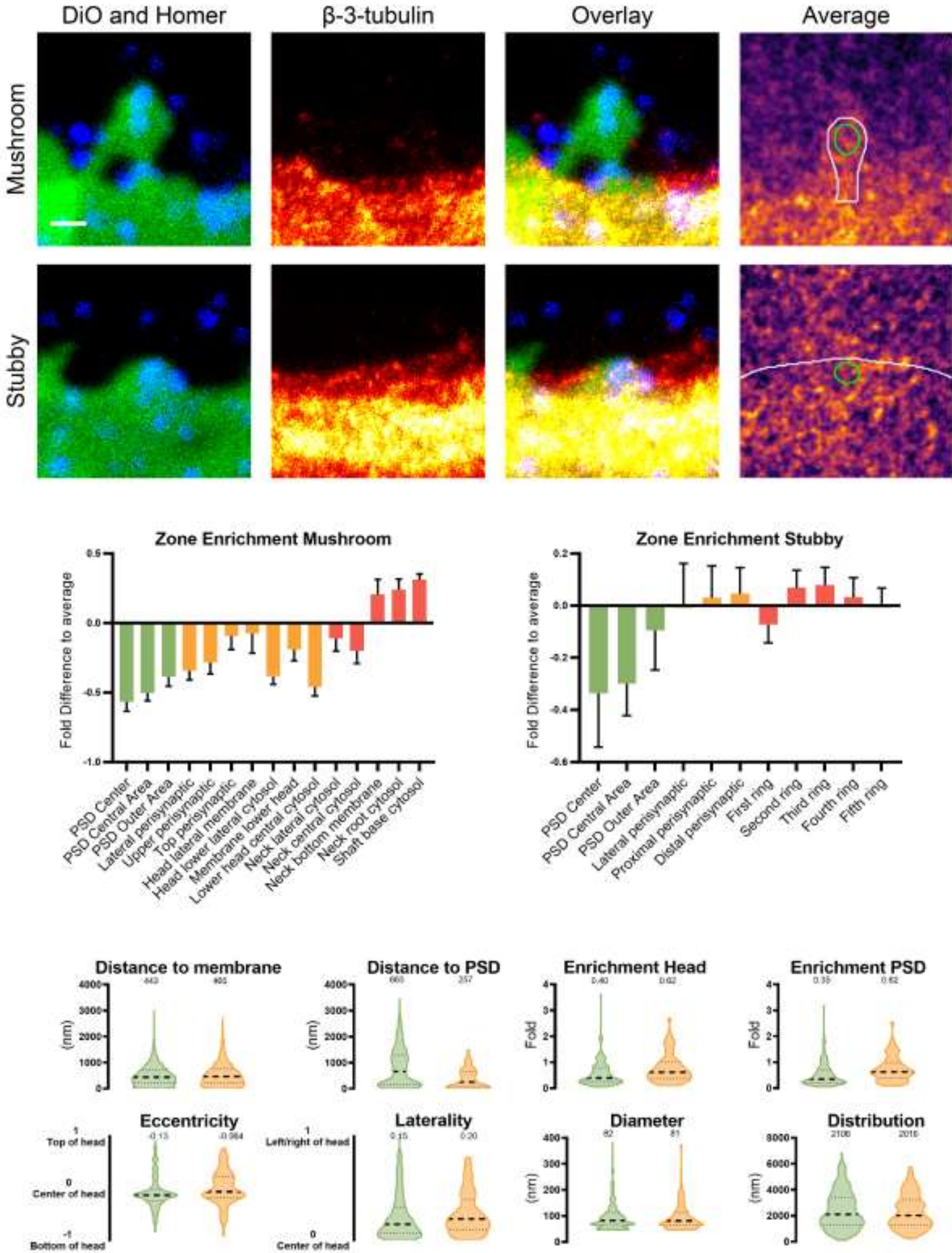

Figure 18: $\beta 3$-tubulin nanoscale localization and abundance. Continued on next page. 


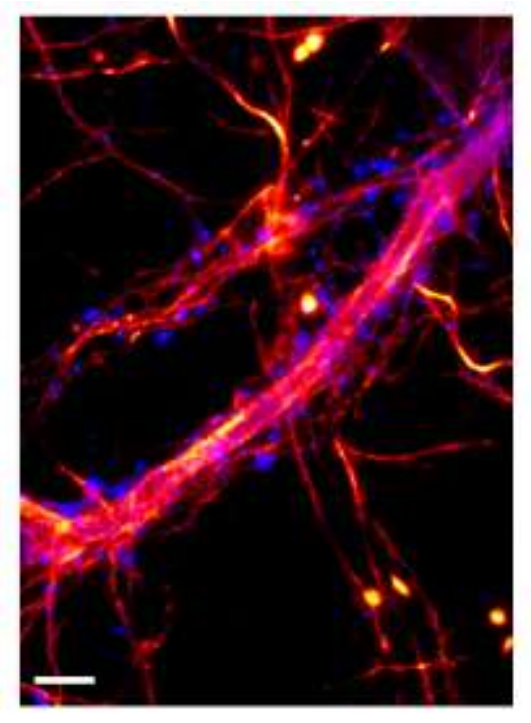

\begin{tabular}{|c|c|c|}
\hline $\begin{array}{c}\text { Whole cell copy } \\
\text { number }\end{array}$ & \multicolumn{2}{|c|}{$135945734.28 \pm 9810384.32$} \\
\hline $\begin{array}{c}\text { Spine copy } \\
\text { number }\end{array}$ & \multicolumn{2}{|c|}{ not detected } \\
\hline$\%$ in PSD & \multicolumn{2}{|c|}{ na } \\
\hline PSD copy number & \multicolumn{2}{|c|}{ na } \\
\hline \begin{tabular}{c|c|} 
Spine copy \\
number
\end{tabular} & na & na \\
\hline PSD copy number & na & na \\
\hline$\%$ of protein & na & na \\
\hline Molarity $[\mu M]$ & na & na \\
\hline
\end{tabular}

\section{References}

Antibody: Cell Signaling 5568

PDB Identifier: $6 \mathrm{e} 7 \mathrm{~b}$

\section{Literature:}

Jaworski et al., 2009; Kaech et al., 2001; Landis and Reese, 1983; Sullivan and Cleveland, 1986 
pan- $\beta$-tubulin (Genes: Tubb1, Tubb2a, Tubb2b, Tubb3, Tubb4a, Tubb4b, Tubb5, Tubb6, Uniprot IDs: M0R8B6, P85108, Q3KRE8, Q4QRB4, B4F7C2, Q6P9T8, P69897, Q4QQV0)

Known function: Forms microtubules

Known organization: Cytosolic, Forms hollow microtubule

Known Interactions: $\alpha$-tubulins, $\alpha$-internexin
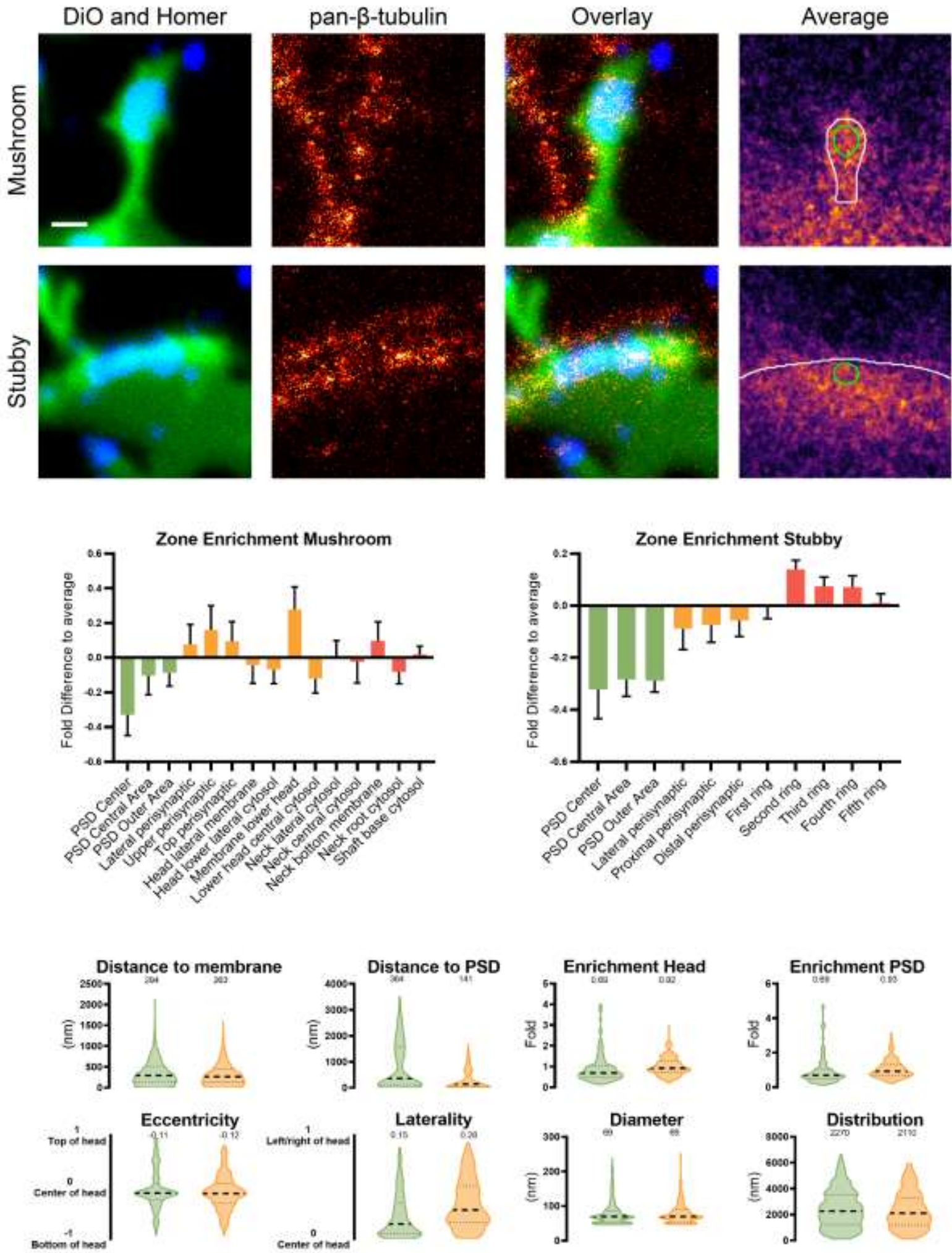

Figure 19:pan- $\beta$-tubulin nanoscale localization and abundance. Continued on next page. 


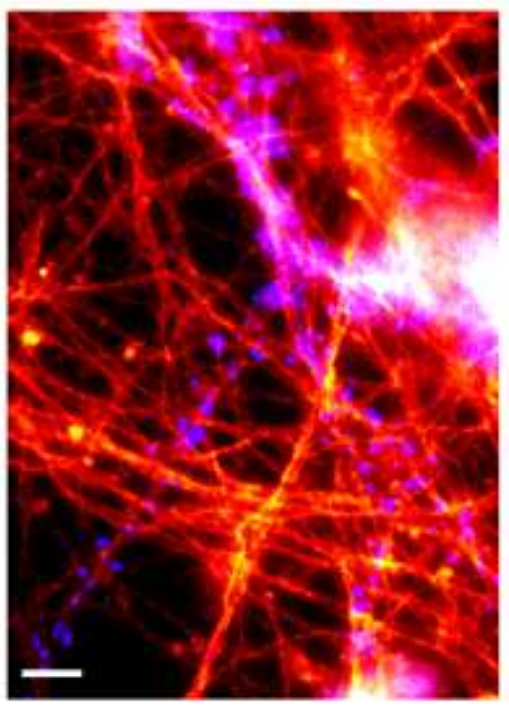

\begin{tabular}{|c|}
$\begin{array}{c}\text { Whole cell copy } \\
\text { number }\end{array}$ \\
\hline $\begin{array}{c}\text { Spine copy } \\
\text { number }\end{array}$ \\
\hline$\%$ in PSD \\
\hline PSD copy number
\end{tabular}

$1265162945.93 \pm 173906492.85$

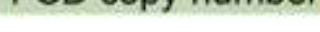

\begin{tabular}{|c|}
\hline $103699.83 \pm 45668.09$ \\
\hline 14.27 \\
\hline $12949.43 \pm 5702.76$ \\
\hline
\end{tabular}

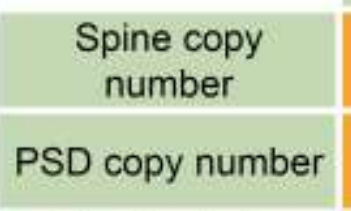

\begin{tabular}{|c|c|}
\hline Mushroom & Stubby \\
\hline $\begin{array}{c}77541.46 \\
\pm 34148.28\end{array}$ & $\begin{array}{l}135616.59 \\
59723.83\end{array}$ \\
\hline 9682.92 & 16935.01 \\
\pm 4264.24 & \pm 7457.96 \\
\hline $64.62 \pm 28.46 \%$ & $72.45 \pm 31.91 \%$ \\
\hline $649.90 \pm 286.21$ & $727.03 \pm 320.17$ \\
\hline
\end{tabular}

Mushroom molecular model

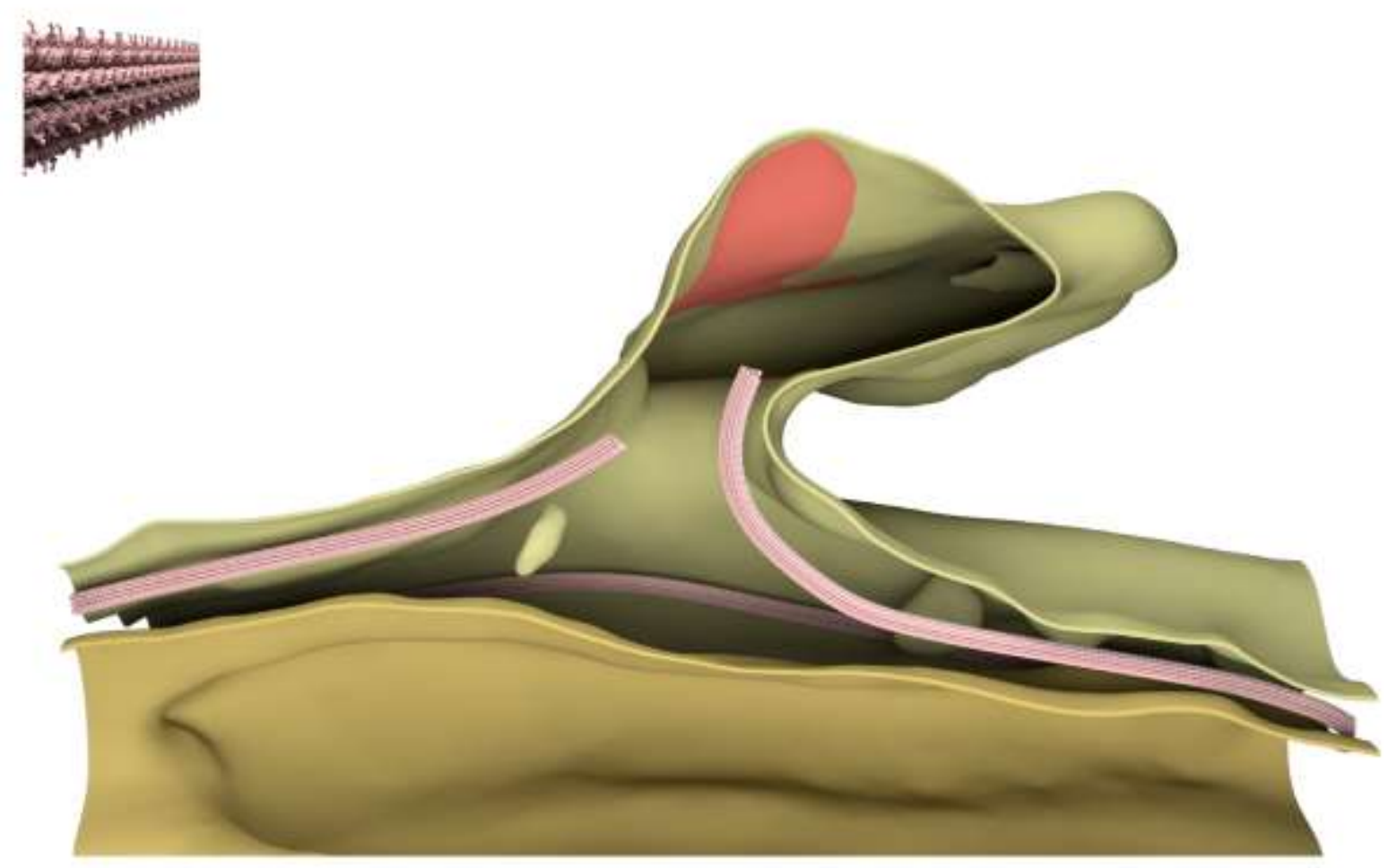

\section{References}

Antibody: Self-made nanobody (Mikhalyova et al. 2015) PDB Identifier: 1tub Literature:

Jaworski et al., 2009; Kaech et al., 2001; Landis and Reese, 1983 


\section{Cortactin (Gene: Cttn, Uniprot ID: Q66HL2)}

Known function: Branching and Stabilization of Actin, Involved in PSD95 clustering Known organization: Cytosolic

Known Interactions: Actin, Shank proteins, PSD95
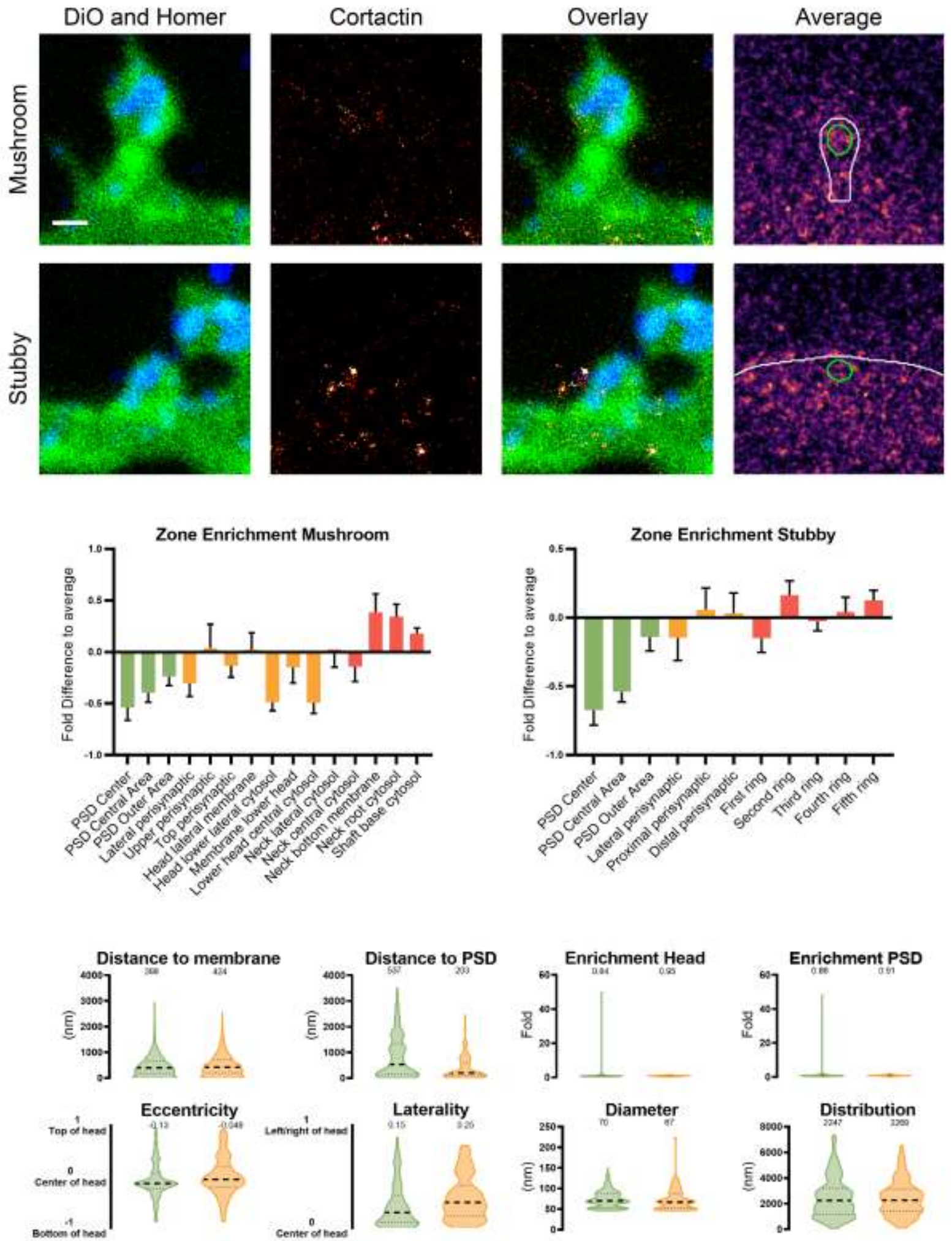

Figure 20: Cortactin nanoscale localization and abundance. Continued on next page. 

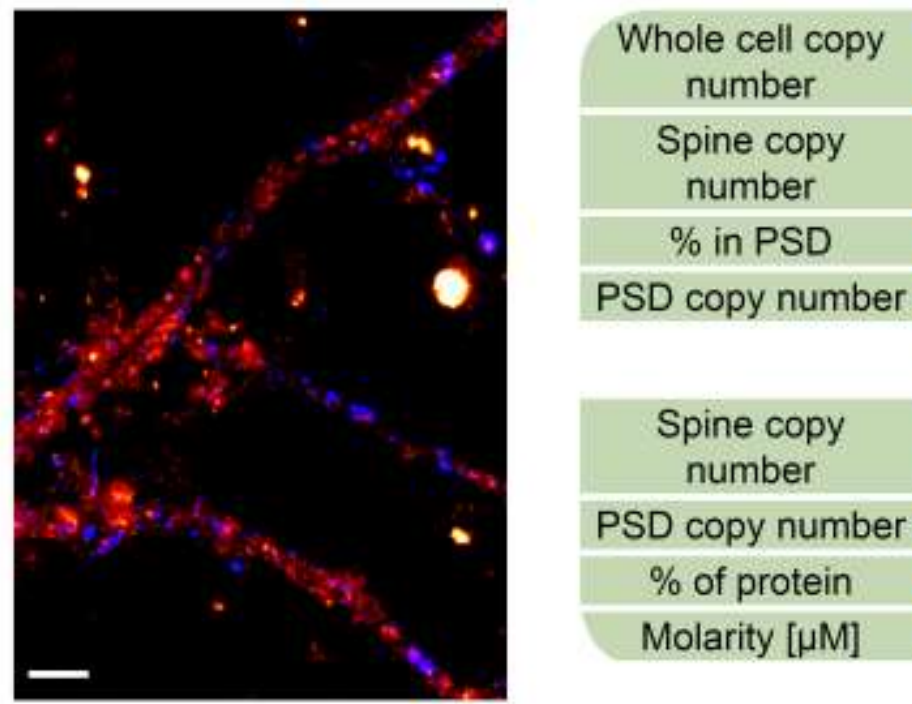

$4501573.83 \pm 264697.54$

number

$39 \pm 21.29$

\begin{tabular}{|c|c|c|}
\hline $\begin{array}{c}\text { Spine copy } \\
\text { number }\end{array}$ & $322.71 \pm 128.71$ & $601.58 \pm 239.93$ \\
\hline PSD copy number & $42.61 \pm 16.99$ & $79.43 \pm 31.68$ \\
\hline$\%$ of protein & $0.04 \pm 0.02 \%$ & $0.05 \pm 0.02$ \\
\hline Molarity $[\mu \mathrm{M}]$ & $2.70 \pm 1.08$ & $3.23 \pm 1.29$ \\
\hline
\end{tabular}

Mushroom molecular model

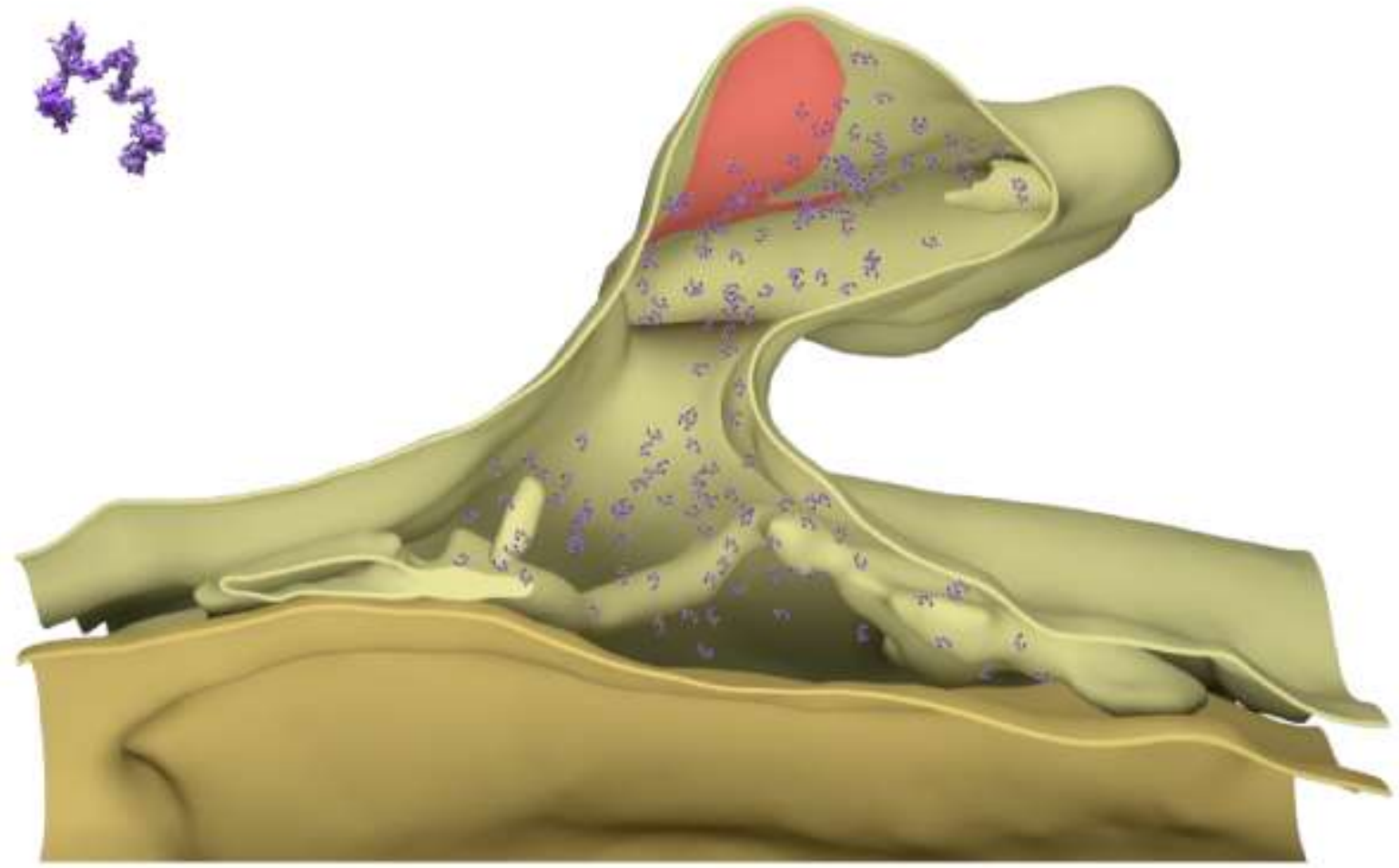

\section{References}

Antibody: Synaptic Systems 313111

PDB Identifier: 3ulr, 2d1x

\section{Literature:}

Catarino et al., 2013; Du et al., 1998; MacGillavry et al., 2016; Naisbitt et al., 1999; Ren et al., 2009; Rostaing et al., 2006; Wu and Parsons, 1993 


\section{Drebrin (Gene: Dbn1, Uniprot ID: Q07266)}

Known function: Stabilizes Actin, Regulates microtubule entry into spines

Known organization: Cytosolic

Known Interactions: Actin
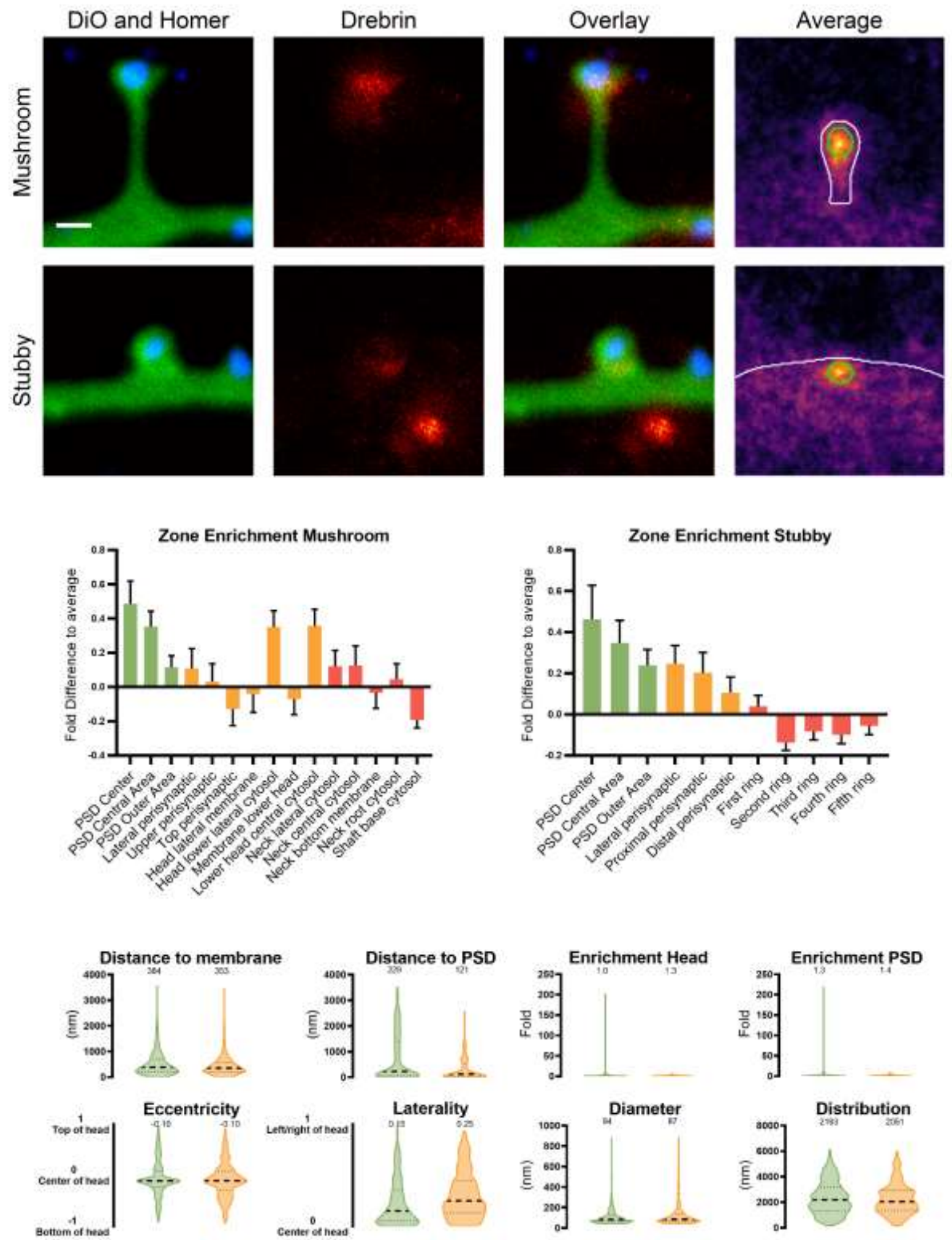

Figure 21: Drebrin nanoscale localization and abundance. Continued on next page. 


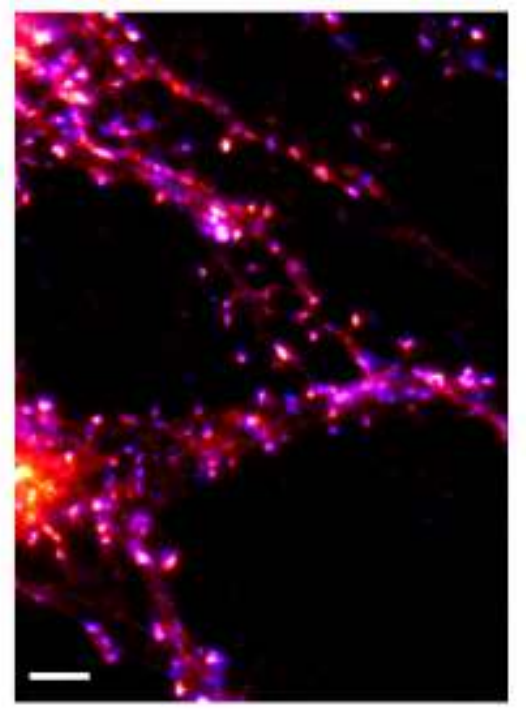

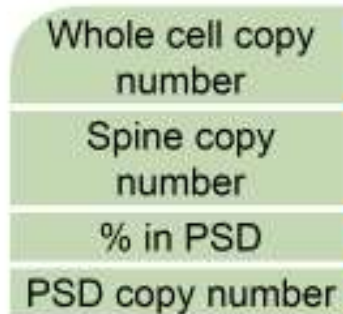

\begin{tabular}{|c|}
\hline $7638873.40 \pm 728504.99$ \\
\hline $6790.52 \pm 991.60$ \\
\hline 26.82 \\
\hline $1435.93 \pm 209.68$ \\
\hline
\end{tabular}

\begin{tabular}{|c|c|c|}
\hline & Mushroom & Stubby \\
\hline $\begin{array}{c}\text { Spine copy } \\
\text { number }\end{array}$ & $\begin{array}{c}\text { 6709.58 } \\
\pm 979.78\end{array}$ & $\begin{array}{c}7124.02 \\
\pm 1040.30\end{array}$ \\
\hline PSD copy number & 1418.81 & 1506.45 \\
\pm 207.18 & \pm 219.98 \\
\hline$\%$ of protein & $1.20 \pm 0.18 \%$ & $0.82 \pm 0.12 \%$ \\
\hline Molarity $[\mu \mathrm{M}]$ & $56.23 \pm 8.21$ & $38.19 \pm 5.58$ \\
\hline
\end{tabular}

Mushroom molecular model

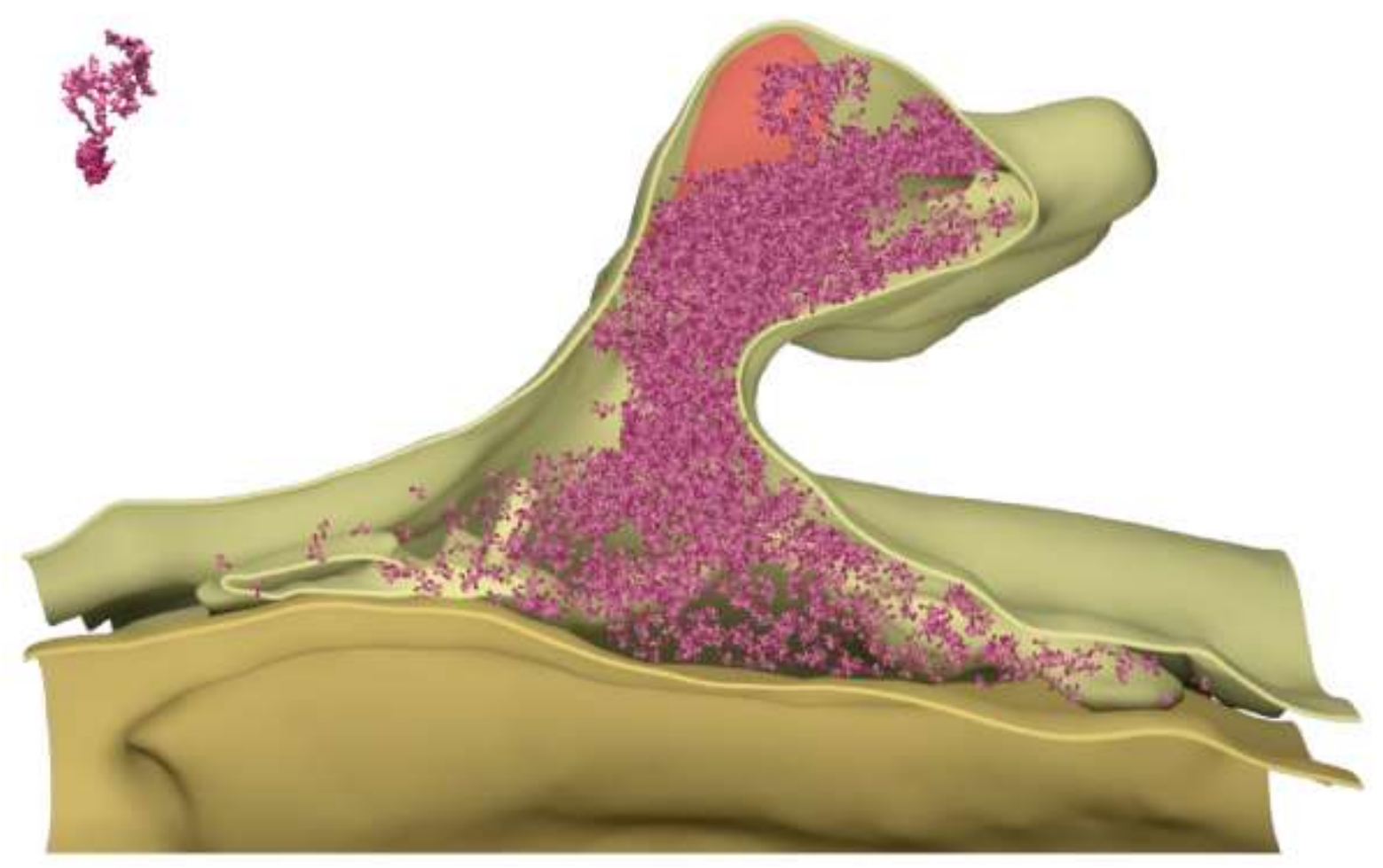

\section{References}

Antibody: Novus Biologicals NB100-1951

PDB Identifier: $5 y 1 z$

\section{Literature:}

Hayashi et al., 1996; Ishikawa et al., 1994; Merriam et al., 2013; Mikati et al., 2013 


\section{MAP2 (Gene: Map2, Uniprot ID: P15146)}

Known function: Stabilizes microtubules, Crosslinks microtubules with neurofilaments Known organization: Cytosolic

Known Interactions: Microtubules, Neurofilaments
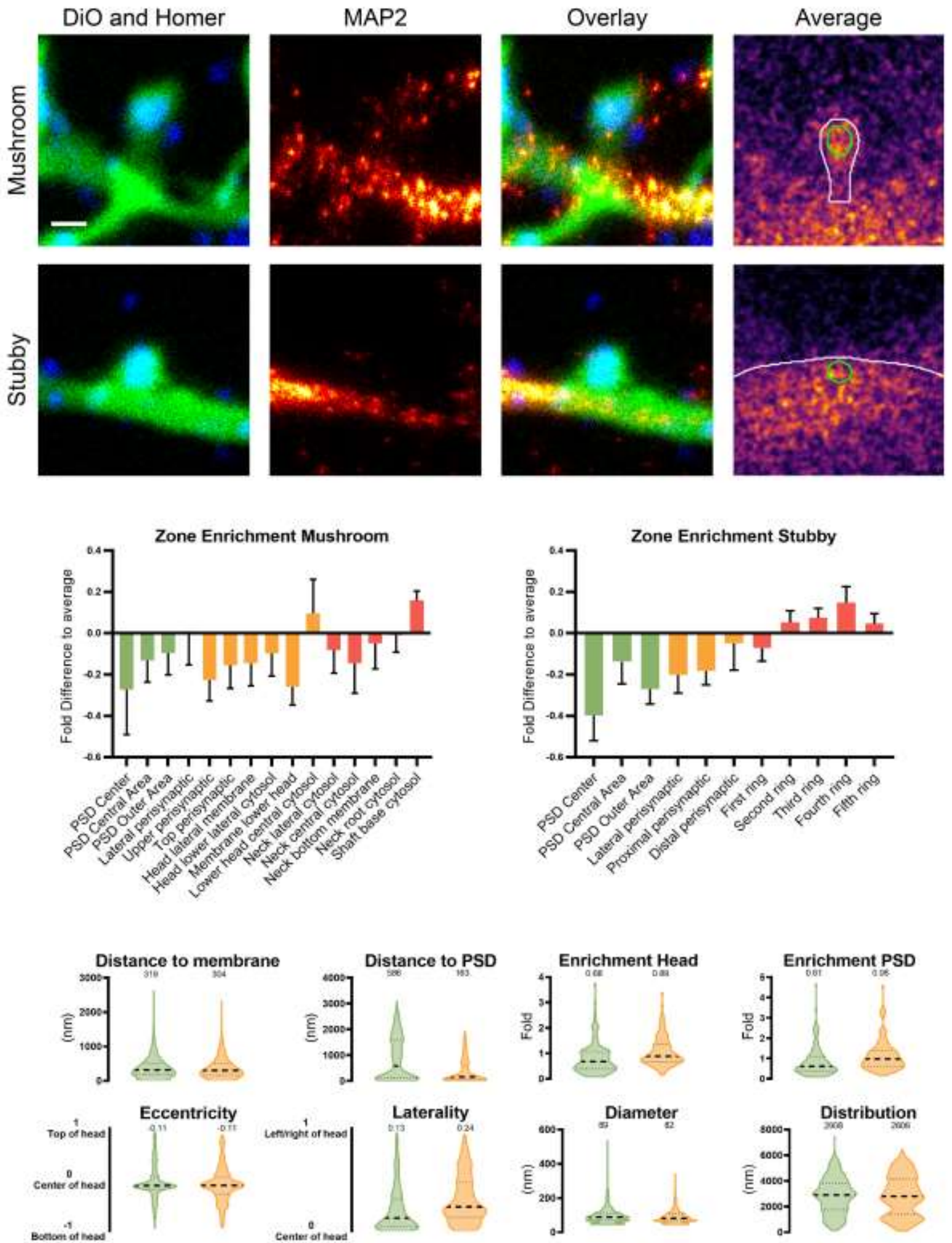

Figure 22: MAP2 nanoscale localization and abundance. Continued on next page. 


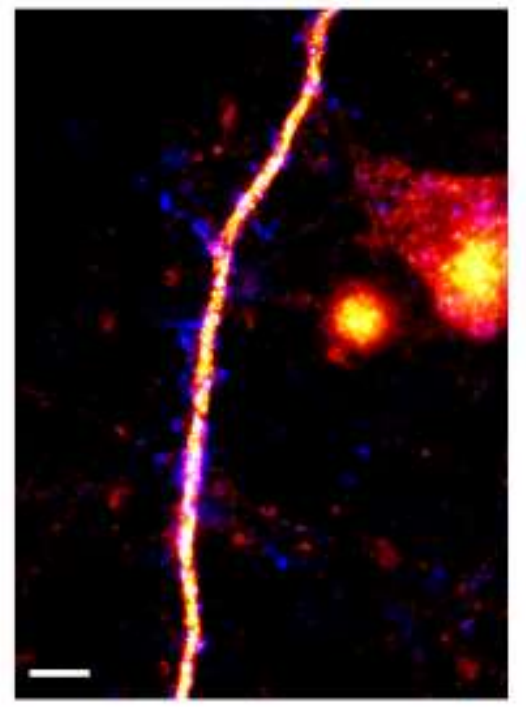

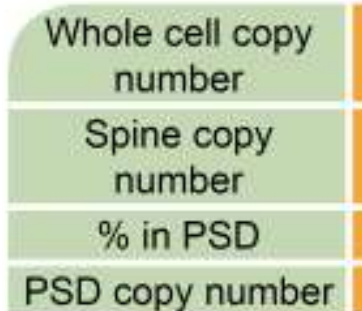

Spine copy number

PSD copy number

$\%$ of protein

Molarity [ $\mu \mathrm{M}]$
$2724287.06 \pm 305050.06$

$$
679.88 \pm 162.65
$$

14.42

$85.71 \pm 20.50$

\begin{tabular}{|c|c|}
\hline Mushroom & Stubby \\
\hline $481.55 \pm 115.20$ & $929.61 \pm 222.39$ \\
\hline $60.71 \pm 14.52$ & $117.19 \pm 28.03$ \\
\hline $0.22 \pm 0.05 \%$ & $0.27 \pm 0.07 \%$ \\
\hline $4.04 \pm 0.97$ & $4.98 \pm 1.19$ \\
\hline
\end{tabular}

Mushroom molecular model

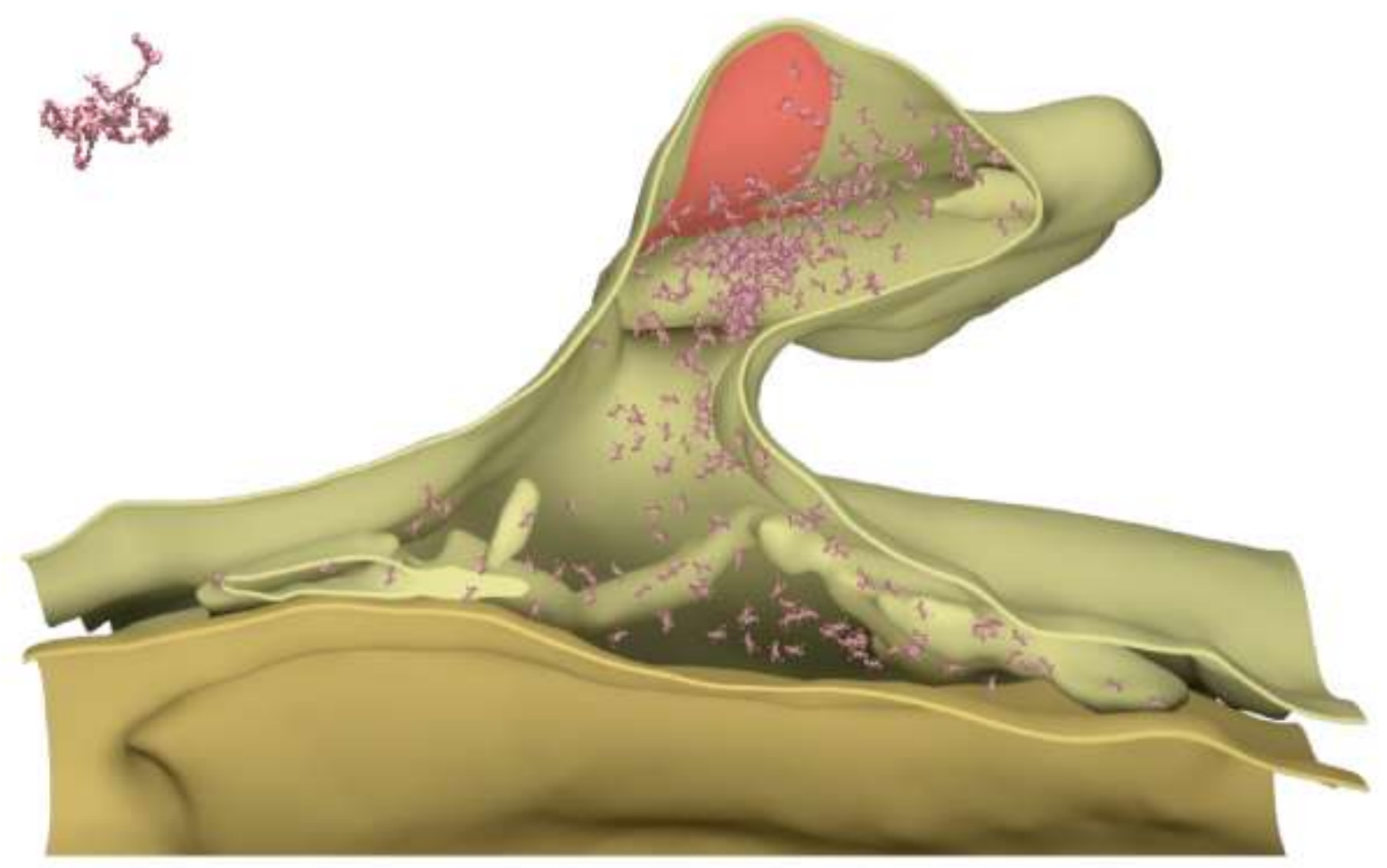

\section{References}

Antibody: Synaptic Systems 188002

PDB Identifier: $6 \mathrm{cvn}$

\section{Literature:}

Farah and Leclerc, 2008; Frappier et al., 1991; Hirokawa et al., 1996 


\section{Myosin5a (Gene: Myo5a, Uniprot ID: Q9QYF3)}

Known function: Transport of GluR1 and GluR2 during LTP, Regulates ER localization, Transport of various other cargos

Known organization: Cytosolic

Known Interactions: Actin
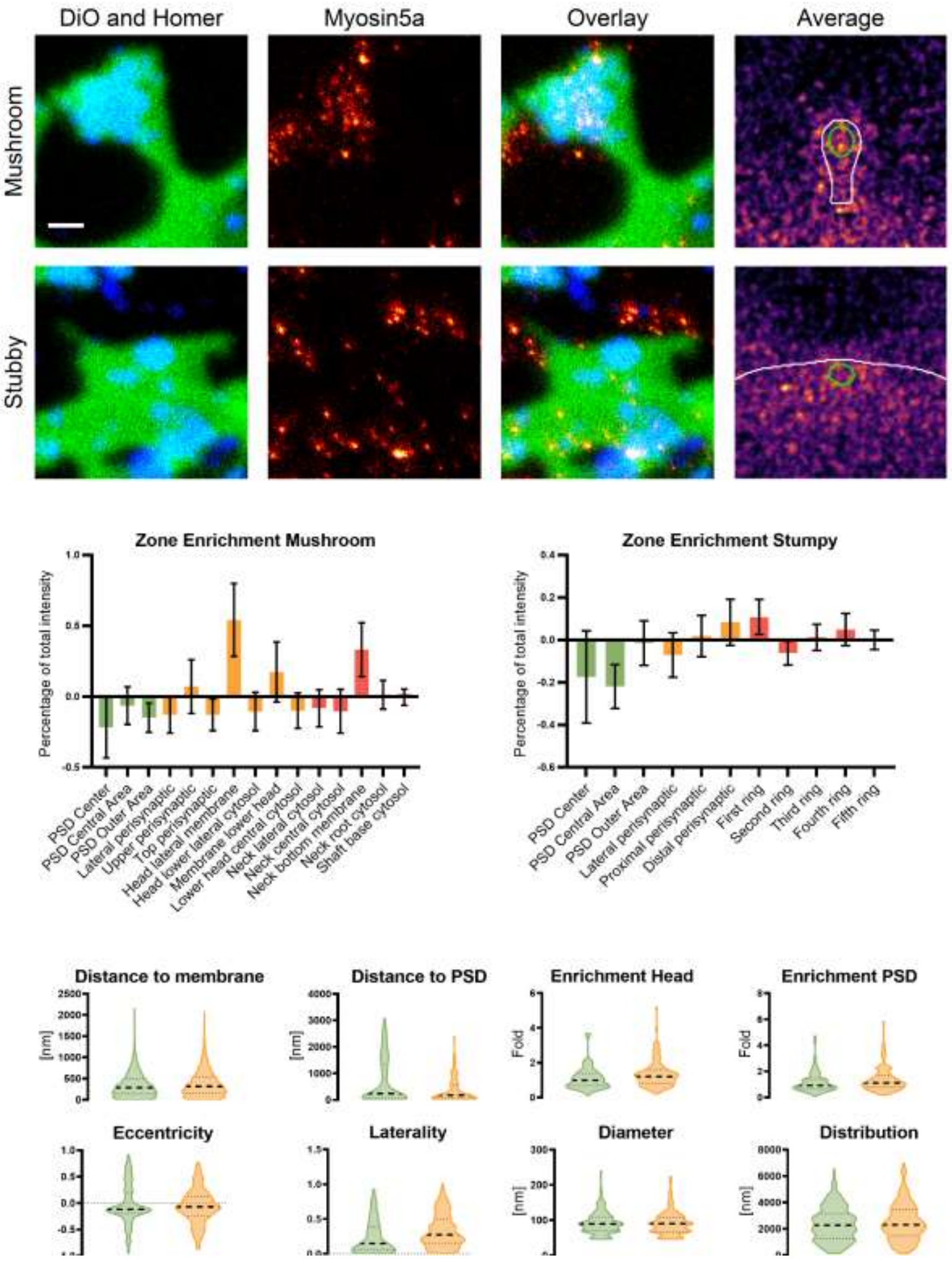

Figure 23: Myosin5a nanoscale localization and abundance. Continued on next page. 


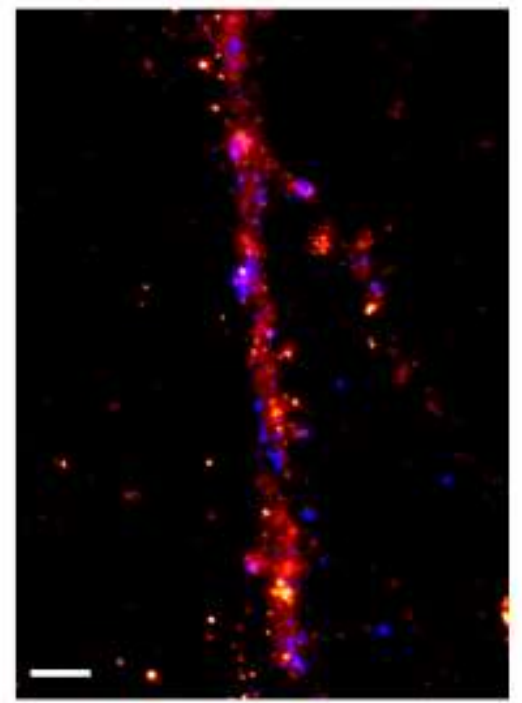

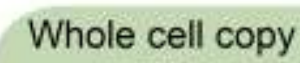
number

Spine copy
number
$\%$ in PSD

PSD copy number

Spine copy
number

PSD copy number

$\%$ of protein

Molarity [ $\mu \mathrm{M}]$

\begin{tabular}{|c|}
\hline $1525288.86 \pm 164897.19$ \\
\hline $3809.79 \pm 3109.92$ \\
\hline 16.78 \\
\hline $547.38 \pm 446.82$ \\
\hline
\end{tabular}

\begin{tabular}{|c|c|}
\hline Mushroom & Stubby \\
\hline $\begin{array}{c}2544.50 \\
\pm 2077.06\end{array}$ & $\begin{array}{c}5517.38 \\
\pm 4503.82\end{array}$ \\
\hline $365.58 \pm 298.43$ & $792.72 \pm 647.09$ \\
\hline $1.24 \pm 1.02 \%$ & $1.73 \pm 1.41 \%$ \\
\hline $21,33 \pm 17.41$ & $29.58 \pm 24.1$ \\
\hline
\end{tabular}

Mushroom molecular model

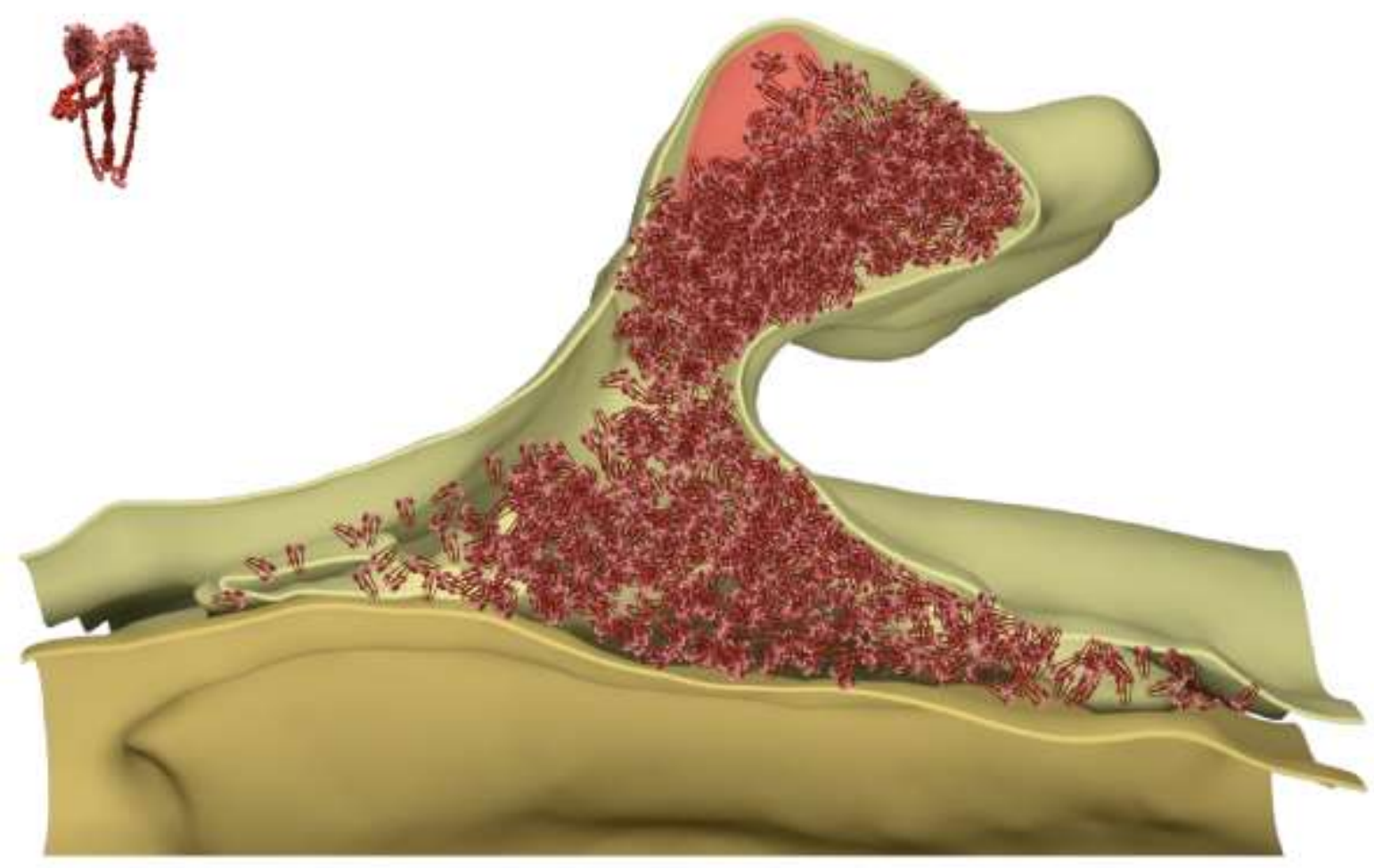

\section{References}

Antibody: Sigma-Aldrich M5062

PDB Identifier: 2 dfs

\section{Literature:}

Correia et al., 2008; Lisé et al., 2006; Miyata et al., 2000; Rudolf et al., 2011 


\section{Neurofilament H (Gene: Nefh, Uniprot ID: P16884)}

Known function: Forms neurofilaments, Regulates ER and Endosome location Known organization: Cytosolic, Forms triplet with Neurofilament $L$ and $M$

Known Interactions: Neurofilament $L$ and $M, \alpha$-internexin, DLGAP1, Myosin5a, Dopamine D1 receptor
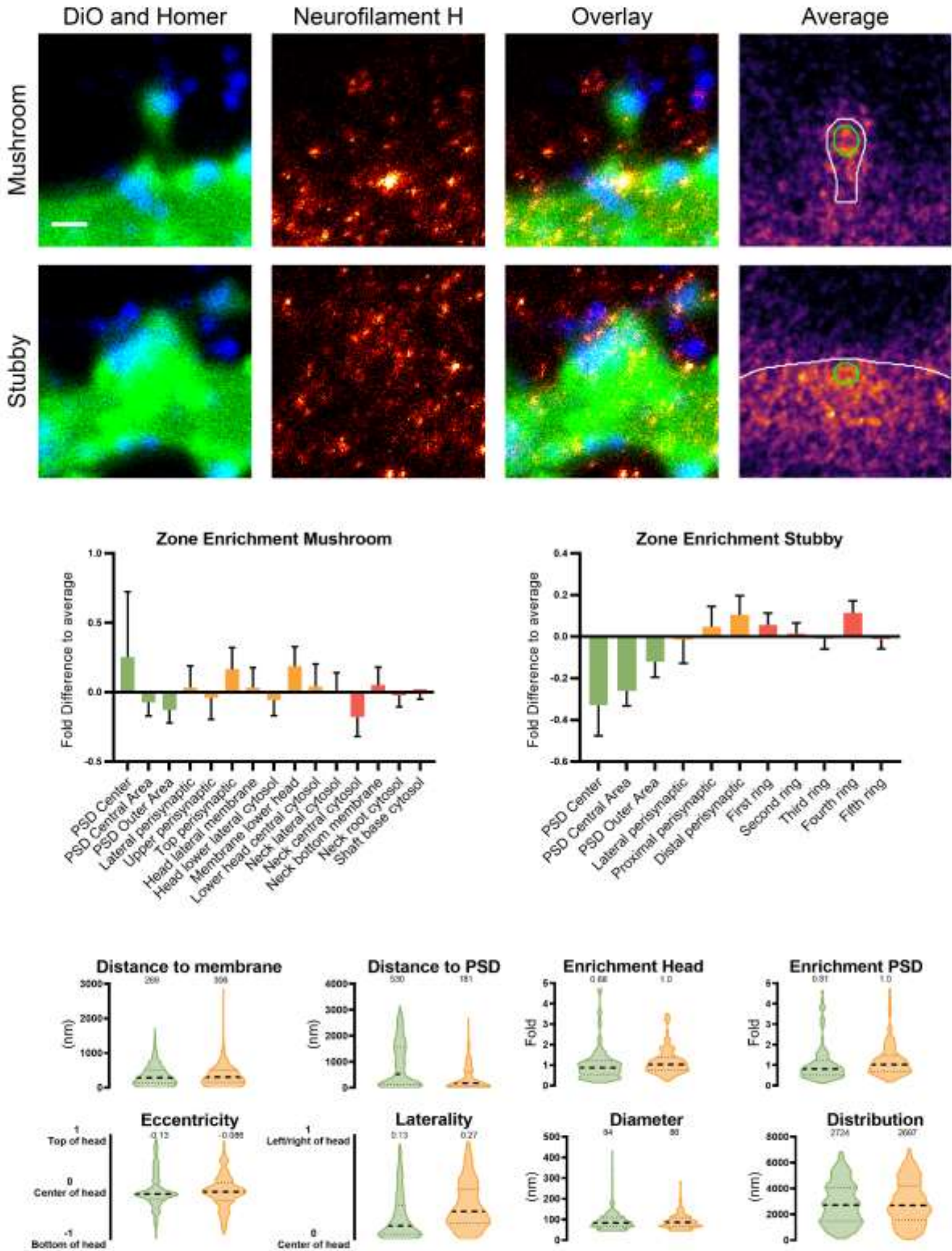

Figure 24: Neurofilament heavy nanoscale localization and abundance. Continued on next page. 


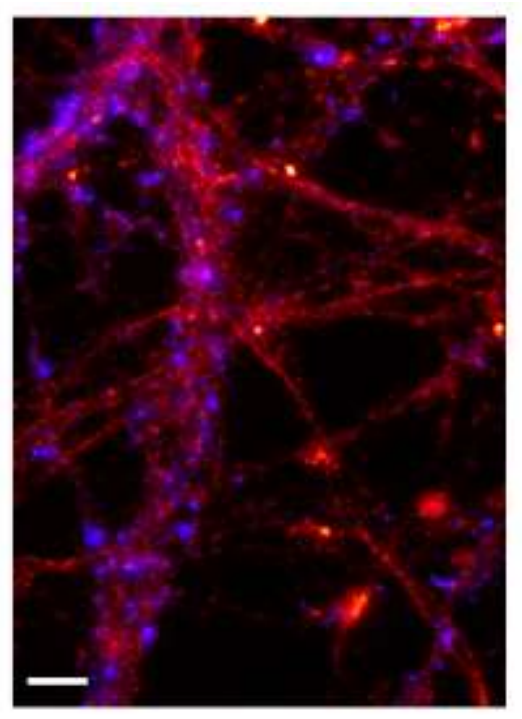

\begin{tabular}{|c|c|c|}
\hline $\begin{array}{c}\text { Whole cell copy } \\
\text { number }\end{array}$ & \multicolumn{2}{|c|}{$624802.88 \pm 189533.54$} \\
\hline $\begin{array}{c}\text { Spine copy } \\
\text { number }\end{array}$ & \multicolumn{2}{|c|}{$69.40 \pm 33.98$} \\
\hline$\%$ in PSD & \multicolumn{2}{|c|}{17.31} \\
\hline PSD copy number & \multicolumn{2}{|c|}{$10.24 \pm 5.01$} \\
\hline Mushroom & Stubby \\
\hline $\begin{array}{c}\text { Spine copy } \\
\text { number }\end{array}$ & $60.88 \pm 29.81$ & $77.28 \pm 37.84$ \\
\hline PSD copy number & $8.98 \pm 4.40$ & $11.40 \pm 5.58$ \\
\hline$\%$ of protein & $0.02 \pm 0.01 \%$ & $0.01 \pm 0.01 \%$ \\
\hline Molarity $[\mu \mathrm{M}]$ & $0.51 \pm 0.25$ & $0.41 \pm 0.20$ \\
\hline
\end{tabular}

\section{References}

Antibody: LS Bio LS-C143052

PDB Identifier: 3s4r, 3swk, 3uf1, 1gk4, 3tnu

\section{Literature:}

Hirao et al., 2000; Rao et al., 2011; Yuan et al., 2012, 2015b, 2015a 


\section{Neurofilament L (Gene: Nefl, Uniprot ID: P19527)}

Known function: Forms neurofilaments, Regulates ER and Endosome location

Known organization: Cytosolic, Forms triplet with Neurofilament $L$ and $M$

Known Interactions: Neurofilament $L$ and $M, \alpha$-internexin, DLGAP1, Myosin5a,

Dopamine D1 receptor
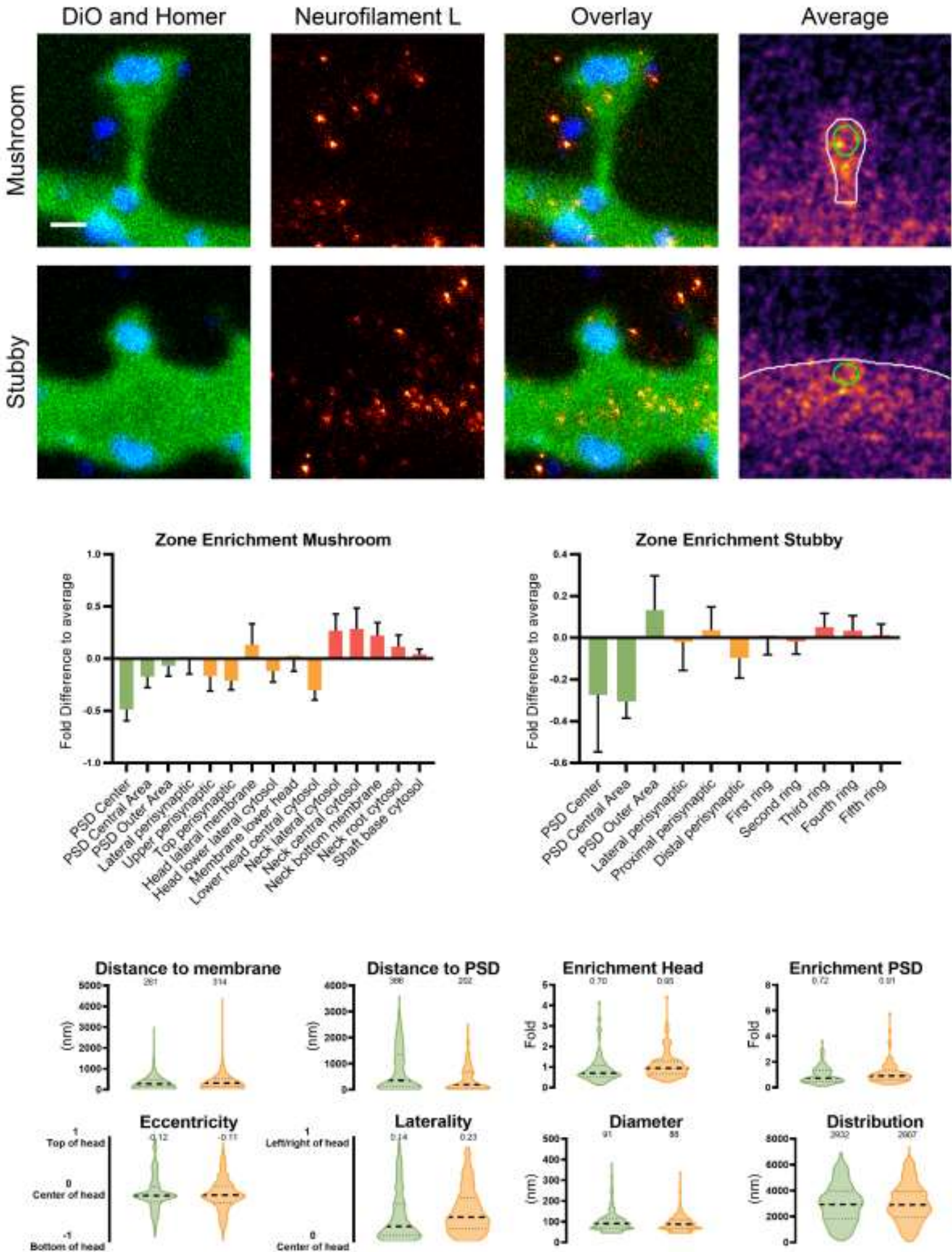

Figure 25: Neurofilament light nanoscale localization and abundance. Continued on next page. 


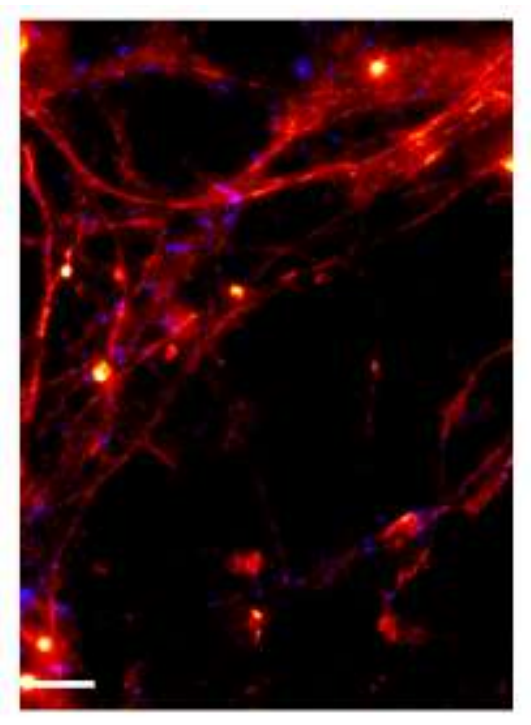

\begin{tabular}{|c|c|c|}
\hline $\begin{array}{c}\text { Whole cell copy } \\
\text { number }\end{array}$ & \multicolumn{2}{|c|}{$8292242.66 \pm 711027.11$} \\
\hline $\begin{array}{c}\text { Spine copy } \\
\text { number }\end{array}$ & \multicolumn{2}{|c|}{$664.29 \pm 325.02$} \\
\hline$\%$ in PSD & \multicolumn{2}{|c|}{16.52} \\
\hline PSD copy number & \multicolumn{2}{|c|}{$94.16 \pm 46.07$} \\
\hline & Mushroom & Stubby \\
\hline \begin{tabular}{c|c|} 
Spine copy \\
number
\end{tabular} & 545.10 & 802.93 \\
\hline PSD copy number & $77.27 \pm 37.70$ & \pm 392.85 \\
\hline$\%$ of protein & $0.08 \pm 0.04 \%$ & $0.07 \pm 0.04 \%$ \\
\hline Molarity $[\mu \mathrm{M}]$ & $4.57 \pm 2.24$ & $4.30 \pm 2.11$ \\
\hline
\end{tabular}

\section{References}

Antibody: Synaptic Systems 171002

PDB Identifier: modified NF-H

\section{Literature:}

Hirao et al., 2000; Rao et al., 2011; Yuan et al., 2012, 2015b, 2015a 


\section{Septin7 (Gene: Sept7, Uniprot ID: Q9WVC0)}

Known function: Restricts diffussion at spine neck, Regulates spine morphology, Stabilizes PSD95 in spines

Known organization: Cytosolic, Forms arc or ring-like structures

Known Interactions: None
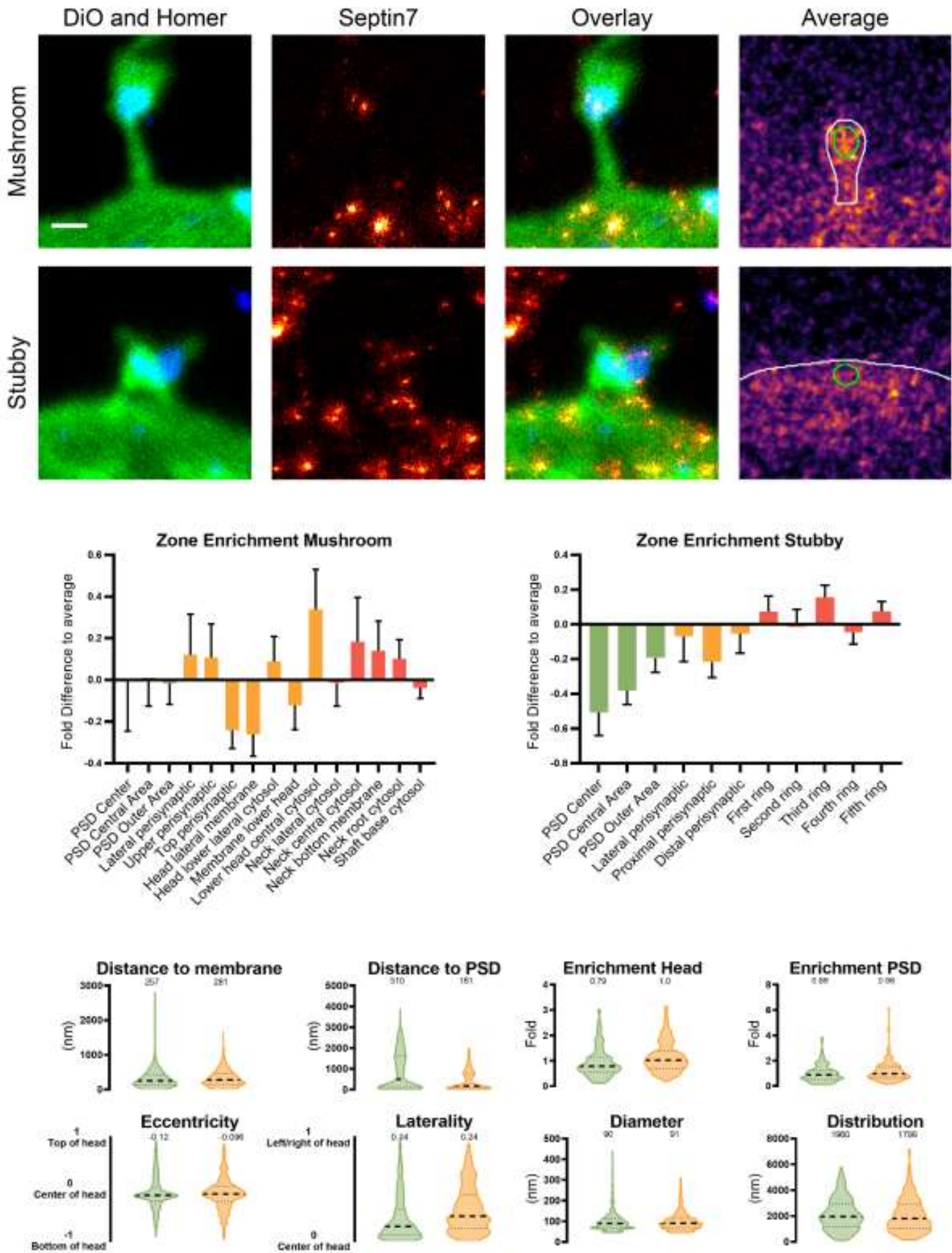

Figure 26: Septin7 nanoscale localization and abundance. Continued on next page. 


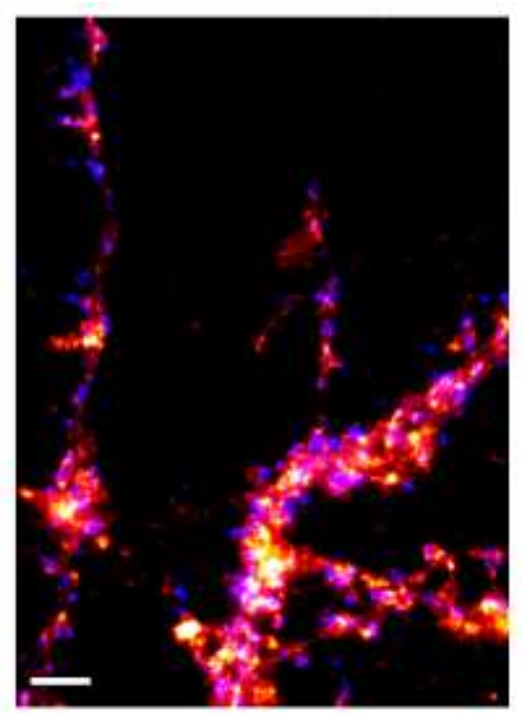

\begin{tabular}{|c|c|c|}
\hline $\begin{array}{c}\text { Whole cell copy } \\
\text { number }\end{array}$ & \multicolumn{2}{|c|}{$1638159.43 \pm 260844.08$} \\
\hline $\begin{array}{c}\text { Spine copy } \\
\text { number }\end{array}$ & \multicolumn{2}{|c|}{$331.12 \pm 98.61$} \\
\hline$\%$ in PSD & \multicolumn{2}{|c|}{15.72} \\
\hline PSD copy number & \multicolumn{2}{|c|}{$44.98 \pm 13.40$} \\
\hline & Mushroom & Stubby \\
\hline $\begin{array}{c}\text { Spine copy } \\
\text { number }\end{array}$ & $263.18 \pm 78.37$ & $375.50 \pm 111.82$ \\
\hline PSD copy number & $35.75 \pm 10.65$ & $51.01 \pm 15.19$ \\
\hline$\%$ of protein & $0.03 \pm 0.01 \%$ & $0.03 \pm 0.01 \%$ \\
\hline Molarity $[\mu \mathrm{M}]$ & $2.21 \pm 0.66$ & $2.01 \pm 0.60$ \\
\hline
\end{tabular}

\section{References}

Antibody: Atlas Antibodies HPA029524

PDB Identifier: 2qag

\section{Literature:}

Brand et al., 2012; Ewers et al., 2014; Kinoshita et al., 2002; Yadav et al., 2017 


\section{Scaffold proteins}

Scaffold proteins are essential to the construction of the PSD density. In this section, I study members of the Membrane-associated guanylate kinase family, Homer and Shank proteins, as well as DLGAP1.

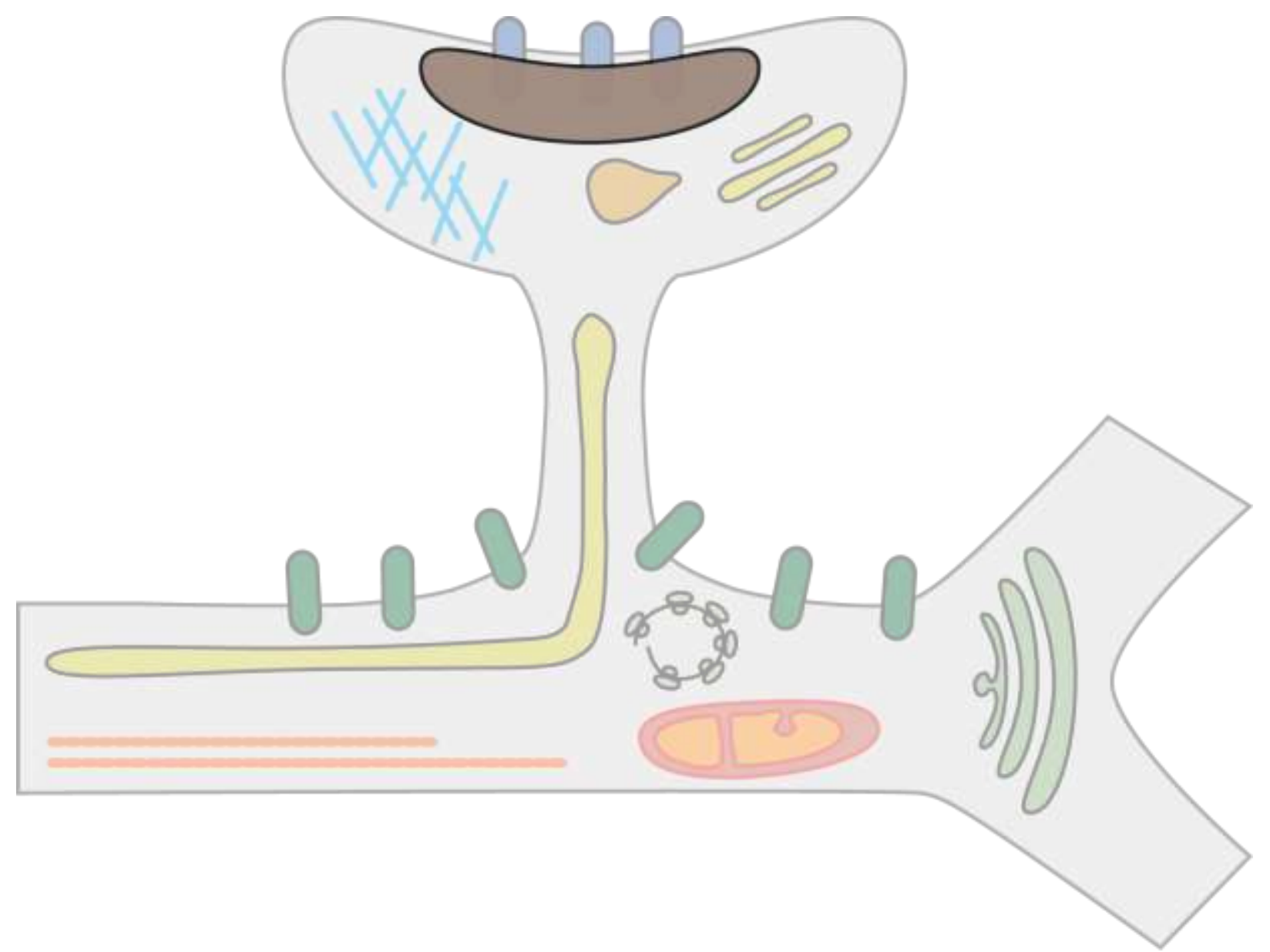

Figure 27: Depiction of scaffold proteins in dendritic spines. 
DLGAP1 (GKAP1, SAPAP1, DAP-1, Gene: Dlgap1, Uniprot ID: P97836)

Known function: Links PSD95 to Shank proteins

Known organization: Cytosolic, Forms clusters within PSD

Known Interactions: PSD95, SAP97, Shank proteins
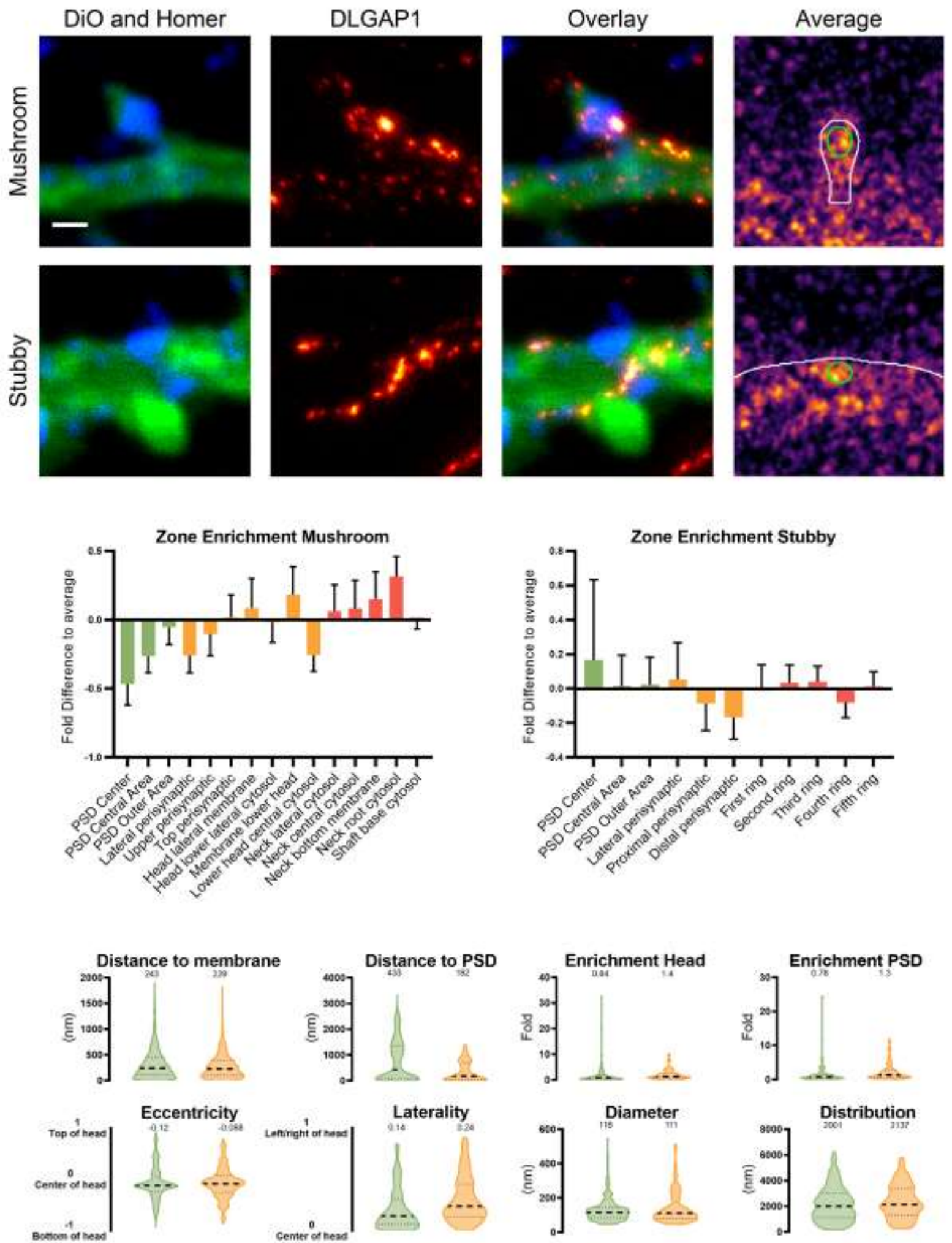

Figure 28: DLGAP1 nanoscale localization and abundance. Continued on next page. 


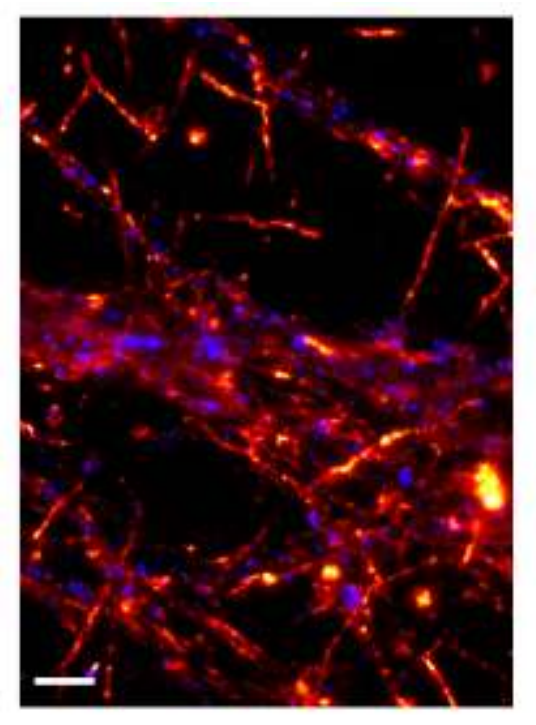

\begin{tabular}{|c|c|c|}
\hline $\begin{array}{c}\text { Whole cell copy } \\
\text { number }\end{array}$ & \multicolumn{2}{|c|}{$105560.36 \pm 11306.76$} \\
\hline $\begin{array}{c}\text { Spine copy } \\
\text { number }\end{array}$ & \multicolumn{2}{|c|}{$208.36 \pm 52.87$} \\
\hline$\%$ in PSD & \multicolumn{2}{|c|}{18.85} \\
\hline PSD copy number & \multicolumn{2}{|c|}{$33.04 \pm 8.38$} \\
\hline & Mushroom & Stubby \\
\hline $\begin{array}{c}\text { Spine copy } \\
\text { number }\end{array}$ & $142.54 \pm 36.17$ & $291.93 \pm 74.08$ \\
\hline PSD copy number & $22.60 \pm 5.74$ & $46.29 \pm 11.75$ \\
\hline$\%$ synaptic protein & $0.04 \pm 0.01 \%$ & $0.05 \pm 0.01 \%$ \\
\hline Molarity $[\mu \mathrm{M}]$ & $0.00 \pm 0.00$ & $0.00 \pm 0.00$ \\
\hline
\end{tabular}

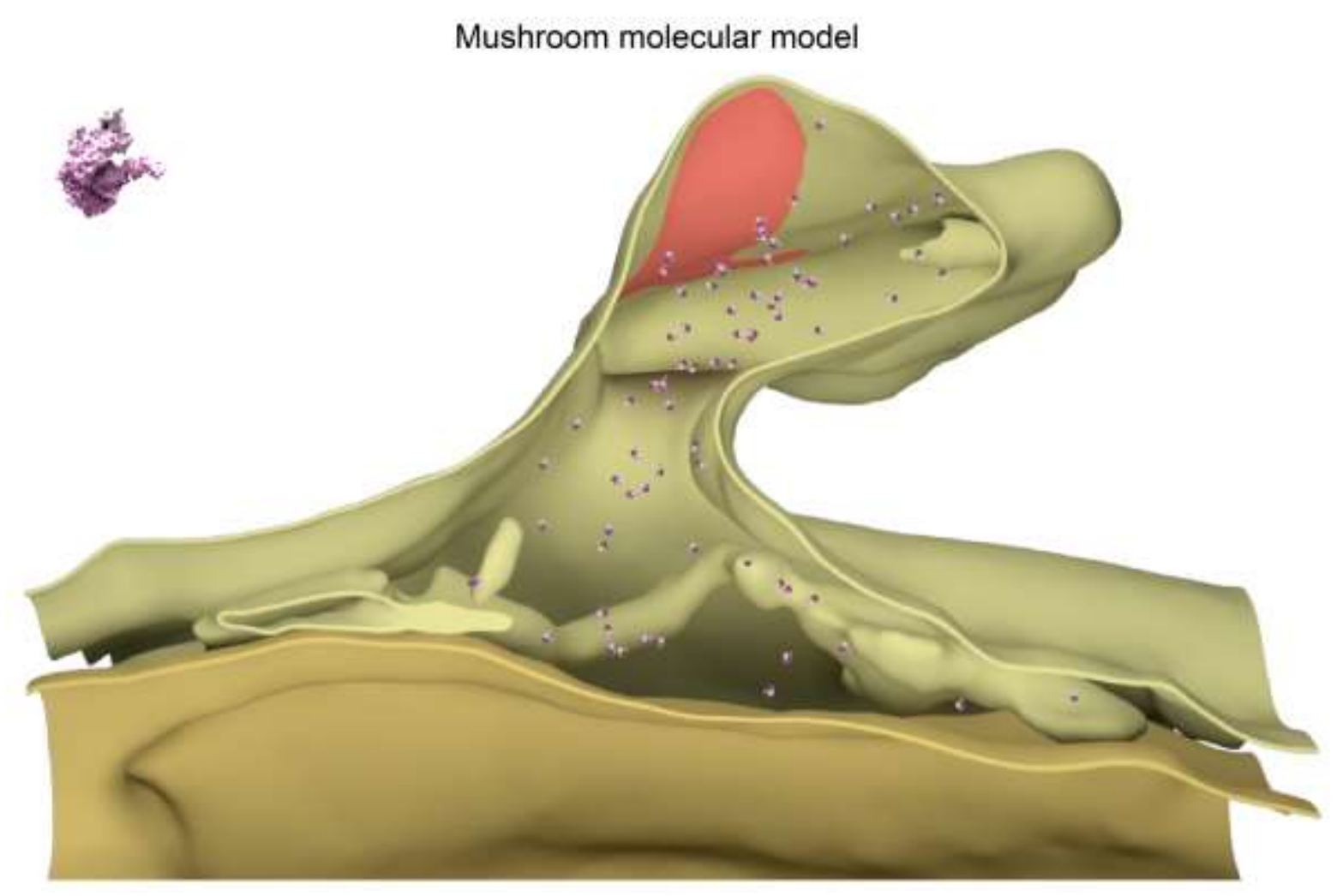

References

Antibody: Novus Biologicals NBP1-76911

PDB Identifier: $4 \mathrm{rOy}$

\section{Literature:}

Boeckers et al., 1999a; Kim et al., 1997; MacGillavry et al., 2013; Naisbitt et al., 1999; Satoh et al., 1997; Takeuchi et al., 1997; Wu, 2000; Yao, 1999 


\section{Homer1 (PSD-Zip45, VesI1, Gene: Homer1, Uniprot: Q9Z214)}

Known function: Scaffold protein, Links mGluR to downstream targets, Homer1a regulates this interaction as a dominant negative isoform

Known organization: Cytosolic, Directly below PSD, Selfmultimerizes (Dimer of dimers), Forms mesh like structure with Shank proteins

Known Interactions: mGluR, Shank proteins, Dynamin 3
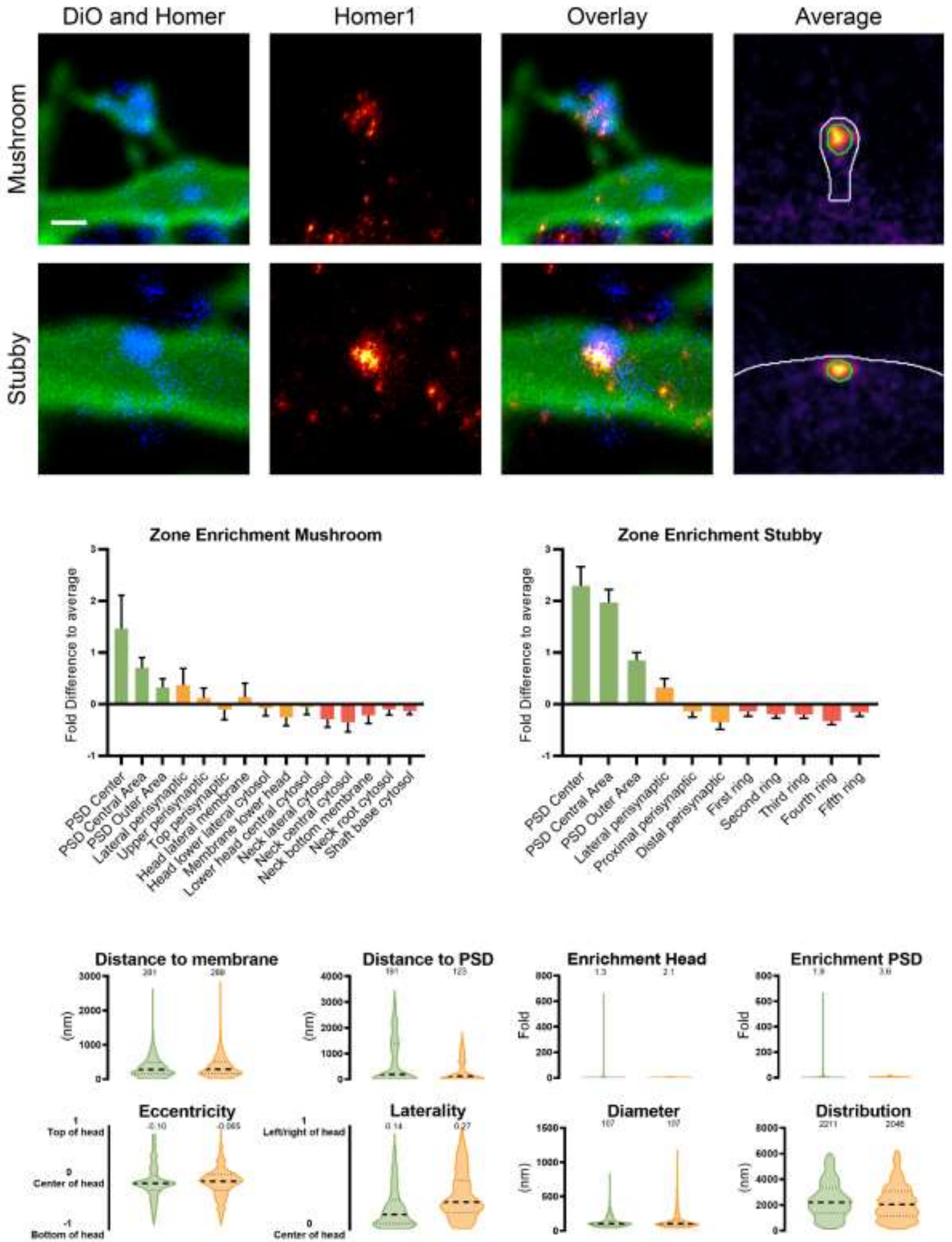

Figure 29: Homer1 nanoscale localization and abundance. Continued on next page. 


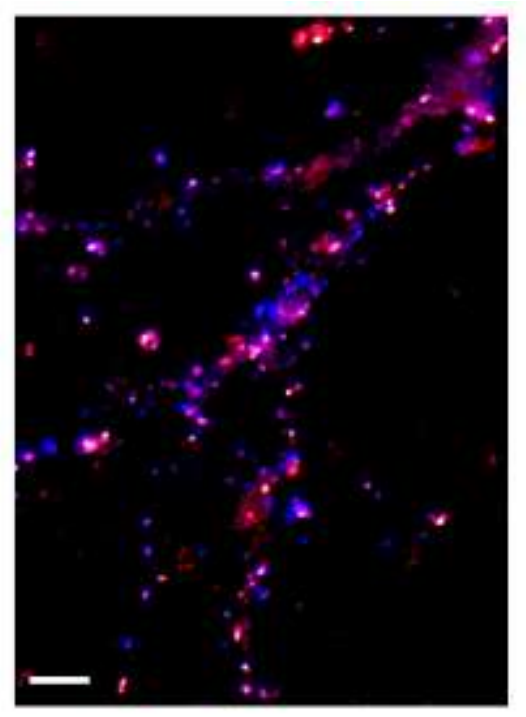

\begin{tabular}{|c|}
\hline $\begin{array}{c}\text { Whole cell copy } \\
\text { number }\end{array}$ \\
\hline $\begin{array}{c}\text { Spine copy } \\
\text { number }\end{array}$ \\
\hline$\%$ in PSD \\
\hline PSD copy number \\
\hline
\end{tabular}

$2156858.01 \pm 207146.63$

\begin{tabular}{|c|c|c|}
\hline & Mushroom & Stubby \\
\hline $\begin{array}{c}\text { Spine copy } \\
\text { number }\end{array}$ & $\begin{array}{c}1698.57 \\
\pm 209.96\end{array}$ & $\begin{array}{l}3779.09 \\
\pm 467.13\end{array}$ \\
\hline PSD copy number & $631.04 \pm 78.00$ & $1403.99 \pm 173.55$ \\
\hline$\%$ of protein & $0.16 \pm 0.02 \%$ & $0.23 \pm 0.03 \%$ \\
\hline Molarity $[\mu \mathrm{M}]$ & $14.24 \pm 1.76$ & $20.26 \pm 2.50$ \\
\hline
\end{tabular}

Mushroom molecular model

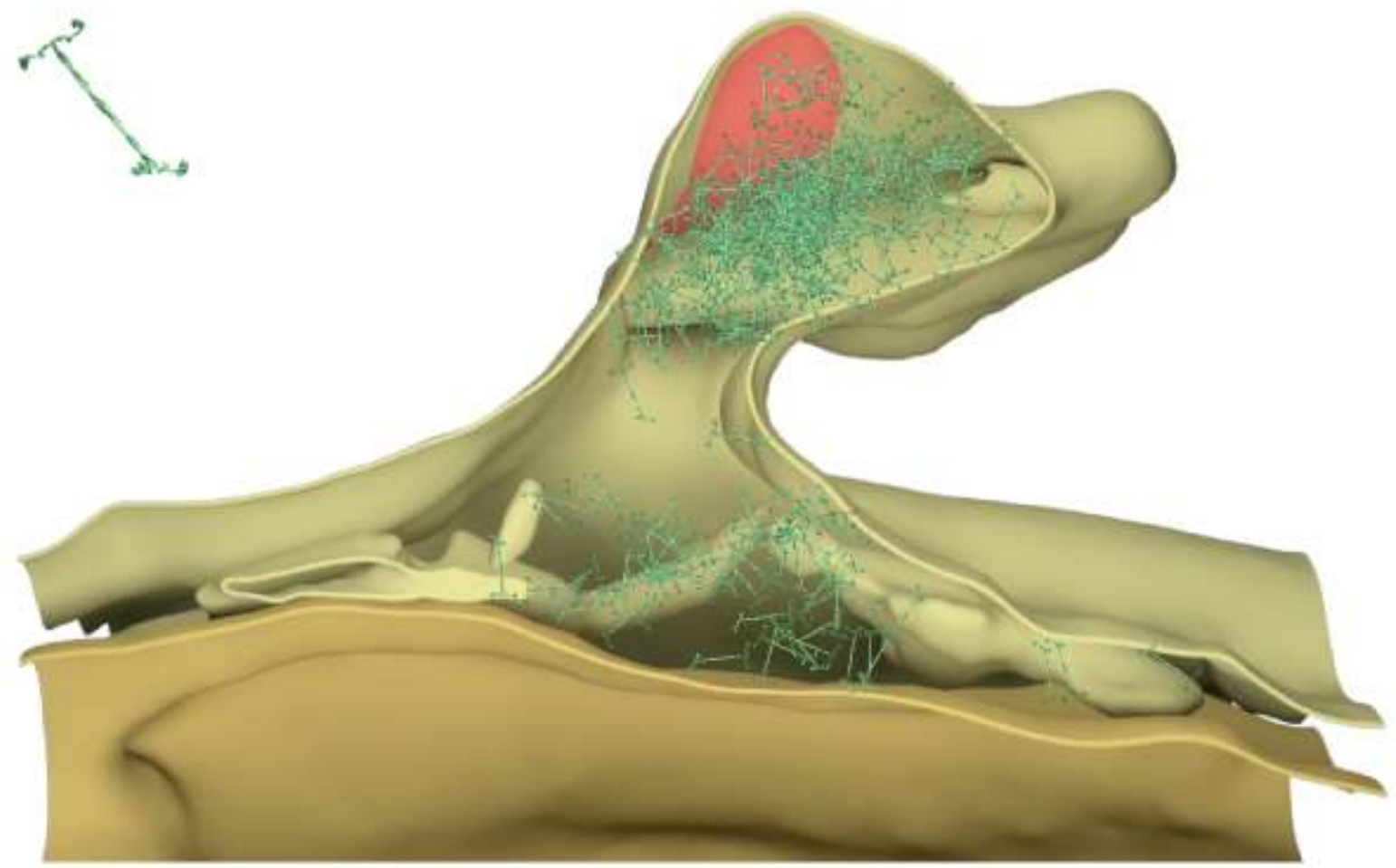

References

Antibody: Synaptic Systems 160011 and 160003 PDB Identifier: 3cve Literature:

Dani et al., 2010; Hayashi et al., 2006, 2009; Kato et al., 1998; Lu et al., 2007; MacGillavry et al., 2013; Newpher and Ehlers, 2008; Roche et al., 1999; Tao-Cheng et al., 2014; Tu et al., 1998; Xiao et al., 1998 


\section{Homer2 (Ves12, Cupidin, Gene: Homer2, Uniprot ID: 088801)}

Known function: Scaffold protein, Links mGluR to downstream targets

Known organization: Cytosolic, Directly below PSD, Selfmultimerizes (Dimer of dimers),

Forms mesh like structure with Shank proteins

Known Interactions: mGluR, Shank proteins, Dynamin 3
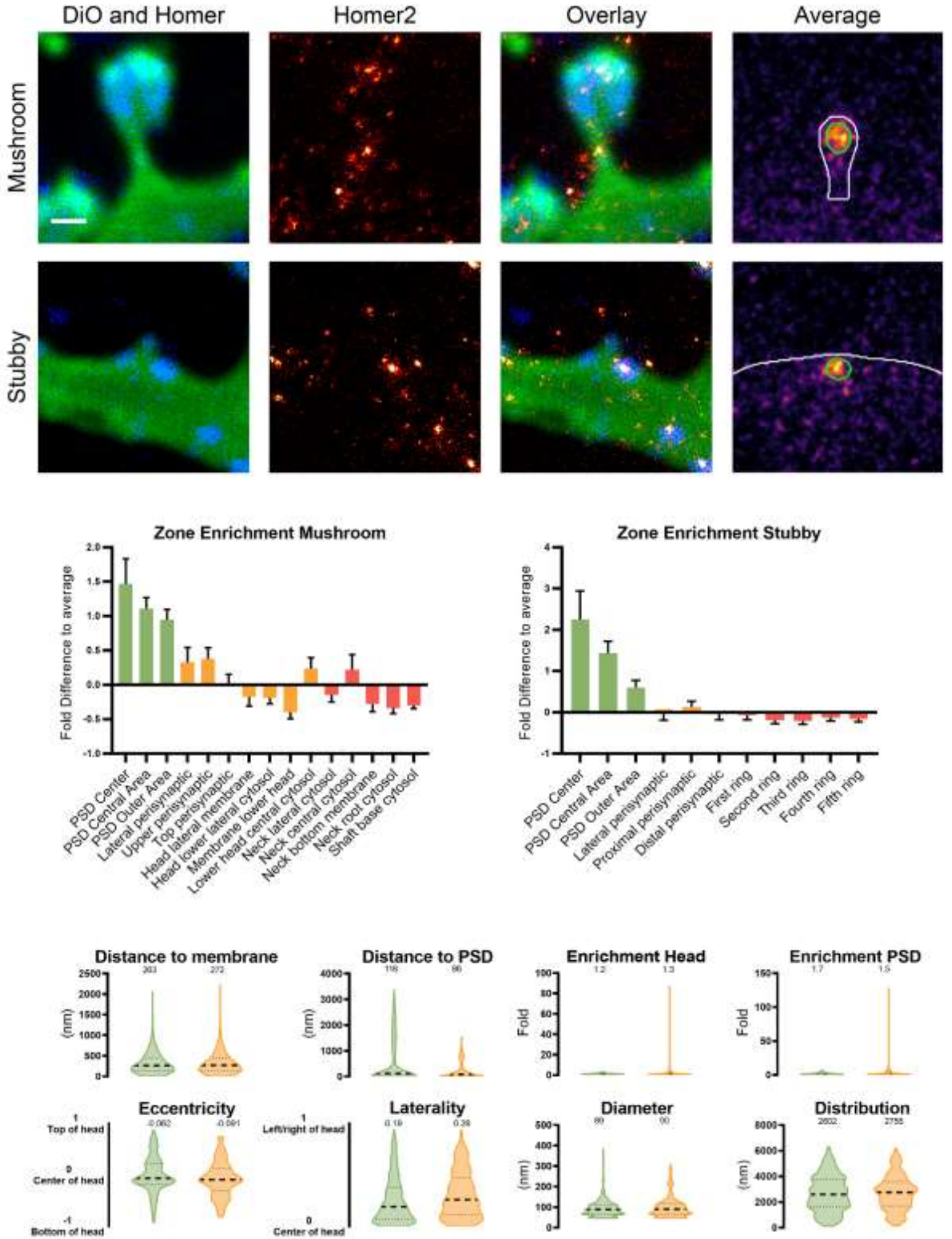

Figure 30: Homer2 nanoscale localization and abundance. Continued on next page. 


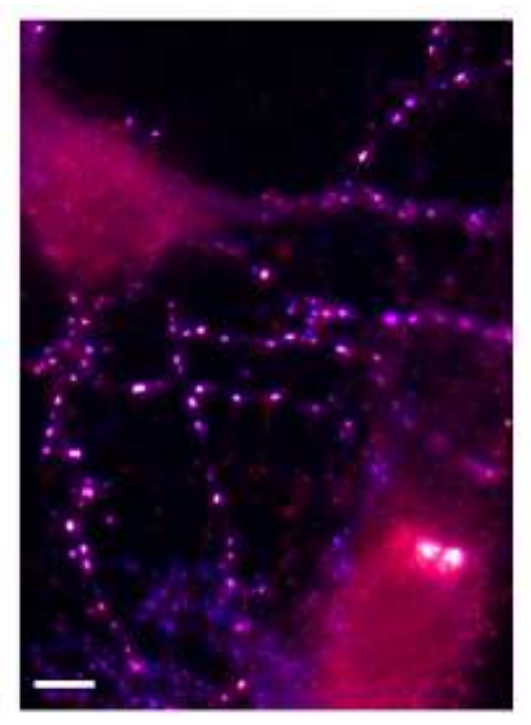

\begin{tabular}{|c|c|c|}
\hline $\begin{array}{c}\text { Whole cell copy } \\
\text { number }\end{array}$ & \multicolumn{2}{|c|}{$57216.45 \pm 17569.93$} \\
\hline $\begin{array}{c}\text { Spine copy } \\
\text { number }\end{array}$ & \multicolumn{2}{|c|}{$18.20 \pm 5.85$} \\
\hline$\%$ in PSD & \multicolumn{2}{|c|}{$49.34 \%$} \\
\hline PSD copy number & \multicolumn{2}{|c|}{$6.01 \pm 1.93$} \\
\hline & Mushroom & Stubby \\
\hline $\begin{array}{c}\text { Spine copy } \\
\text { number }\end{array}$ & $16.06 \pm 5.16$ & $22.76 \pm 7.32$ \\
\hline PSD copy number & $5.31 \pm 1.71$ & $7.52 \pm 2.42$ \\
\hline$\%$ of protein & 0.00 & 0.00 \\
\hline Molarity $[\mu \mathrm{M}]$ & $0.13 \pm 0.04$ & $0.12 \pm 0.04$ \\
\hline
\end{tabular}

\section{References}

Antibody: Synaptic Systems 160203

PDB Identifier: 3cve (modified)

\section{Literature:}

Dani et al., 2010; Hayashi et al., 2006, 2009; Kato et al., 1998; Lu et al., 2007; MacGillavry et al., 2013; Newpher and Ehlers, 2008; Roche et al., 1999; Tao-Cheng et al., 2014; Tu et al., 1998; Xiao et al., 1998 


\section{Homer3 (VesI3, Gene: Homer3, Uniprot ID: Q9Z2X5)}

Known function: Scaffold protein, Links mGluR to downstream targets

Known organization: Cytosolic, Directly below PSD, Selfmultimerizes (Dimer of dimers),

Forms mesh like structure with Shank proteins

Known Interactions: mGluR, IP3R, RyR, Shank proteins, Dynamin 3
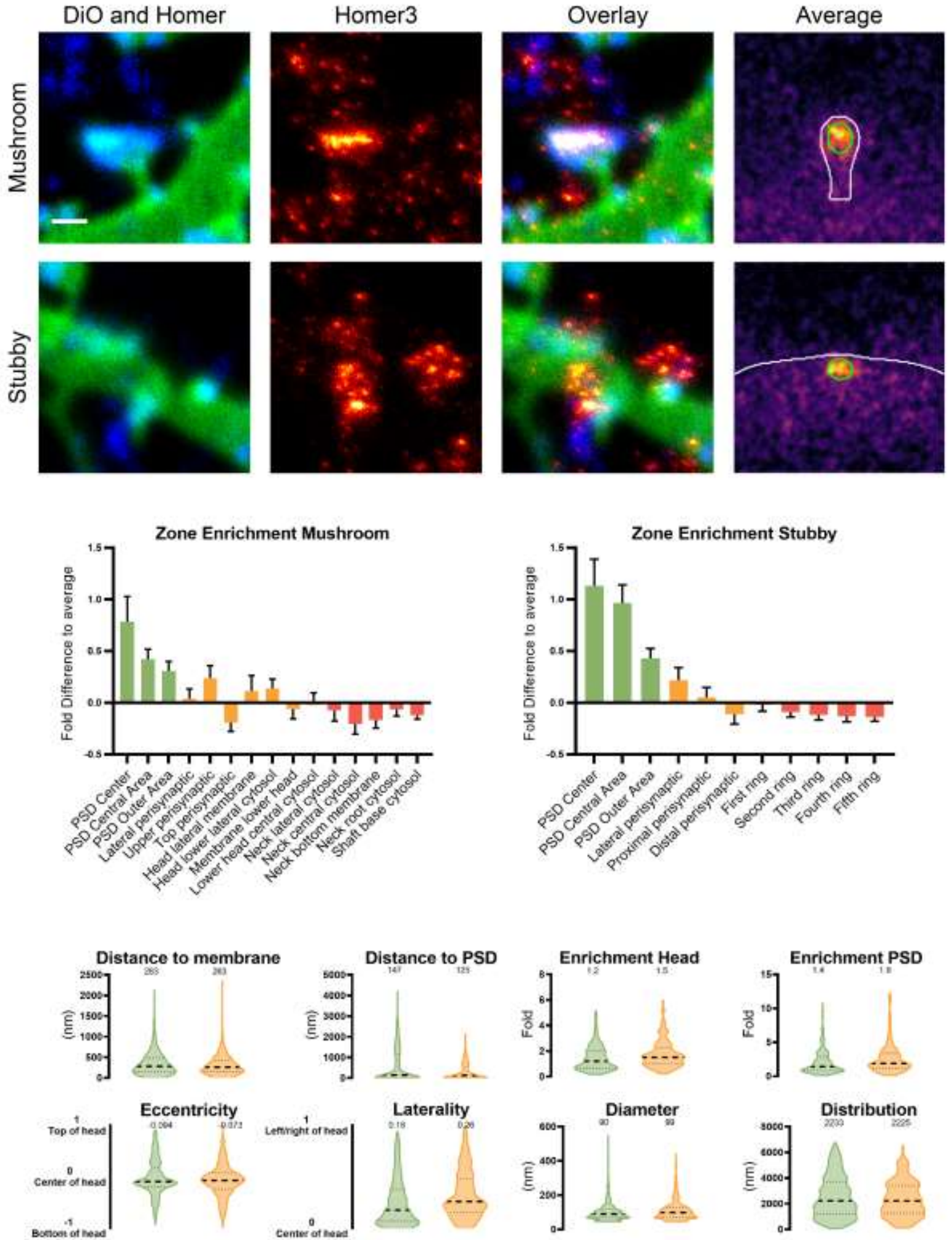

Figure 31: Homer3 nanoscale localization and abundance. Continued on next page. 


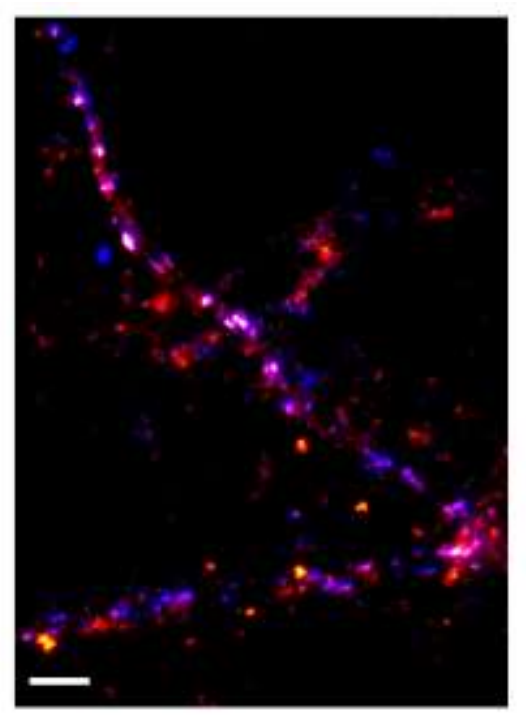

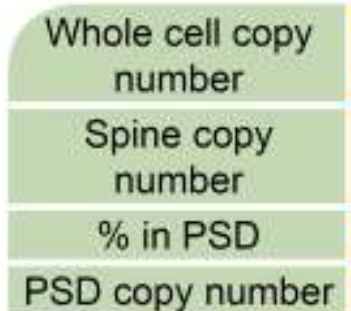

PSD copy number

Spine copy
number

PSD copy number

$\%$ of protein

Molarity $[\mu \mathrm{M}]$

\begin{tabular}{|c|}
\hline $372633.47 \pm 69292.96$ \\
\hline $237.38 \pm 48.26$ \\
\hline 31.73 \\
\hline $57.18 \pm 11.63$ \\
\hline
\end{tabular}

\begin{tabular}{|c|c|}
\hline Mushroom & Stubby \\
\hline $215.49 \pm 43.81$ & $262.55 \pm 53.38$ \\
\hline $51.91 \pm 10.55$ & $63.25 \pm 12.86$ \\
\hline $0.02 \pm 0.00$ & $0.02 \pm 0.00$ \\
\hline $1.81 \pm 0.47$ & $2.22 \pm 0.60$ \\
\hline
\end{tabular}

Mushroom molecular model

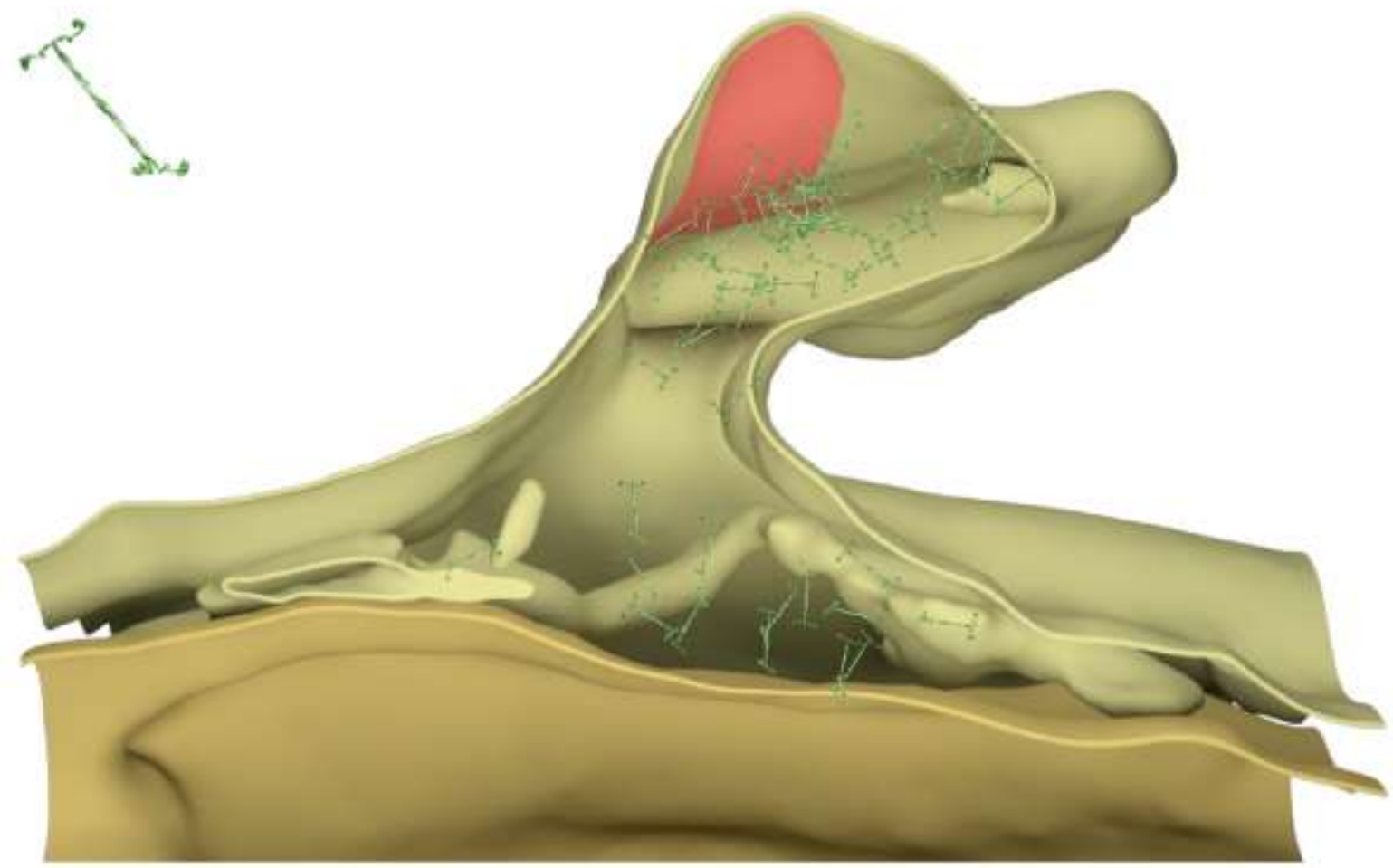

\section{References}

Antibody: Synaptic Systems 160303

PDB Identifier: $3 c v f$

\section{Literature:}

Dani et al., 2010; Hayashi et al., 2006, 2009; Kato et al., 1998; Lu et al., 2007; MacGillavry et al., 2013; Newpher and Ehlers, 2008; Roche et al., 1999; Tao-Cheng et al., 2014; Tu et al., 1998; Xiao et al., 1998 


\section{PSD93 (Dlg2, Chapsyn-110, Gene: Dlg2, Uniprot ID: Q63622)}

Known function: Major organizer of the PSD

Known organization: Membrane-associated, Forms supercomplexes with other scaffolds, receptors and other proteins

Known Interactions: PSD95, NMDA receptors, nNOS, Kir2.1
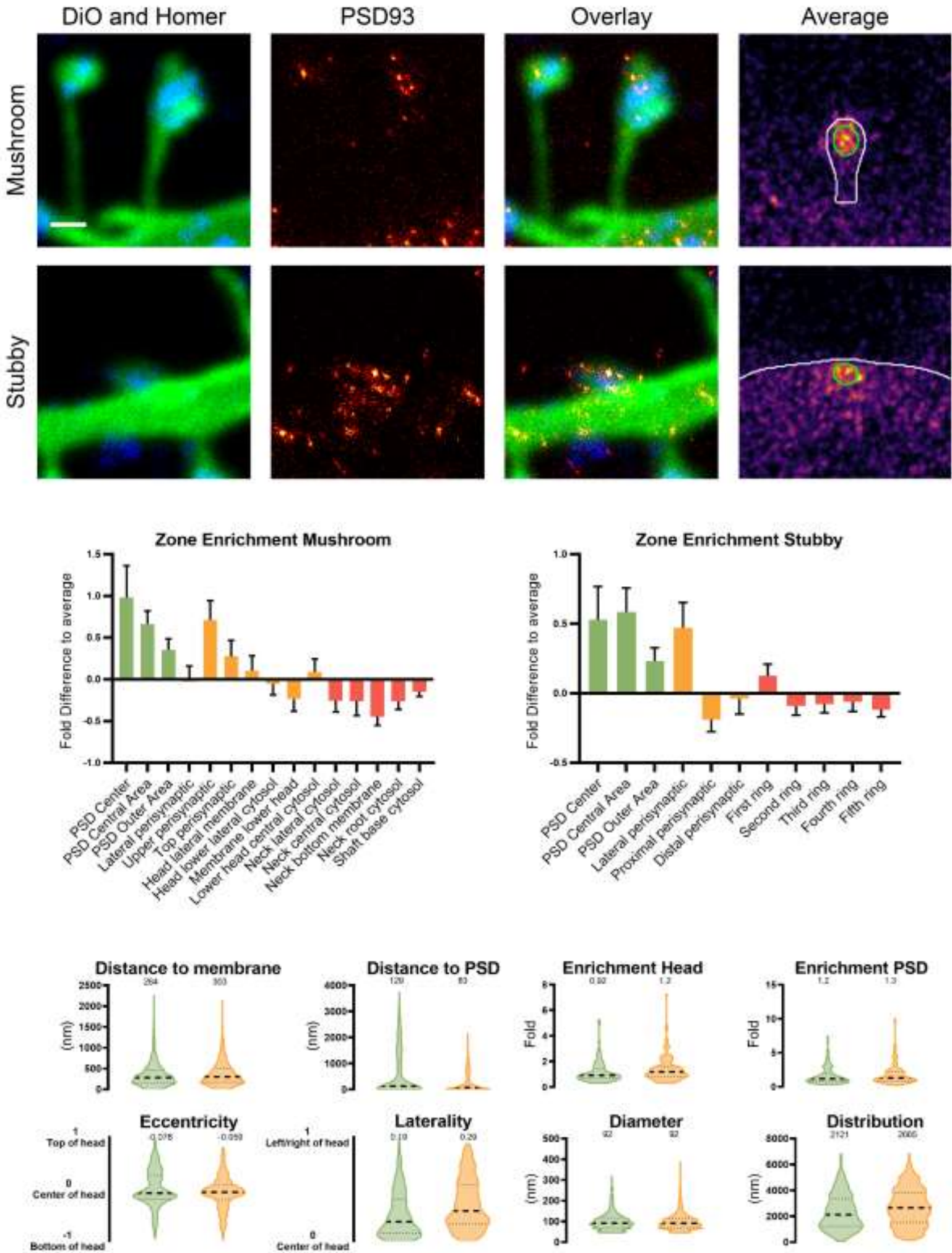

Figure 32: PSD93 nanoscale localization and abundance. Continued on next page. 


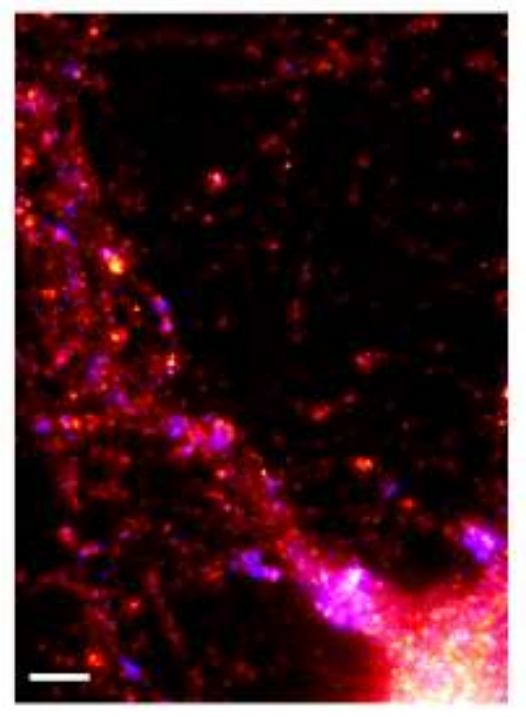

\begin{tabular}{|c|c|c|}
\hline $\begin{array}{c}\text { Whole cell copy } \\
\text { number }\end{array}$ & \multicolumn{2}{|c|}{$2090886.95 \pm 76779.43$} \\
\hline $\begin{array}{c}\text { Spine copy } \\
\text { number }\end{array}$ & \multicolumn{2}{|c|}{$237.38 \pm 48.26$} \\
\hline$\%$ in PSD & \multicolumn{2}{|c|}{29.66} \\
\hline PSD copy number & \multicolumn{2}{|c|}{$159.91 \pm 14.96$} \\
\hline & Mushroom & Stubby \\
\hline $\begin{array}{c}\text { Spine copy } \\
\text { number }\end{array}$ & $645.93 \pm 60.42$ & $791.50 \pm 74.04$ \\
\hline PSD copy number & $147.77 \pm 13.82$ & $181.07 \pm 16.94$ \\
\hline$\%$ of protein & $0.14 \pm 0.01$ & $0.11 \pm 0.01$ \\
\hline Molarity $[\mu M]$ & $5.41 \pm 0.51$ & $4.24 \pm 0.40$ \\
\hline
\end{tabular}

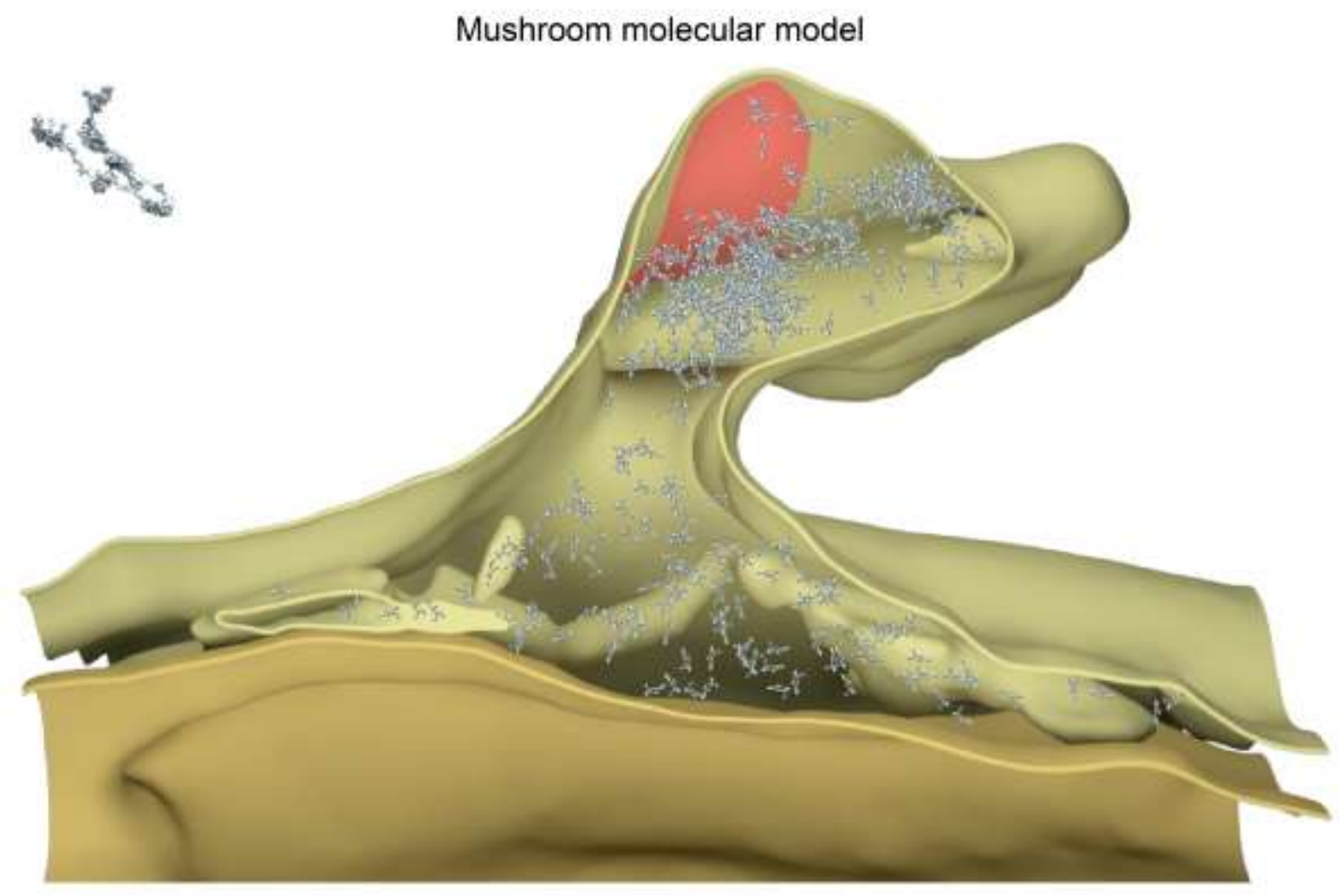

\section{References}

Antibody: Invitrogen 34-4700

PDB Identifier: 2 wl7

\section{Literature:}

Brenman et al., 1996a; Dakoji et al., 2003; Frank et al., 2016; Leyland and Dart, 2004; Niethammer et al., 1996; Parker, 2004 
PSD95 (DIg4, Sap90, Gene: DIg4, Uniprot ID: P31016)

Known function: Major organizer of the PSD

Known organization: Membrane-associated and cytosolic, Forms supercomplexes with other scaffolds, receptors and other proteins, Forms Nanodomains

Known Interactions: PSD93, NMDA receptors, nNOS, Potassium channels
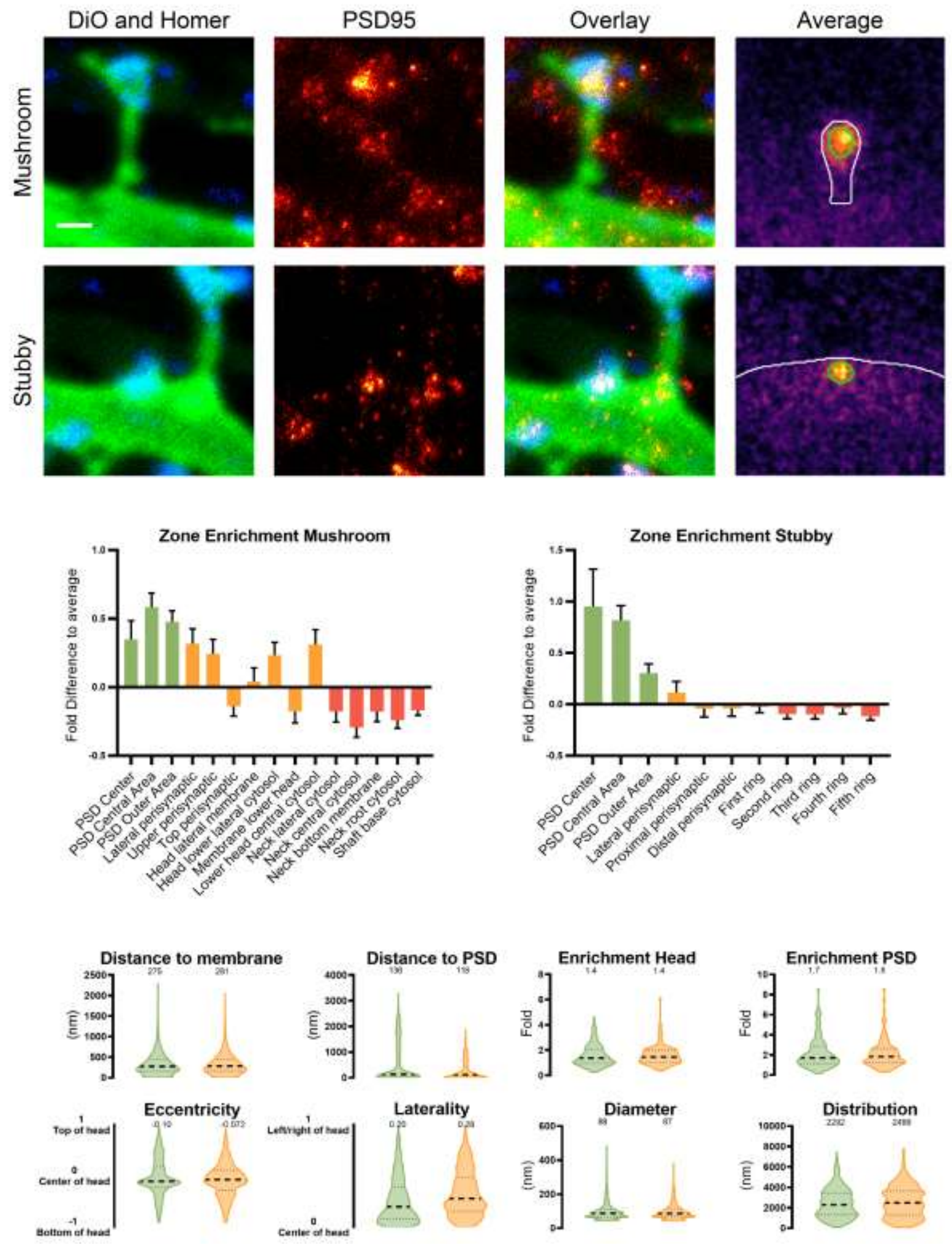

Figure 33: PSD95 nanoscale localization and abundance. Continued on next page. 


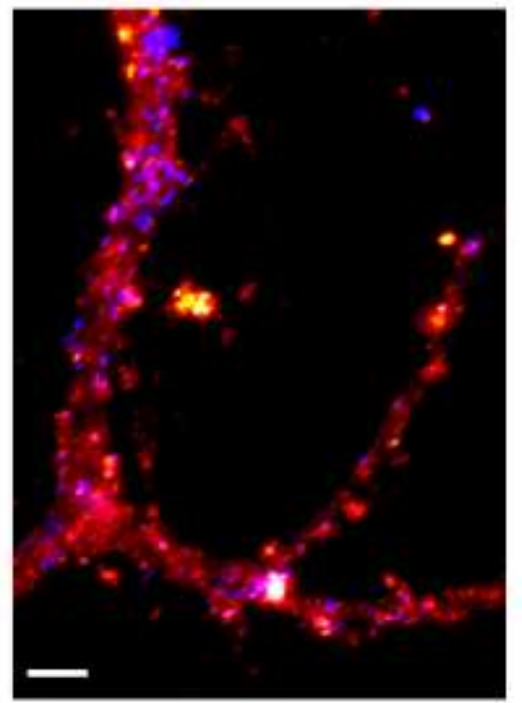

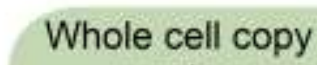
number

Spine copy

number

$\%$ in PSD

PSD copy number

PSD copy number

Spine copy number

PSD copy number

$\%$ of protein

Molarity $[\mu \mathrm{M}]$

\begin{tabular}{|c|}
\hline $2445674.62 \pm 138415.13$ \\
\hline $1123.34 \pm 134.46$ \\
\hline 34.51 \\
\hline $288.21 \pm 34.50$ \\
\hline
\end{tabular}

\begin{tabular}{|c|c|}
\hline Mushroom & Stubby \\
\hline 1133.42 & $1187.37 \pm$ \\
\pm 135.67 & 142.12 \\
\hline $290.79 \pm 34.81$ & $304.63 \pm 36.46$ \\
\hline $0.21 \pm 0.03$ & $0.14 \pm 0.02$ \\
\hline $9.50 \pm 1.14$ & $6.37 \pm 0.76$ \\
\hline
\end{tabular}

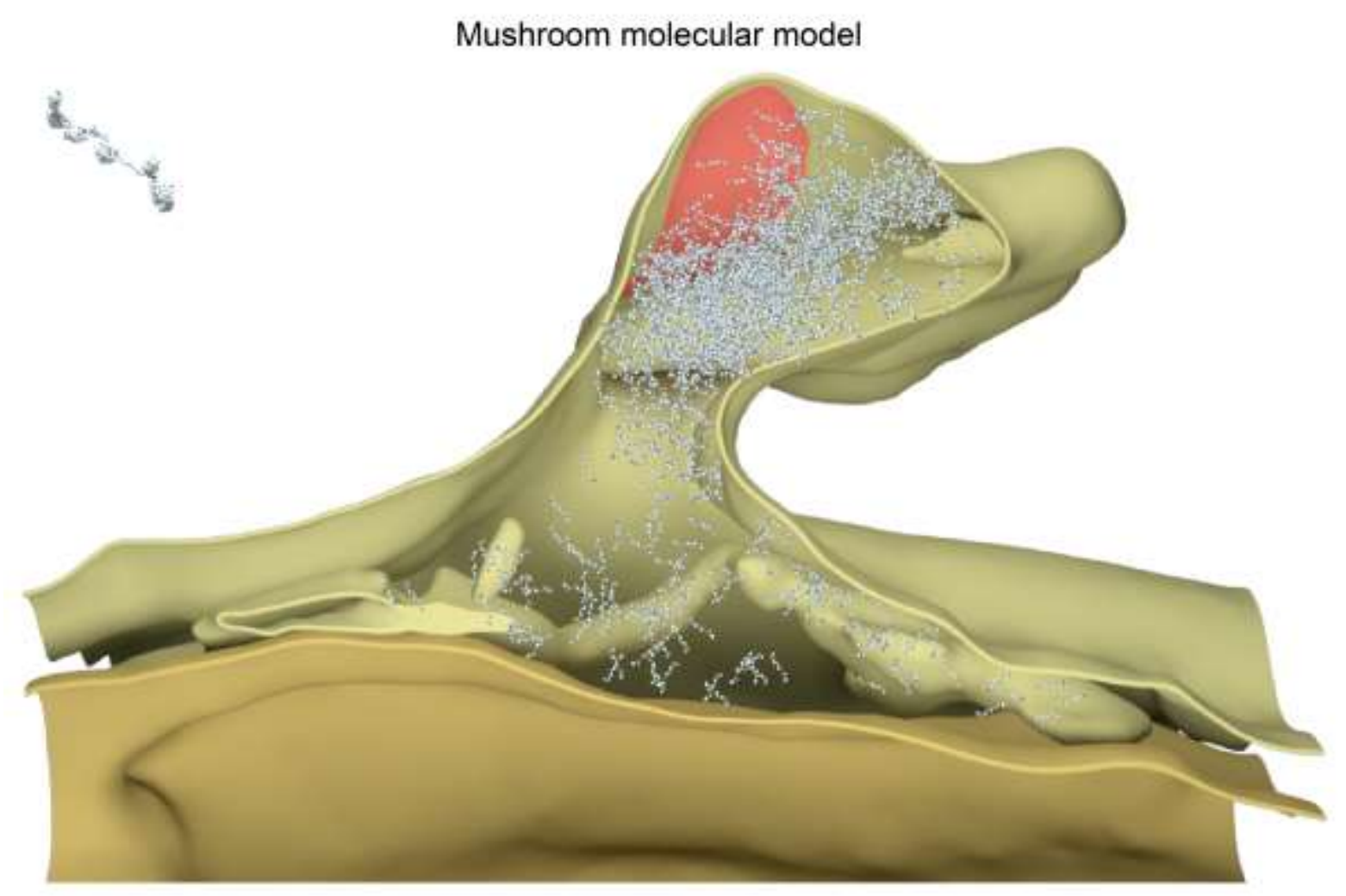

\section{References}

Antibody: Cell Signaling 3450

PDB Identifier: $2 \times k x, 5 j \times b$

\section{Literature:}

Bhattacharyya et al., 2009; Broadhead et al., 2016; Dani et al., 2010; Frank et al., 2017; Garner et al., 2000; Hruska et al., 2018; Inagaki et al., 2001; Kim et al., 1997; MacGillavry et al., 2013; Niethammer et al., 1996; Schultze et al., 2001; Takeuchi et al., 1997; Tang et al., 2016 


\section{Shank1 (Synamon, Gene Shank1, Uniprot ID: Q9WV48)}

Known function: Links the PSD to the cytoskeleton

Known organization: Cytosolic, Selfmultimerizes, Forms mesh like structure with Homer proteins, Axially more distal to PSD than PSD95, Forms clusters

Known Interactions: Homer proteins, DLGAP1, GluR1
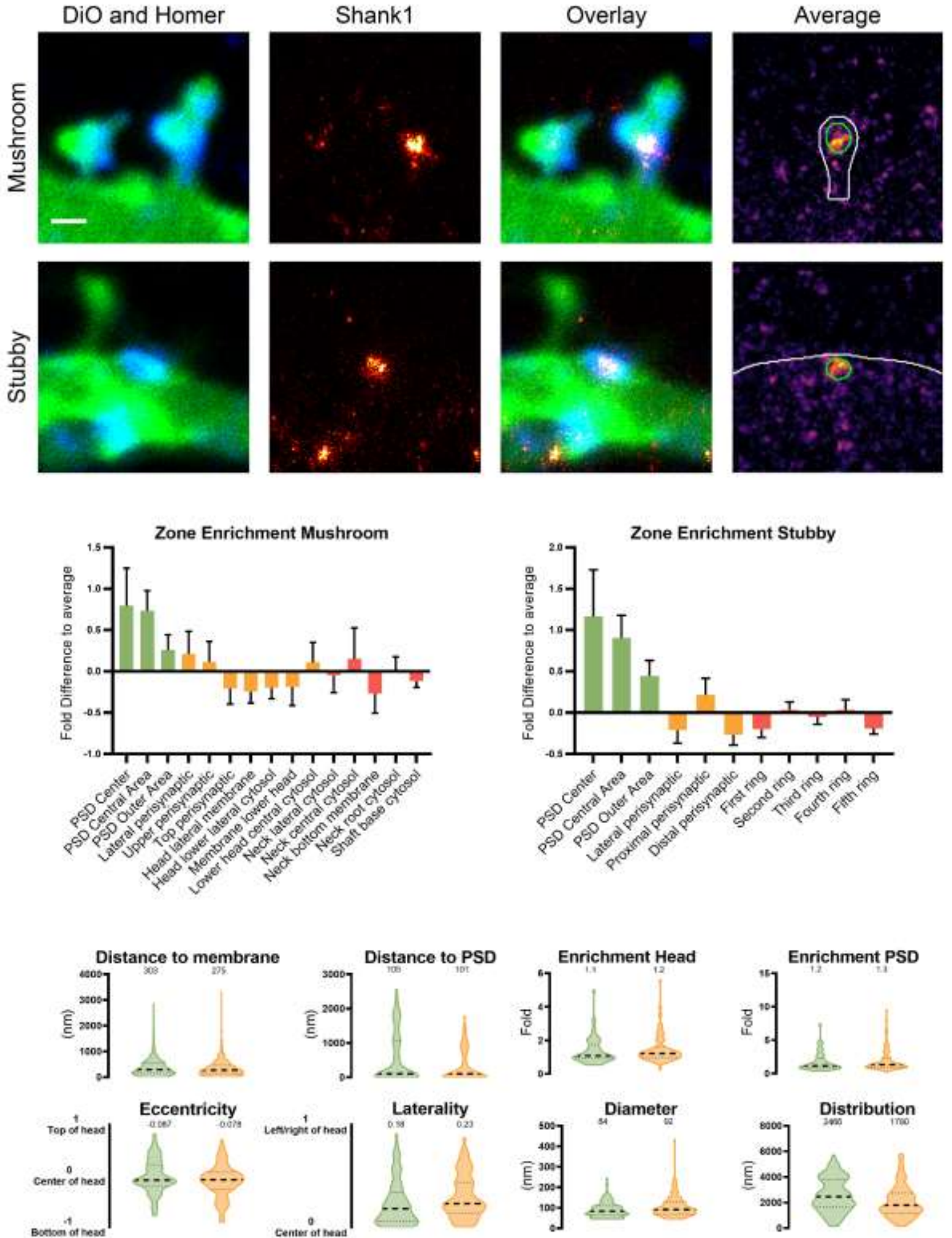

Figure 34: Shank 1 nanoscale localization and abundance. Continued on next page. 


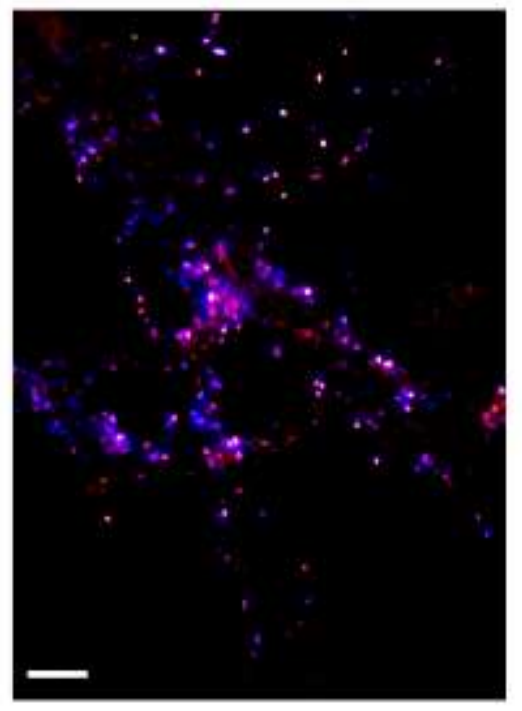

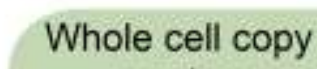
number

Spine copy
number

$\%$ in PSD

PSD copy number

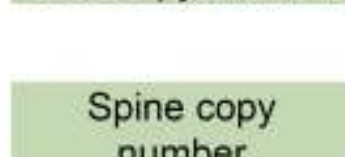
number

PSD copy number

$\%$ of protein

Molarity [ $\mu \mathrm{M}]$

\begin{tabular}{|c|}
\hline $45698.29 \pm 1849.10$ \\
\hline $179.66 \pm 17.15$ \\
\hline 47.08 \\
\hline $57.51 \pm 5.49$ \\
\hline
\end{tabular}

\begin{tabular}{|c|c|}
\hline Mushroom & Stubby \\
\hline $139.91 \pm 13.36$ & $232.08 \pm 22.16$ \\
\hline $44.78 \pm 4.28$ & $74.29 \pm 7.09$ \\
\hline $0.07 \pm 0.01$ & $0.08 \pm 0.01$ \\
\hline $1.17 \pm 0.11$ & $1.24 \pm 0.12$ \\
\hline
\end{tabular}

Mushroom molecular model

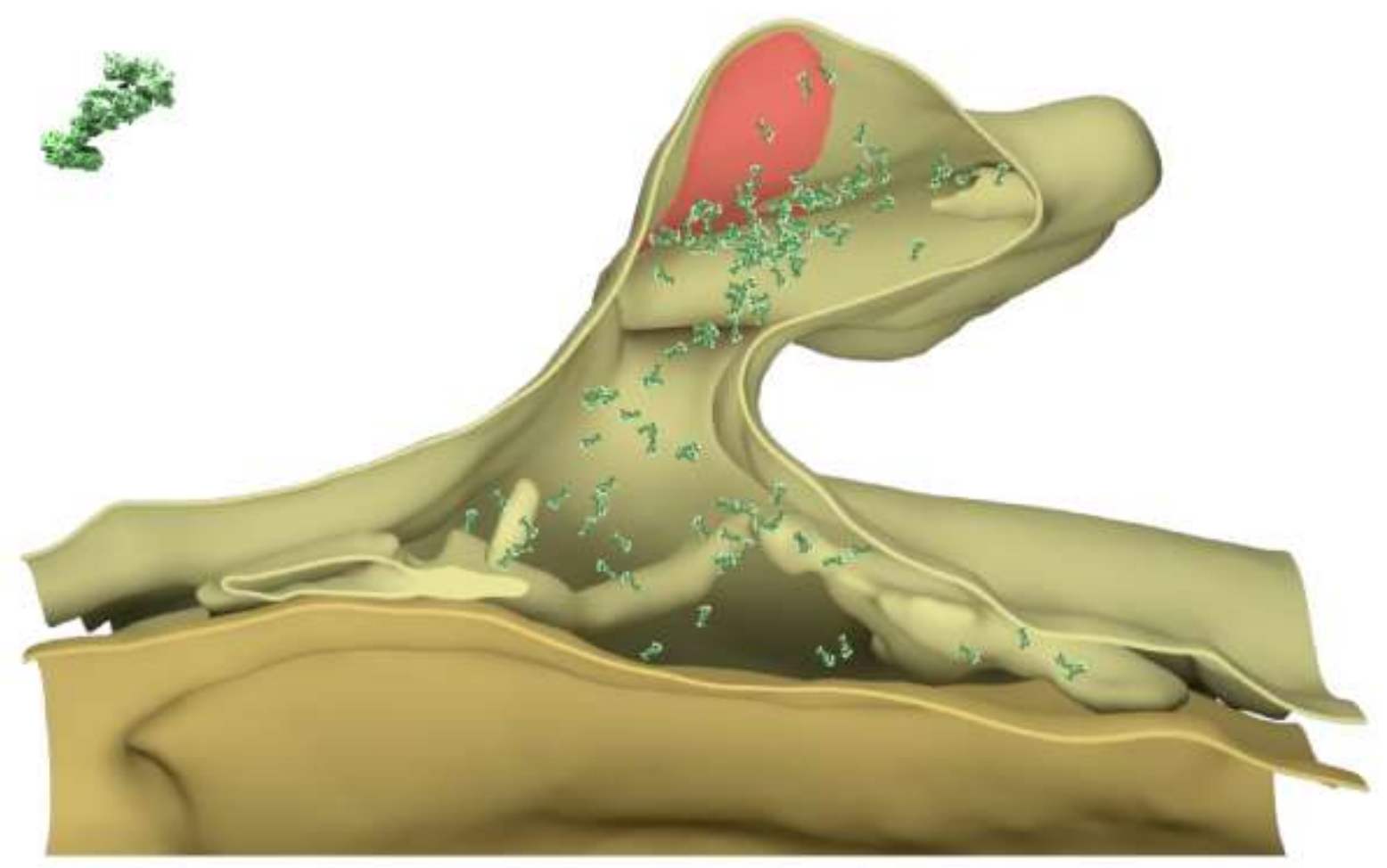

\section{References}

Antibody: Synaptic Systems 162013

PDB Identifier: $1 q 30,1 w s x, 2 f 3 n$

\section{Literature:}

Boeckers et al., 1999b; Dani et al., 2010; Hayashi et al., 2006, 2009; MacGillavry et al., 2013; Naisbitt et al., 1999; Tao-Cheng et al., 2015; Tu et al., 1999; Uchino et al., 2006; Valtschanoff and Weinberg, 2001 
Shank2 (ProSAP1, CortBP1, Gene: Shank2, Uniprot ID: Q9QX74)

Known function: Links the PSD to the cytoskeleton

Known organization: Cytosolic, Selfmultimerizes, Forms mesh like structure with Homer proteins, Axially more distal to PSD than PSD95, Forms clusters

Known Interactions: Homer proteins, DLGAP1, Cortactin, GluR1
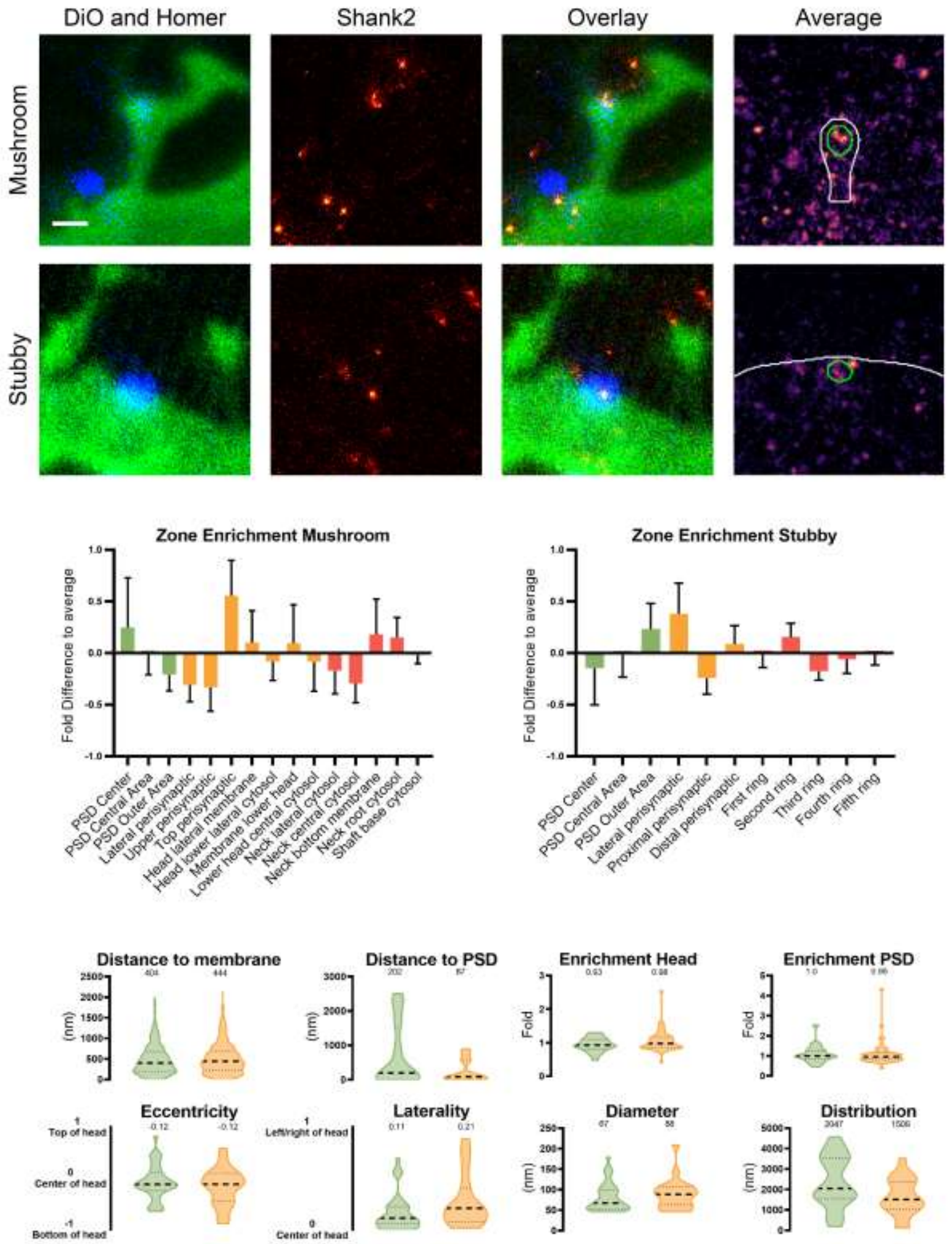

Figure 35: Shank2 nanoscale localization and abundance. Continued on next page. 


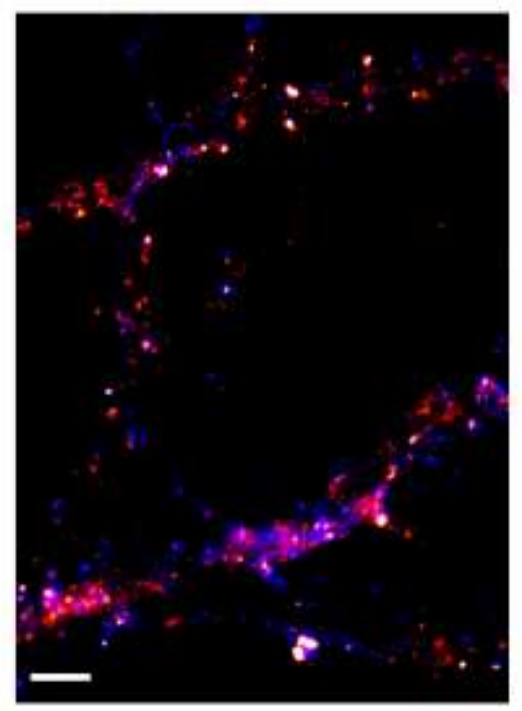

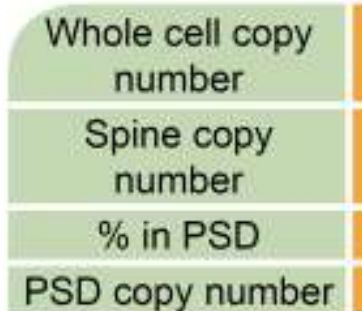

\begin{tabular}{|c|}
\hline $98061.44 \pm 12305.69$ \\
\hline $241.61 \pm 80.59$ \\
\hline 28.51 \\
\hline $53.61 \pm 17.88$ \\
\hline
\end{tabular}

\begin{tabular}{|c|c|c|}
\hline $\begin{array}{c}\text { Spine copy } \\
\text { number }\end{array}$ & Mushroom & Stubby \\
\hline PSD copy number & $61.54 \pm 20.52 \pm 92.50$ & $226.87 \pm 75.67$ \\
\hline$\%$ of protein & $0.13 \pm 0.04$ & $50.34 \pm 16.79$ \\
\hline Molarity $[\mu \mathrm{M}]$ & $2.32 \pm 0.78$ & $1.22 \pm 0.41$ \\
\hline
\end{tabular}

Mushroom molecular model

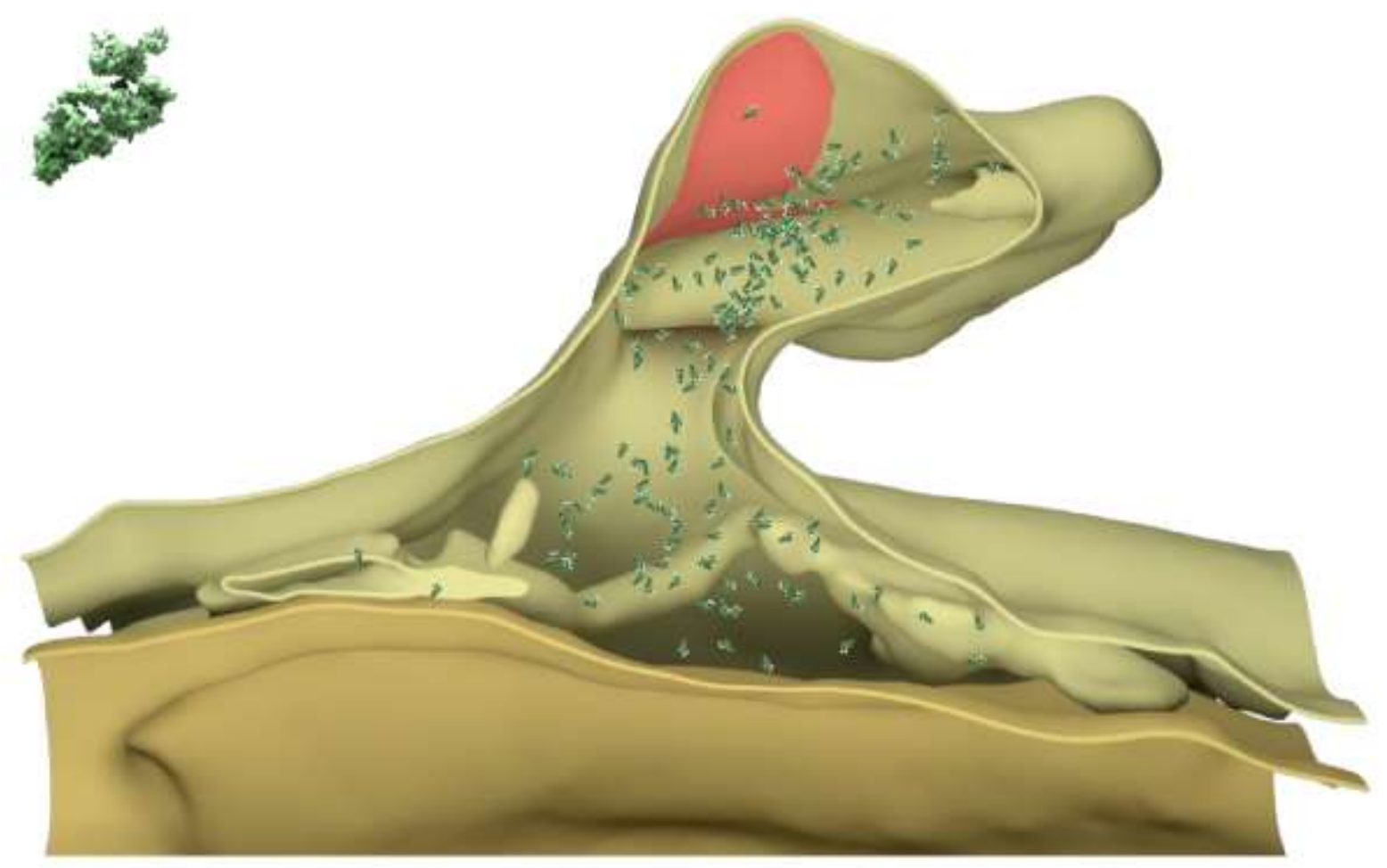

\section{References}

Antibody: Synaptic Systems 162202

PDB Identifier: modified Shank1

\section{Literature:}

Boeckers et al., 1999b; Dani et al., 2010; Hayashi et al., 2006, 2009; MacGillavry et al., 2013; Naisbitt et al., 1999; Tao-Cheng et al., 2015; Tu et al., 1999; Uchino et al., 2006; Valtschanoff and Weinberg, 2001 


\section{Shank3 (ProSAP2 Gene: Shank2, Uniprot ID: Q9JLU4)}

Known function: Links the PSD to the cytoskeleton

Known organization: Cytosolic, Selfmultimerizes, Forms mesh like structure with Homer proteins, Axially more distal to PSD than PSD95, Forms clusters Known Interactions: Homer proteins, DLGAP1, Cortactin, GluR1
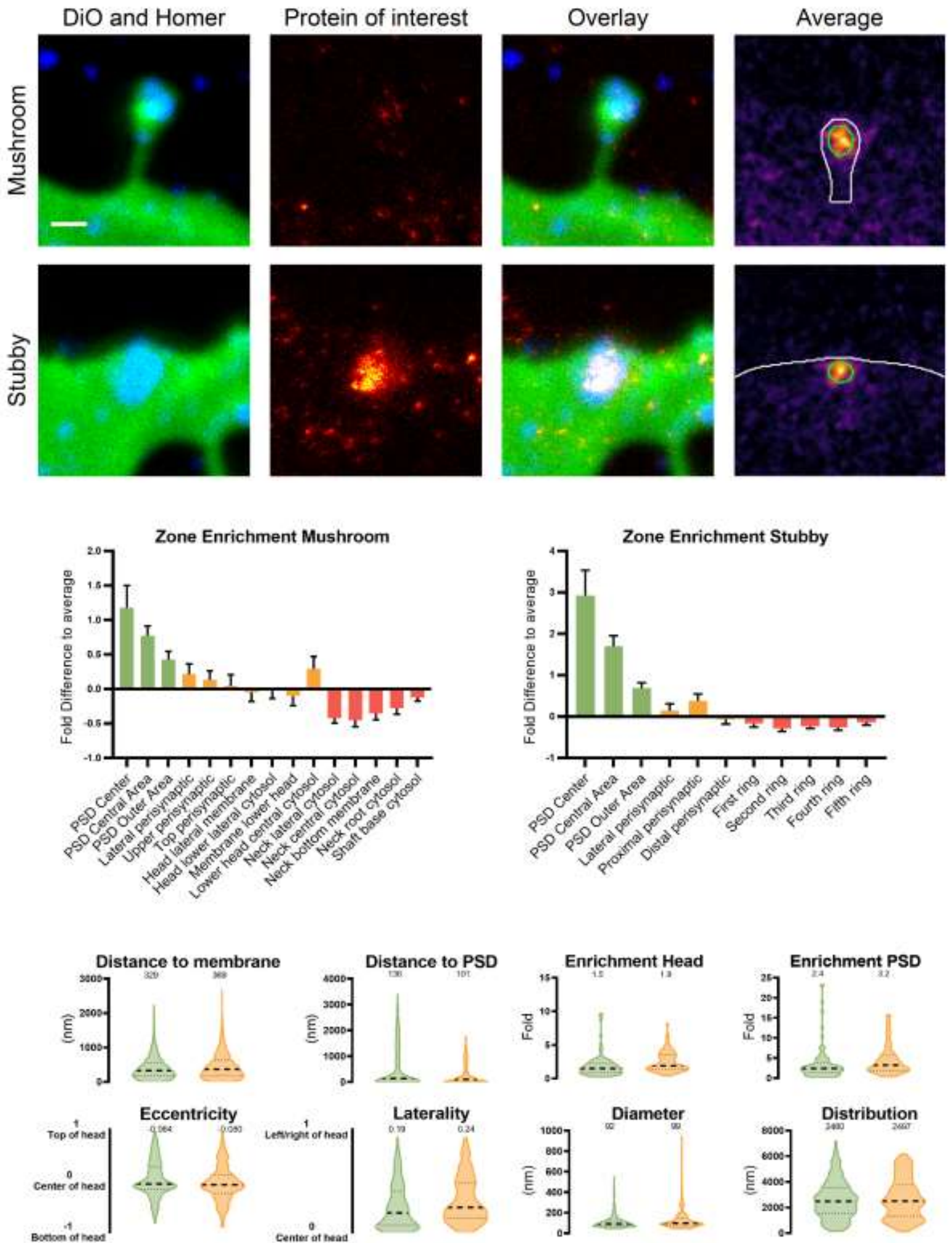

Figure 36: Shank3 nanoscale localization and abundance. Continued on next page. 

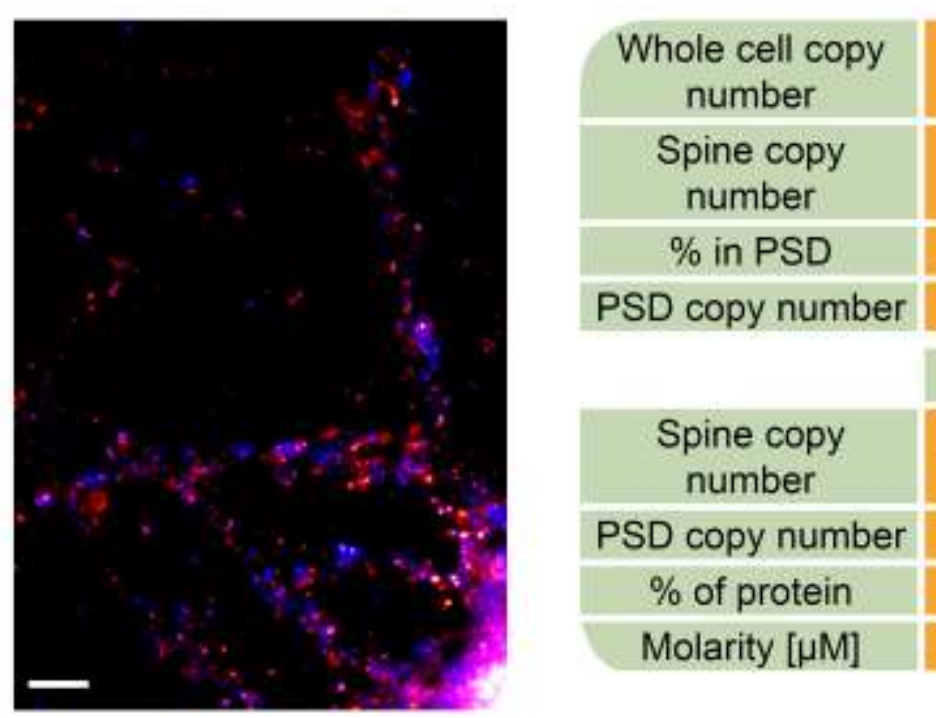

\begin{tabular}{|c|}
\hline $51944.33 \pm 7495.61$ \\
\hline $130.83 \pm 12.92$ \\
49.89 \\
\hline $43.54 \pm 4.30$ \\
\hline
\end{tabular}

\begin{tabular}{|c|c|c|}
\hline $\begin{array}{c}\text { Spine copy } \\
\text { number }\end{array}$ & Mushroom & Stubby \\
\hline PSD copy number & $368.44 \pm 10.71$ & $159.51 \pm 15.75$ \\
\hline$\%$ of protein & $0.05 \pm 0.00$ & $0.04 \pm 0.00$ \\
\hline Molarity $[\mu \mathrm{M}]$ & $0.91 \pm 0.09$ & $0.86 \pm 0.08$ \\
\hline
\end{tabular}

Mushroom molecular model

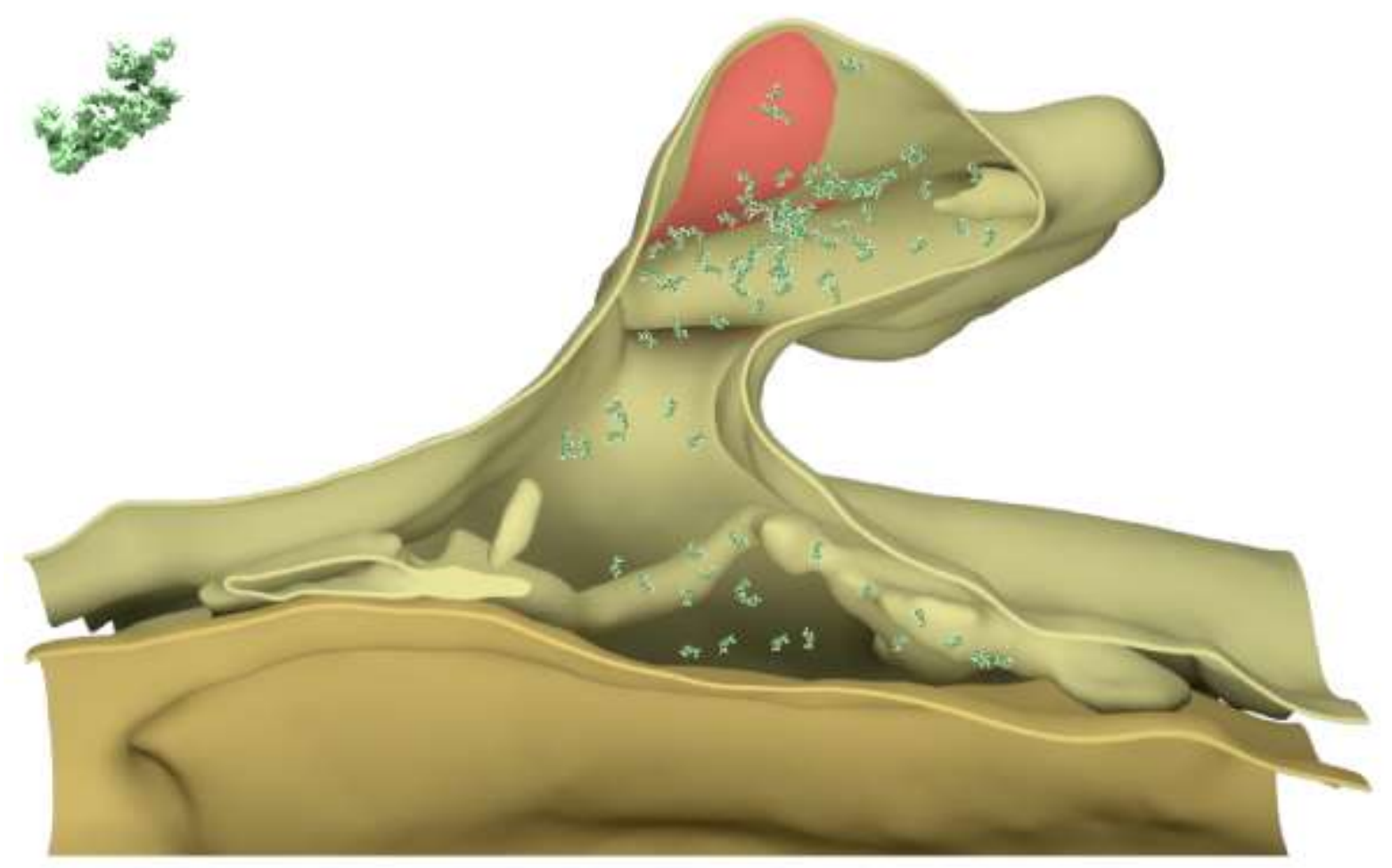

\section{References}

Antibody: Synaptic Systems 162302

PDB Identifier: $5 q 4 x$

\section{Literature:}

Boeckers et al., 1999b; Dani et al., 2010; Hayashi et al., 2006, 2009; MacGillavry et al., 2013; Naisbitt et al., 1999; Tao-Cheng et al., 2015; Tu et al., 1999; Uchino et al., 2006; Valtschanoff and Weinberg, 2001 


\section{Receptors}

The most important function of dendritic spines is the recognition of neurotransmitter. This is brought about by several different types of receptors, AMPAR, NMDAR, kainate and metabotropic glutamate receptors. Several other receptor types exist on dendritic spines, here I investigated also dopamine and neurotrophin receptors.

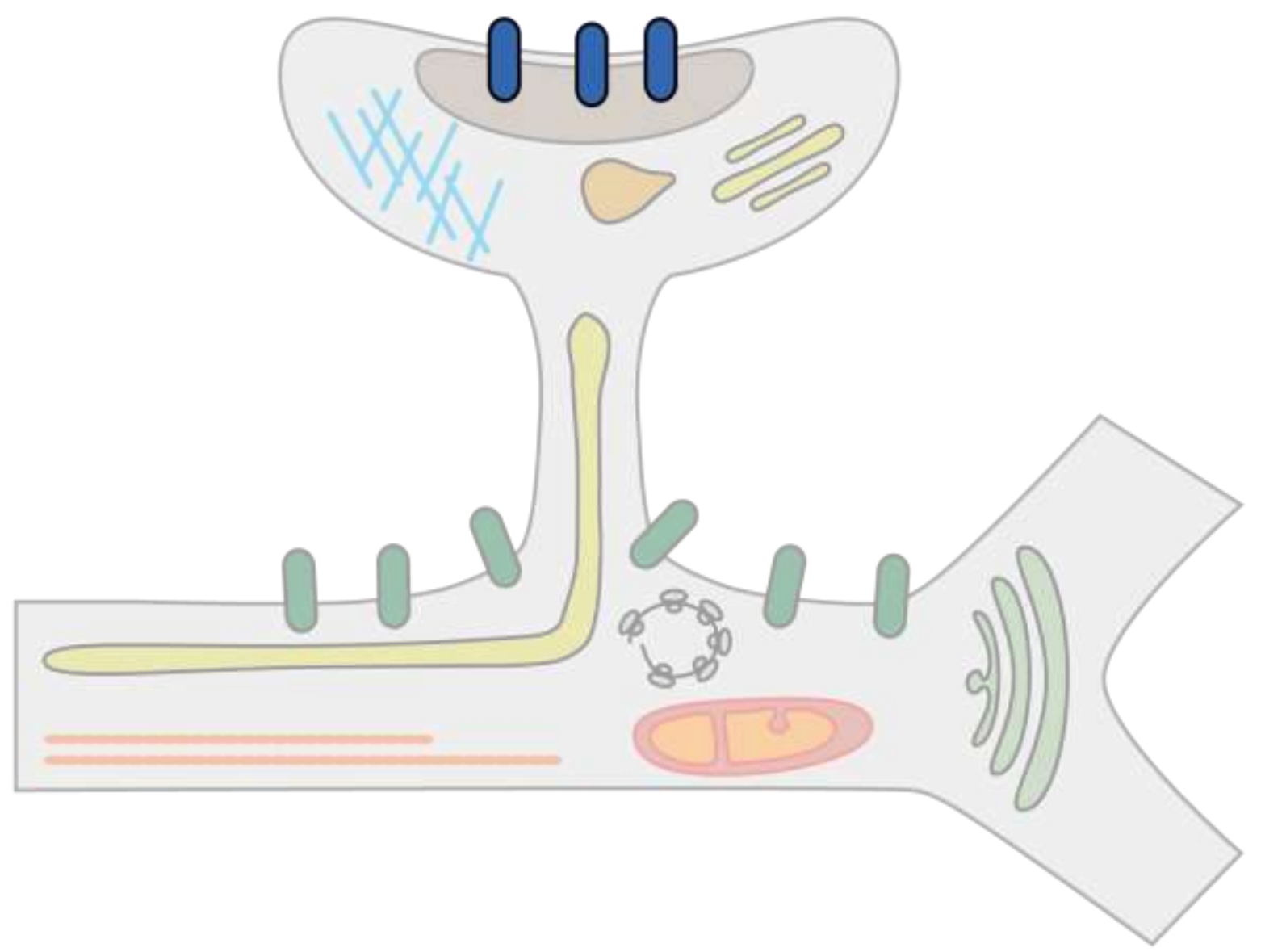

Figure 37: Depiction of receptors in dendritic spines. 


\section{Dopamine Receptor D1 (Gene: Drd1, Uniprot ID: P18901)}

Known function: Activation of Adenylyl cyclase, Enhances NMDAR response

Known organization: Transmembrane proteine, Present in clusters, Predominantly on shaft and perisynaptic regions

Known Interactions: Dopamine Receptor D2, NMDA Receptors
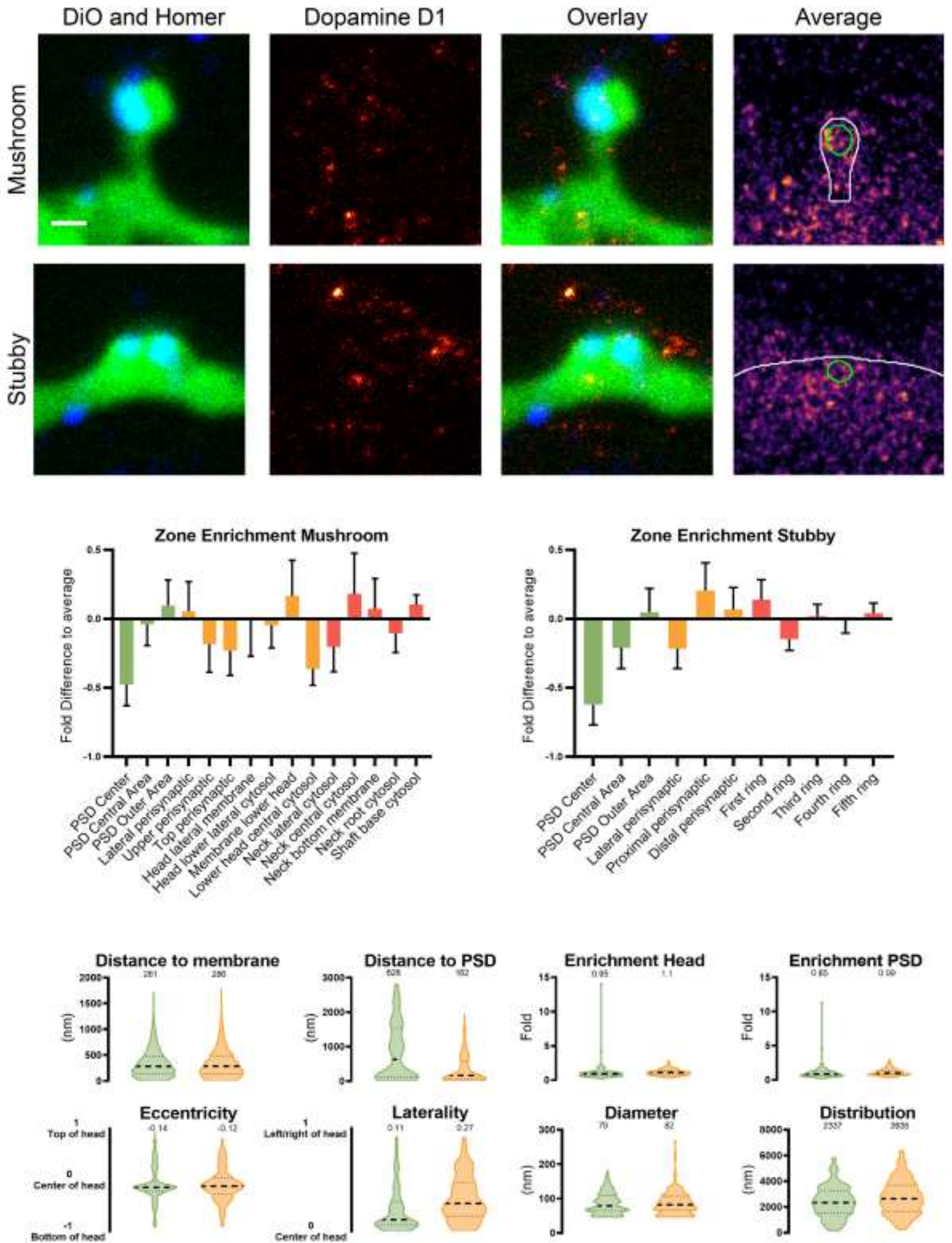

Figure 38: Dopamine Receptor D1 nanoscale localization and abundance. Continued on next page. 


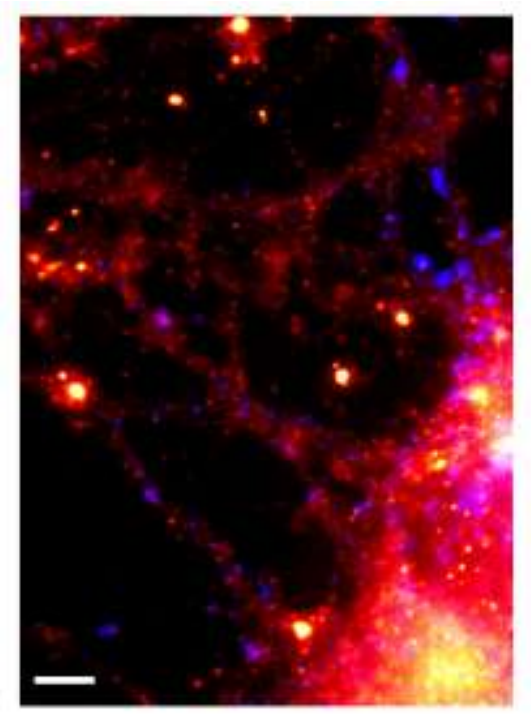

\begin{tabular}{|c|c|c|}
\hline $\begin{array}{c}\text { Whole cell copy } \\
\text { number }\end{array}$ & \multicolumn{2}{|c|}{ not detected } \\
\hline $\begin{array}{c}\text { Spine copy } \\
\text { number }\end{array}$ & \multicolumn{2}{|c|}{ na } \\
\hline$\%$ in PSD & \multicolumn{2}{|c|}{ na } \\
\hline PSD copy number & \multicolumn{2}{|c|}{ na } \\
\hline \begin{tabular}{c|c|} 
Spine copy \\
number
\end{tabular} & na & na \\
\hline PSD copy number & na & na \\
\hline$\%$ of protein & na & na \\
\hline Molarity $[\mu M]$ & na & na \\
\hline
\end{tabular}

\section{References}

Antibody: Abcam ab40653

PDB Identifier: $6 \mathrm{~cm} 4$

\section{Literature:}

Agnati et al., 2016; Beaulieu et al., 2015; Ladepeche et al., 2013a, 2013b; Lee et al., 2002; Pei, 2004 


\section{Dopamine Receptor D2 (Gene: Drd2, Uniprot ID: P61169)}

Known function: Inhibition of Adenylyl cyclase, Decreases NMDAR response and GluR1 insertion

Known organization: Transmembrane proteine, Close to or within PSD

Known Interactions: Other Dopamine receptor isoforms, NMDA receptors
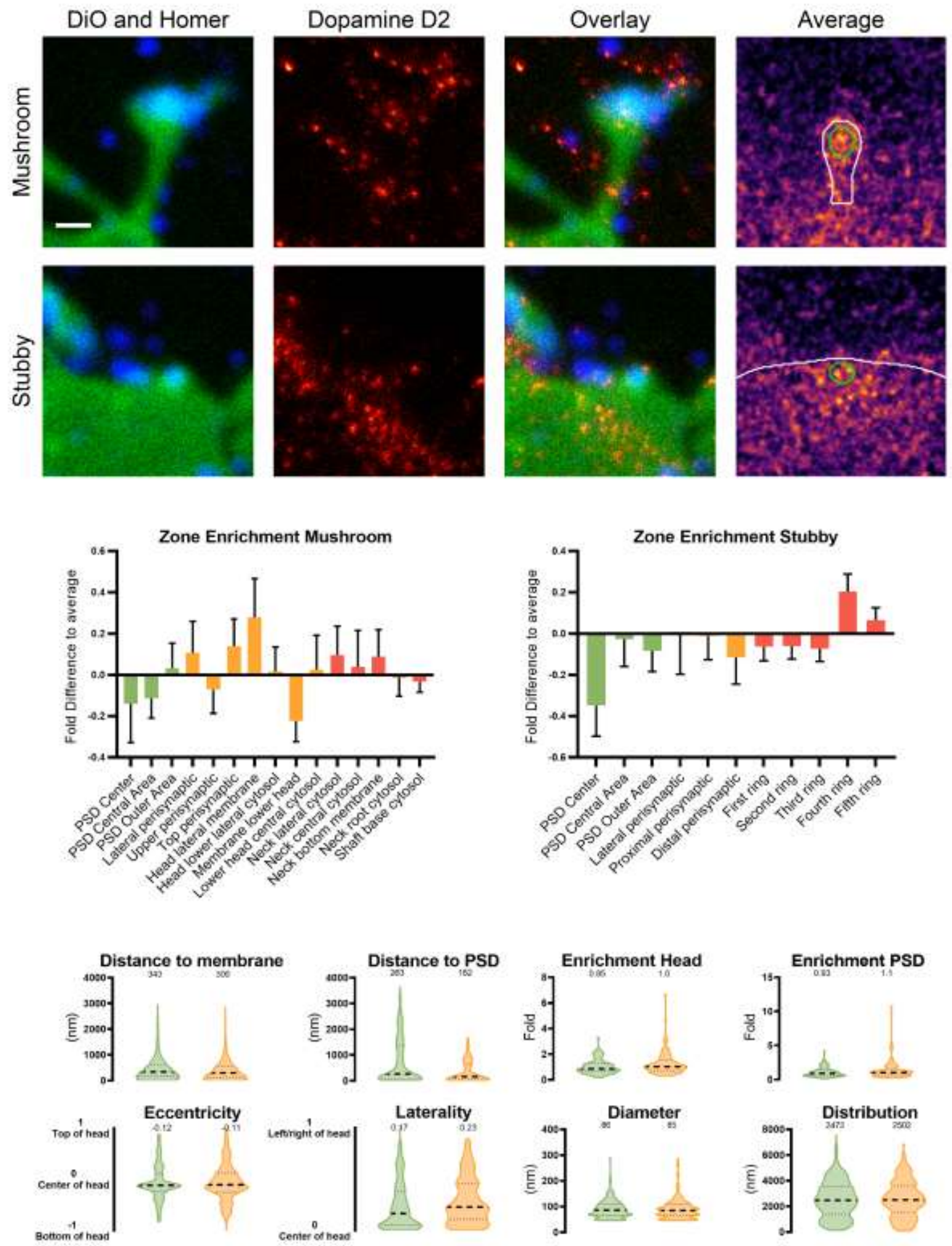

Figure 39: Dopamine Receptor D2 nanoscale localization and abundance. Continued on next page. 


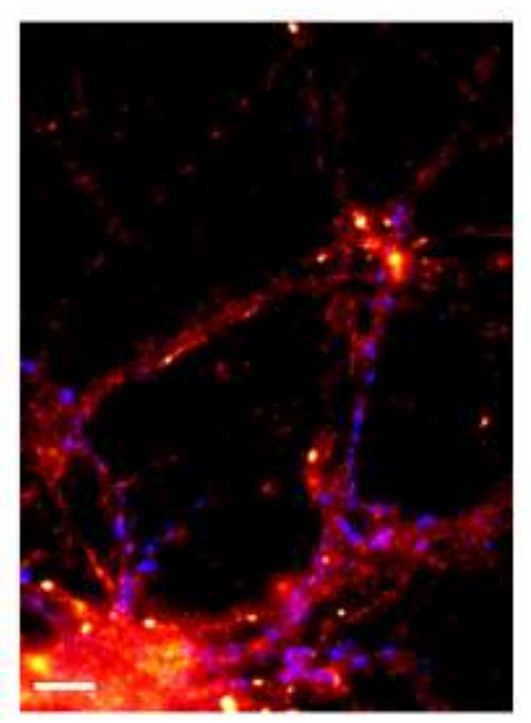

\begin{tabular}{|c|c|c|}
\hline $\begin{array}{c}\text { Whole cell copy } \\
\text { number }\end{array}$ & \multicolumn{2}{|c|}{ not detected } \\
\hline $\begin{array}{c}\text { Spine copy } \\
\text { number }\end{array}$ & \multicolumn{2}{|c|}{ na } \\
\hline$\%$ in PSD & \multicolumn{2}{|c|}{ na } \\
\hline PSD copy number & \multicolumn{2}{|c|}{ na } \\
\hline \begin{tabular}{c|c|} 
Spine copy \\
number
\end{tabular} & na & na \\
\hline PSD copy number & na & na \\
\hline$\%$ of protein & na & na \\
\hline Molarity $[\mu M]$ & na & na \\
\hline
\end{tabular}

\section{References}

Antibody: Merck Millipore AB5084P

PDB Identifier: $6 \mathrm{~cm} 4$

\section{Literature:}

Agnati et al., 2016; Beaulieu et al., 2015; Garzón et al., 2013; Liu et al., 2006; Sun et al., 2005 
GluK1 (GluR5, Grik1, Gene: Grik1, Uniprot ID: P22756)

Known function: Involved in excitatory transmission, Limited role in plasticity

Known organization: Transmembrane protein

Known Interactions: PSD95
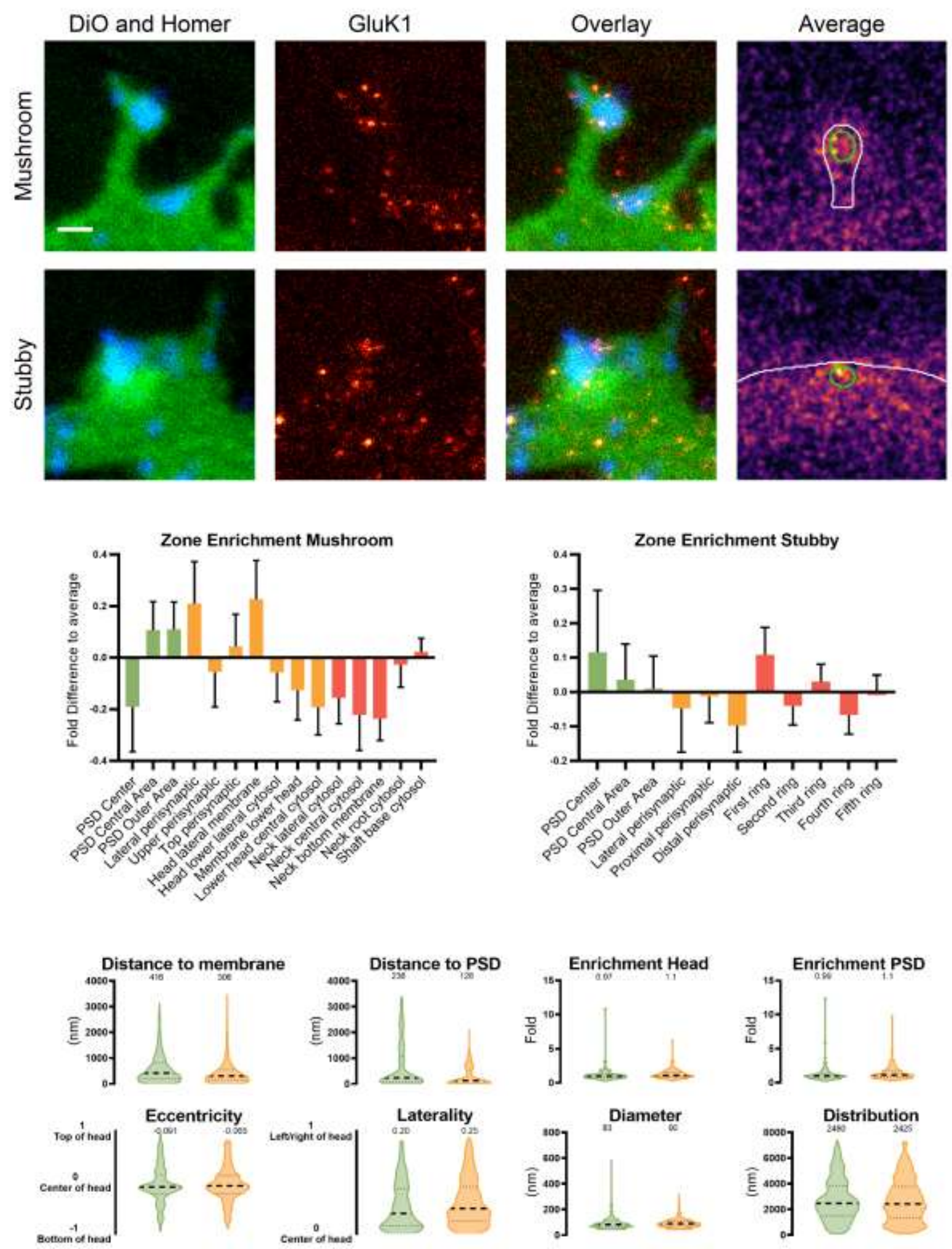

Figure 40: GluK1 nanoscale localization and abundance. Continued on next page. 


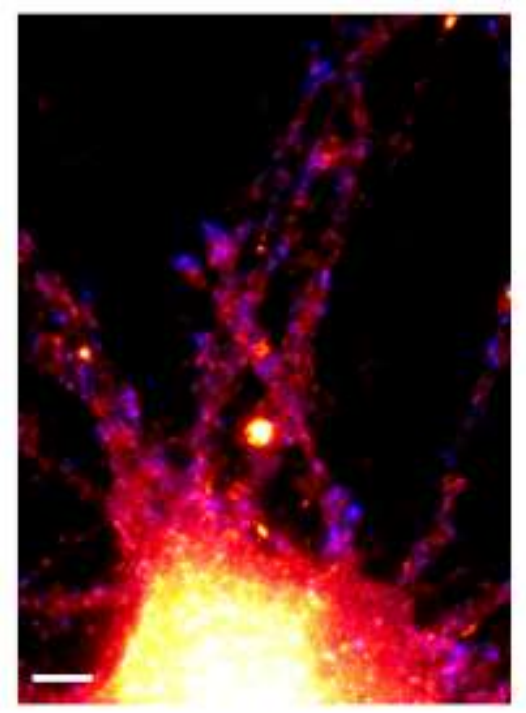

\begin{tabular}{|c|c|c|}
\hline $\begin{array}{c}\text { Whole cell copy } \\
\text { number }\end{array}$ & \multicolumn{2}{|c|}{ not detected } \\
\hline $\begin{array}{c}\text { Spine copy } \\
\text { number }\end{array}$ & \multicolumn{2}{|c|}{ na } \\
\hline$\%$ in PSD & \multicolumn{2}{|c|}{ na } \\
\hline PSD copy number & \multicolumn{2}{|c|}{ na } \\
\hline \begin{tabular}{c|c|} 
Spine copy \\
number
\end{tabular} & na & na \\
\hline PSD copy number & na & na \\
\hline$\%$ of protein & na & na \\
\hline Molarity $[\mu M]$ & na & na \\
\hline
\end{tabular}

\section{References}

Antibody: Alomone Labs AGC-008

PDB Identifier: 5 kuv

\section{Literature:}

Contractor et al., 2011; Hirbec et al., 2003; Schmitz et al., 2001; Song and Huganir, 2002 
GluN1 (NMDAR1, NR1, Gene: Grin1, Uniprot ID: P35439)

Known function: Critical for synaptic plasticity and learning

Known organization: Transmembrane proteine, Highly concentrated in PSD, Part of supercomplexes with PSD95 and other proteins, Often close to dopamine receptors

Known Interactions: mGluR5, Dopamine receptor D1
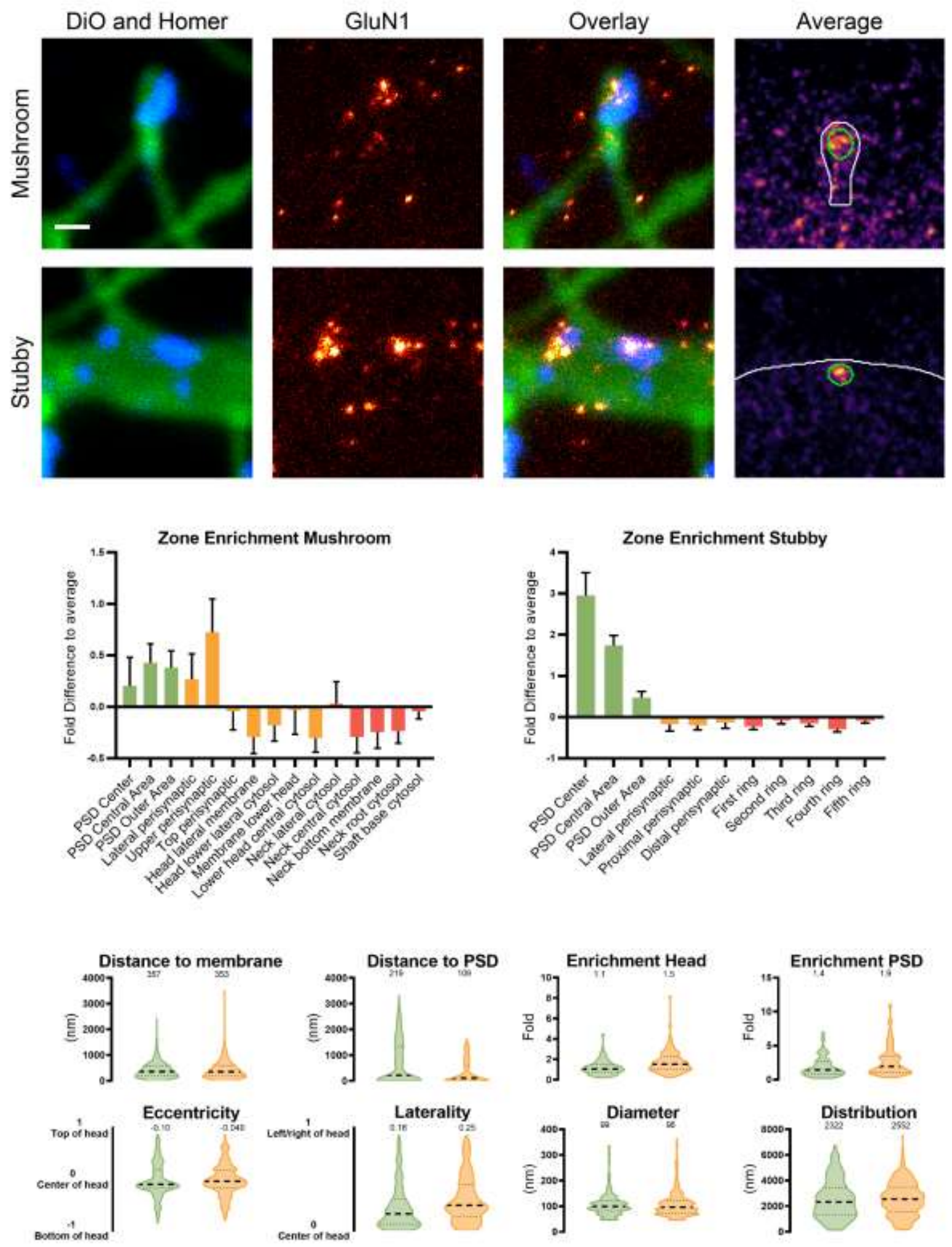

Figure 41: GluN1 nanoscale localization and abundance. Continued on next page. 


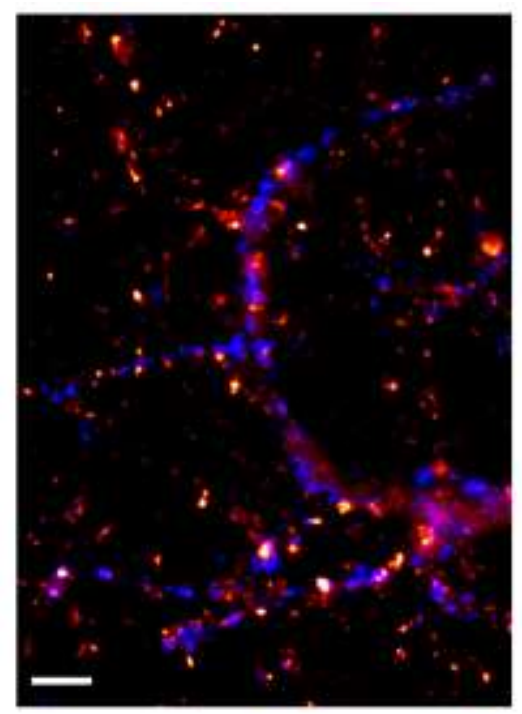

\begin{tabular}{|c|c|}
\hline $\begin{array}{c}\text { Whole cell copy } \\
\text { number }\end{array}$ & $290430.79 \pm 10595.00$ \\
\hline $\begin{array}{c}\text { Spine copy } \\
\text { number }\end{array}$ & $120.70 \pm 11.51$ \\
\hline$\%$ in PSD & 37.10 \\
\hline PSD copy number & $32.66 \pm 3.12$ \\
\hline
\end{tabular}

\begin{tabular}{|c|c|c|}
\hline & Mushroom & Stubby \\
\hline $\begin{array}{c}\text { Spine copy } \\
\text { number }\end{array}$ & $112.01 \pm 10.68$ & $133.67 \pm 12.75$ \\
\hline PSD copy number & $30.31 \pm 2.89$ & $36.17 \pm 3.45$ \\
\hline$\%$ of protein & $0.03 \pm 0.00$ & $0.02 \pm 0.00$ \\
\hline Molarity $[\mu \mathrm{M}]$ & $0.94 \pm 0.09$ & $0.72 \pm 0.07$ \\
\hline
\end{tabular}

\section{References}

Antibody: Synaptic Systems 114011

PDB Identifier: 4pe5

\section{Literature:}

Dani et al., 2010; Frank et al., 2016; Lee et al., 2002; MacGillavry et al., 2013; Pei, 2004; Perroy et al., 2008 
GluN2A (NMDAR2A, NR2A, Gene: Grin2a, Uniprot ID: Q00959)

Known function: Critical for synaptic plasticity and learning, Postnatal dominant isoform.

Known organization: Transmembrane proteine, Highly concentrated in PSD, Part of supercomplexes with PSD95 and other proteins, Often close to Dopamine receptors Known Interactions: PSD93, PSD95, Dopamine Receptor D1, Calmodulin, CaMKII
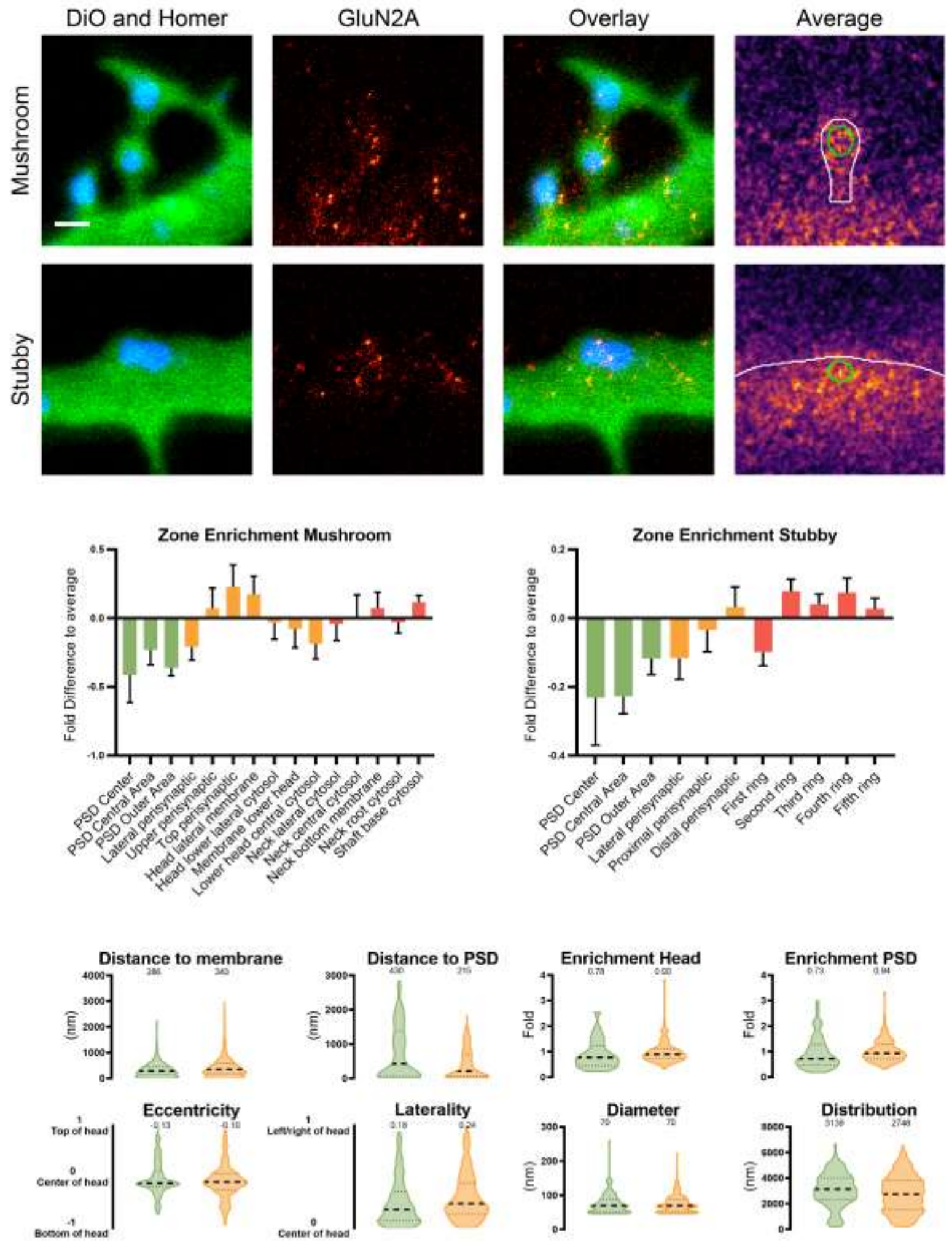

Figure 42: GluN2A nanoscale localization and abundance. Continued on next page. 


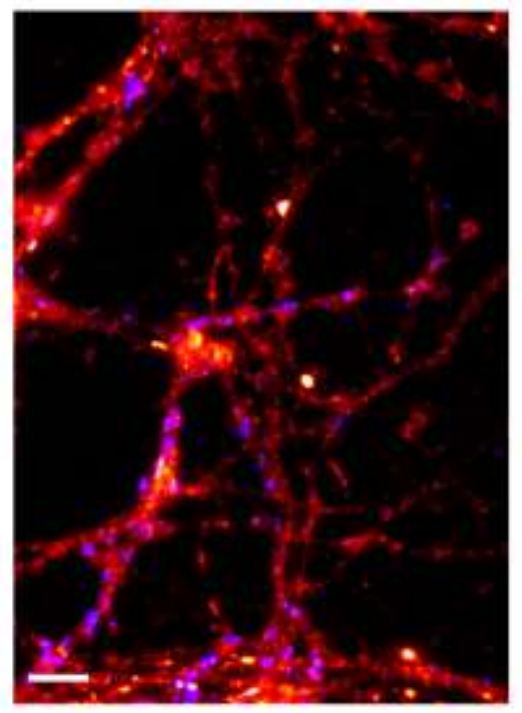

\begin{tabular}{|c|c|c|}
\hline $\begin{array}{c}\text { Whole cell copy } \\
\text { number }\end{array}$ & \multicolumn{2}{|c|}{$\begin{array}{l}23076.60 \\
\text { (only detected in } 1 \text { replicate) }\end{array}$} \\
\hline $\begin{array}{l}\text { Spine copy } \\
\text { number }\end{array}$ & \multicolumn{2}{|c|}{2.43} \\
\hline$\%$ in PSD & \multicolumn{2}{|c|}{15.00} \\
\hline \multirow[t]{2}{*}{ PSD copy number } & \multicolumn{2}{|c|}{0.32} \\
\hline & Mushroom & Stubby \\
\hline $\begin{array}{l}\text { Spine copy } \\
\text { number }\end{array}$ & 2.06 & 2.71 \\
\hline PSD copy number & 0.27 & 0.35 \\
\hline$\%$ of protein & 0.00 & 0.00 \\
\hline Molarity $[\mu \mathrm{M}]$ & 0.02 & 0.01 \\
\hline
\end{tabular}

\section{References}

Antibody: NeuroMab 75-288

PDB Identifier: 5uow

\section{Literature:}

Al-Hallaq et al., 2007; Bard et al., 2010; Ehlers et al., 1996; Elias and Nicoll, 2007; Frank et al., 2016; Krapivinsky et al., 2003; Lee et al., 2002; MacGillavry et al., 2013; Pei, 2004; Racca et al., 2000; Sans et al., 2000; Sheng et al., 1994; Shinohara et al., 2008; Wyszynski et al., 1997 
GluN2B (NR2B, NMDAR2B, Gene: Grin2b, Uniprot ID: Q00960)

Known function: Critical for synaptic plasticity and learning, Prenatal dominant isoform Known organization: Transmembrane proteine, Highly concentrated in PSD, Part of supercomplexes with PSD95 and other proteins, Often colocalized with Dopamine receptors

Known Interactions: PSD93, PSD95, mGluR5, Dopamine Receptor D2, CaM, CaMKII
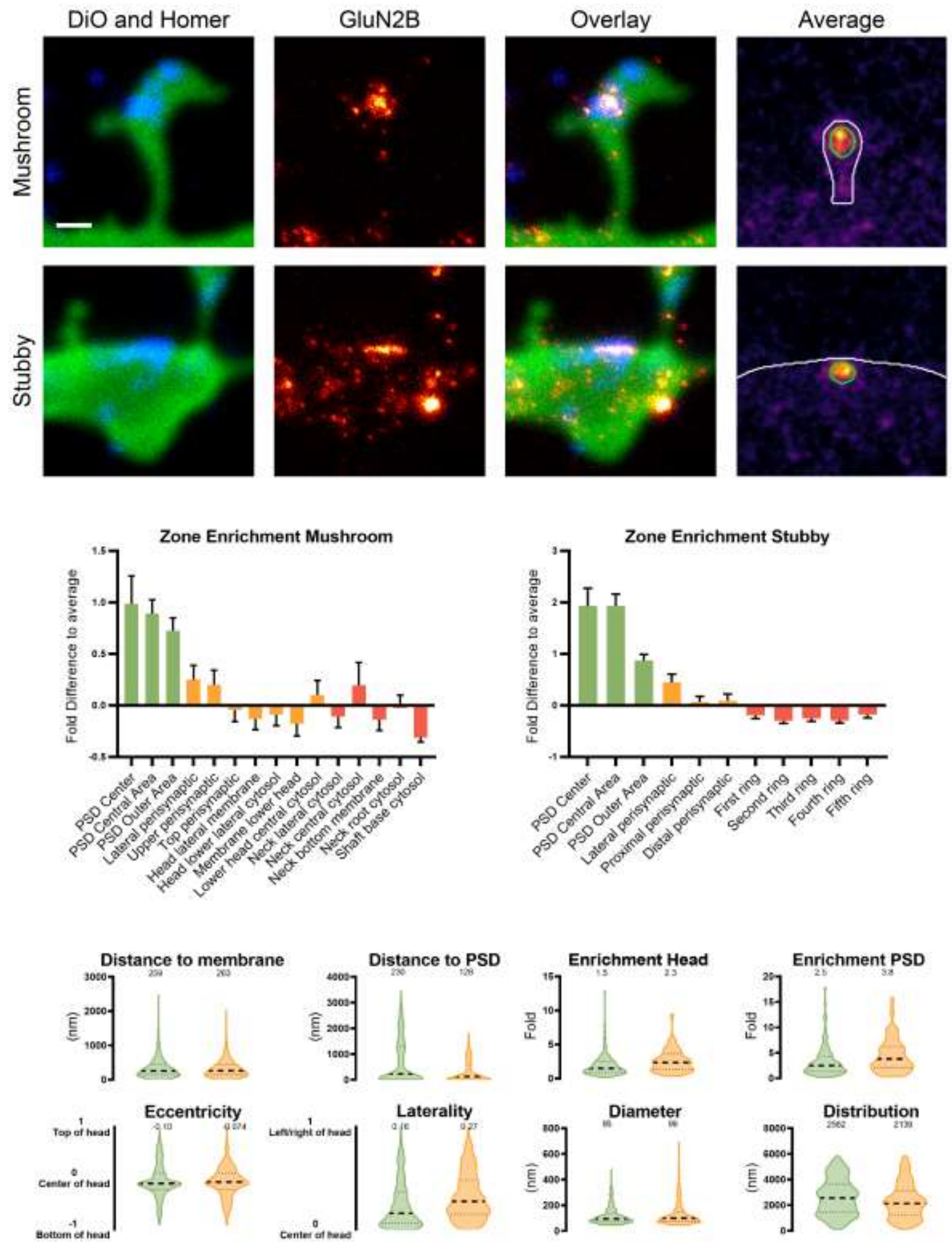

Figure 43: GluN2B nanoscale localization and abundance. Continued on next page. 


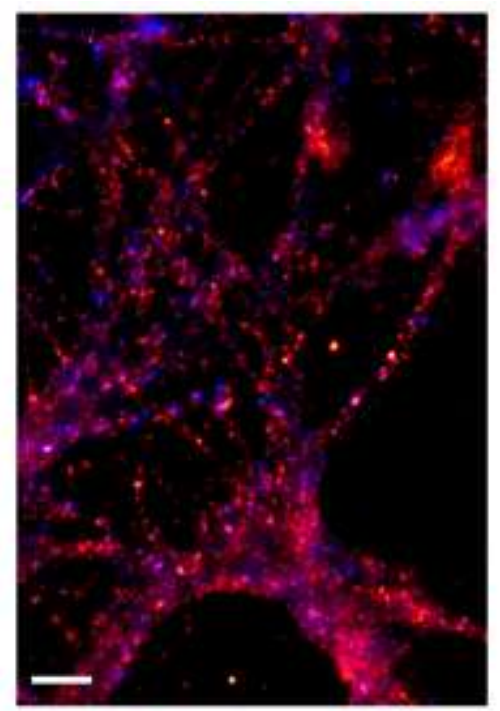

\begin{tabular}{|c|c|}
\hline $\begin{array}{c}\text { Whole cell copy } \\
\text { number }\end{array}$ & $117169.17 \pm 18564.25$ \\
\hline $\begin{array}{c}\text { Spine copy } \\
\text { number }\end{array}$ & $55.07 \pm 9.77$ \\
\hline$\%$ in PSD & 47.07 \\
\hline PSD copy number & $17.63 \pm 3.13$ \\
\hline
\end{tabular}

\begin{tabular}{|c|c|c|}
\hline $\begin{array}{c}\text { Spine copy } \\
\text { number }\end{array}$ & Mushroom & Stubby \\
\hline PSD copy number & $15.24 \pm 2.71$ & $21.51 \pm 3.82$ \\
\hline$\%$ of protein & $0.02 \pm 0.00$ & $0.02 \pm 0.00$ \\
\hline Molarity [ $\mu \mathrm{M}]$ & $0.40 \pm 0.07$ & $0.36 \pm 0.06$ \\
\hline
\end{tabular}

\section{References}

Antibody: Neuromab 75-101

PDB Identifier: $6 \mathrm{cna}$

\section{Literature:}

Al-Hallaq et al., 2007; Bard et al., 2010; Dani et al., 2010; Ehlers et al., 1996; Elias and Nicoll, 2007; Frank et al., 2016; Halt et al., 2012; Krapivinsky et al., 2003; Liu et al., 2006; MacGillavry et al., 2013; Perroy et al., 2008; Racca et al., 2000; Sans et al., 2000; Sheng et al., 1994; Shinohara et al., 2008; Wyszynski et al., 1997; Zhang and Diamond, 2009; Zhou et al., 2007 


\section{GluR1 (GluA1, Gene: Gria1, Uniprot ID: P19490)}

Known function: Part of primary glutamate receptor, Important for plasticity, Rapidly delivered to synapse upon activation, Stable localization at synapse

Known organization: Transmembrane proteine, Heterotetramers, Forms $80 \mathrm{~nm}$ clusters, Known Interactions: None
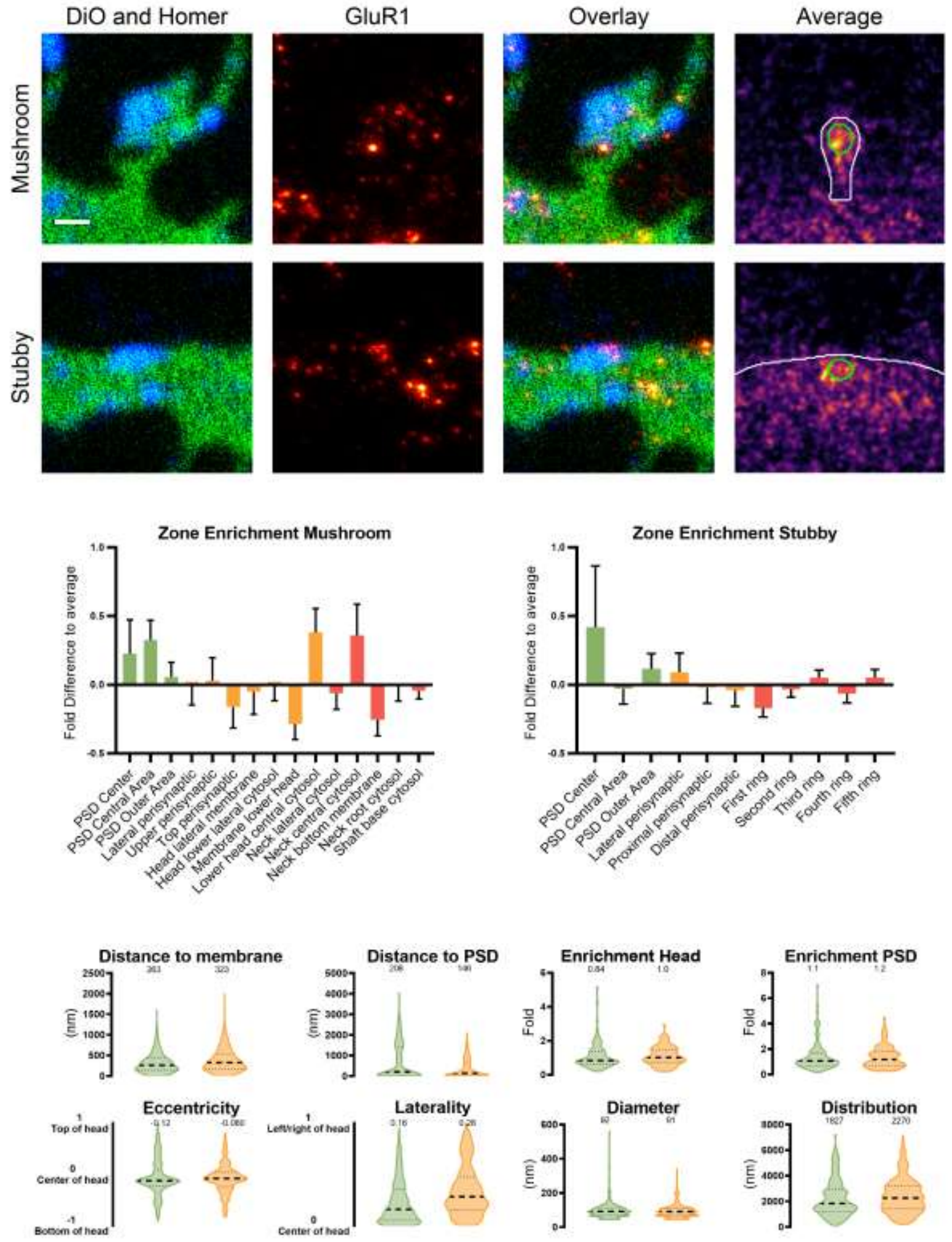

Figure 44: GluR1 nanoscale localization and abundance. Continued on next page. 


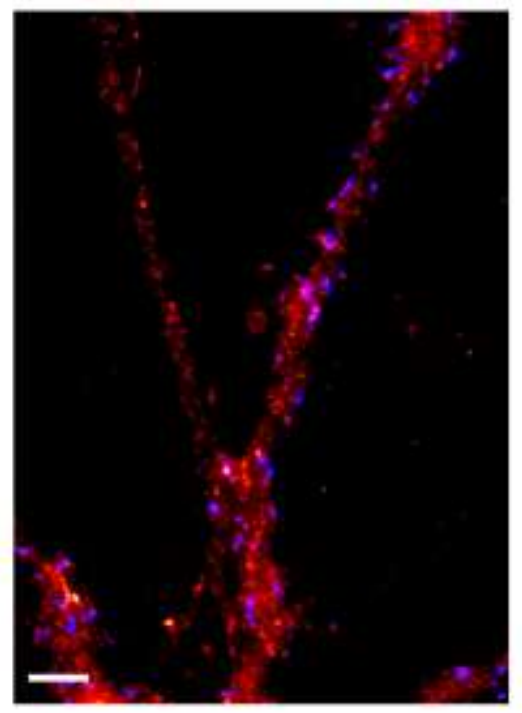

\begin{tabular}{|c|c|c|}
\hline $\begin{array}{c}\text { Whole cell copy } \\
\text { number }\end{array}$ & \multicolumn{2}{|c|}{$275371.42 \pm 23163.08$} \\
\hline $\begin{array}{c}\text { Spine copy } \\
\text { number }\end{array}$ & \multicolumn{2}{|c|}{$79.24 \pm 12.62$} \\
\hline$\%$ in PSD & \multicolumn{2}{|c|}{23.49} \\
\hline PSD copy number & \multicolumn{2}{|c|}{$15.07 \pm 2.40$} \\
\hline & Mushroom & Stubby \\
\hline $\begin{array}{c}\text { Spine copy } \\
\text { number }\end{array}$ & $63.78 \pm 10.16$ & $100.18 \pm 15.96$ \\
\hline PSD copy number & $12.13 \pm 1.93$ & $19.06 \pm 3.04$ \\
\hline$\%$ of protein & $0.01 \pm 0.00$ & $0.02 \pm 0.00$ \\
\hline Molarity [MM] & $0.53 \pm 0.09$ & $0.54 \pm 0.09$ \\
\hline
\end{tabular}

\section{References}

Antibody: Synaptic Systems 182003

PDB Identifier: modified GluR2

\section{Literature:}

MacGillavry et al., 2013; Nair et al., 2013 


\section{GluR2 (GluA2, Gene: Gria2, Uniprot ID: P19491)}

Known function: Part of primary glutamate receptor, Important for plasticity

Known organization: Transmembrane proteine, Heterotetramers, Forms $80 \mathrm{~nm}$ clusters

Known Interactions: GRIP, NSF, alpha-SNAP, beta-SNAP, Syntaxin 13
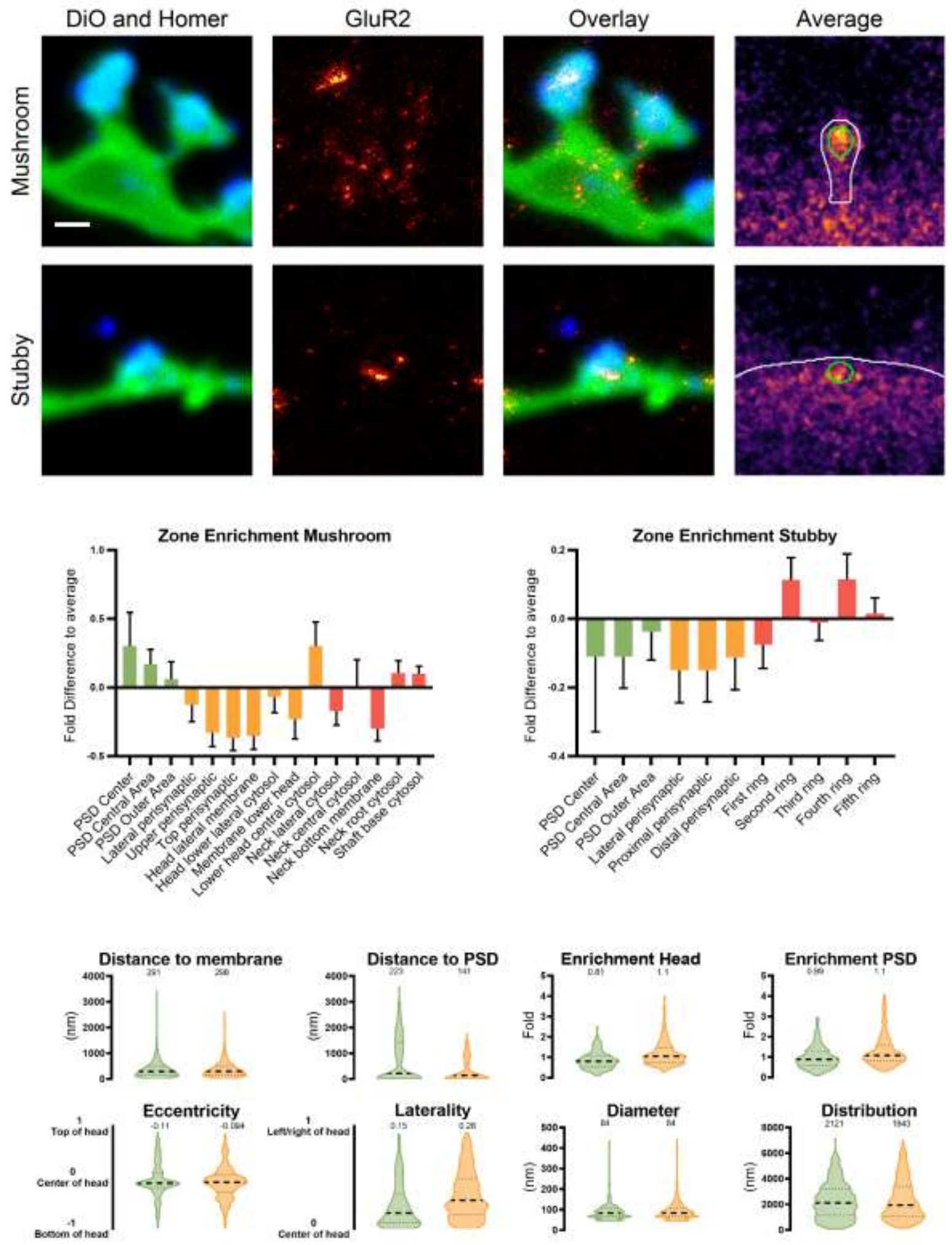

Figure 45: GluR2 nanoscale localization and abundance. Continued on next page. 


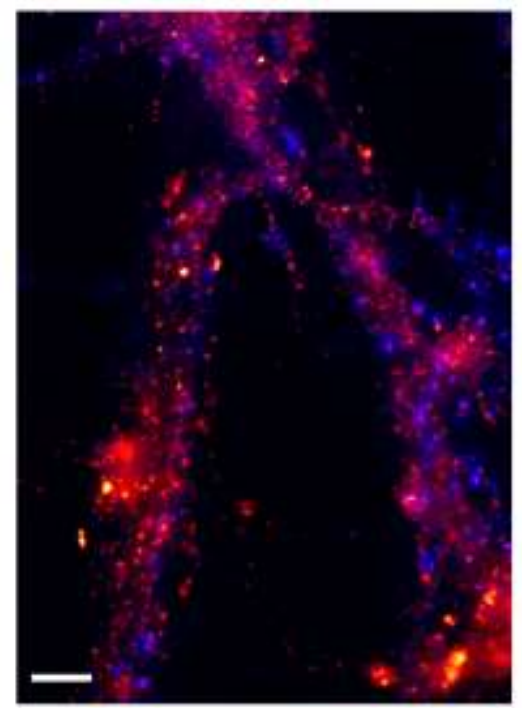

\begin{tabular}{|c|c|c|}
\hline $\begin{array}{c}\text { Whole cell copy } \\
\text { number }\end{array}$ & \multicolumn{2}{|c|}{$1447843.11 \pm 131972.07$} \\
\hline $\begin{array}{c}\text { Spine copy } \\
\text { number }\end{array}$ & \multicolumn{2}{|c|}{$372.01 \pm 53.93$} \\
\hline$\%$ in PSD & \multicolumn{2}{|c|}{$58.34 \pm 8.46$} \\
\hline PSD copy number & \multicolumn{2}{|c|}{ Stubby } \\
\hline & Mushroom & $480.63 \pm 69.67$ \\
\hline $\begin{array}{c}\text { Spine copy } \\
\text { number }\end{array}$ & $303.87 \pm 44.05$ & $45.38 \pm 10.93$ \\
\hline PSD copy number & $47.66 \pm 6.91$ & $0.07 \pm 0.01$ \\
\hline$\%$ of protein & $0.07 \pm 0.01$ & $0.58 \pm 0.37$ \\
\hline Molarity $[\mu M]$ & $2.55 \pm 0.37$ & 2.58 \\
\hline
\end{tabular}

\section{References}

Antibody: Alomone Labs AGC-005

PDB Identifier: $511 \mathrm{~h}$

\section{Literature:}

Hirbec et al., 2003; Kato et al., 2008; Lu and Ziff, 2005; MacGillavry et al., 2013; Nair et al., 2013; Osten et al., 1998 


\section{GluR3 (GluA3, Gene: Gria3, Uniprot ID: P19492)}

Known function: Part of primary glutamate receptor, Important for plasticity,

Constitutively trafficks between synaptic membrane and endosomes

Known organization: Transmembrane proteine, Heterotetramers, Forms $80 \mathrm{~nm}$ clusters Known Interactions: GRIP
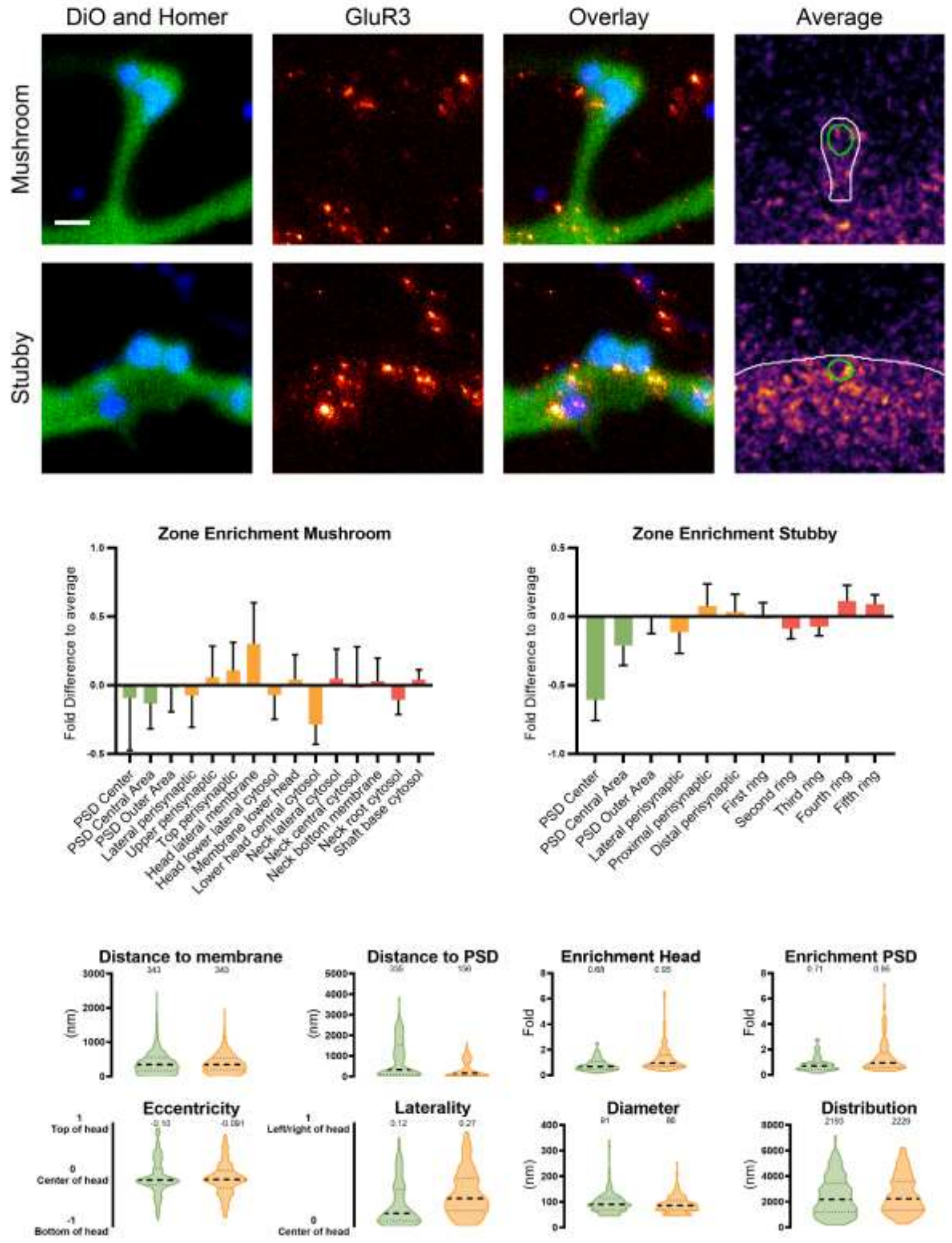

Figure 46: GluR3 nanoscale localization and abundance. Continued on next page. 


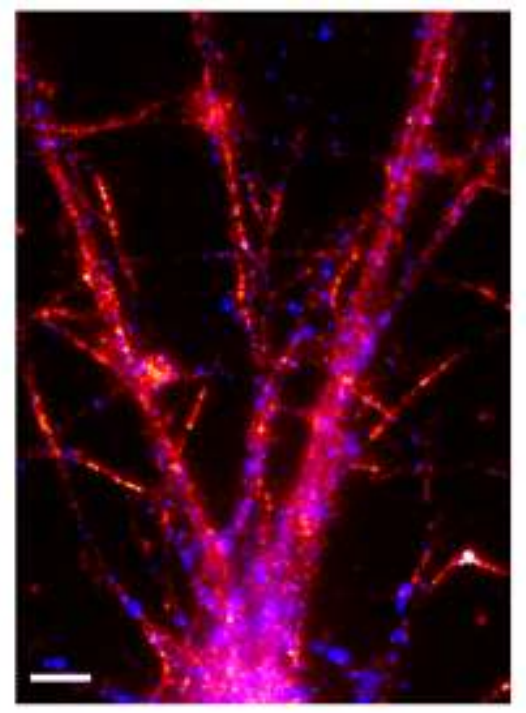

\begin{tabular}{|c|c|c|}
\hline $\begin{array}{c}\text { Whole cell copy } \\
\text { number }\end{array}$ & \multicolumn{2}{|c|}{$88892.02 \pm 14641.22$} \\
\hline $\begin{array}{c}\text { Spine copy } \\
\text { number }\end{array}$ & \multicolumn{2}{|c|}{$9.05 \pm 3.13$} \\
\hline \% in PSD & \multicolumn{2}{|c|}{13.90} \\
\hline PSD copy number & \multicolumn{2}{|c|}{$1.10 \pm 0.38$} \\
\hline & Mushroom & Stubby \\
\hline $\begin{array}{c}\text { Spine copy } \\
\text { number }\end{array}$ & $6.69 \pm 2.31$ & $11.75 \pm 4.06$ \\
\hline PSD copy number & $0.82 \pm 0.28$ & $1.43 \pm 0.50$ \\
\hline$\%$ of protein & $0.00 \pm 0.00$ & $0.00 \pm 0.00$ \\
\hline Molarity $[\mu M]$ & $0.06 \pm 0.02$ & $0.06 \pm 0.02$ \\
\hline
\end{tabular}

\section{References}

Antibody: Invitrogen 32-0400

PDB Identifier: modified GluR2

\section{Literature:}

Hirbec et al., 2003; MacGillavry et al., 2013; Nair et al., 2013; Xia et al., 1999 


\section{IGF1R (CD221, Gene: Igf1r, Uniprot ID: P24062)}

Known function: Required for development, growth and survival, Influences lifespan, Potentiates excitatory synaptic transmission, Regulates mitochondria

Known organization: Transmembrane proteine, Homodimers

Known Interactions: None
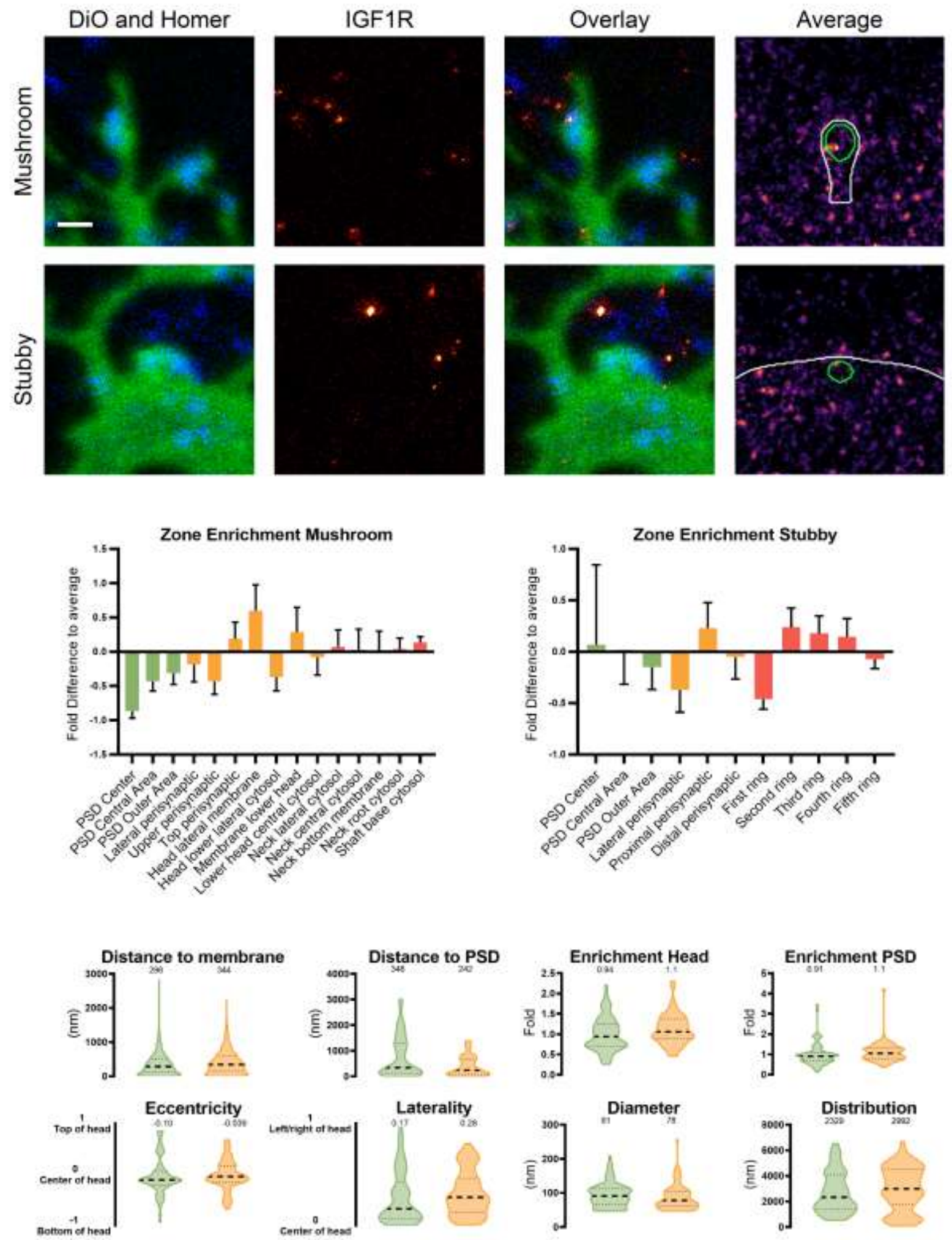

Figure 47: IGF1R nanoscale localization and abundance. Continued on next page. 


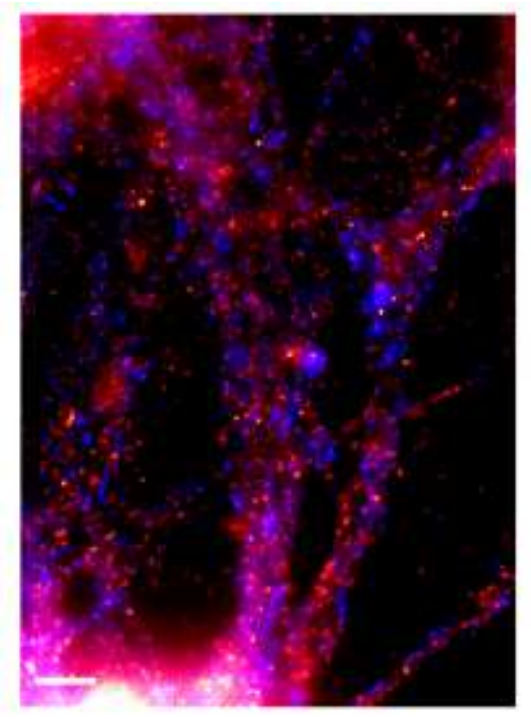

\begin{tabular}{|c|c|c|}
\hline $\begin{array}{c}\text { Whole cell copy } \\
\text { number }\end{array}$ & \multicolumn{2}{|c|}{ not detected } \\
\hline $\begin{array}{c}\text { Spine copy } \\
\text { number }\end{array}$ & \multicolumn{2}{|c|}{ na } \\
\hline \% in PSD & \multicolumn{2}{|c|}{ na } \\
\hline PSD copy number & \multicolumn{2}{|c|}{ na } \\
\hline \begin{tabular}{c|c|} 
Spine copy \\
number
\end{tabular} & na & na \\
\hline PSD copy number & na & na \\
\hline$\%$ of protein & na & na \\
\hline Molarity $[\mu M]$ & na & na \\
\hline
\end{tabular}

\section{References}

Antibody: Cell Signaling 3027

PDB Identifier: $4 z x b$

\section{Literature:}

D'Ercole et al., 1996; Gazit et al., 2016; Kenyon, 2010; Lemmon and Schlessinger, 2010; Ramsey et al., 2005; Suh et al., 2008 


\section{LNGFR (p75NTR Gene: Ngfr, Uniprot ID: P07174)}

Known function: Neurotrophic signaling, Recognizes all neurotrophins, Involved in LTD Known organization: Transmembrane protein

Known Interactions: TrkB, BDNF
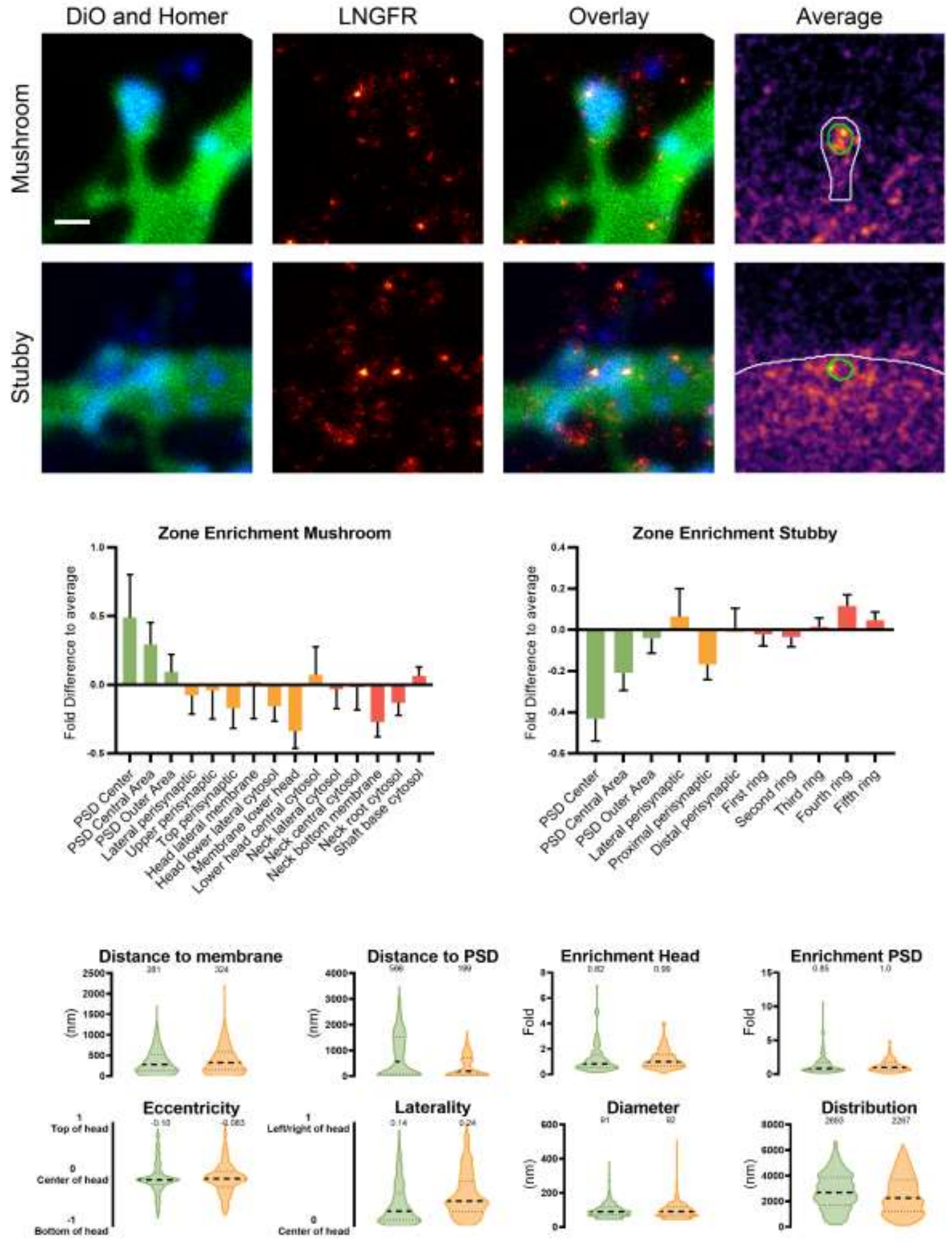

Figure 48: LNGFR nanoscale localization and abundance. Continued on next page. 


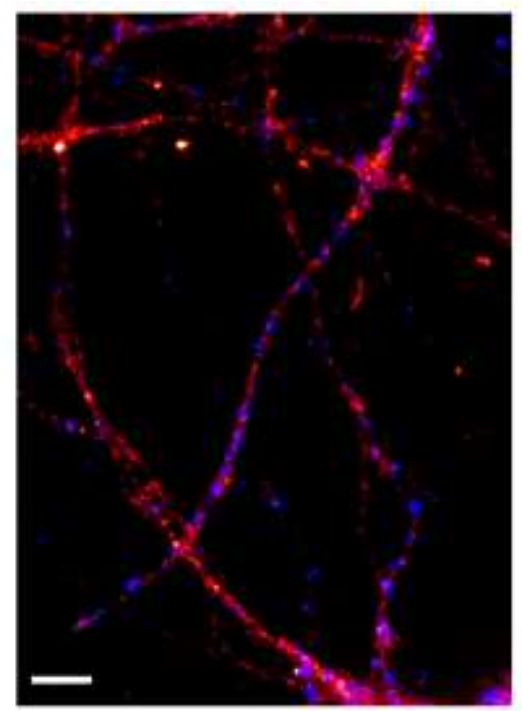

\begin{tabular}{|c|c|c|}
\hline $\begin{array}{c}\text { Whole cell copy } \\
\text { number }\end{array}$ & \multicolumn{2}{|c|}{ not detected } \\
\hline $\begin{array}{l}\text { Spine copy } \\
\text { number }\end{array}$ & \multicolumn{2}{|c|}{ na } \\
\hline$\%$ in PSD & \multicolumn{2}{|c|}{ na } \\
\hline \multirow[t]{2}{*}{ PSD copy number } & \multicolumn{2}{|c|}{ na } \\
\hline & Mushroom & Stubby \\
\hline $\begin{array}{l}\text { Spine copy } \\
\text { number }\end{array}$ & na & na \\
\hline PSD copy number & na & na \\
\hline$\%$ of protein & na & na \\
\hline Molarity $[\mu \mathrm{M}]$ & na & na \\
\hline
\end{tabular}

\section{References}

Antibody: Cell Signaling 8238

PDB Identifier: $2 \mathrm{mic}, 4 \mathrm{f} 44$, 3buk

\section{Literature:}

Barker, 1998; Ernfors et al., 1990; Leal et al., 2015; Rodriguez-Tébar et al., 1990; Yang et al., 2009,2014 
mAChR1 (HM1, Gene: Chrm1, Uniprot ID: P08482)

Known function: Activates PLC, Regulates intracellular calcium release, Reduces potassium conductance

Known organization: Transmembrane protein, Enriched at dendrites and PSDs

Known Interactions: $G_{q}$ coupled
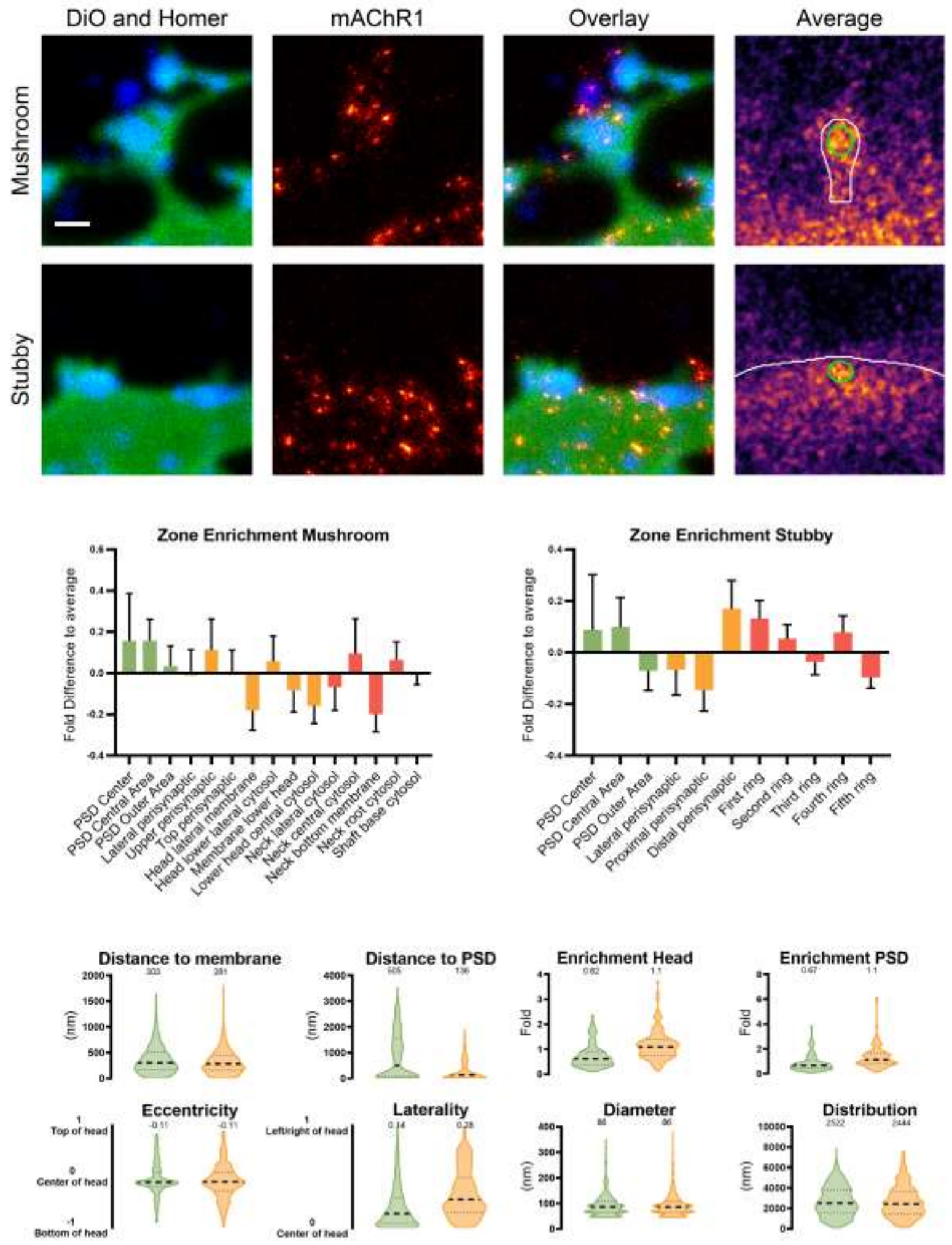

Figure 49: mAChR1 nanoscale localization and abundance. Continued on next page. 


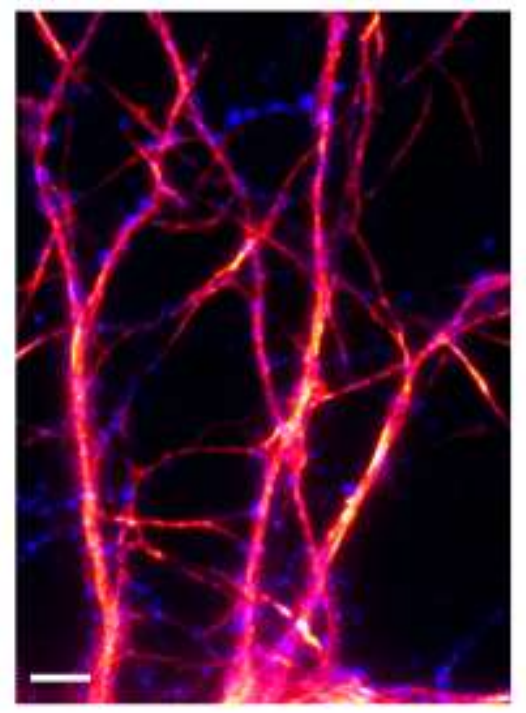

\begin{tabular}{|c|c|}
\hline $\begin{array}{c}\text { Whole cell copy } \\
\text { number }\end{array}$ & $38121.70 \pm 10040.53$ \\
\hline $\begin{array}{c}\text { Spine copy } \\
\text { number }\end{array}$ & $7.76 \pm 2.23$ \\
\hline$\%$ in PSD & 17.99 \\
\hline PSD copy number & $1.18 \pm 0.34$ \\
\hline
\end{tabular}

\begin{tabular}{|c|c|c|}
\hline & Mushroom & Stubby \\
\hline $\begin{array}{l}\text { Spine copy } \\
\text { number }\end{array}$ & $6.21 \pm 1.79$ & $8.78 \pm 2.52$ \\
\hline PSD copy number & $0.95 \pm 0.27$ & $1.34 \pm 0.38$ \\
\hline$\%$ of protein & $0.00 \pm 0.00$ & $0.00 \pm 0.00$ \\
\hline Molarity $[\mu \mathrm{M}]$ & $0.05 \pm 0.01$ & $0.05 \pm 0.01$ \\
\hline
\end{tabular}

\section{References}

Antibody: Novus Biologicals NBP1-87466

PDB Identifier: $5 c x v$

\section{Literature:}

Egorov et al., 2002; Hersch et al., 1994; Smith and Araneda, 2010; Uchimura and North, 1990 


\section{mGluR1 (Gene: Grm1, Uniprot ID: P23385)}

Known function: Activates PKC and PLC, Regulates intracellular calcium release, Involved in LTP and homeostatic plasticity, Activates cannabinoid synthesis Known organization: Transmembrane protein, Perisynaptic

Known Interactions: $G_{q}$ coupled, mGluR5, Homer proteins
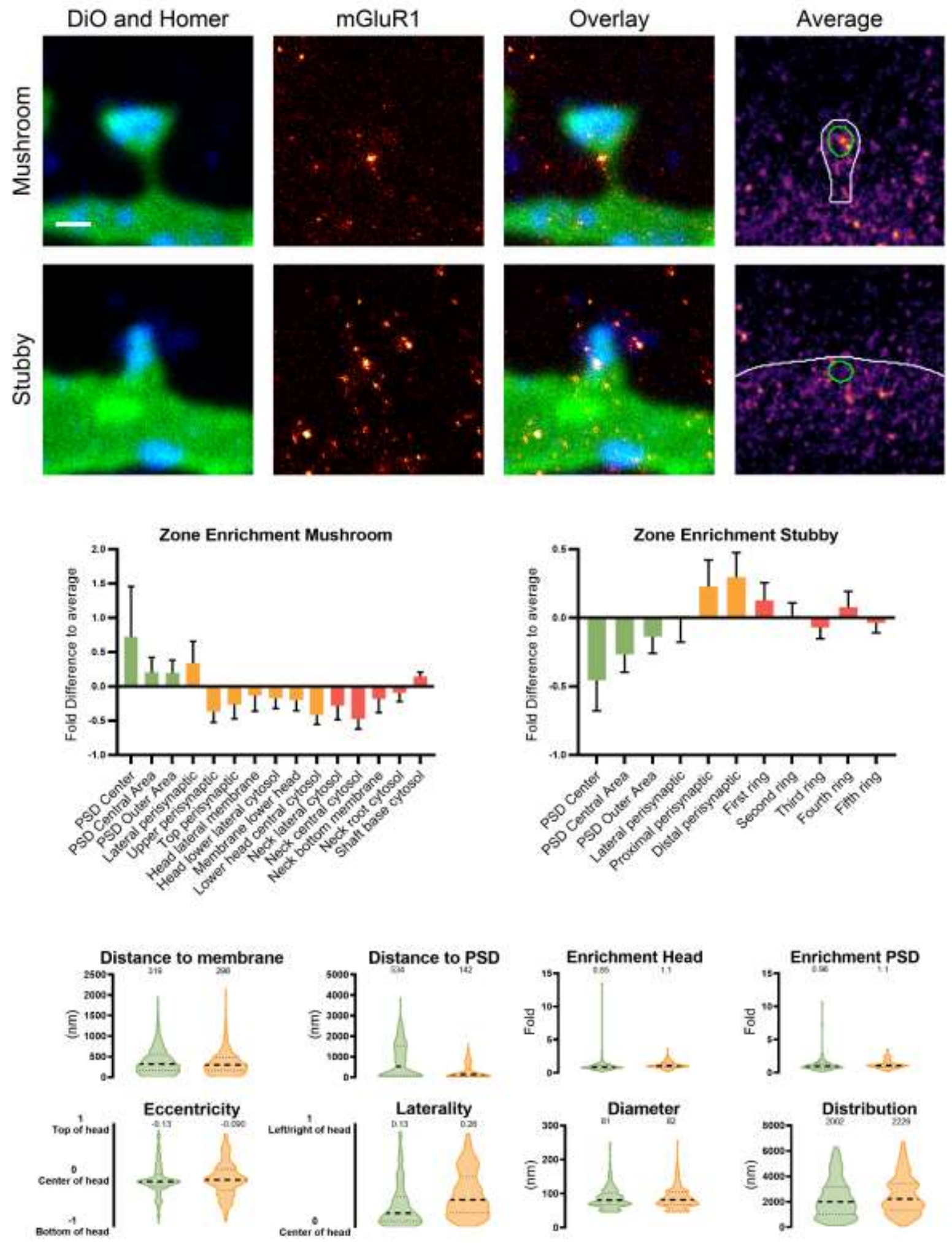

Figure 50: mGluR1 nanoscale localization and abundance. Continued on next page. 


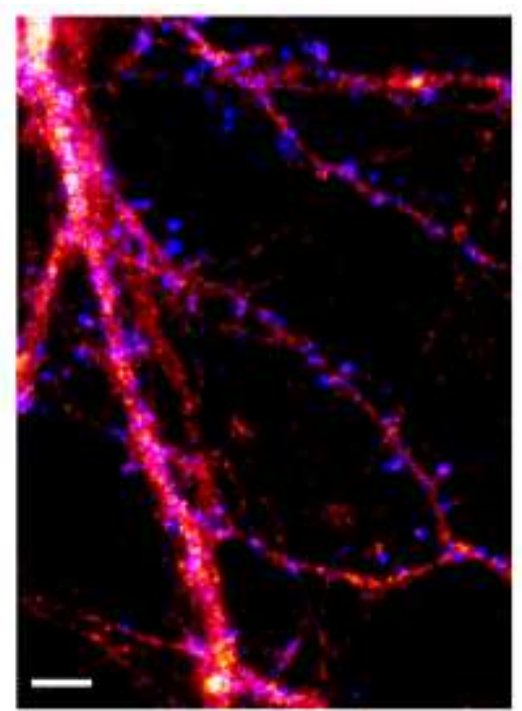

\begin{tabular}{|c|c|}
\hline $\begin{array}{c}\text { Whole cell copy } \\
\text { number }\end{array}$ & $\begin{array}{c}3374.86 \\
\text { (only detected in 1 replicate) }\end{array}$ \\
\hline $\begin{array}{c}\text { Spine copy } \\
\text { number }\end{array}$ & 0.18 \\
\hline$\%$ in PSD & 19.30 \\
\hline PSD copy number & 0.03 \\
\hline
\end{tabular}

\begin{tabular}{|c|c|c|}
\hline & Mushroom & Stubby \\
\hline $\begin{array}{c}\text { Spine copy } \\
\text { number }\end{array}$ & 0.16 & 0.19 \\
\hline PSD copy number & 0.03 & 0.03 \\
\hline$\%$ of protein & 0.00 & 0.00 \\
\hline Molarity $[\mu \mathrm{M}]$ & 0.00 & 0.00 \\
\hline
\end{tabular}

\section{References}

Antibody: Abcam ab51314 PDB Identifier: 1ewk, 1ewt, 2e4u, 4or2, 3ks9

\section{Literature:}

Feng et al., 2002; Hu et al., 2010; Kato et al., 1998; Kitano et al., 2003; Maejima et al., 2001; Mansouri et al., 2015; Pandya et al., 2016; Park et al., 2008; Roche et al., 1999; Skeberdis et al., 2001; Techlovská et al., 2014; Tu et al., 1998; Xiao et al., 1998; Yuan et al., 2003 


\section{mGluR2 (Gene: Grm2, Uniprot ID: P31421)}

Known function: Inhibits PLC, Regulates intracellular calcium release

Known organization: Poorly understood

Known Interactions: $G_{i}$ coupled, GRIP
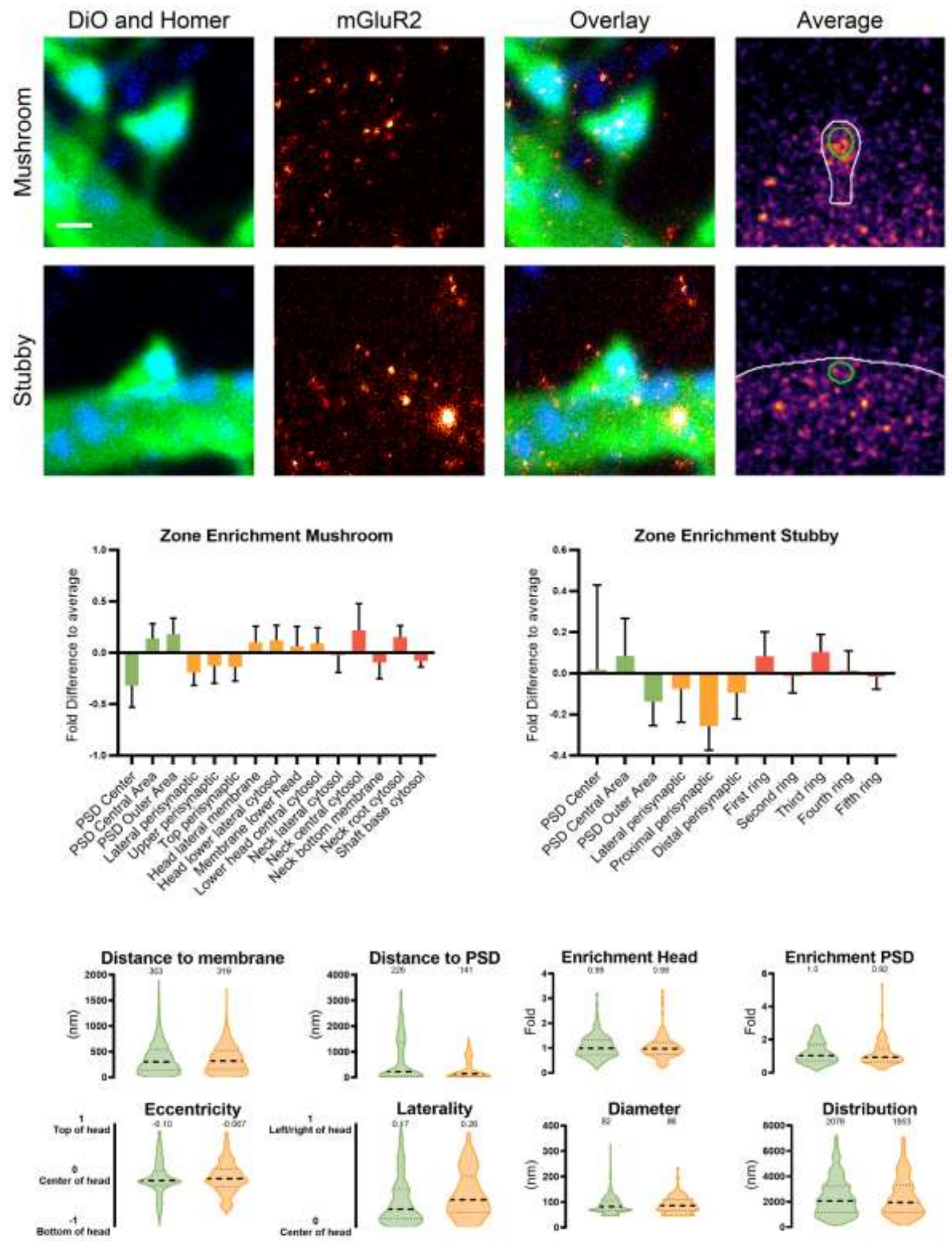

Figure 51: mGluR2 nanoscale localization and abundance. Continued on next page. 


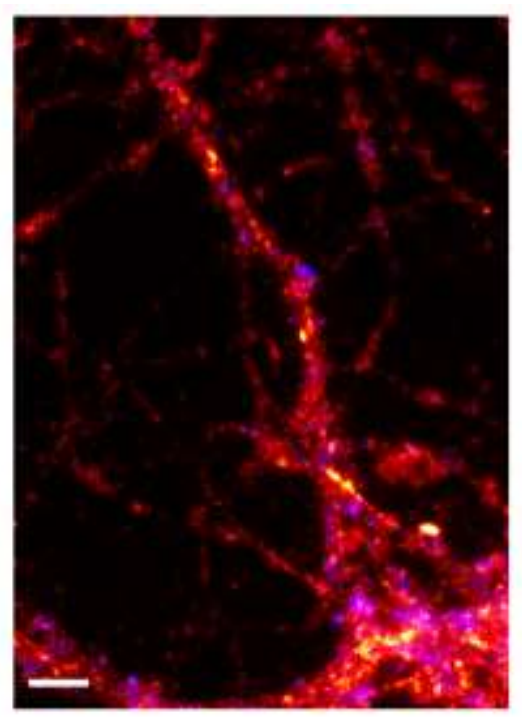

\begin{tabular}{|c|c|c|}
\hline $\begin{array}{l}\text { Whole cell copy } \\
\text { number }\end{array}$ & \multicolumn{2}{|c|}{ not detected } \\
\hline $\begin{array}{l}\text { Spine copy } \\
\text { number }\end{array}$ & \multicolumn{2}{|c|}{$\begin{array}{c}101.32 \pm 1.67 \\
\text { from comparative imaging }\end{array}$} \\
\hline$\%$ in PSD & \multicolumn{2}{|c|}{20.28} \\
\hline \multirow[t]{2}{*}{ PSD copy number } & \multicolumn{2}{|c|}{$17.08 \pm 0.28$} \\
\hline & Mushroom & Stubby \\
\hline $\begin{array}{l}\text { Spine copy } \\
\text { number }\end{array}$ & $79.95 \pm 1.32$ & $112.26 \pm 1.85$ \\
\hline PSD copy number & $13.48 \pm 0.22$ & $18.93 \pm 0.31$ \\
\hline$\%$ of protein & \pm & \pm \\
\hline Molarity $[\mu \mathrm{M}]$ & $0.67 \pm 0.01$ & $0.60 \pm 0.01$ \\
\hline
\end{tabular}

\section{References}

Antibody: Abcam ab150387

PDB Identifier: modified mGluR1

\section{Literature:}

Ambrosini et al., 1995; Cai et al., 2001; Hirbec et al., 2002; Jin et al., 2017; Schaffhauser et al., 2000 


\section{mGluR5 (Gene: Grm5, Uniprot ID: P31424)}

Known function: Activates PKC and PLC, Regulates intracellular calcium release, Involved in LTP and homeostatic plasticity, Activates cannabinoid synthesis Known organization: Transmembrane protein, Perisynaptic

Known Interactions: $\mathrm{G}_{q}$ coupled, mGluR1, Homer proteins
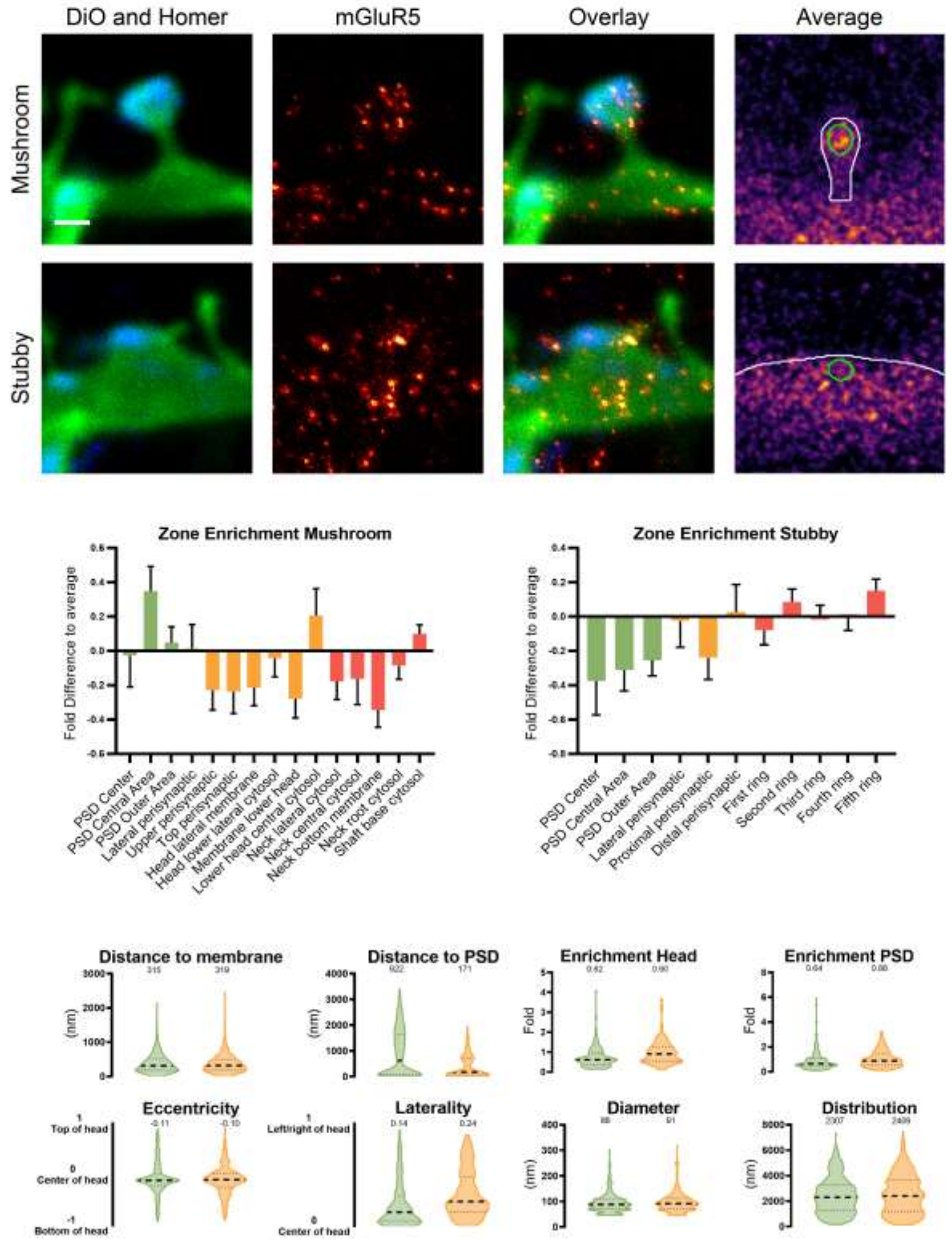

Figure 52: mGluR5 nanoscale localization and abundance. Continued on next page. 


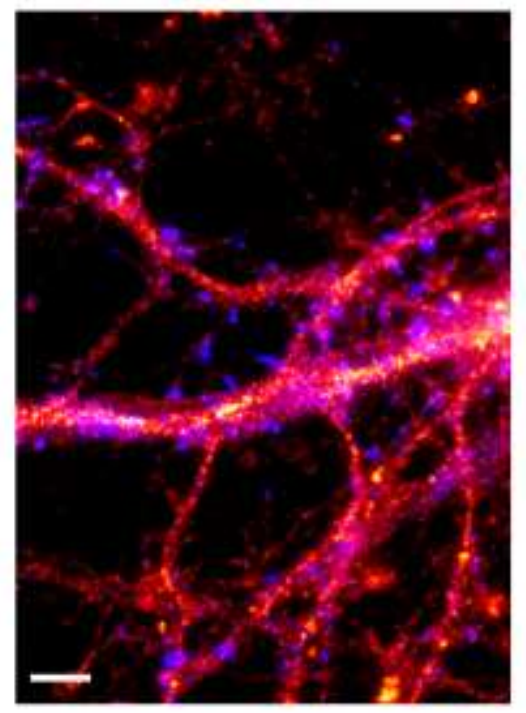

\begin{tabular}{|c|c|c|}
\hline $\begin{array}{c}\text { Whole cell copy } \\
\text { number }\end{array}$ & \multicolumn{2}{|c|}{$119431.79 \pm 17501.53$} \\
\hline $\begin{array}{c}\text { Spine copy } \\
\text { number }\end{array}$ & \multicolumn{2}{|c|}{$32.76 \pm 7.95$} \\
\hline \% in PSD & \multicolumn{2}{|c|}{17.26} \\
\hline PSD copy number & \multicolumn{2}{|c|}{$4.82 \pm 1.17$} \\
\hline & Mushroom & Stubby \\
\hline \begin{tabular}{c|c|} 
Spine copy \\
number
\end{tabular} & $23.59 \pm 5.72$ & $46.29 \pm 11.23$ \\
\hline PSD copy number & $3.47 \pm 0.84$ & $6.81 \pm 1.65$ \\
\hline$\%$ of protein & $0.01 \pm 0.00$ & $0.01 \pm 0.00$ \\
\hline Molarity [ $\mu \mathrm{M}]$ & $0.20 \pm 0.05$ & $0.25 \pm 0.06$ \\
\hline
\end{tabular}

\section{References}

Antibody: Abcam ab76316

PDB Identifier: 3 lmk, 5cgd

\section{Literature:}

Feng et al., 2002; Hu et al., 2010; Kato et al., 1998; Kitano et al., 2003; Maejima et al., 2001; Mansouri et al., 2015; Pandya et al., 2016; Park et al., 2008; Roche et al., 1999; Skeberdis et al., 2001; Techlovská et al., 2014; Tu et al., 1998; Xiao et al., 1998; Yuan et al., 2003 


\section{TrkB (Gene: Ntrk2, Uniprot ID: Q63604)}

Known function: Activates PI3K, PLC and MAP/ERK pathways, Important for dendritic growth, spine maturation and LTP

Known organization: Transmembrane protein, Forms small clusters

Known Interactions: BDNF, LNGFR
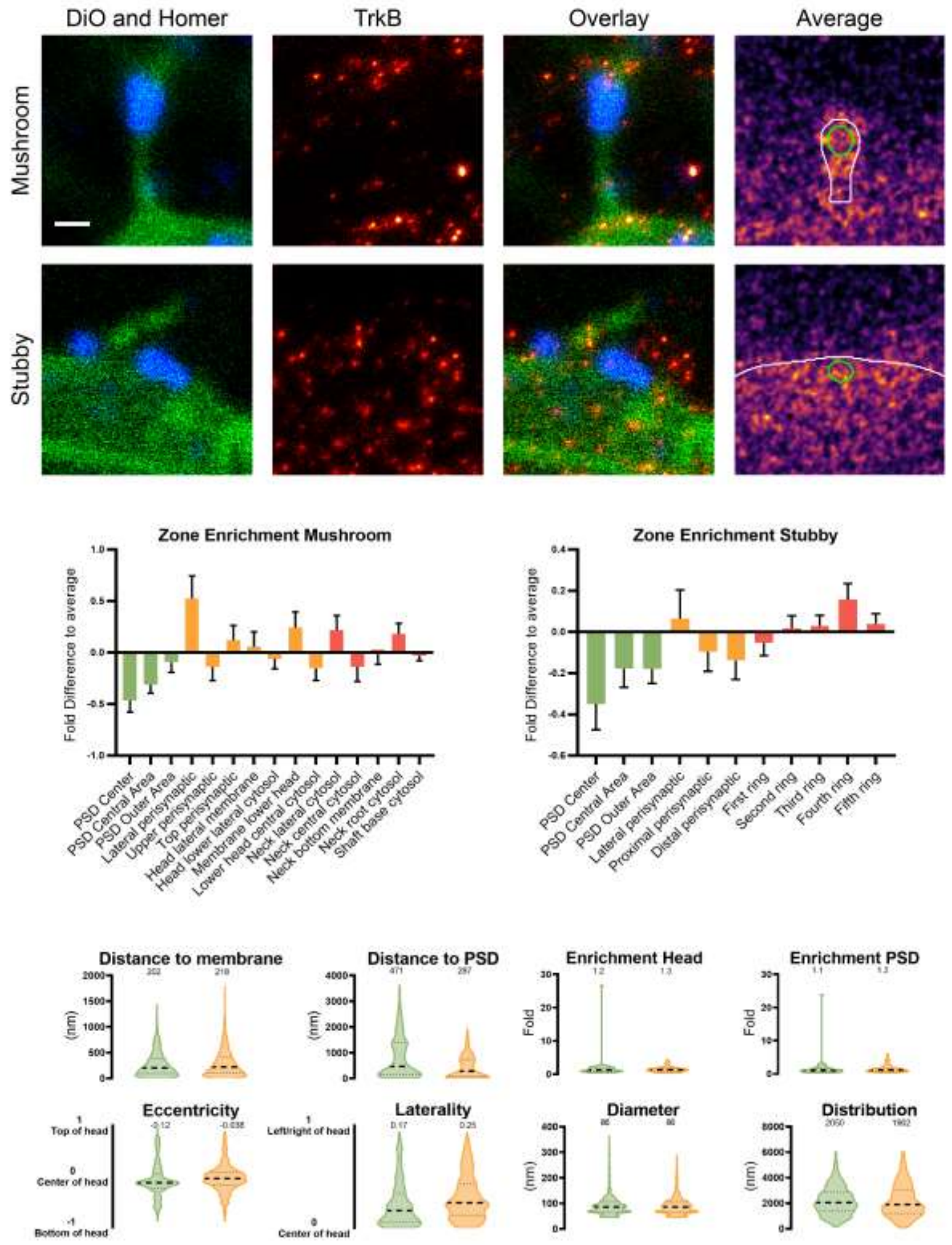

Figure 53: TrkB nanoscale localization and abundance. Continued on next page. 


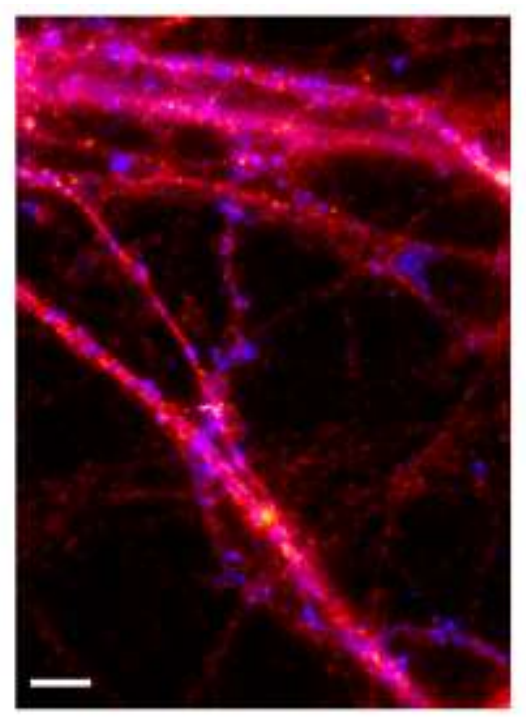

\begin{tabular}{|c|c|c|}
\hline $\begin{array}{c}\text { whole cell copy } \\
\text { number }\end{array}$ & \multicolumn{2}{|c|}{$241083.43 \pm 23652.73$} \\
\hline $\begin{array}{c}\text { Spine copy } \\
\text { number }\end{array}$ & \multicolumn{2}{|c|}{$17.14 \pm 10.78$} \\
\hline$\%$ in PSD & \multicolumn{2}{|c|}{14.77} \\
\hline PSD copy number & \multicolumn{2}{|c|}{$2.21 \pm 1.39$} \\
\hline & Mushroom & Stubby \\
\hline $\begin{array}{c}\text { Spine copy } \\
\text { number }\end{array}$ & $15.40 \pm 9.68$ & $16.61 \pm 10.44$ \\
\hline PSD copy number & $1.98 \pm 1.25$ & $2.14 \pm 1.34$ \\
\hline$\%$ of protein & $0.00 \pm 0.00$ & $0.00 \pm 0.00$ \\
\hline Molarity [MM] & $0.13 \pm 0.08$ & $0.09 \pm 0.06$ \\
\hline
\end{tabular}

\section{References}

Antibody: Abcam ab33655

PDB Identifier: 1hcf, 1www, 4asz

\section{Literature:}

Angelov and Angelova, 2017; Bibel et al., 1999; Gomes et al., 2006; Gorski et al., 2003; Kang and Schuman, 1995; Soppet et al., 1991; Tanaka et al., 1997; Wirth et al., 2003; Xu et al., 2000 


\section{Ion Channels}

Ion channels are important for the maintenance of the neuronal resting potential. They are involved in the propagation of electrical signal as well as calcium entry.

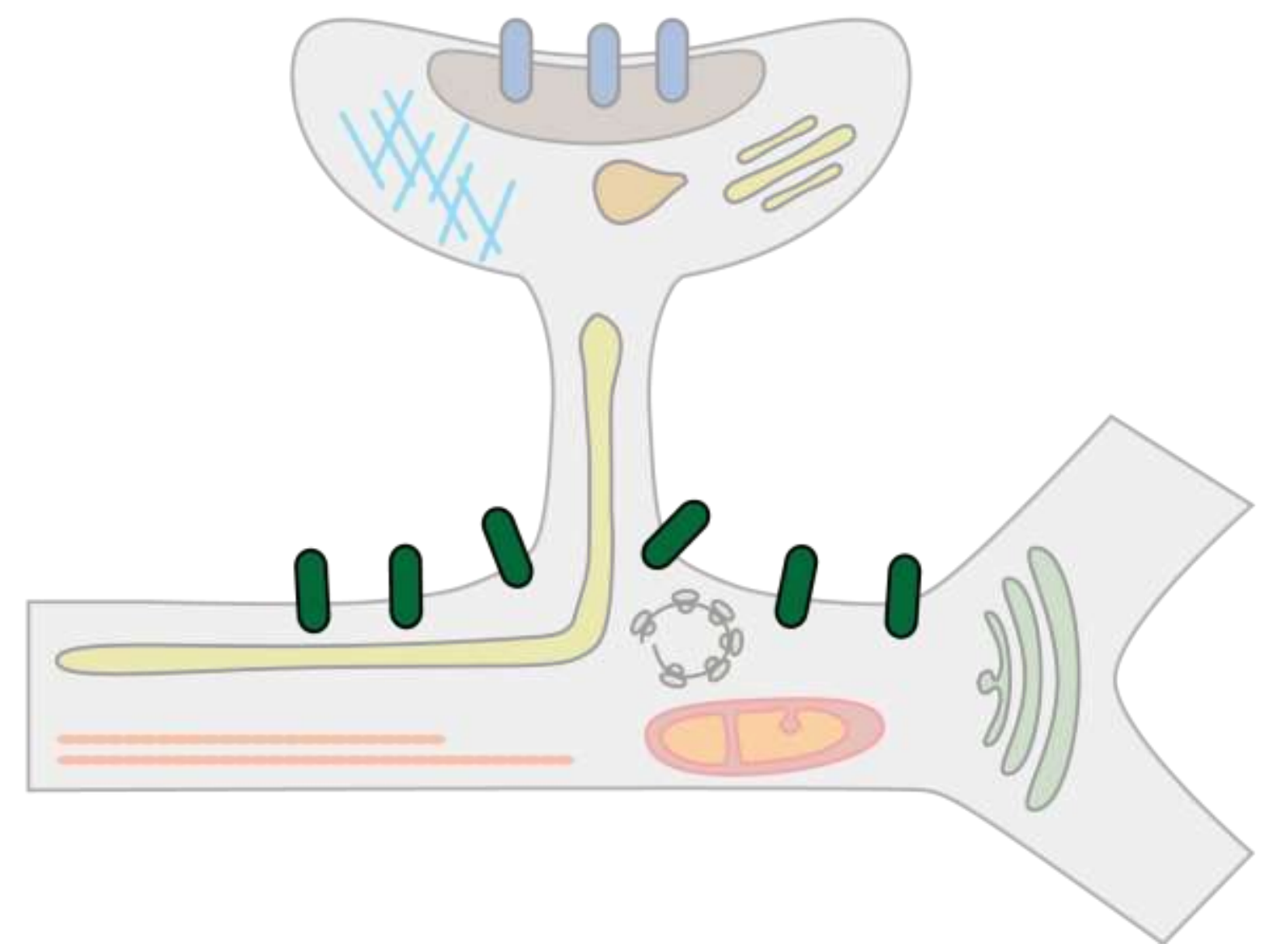

Figure 54: Depiction of ion channels in dendritic spines. 


\section{$\mathrm{Ca}_{\mathrm{v}} 1.3$ (Gene: Cacna1d, Uniprot ID: P27732)}

Known function: Calcium channel, Involved in plasticity, scaling and spine morphology.

Known organization: Transmembrane protein

Known Interactions: Shank1
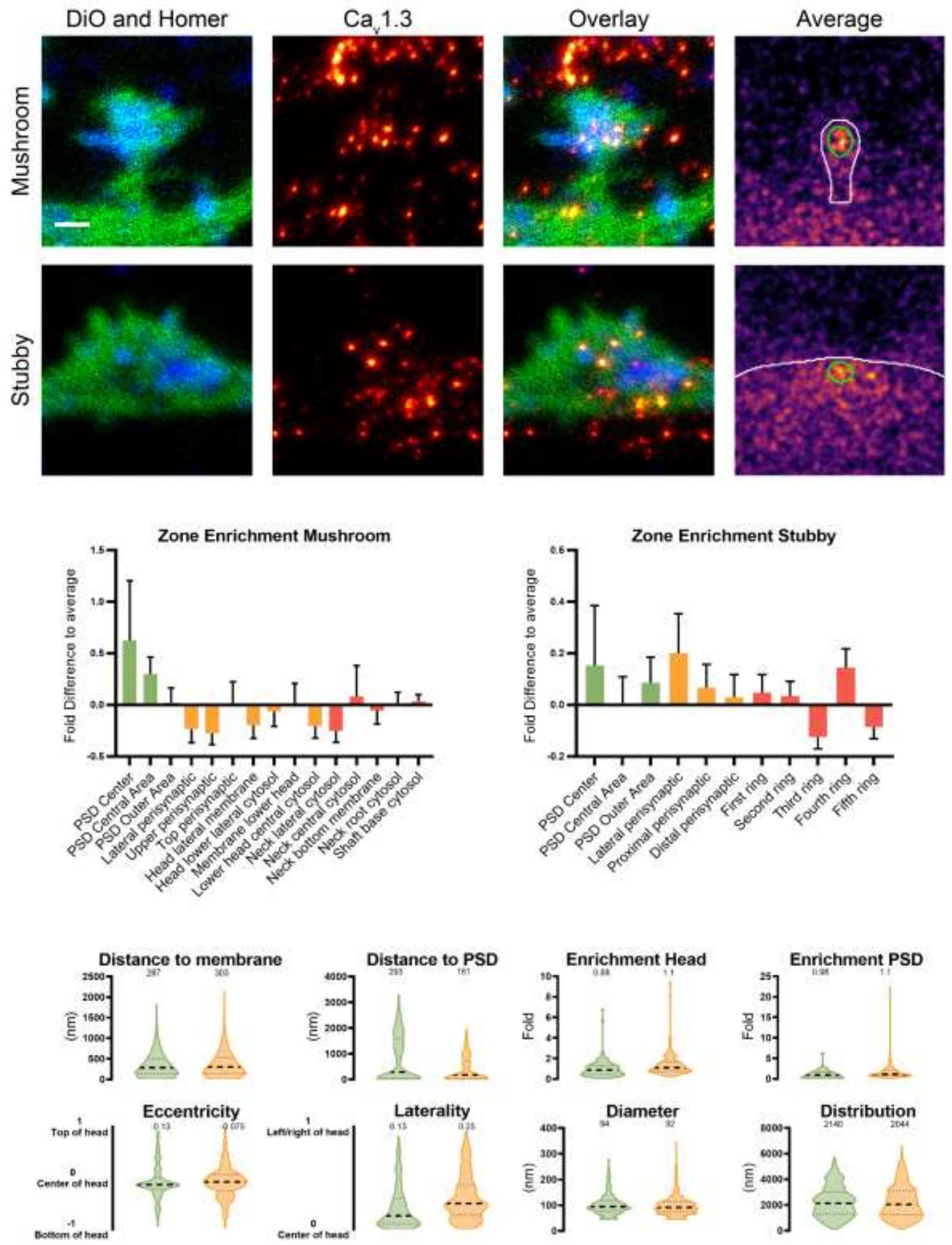

Figure 55: Cav1.3 nanoscale localization and abundance. Continued on next page. 


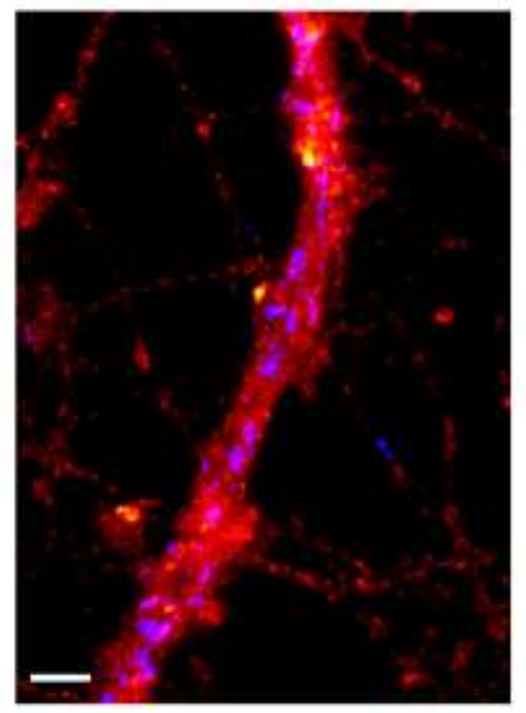

\begin{tabular}{|c|c|c|}
\hline $\begin{array}{c}\text { Whole cell copy } \\
\text { number }\end{array}$ & \multicolumn{2}{|c|}{ not detected } \\
\hline $\begin{array}{c}\text { Spine copy } \\
\text { number }\end{array}$ & \multicolumn{2}{|c|}{ na } \\
\hline$\%$ in PSD & \multicolumn{2}{|c|}{ na } \\
\hline PSD copy number & \multicolumn{2}{|c|}{ na } \\
\hline \begin{tabular}{c|c|} 
Spine copy \\
number
\end{tabular} & na & na \\
\hline PSD copy number & na & na \\
\hline$\%$ of protein & na & na \\
\hline Molarity [MM] & na & na \\
\hline
\end{tabular}

\section{References}

Antibody: Alomone labs ACC-311

PDB Identifier: $5 \mathrm{gjw}$

\section{Literature:}

Greer and Greenberg, 2008; Ibata et al., 2008; Jenkins et al., 2010; Moosmang et al., 2005; Stanika et al., 2016; Wang et al., 2017; Zhang et al., 2005 


\section{$\mathrm{Ca}_{\mathrm{v}} 2.1$ (Gene: Cacna1a, Uniprot ID: P54282)}

Known function: Calcium channel, Involved in LTP

Known organization: Transmembrane protein

Known Interactions: CaMKII
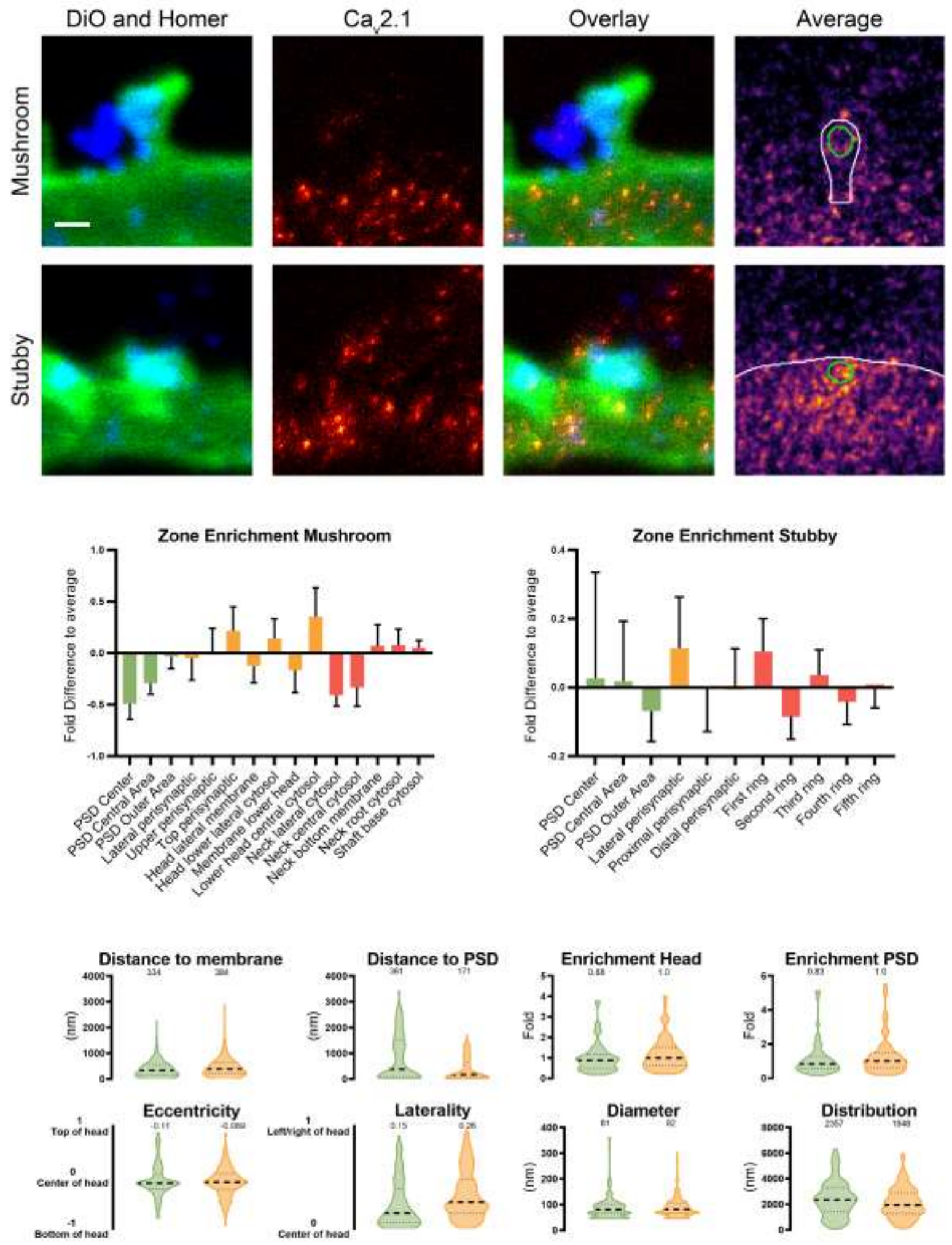

Figure 56: Cav2.1 nanoscale localization and abundance. Continued on next page. 


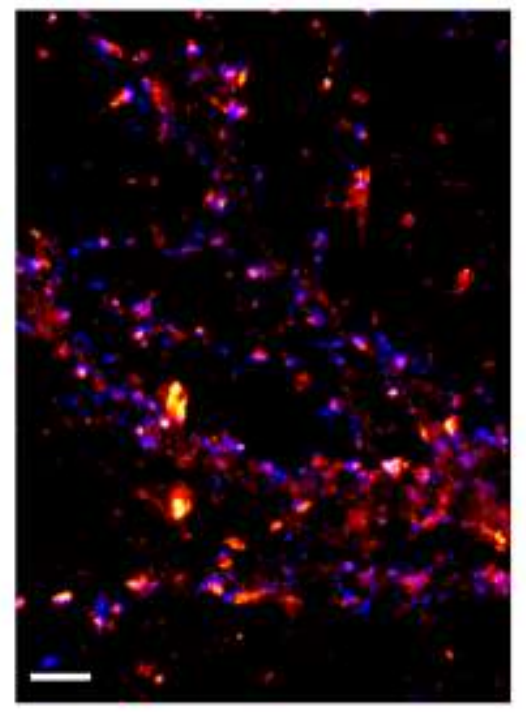

\begin{tabular}{|c|c|c|}
\hline $\begin{array}{c}\text { Whole cell copy } \\
\text { number }\end{array}$ & \multicolumn{2}{|c|}{$10296.87 \pm 2660.19$} \\
\hline $\begin{array}{c}\text { Spine copy } \\
\text { number }\end{array}$ & \multicolumn{2}{|c|}{$43.61 \pm 8.42$} \\
\hline$\%$ in PSD & \multicolumn{2}{|c|}{16.29} \\
\hline PSD copy number & \multicolumn{2}{|c|}{$6.11 \pm 1.18$} \\
\hline & Mushroom & Stubby \\
\hline $\begin{array}{c}\text { Spine copy } \\
\text { number }\end{array}$ & $35.31 \pm 6.82$ & $49.40 \pm 9.54$ \\
\hline PSD copy number & $4.95 \pm 0.96$ & $6.92 \pm 1.34$ \\
\hline$\%$ of protein & $0.02 \pm 0.00 \%$ & $0.02 \pm 0.00 \%$ \\
\hline Molarity [MM] & $0.30 \pm 0.06$ & $0.26 \pm 0.05$ \\
\hline
\end{tabular}

\section{References}

Antibody: Synaptic Systems 152203

PDB Identifier: modified Cav1.3

\section{Literature:}

Jiang et al., 2008; Magupalli et al., 2013; Nanou et al., 2016; Westenbroek et al., 1995; Wu et al., 1999 
Kir2.1 (IRK-1, Gene: Kcnj2, Uniprot ID: Q64273)

Known function: Inwardly recitifying potassium channel

Known organization: Transmembrane protein, Present in PSD95 supercomplexes

Known Interactions: PSD95
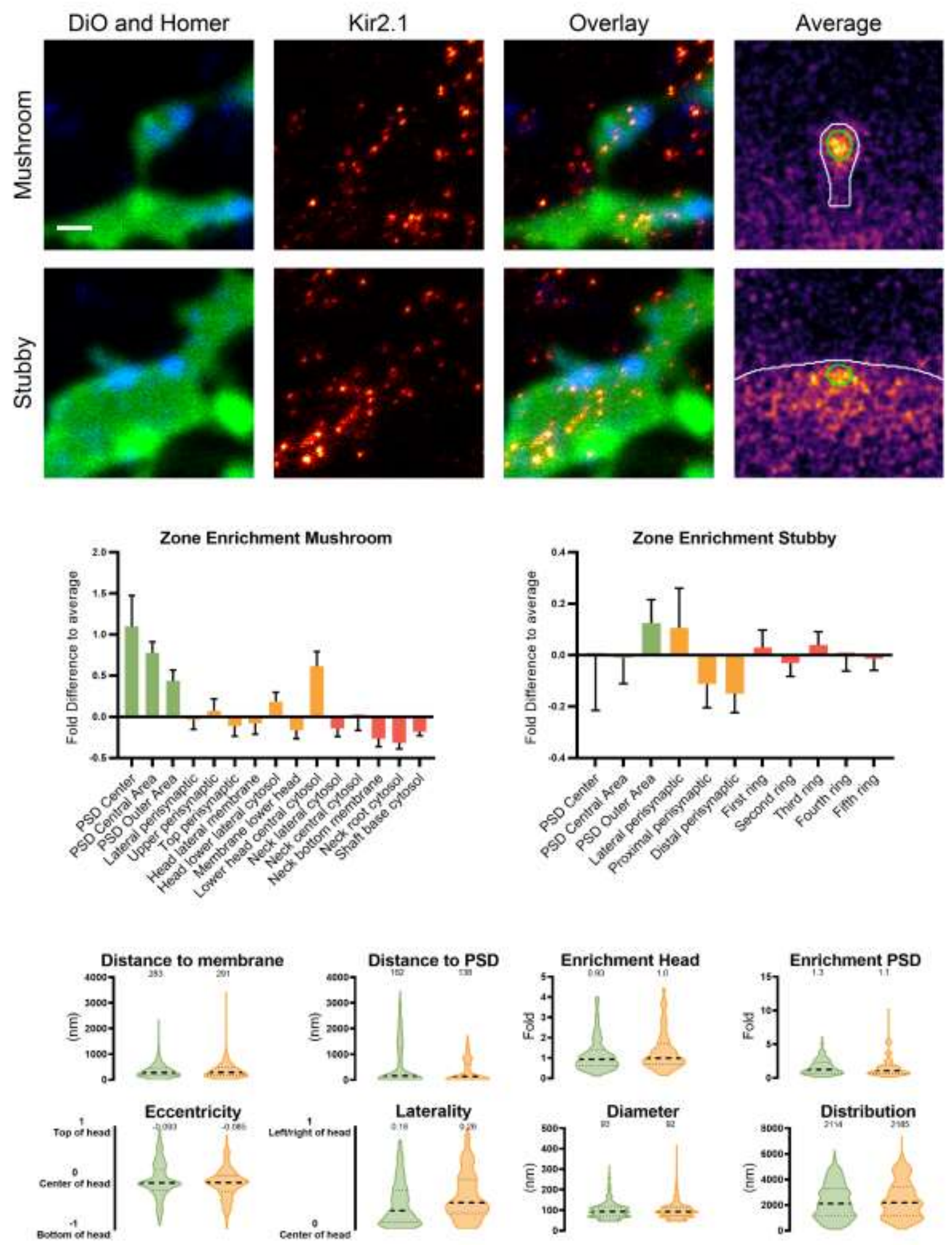

Figure 57: Kir2.1 nanoscale localization and abundance. Continued on next page. 


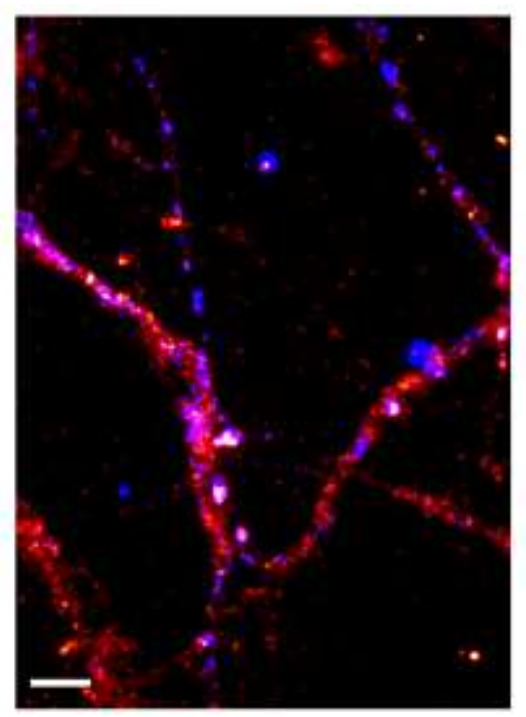

\begin{tabular}{|c|c|c|}
\hline $\begin{array}{c}\text { Whole cell copy } \\
\text { number }\end{array}$ & \multicolumn{2}{|c|}{ not detected } \\
\hline $\begin{array}{c}\text { Spine copy } \\
\text { number }\end{array}$ & \multicolumn{2}{|c|}{ na } \\
\hline$\%$ in PSD & \multicolumn{2}{|c|}{ na } \\
\hline PSD copy number & \multicolumn{2}{|c|}{ na } \\
\hline \begin{tabular}{c|c|} 
Spine copy \\
number
\end{tabular} & na & na \\
\hline PSD copy number & na & na \\
\hline$\%$ of protein & na & na \\
\hline Molarity [MM] & na & na \\
\hline
\end{tabular}

\section{References}

Antibody: Novus Biologicals NBP1-95482

PDB Identifier: 3spi

\section{Literature:}

Fomina et al., 2011; Frank et al., 2016; Vikstrom et al., 2009 


\section{K 1.1 (Gene: Kcna1, Uniprot ID: P10499)}

Known function: Delayed rectifier potassium channel

Known organization: Transmembrane protein

Known Interactions: PSD95
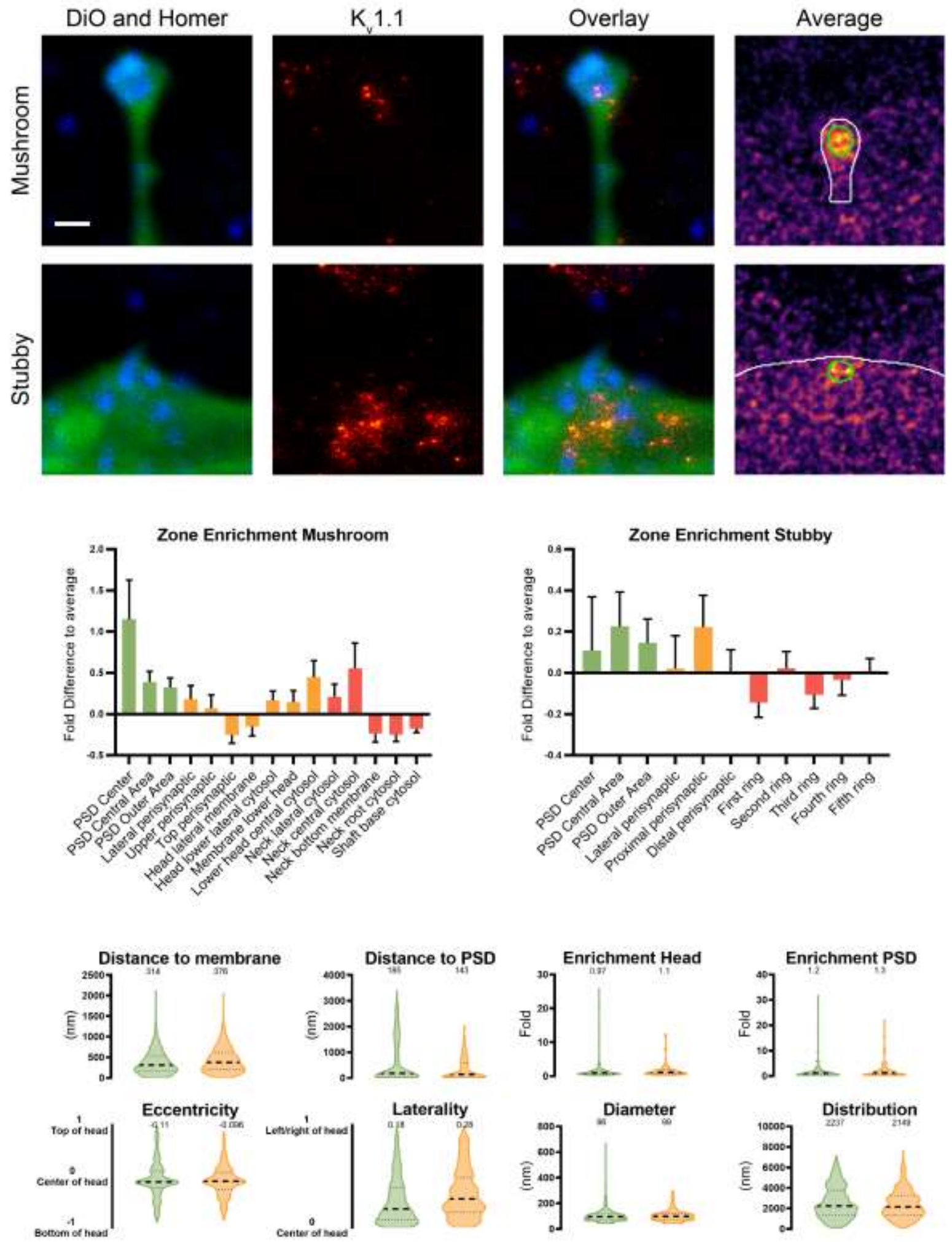

Figure 58: $\mathrm{K}_{\mathrm{v}} 1.1$ nanoscale localization and abundance. Continued on next page. 


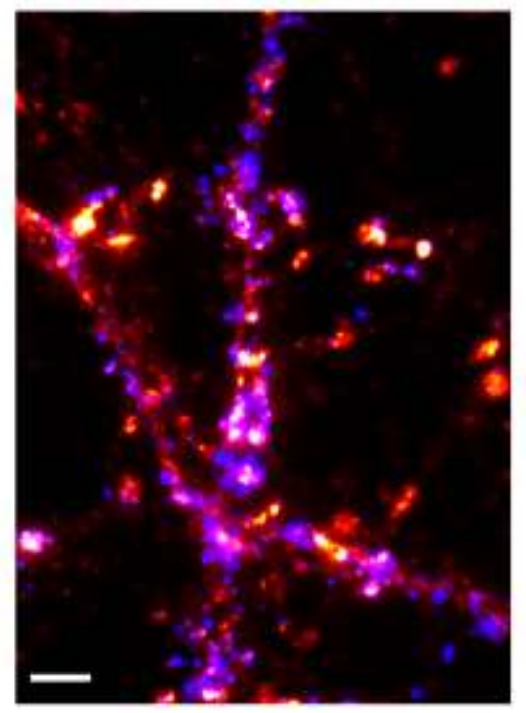

\begin{tabular}{|c|c|c|}
\hline $\begin{array}{c}\text { Whole cell copy } \\
\text { number }\end{array}$ & \multicolumn{2}{|c|}{ not detected } \\
\hline $\begin{array}{c}\text { Spine copy } \\
\text { number }\end{array}$ & \multicolumn{2}{|c|}{ na } \\
\hline \% in PSD & \multicolumn{2}{|c|}{ na } \\
\hline PSD copy number & \multicolumn{2}{|c|}{ na } \\
\hline \begin{tabular}{c|c|} 
Spine copy \\
number
\end{tabular} & na & na \\
\hline PSD copy number & na & na \\
\hline$\%$ of protein & na & na \\
\hline Molarity $[\mu M]$ & na & na \\
\hline
\end{tabular}

\section{References}

Antibody: Thermo Scientific PA5-19593

PDB Identifier: 2a79

\section{Literature:}

Kim et al., 1995; Tiffany et al., 2000 


\section{K 2.1 (Gene: Kcnb1, Uniprot ID: P15387)}

Known function: Delayed rectifyer potassium channel, Dominant type in hippocampus, Regulates gene transcription

Known organization: Transmembrane protein, Forms clusters on soma

Known Interactions: Syntaxin1
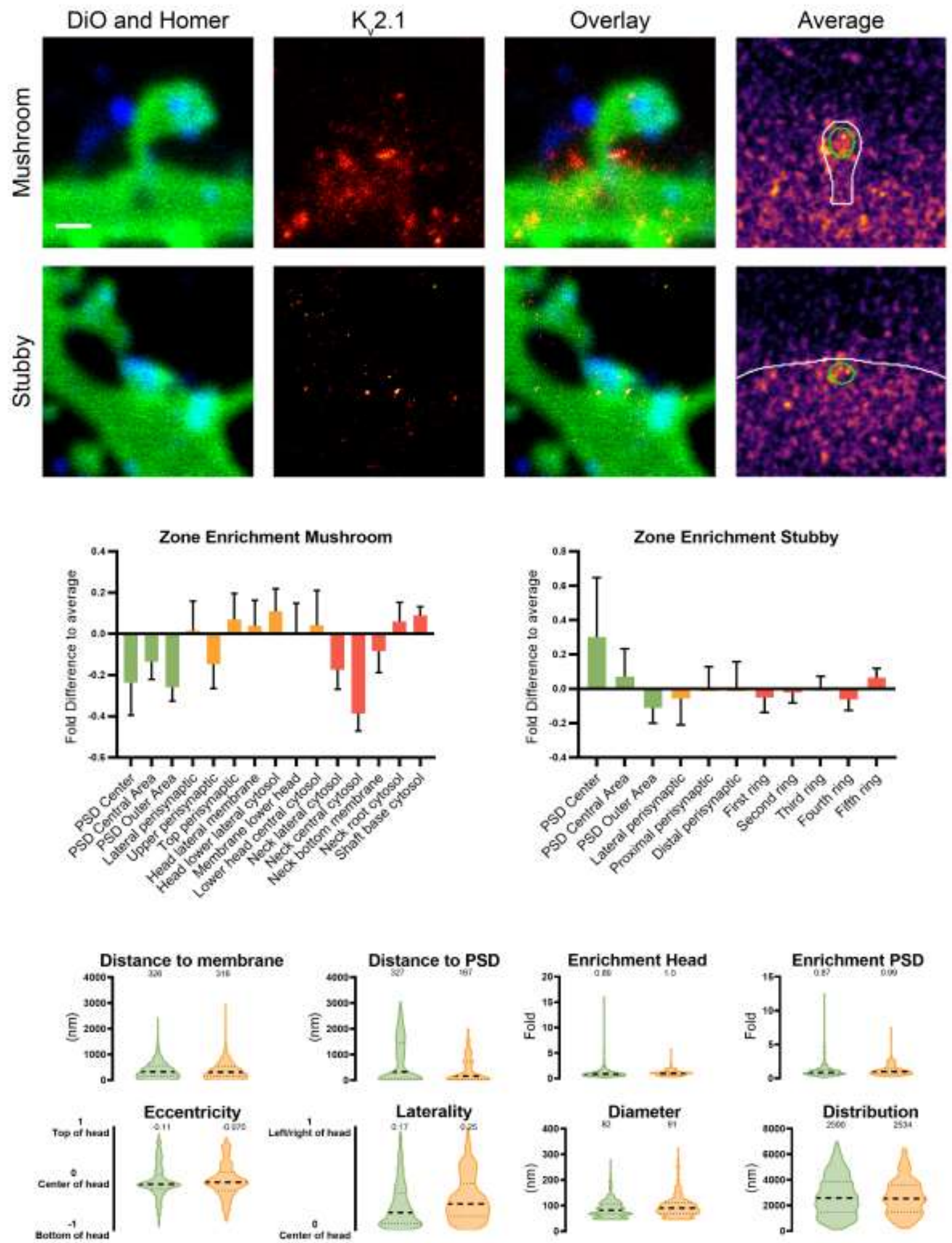

Figure 59: $\mathrm{K}_{\mathrm{v}} 2.1$ nanoscale localization and abundance. Continued on next page. 


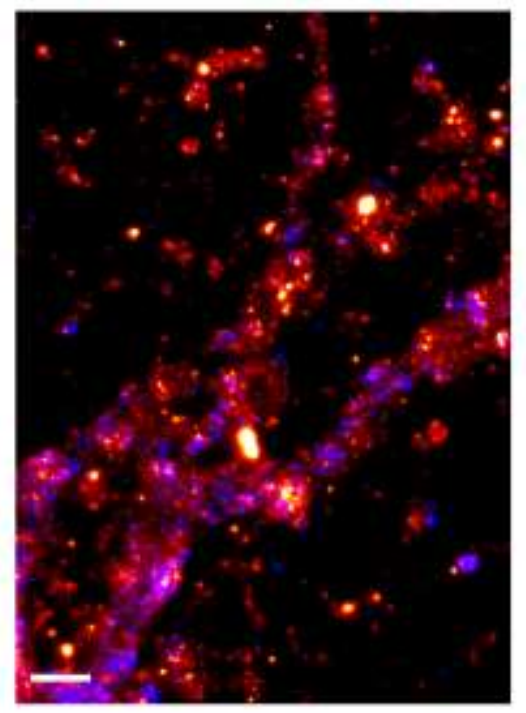

\begin{tabular}{|c|c|c|}
\hline $\begin{array}{c}\text { Whole cell copy } \\
\text { number }\end{array}$ & \multicolumn{2}{|c|}{$\begin{array}{c}767.94 \\
\text { (only detected in 1 replicate) }\end{array}$} \\
\hline $\begin{array}{c}\text { Spine copy } \\
\text { number }\end{array}$ & \multicolumn{2}{|c|}{0.09} \\
\hline$\%$ in PSD & \multicolumn{2}{|c|}{18.83} \\
\hline PSD copy number & \multicolumn{2}{|c|}{0.01} \\
\hline & Mushroom & Stubby \\
\hline $\begin{array}{c}\text { Spine copy } \\
\text { number }\end{array}$ & 0.08 & 0.10 \\
\hline PSD copy number & 0.01 & 0.02 \\
\hline$\%$ of protein & $0.00 \pm 0.00 \%$ & $0.00 \pm 0.00 \%$ \\
\hline Molarity [ $\mu \mathrm{M}]$ & $0.00 \pm 0.00$ & $0.00 \pm 0.00$ \\
\hline
\end{tabular}

\section{References}

Antibody: Synaptic Systems 231002

PDB Identifier: 4jtc

\section{Literature:}

Du et al., 2000; Feinshreiber et al., 2009; Fox et al., 2013; Lai and Jan, 2006; Leung et al., 2007; Misonou et al., 2005; Murakoshi and Trimmer, 1999; O'Connell et al., 2010; Scannevin et al., 1996; Trimmer, 1991 


\section{Na $\beta 1$ (Gene: Scnb1, Uniprot ID: Q00954)}

Known function: Modulates sodium channel properties, Acts as adhesion molecule Known organization: Transmembrane protein

Known Interactions: $\mathrm{Na}_{v} 1.1, \mathrm{Na}_{\mathrm{v}} 1.3$
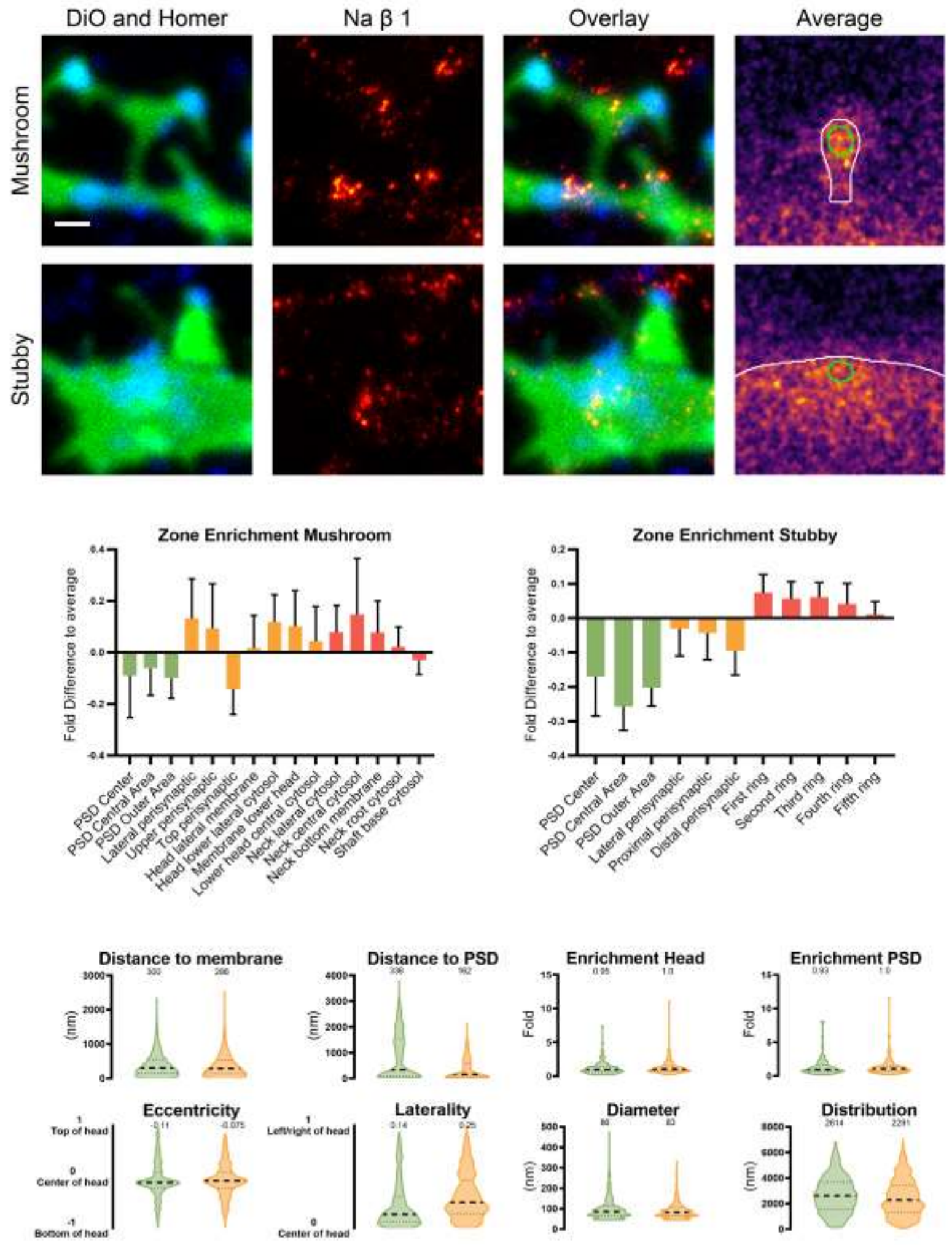

Figure 60: Na $\beta 1$ nanoscale localization and abundance. Continued on next page. 


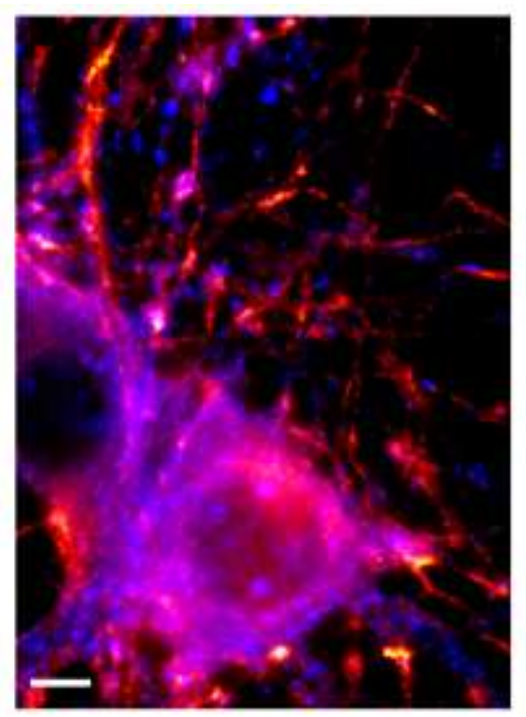

\begin{tabular}{|c|c|c|}
\hline $\begin{array}{c}\text { Whole cell copy } \\
\text { number }\end{array}$ & \multicolumn{2}{|c|}{$1093309.68 \pm 323561.33$} \\
\hline $\begin{array}{c}\text { Spine copy } \\
\text { number }\end{array}$ & \multicolumn{2}{|c|}{$434.97 \pm 145.55$} \\
\hline$\%$ in PSD & \multicolumn{2}{|c|}{15.47} \\
\hline PSD copy number & \multicolumn{2}{|c|}{$58.28 \pm 19.50$} \\
\hline & Mushroom & Stubby \\
\hline $\begin{array}{c}\text { Spine copy } \\
\text { number }\end{array}$ & $\begin{array}{c}375.03 \\
\pm 125.49\end{array}$ & 500.31 \\
\hline PSD copy number & $50.25 \pm 16.82$ & $67.04 \pm 22.43$ \\
\hline$\%$ of protein & $0.02 \pm 0.01 \%$ & $0.02 \pm 0.01 \%$ \\
\hline Molarity [ $\mu \mathrm{M}]$ & $3.14 \pm 1.05$ & $2.68 \pm 0.90$ \\
\hline
\end{tabular}

\section{References}

Antibody: Alomone labs ASC-041

PDB Identifier: 6agf

\section{Literature:}

Kazarinova-Noyes et al., 2001; Malhotra et al., 2000 


\section{$\mathrm{Na}_{v} 1.1$ (Gene: Scn1a, Uniprot ID: P04774)}

Known function: Sodium channel

Known organization: Transmembrane protein

Known Interactions: $\mathrm{Na} \beta 1$
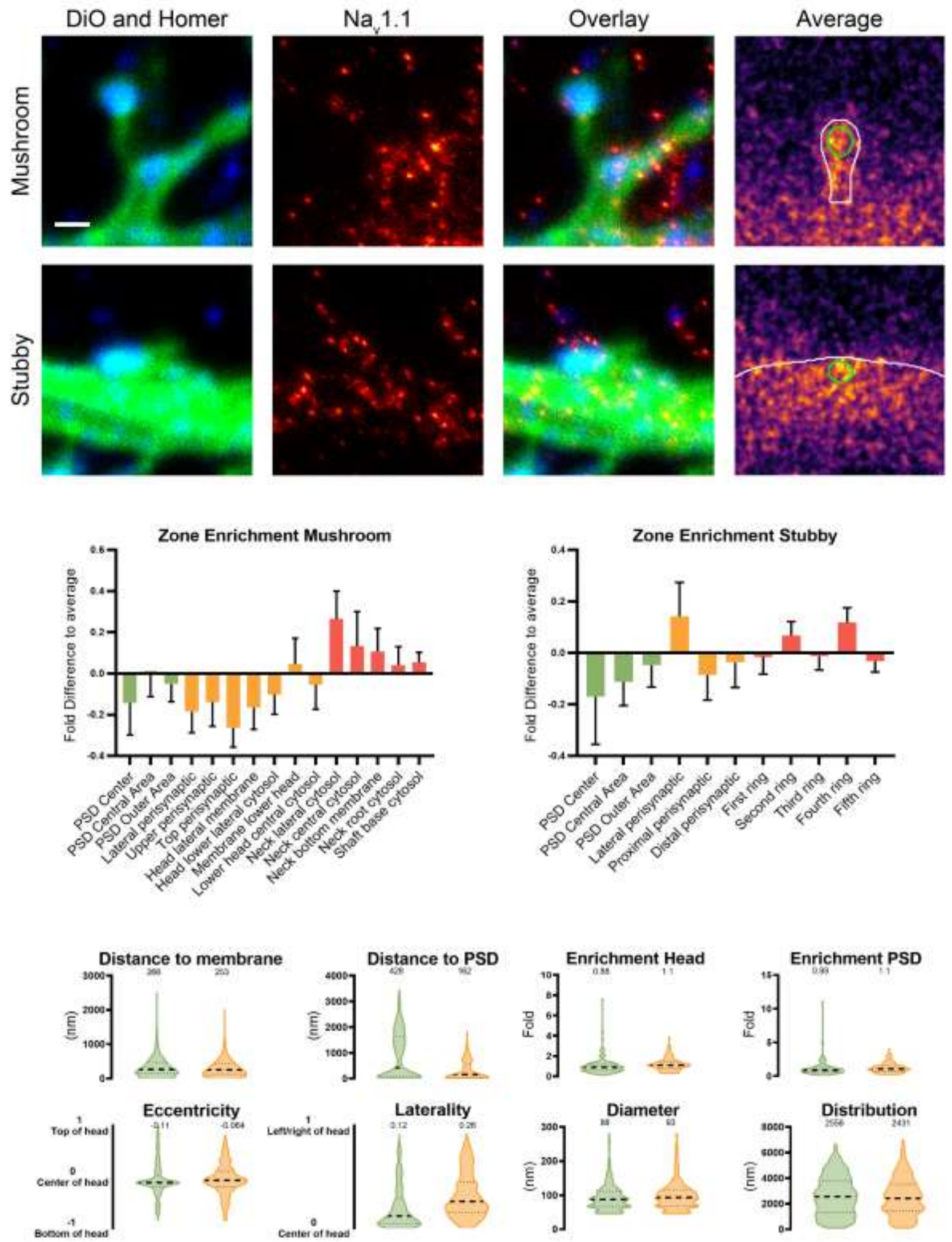

Figure 61: Nav1.1 nanoscale localization and abundance. Continued on next page. 


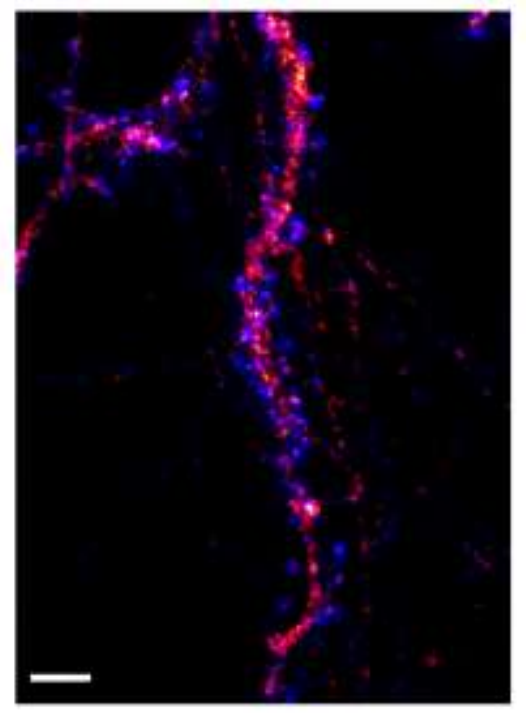

\begin{tabular}{|c|c|c|}
\hline $\begin{array}{c}\text { Whole cell copy } \\
\text { number }\end{array}$ & \multicolumn{2}{|c|}{$135451.27 \pm 10480.00$} \\
\hline $\begin{array}{c}\text { Spine copy } \\
\text { number }\end{array}$ & \multicolumn{2}{|c|}{$29.47 \pm 5.11$} \\
\hline$\%$ in PSD & \multicolumn{2}{|c|}{16.18} \\
\hline PSD copy number & \multicolumn{2}{|c|}{$4.10 \pm 0.71$} \\
\hline & Mushroom & Stubby \\
\hline $\begin{array}{c}\text { Spine copy } \\
\text { number }\end{array}$ & $25.31 \pm 4.39$ & $33.91 \pm 5.88$ \\
\hline PSD copy number & $3.53 \pm 0.61$ & $4.72 \pm 0.82$ \\
\hline$\%$ of protein & $0.01 \pm 0.00 \%$ & $0.01 \pm 0.00 \%$ \\
\hline Molarity [MM] & $0.21 \pm 0.04$ & $0.18 \pm 0.03$ \\
\hline
\end{tabular}

\section{References}

Antibody: Merck Millipore 06-811

PDB Identifier: 6agf

\section{Literature:}

Westenbroek et al., 1989 


\section{$\mathrm{Na}_{v} 1.3$ (Gene: Scn3a, Uniprot ID: P08104)}

Known function: Sodium channel

Known organization: Transmembrane protein

Known Interactions: $\mathrm{Na} \beta 1$

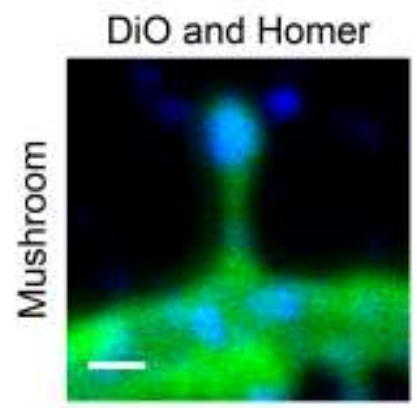

$\mathrm{Na}_{\mathbf{y}} 1.3$
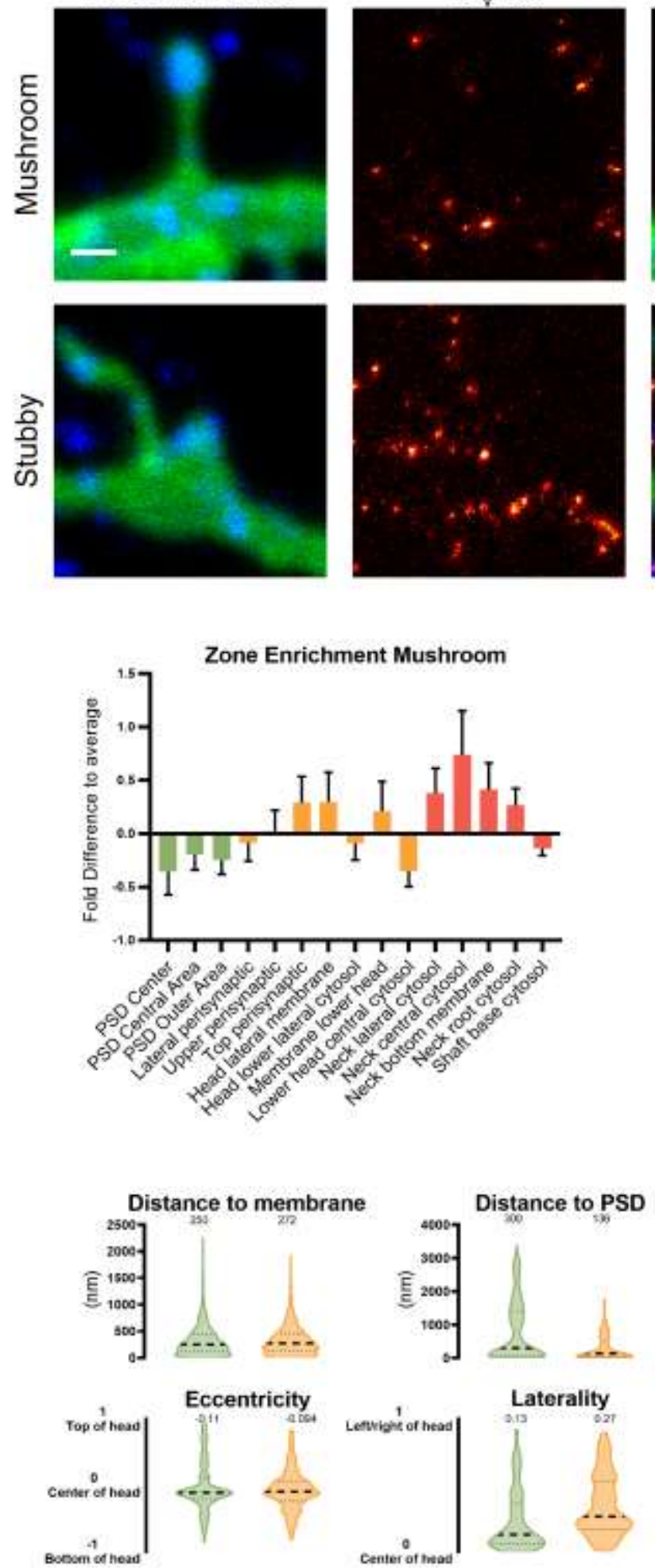
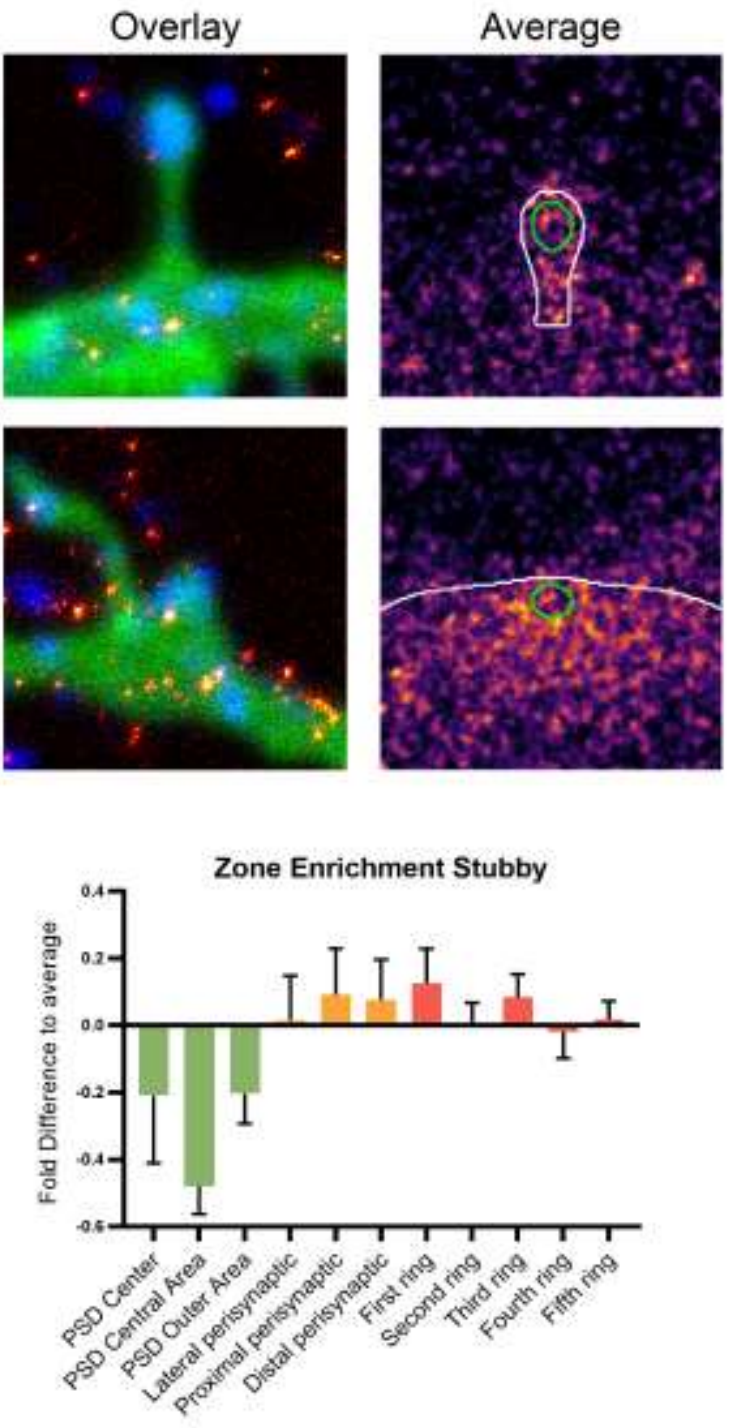

Figure 62: Nav1.3 nanoscale localization and abundance. Continued on next page. 


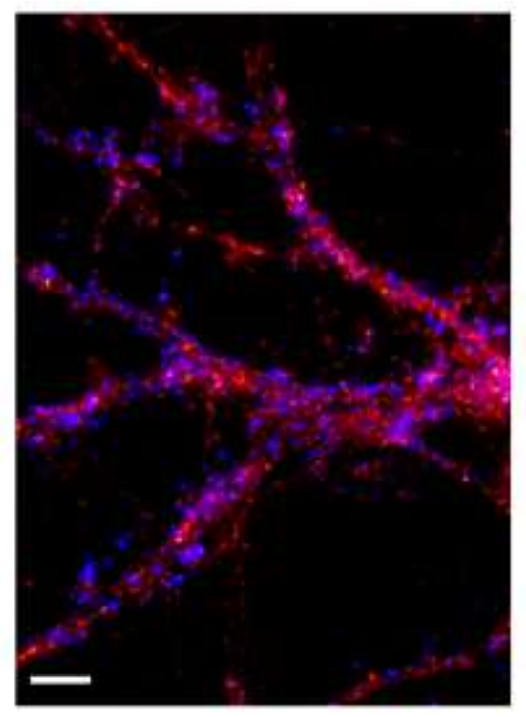

\begin{tabular}{|c|c|c|}
\hline $\begin{array}{c}\text { Whole cell copy } \\
\text { number }\end{array}$ & \multicolumn{2}{|c|}{$\begin{array}{c}2602.88 \\
\text { (only detected in } 1 \text { replicate) }\end{array}$} \\
\hline $\begin{array}{l}\text { Spine copy } \\
\text { number }\end{array}$ & \multicolumn{2}{|c|}{0.08} \\
\hline$\%$ in PSD & \multicolumn{2}{|c|}{14.18} \\
\hline \multirow[t]{2}{*}{ PSD copy number } & \multicolumn{2}{|c|}{0.01} \\
\hline & Mushroom & Stubby \\
\hline $\begin{array}{l}\text { Spine copy } \\
\text { number }\end{array}$ & 0.06 & 0.09 \\
\hline PSD copy number & 0.01 & 0.01 \\
\hline$\%$ of protein & $0.00 \%$ & $0.00 \%$ \\
\hline Molarity $[\mu \mathrm{M}]$ & 0.00 & 0.00 \\
\hline
\end{tabular}

\section{References}

Antibody:

PDB Identifier: 6agf

\section{Literature:}

Westenbroek et al., 1989; Whitaker et al., 2001 


\section{$\mathrm{Na}^{+} / \mathrm{K}^{+}$ATPase (Gene: Atp1a3, Uniprot ID: P06687)}

Known function: Maintains resting potential

Known organization: Transmembrane protein, Forms clusters

Known Interactions: None
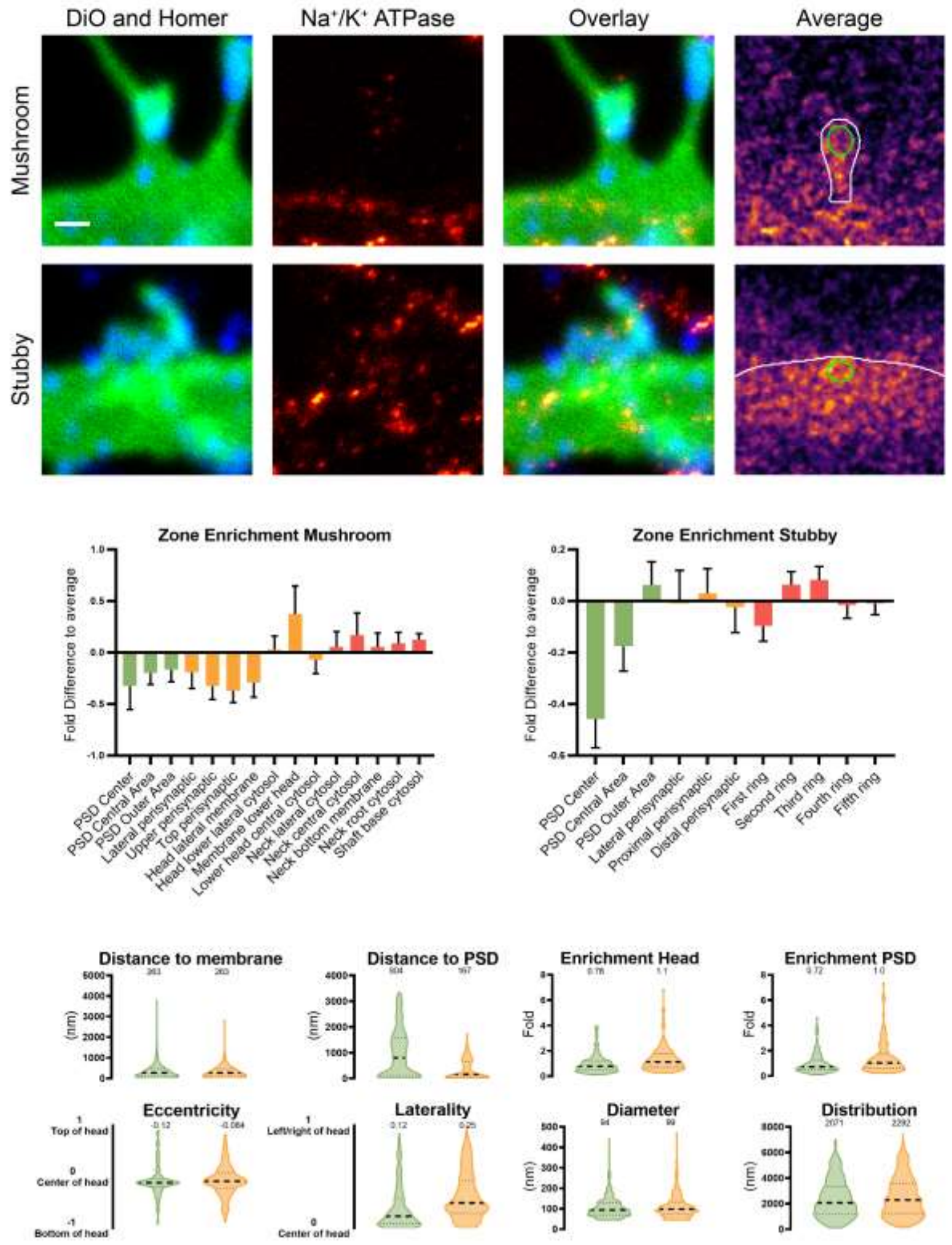

Figure 63: $\mathrm{Na}^{+} / \mathrm{K}^{+}$ATPase nanoscale localization and abundance. Continued on next page. 


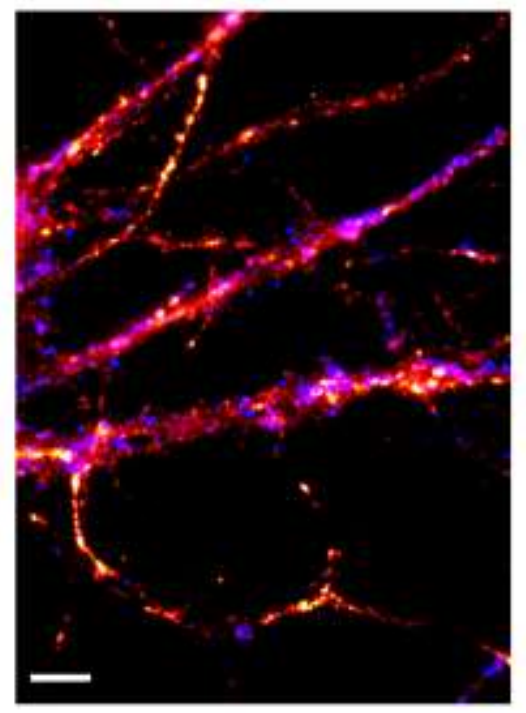

\begin{tabular}{|c|c|c|}
\hline $\begin{array}{c}\text { Whole cell copy } \\
\text { number }\end{array}$ & \multicolumn{2}{|c|}{$34823381.81 \pm 2325248.32$} \\
\hline $\begin{array}{c}\text { Spine copy } \\
\text { number }\end{array}$ & \multicolumn{2}{|c|}{$7204.25 \pm 1749.03$} \\
\hline$\%$ in PSD & \multicolumn{2}{|c|}{16.06} \\
\hline PSD copy number & \multicolumn{2}{|c|}{$997.01 \pm 242.05$} \\
\hline & Mushroom & Stubby \\
\hline $\begin{array}{c}\text { Spine copy } \\
\text { number }\end{array}$ & $\begin{array}{c}5287.76 \\
\pm 1283.75\end{array}$ & 9447.61 \\
\hline PSD copy number & $731.78 \pm 177.66$ & $1307.47 \pm 317.43$ \\
\hline$\%$ of protein & $1.36 \pm 0.33 \%$ & $1.56 \pm 0.38 \%$ \\
\hline Molarity [ $\mu \mathrm{M}]$ & $44.32 \pm 10.76$ & $50.65 \pm 12.30$ \\
\hline
\end{tabular}

\section{References}

Antibody: Thermo Scientific MA3-915

PDB Identifier: 4 xe5

\section{Literature:}

(Blom et al., 2011, 2012, 2016; Skou, 1957) 


\section{Signaling proteins}

Within dendritic spines, many signaling cascades are operating in parallel. These are often kinase/phosphatase networks, but the spine is also actively sending retrograde signals to the presynapse.

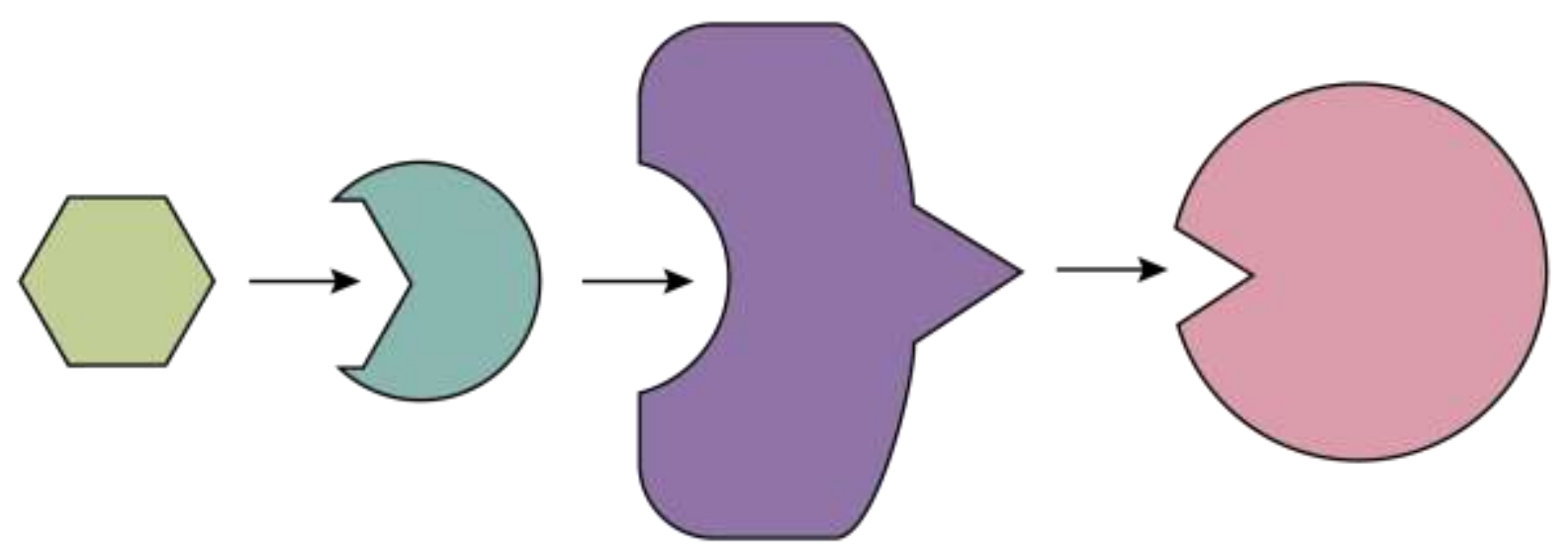

Figure 64: Depiction of a signaling cascade 


\section{ADAM22 (Gene: Adam22, Uniprot ID: M0R5P8)}

Known function: Catalytically inactive metalloprotease, Involved in cell adhesion,

Regulation of AMPAR, $\mathrm{K}_{\vee} 1$ channels and PSD95

Known organization: Transmembrane protein, On PM

Known Interactions: $\mathrm{K}_{\downarrow} 1.1, \mathrm{PSD} 95, \mathrm{AMPA}$ receptors
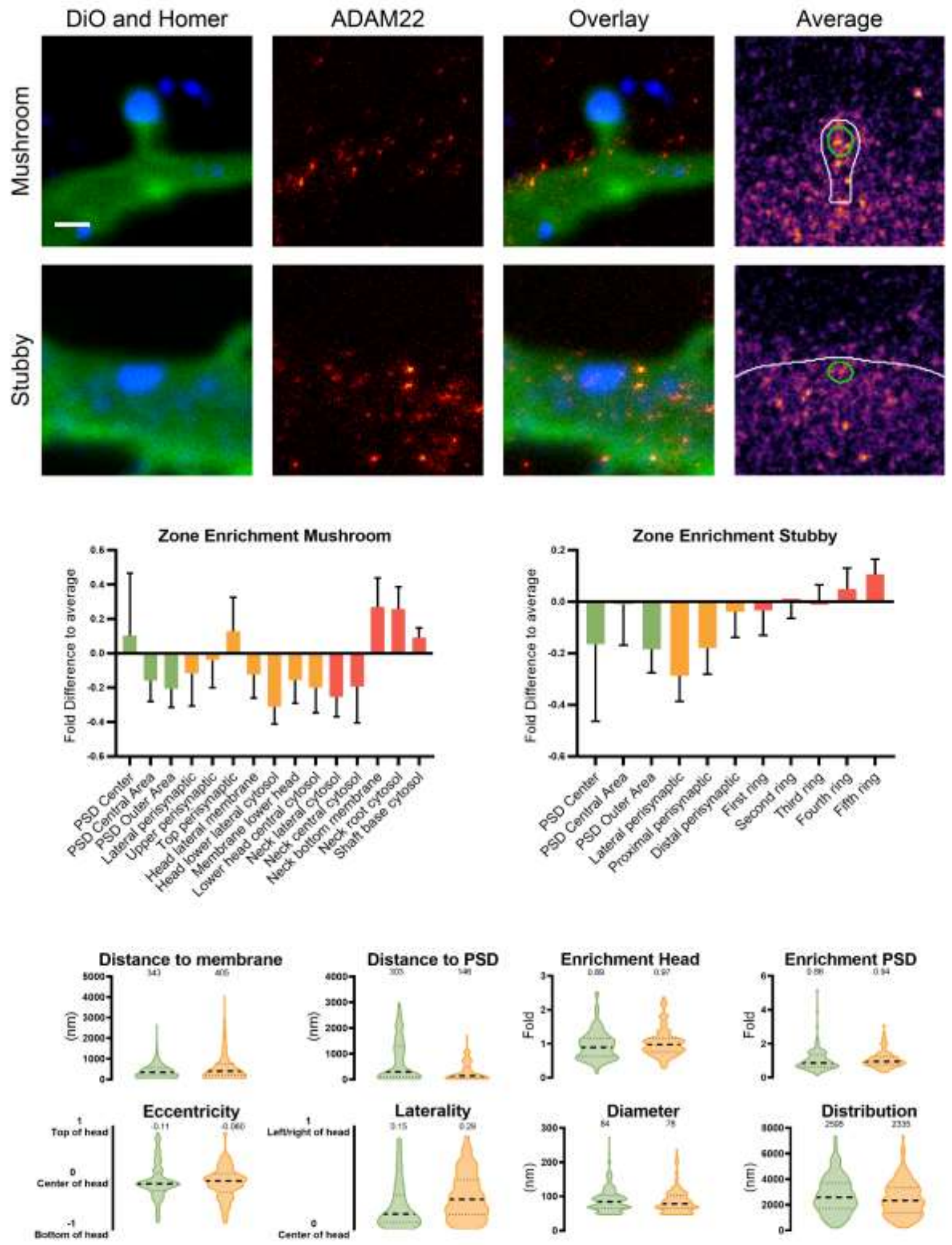

Figure 65: ADAM22 nanoscale localization and abundance. Continued on next page. 


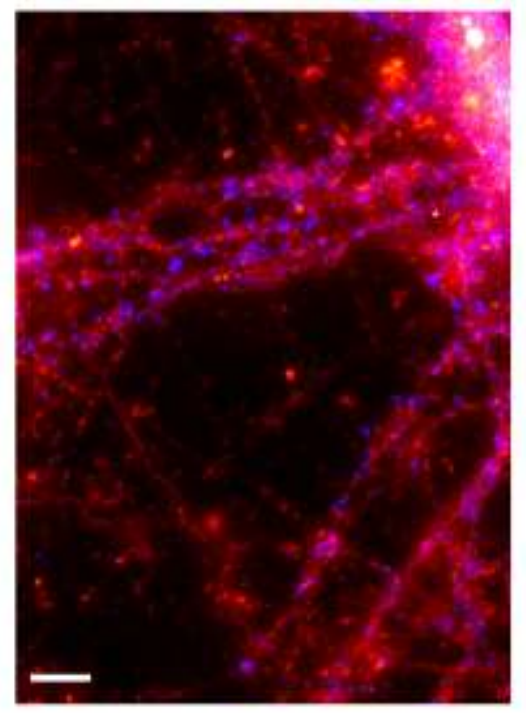

\begin{tabular}{|c|c|c|}
\hline $\begin{array}{c}\text { Whole cell copy } \\
\text { number }\end{array}$ & \multicolumn{2}{|c|}{$141288.07 \pm 23437.74$} \\
\hline $\begin{array}{c}\text { Spine copy } \\
\text { number }\end{array}$ & \multicolumn{2}{|c|}{$9.57 \pm 4.21$} \\
\hline$\%$ in PSD & \multicolumn{2}{|c|}{15.83} \\
\hline PSD copy number & \multicolumn{2}{|c|}{$1.31 \pm 0.58$} \\
\hline & Mushroom & Stubby \\
\hline $\begin{array}{c}\text { Spine copy } \\
\text { number }\end{array}$ & $7.82 \pm 3.44$ & $11.16 \pm 4.91$ \\
\hline PSD copy number & $1.07 \pm 0.47$ & $1.53 \pm 0.67$ \\
\hline$\%$ of protein & $0.00 \pm 0.00 \%$ & $0.00 \pm 0.00 \%$ \\
\hline Molarity [ $\mu \mathrm{M}]$ & $0.07 \pm 0.03$ & $0.06 \pm 0.03$ \\
\hline
\end{tabular}

\section{References}

Antibody: Novus Biologicals NBP2-22425

PDB Identifier: $5 y 2 z$

\section{Literature:}

D’Abaco et al., 2006; Fukata et al., 2006; Lovero et al., 2015; Ogawa et al., 2010; Poindexter et al., 1999; Sagane et al., 1998 
pan-Akt (PKB, Genes: Akt1, Akt2, Akt3, Uniprot ID: P47196, P47196,

\section{P47196)}

Known function: Mediates PI3-K signaling, Activates nNOS, Regulates AMPAR expression, Is activated by NMDA receptors

Known organization: Cytosolic

Known Interactions: nNOS
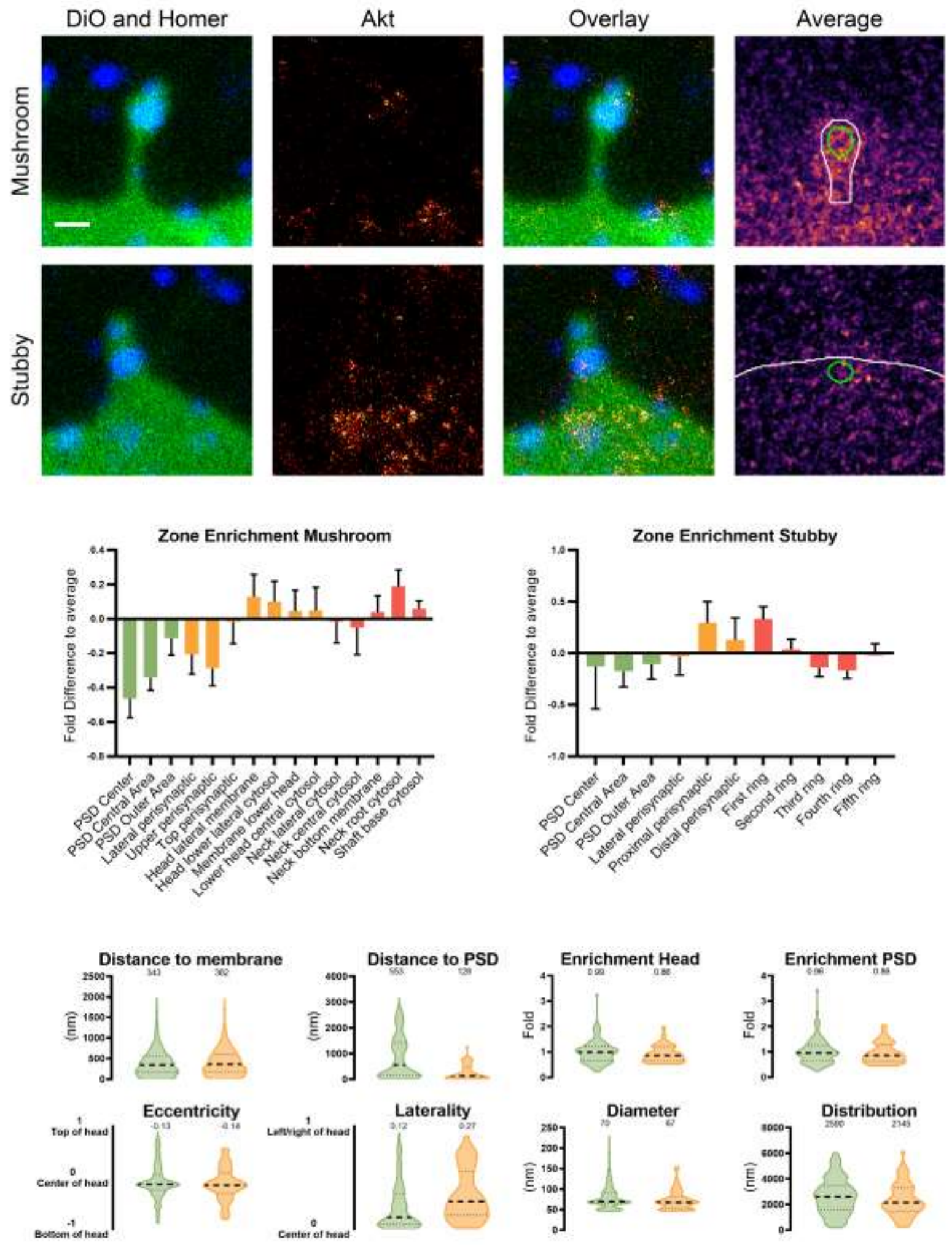

Figure 66: Akt nanoscale localization and abundance. Continued on next page. 


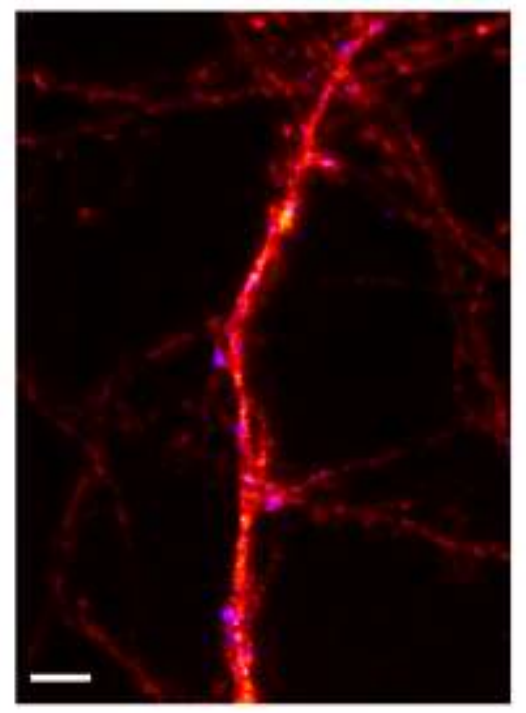

\begin{tabular}{|c|c|}
\hline $\begin{array}{c}\text { Whole cell copy } \\
\text { number }\end{array}$ & $1525938.29 \pm 138993.59$ \\
\hline $\begin{array}{c}\text { Spine copy } \\
\text { number }\end{array}$ & $94.94 \pm 41.29$ \\
\hline$\%$ in PSD & 17.12 \\
\hline PSD copy number & $13.88 \pm 6.04$ \\
\hline
\end{tabular}

\begin{tabular}{|c|c|c|}
\hline $\begin{array}{c}\text { Spine copy } \\
\text { number }\end{array}$ & Mushroom & Stubby \\
\hline PSD copy number & $12.55 \pm 5.46$ & $13.63 \pm 5.93$ \\
\hline$\%$ of protein & $0.02 \pm 0.01 \%$ & $0.02 \pm 0.01 \%$ \\
\hline Molarity $[\mu \mathrm{M}]$ & $0.72 \pm 0.31$ & $0.50 \pm 0.22$ \\
\hline
\end{tabular}

Mushroom molecular model

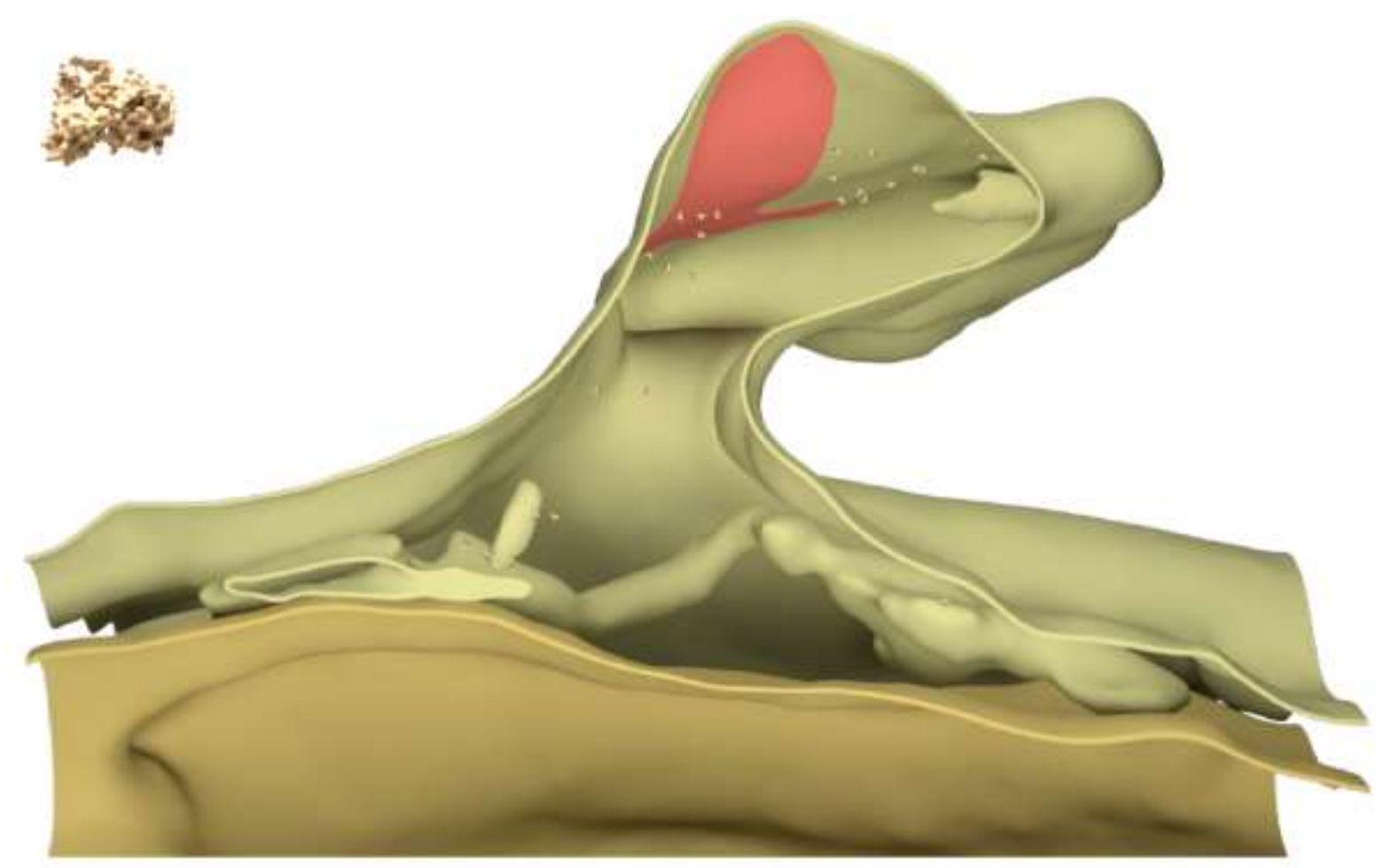

\section{References}

Antibody: Cell Signaling 4691

PDB Identifier: 5 buu

\section{Literature:}

Dimmeler et al., 1999; Fulton et al., 1999; Gobert et al., 2008; Karpova et al., 2006; Kennedy et al., 1997, 1999; Man et al., 2003; Pen et al., 2016; Qin et al., 2005; Schratt et al., 2004; Wang et al., 2011; Znamensky et al., 2003 


\section{APP (Gene: App, Uniprot ID: P08592)}

Known function: Exact function unknown, involved in neurite growth and LTP, Precursor protein of $A \beta$, the hallmark of Alzheimer's disease

Known organization: Transmembrane protein, Also secreted to extracellular matrix Known Interactions: None
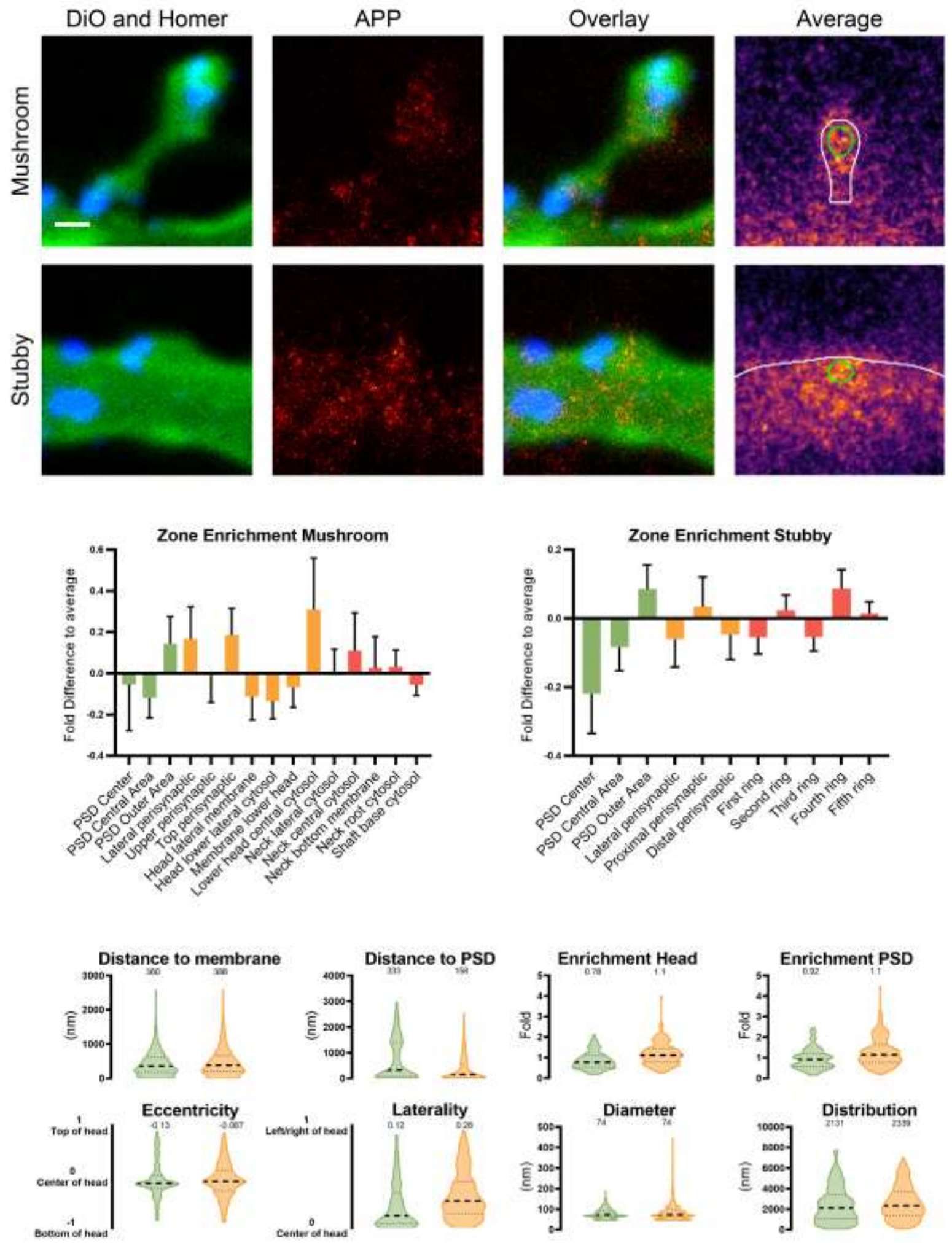

Figure 67: APP nanoscale localization and abundance. Continued on next page. 


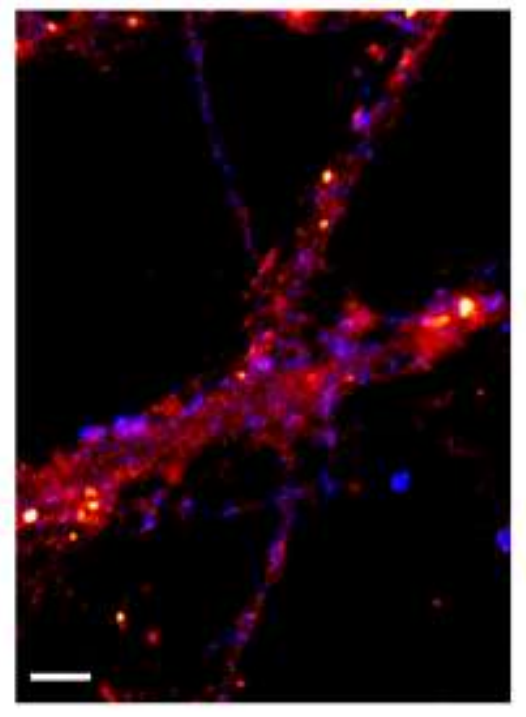

\begin{tabular}{|c|c|c|}
\hline $\begin{array}{c}\text { Whole cell copy } \\
\text { number }\end{array}$ & \multicolumn{2}{|c|}{$2033416.20 \pm 153706.96$} \\
\hline $\begin{array}{l}\text { Spine copy } \\
\text { number }\end{array}$ & \multicolumn{2}{|c|}{$320.20 \pm 76.75$} \\
\hline$\%$ in PSD & \multicolumn{2}{|c|}{17.33} \\
\hline \multirow[t]{2}{*}{ PSD copy number } & \multicolumn{2}{|c|}{$47.28 \pm 11.33$} \\
\hline & Mushroom & Stubby \\
\hline $\begin{array}{l}\text { Spine copy } \\
\text { number }\end{array}$ & $260.74 \pm 62.50$ & $367.97 \pm 88.20$ \\
\hline PSD copy number & $38.50 \pm 9.23$ & $54.34 \pm 13.02$ \\
\hline$\%$ of protein & $0.05 \pm 0.01 \%$ & $0.05 \pm 0.01 \%$ \\
\hline Molarity $[\mu \mathrm{M}]$ & $2.19 \pm 0.52$ & $1.97 \pm 0.47$ \\
\hline
\end{tabular}

\section{References}

Antibody: Merck-Millipore MAB-348

PDB Identifier: $2 \mathrm{fkl}, 4 \mathrm{yno}$

\section{Literature:}

Müller and Zheng, 2012; Perez et al., 1997; Simons et al., 1996; Slunt et al., 1994; Taylor et al., 2008; Young-Pearse et al., 2008 
BDNF (Gene: Bdnf, Uniprot ID: P23363)

Known function: Important neurotrophin, Involved in neuronal growth and plasticity Known organization: In ER and secretory granules

Known Interactions: TrkB, LNGFR
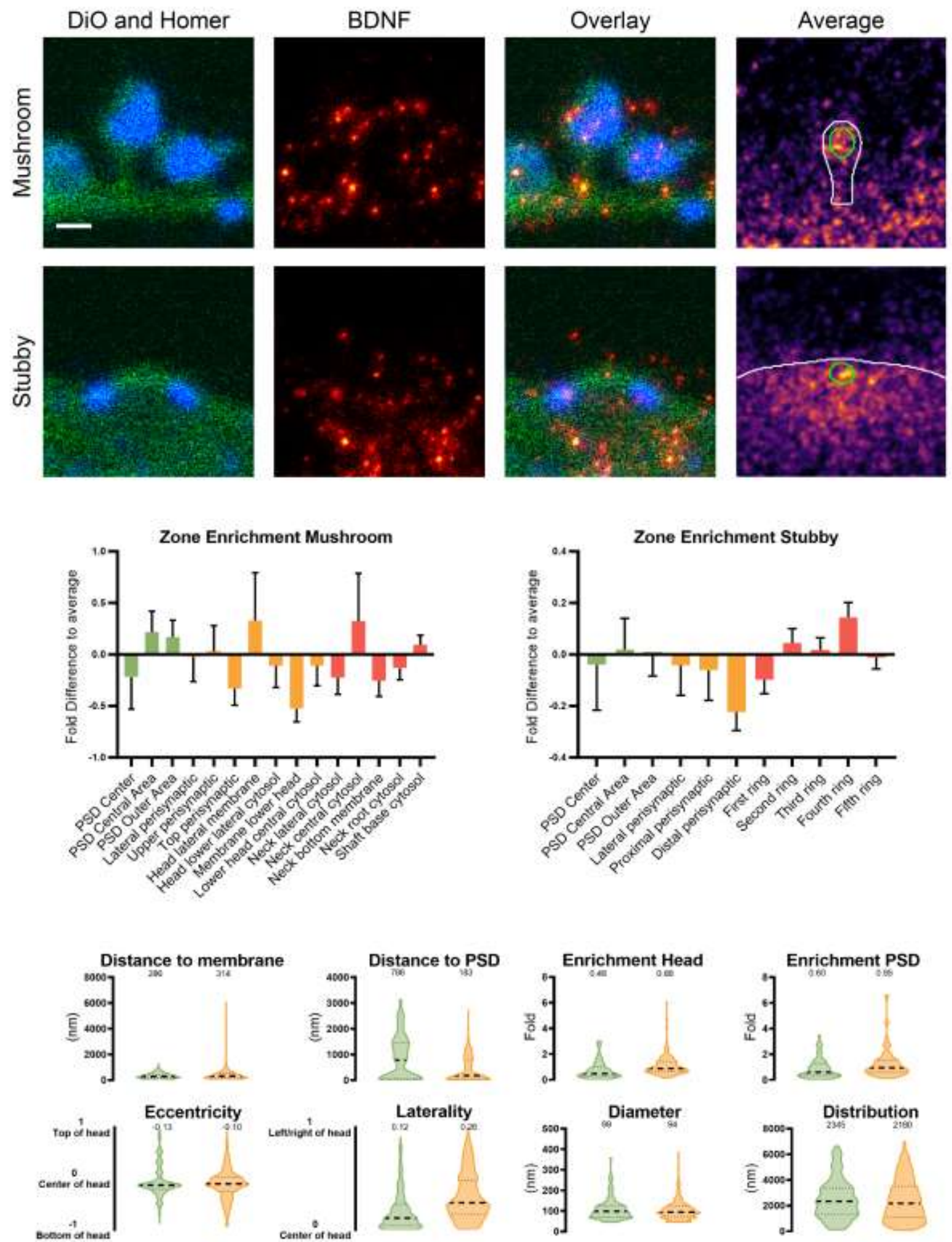

Figure 68: BDNF nanoscale localization and abundance. Continued on next page. 


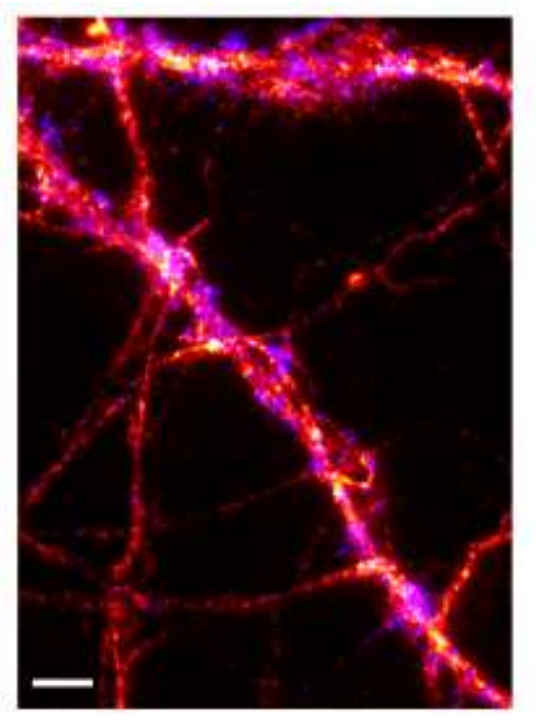

\begin{tabular}{|c|c|c|}
\hline $\begin{array}{c}\text { Whole cell copy } \\
\text { number }\end{array}$ & \multicolumn{2}{|c|}{$158815.27 \pm 30648.19$} \\
\hline $\begin{array}{c}\text { Spine copy } \\
\text { number }\end{array}$ & \multicolumn{2}{|c|}{$35.65 \pm 8.51$} \\
\hline$\%$ in PSD & \multicolumn{2}{|c|}{16.76} \\
\hline PSD copy number & \multicolumn{2}{|c|}{$5.12 \pm 1.22$} \\
\hline & Mushroom & Stubby \\
\hline $\begin{array}{c}\text { Spine copy } \\
\text { number }\end{array}$ & $28.52 \pm 6.81$ & $44.87 \pm 10.71$ \\
\hline PSD copy number & $4.09 \pm 0.98$ & $6.44 \pm 1.54$ \\
\hline$\%$ of protein & $0.00 \pm 0.00 \%$ & $0.00 \pm 0.00 \%$ \\
\hline Molarity [MM] & $0.24 \pm 0.06$ & $0.24 \pm 0.06$ \\
\hline
\end{tabular}

\section{References}

Antibody: Biorbyt orb38809

PDB Identifier: $1 \mathrm{~b} 8 \mathrm{~m}$

\section{Literature:}

Adachi et al., 2005; Hartmann et al., 2001; Kohara et al., 2001; Korte et al., 1995; Leal et al., 2015; Minichiello et al., 2002; Patterson et al., 1996; Soppet et al., 1991 


\section{Calbindin-D28K (Gene: Calb1, Uniprot ID: P07171)}

Known function: Calcium buffer, Only found in a subset of hippocampal neurons Known organization: Cytosolic

Known Interactions: None
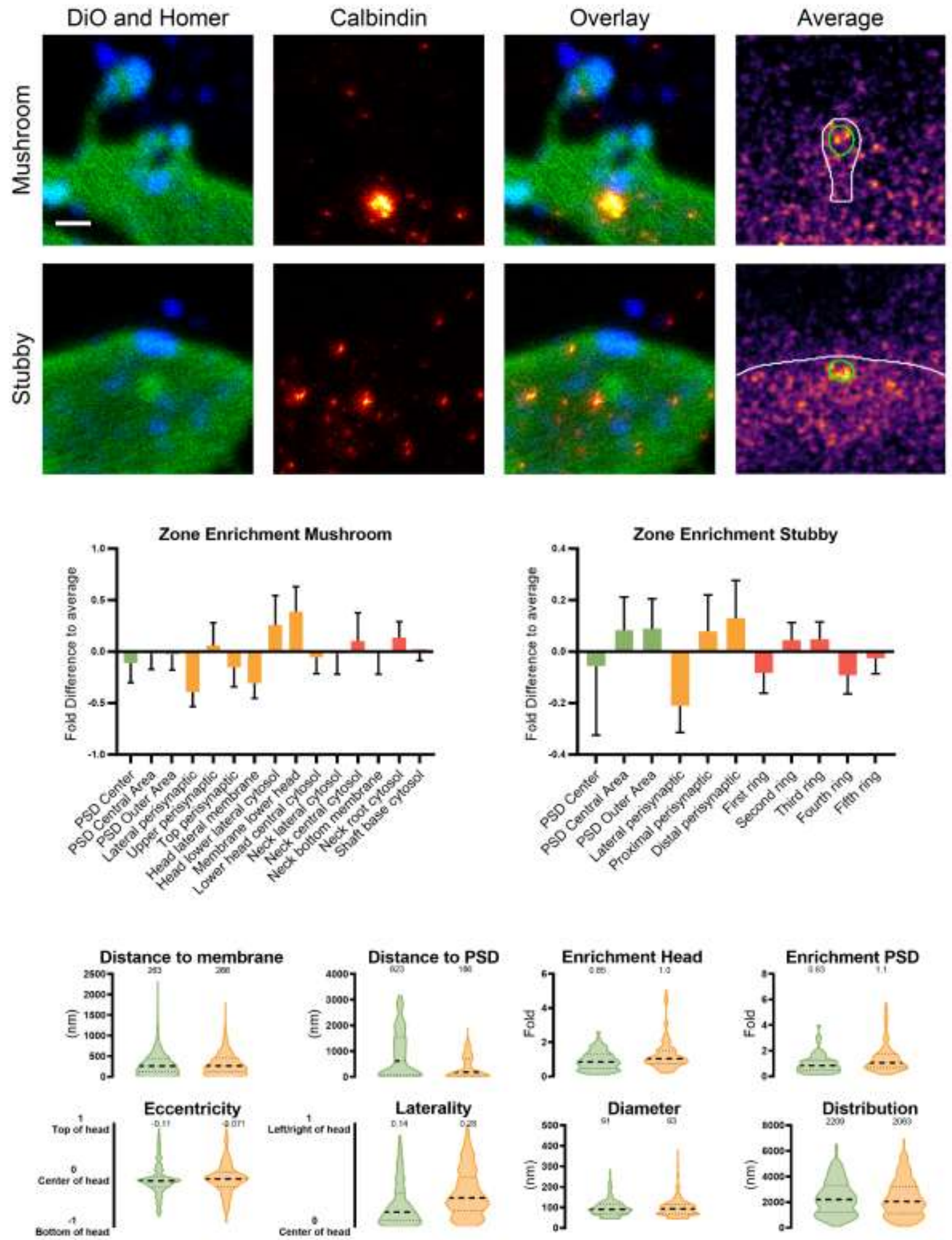

Figure 69: Calbindin nanoscale localization and abundance. Continued on next page. 


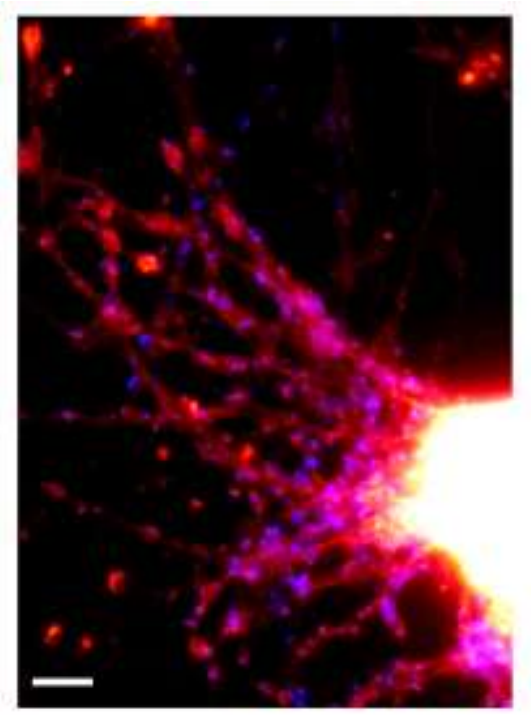

\begin{tabular}{|c|c|c|}
\hline $\begin{array}{c}\text { Whole cell copy } \\
\text { number }\end{array}$ & \multicolumn{2}{|c|}{$4366931.71 \pm 1085798.02$} \\
\hline $\begin{array}{c}\text { Spine copy } \\
\text { number }\end{array}$ & \multicolumn{2}{|c|}{$627.61 \pm 207.65$} \\
\hline$\%$ in PSD & \multicolumn{2}{|c|}{20.36} \\
\hline PSD copy number & \multicolumn{2}{|c|}{$106.14 \pm 35.12$} \\
\hline & Mushroom & Stubby \\
\hline $\begin{array}{c}\text { Spine copy } \\
\text { number }\end{array}$ & 554.49 & 699.78 \\
\hline PSD copy number & $93.78 \pm 31.03$ & $118.35 \pm 39.16$ \\
\hline$\%$ of protein & $0.04 \pm 0.01 \%$ & $0.03 \pm 0.01 \%$ \\
\hline Molarity [MM] & $4.65 \pm 1.54$ & $3.75 \pm 1.24$ \\
\hline
\end{tabular}

Mushroom molecular model

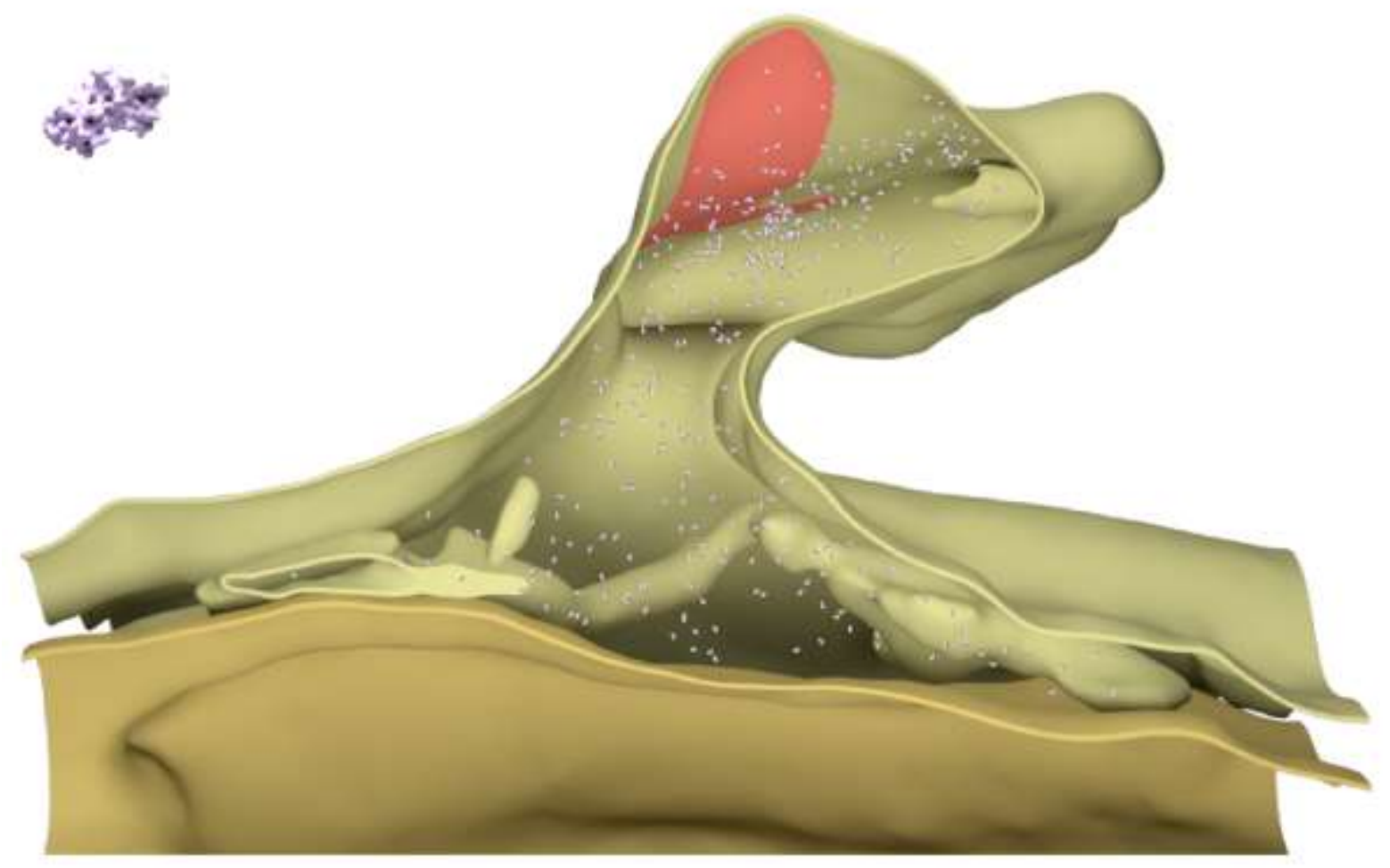

\section{References}

Antibody: Synaptic Systems 214002

PDB Identifier: 2g9b

\section{Literature:}

Berggard et al., 2002; Veenstra et al., 1997; Villa et al., 1994 


\section{Calmodulin (Gene: Calm, Uniprot ID: P0DP29)}

Known function: Important calcium binding protein, Regulates over 100 target proteins. Known organization: Cytosolic. Also membrane-attached via interaction with neurogranin Known Interactions: CaMKII, Calcineurin, Calcium channels, nNOS, PSD95
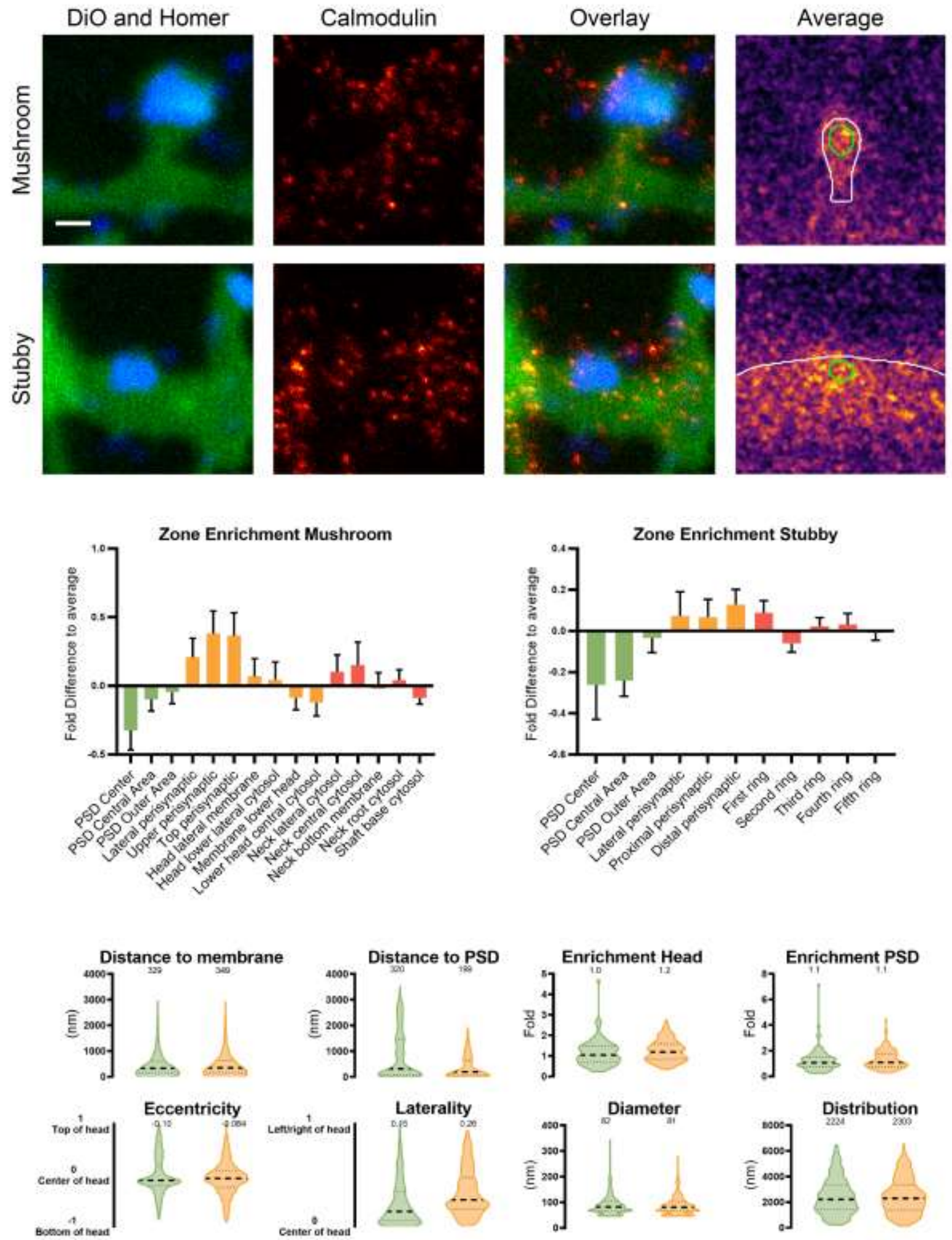

Figure 70: Calmodulin nanoscale localization and abundance. Continued on next page. 

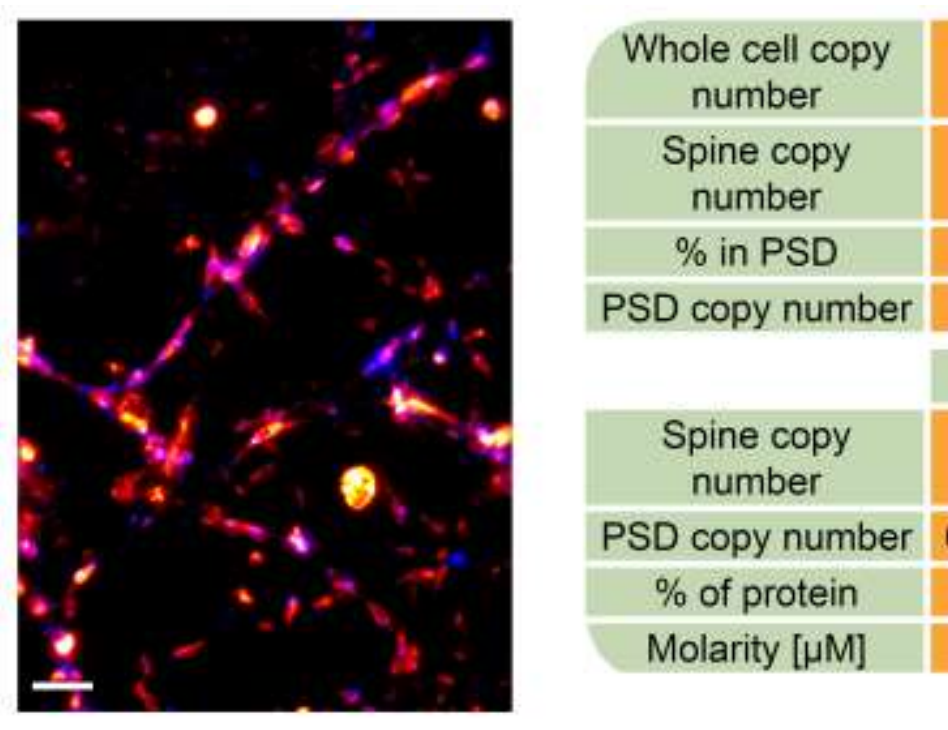

$37553352.54 \pm 6052717.99$

\begin{tabular}{|c|c|c|}
\hline & Mushroom & Stubby \\
\hline $\begin{array}{l}\text { Spine copy } \\
\text { number }\end{array}$ & $\begin{array}{c}4121.88 \\
\pm 1839.86\end{array}$ & $\begin{array}{c}5139.52 \\
\pm 2294.10 \\
\end{array}$ \\
\hline PSD copy number & $624.72 \pm 278.85$ & $778.96 \pm 347.70$ \\
\hline$\%$ of protein & $0.16 \pm 0.07 \%$ & $0.13 \pm 0.06 \%$ \\
\hline Molarity $[\mu \mathrm{M}]$ & $34.55 \pm 15.42$ & $27.55 \pm 12.30$ \\
\hline
\end{tabular}

Mushroom molecular model

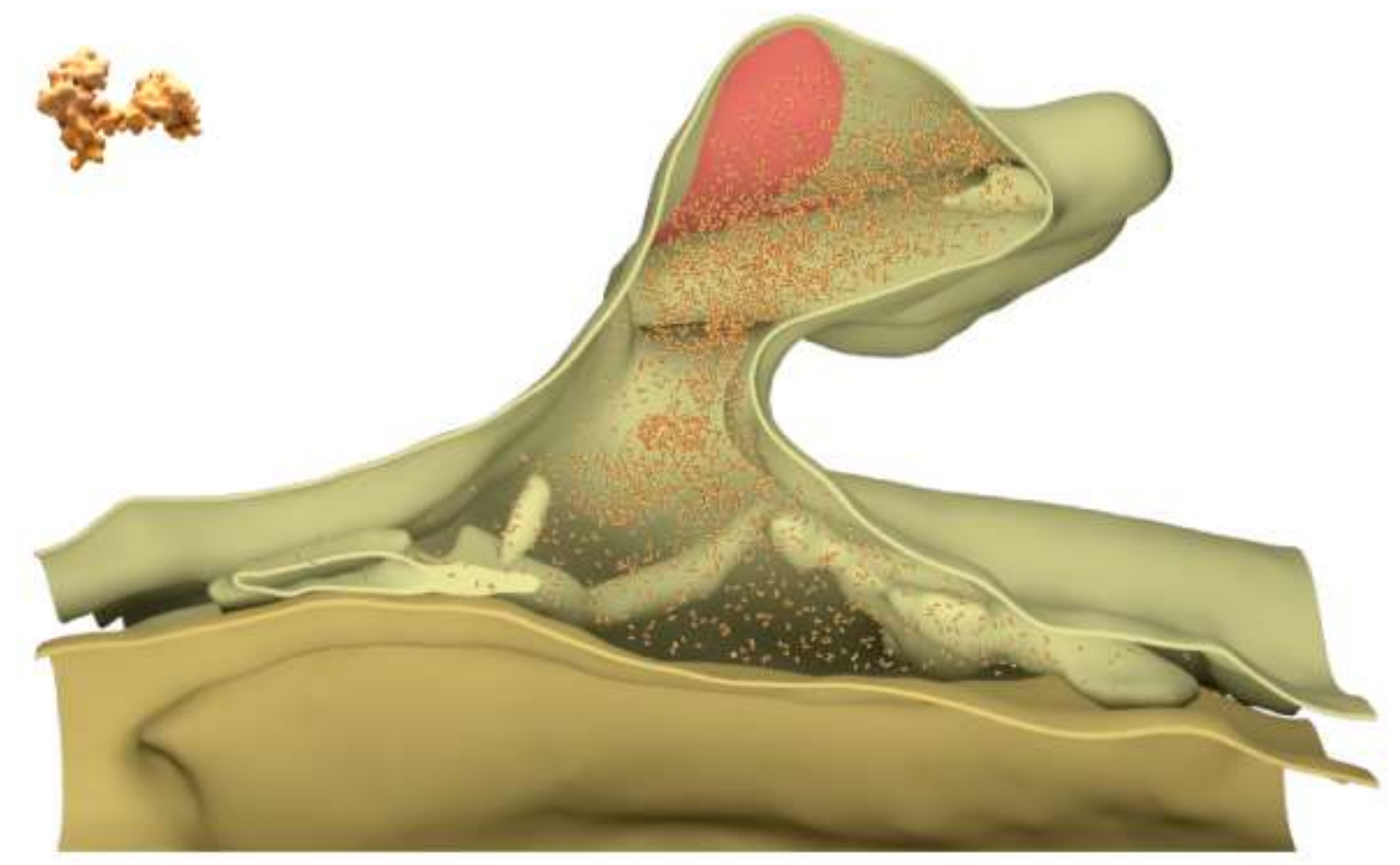

\section{References}

Antibody: Abcam ab45689

PDB Identifier: 1up5

\section{Literature:}

Bartos et al., 2010; Baudier et al., 1991; Chowdhury et al., 2017; Fukunaga et al., 2005; Olwin et al., 1984; Prichard et al., 1999; Xia and Storm, 2005; Zhang et al., 2014 


\section{Calretinin (Gene: Calb2, Uniprot ID: P47728)}

Known function: Calcium binding protein

Known organization: Cytosolic

Known Interactions: $\mathrm{Ca}_{2} 2.1$
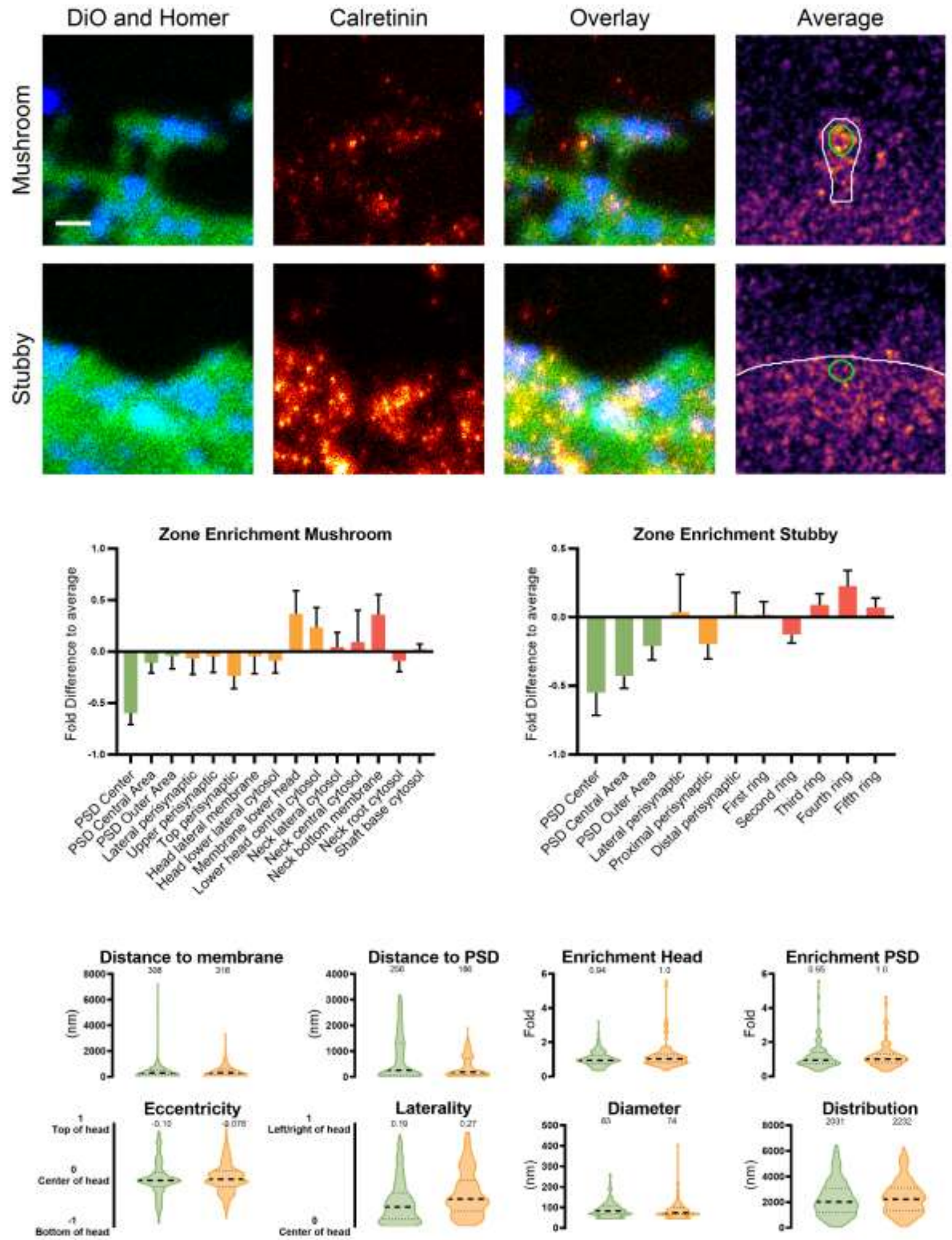

Figure 71: Calretinin nanoscale localization and abundance. Continued on next page. 


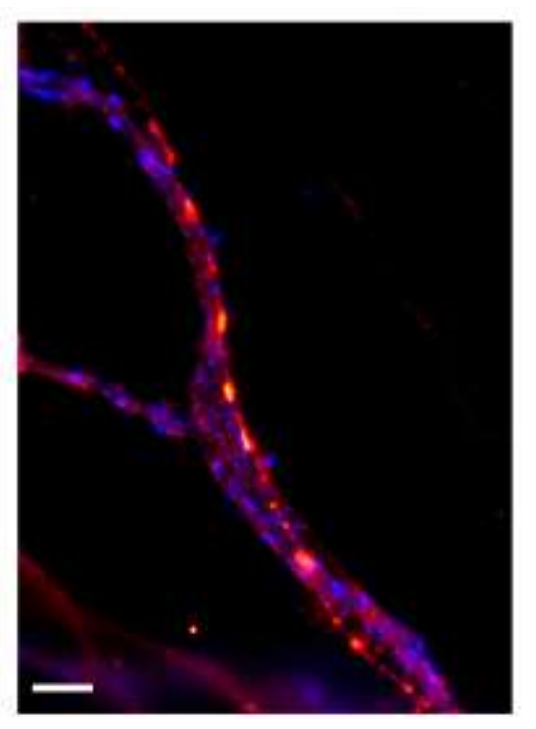

\begin{tabular}{|c|c|c|}
\hline $\begin{array}{c}\text { Whole cell copy } \\
\text { number }\end{array}$ & \multicolumn{2}{|c|}{$374551.58 \pm 90506.69$} \\
\hline $\begin{array}{c}\text { Spine copy } \\
\text { number }\end{array}$ & \multicolumn{2}{|c|}{$27.23 \pm 11.05$} \\
\hline$\%$ in PSD & \multicolumn{2}{|c|}{15.74} \\
\hline PSD copy number & \multicolumn{2}{|c|}{$3.70 \pm 1.50$} \\
\hline & Mushroom & Stubby \\
\hline $\begin{array}{c}\text { Spine copy } \\
\text { number }\end{array}$ & $21.91 \pm 8.89$ & $34.33 \pm 13.93$ \\
\hline PSD copy number & $2.98 \pm 1.21$ & $4.67 \pm 1.89$ \\
\hline$\%$ of protein & $0.00 \pm 0.00 \%$ & $0.00 \pm 0.00 \%$ \\
\hline Molarity $[\mu \mathrm{M}]$ & $0.18 \pm 0.07$ & $0.18 \pm 0.07$ \\
\hline
\end{tabular}

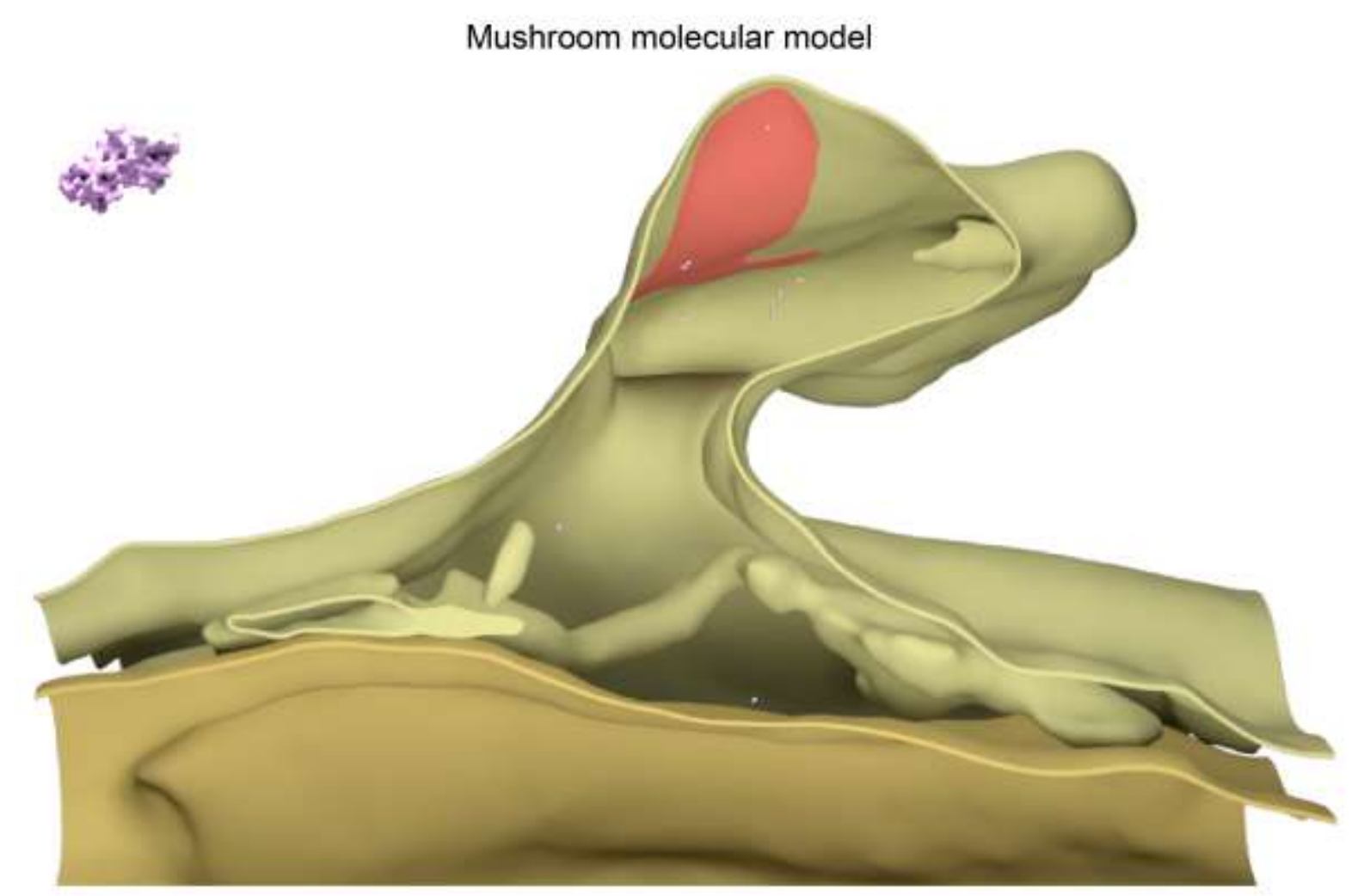

\section{References}

Antibody: Novus Biologicals NBP1-88220 Structure: Modelled by Burkhard Rammner Literature:

Christel et al., 2012; Dargan et al., 2004; Molgaard et al., 2014 
CaMKII $\alpha / \beta$ (Genes: Camk2a, Camk2b Uniprot ID: P11275, P08413)

Known function: Central kinase in dendritic spines, Inolved in synaptic plasticity Known organization: Cytosolic, Forms dodecamer

Known Interactions: AMPA receptors, Calmodulin, $\mathrm{Ca}_{v} 2.1, \mathrm{CDC} 42, \mathrm{GluN2B}, \mathrm{Homer}$ proteins, PSD95, F-actin
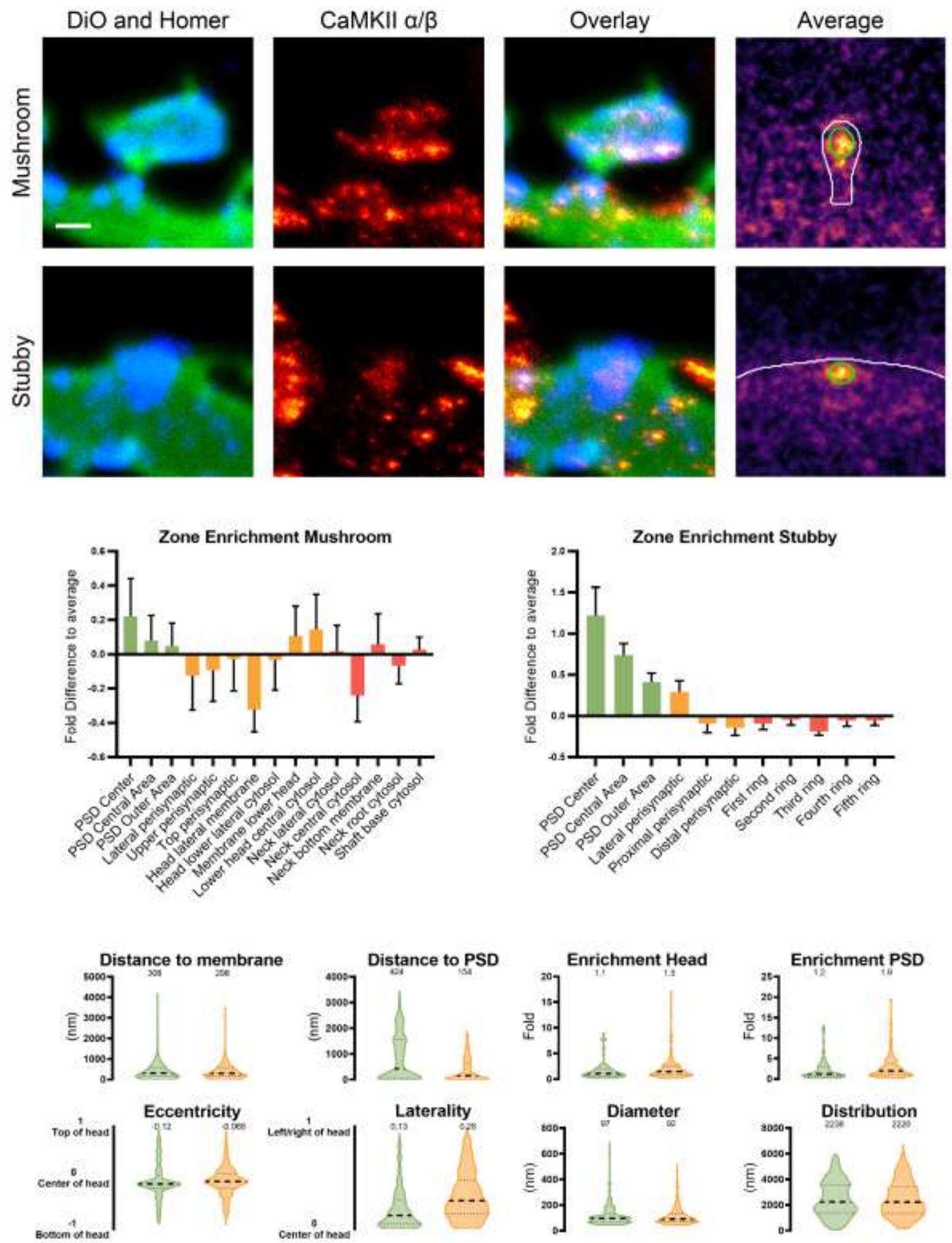

Figure 72: CaMKII nanoscale localization and abundance. Continued on next page. 

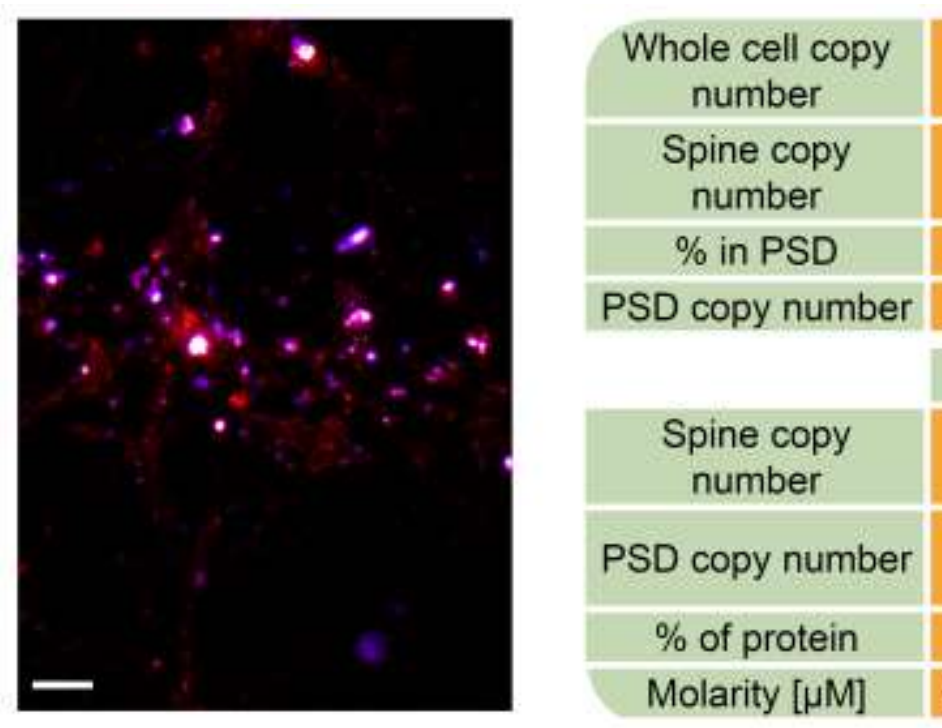

\begin{tabular}{|c|}
\hline $55667540.57 \pm 3269411.75$ \\
\hline $44582.29 \pm 5110.66$ \\
\hline 28.14 \\
\hline $9789.92 \pm 1122.26$ \\
\hline
\end{tabular}

\begin{tabular}{|c|c|c|}
\hline & Mushroom & Stubby \\
\hline $\begin{array}{c}\text { Spine copy } \\
\text { number }\end{array}$ & $\begin{array}{c}\text { 35398.75 } \\
\pm 4057.91\end{array}$ & $\begin{array}{l}58510.72 \\
\pm 6707.34\end{array}$ \\
\hline PSD copy number & $\begin{array}{l}7773.29 \\
\pm 891.09\end{array}$ & $\begin{array}{c}12848.49 \\
\pm 1472.88\end{array}$ \\
\hline \% of protein & $4.43 \pm 0.51 \%$ & $4.69 \pm 0.54 \%$ \\
\hline Molarity $[\mu \mathrm{M}]$ & $296.69 \pm 34.01$ & $313.67 \pm 35.96$ \\
\hline
\end{tabular}

Mushroom molecular model

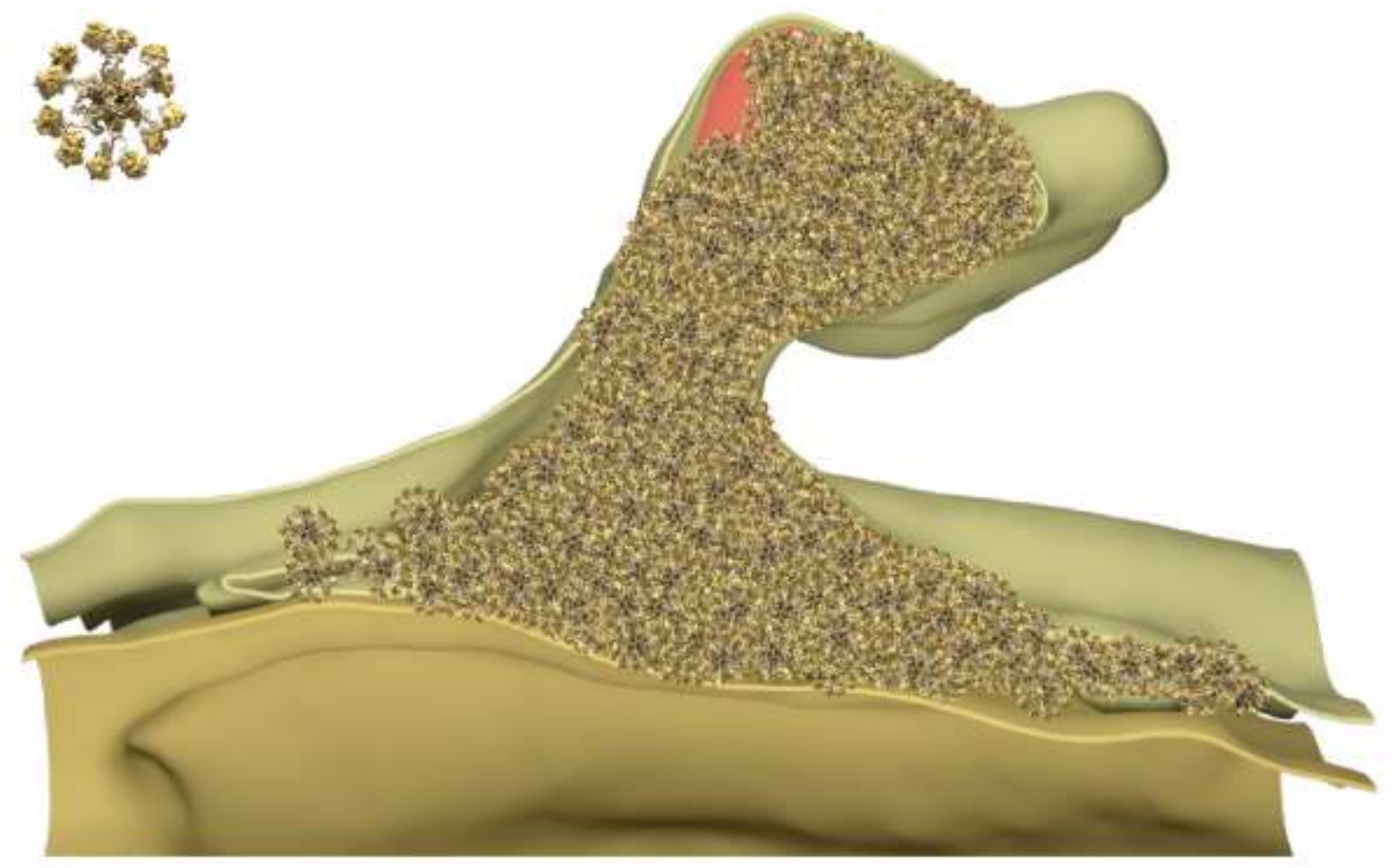

\section{References}

Antibody: Abnova MAB6627

PDB Identifier: 5u6y

\section{Literature:}

Barria and Malinow, 2005; Braun and Schulman, 1995; Chao et al., 2011; Colbran and Brown, 2004; Fink et al., 2003; Gardoni et al., 2006; Halt et al., 2012; Hell, 2014; Jiang et al., 2008; Matsuzaki et al., 2004; McGlade-McCulloh et al., 1993; Mizutani et al., 2008; Murakoshi et al., 2011; Shen and Meyer, 1999; Zhou et al., 2007 
CAPS1 (Gene: Cadps, Uniprot ID: Q62717)

Known function: Regulates LDCV and secretory granule exocytosis

Known organization: Cytosolic

Known Interactions: None
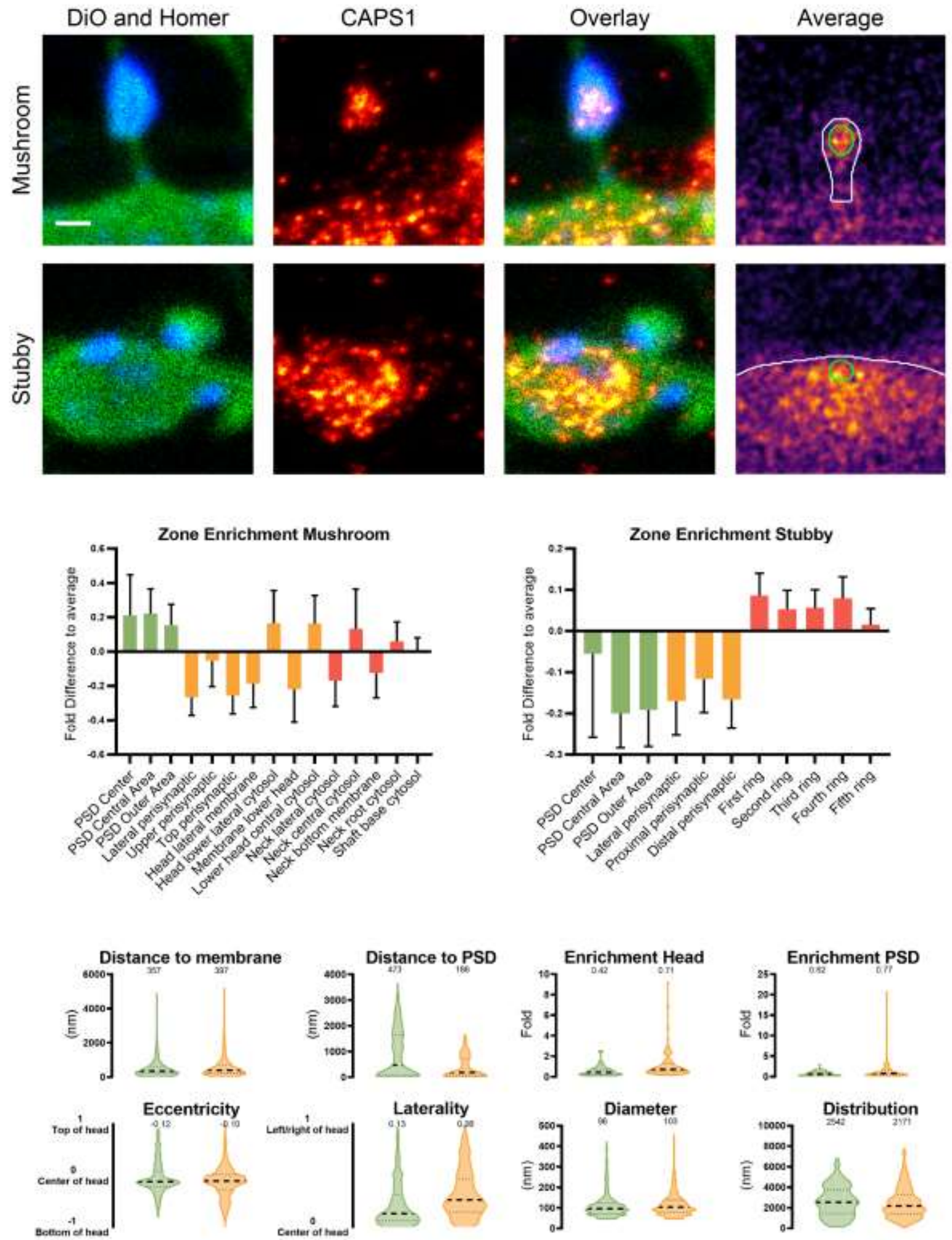

Figure 73: CAPS1 nanoscale localization and abundance. Continued on next page. 


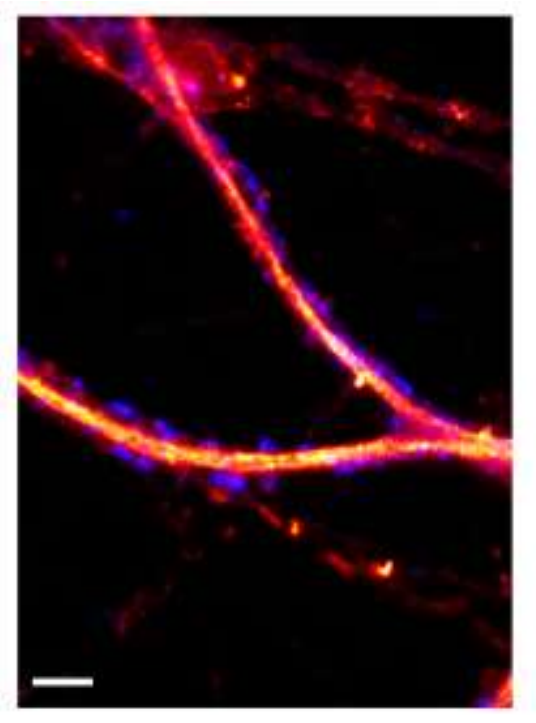

\begin{tabular}{|c|c|c|}
\hline $\begin{array}{c}\text { Whole cell copy } \\
\text { number }\end{array}$ & \multicolumn{2}{|c|}{$690565.64 \pm 120801.38$} \\
\hline $\begin{array}{c}\text { Spine copy } \\
\text { number }\end{array}$ & \multicolumn{2}{|c|}{$264.48 \pm 62.47$} \\
\hline$\%$ in PSD & \multicolumn{2}{|c|}{14.78} \\
\hline PSD copy number & \multicolumn{2}{|c|}{$34.06 \pm 8.05$} \\
\hline & Mushroom & Stubby \\
\hline $\begin{array}{c}\text { Spine copy } \\
\text { number }\end{array}$ & $217.41 \pm 51.35$ & $349.83 \pm 82.63$ \\
\hline PSD copy number & $28.00 \pm 6.61$ & $45.06 \pm 10.64$ \\
\hline$\%$ of protein & $0.07 \pm 0.02 \%$ & $0.08 \pm 0.02 \%$ \\
\hline Molarity $[\mu \mathrm{M}]$ & $1.82 \pm 0.43$ & $1.88 \pm 0.44$ \\
\hline
\end{tabular}

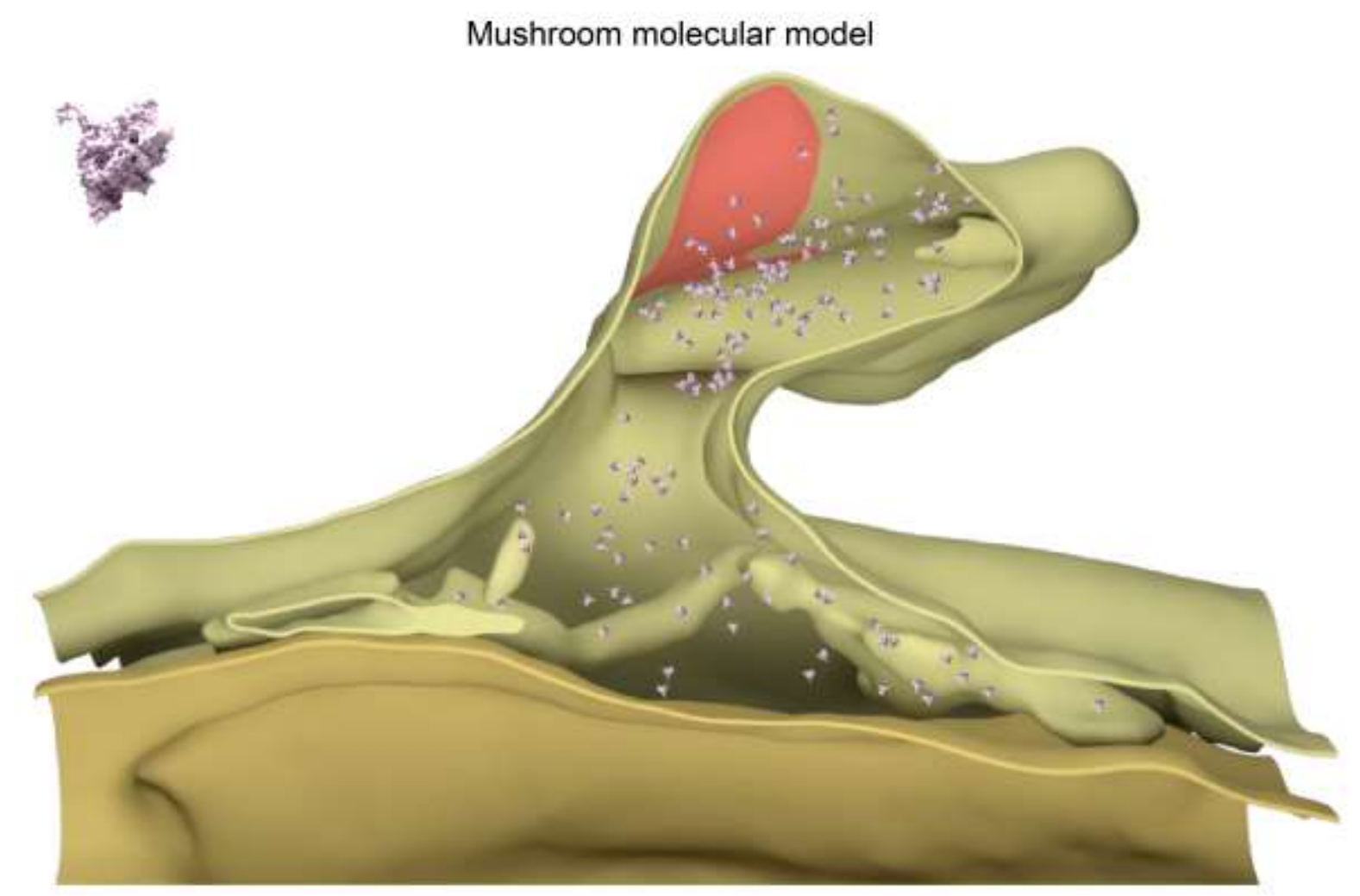

\section{References}

Antibody: Abcam ab69797

Structure: Modelled by Burkhard Rammner

\section{Literature:}

Eckenstaler et al., 2016; Farina et al., 2015; Sadakata et al., 2013 


\section{CDC42 (Gene: Cdc42, Uniprot ID: Q8CFN2)}

Known function: Rho type GTPase, Regulates actin cytoskeleton

Known organization: Cytosolic

Known Interactions: CaMKII, Homer2
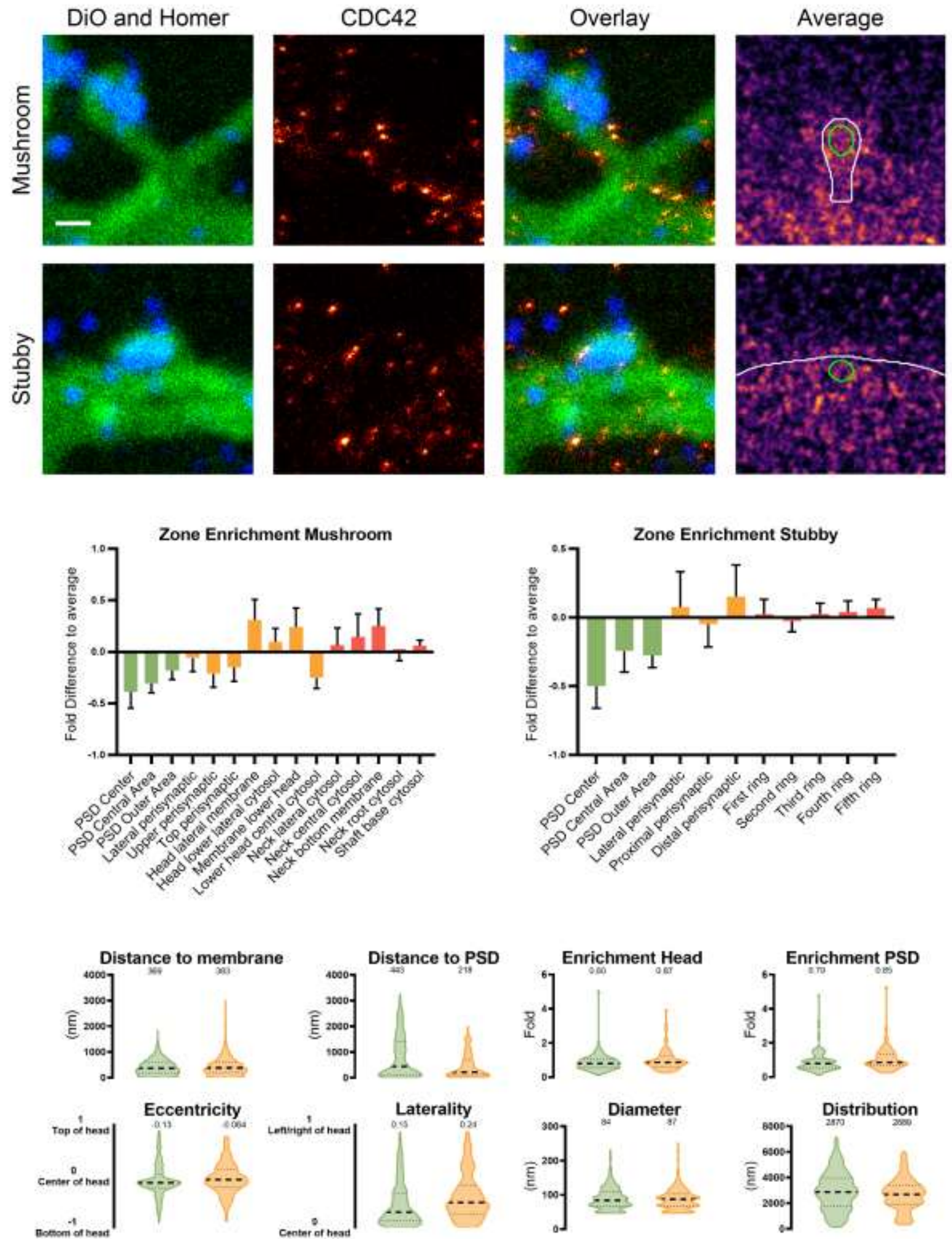

Figure 74: CDC42 nanoscale localization and abundance. Continued on next page. 

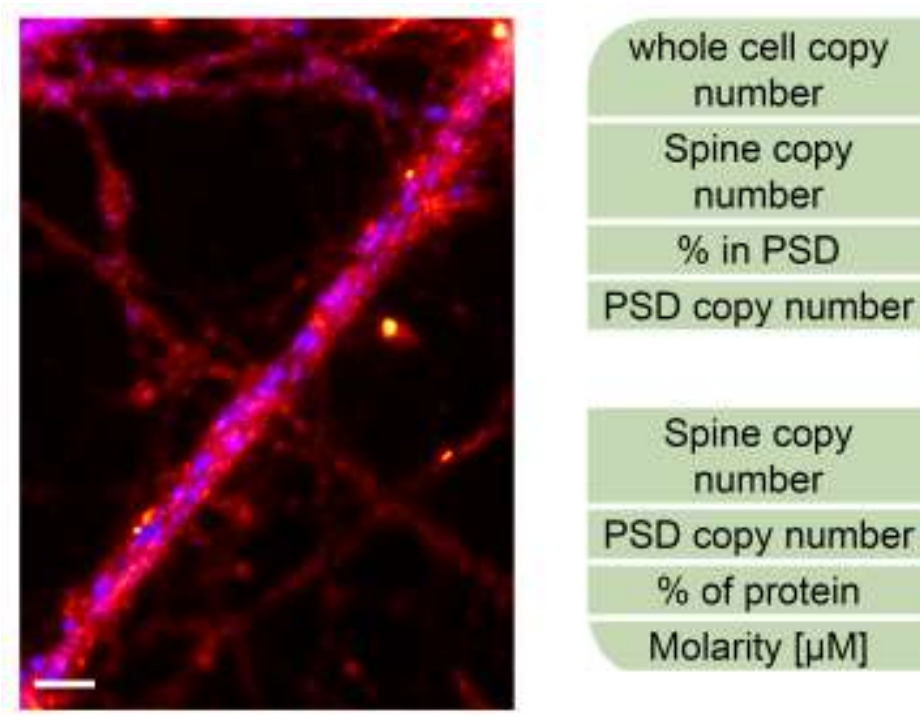

\begin{tabular}{|c|}
\hline $46617474.99 \pm 3970051.86$ \\
\hline $2179.68 \pm 2134.74$ \\
\hline 15.25 \\
\hline $288.42 \pm 282.48$ \\
\hline
\end{tabular}

\begin{tabular}{|c|c|c|}
\hline & Mushroom & Stubby \\
\hline $\begin{array}{c}\text { Spine copy } \\
\text { number }\end{array}$ & $\begin{array}{c}1595.00 \\
\pm 1562.12\end{array}$ & $\begin{array}{c}3014.07 \\
\pm 2951.93\end{array}$ \\
\hline PSD copy number & $211.06 \pm 206.71$ & $398.83 \pm 390.61$ \\
\hline$\%$ of protein & $0.08 \pm 0.08 \%$ & $0.09 \pm 0.09 \%$ \\
\hline Molarity $[\mu \mathrm{M}]$ & $13.37 \pm 13.09$ & $16.16 \pm 15.82$ \\
\hline
\end{tabular}

Mushroom molecular model

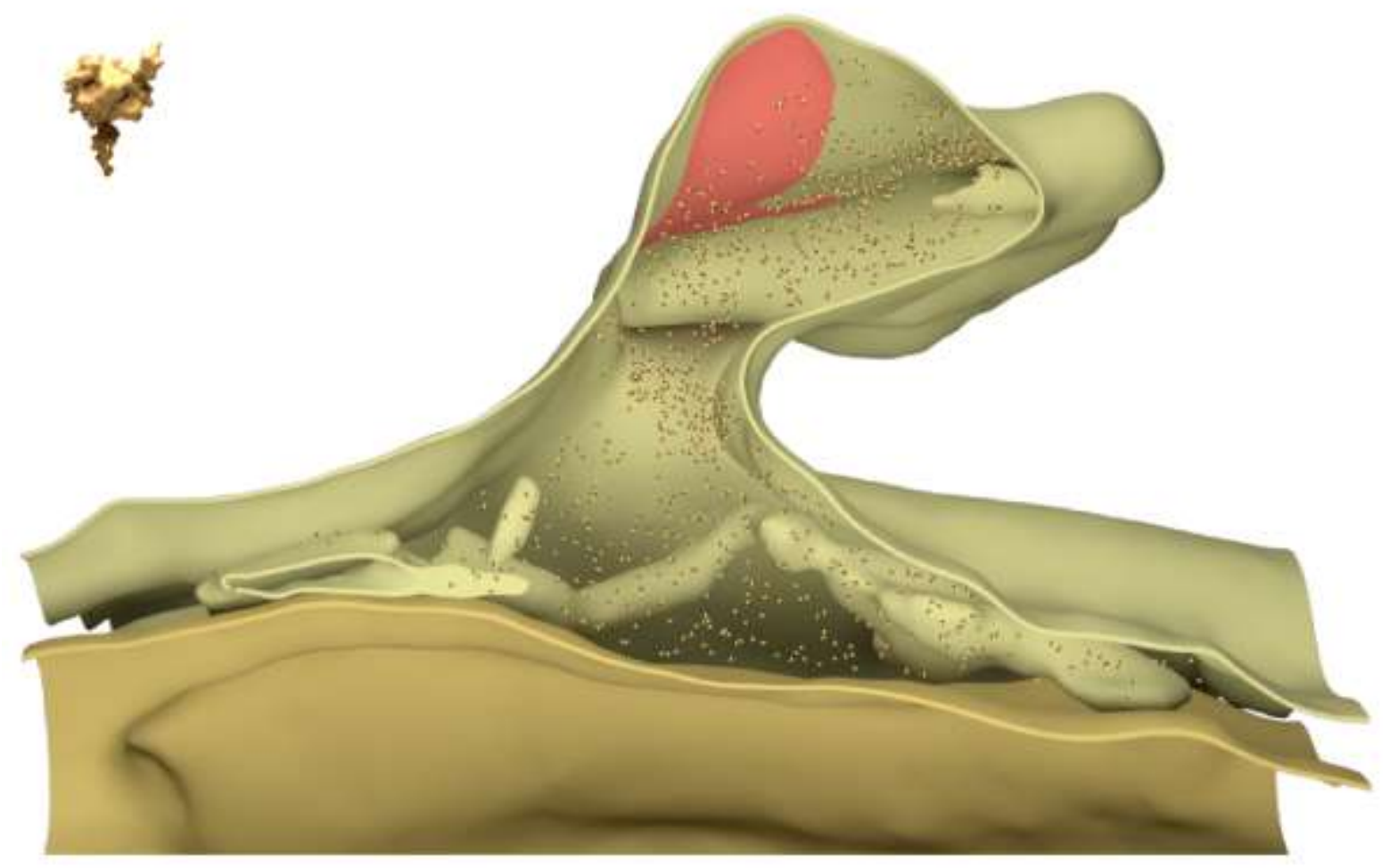

\section{References}

Antibody: Thermo Scientific PA1-092

PDB Identifier: $1 \mathrm{ki} 1$

\section{Literature:}

Choi et al., 2005; Murakoshi et al., 2011; Shiraishi-Yamaguchi et al., 2009 


\section{Chromogranin A (Beta-granin, Gene: Chga, Uniprot ID: P10354)}

Known function: Acts as pro-hormone, Involved in LDCV biogenesis

Known organization: In LDCV lumen

Known Interactions: Chromogranin B
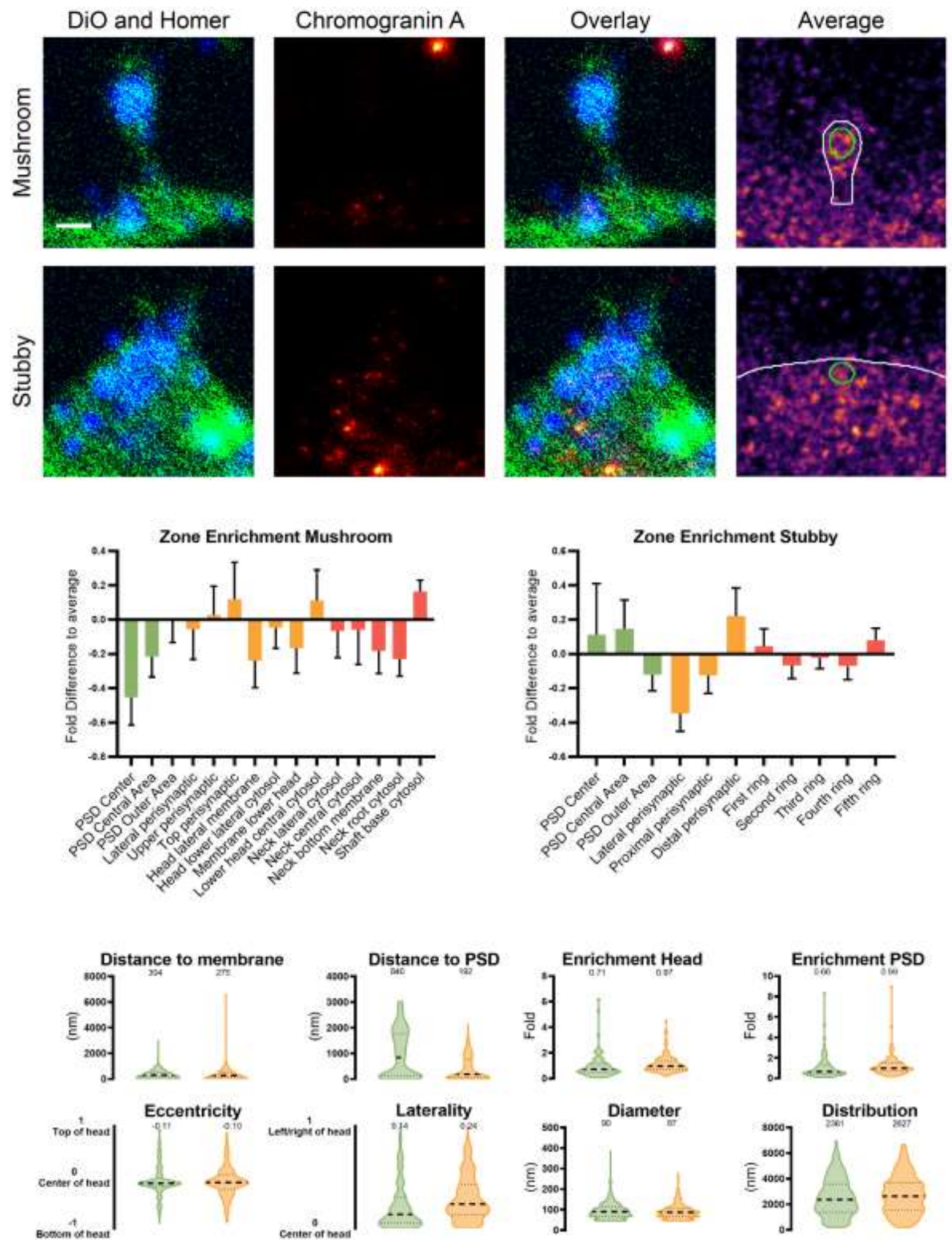

Figure 75: Chromogranin A nanoscale localization and abundance. Continued on next page. 


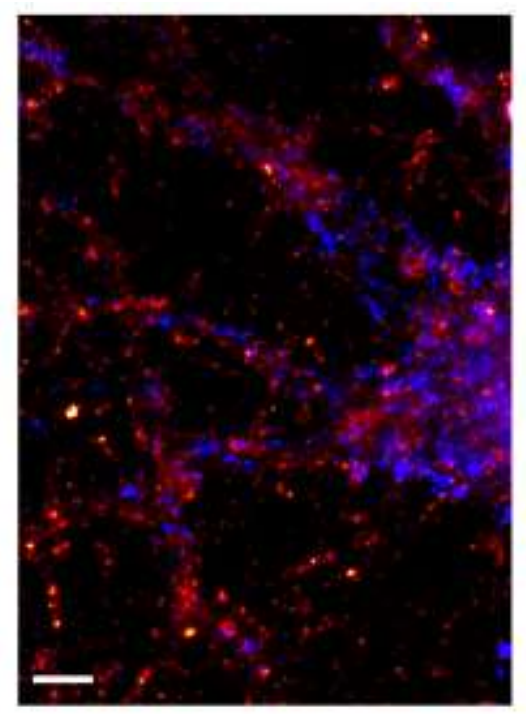

\begin{tabular}{|c|c|}
\hline $\begin{array}{c}\text { Whole cell copy } \\
\text { number }\end{array}$ & $\begin{array}{c}15200.85 \\
\text { (only detected in 1 replicate) }\end{array}$ \\
\hline $\begin{array}{c}\text { Spine copy } \\
\text { number }\end{array}$ & 2.41 \\
\hline$\%$ in PSD & 16.54 \\
\hline PSD copy number & 0.34 \\
\hline
\end{tabular}

\begin{tabular}{|c|c|c|}
\hline & Mushroom & Stubby \\
\hline $\begin{array}{l}\text { Spine copy } \\
\text { number }\end{array}$ & 2.16 & 2.61 \\
\hline PSD copy number & 0.31 & 0.37 \\
\hline$\%$ of protein & $0.00 \%$ & $0.00 \%$ \\
\hline Molarity $[\mu \mathrm{M}]$ & 0.02 & 0.01 \\
\hline
\end{tabular}

\section{References}

Antibody: Synaptic Systems $259003 \quad$ PDB Identifier: Modelled with I-TASSER

\section{Literature:}

Krömer et al., 1998; Montero-Hadjadje et al., 2009; Natori and Huttner, 1996; Sun et al., 2013 


\section{Chromogranin B (Secretogranin I, Gene: Chgb, Uniprot ID: 035314)}

Known function: Acts as pro-hormone, Involved in LDCV biogenesis

Known organization: In LDCV lumen

Known Interactions: Chromogranin A
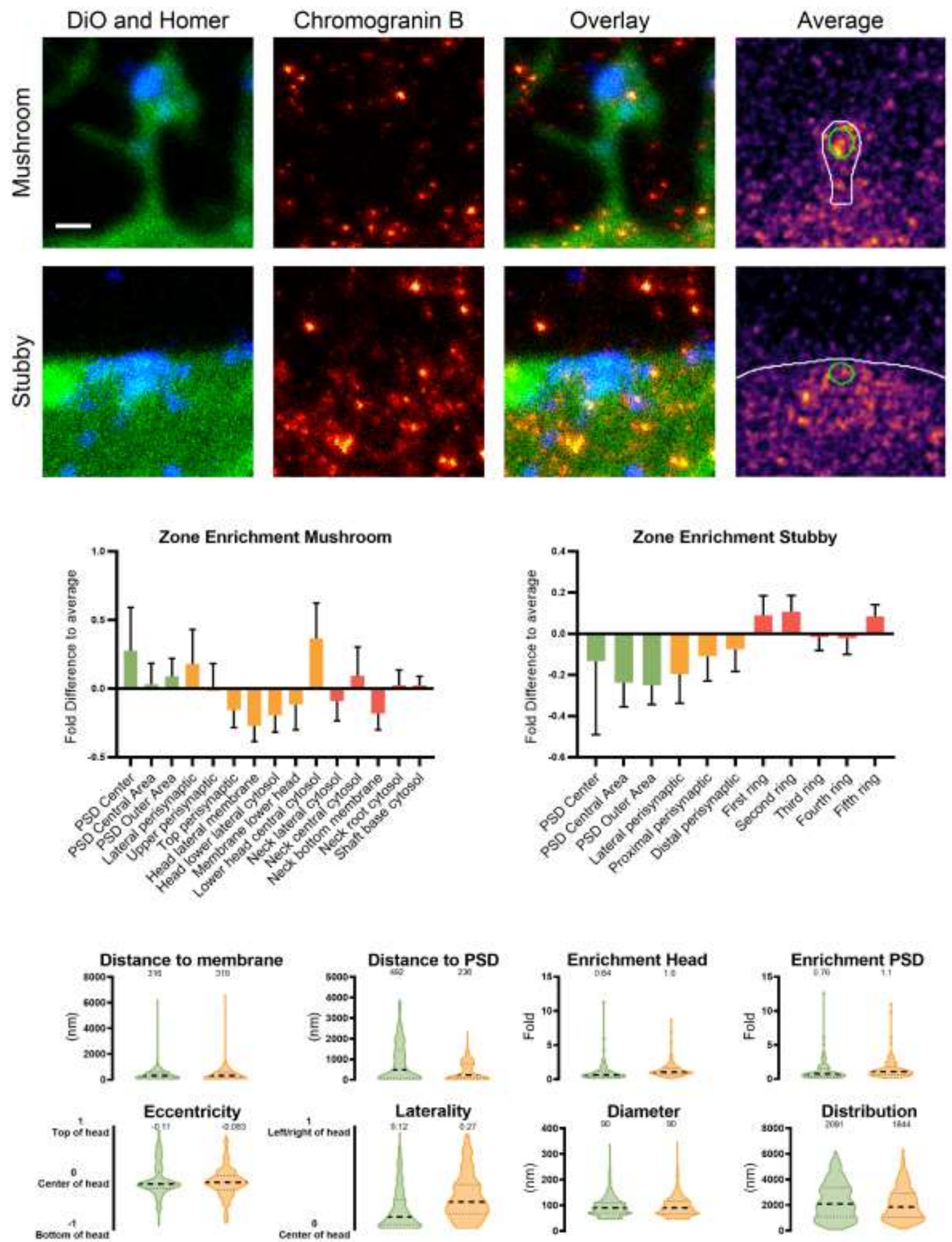

Figure 76: Chromogranin B nanoscale localization and abundance. Continued on next page. 


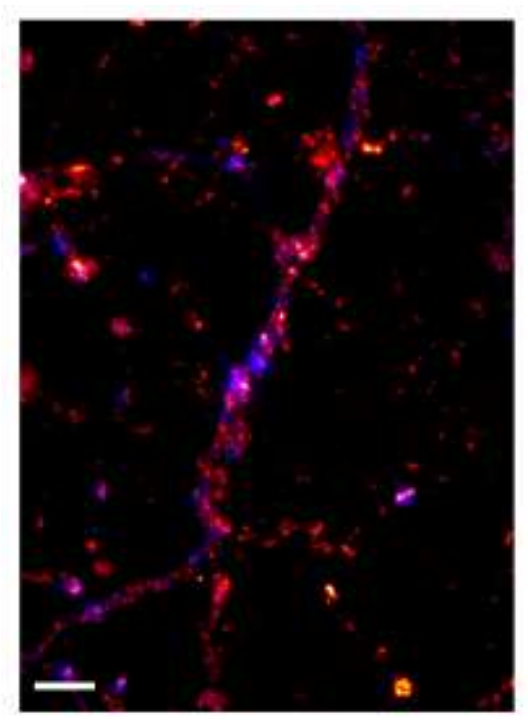

\begin{tabular}{|c|c|c|}
\hline $\begin{array}{c}\text { Whole cell copy } \\
\text { number }\end{array}$ & \multicolumn{2}{|c|}{$197749.76 \pm 40652.97$} \\
\hline $\begin{array}{c}\text { Spine copy } \\
\text { number }\end{array}$ & \multicolumn{2}{|c|}{$40.39 \pm 11.76$} \\
\hline$\%$ in PSD & \multicolumn{2}{|c|}{15.59} \\
\hline PSD copy number & \multicolumn{2}{|c|}{$5.45 \pm 1.59$} \\
\hline & Mushroom & Stubby \\
\hline \begin{tabular}{c|c|} 
Spine copy \\
number
\end{tabular} & $40.33 \pm 11.74$ & $40.58 \pm 11.81$ \\
\hline PSD copy number & $5.44 \pm 1.58$ & $5.47 \pm 1.59$ \\
\hline$\%$ of protein & $0.01 \pm 0.00 \%$ & $0.00 \pm 0.00 \%$ \\
\hline Molarity $[\mu \mathrm{M}]$ & $0.34 \pm 0.10$ & $0.22 \pm 0.06$ \\
\hline
\end{tabular}

\section{References}

Antibody: Synaptic Systems 259103

Structure: Modelled with I-TASSER

\section{Literature:}

Krömer et al., 1998; Montero-Hadjadje et al., 2009; Natori and Huttner, 1996; Sun et al., 2013 
Neuronal NO synthethase (nNOS, Gene: Nos1, Uniprot ID: P29476)

Known function: Production of NO, Activates cGMP production and Akt, Has

neuroprotective functions by regulating glutamate and GABA release as well as NMDA receptors, Acts as retrograde signalling molecule

Known organization: Cytosolic

Known Interactions: Calmodulin, NMDA receptors, PSD93, PSD95
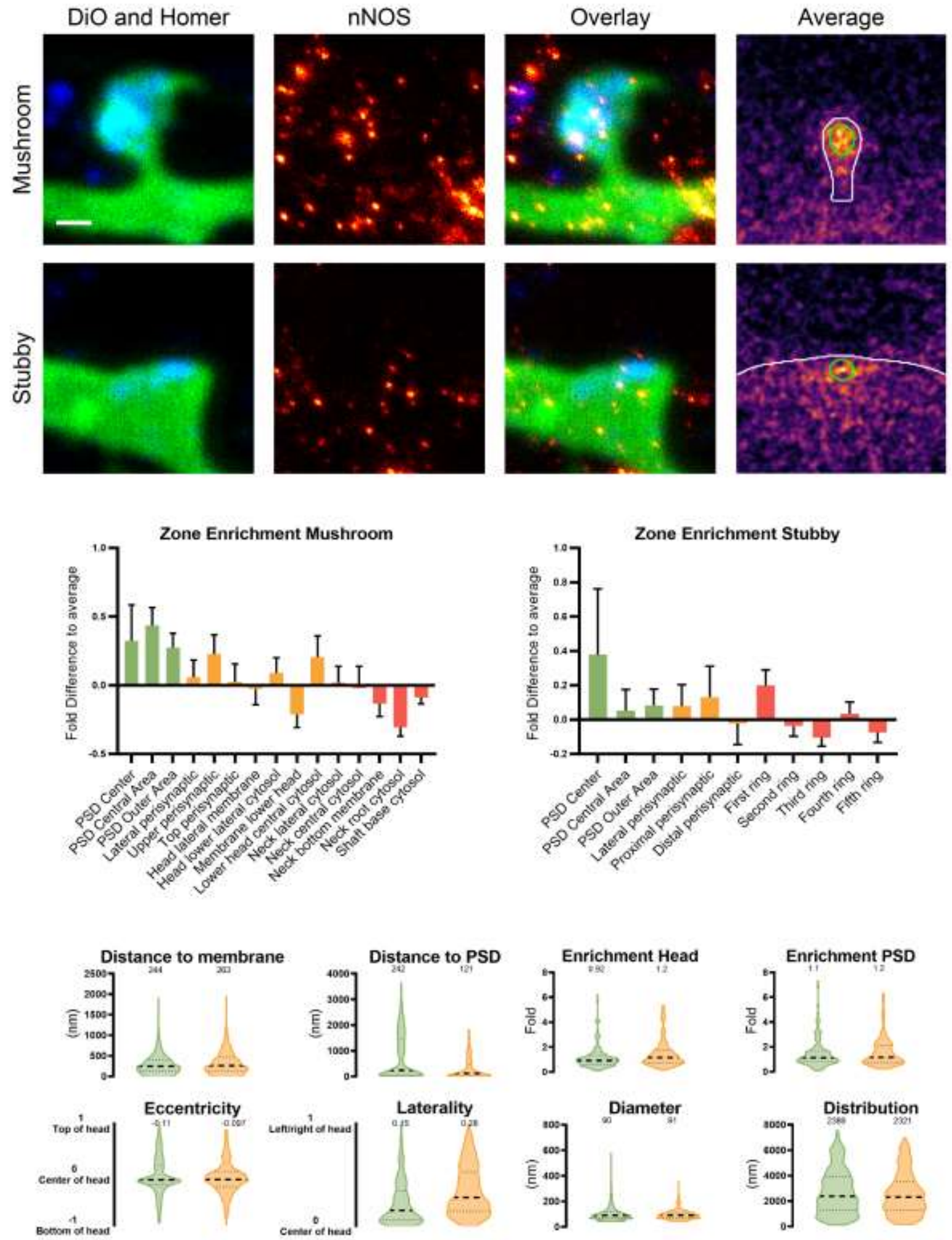

Figure 77: nNOS nanoscale localization and abundance. Continued on next page. 


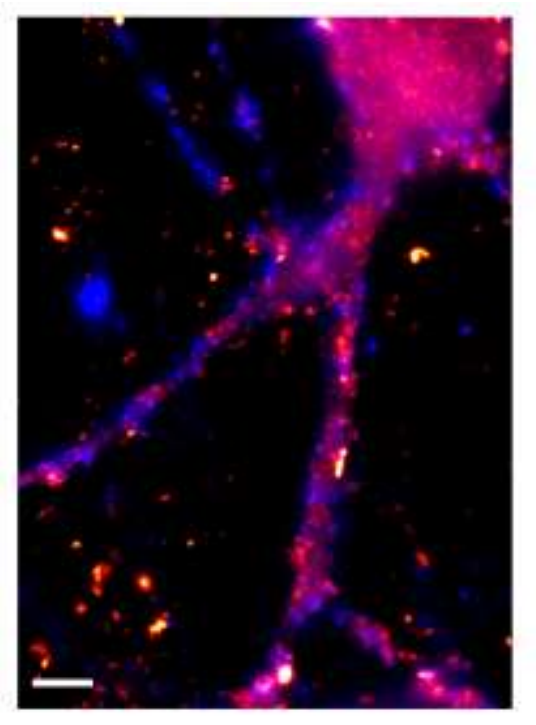

\begin{tabular}{|c|c|}
\hline $\begin{array}{c}\text { Whole cell copy } \\
\text { number }\end{array}$ & $162115.34 \pm 39288.49$ \\
\hline $\begin{array}{c}\text { Spine copy } \\
\text { number }\end{array}$ & $43.56 \pm 11.70$ \\
\hline$\%$ in PSD & 24.82 \\
\hline PSD copy number & $8.66 \pm 2.33$ \\
\hline
\end{tabular}

\begin{tabular}{|c|c|c|}
\hline & Mushroom & Stubby \\
\hline $\begin{array}{l}\text { Spine copy } \\
\text { number }\end{array}$ & $36.90 \pm 9.91$ & $51.29 \pm 13.78$ \\
\hline PSD copy number & $7.34 \pm 1.97$ & $10.20 \pm 2.74$ \\
\hline$\%$ of protein & $0.01 \pm 0.00 \%$ & $0.01 \pm 0.00 \%$ \\
\hline Molarity $[\mu \mathrm{M}]$ & $0.31 \pm 0.08$ & $0.27 \pm 0.07$ \\
\hline
\end{tabular}

Mushroom molecular model

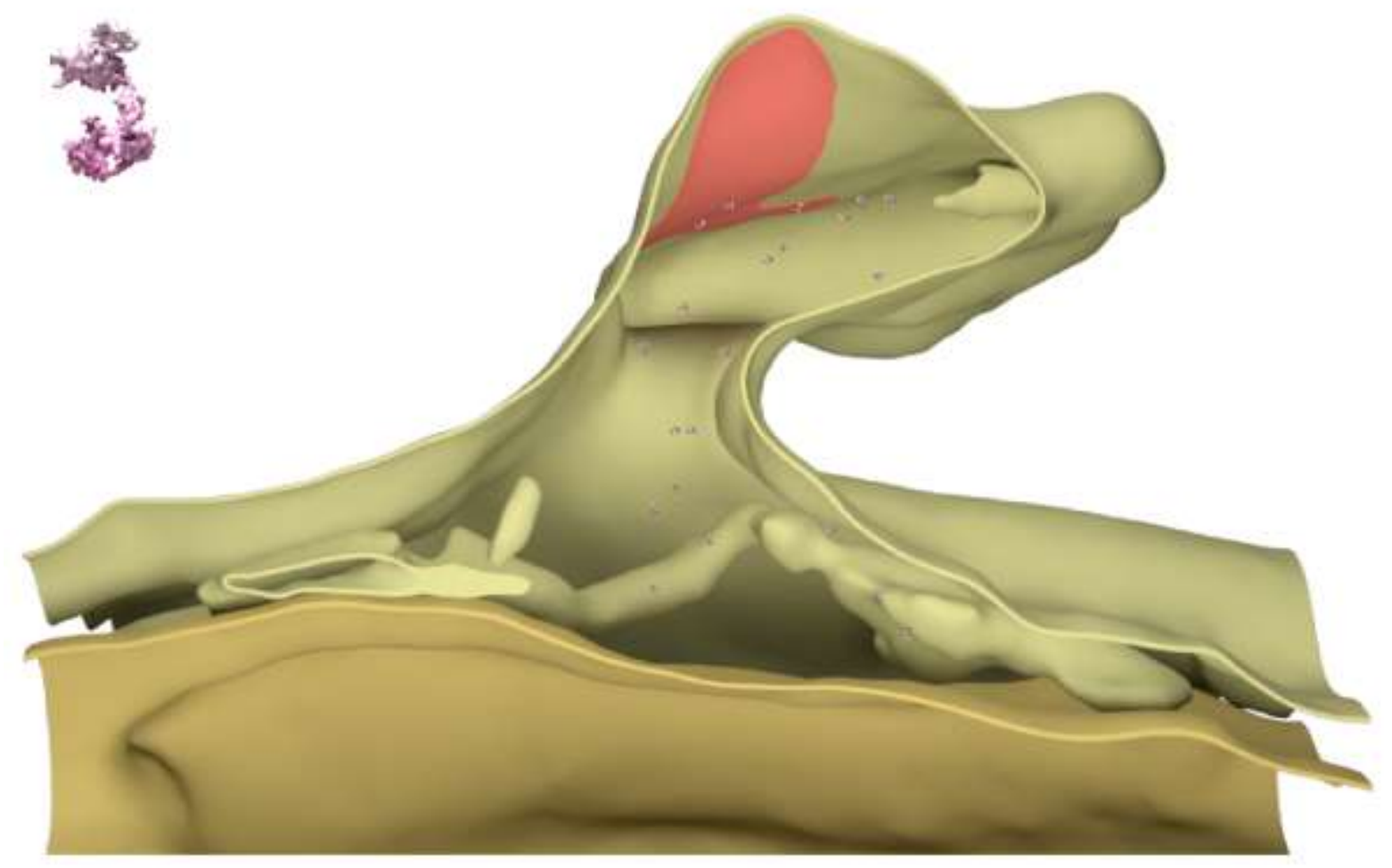

\section{References}

Antibody: Thermo Scientific PA1-033

PDB Identifier: $1 \mathrm{~b} 8 \mathrm{q}, 1 \mathrm{f} 20$

\section{Literature:}

Aarts et al., 2002; Aoki et al., 1997; Arnold et al., 1977; Bon and Garthwaite, 2003; Brenman et al., 1996b; Calabrese et al., 2007; Choi et al., 2000; Getting et al., 1996; Hopper et al., 2004; Jaffrey et al., 1998; Lonart et al., 1992; Ohkuma et al., 1996; Riccio et al., 2006 
Secretogranin II (Chromogranin C, Gene: Scg2, Uniprot ID: P10362)

Known function: Acts as pro-hormone, Involved in LDCV biogenesis

Known organization: In LDCV lumen

Known Interactions: None
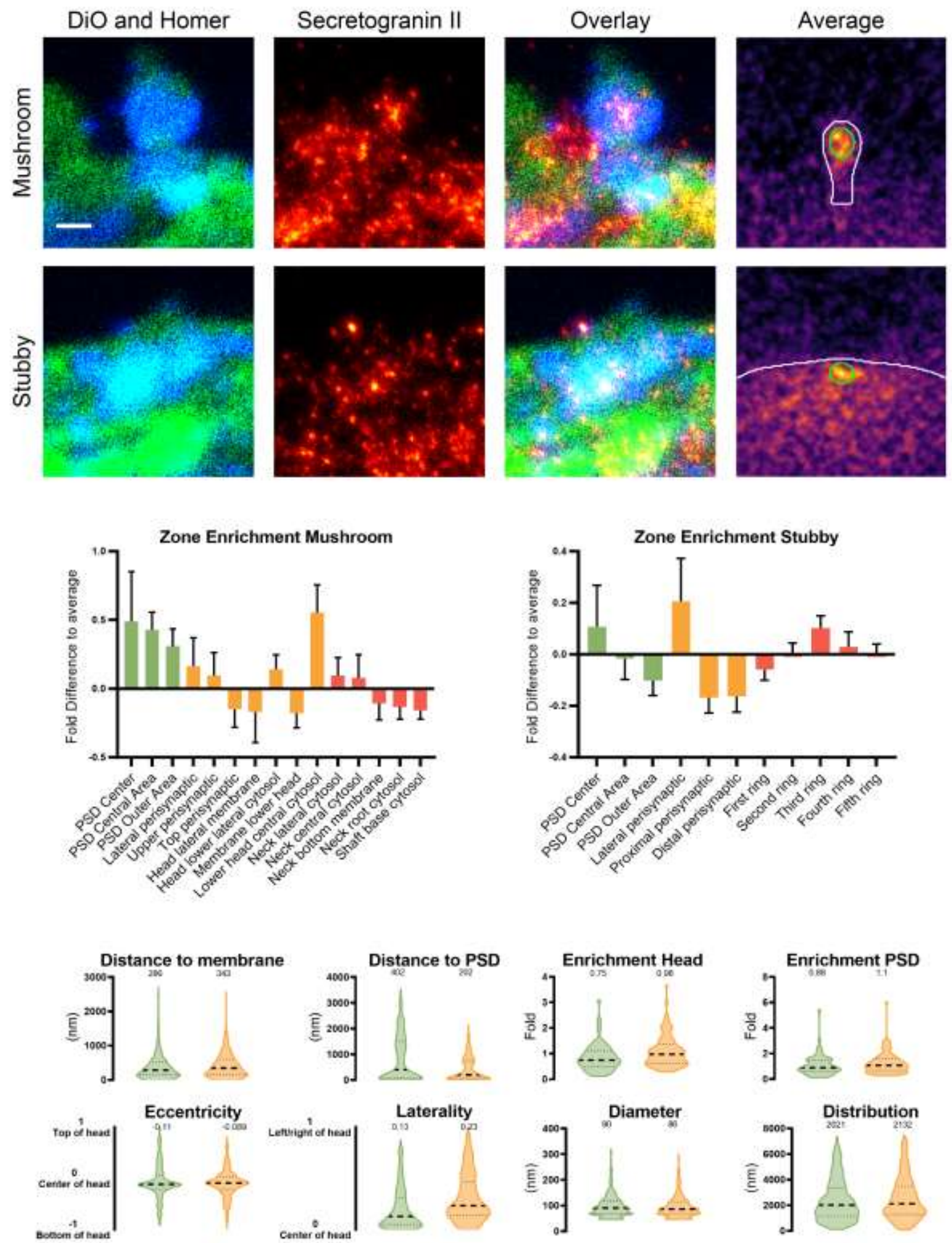

Figure 78: Secretogranin II nanoscale localization and abundance. Continued on next page. 


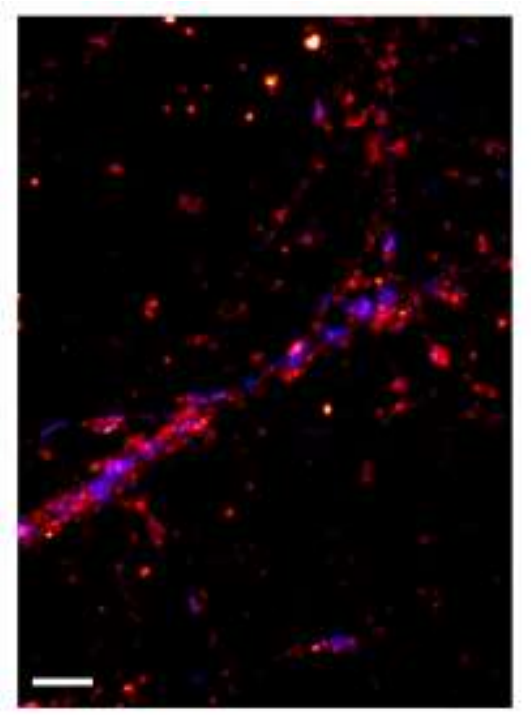

\begin{tabular}{|c|c|c|}
\hline $\begin{array}{c}\text { Whole cell copy } \\
\text { number }\end{array}$ & \multicolumn{2}{|c|}{$685949.02 \pm 46298.31$} \\
\hline $\begin{array}{c}\text { Spine copy } \\
\text { number }\end{array}$ & \multicolumn{2}{|c|}{$318.94 \pm 44.17$} \\
\hline$\%$ in PSD & \multicolumn{2}{|c|}{20.07} \\
\hline PSD copy number & \multicolumn{2}{|c|}{$53.31 \pm 7.38$} \\
\hline & Mushroom & Stubby \\
\hline \begin{tabular}{c|c|} 
Spine copy \\
number
\end{tabular} & $263.16 \pm 36.45$ & $406.30 \pm 56.27$ \\
\hline PSD copy number & $43.99 \pm 6.09$ & $67.92 \pm 9.41$ \\
\hline$\%$ of protein & $0.04 \pm 0.01 \%$ & $0.04 \pm 0.01 \%$ \\
\hline Molarity $[\mu \mathrm{M}]$ & $2.21 \pm 0.31$ & $2.18 \pm 0.30$ \\
\hline
\end{tabular}

\section{References}

Antibody: Abcam ab12241

Structure: Modelled with I-Tasser

\section{Literature:}

Courel et al., 2010; Fischer-Colbrie et al., 1995 


\section{Trafficking proteins}

The manifold subcellular compartments within the dendrite require highly specialized trafficking. Here I study mostly endocytosis-related proteins as well as rab proteins as markers for endosomal compartments.

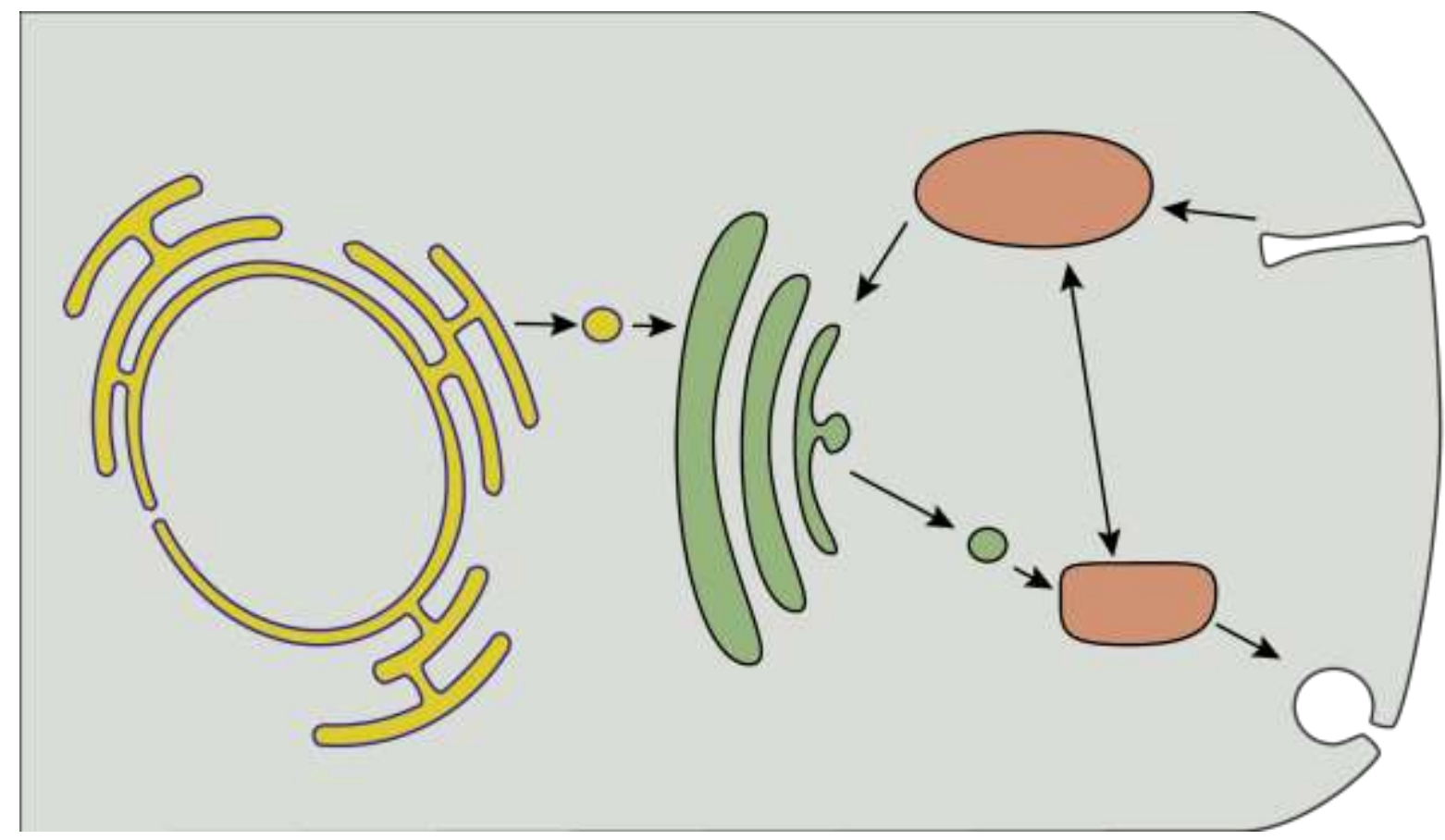

Figure 79: Depiction trafficking. 


\section{AP180 (Gene: Snap91, Uniprot ID: Q05140)}

Known function: Initiation of clathrin coat formation, AMPAR endocytosis

Known organization: Cytosolic or membrane-associated

Known Interactions: Clathrin
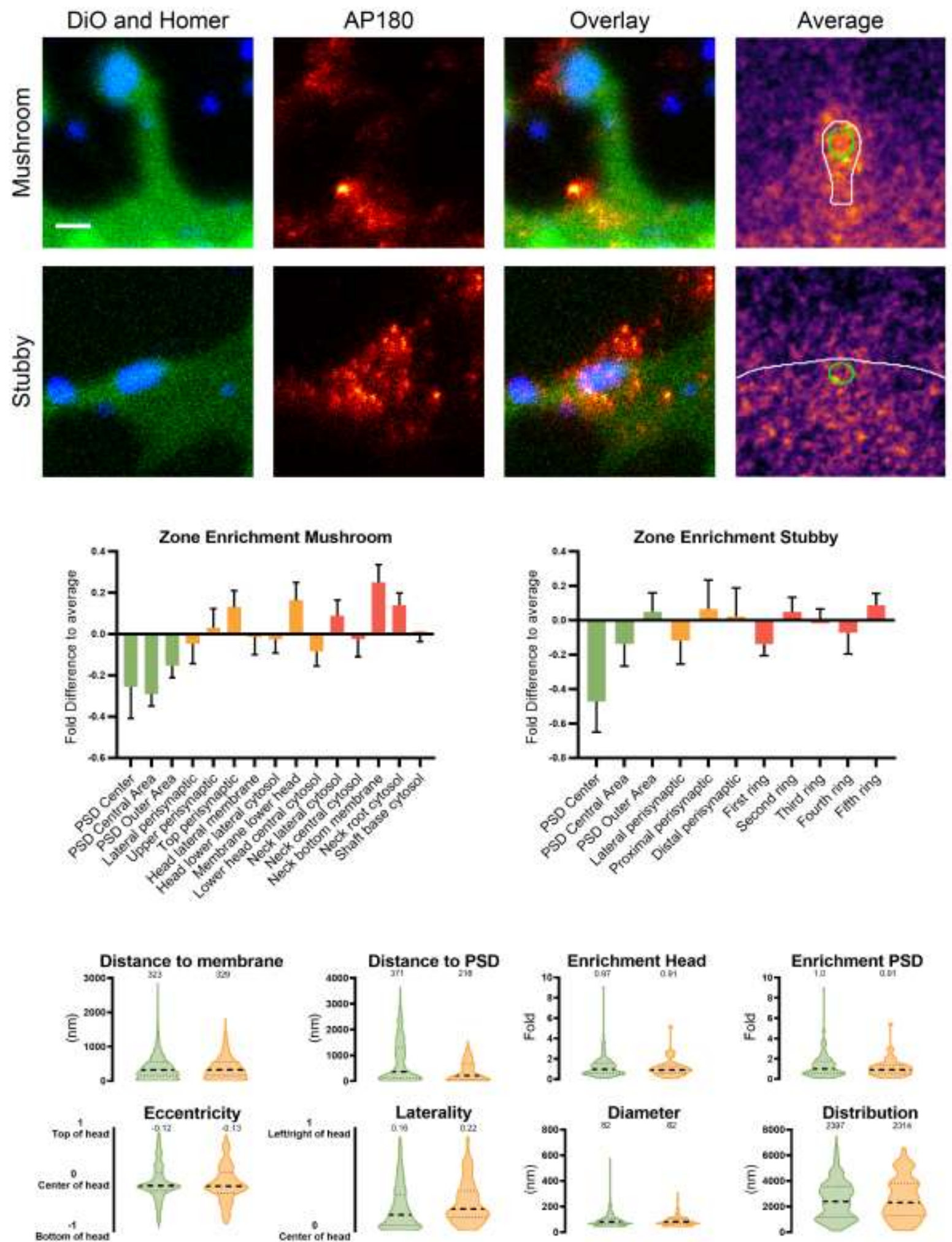

Figure 80: AP180 nanoscale localization and abundance. Continued on next page. 


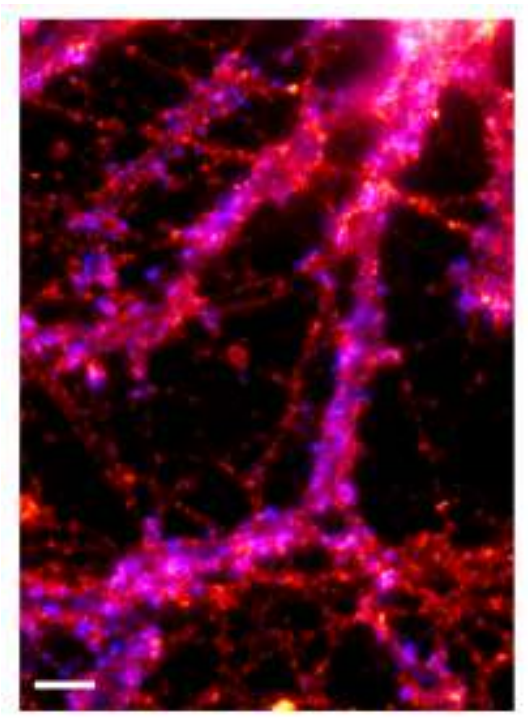

\begin{tabular}{|c|c|c|}
\hline $\begin{array}{c}\text { Whole cell copy } \\
\text { number }\end{array}$ & \multicolumn{2}{|c|}{$5230931.33 \pm 318694.48$} \\
\hline $\begin{array}{c}\text { Spine copy } \\
\text { number }\end{array}$ & \multicolumn{2}{|c|}{$404.49 \pm 170.90$} \\
\hline$\%$ in PSD & \multicolumn{2}{|c|}{18.13} \\
\hline PSD copy number & \multicolumn{2}{|c|}{$62.07 \pm 26.23$} \\
\hline & Mushroom & Stubby \\
\hline Spine copy & 370.91 & 428.21 \\
\hline number & \pm 156.71 & \pm 180.91 \\
\hline PSD copy number & $56.92 \pm 24.05$ & $65.71 \pm 27.76$ \\
\hline$\%$ of protein & $0.08 \pm 0.03 \%$ & $0.06 \pm 0.03 \%$ \\
\hline Molarity $[\mu \mathrm{M}]$ & $3.11 \pm 1.31$ & $2.30 \pm 0.97$ \\
\hline
\end{tabular}

\section{References}

Antibody: Synaptic Systems $155003 \quad$ Structure: Modelled by Burkhard Rammner

\section{Literature:}

Ahle and Ungewickell, 1986; Burbea et al., 2002; Hering et al., 2003; Keen, 1987; Lindner and Ungewickell, 1992; Murphy et al., 1991; Norris et al., 1995; Sousa et al., 1992; Ye and Lafer, 1995; Zhou et al., 1992 


\section{Clathrin heavy chain (Gene: Cltc, Uniprot ID: Q05140)}

Known function: Endocytosis

Known organization: Cytosolic or membrane-associated, Forms triskelia together with clathrin light chains, Endocytic zones lateral to PSD or on shaft

Known Interactions: Clathrin light chains, Adaptor proteins
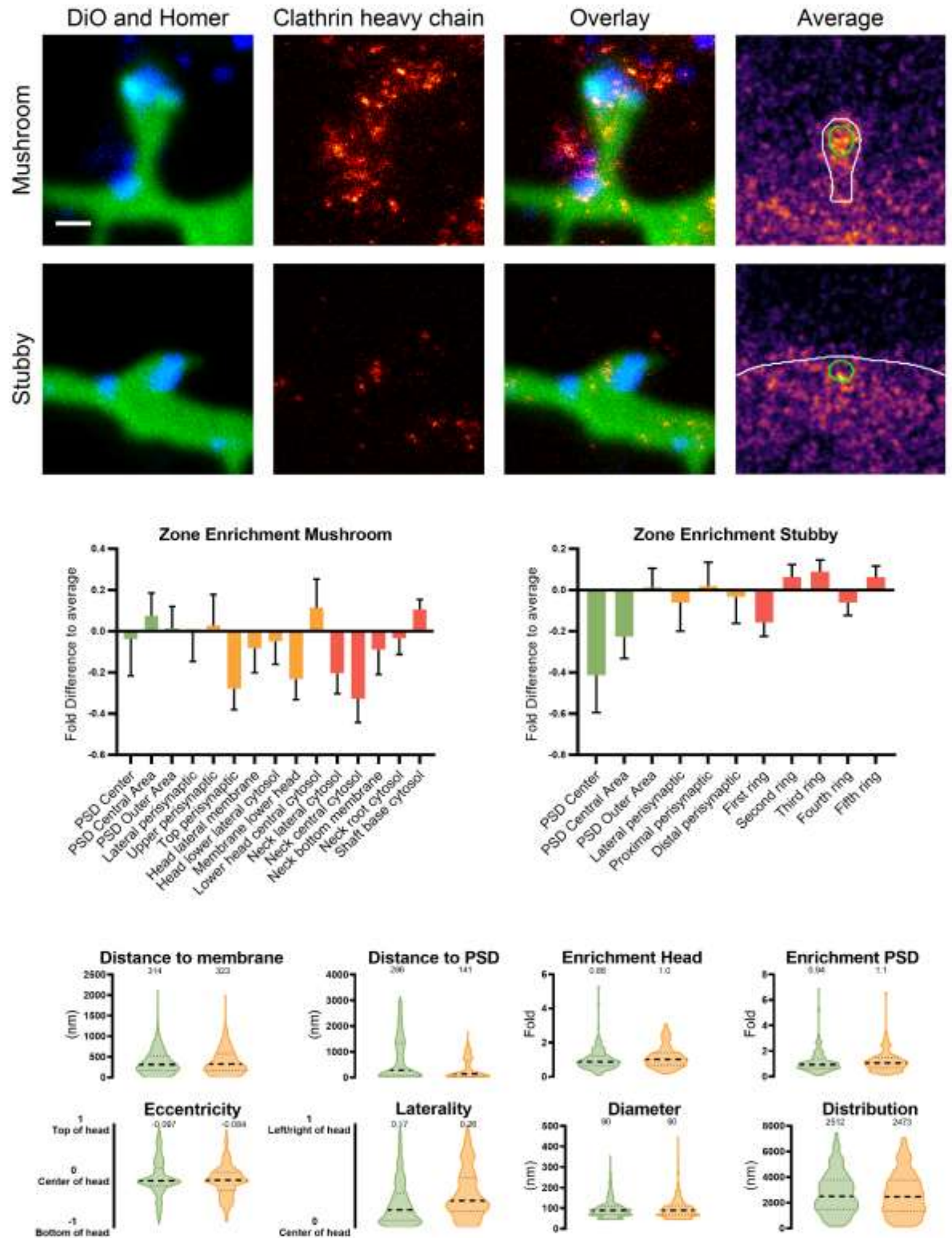

Figure 81: Clathrin heavy chain nanoscale localization and abundance. Continued on next page. 


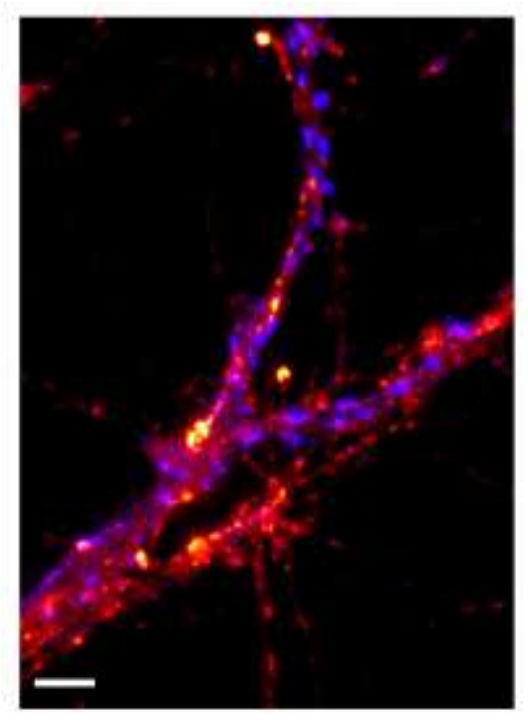

\begin{tabular}{|c|c|c|}
\hline $\begin{array}{c}\text { Whole cell copy } \\
\text { number }\end{array}$ & \multicolumn{2}{|c|}{$9863953.46 \pm 1883721.98$} \\
\hline $\begin{array}{c}\text { Spine copy } \\
\text { number }\end{array}$ & \multicolumn{2}{|c|}{$2065.78 \pm 525.15$} \\
\hline$\%$ in PSD & \multicolumn{2}{|c|}{18.41} \\
\hline PSD copy number & \multicolumn{2}{|c|}{$321.17 \pm 81.65$} \\
\hline & Mushroom & Stubby \\
\hline $\begin{array}{c}\text { Spine copy } \\
\text { number }\end{array}$ & 1335.87 & 3068.14 \\
\hline PSD copy number & $207.69 \pm 52.60$ & \pm 779.96 \\
\hline$\%$ of protein & $0.59 \pm 0.15 \%$ & $0.87 \pm 0.22 \%$ \\
\hline Molarity $[\mu M]$ & $11.20 \pm 2.85$ & $16.45 \pm 4.18$ \\
\hline
\end{tabular}

\section{References}

Antibody: BD Biosciences 610499

PDB Identifier: $1 \times 14$

\section{Literature:}

Blanpied et al., 2002; Boehm et al., 2006; Cheng et al., 2007; Cooney et al., 2002; Loebrich et al., 2013; Lu et al., 2007; Musacchio et al., 1999; Park et al., 2006; Rácz et al., 2004; Rosendale et al., 2017; Tao-Cheng et al., 2011; Ungewickell and Branton, 1981; Yudowski et al., 2007 


\section{Clathrin light chain a \& b (Genes: Clta \& Cltb , Uniprot IDs: P08081 \& P08082)}

Known function: Endocytosis

Known organization: Cytosolic or membrane-associated, Forms triskelia together with clathrin heavy hains, On endocytic zones lateral to PSD or on shaft

Known Interactions: Clathrin light chains, Adaptor proteins
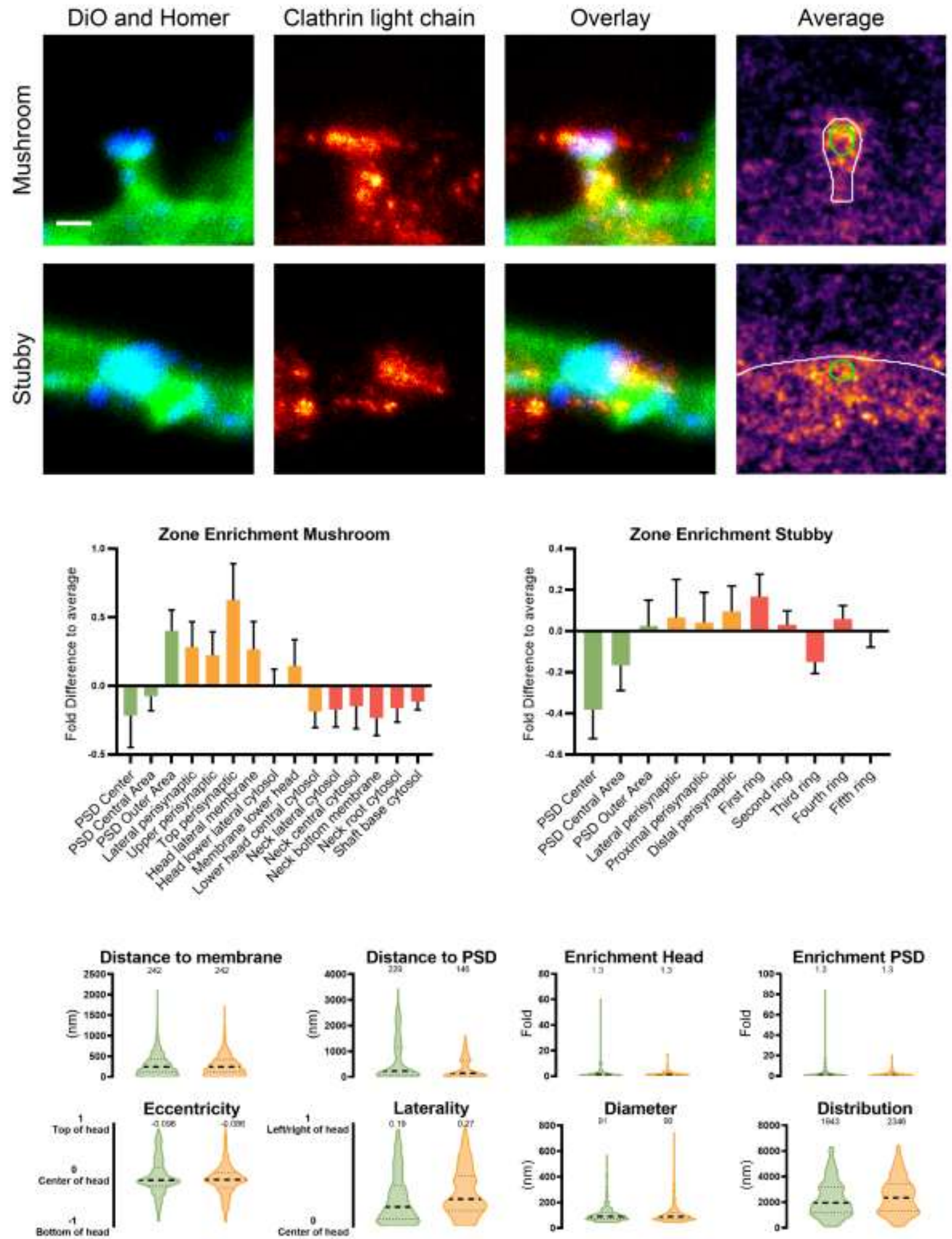

Figure 82: Clathrin light chain nanoscale localization and abundance. Continued on next page. 


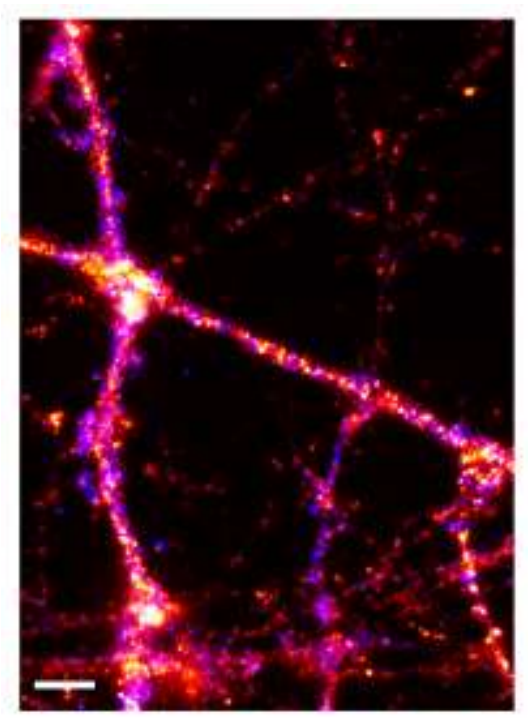

\begin{tabular}{|c|c|c|}
\hline $\begin{array}{c}\text { Whole cell copy } \\
\text { number }\end{array}$ & \multicolumn{2}{|c|}{$61754332.85 \pm 7545013.76$} \\
\hline $\begin{array}{l}\text { Spine copy } \\
\text { number }\end{array}$ & \multicolumn{2}{|c|}{$19493.93 \pm 4273.72$} \\
\hline$\%$ in PSD & \multicolumn{2}{|c|}{21.63} \\
\hline \multirow[t]{2}{*}{ PSD copy number } & \multicolumn{2}{|c|}{$3466.93 \pm 760.06$} \\
\hline & Mushroom & Stubby \\
\hline $\begin{array}{l}\text { Spine copy } \\
\text { number }\end{array}$ & $\begin{array}{r}16909.44 \\
\pm 3707.11\end{array}$ & $\begin{array}{l}21932.81 \\
\pm 4808.40\end{array}$ \\
\hline PSD copy number & $\begin{array}{l}3007.28 \\
\pm 659.30\end{array}$ & $\begin{array}{l}3900.67 \\
\pm 855.16\end{array}$ \\
\hline$\%$ of protein & $1.98 \pm 0.43 \%$ & $1.65 \pm 0.36 \%$ \\
\hline Molarity $[\mu \mathrm{M}]$ & $141.72 \pm 31.07$ & $117.58 \pm 25.78$ \\
\hline
\end{tabular}

\section{References}

Antibody: Synaptic Systems 113011

PDB Identifier: $1 \times 14$

\section{Literature:}

Blanpied et al., 2002; Boehm et al., 2006; Cheng et al., 2007; Cooney et al., 2002; Loebrich et al., 2013; Lu et al., 2007; Musacchio et al., 1999; Park et al., 2006; Rácz et al., 2004; Rosendale et al., 2017; Tao-Cheng et al., 2011; Ungewickell and Branton, 1981; Yudowski et al., 2007 


\section{Dynamin1-3 (Genes: Dnm1, Dnm2, Dnm3, Uniprot IDs: P21575, P39052, Q08877)}

Known function: Membrane fission in endocytosis, AMPAR and NMDAR endocytosis Known organization: Cytosolic, Forms dimers and helices

Known Interactions: Homer and Shank proteins, mGluR5
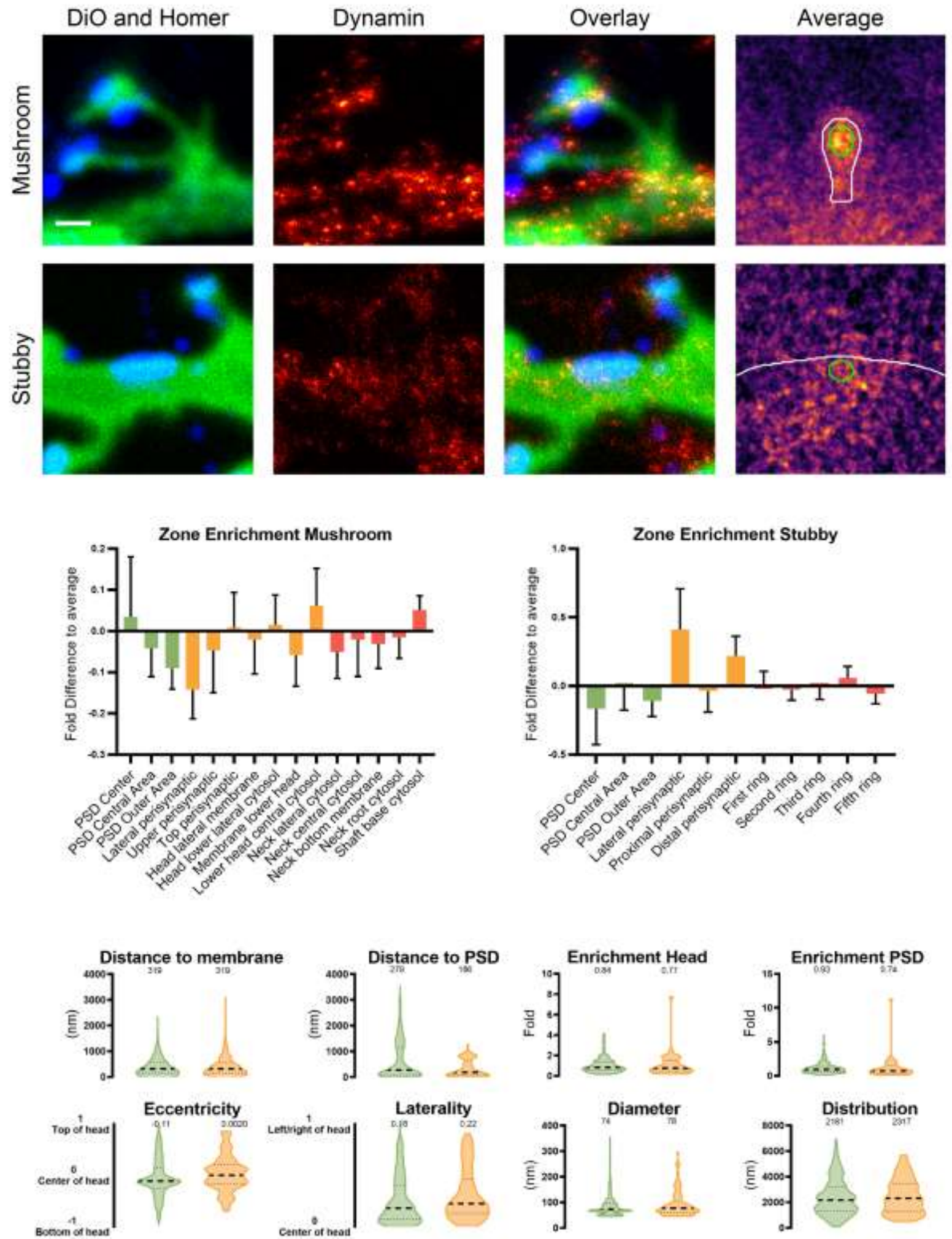

Figure 83: Dynamin1-3 nanoscale localization and abundance. Continued on next page. 


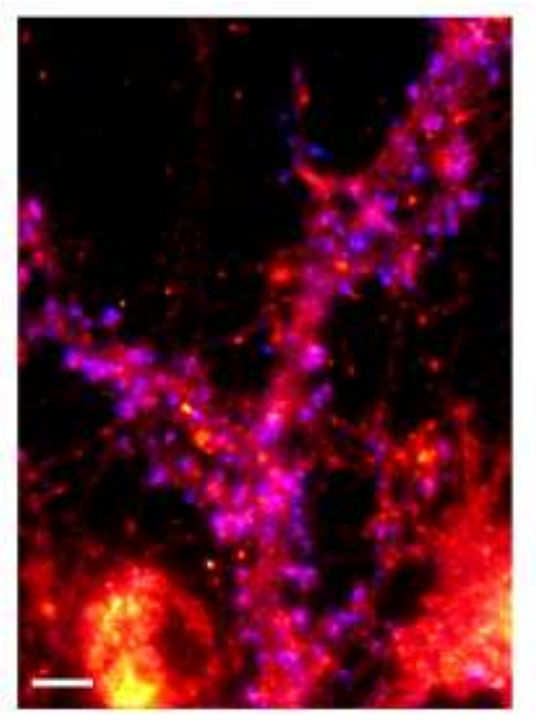

\begin{tabular}{|c|c|}
\hline $\begin{array}{c}\text { Whole cell copy } \\
\text { number }\end{array}$ & $9257216.57 \pm 629499.27$ \\
\hline $\begin{array}{c}\text { Spine copy } \\
\text { number }\end{array}$ & $2152.21 \pm 447.29$ \\
\hline$\%$ in PSD & 19.71 \\
\hline PSD copy number & $354.30 \pm 73.63$ \\
\hline
\end{tabular}

\begin{tabular}{|c|c|c|}
\hline & Mushroom & Stubby \\
\hline $\begin{array}{c}\text { Spine copy } \\
\text { number }\end{array}$ & $\begin{array}{r}1942.26 \\
\pm 403.66\end{array}$ & $\begin{array}{r}2233.12 \\
\pm 464.11\end{array}$ \\
\hline PSD copy number & $319.74 \pm 66.45$ & $367.62 \pm 76.40$ \\
\hline$\%$ of protein & $1.31 \pm 0.27 \%$ & $0.97 \pm 0.20 \%$ \\
\hline Molarity $[\mu \mathrm{M}]$ & $16.28 \pm 3.38$ & $11.97 \pm 2.49$ \\
\hline
\end{tabular}

Mushroom molecular model

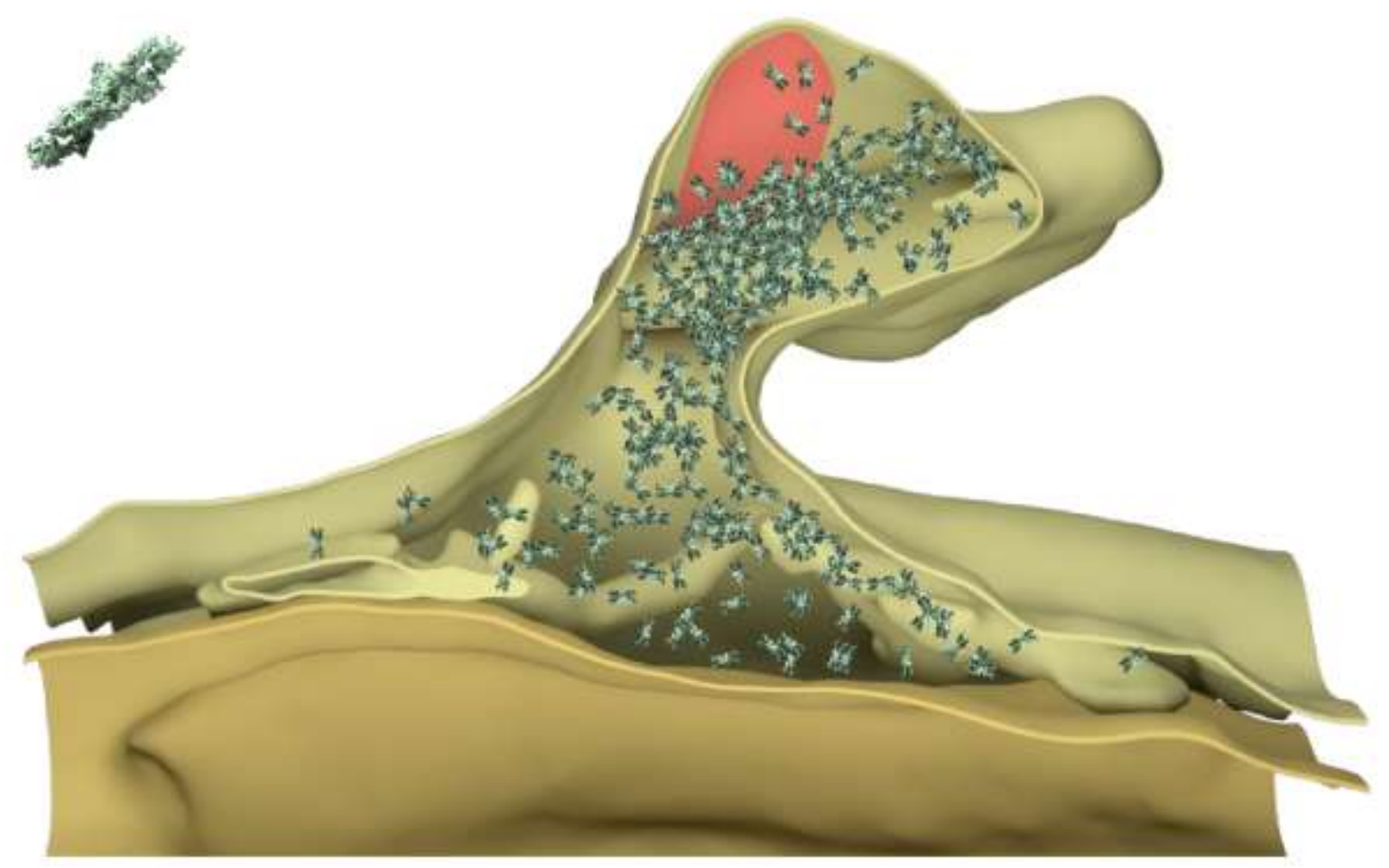

\section{References}

Antibody: BD Biosciences 610245

PDB Identifier: $5 \mathrm{a} 3 \mathrm{f}$

\section{Literature:}

Carroll et al., 1999; Damke et al., 1994; Faelber et al., 2011; Gray et al., 2003; Lu et al., 2007; Pérez-Otaño et al., 2006; Raimondi et al., 2011; Takei et al., 1996 
GRIP1/2 (Genes: Grip1, Grip2, Uniprot ID: P97879, Q9WTW1)

Known function: Controls AMPA and kainate receptor trafficking

Known organization: Cytosolic

Known Interactions: AMPA and kainate receptors
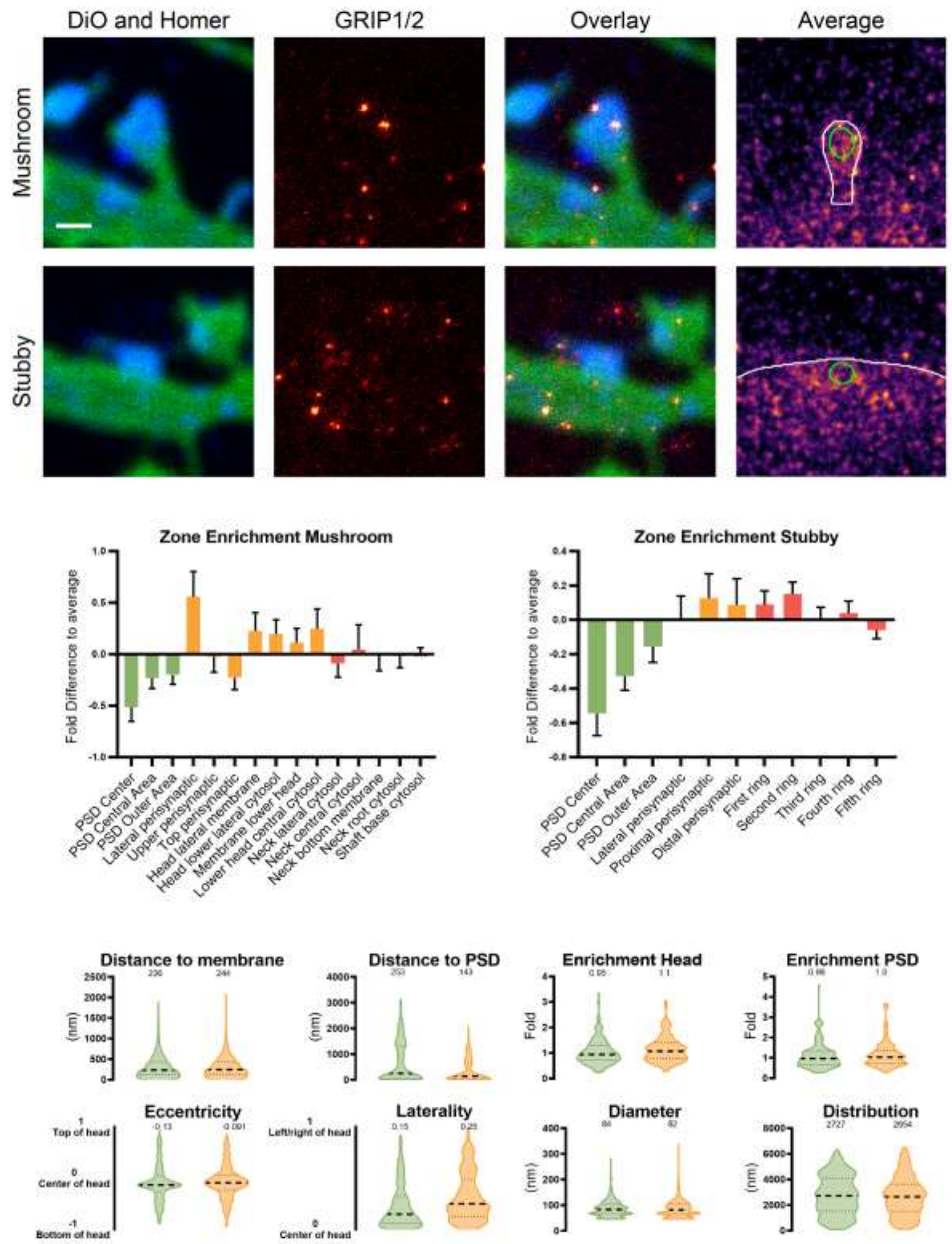

Figure 84: GRIP1/2 nanoscale localization and abundance. Continued on next page. 


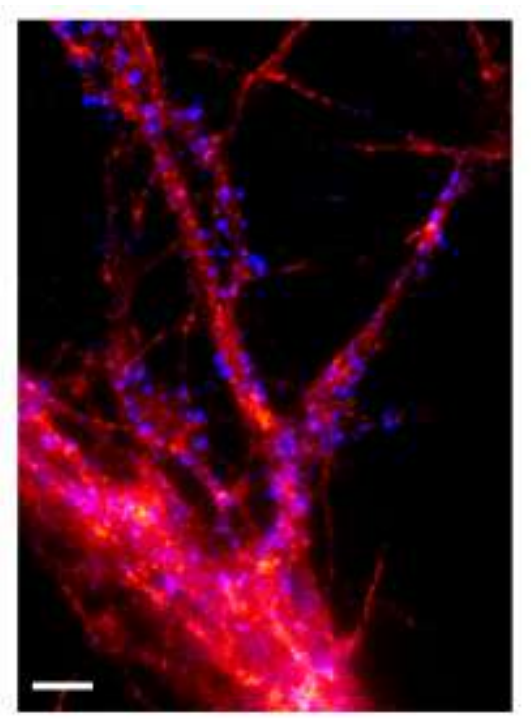

\begin{tabular}{|c|c|c|}
\hline $\begin{array}{c}\text { Whole cell copy } \\
\text { number }\end{array}$ & \multicolumn{2}{|c|}{$266832.89 \pm 38077.24$} \\
\hline $\begin{array}{c}\text { Spine copy } \\
\text { number }\end{array}$ & \multicolumn{2}{|c|}{$17.18 \pm 5.35$} \\
\hline$\%$ in PSD & \multicolumn{2}{|c|}{16.22} \\
\hline PSD copy number & \multicolumn{2}{|c|}{$2.40 \pm 0.75$} \\
\hline & Mushroom & Stubby \\
\hline $\begin{array}{c}\text { Spine copy } \\
\text { number }\end{array}$ & $16.41 \pm 5.11$ & $19.42 \pm 6.05$ \\
\hline PSD copy number & $2.29 \pm 0.71$ & $2.71 \pm 0.84$ \\
\hline$\%$ of protein & $0.00 \pm 0.00 \%$ & $0.00 \pm 0.00 \%$ \\
\hline Molarity $[\mu \mathrm{M}]$ & $0.14 \pm 0.04$ & $0.10 \pm 0.03$ \\
\hline
\end{tabular}

\section{References}

Antibody: Synaptic Systems 151003 PDB Identifier: 2qt5, 1p1d, 1n7e, 1m5z

\section{Literature:}

Braithwaite et al., 2002; Dong et al., 1997, 1999; Hanley and Henley, 2010 


\section{HSC70 (Gene: Hspa8, Uniprot ID: P63018)}

Known function: Clathrin uncoating, ERAD associated chaperone

Known organization: Cytosolic

Known Interactions: Clathrin (via auxilin)
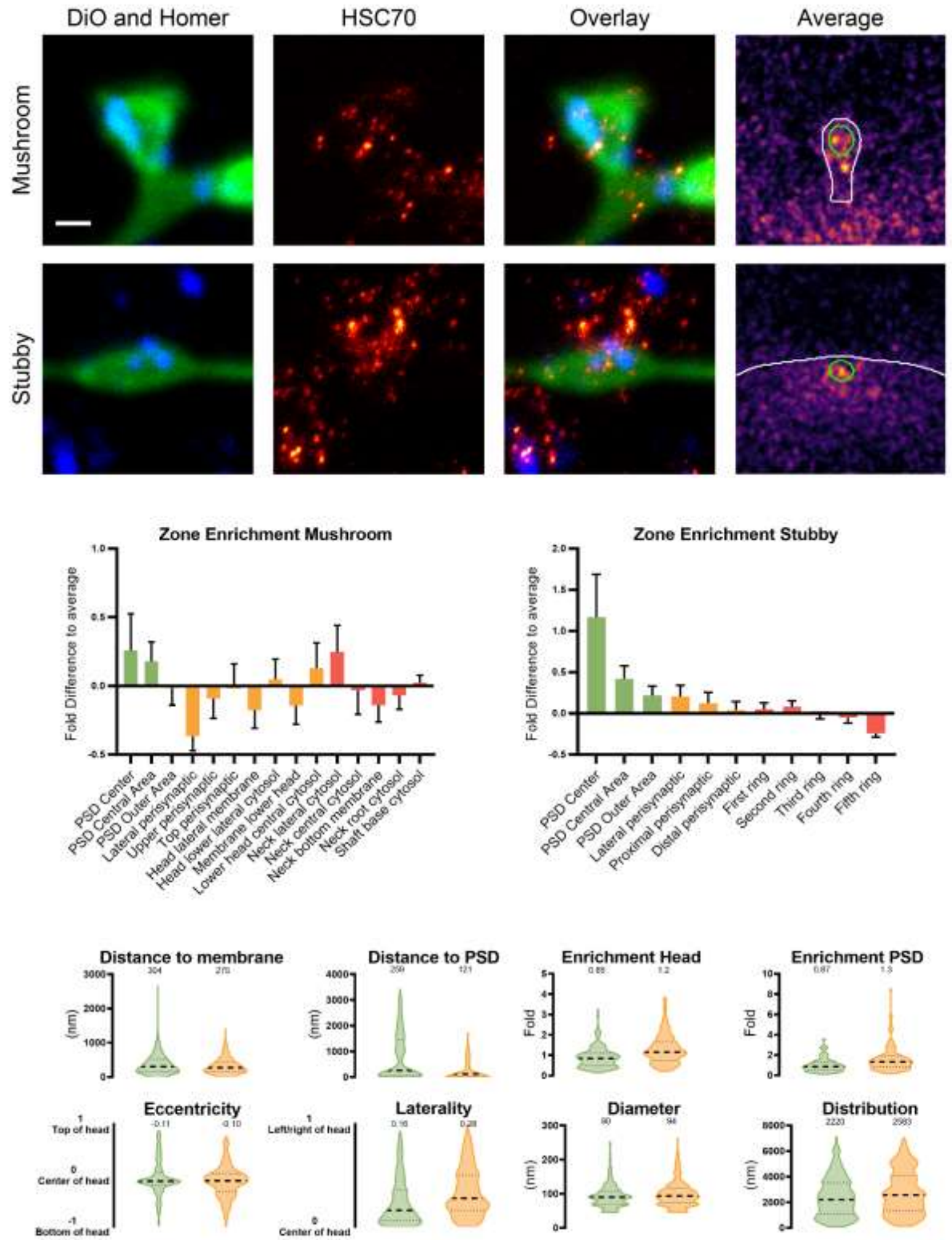

Figure 85: HSC70 nanoscale localization and abundance. Continued on next page. 


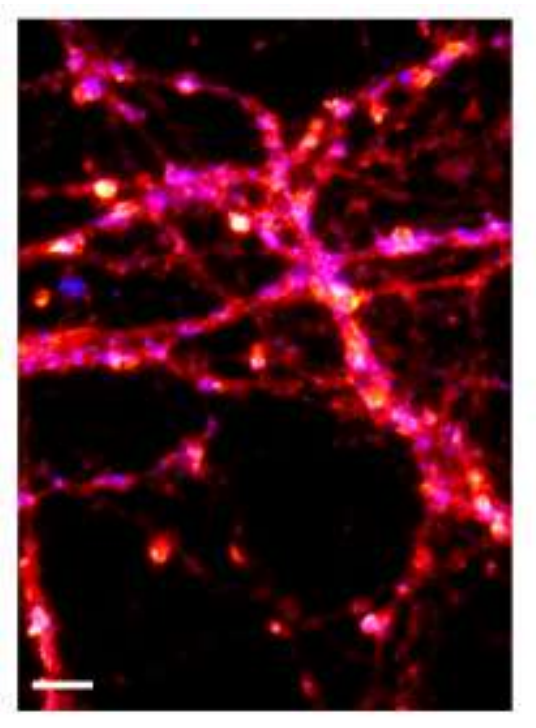

\begin{tabular}{|c|c|c|}
\hline $\begin{array}{c}\text { Whole cell copy } \\
\text { number }\end{array}$ & \multicolumn{2}{|c|}{$825687.65 \pm 200691.43$} \\
\hline $\begin{array}{c}\text { Spine copy } \\
\text { number }\end{array}$ & \multicolumn{2}{|c|}{$298.71 \pm 80.02$} \\
\hline$\%$ in PSD & \multicolumn{2}{|c|}{20.34} \\
\hline PSD copy number & \multicolumn{2}{|c|}{$50.48 \pm 13.52$} \\
\hline & Mushroom & Stubby \\
\hline $\begin{array}{c}\text { Spine copy } \\
\text { number }\end{array}$ & $210.16 \pm 56.30$ & $414.68 \pm 111.09$ \\
\hline PSD copy number & $35.52 \pm 9.51$ & $70.08 \pm 18.77$ \\
\hline$\%$ of protein & $0.03 \pm 0.01 \%$ & $0.04 \pm 0.01 \%$ \\
\hline Molarity [ $[\mu \mathrm{M}]$ & $1.76 \pm 0.47$ & $2.22 \pm 0.60$ \\
\hline
\end{tabular}

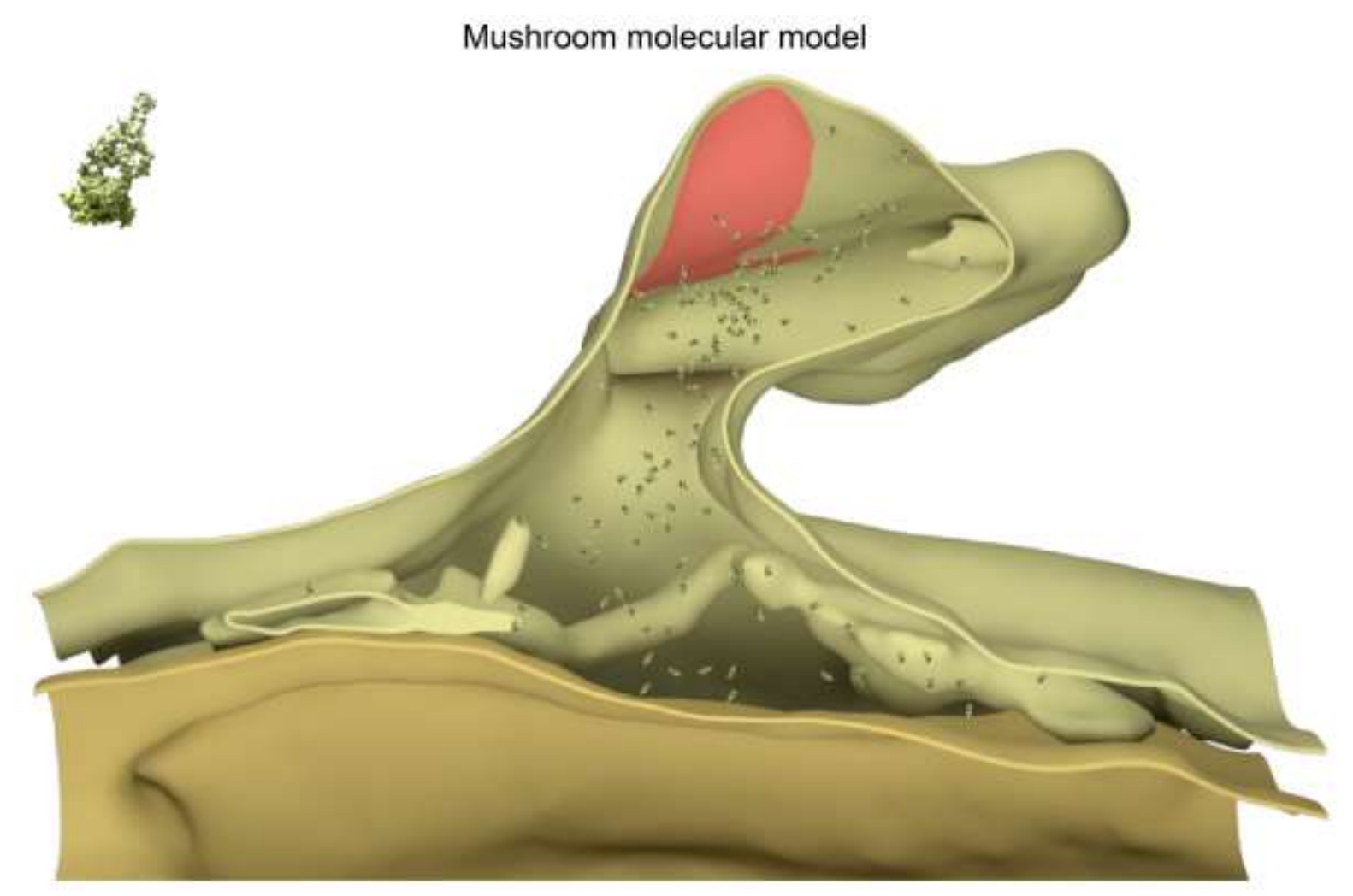

\section{References}

Antibody: Santa Cruz sc-7298 PDB Identifier: $4 \mathrm{~h} 5 \mathrm{t}, 1 \mathrm{~h} \times 1$, 3ldq, 1 udo

\section{Literature:}

Eisenberg and Greene, 2007; Grove et al., 2011; Huang et al., 1993; Rothnie et al., 2011; Schlossman et al., 1984; Ungewickell et al., 1997; Yamamoto et al., 2010 


\section{Rab3 (Gene: Rab3a, Uniprot ID: P63012)}

Known function: SV and neuroendocrine exocytosis

Known organization: Membrane-associated and cytosolic

Known Interactions: None
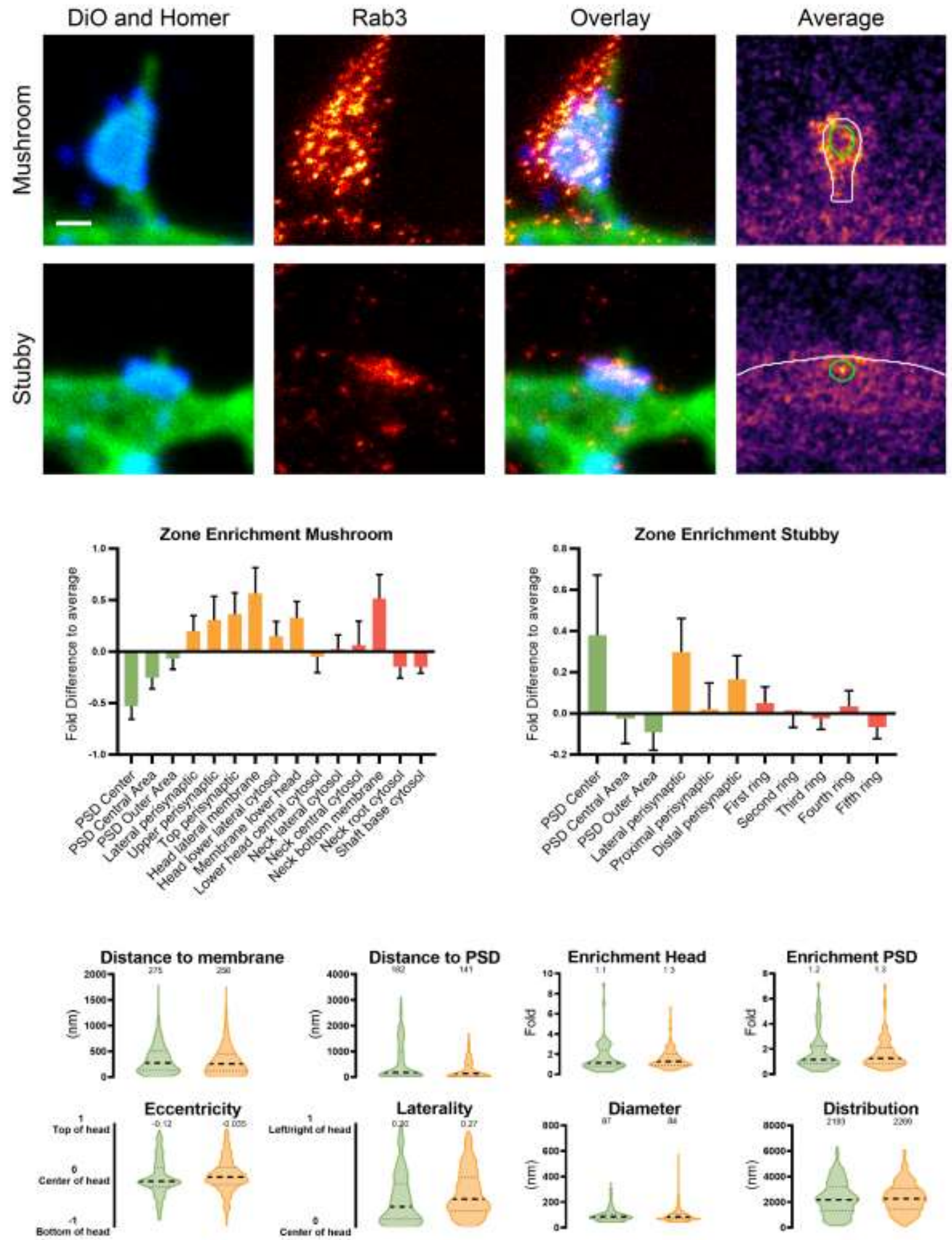

Figure 86: Rab3 nanoscale localization and abundance. Continued on next page. 


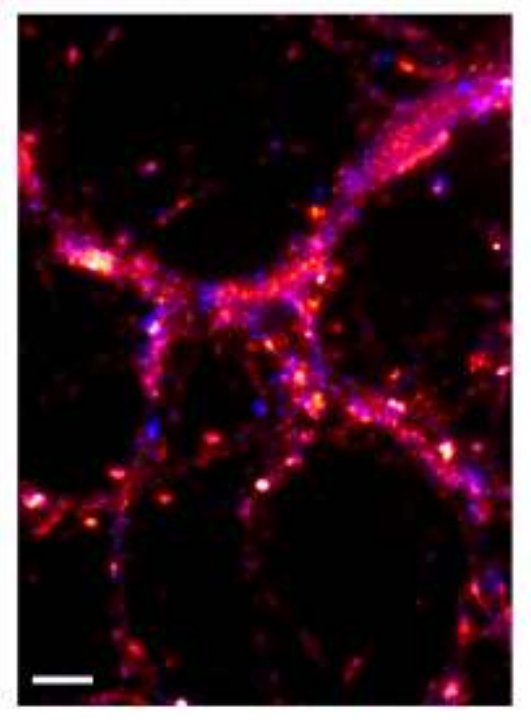

\begin{tabular}{|c|c|c|}
\hline $\begin{array}{c}\text { Whole cell copy } \\
\text { number }\end{array}$ & \multicolumn{2}{|c|}{$77049113.98 \pm 7460252.12$} \\
\hline $\begin{array}{c}\text { Spine copy } \\
\text { number }\end{array}$ & \multicolumn{2}{|c|}{$10233.92 \pm 3725.36$} \\
\hline$\%$ in PSD & \multicolumn{2}{|c|}{18.86} \\
\hline PSD copy number & \multicolumn{2}{|c|}{$1623.63 \pm 591.04$} \\
\hline Spine copy & 9717.88 & 11426.00 \\
\hline number & \pm 3537.51 & \pm 4159.30 \\
\hline PSD copy number & 1541.76 & 1812.76 \\
\pm 561.23 & \pm 659.88 \\
\hline$\%$ of protein & $2.24 \pm 0.82 \%$ & $1.69 \pm 0.62 \%$ \\
\hline Molarity [MM] & $81.45 \pm 29.65$ & $61.25 \pm 22.30$ \\
\hline
\end{tabular}

\section{References}

Antibody: BD Biosciences 610379

PDB Identifier: 3rab

\section{Literature:}

Fischer von Mollard et al., 1990; von Mollard et al., 1991; Schlüter et al., 2002 


\section{Rab4 (Gene: Rab4a, Uniprot ID: P05714)}

Known function: Early/recycling endosomes to PM traffick, Basal AMPAR recycling Known organization: Membrane-associated and cytosolic Known Interactions: None
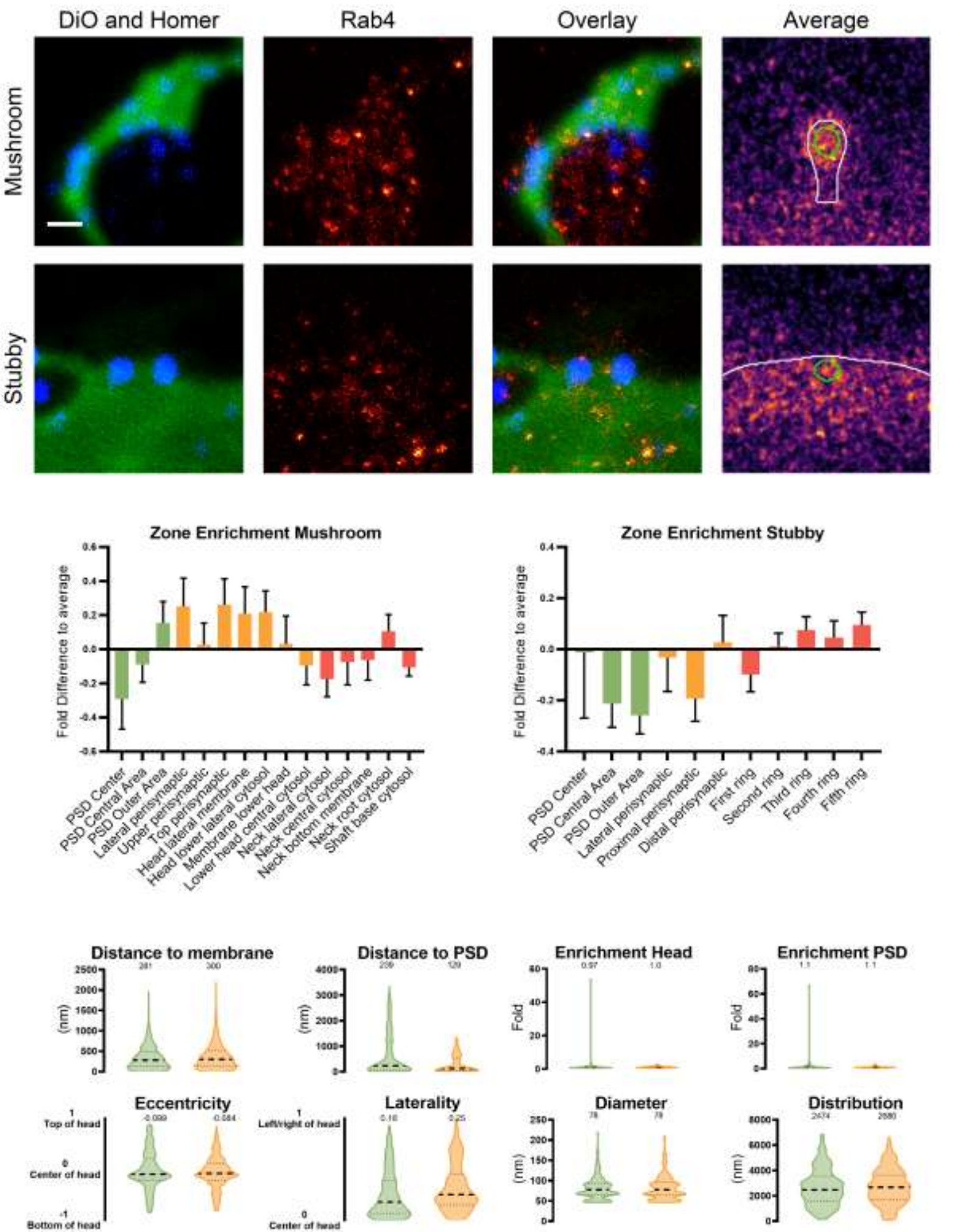


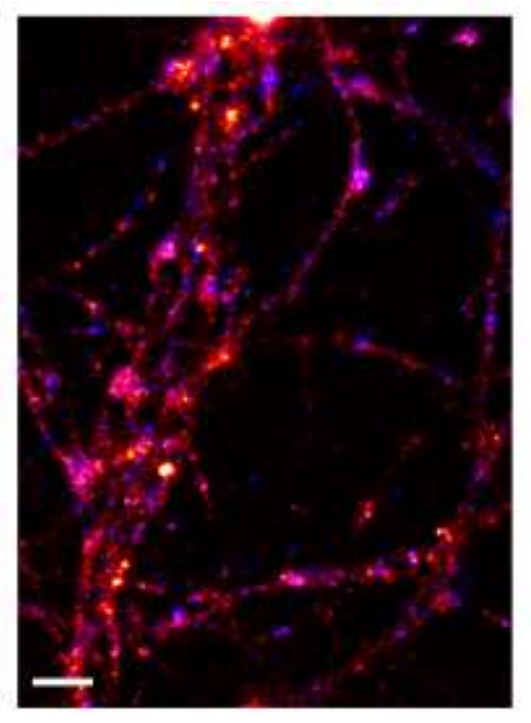

\begin{tabular}{|c|c|c|}
\hline $\begin{array}{c}\text { Whole cell copy } \\
\text { number }\end{array}$ & \multicolumn{2}{|c|}{$4658790.90 \pm 406145.95$} \\
\hline $\begin{array}{c}\text { Spine copy } \\
\text { number }\end{array}$ & \multicolumn{2}{|c|}{$419.81 \pm 108.01$} \\
\hline$\%$ in PSD & \multicolumn{2}{|c|}{17.67} \\
\hline PSD copy number & \multicolumn{2}{|c|}{$63.04 \pm 16.22$} \\
\hline & Mushroom & Stubby \\
\hline $\begin{array}{c}\text { Spine copy } \\
\text { number }\end{array}$ & $387.00 \pm 99.57$ & $456.07 \pm 117.34$ \\
\hline PSD copy number & $58.11 \pm 14.95$ & $68.48 \pm 17.62$ \\
\hline$\%$ of protein & $0.04 \pm 0.01 \%$ & $0.03 \pm 0.01 \%$ \\
\hline Molarity [MM] & $3.24 \pm 0.83$ & $2.44 \pm 0.63$ \\
\hline
\end{tabular}

\section{References}

Antibody: BD Biosciences 610888

PDB Identifier: 1yu9

\section{Literature:}

Brown et al., 2007; Gu and Huganir, 2016; Hoogenraad et al., 2010; Mohrmann et al., 2002; de Wit et al., 2001 


\section{Rab5 (Gene: Rab5a, Uniprot ID: M0RC99)}

Known function: Early endosome trafficking, Basal AMPAR recycling

Known organization: Membrane-associated and cytosolic

Known Interactions: Syntaxin 13
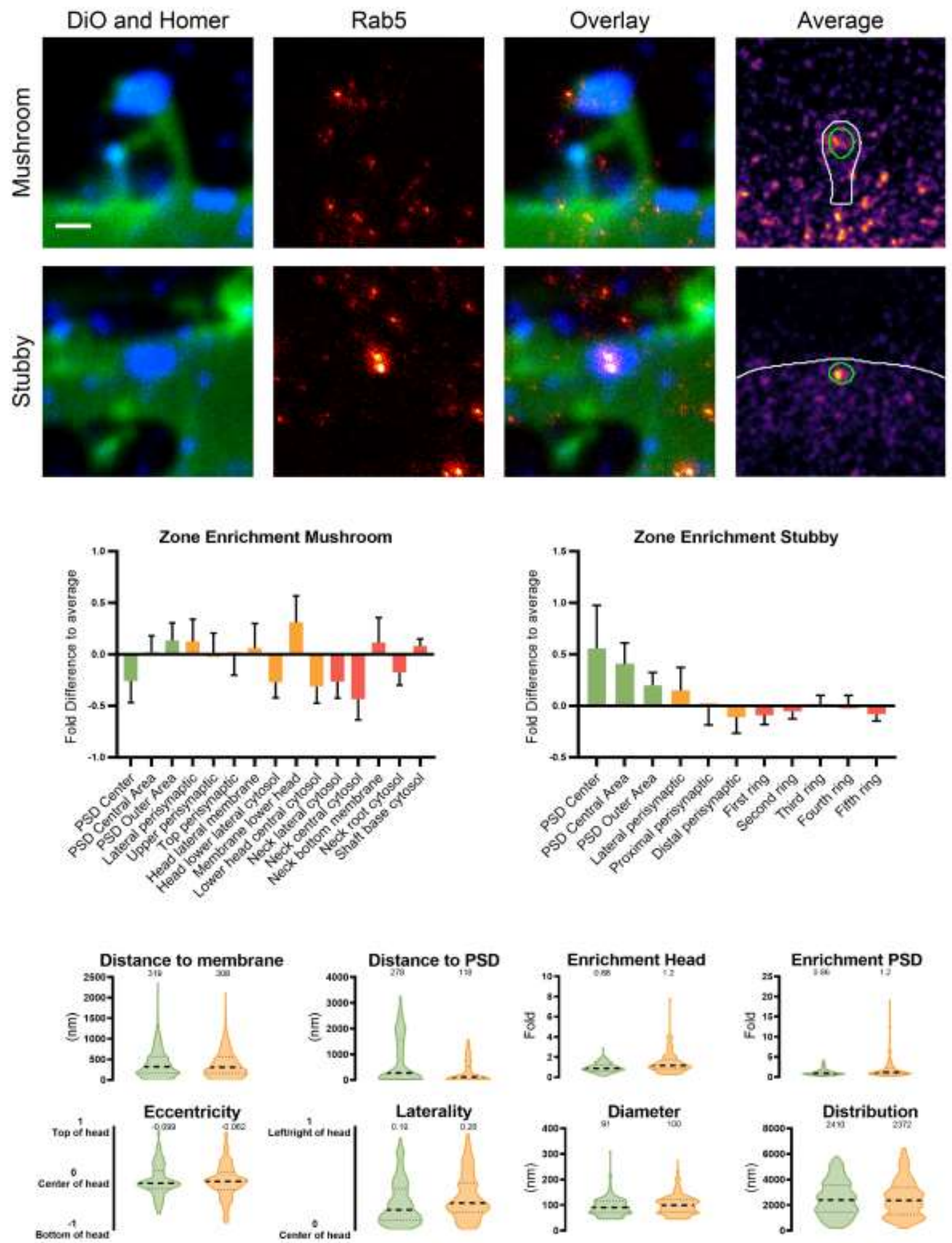

Figure 88: Rab5 nanoscale localization and abundance. Continued on next page. 


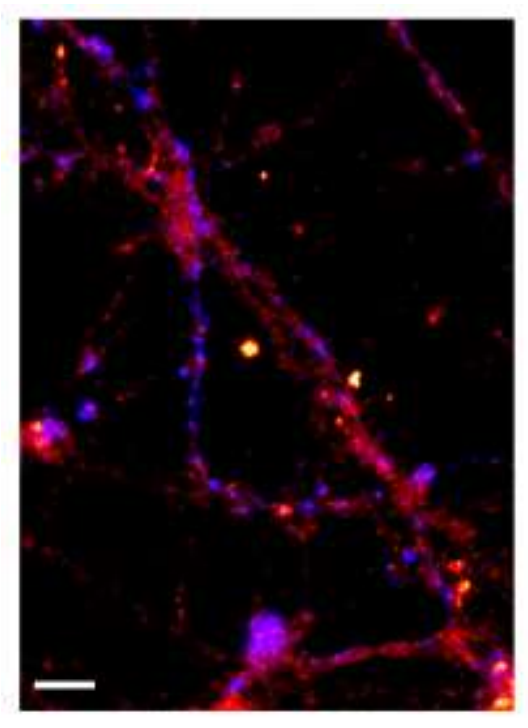

\begin{tabular}{|c|c|c|}
\hline $\begin{array}{c}\text { Whole cell copy } \\
\text { number }\end{array}$ & \multicolumn{2}{|c|}{$33181430.46 \pm 3456407.25$} \\
\hline $\begin{array}{l}\text { Spine copy } \\
\text { number }\end{array}$ & \multicolumn{2}{|c|}{$7273.26 \pm 1333.41$} \\
\hline$\%$ in PSD & \multicolumn{2}{|c|}{24.14} \\
\hline \multirow[t]{2}{*}{ PSD copy number } & \multicolumn{2}{|c|}{$1414.50 \pm 259.32$} \\
\hline & Mushroom & Stubby \\
\hline $\begin{array}{l}\text { Spine copy } \\
\text { number }\end{array}$ & $\begin{array}{r}5160.37 \\
\pm 946.05\end{array}$ & $\begin{array}{c}9574.90 \\
\pm 1755.37\end{array}$ \\
\hline PSD copy number & $\begin{array}{r}1003.59 \\
\pm 183.99\end{array}$ & $\begin{array}{r}1862.13 \\
\pm 341.39 \\
\end{array}$ \\
\hline$\%$ of protein & $1.13 \pm 0.21 \%$ & $1.34 \pm 0.25 \%$ \\
\hline Molarity $[\mu \mathrm{M}]$ & $43.25 \pm 7.93$ & $51.33 \pm 9.41$ \\
\hline
\end{tabular}

\section{References}

Antibody: Reinhard Jahn laboratory cl. $621.3 \quad$ PDB Identifier: 1 tu4

\section{Literature:}

Brown et al., 2005; Bucci et al., 1992; Deinhardt et al., 2006; Fischer von Mollard et al., 1994; Gu and Huganir, 2016; McBride et al., 1999; Szíber et al., 2017 


\section{Rab7 (Gene: Rab7a, Uniprot ID: P09527)}

Known function: Late endosome to lysosome trafficking, Involved in AMPAR trafficking Known organization: Membrane-associated and cytosolic

Known Interactions: None
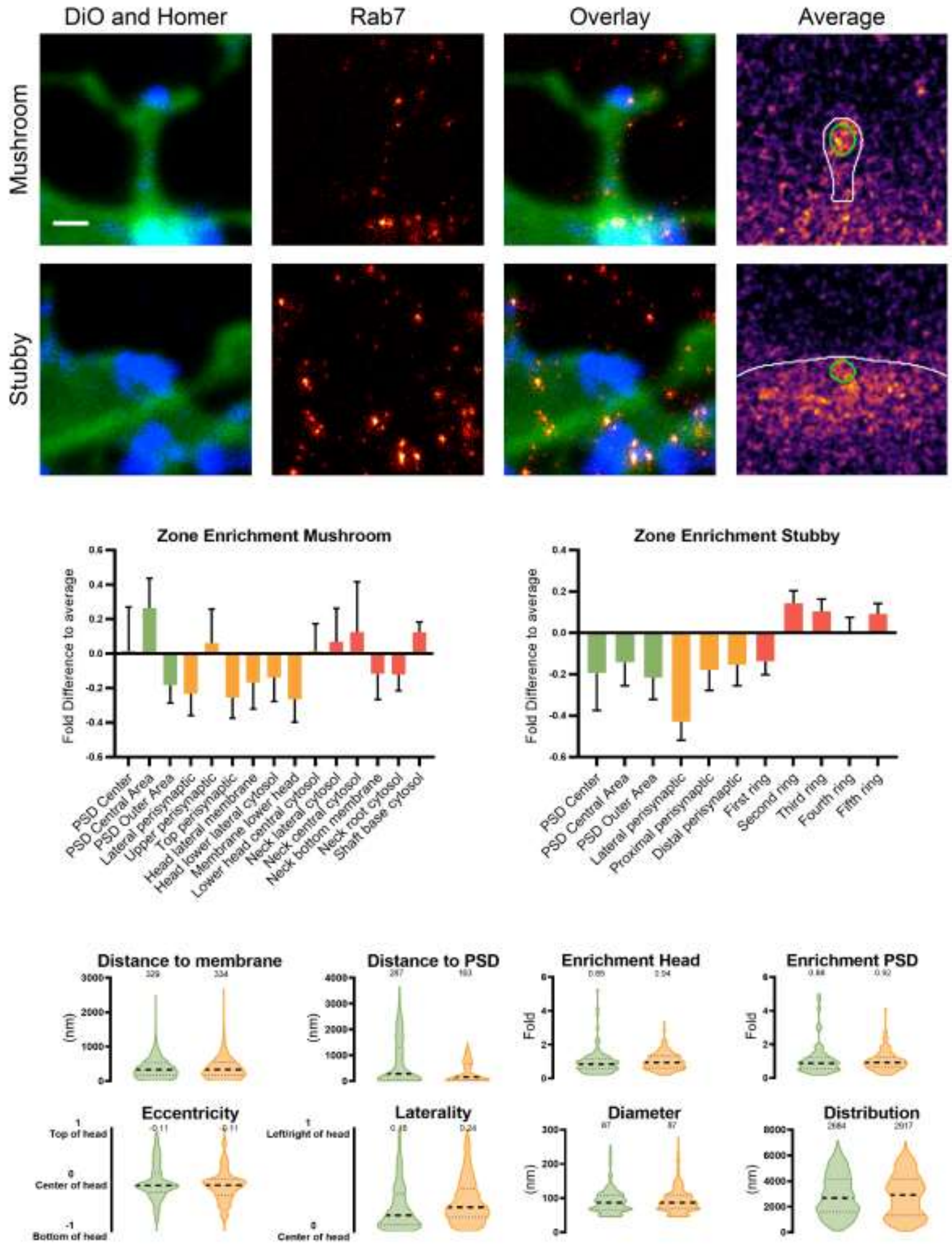

Figure 89: Rab7 nanoscale localization and abundance. Continued on next page. 


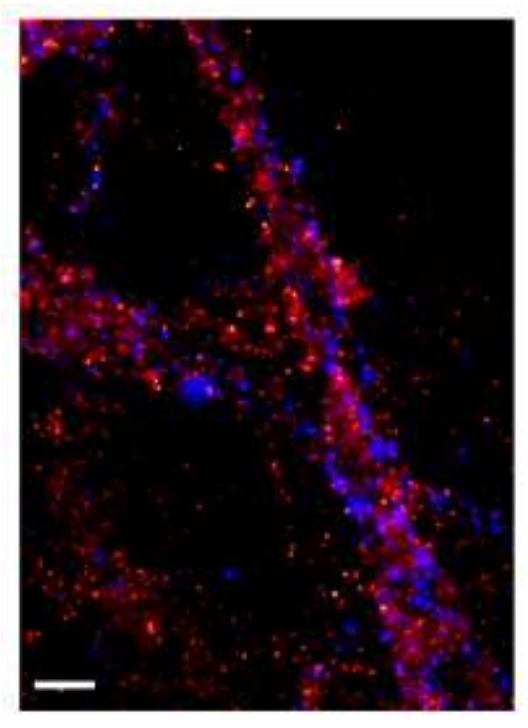

\begin{tabular}{|c|c|c|}
\hline $\begin{array}{c}\text { Whole cell copy } \\
\text { number }\end{array}$ & \multicolumn{2}{|c|}{$53718587.87 \pm 4160685.05$} \\
\hline $\begin{array}{c}\text { Spine copy } \\
\text { number }\end{array}$ & \multicolumn{2}{|c|}{$10958.99 \pm 1937.38$} \\
\hline$\%$ in PSD & \multicolumn{2}{|c|}{15.60} \\
\hline PSD copy number & \multicolumn{2}{|c|}{$1478.74 \pm 261.42$} \\
\hline & Mushroom & Stubby \\
\hline Spine copy & 8519.68 & 13714.62 \\
number & \pm 1506.15 & \pm 2424.53 \\
\hline PSD copy number & $1149.60 \pm$ & 1850.57 \\
\hline$\%$ of protein & 203.23 & \pm 327.15 \\
\hline Molarity $[\mu M]$ & $0.46 \pm 0.08 \%$ & $0.48 \pm 0.08 \%$ \\
\hline
\end{tabular}

\section{References}

Antibody: Cell Signaling 9367

PDB Identifier: $1 \mathrm{vg} 1$

\section{Literature:}

Deinhardt et al., 2006; Gutierrez et al., 2004; Lee et al., 2013 


\section{Rab9 (Gene: Rab9a, Uniprot ID: Q99P75)}

Known function: Late endosome to TGN trafficking

Known organization: Membrane-associated and cytosolic

Known Interactions: None
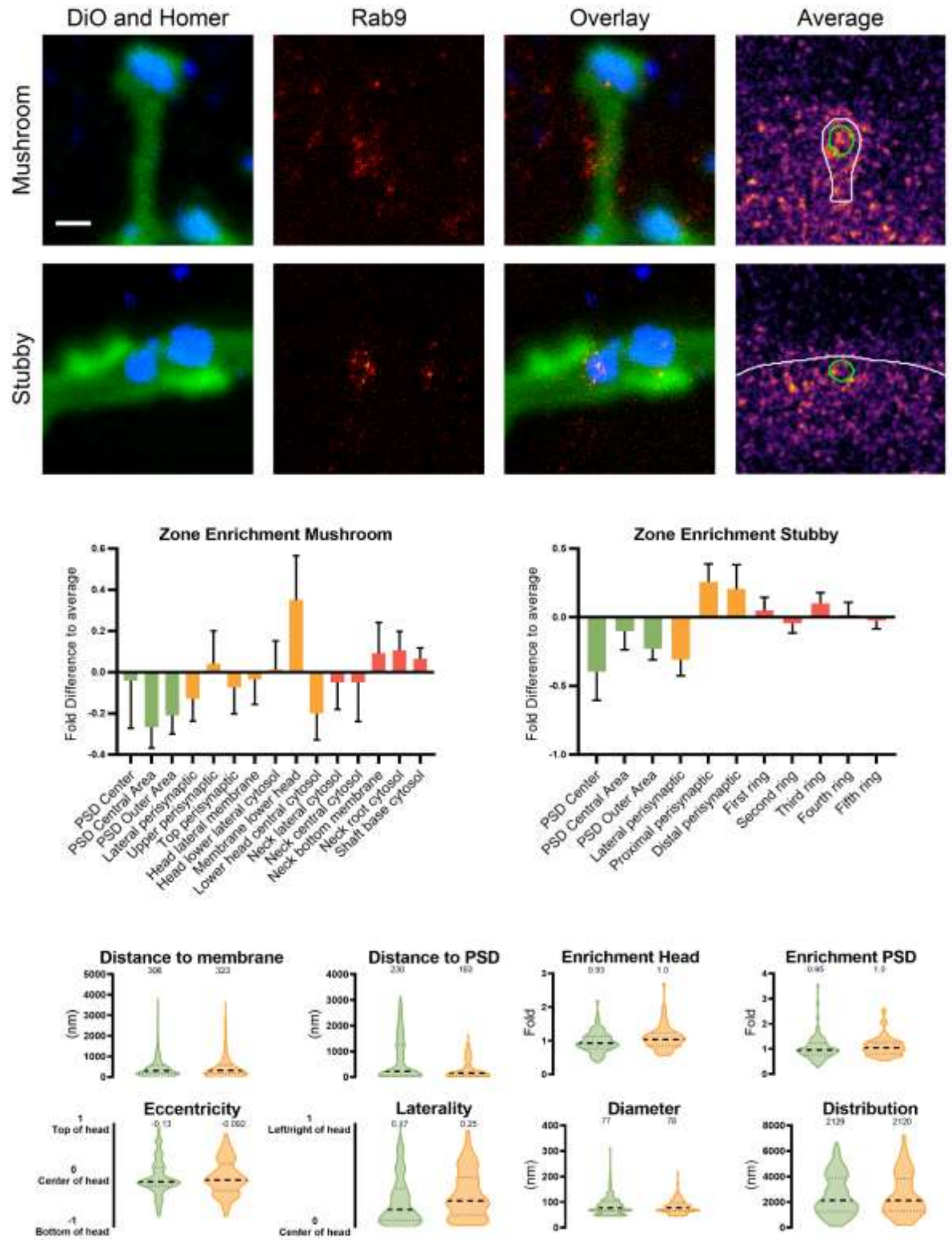

Figure 90: Rab9 nanoscale localization and abundance. Continued on next page. 


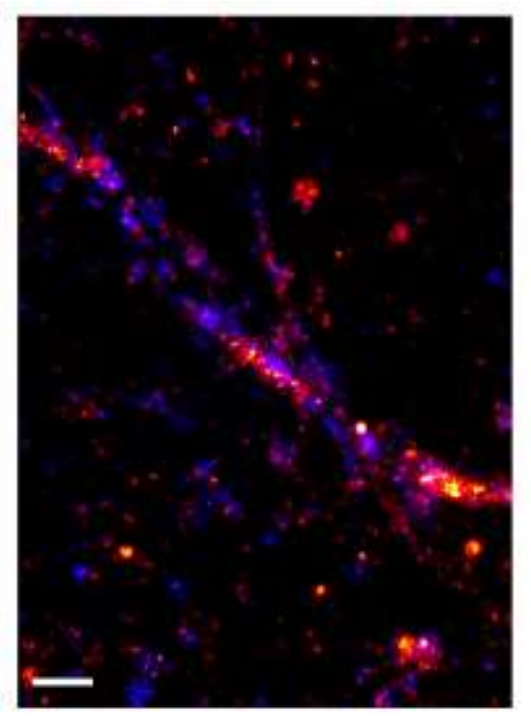

\begin{tabular}{|c|c|c|}
\hline $\begin{array}{c}\text { Whole cell copy } \\
\text { number }\end{array}$ & \multicolumn{2}{|c|}{$4453835.81 \pm 312494.67$} \\
\hline $\begin{array}{c}\text { Spine copy } \\
\text { number }\end{array}$ & \multicolumn{2}{|c|}{$62.77 \pm 120.83$} \\
\hline$\%$ in PSD & \multicolumn{2}{|c|}{18.47} \\
\hline PSD copy number & \multicolumn{2}{|c|}{$9.79 \pm 18.84$} \\
\hline & Mushroom & Stubby \\
\hline $\begin{array}{c}\text { Spine copy } \\
\text { number }\end{array}$ & $54.23 \pm 104.39$ & $71.81 \pm 138.24$ \\
\hline PSD copy number & $8.45 \pm 16.28$ & $11.20 \pm 21.55$ \\
\hline$\%$ of protein & $0.01 \pm 0.01 \%$ & $0.00 \pm 0.01 \%$ \\
\hline Molarity [MM] & $0.45 \pm 0.87$ & $0.38 \pm 0.74$ \\
\hline
\end{tabular}

\section{References}

Antibody: Cell Signaling 5118 PDB Identifier: 4qxa

\section{Literature:}

Díaz et al., 1997; Ganley et al., 2004; Lombardi et al., 1993 


\section{Rab11 (Gene: Rab11a, Uniprot ID: P62494)}

Known function: TGN to PM trafficking, Involved in basal and regulated AMPAR and TrkB trafficking, Involved in neurite growth

Known organization: Membrane-associated and cytosolic

Known Interactions: None
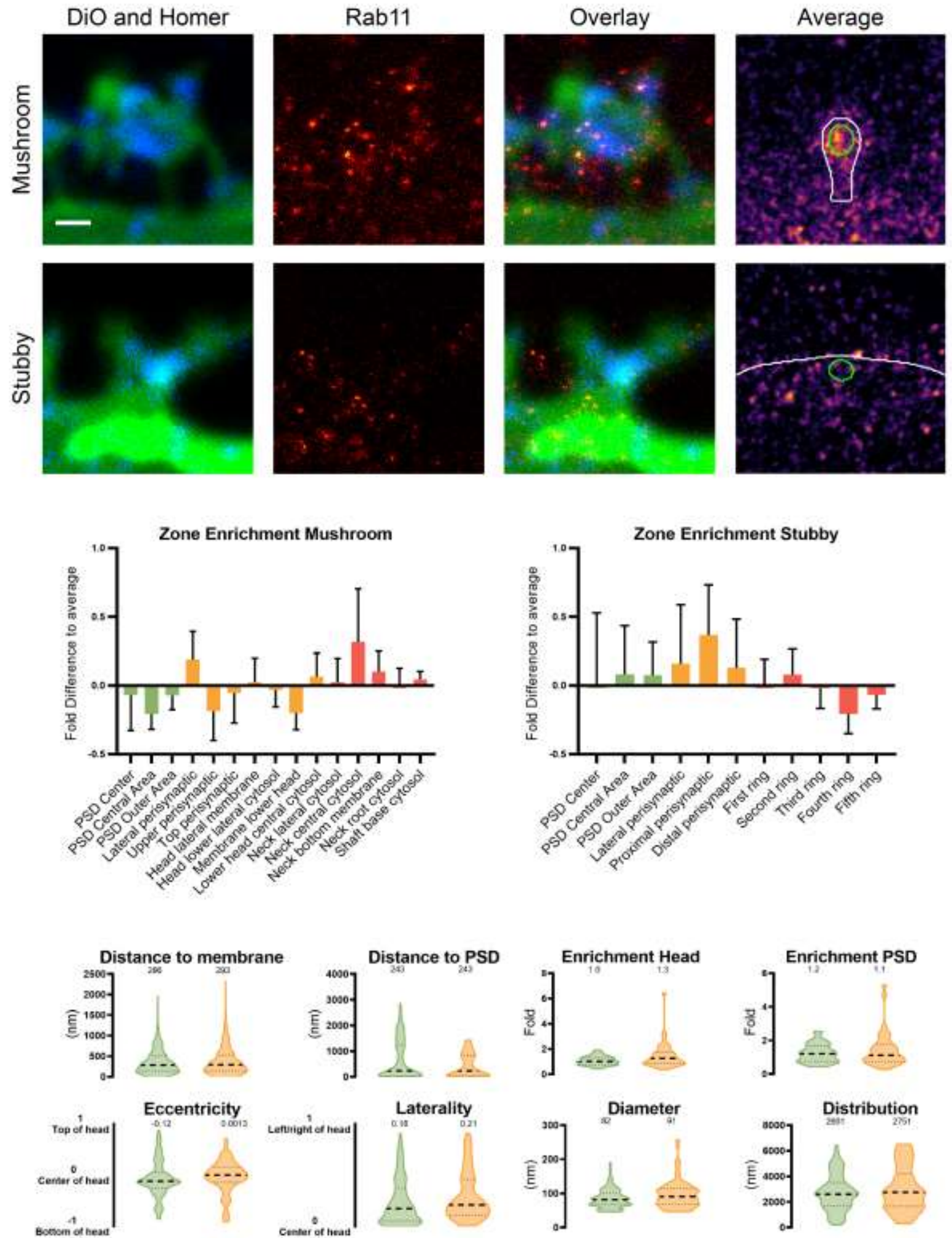

Figure 91: Rab11 nanoscale localization and abundance. Continued on next page. 


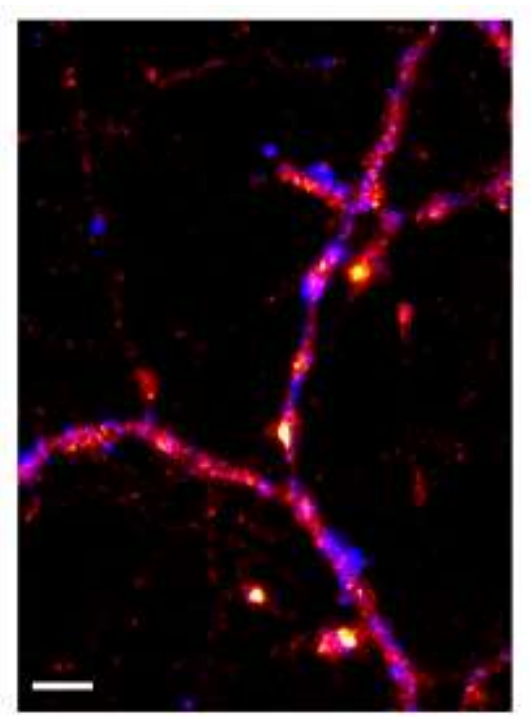

\begin{tabular}{|c|c|c|}
\hline $\begin{array}{c}\text { Whole cell copy } \\
\text { number }\end{array}$ & \multicolumn{2}{|c|}{$20073729.36 \pm 2350097.34$} \\
\hline $\begin{array}{c}\text { Spine copy } \\
\text { number }\end{array}$ & \multicolumn{2}{|c|}{$955.81 \pm 563.49$} \\
\hline$\%$ in PSD & \multicolumn{2}{|c|}{23.89} \\
\hline PSD copy number & \multicolumn{2}{|c|}{$184.30 \pm 108.65$} \\
\hline & Mushroom & Stubby \\
\hline \begin{tabular}{c|c|} 
Spine copy \\
number
\end{tabular} & 1033.21 & 802.74 \\
\hline PSD copy number & $199.22 \pm 117.45$ & $154.78 \pm 91.25$ \\
\hline$\%$ of protein & $0.06 \pm 0.03 \%$ & $0.03 \pm 0.02 \%$ \\
\hline Molarity $[\mu \mathrm{M}]$ & $8.66 \pm 5.11$ & $4.30 \pm 2.54$ \\
\hline
\end{tabular}

\section{References}

Antibody: Cell Signaling 3539

PDB Identifier: 1yzk

\section{Literature:}

Bacaj et al., 2015; Brown et al., 2007; Chen et al., 1998; Gu et al., 2016; Huang et al., 2013; Khvotchev et al., 2003; Lazo et al., 2013; Shirane and Nakayama, 2006 


\section{SNAREs and associated proteins}

Transport between organelles requires fusion, which is often brought about by SNARE proteins. In this section, I determine the localization and abundance of almost every known SNARE protein known to be present in rat neurons.

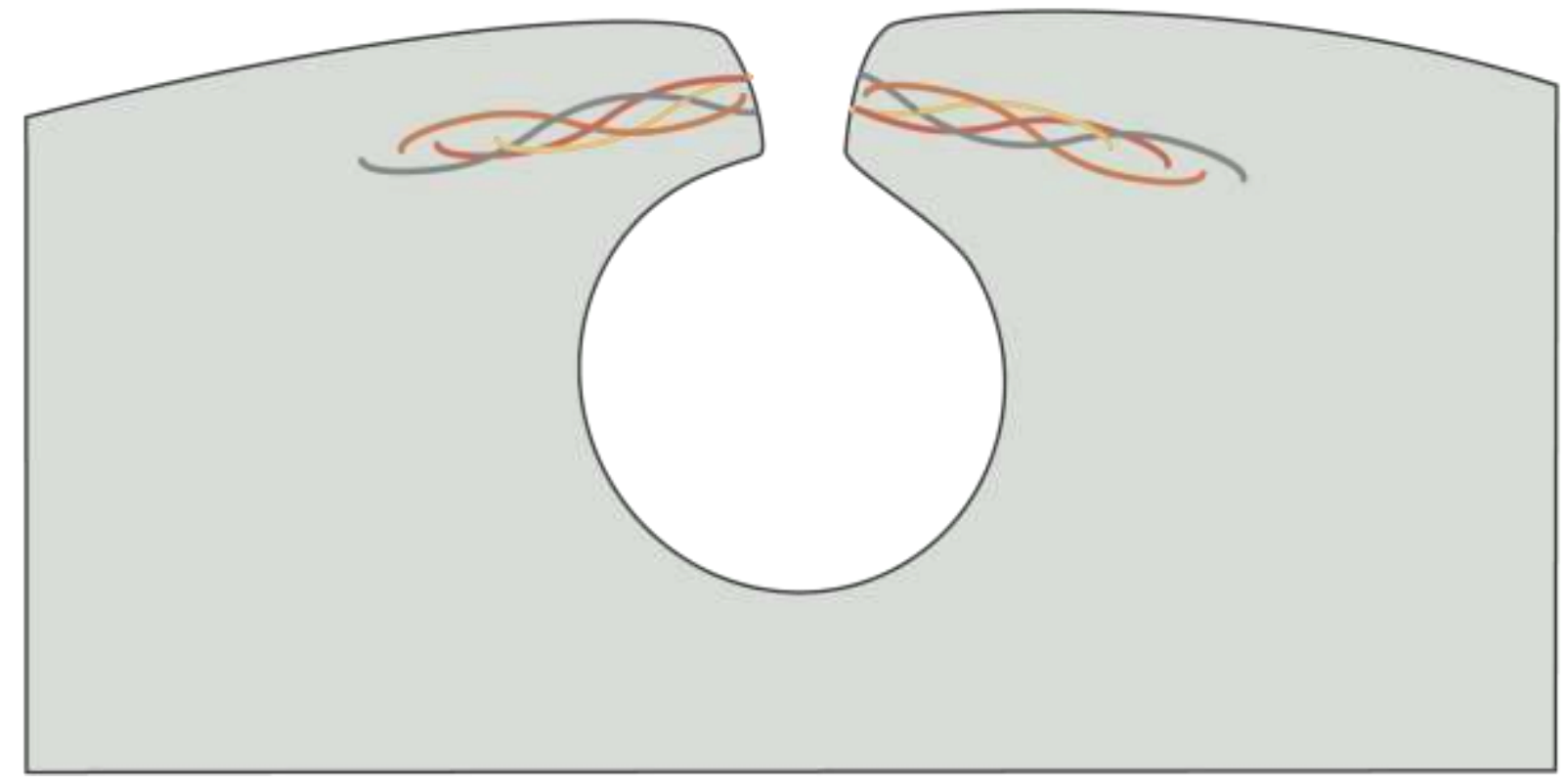

Figure 92: Depiction of SNARE proteins. 
a/ß-SNAP (Genes: Napa and Napb, Uniprot ID: P54921 and P85969)

Known function: Bring SNARE complexes to NSF for disassembly

Known organization: Cytosolic

Known Interactions: SNARE complexes of different compositions, NSF
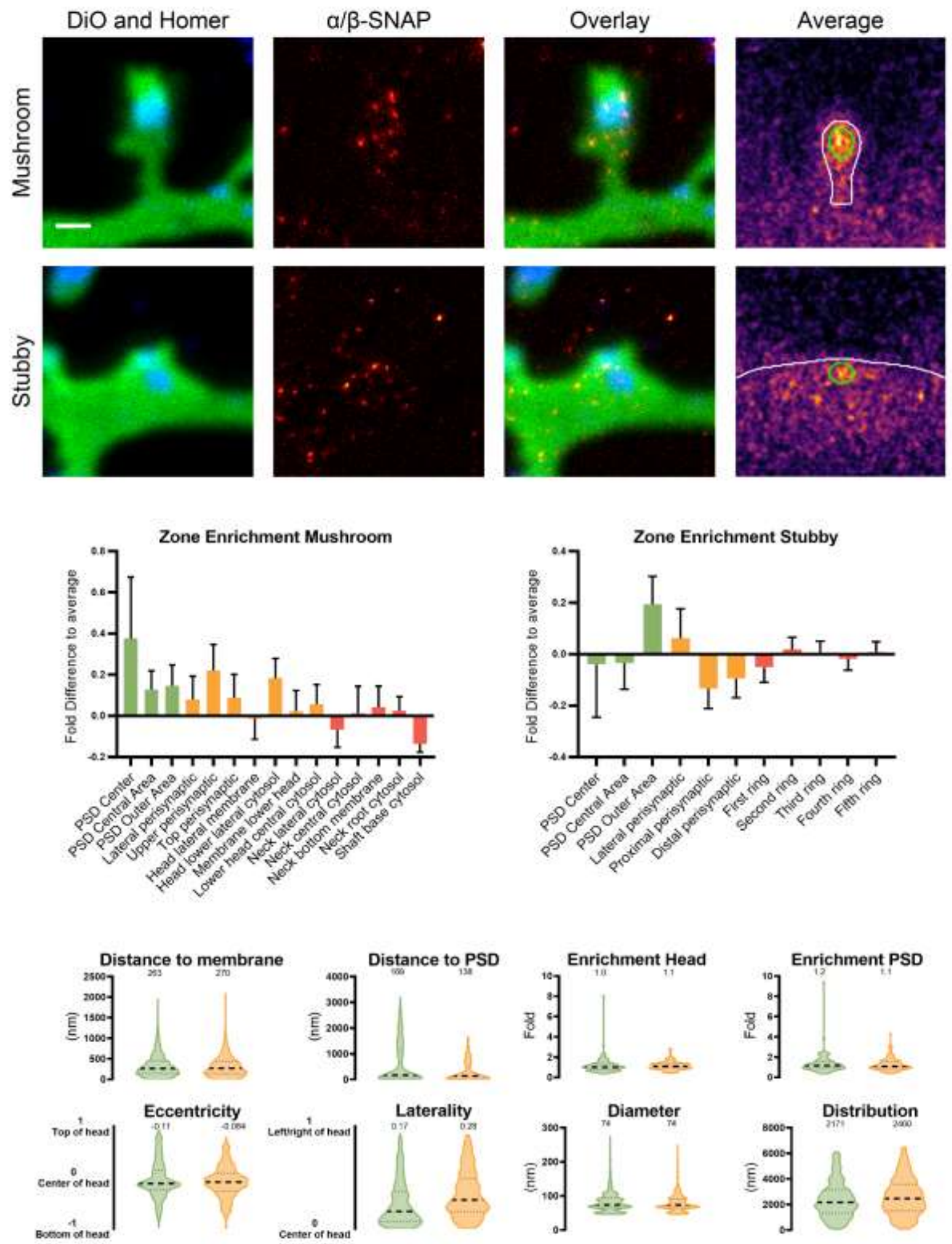

Figure 93: $\alpha / \beta$ SNAP nanoscale localization and abundance. Continued on next page. 


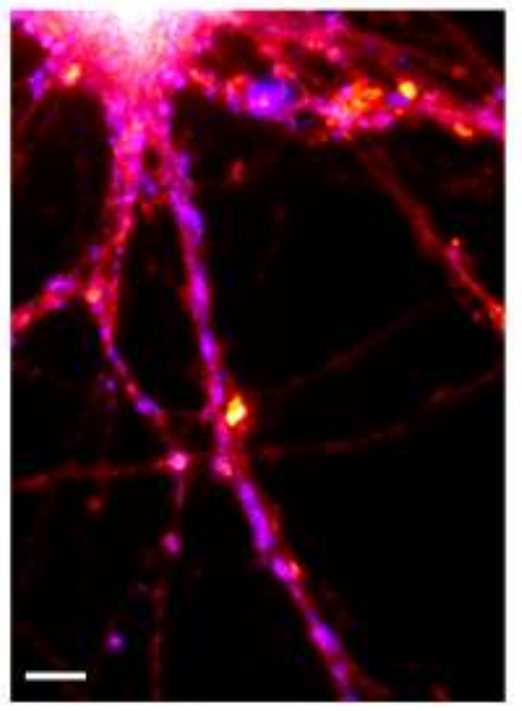

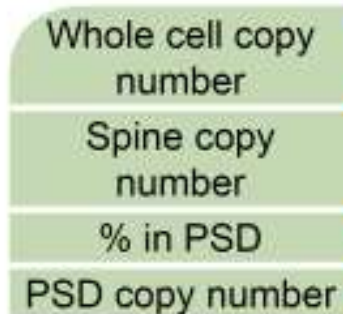

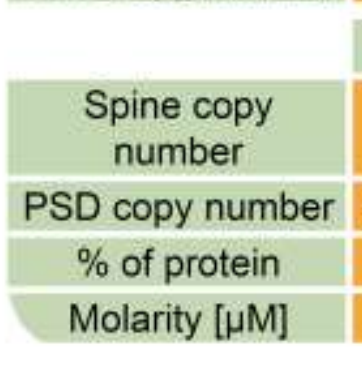

\begin{tabular}{|c|}
\hline $14019738.16 \pm 1687591.28$ \\
\hline $2878.33 \pm 590.12$ \\
\hline 22.03 \\
\hline $519.59 \pm 106.53$ \\
\hline
\end{tabular}

\begin{tabular}{|c|c|}
\hline Mushroom & Stubby \\
\hline 2531.67 & 3357.24 \\
\pm 519.05 & \pm 688.31 \\
\hline $457.01 \pm 93.70$ & $606.04 \pm 124.25$ \\
\hline $0.39 \pm 0.08 \%$ & $0.33 \pm 0.07 \%$ \\
\hline $21.22 \pm 4.35$ & $18.00 \pm 3.69$ \\
\hline
\end{tabular}

Mushroom molecular model

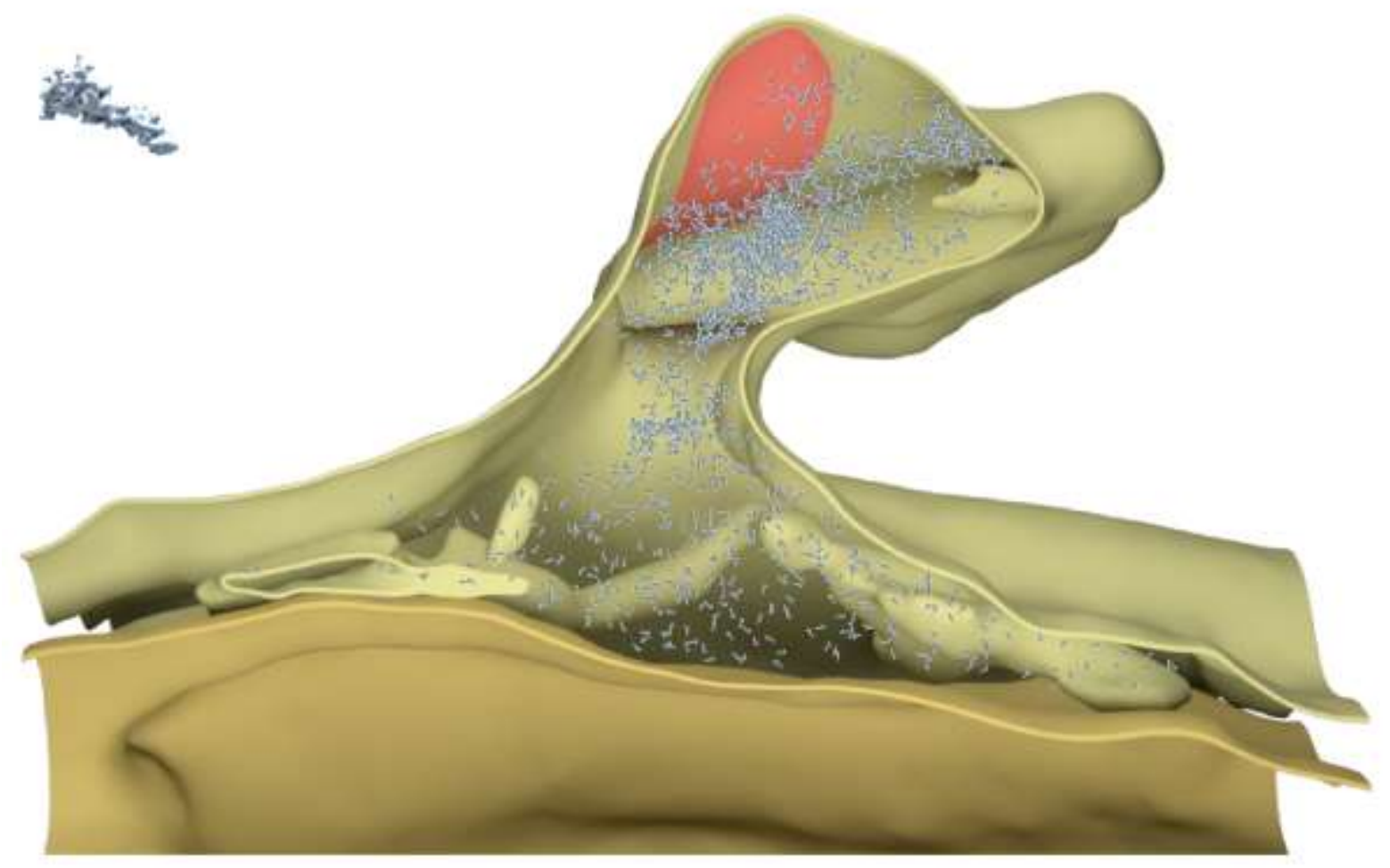

\section{References}

Antibody: Reinhard Jahn, clone 77.2

PDB Identifier: $6 \mathrm{mdm}$

\section{Literature:}

Clary et al., 1990; Hanson et al., 1995; Hohl et al., 1998; Söllner et al., 1993a, 1993b; Weidman et al., 1989; Whiteheart et al., 1993; Wilson et al., 1989 
NSF (Vesicle-fusing ATPase, Gene: Nsf, Uniprot ID: Q9QUL6)

Known function: Disassembly of trans-SNARE complexes

Known organization: Cytosolic, Homohexamer

Known Interactions: $\alpha$ and $\beta$ SNAP, GluR2
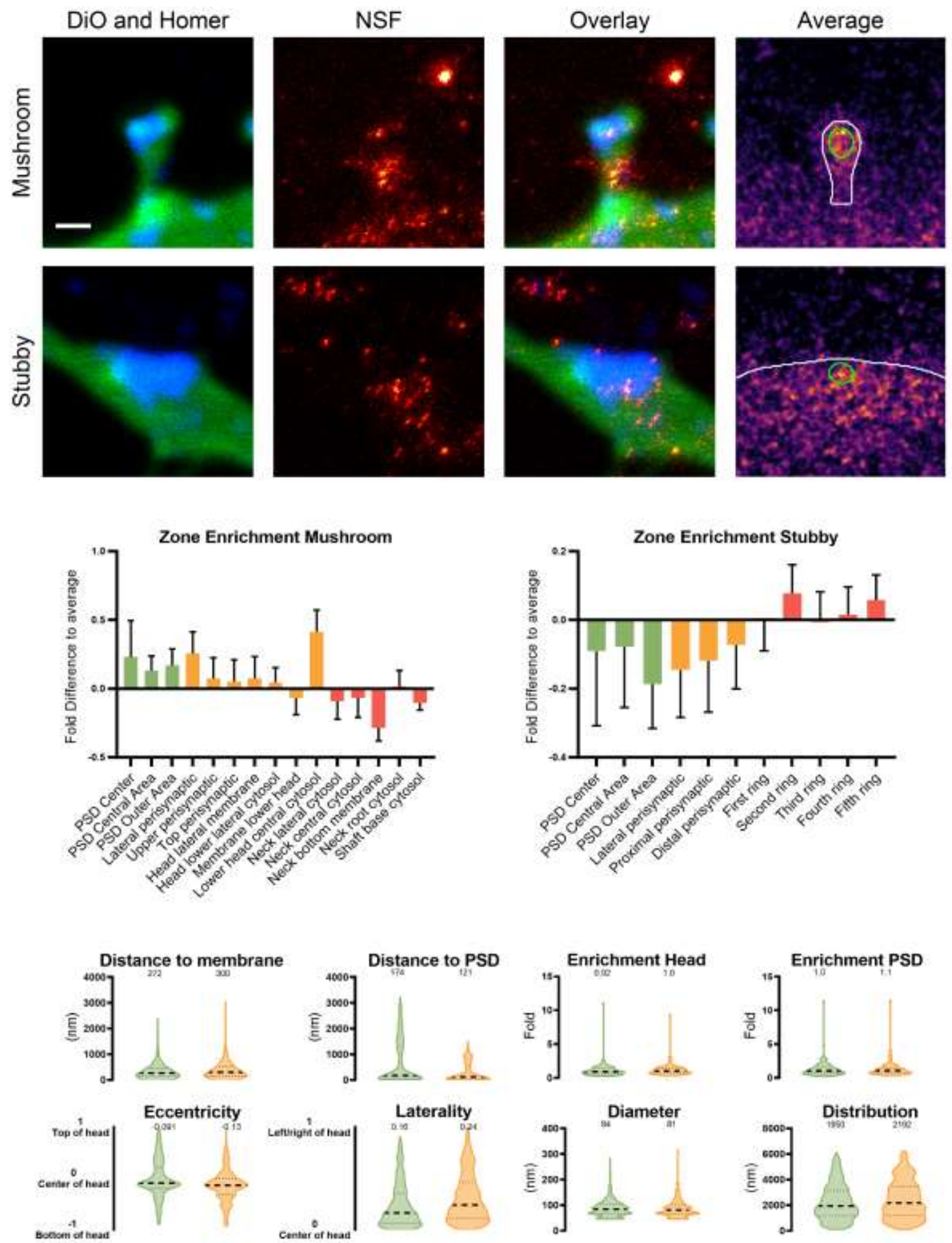

Figure 94: NSF nanoscale localization and abundance. Continued on next page. 


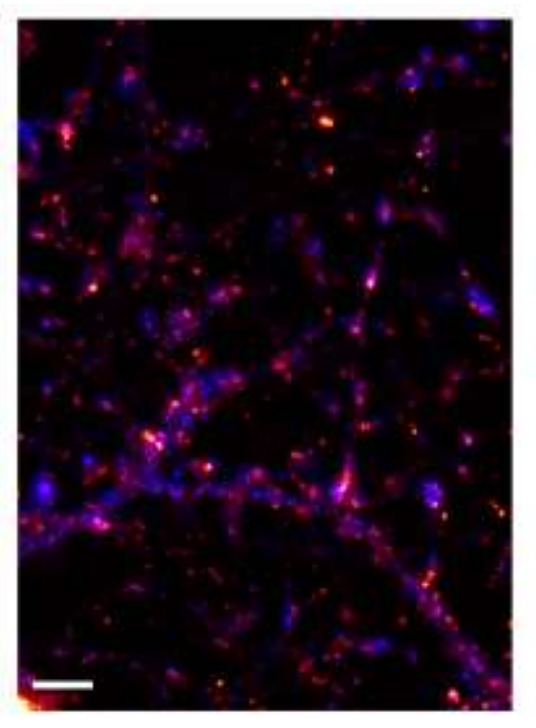

\begin{tabular}{|c|c|}
\hline $\begin{array}{c}\text { Whole cell copy } \\
\text { number }\end{array}$ & $15903989.38 \pm 1635617.00$ \\
\hline $\begin{array}{c}\text { Spine copy } \\
\text { number }\end{array}$ & $4442.98 \pm 753.56$ \\
\hline$\%$ in PSD & 20.68 \\
\hline PSD copy number & $761.45 \pm 129.15$ \\
\hline
\end{tabular}

\begin{tabular}{|c|c|c|}
\hline & Mushroom & Stubby \\
\hline $\begin{array}{l}\text { Spine copy } \\
\text { number }\end{array}$ & $\begin{array}{c}3523.11 \pm \\
597.55\end{array}$ & $\begin{array}{l}5619.24 \\
+953.07\end{array}$ \\
\hline PSD copy number & $603.80 \pm 102.41$ & $963.04 \pm 163.34$ \\
\hline$\%$ of protein & $0.67 \pm 0.11 \%$ & $0.69 \pm 0.12 \%$ \\
\hline Molarity $[\mu \mathrm{M}]$ & $29.53 \pm 5.01$ & $30.12 \pm 5.11$ \\
\hline
\end{tabular}

Mushroom molecular model

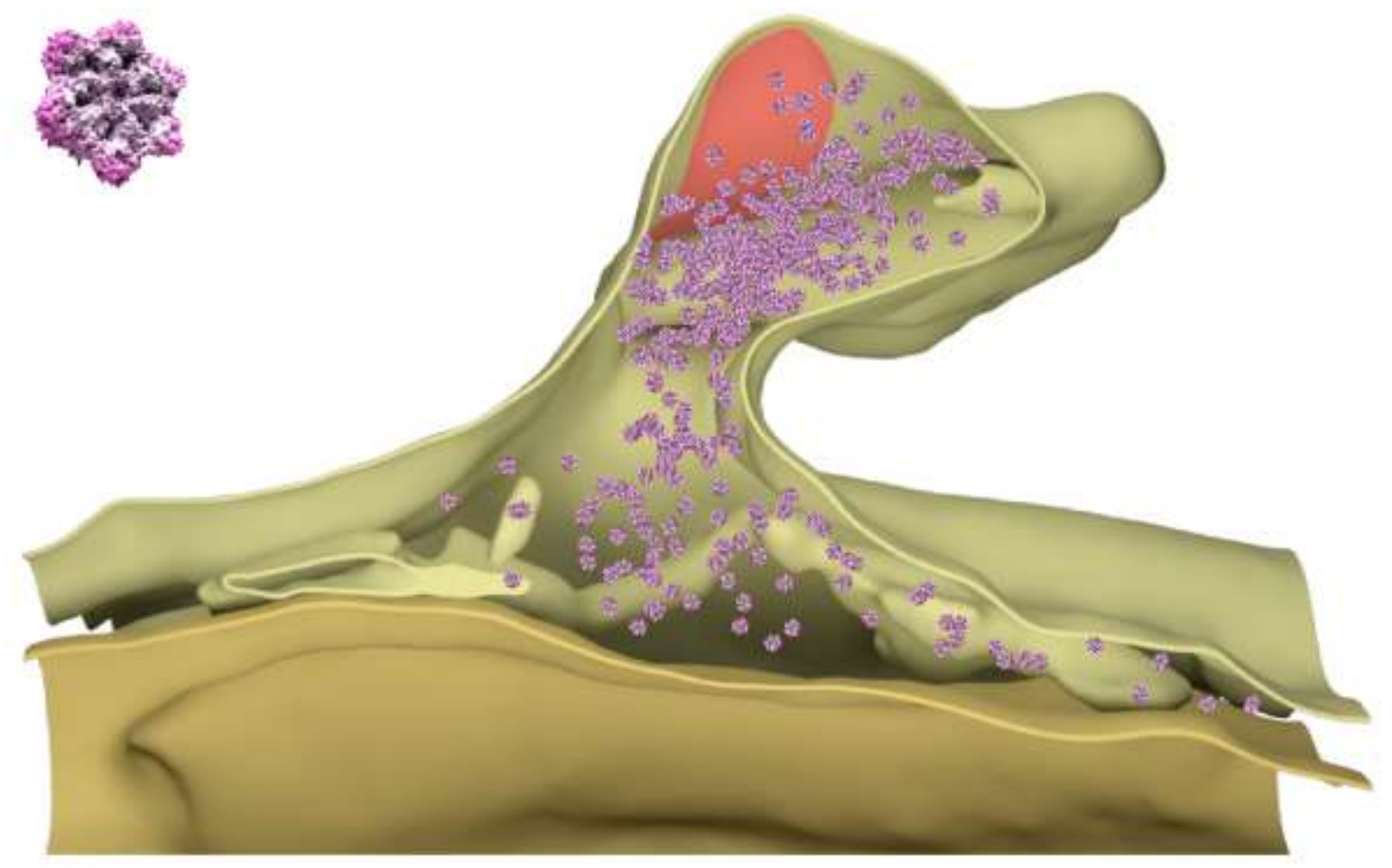

\section{References}

Antibody: Synaptic Systems 132002

PDB Identifier: 3j94

\section{Literature:}

Block et al., 1988; Clary et al., 1990; Evers et al., 2010; Hanson et al., 1995; Hohl et al., 1998;

Söllner et al., 1993a, 1993b; Wilson et al., 1989 


\section{Sec22b (ERS24, Gene: Sec22b, Uniprot ID: Q4KM74)}

Known function: R-SNARE, Antero- and retrograde ER-Golgi transport, Homotypic COPII vesicle fusion, Delivery of NMDAR and GABAAR to PM

Known organization: Transmembrane proteine, ER, Golgi and ERGIC

Known Interactions: Syntaxin1, Syntaxin5
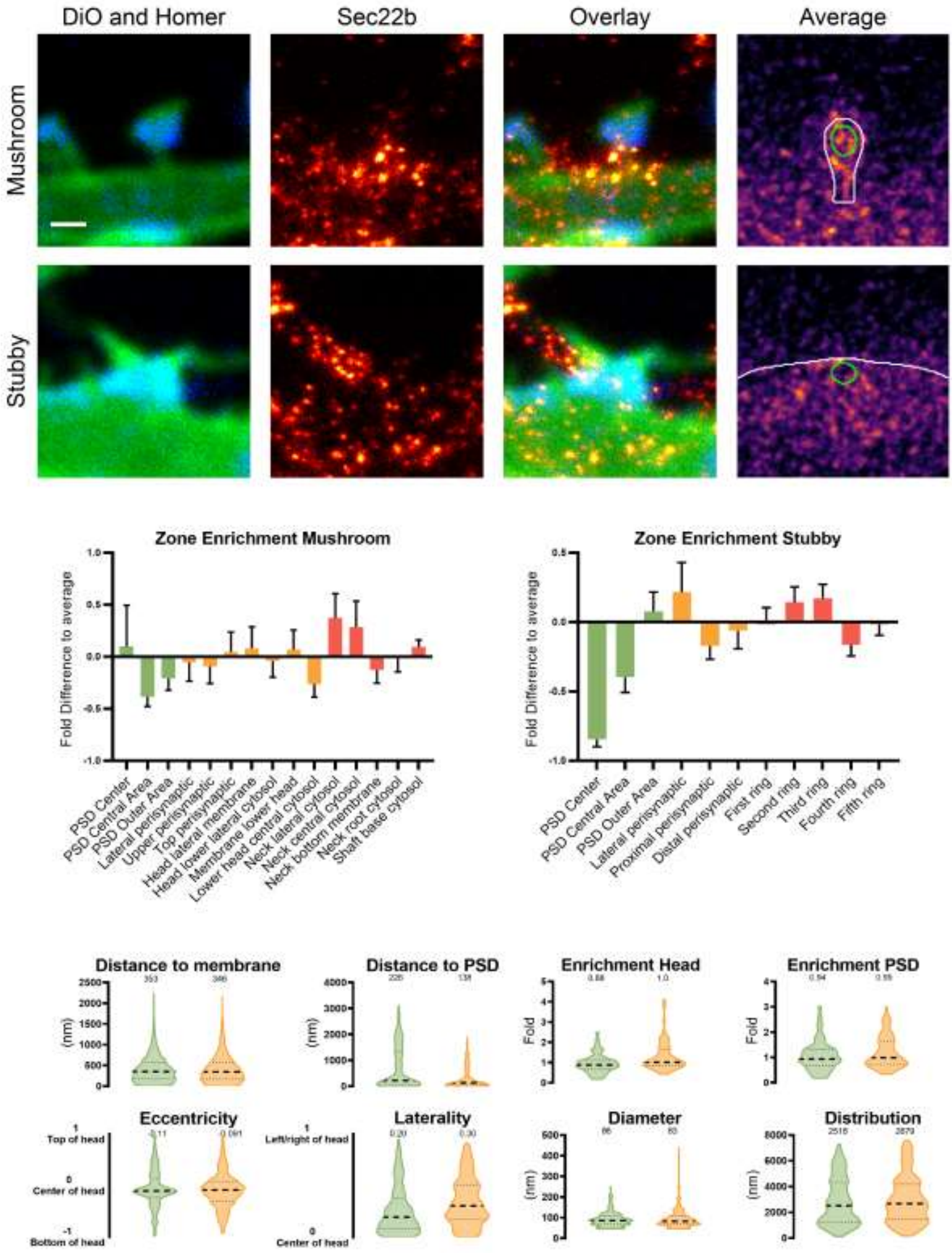

Figure 95: Sec22b nanoscale localization and abundance. Continued on next page. 


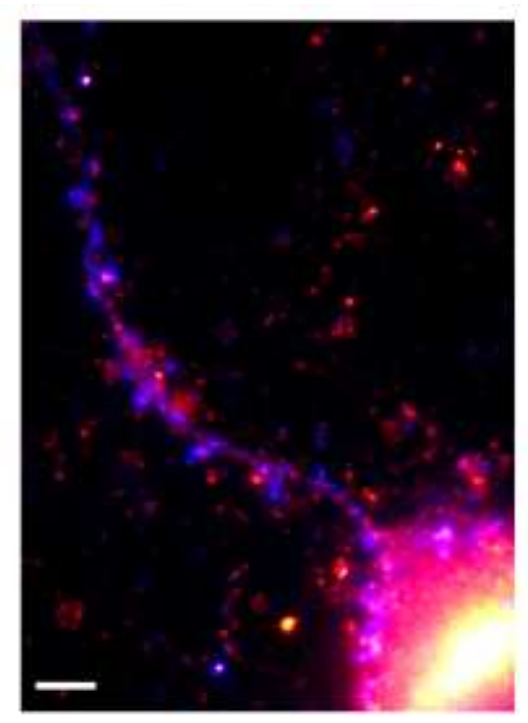

\begin{tabular}{|c|c|c|}
\hline $\begin{array}{c}\text { Whole cell copy } \\
\text { number }\end{array}$ & \multicolumn{2}{|c|}{$13943250.47 \pm 1470099.36$} \\
\hline $\begin{array}{c}\text { Spine copy } \\
\text { number }\end{array}$ & \multicolumn{2}{|c|}{$558.32 \pm 375.29$} \\
\hline$\%$ in PSD & \multicolumn{2}{|c|}{17.18} \\
\hline PSD copy number & \multicolumn{2}{|c|}{$81.86 \pm 55.02$} \\
\hline & Mushroom & Stubby \\
\hline \begin{tabular}{c|c|} 
Spine copy \\
number
\end{tabular} & 543.09 & 603.08 \\
\hline PSD copy number & \pm 365.05 & \pm 405.38 \\
\hline$\%$ of protein & $0.03 \pm 0.02 \%$ & $0.02 \pm 0.01 \%$ \\
\hline Molarity $[\mu \mathrm{M}]$ & $4.55 \pm 3.06$ & $3.23 \pm 2.17$ \\
\hline
\end{tabular}

\section{References}

Antibody: Synaptic Systems 186003

PDB Identifier: $5 \mathrm{vno}$

\section{Literature:}

Burri et al., 2003; Gu et al., 2016; Hay et al., 1997; Lewis et al., 1997; McNew et al., 2000; Washbourne, 2004; Zhang et al., 1997, 1999 


\section{SNAP23 (Gene: Snap23, Uniprot ID: 070377)}

Known function: Qbc SNARE, Lysosome and granule secretion, Asynchronous SV release, Delivery of NMDAR to PM

Known organization: Membrane associated, On PM, Enriched in spines, Perisynaptic Known Interactions: Syntaxin2, Syntaxin4, VAMP2, VAMP7, Synaptotagmin 7
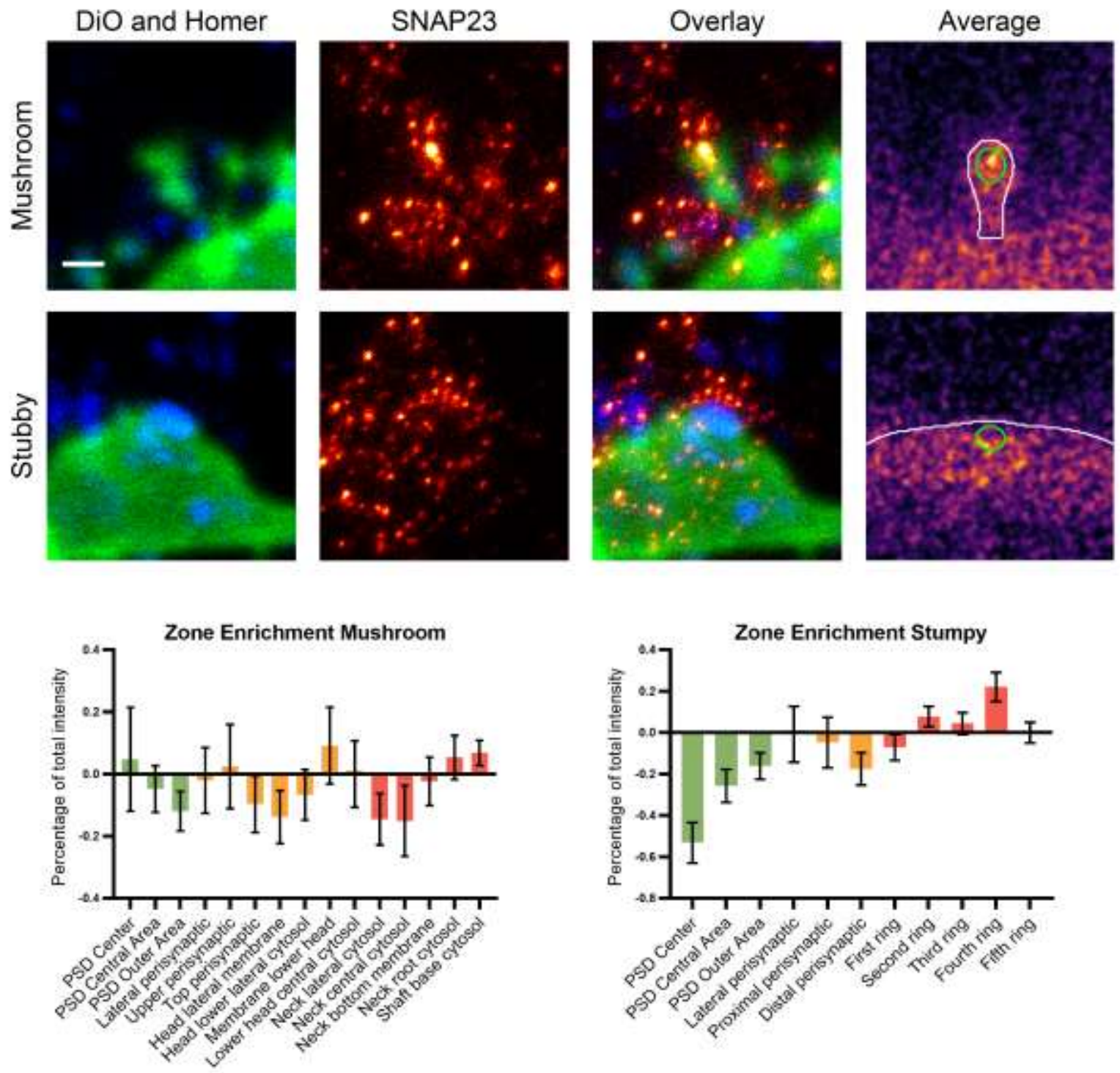
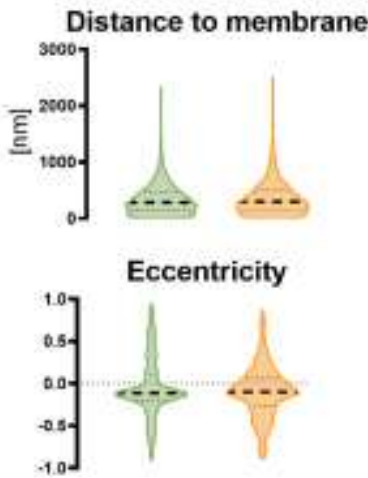

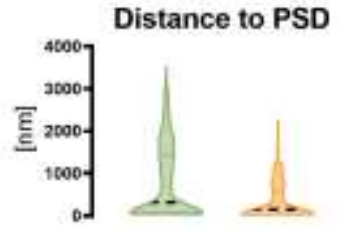

Laterality

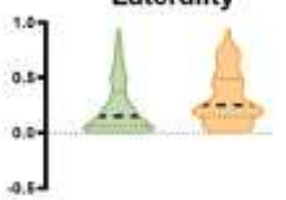

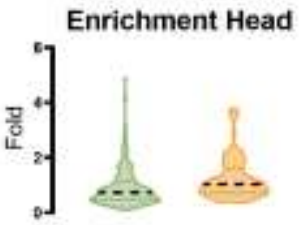

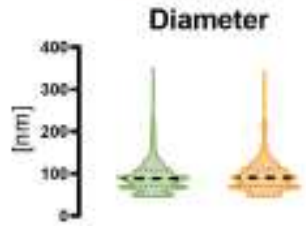

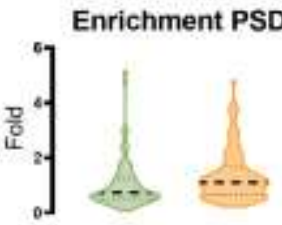

Distribution

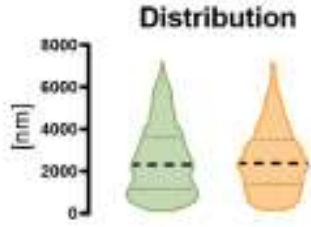

Figure 96: SNAP23 nanoscale localization and abundance. Continued on next page. 


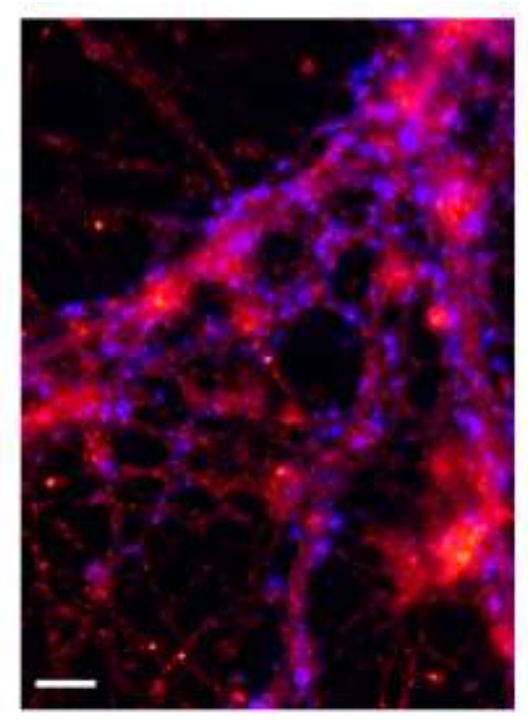

\begin{tabular}{|c|c|c|}
\hline $\begin{array}{c}\text { Whole cell copy } \\
\text { number }\end{array}$ & \multicolumn{2}{|c|}{$180258121.35 \pm 17287591.15$} \\
\hline $\begin{array}{l}\text { Spine copy } \\
\text { number }\end{array}$ & \multicolumn{2}{|c|}{$47706.77 \pm 8477.64$} \\
\hline$\%$ in PSD & \multicolumn{2}{|c|}{16.78} \\
\hline \multirow[t]{2}{*}{ PSD copy number } & \multicolumn{2}{|c|}{$6855.27 \pm 1218.20$} \\
\hline & Mushroom & Stubby \\
\hline $\begin{array}{l}\text { Spine copy } \\
\text { number }\end{array}$ & $\begin{array}{r}39266.18 \\
\pm 6977.72\end{array}$ & $\begin{array}{c}61336.28 \\
\pm 10899.65\end{array}$ \\
\hline PSD copy number & $\begin{array}{c}5642.40 \pm \\
1002.67\end{array}$ & $\begin{array}{c}8813.78 \\
\pm 1566.24\end{array}$ \\
\hline$\%$ of protein & $\pm \%$ & $\pm \%$ \\
\hline Molarity $[\mu \mathrm{M}]$ & $329.10 \pm 58.48$ & $328.82 \pm 58.43$ \\
\hline
\end{tabular}

\section{References}

Antibody: Synaptic Systems 111202

PDB Identifier: modified SNAP25

\section{Literature:}

Flaumenhaft et al., 1999; Rao et al., 2004; Suh et al., 2010; Weber et al., 2014 


\section{SNAP25 (Gene: Snap25, Uniprot ID: P60881)}

Known function: Qbc SNARE, SV release, regulates PSD95 mobility, Delivery of AMPAR and NMDAR to PM

Known organization: Membrane-associated, On PM, Slightly on endosomes and TGN Known Interactions: Syntaxin1, Syntaxin 4, Syntaxin 13, VAMP1, VAMP2, GRIP1
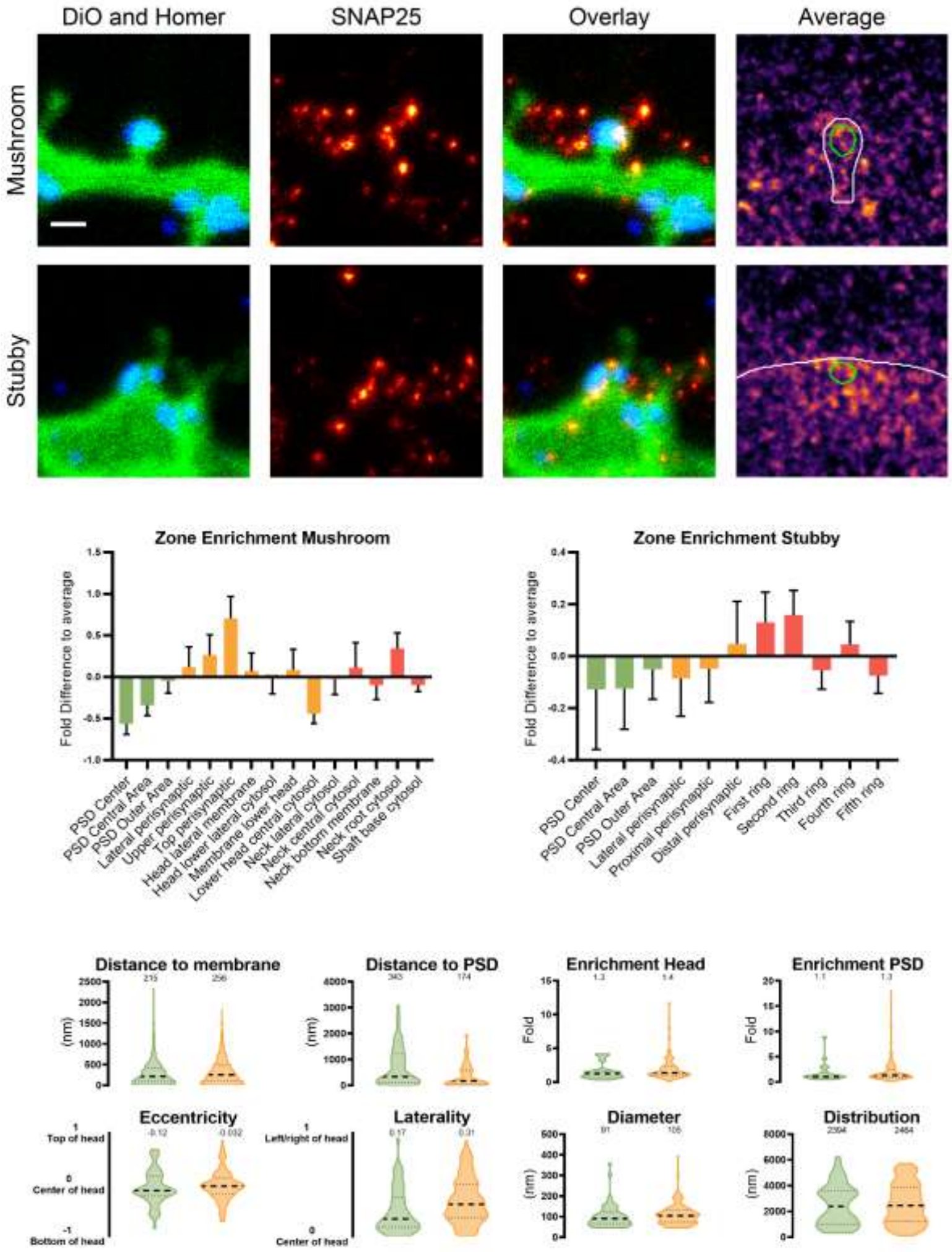

Figure 97: SNAP25 nanoscale localization and abundance. Continued on next page. 


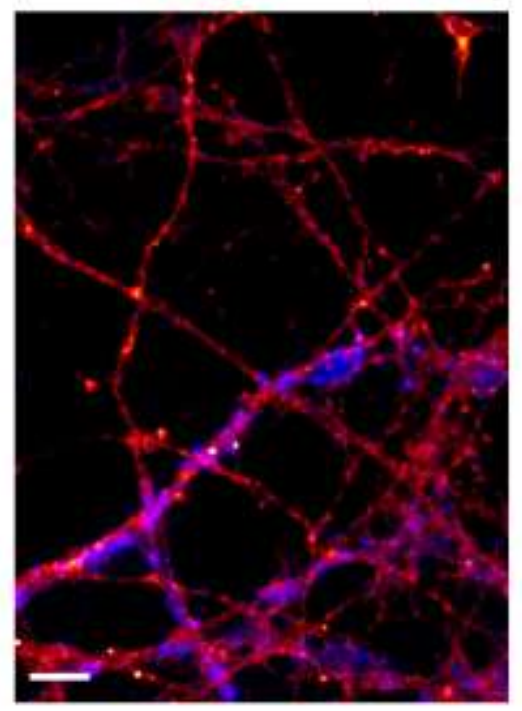

\begin{tabular}{|c|c|c|}
\hline $\begin{array}{c}\text { Whole cell copy } \\
\text { number }\end{array}$ & \multicolumn{2}{|c|}{$180258121.35 \pm 17287591.15$} \\
\hline $\begin{array}{l}\text { Spine copy } \\
\text { number }\end{array}$ & \multicolumn{2}{|c|}{$4897.96 \pm 9042.82$} \\
\hline$\%$ in PSD & \multicolumn{2}{|c|}{18.53} \\
\hline \multirow[t]{2}{*}{ PSD copy number } & \multicolumn{2}{|c|}{$765.78 \pm 1413.82$} \\
\hline & Mushroom & Stubby \\
\hline $\begin{array}{l}\text { Spine copy } \\
\text { number }\end{array}$ & $\begin{array}{r}4037.53 \\
\pm 7454.26\end{array}$ & $\begin{array}{c}6766.23 \\
\pm 12492.10\end{array}$ \\
\hline PSD copy number & $\begin{array}{c}631.26 \\
\pm 1165.45\end{array}$ & $\begin{array}{c}1057.88 \\
\pm 1953.11\end{array}$ \\
\hline$\%$ of protein & $\pm \%$ & $\pm \%$ \\
\hline Molarity $[\mu \mathrm{M}]$ & $33.84 \pm 62.48$ & $36.27 \pm 66.97$ \\
\hline
\end{tabular}

\section{References}

Antibody: Synaptic Systems 111011

Structure: From Takamori et al. 2006

\section{Literature:}

Aikawa et al., 2006; Gu and Huganir, 2016; Gu et al., 2016; Lau et al., 2010; McMahon and

Südhof, 1995; Söllner et al., 1993b; Veit et al., 1996 
SNAP29 (Gs32, Gene: Snap29, Uniprot ID: Q9Z2P6)

Known function: Qbc SNARE, Autophagosome-lysosome fusion, Inhibition of SNARE complex disassembly

Known organization: Membrane-associated, On Golgi, Endosomes, SV, Also in cytosol Known Interactions: Syntaxin3, Syntaxin6, VAMP7, SNARE complexes in general
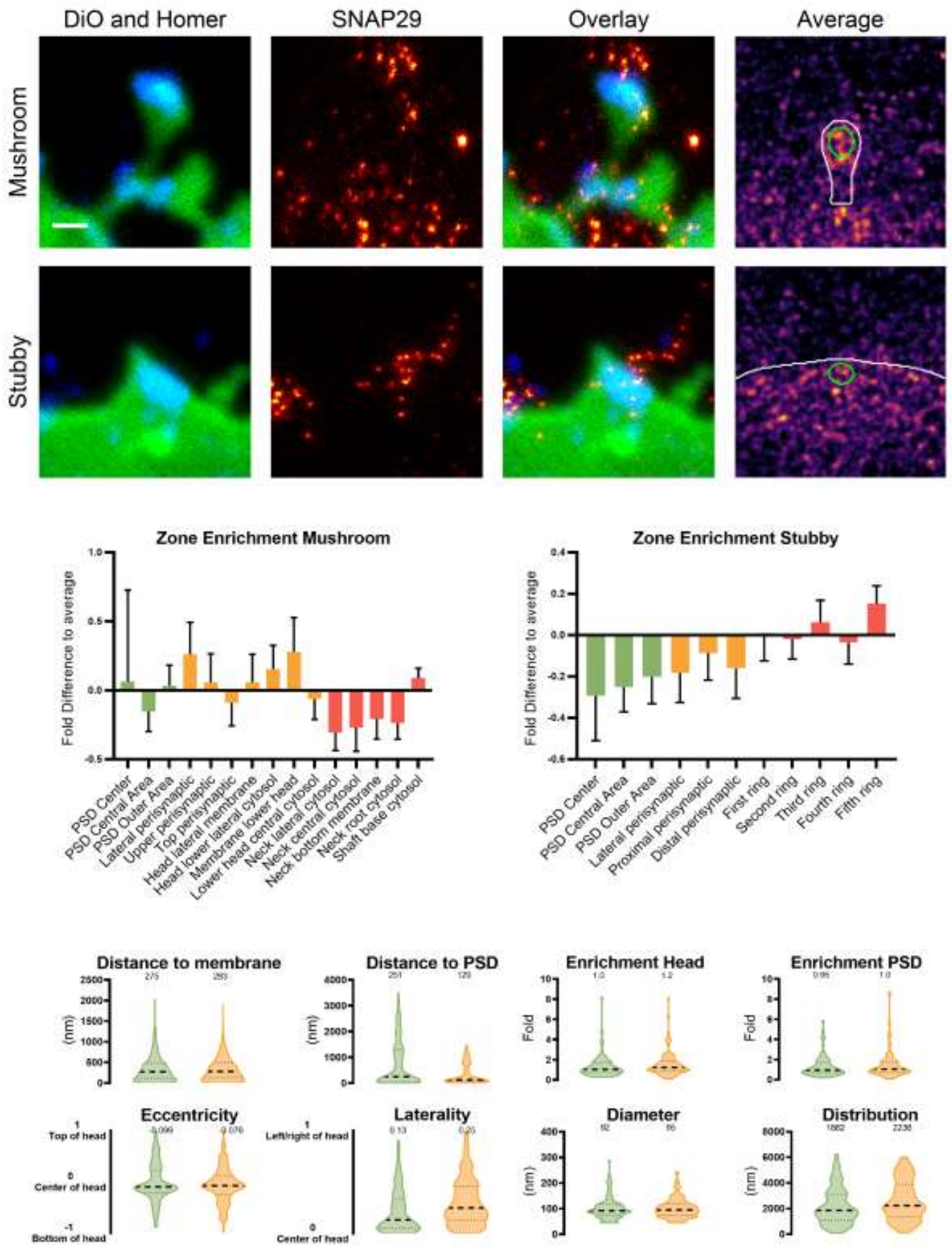

Figure 98: SNAP29 nanoscale localization and abundance. Continued on next page. 


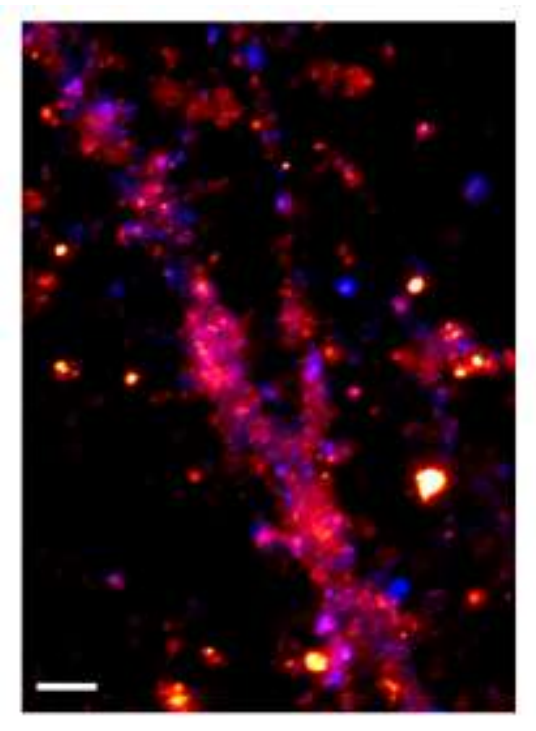

\begin{tabular}{|c|c|c|}
\hline $\begin{array}{c}\text { Whole cell copy } \\
\text { number }\end{array}$ & \multicolumn{2}{|c|}{$1082243.55 \pm 64216.37$} \\
\hline $\begin{array}{c}\text { Spine copy } \\
\text { number }\end{array}$ & \multicolumn{2}{|c|}{$94.89 \pm 48.77$} \\
\hline$\%$ in PSD & \multicolumn{2}{|c|}{17.88} \\
\hline PSD copy number & \multicolumn{2}{|c|}{$14.39 \pm 7.40$} \\
\hline & Mushroom & Stubby \\
\hline $\begin{array}{c}\text { Spine copy } \\
\text { number }\end{array}$ & $85.97 \pm 44.19$ & $112.79 \pm 57.97$ \\
\hline PSD copy number & $13.04 \pm 6.70$ & $17.11 \pm 8.79$ \\
\hline$\%$ of protein & $0.01 \pm 0.00 \%$ & $0.00 \pm 0.00 \%$ \\
\hline Molarity $[\mu \mathrm{M}]$ & $0.72 \pm 0.37$ & $0.60 \pm 0.31$ \\
\hline
\end{tabular}

\section{References}

Antibody: Synaptic Systems 111302

Structure: From Takamori et al. 2006 Literature:

Pan et al., 2005; Steegmaier et al., 1998; Su et al., 2001; Wang et al., 2016; Wong et al., 1999 


\section{SNAP47 (Gene: Snap47, Uniprot ID: Q6P6S0)}

Known function: Qbc SNARE, AMPAR surface delivery during LTP, BDNF release Known organization: Cytosolic, Perisynaptic in spines

Known Interactions: Syntaxin1, Syntaxin3, VAMP2
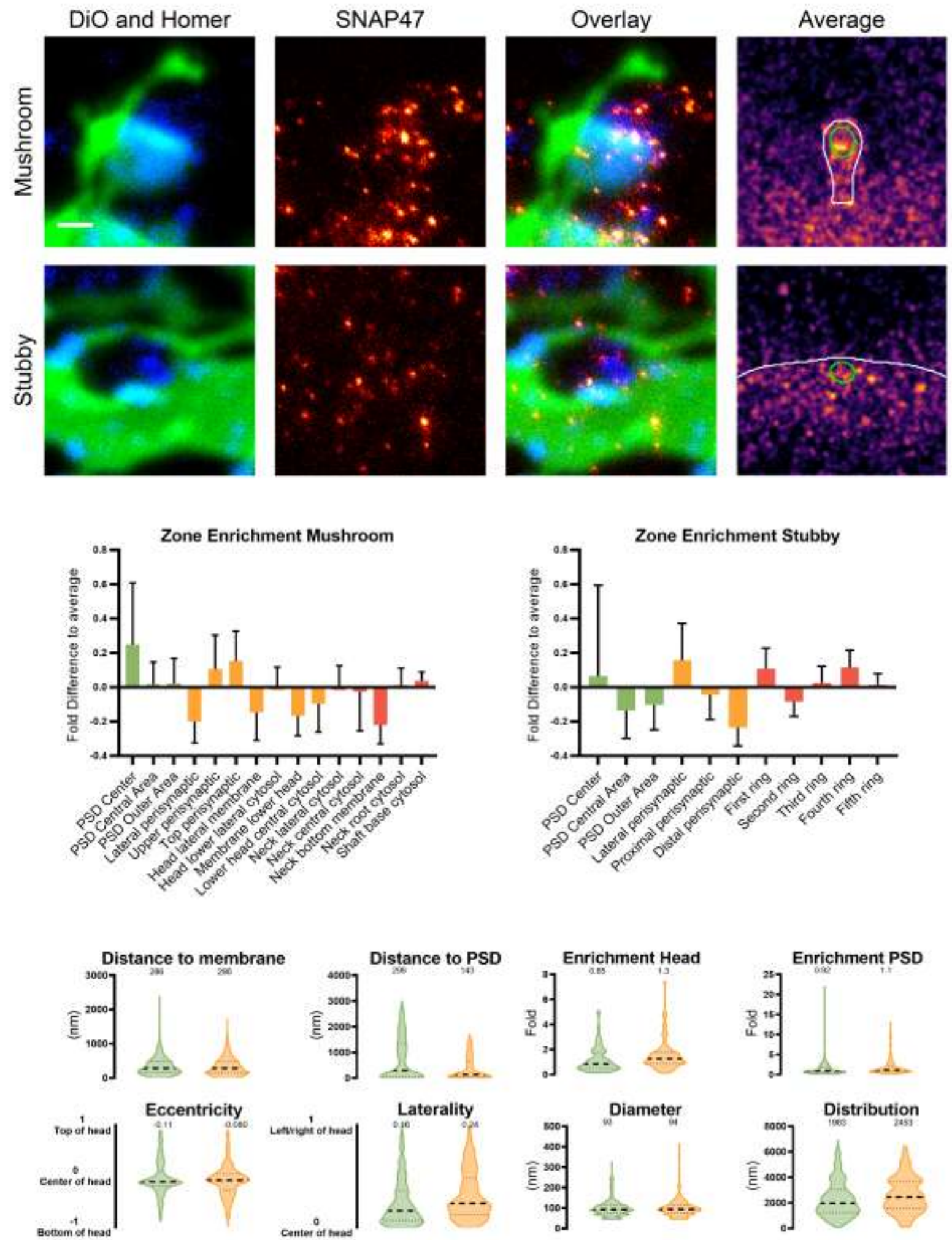

Figure 99: SNAP47 nanoscale localization and abundance. Continued on next page. 


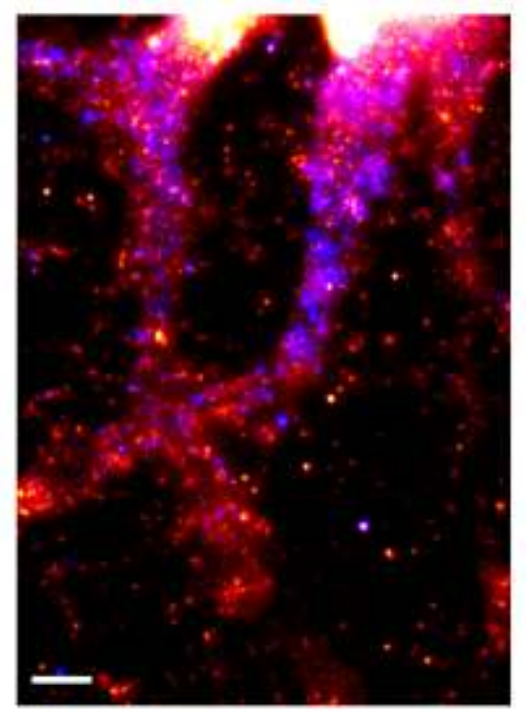

\begin{tabular}{|c|c|c|}
\hline $\begin{array}{c}\text { Whole cell copy } \\
\text { number }\end{array}$ & \multicolumn{2}{|c|}{$70903.80 \pm 20315.38$} \\
\hline $\begin{array}{c}\text { Spine copy } \\
\text { number }\end{array}$ & \multicolumn{2}{|c|}{$13.89 \pm 4.60$} \\
\hline$\%$ in PSD & \multicolumn{2}{|c|}{17.95} \\
\hline PSD copy number & \multicolumn{2}{|c|}{$2.11 \pm 0.70$} \\
\hline Spine copy & Mushroom & Stubby \\
\hline number & $14.74 \pm 4.88$ & $14.22 \pm 4.71$ \\
\hline PSD copy number & $2.24 \pm 0.74$ & $2.16 \pm 0.72$ \\
\hline$\%$ of protein & $0.00 \pm 0.00 \%$ & $0.00 \pm 0.00 \%$ \\
\hline Molarity $[\mu \mathrm{M}]$ & $0.12 \pm 0.04$ & $0.08 \pm 0.03$ \\
\hline
\end{tabular}

\section{References}

Antibody: Synaptic Systems 111403

PDB Identifier: Modified SNAP29

\section{Literature:}

Holt et al., 2006; Jurado et al., 2013; Münster-Wandowski et al., 2017; Shimojo et al., 2015 


\section{Synaptotagmin4 (Gene: Syt4, Uniprot ID: P50232)}

Known function: Retrograde signalling in drosophila

Known organization: Transmembrane protein

Known Interactions: None
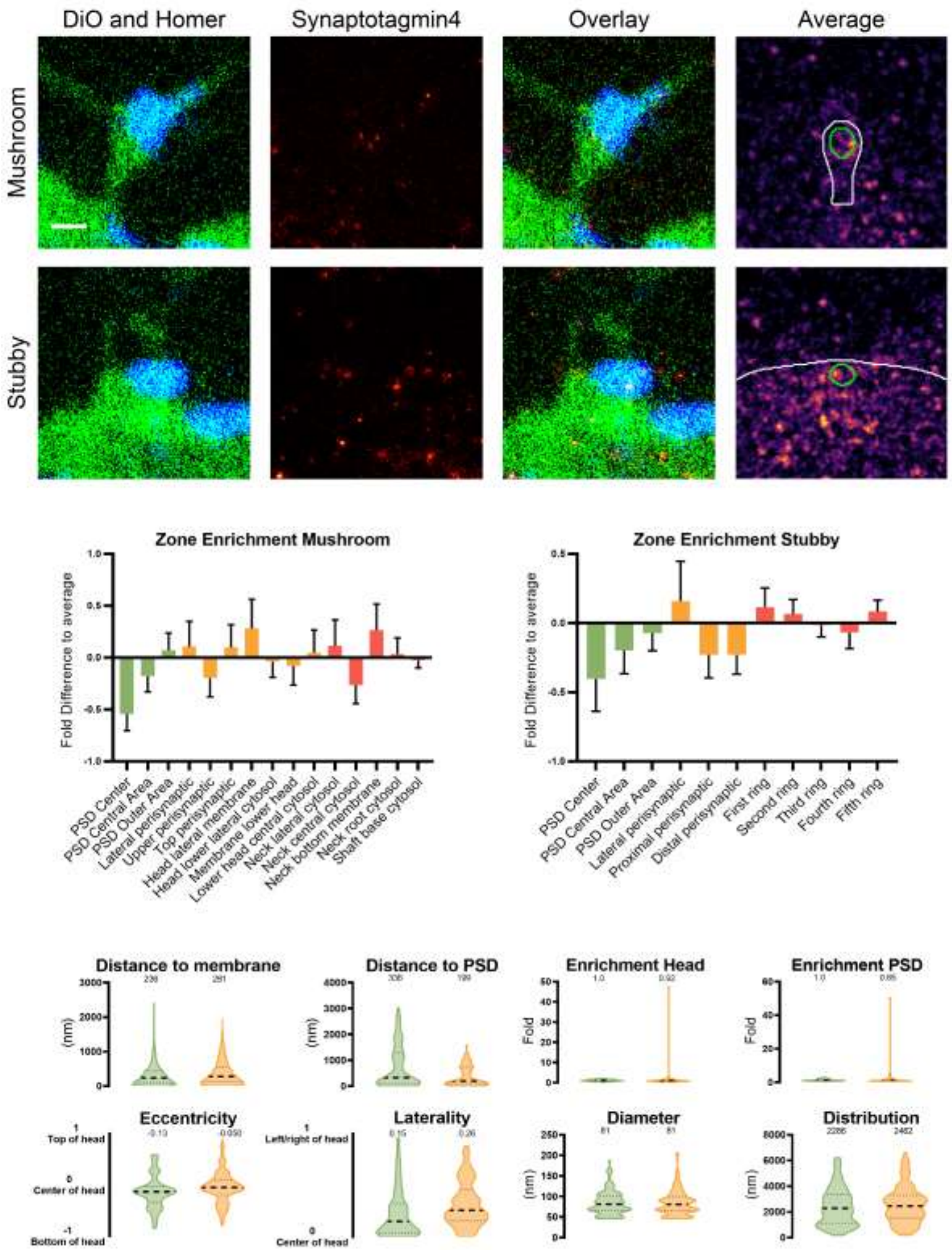

Figure 100: Synaptotagmin 4 nanoscale localization and abundance. Continued on next page. 


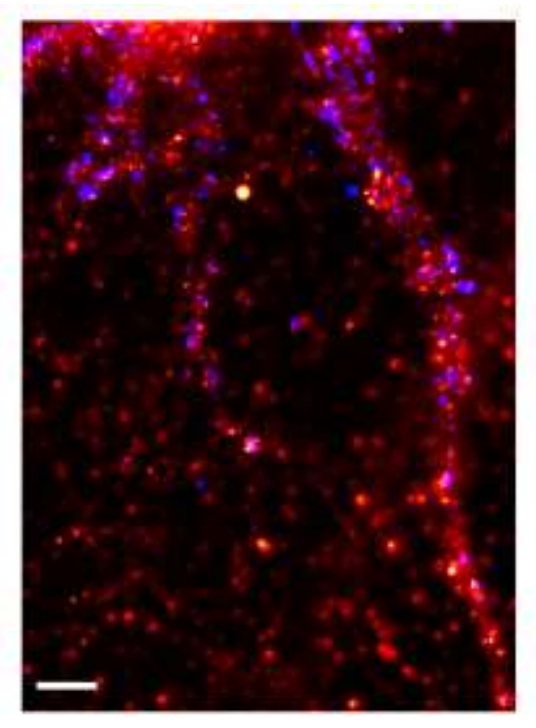

\begin{tabular}{|c|c|c|}
\hline $\begin{array}{c}\text { Whole cell copy } \\
\text { number }\end{array}$ & \multicolumn{2}{|c|}{$329233.65 \pm 63456.19$} \\
\hline $\begin{array}{c}\text { Spine copy } \\
\text { number }\end{array}$ & \multicolumn{2}{|c|}{$14.06 \pm 7.31$} \\
\hline$\%$ in PSD & \multicolumn{2}{|c|}{19.26} \\
\hline PSD copy number & \multicolumn{2}{|c|}{$2.27 \pm 1.18$} \\
\hline & Mushroom & Stubby \\
\hline $\begin{array}{c}\text { Spine copy } \\
\text { number }\end{array}$ & $9.14 \pm 4.75$ & $18.18 \pm 9.46$ \\
\hline PSD copy number & $1.48 \pm 0.77$ & $2.94 \pm 1.53$ \\
\hline$\%$ of protein & $0.00 \pm 0.00 \%$ & $0.00 \pm 0.00 \%$ \\
\hline Molarity $[\mu M]$ & $0.08 \pm 0.04$ & $0.10 \pm 0.05$ \\
\hline
\end{tabular}

\section{References}

Antibody: Synaptic Systems 105143

PDB Identifier: 6ank

\section{Literature:}

Harris et al., 2016; Korkut et al., 2013; Yoshihara et al., 2005 


\section{Synaptotagmin5 (Synaptotagmin9, Gene: Syt5, Uniprot ID: P47861)}

Known function: Involved in dense-core vesicle as well as insulin exocytosis

Known organization: Transmembrane protein, On recycling endosome, LDCV

Known Interactions: None
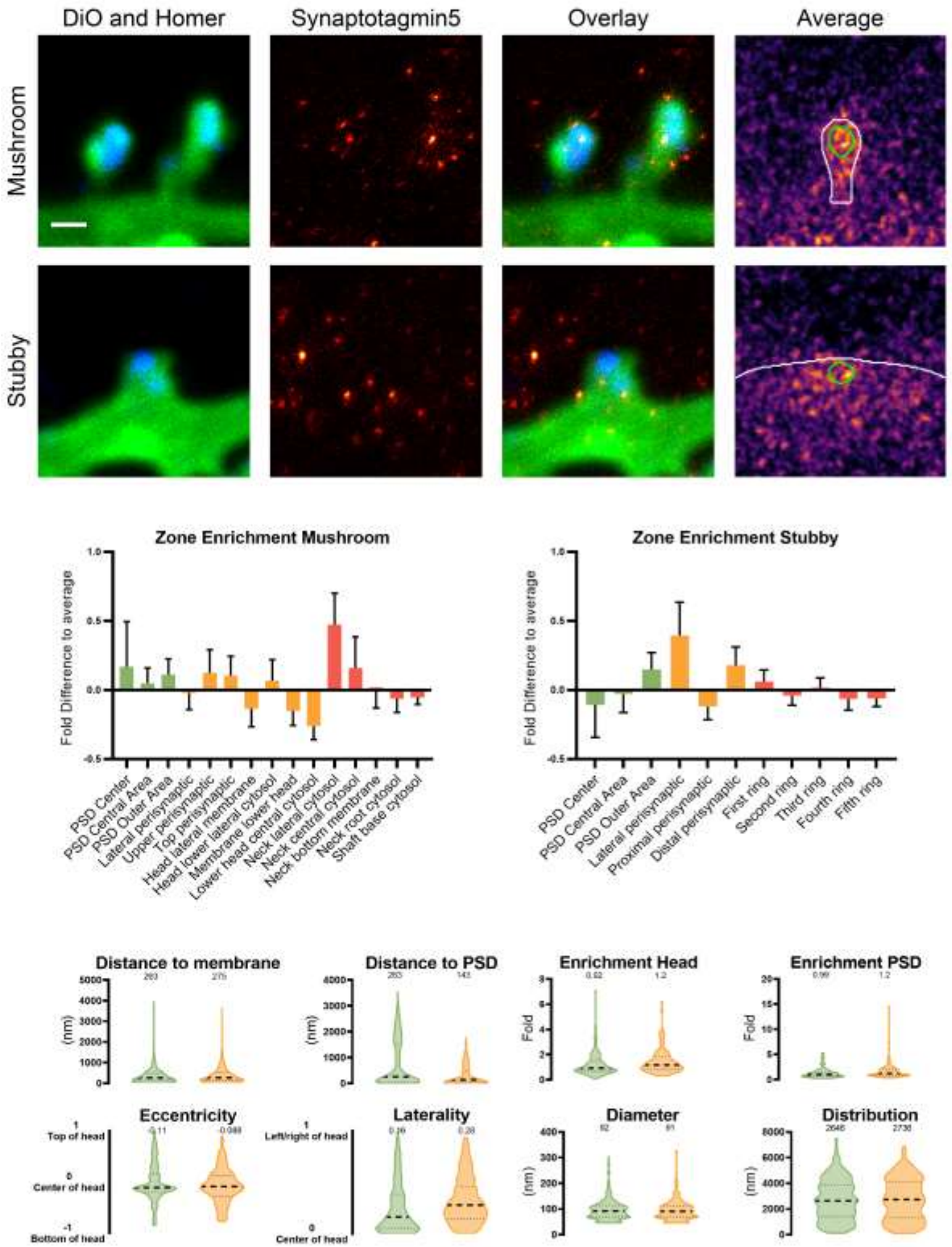

Figure 101: Synaptotagmin 5 nanoscale localization and abundance. Continued on next page. 


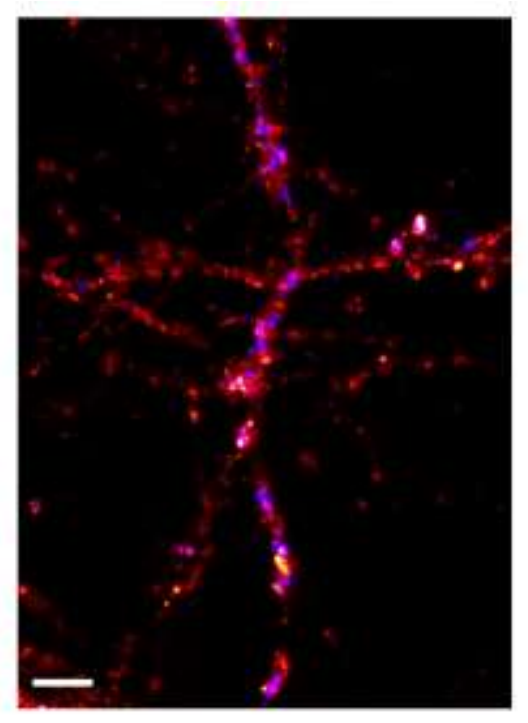

\begin{tabular}{|c|c|c|}
\hline $\begin{array}{c}\text { Whole cell copy } \\
\text { number }\end{array}$ & \multicolumn{2}{|c|}{$1277323.90 \pm 104747.38$} \\
\hline $\begin{array}{c}\text { Spine copy } \\
\text { number }\end{array}$ & \multicolumn{2}{|c|}{$149.54 \pm 47.14$} \\
\hline$\%$ in PSD & \multicolumn{2}{|c|}{21.85} \\
\hline PSD copy number & \multicolumn{2}{|c|}{$26.82 \pm 8.45$} \\
\hline & Mushroom & Stubby \\
\hline $\begin{array}{c}\text { Spine copy } \\
\text { number }\end{array}$ & $124.43 \pm 39.22$ & $177.63 \pm 56.00$ \\
\hline PSD copy number & $22.31 \pm 7.03$ & $31.86 \pm 10.04$ \\
\hline$\%$ of protein & $0.01 \pm 0.00 \%$ & $0.01 \pm 0.00 \%$ \\
\hline Molarity $[\mu \mathrm{M}]$ & $1.04 \pm 0.33$ & $0.95 \pm 0.30$ \\
\hline
\end{tabular}

\section{References}

Antibody: Synaptic Systems 105053

PDB Identifier: 6ank

\section{Literature:}

Hudson and Birnbaum, 1995; lezzi et al., 2004; Saegusa et al., 2002 


\section{Synaptotagmin7 (Gene: Syt7, Uniprot ID: Q62747)}

Known function: Asynchronous SV release, AMPAR exocytosis during LTP

Known organization: Transmembrane protein, Homo- or Heterodimers, On SV,

endosomes, lysosomes

Known Interactions: Synaptotagmin5, Calmodulin
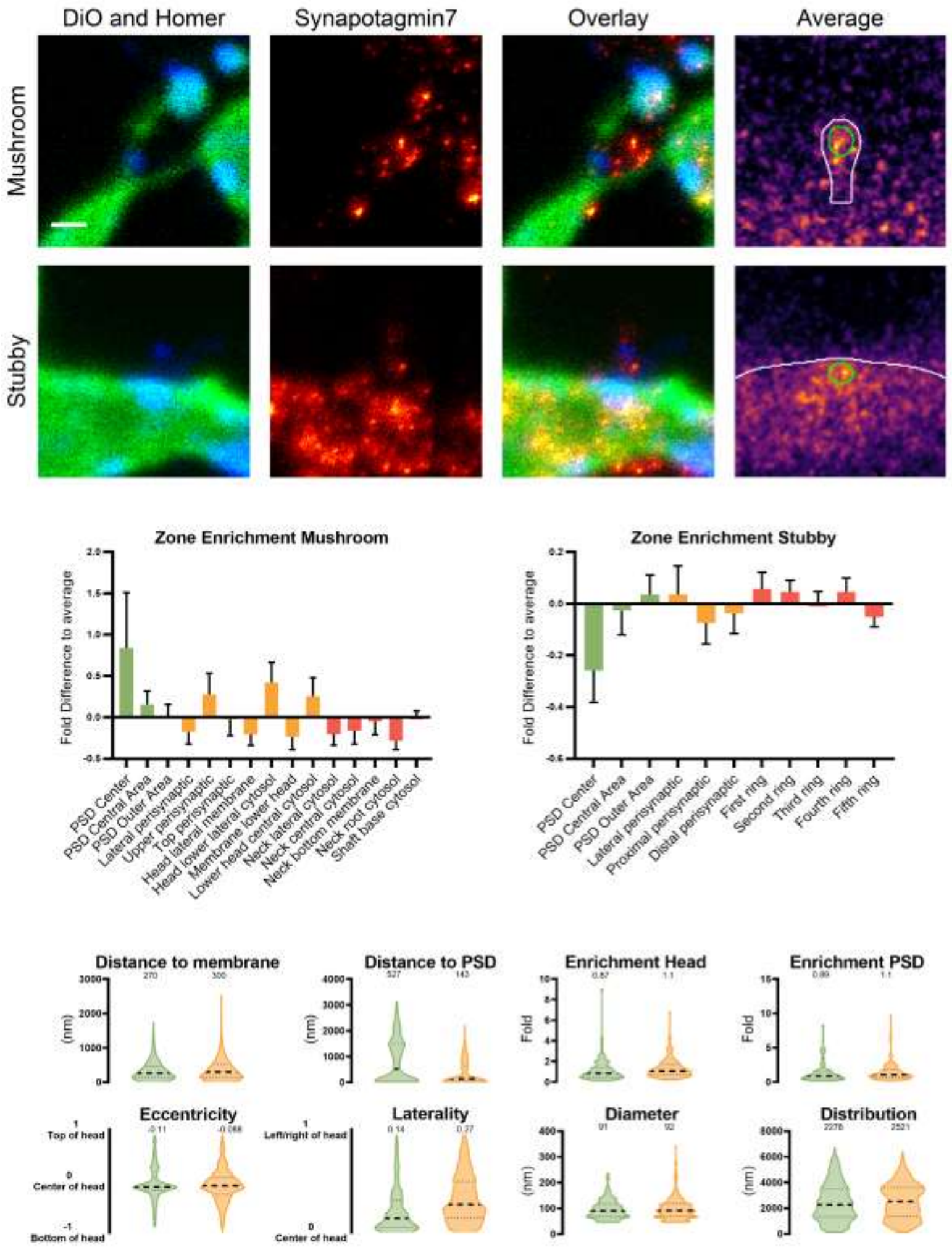

Figure 102: Synaptotagmin 7 nanoscale localization and abundance. Continued on next page. 


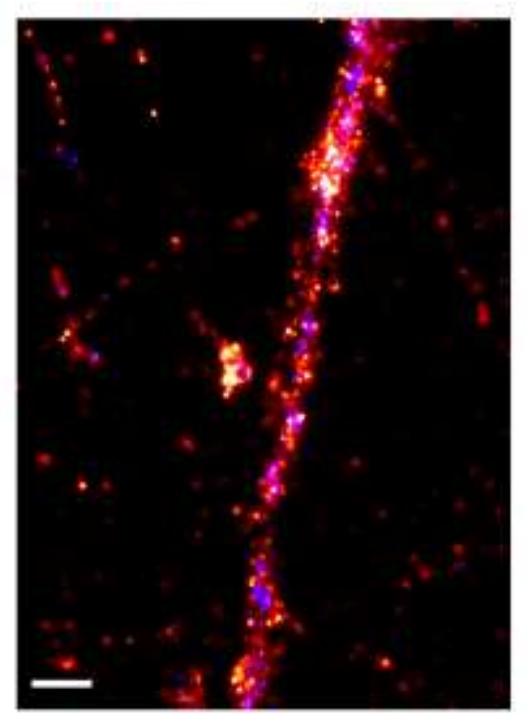

\begin{tabular}{|c|c|c|}
\hline $\begin{array}{c}\text { Whole cell copy } \\
\text { number }\end{array}$ & \multicolumn{2}{|c|}{$87596.66 \pm 11531.36$} \\
\hline $\begin{array}{c}\text { Spine copy } \\
\text { number }\end{array}$ & \multicolumn{2}{|c|}{$26.00 \pm 5.35$} \\
\hline$\%$ in PSD & \multicolumn{2}{|c|}{18.03} \\
\hline PSD copy number & \multicolumn{2}{|c|}{$3.97 \pm 0.82$} \\
\hline \begin{tabular}{c|c|} 
Spine copy \\
number
\end{tabular} & Mushroom & Stubby \\
\hline PSD copy number & $2.85 \pm 0.59$ & $5.30 \pm 1.09$ \\
\hline$\%$ of protein & $0.00 \pm 0.00 \%$ & $0.00 \pm 0.00 \%$ \\
\hline Molarity [MM] & $0.16 \pm 0.03$ & $0.19 \pm 0.04$ \\
\hline
\end{tabular}

\section{References}

Antibody: Synaptic Systems 105173

PDB Identifier: 6ank

\section{Literature:}

Bacaj et al., 2013, 2015; Flannery et al., 2010; Fukuda and Mikoshiba, 2000; Luo et al., 2015; Wen et al., 2010; Wu et al., 2017 


\section{Syntaxin1a (Gene: Stx1a, Uniprot ID: P32851)}

Known function: Qa SNARE, SV fusion, Delivery of AMPAR and GABAAR to PM

Known organization: Transmembrane protein, On PM, Homooligomeric clusters

Known Interactions: VAMP1, VAMP2, SNAP25
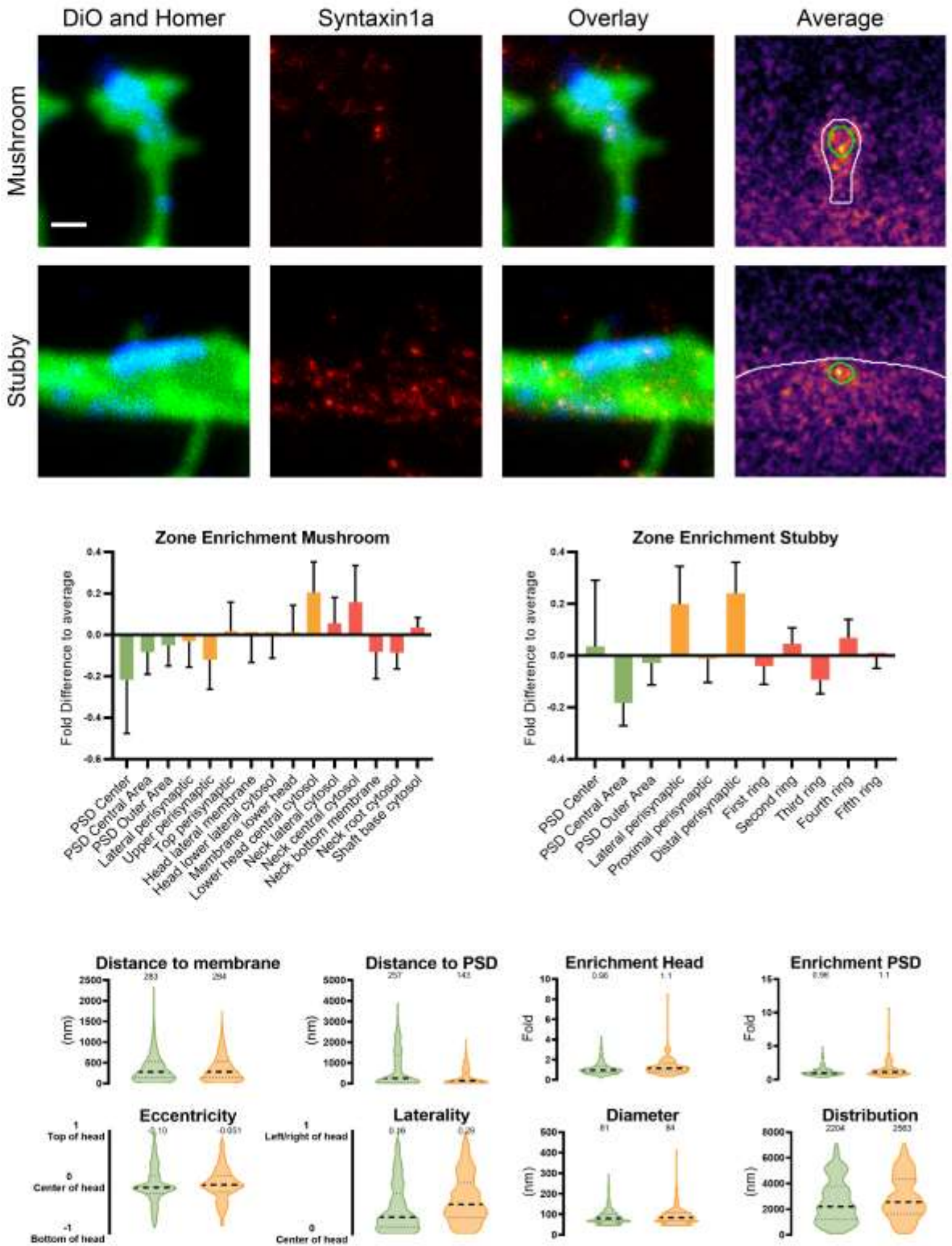

Figure 103: Syntaxin1a nanoscale localization and abundance. Continued on next page. 


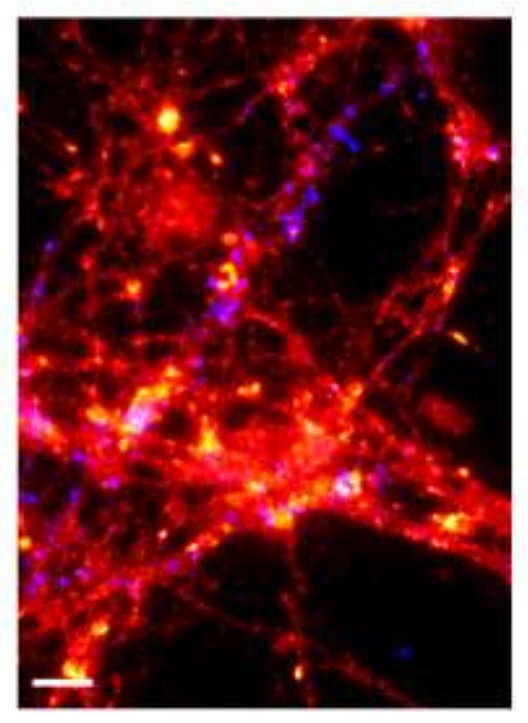

\begin{tabular}{|c|c|c|}
\hline $\begin{array}{c}\text { Whole cell copy } \\
\text { number }\end{array}$ & \multicolumn{2}{|c|}{$16988830.56 \pm 2737062.67$} \\
\hline $\begin{array}{c}\text { spine copy } \\
\text { number }\end{array}$ & \multicolumn{2}{|c|}{$1907.38 \pm 579.27$} \\
\hline$\%$ in PSD & \multicolumn{2}{|c|}{19.37} \\
\hline PSD copy number & \multicolumn{2}{|c|}{$309.53 \pm 94.00$} \\
\hline & Mushroom & Stubby \\
\hline \begin{tabular}{c|c|} 
Spine copy \\
number
\end{tabular} & $\begin{array}{c}1719.53 \\
\pm 522.22\end{array}$ & 2254.22 \\
\hline PSD copy number & $279.05 \pm 84.75$ & $365.81 \pm 111.10$ \\
\hline$\%$ of protein & $0.13 \pm 0.04 \%$ & $0.11 \pm 0.03 \%$ \\
\hline Molarity $[\mu \mathrm{M}]$ & $14.41 \pm 4.38$ & $12.08 \pm 3.67$ \\
\hline
\end{tabular}

\section{References}

Antibody: Synaptic Systems 110011

PDB Identifier: 2m8r, 1 bro

\section{Literature:}

Calakos et al., 1994; Gu et al., 2016; Hussain et al., 2016; Lang et al., 2002; Maidorn, 2017; Oyler et al., 1989; Sieber et al., 2006, 2007; Trimble et al., 1988 
Syntaxin2 (Epimorphin, Gene: Stx2, Uniprot ID: P50279)

Known function: Qa SNARE, Endosome-Recycling Endosome fusion, LDCV release

Known organization: Transmembrane protein, On PM

Known Interactions: VAMP1, VAMP2, VAMP7, SNAP23
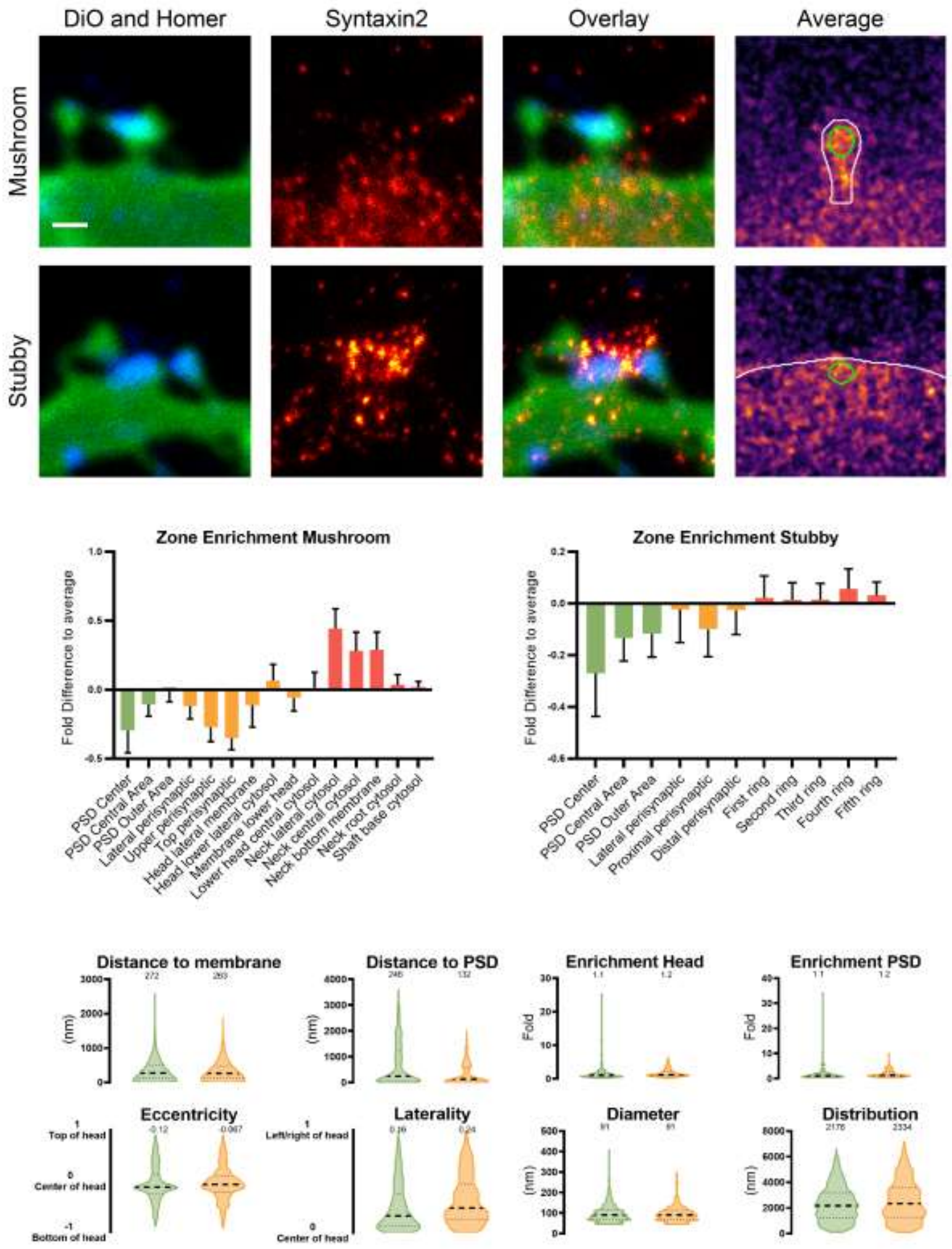

Figure 104: Syntaxin2 nanoscale localization and abundance. Continued on next page. 


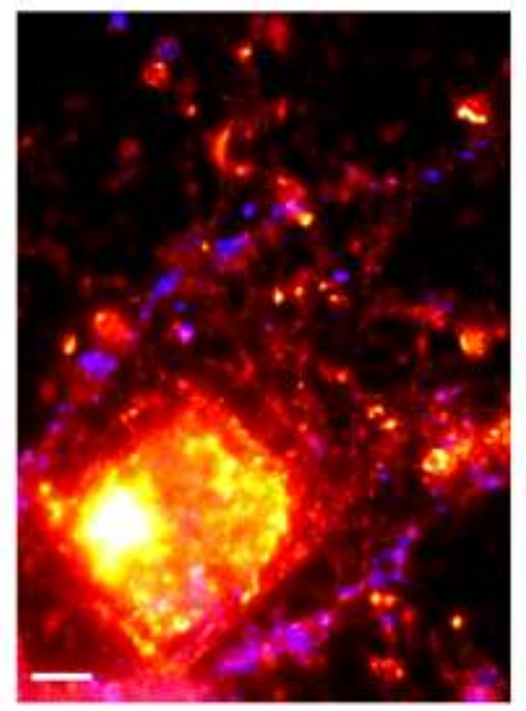

\begin{tabular}{|c|c|c|}
\hline $\begin{array}{c}\text { Whole cell copy } \\
\text { number }\end{array}$ & \multicolumn{2}{|c|}{ not detected } \\
\hline $\begin{array}{c}\text { Spine copy } \\
\text { number }\end{array}$ & \multicolumn{2}{|c|}{ na } \\
\hline$\%$ in PSD & \multicolumn{2}{|c|}{ na } \\
\hline PSD copy number & \multicolumn{2}{|c|}{ na } \\
\hline \begin{tabular}{c|c|} 
Spine copy \\
number
\end{tabular} & na & na \\
\hline PSD copy number & na & na \\
\hline$\%$ of protein & na & na \\
\hline Molarity $[\mu M]$ & na & na \\
\hline
\end{tabular}

\section{References}

Antibody: Synaptic Systems 110022

PDB Identifier: modified Syntaxin1

\section{Literature:}

Calakos et al., 1994; Chen et al., 2000; Kennedy et al., 2010 


\section{Syntaxin3 (Gene: Stx3, Uniprot ID: Q08849)}

Known function: Qa SNARE, Delivery of AMPAR during LTP

Known organization: Transmembrane protein, On PM

Known Interactions: SNAP47, VAMP2
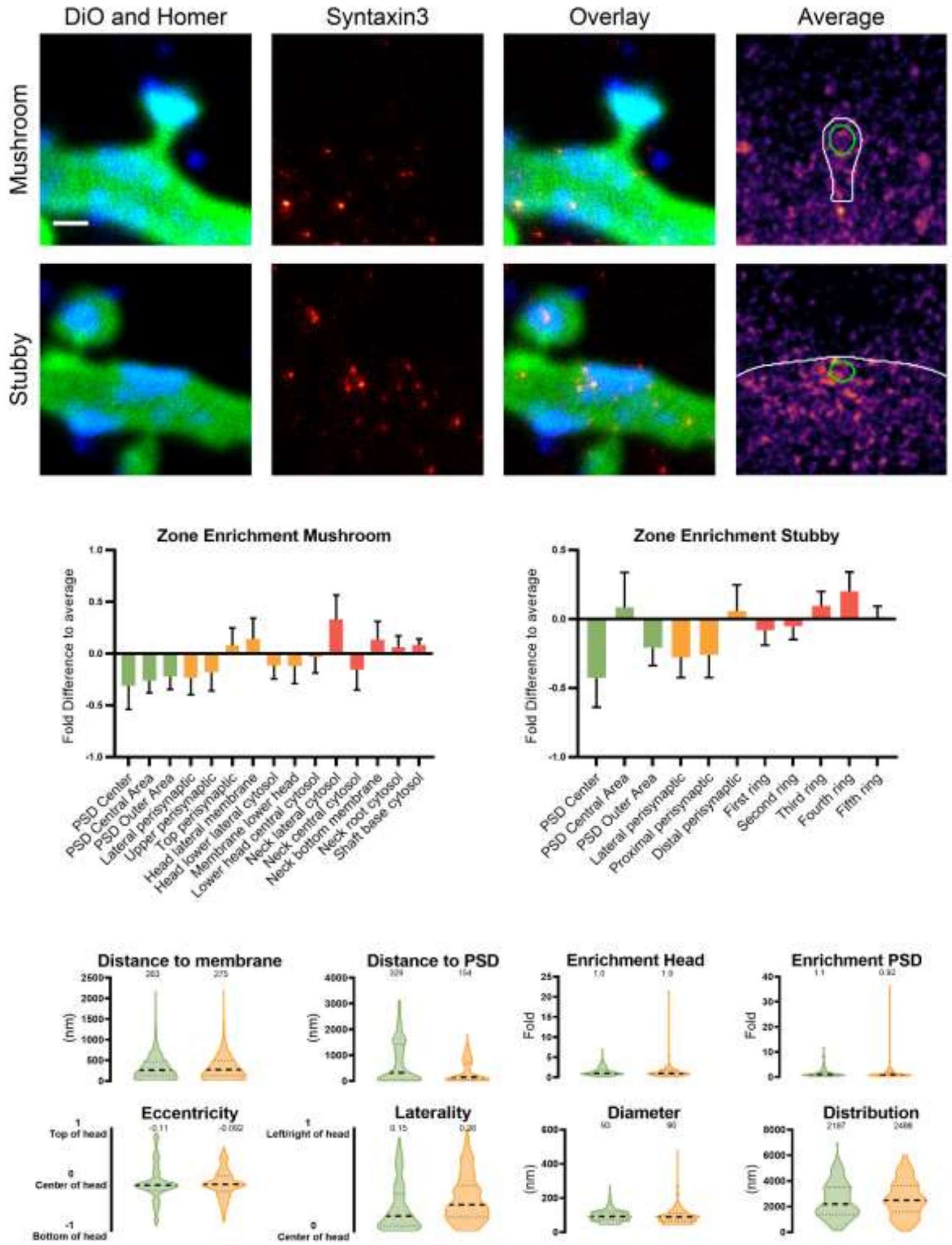

Figure 105: Syntaxin3 nanoscale localization and abundance. Continued on next page. 


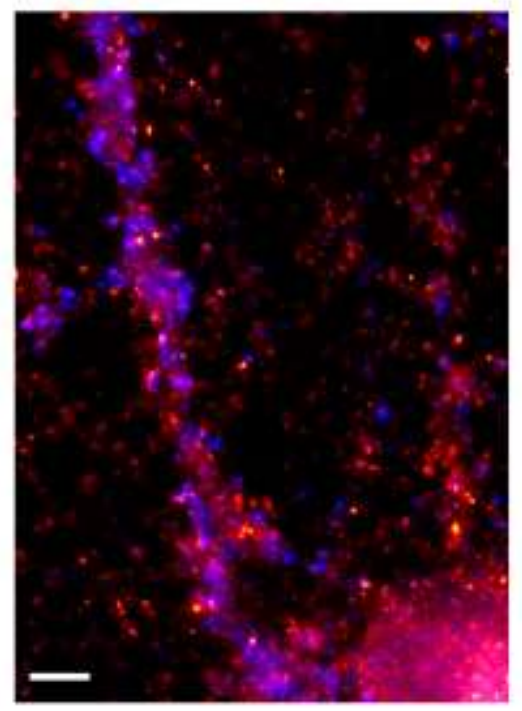

\begin{tabular}{|c|c|c|}
\hline $\begin{array}{c}\text { Whole cell copy } \\
\text { number }\end{array}$ & \multicolumn{2}{|c|}{$98311.82 \pm 19031.44$} \\
\hline $\begin{array}{c}\text { Spine copy } \\
\text { number }\end{array}$ & \multicolumn{2}{|c|}{$17.21 \pm 4.56$} \\
\hline$\%$ in PSD & \multicolumn{2}{|c|}{17.28} \\
\hline PSD copy number & \multicolumn{2}{|c|}{$2.54 \pm 0.67$} \\
\hline \begin{tabular}{c|c|} 
Spine copy \\
number
\end{tabular} & Mushroom & Stubby \\
\hline PSD copy number & $13.17 \pm 3.49$ & $25.46 \pm 6.75$ \\
\hline$\%$ of protein & $0.00 \pm 0.00 \%$ & $0.00 \pm 0.00 \%$ \\
\hline Molarity $[\mu \mathrm{M}]$ & $0.11 \pm 0.03$ & $0.14 \pm 0.04$ \\
\hline
\end{tabular}

\section{References}

Antibody: Synaptic Systems 110033

PDB Identifier: modified Syntaxin1

\section{Literature:}

Arendt et al., 2015; Jurado et al., 2013 


\section{Syntaxin4 (Gene: Stx4, Uniprot ID: Q08850)}

Known function: Qa SNARE, Delivery of AMPAR, NMDAR and Nig1 to PM, Involved in Homeostatic plasticity, Retrograde signaling, Caveolea fusion

Known organization: Transmembrane protein, Homooligomeric clusters, Lateral spine head

Known Interactions: VAMP1, VAMP2, SNAP23, SNAP47
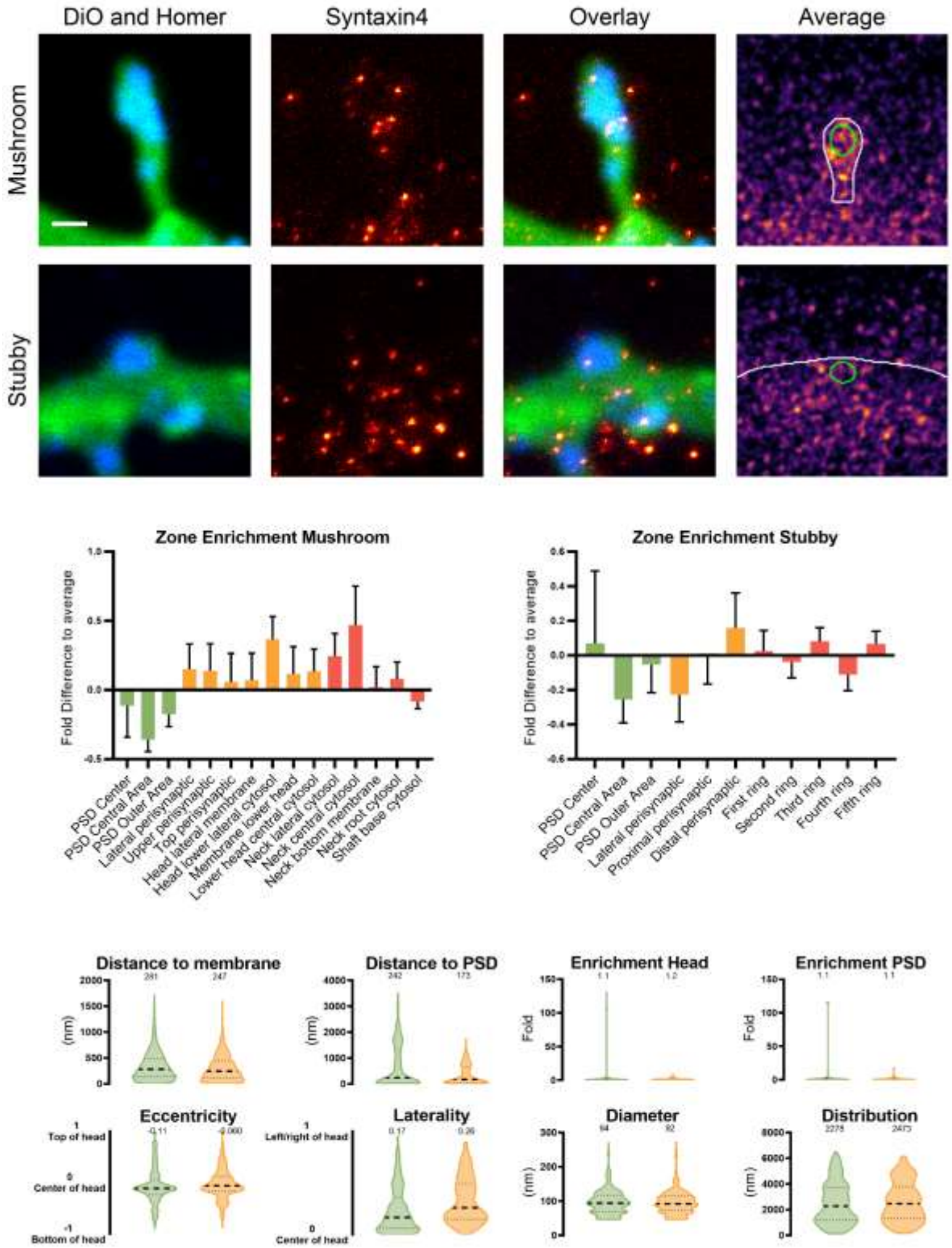

Figure 106: Syntaxin4 nanoscale localization and abundance. Continued on next page. 


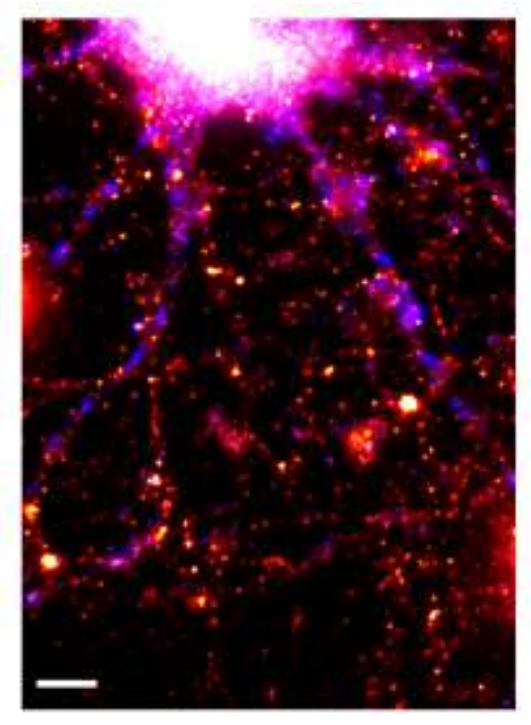

\begin{tabular}{|c|c|c|}
\hline $\begin{array}{c}\text { Whole cell copy } \\
\text { number }\end{array}$ & \multicolumn{2}{|c|}{ not detected } \\
\hline $\begin{array}{c}\text { Spine copy } \\
\text { number }\end{array}$ & \multicolumn{2}{|c|}{ na } \\
\hline$\%$ in PSD & \multicolumn{2}{|c|}{ na } \\
\hline PSD copy number & \multicolumn{2}{|c|}{ na } \\
\hline \begin{tabular}{c|c|} 
Spine copy \\
number
\end{tabular} & na & na \\
\hline PSD copy number & na & na \\
\hline$\%$ of protein & na & na \\
\hline Molarity $[\mu M]$ & na & na \\
\hline
\end{tabular}

\section{References}

Antibody: Synaptic Systems 110042

PDB Identifier: modified Syntaxin1

\section{Literature:}

Arendt et al., 2015; Bin et al., 2018; Gu and Huganir, 2016; Harris et al., 2016; Kennedy et al., 2010; Predescu et al., 2005; Sieber et al., 2006 


\section{Syntaxin5 (Gene: Stx5, Uniprot ID: Q08851)}

Known function: Qa SNARE, ER-Golgi transport

Known organization: Transmembrane protein, On Golgi

Known Interactions: Sec22b
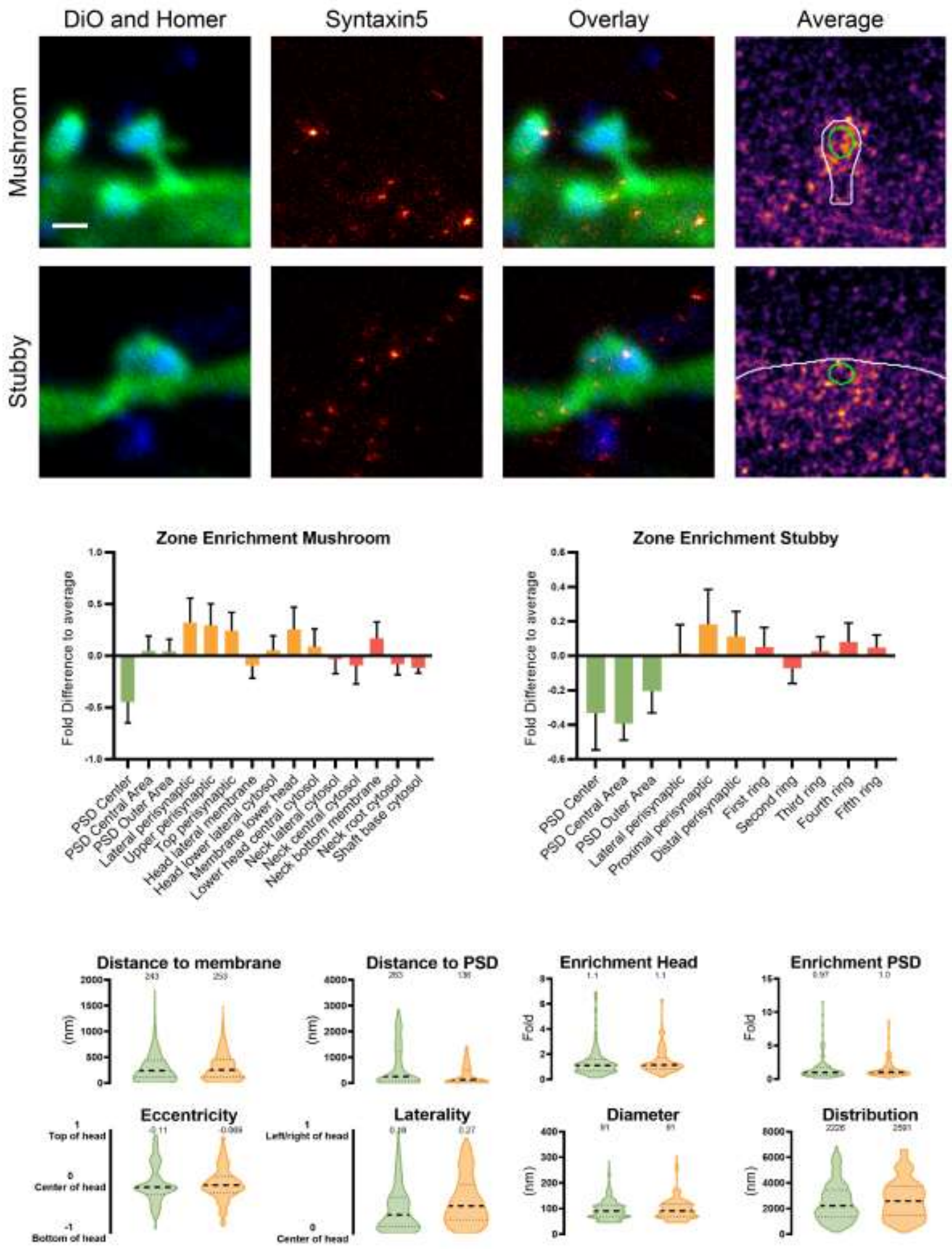

Figure 107: Syntaxin 5 nanoscale localization and abundance. Continued on next page. 


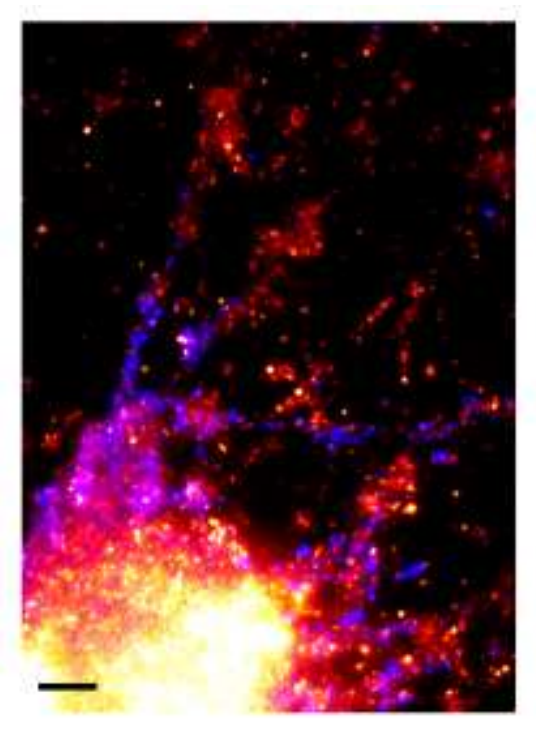

\begin{tabular}{|c|c|c|}
\hline $\begin{array}{c}\text { Whole cell copy } \\
\text { number }\end{array}$ & \multicolumn{2}{|c|}{$137410.70 \pm 40668.37$} \\
\hline $\begin{array}{c}\text { Spine copy } \\
\text { number }\end{array}$ & \multicolumn{2}{|c|}{$9.24 \pm 4.00$} \\
\hline $\begin{array}{c}|2| \\
\% \text { in PSD }\end{array}$ & \multicolumn{2}{|c|}{18.85} \\
\hline PSD copy number & \multicolumn{2}{|c|}{$1.47 \pm 0.63$} \\
\hline Spine copy & Mushroom & Stubby \\
\hline number & $8.63 \pm 3.73$ & $9.87 \pm 4.27$ \\
\hline PSD copy number & $1.37 \pm 0.59$ & $1.57 \pm 0.68$ \\
\hline$\%$ of protein & $0.00 \pm 0.00 \%$ & $0.00 \pm 0.00 \%$ \\
\hline Molarity $[\mu \mathrm{M}]$ & $0.07 \pm 0.03$ & $0.05 \pm 0.02$ \\
\hline
\end{tabular}

\section{References}

Antibody: Synaptic Systems 110053

PDB Identifier: modified Syntaxin1

\section{Literature:}

Hay et al., 1997; Hohl et al., 1998; Hong, 2005; Renna et al., 2011 


\section{Syntaxin6 (Gene: Stx6, Uniprot ID: Q63635)}

Known function: Qc SNARE, Late secretory pathway, Various endosomal fusion events Known organization: Transmembrane protein, On TGN and endosomes Known Interactions: Syntaxin4, Syntaxin16, VAMP2, VAMP7, SNAP23, SNAP25, SNAP29, Vti1a
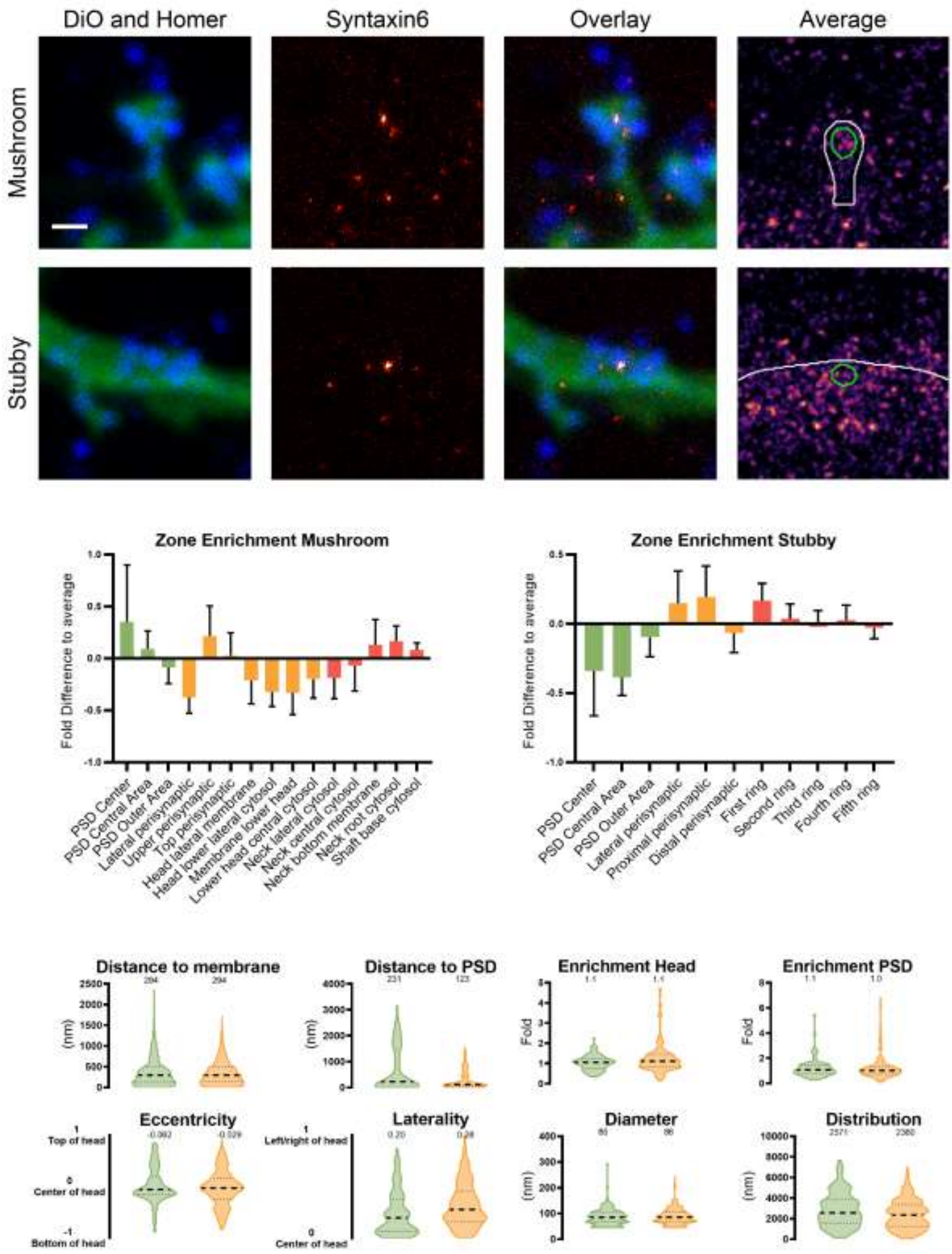

Figure 108: Syntaxin 6 nanoscale localization and abundance. Continued on next page. 


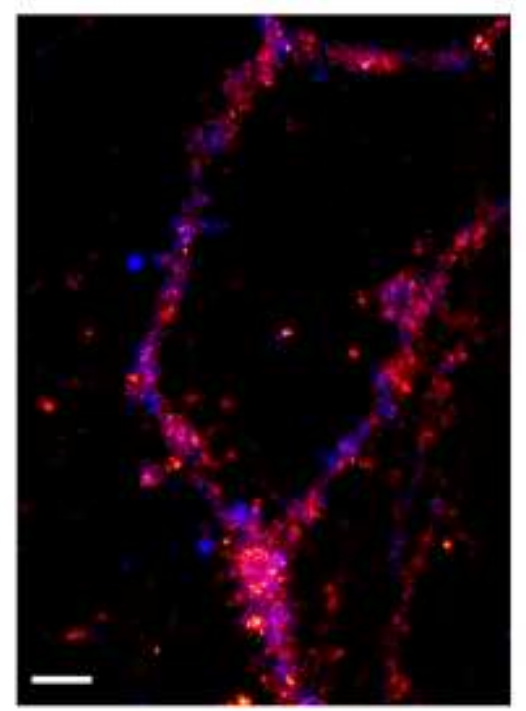

\begin{tabular}{|c|c|c|}
\hline $\begin{array}{c}\text { Whole cell copy } \\
\text { number }\end{array}$ & \multicolumn{2}{|c|}{$931868.73 \pm 208843.91$} \\
\hline $\begin{array}{c}\text { Spine copy } \\
\text { number }\end{array}$ & \multicolumn{2}{|c|}{$23.21 \pm 15.92$} \\
\hline$\%$ in PSD & \multicolumn{2}{|c|}{16.99} \\
\hline PSD copy number & \multicolumn{2}{|c|}{$3.37 \pm 2.31$} \\
\hline $\begin{array}{c}\text { Spine copy } \\
\text { number }\end{array}$ & Mushroom & Stubby \\
\hline PSD copy number & $2.81 \pm 1.93$ & $4.40 \pm 3.02$ \\
\hline$\%$ of protein & $0.00 \pm 0.00 \%$ & $0.00 \pm 0.00 \%$ \\
\hline Molarity $[\mu \mathrm{M}]$ & $0.16 \pm 0.11$ & $0.16 \pm 0.11$ \\
\hline
\end{tabular}

\section{References}

Antibody: Cell Signalin 2869

PDB Identifier: 1Ivf, 4j2c

\section{Literature:}

Antonin et al., 2000; Gerrard et al., 2000; Hanson et al., 1995; Hong, 2005; McMahon et al., 1993; Wade et al., 2001; Watson and Pessin, 2000; Wendler and Tooze, 2001 


\section{Syntaxin8 (Gene: Stx8, Uniprot ID: Q9Z2Q7)}

Known function: Qc SNARE, Late endosome fusion, Delivery of TrkA transport to PM Known organization: Transmembrane protein, Also palmitoylated, On Golgi, early and late endosome

Known Interactions: None
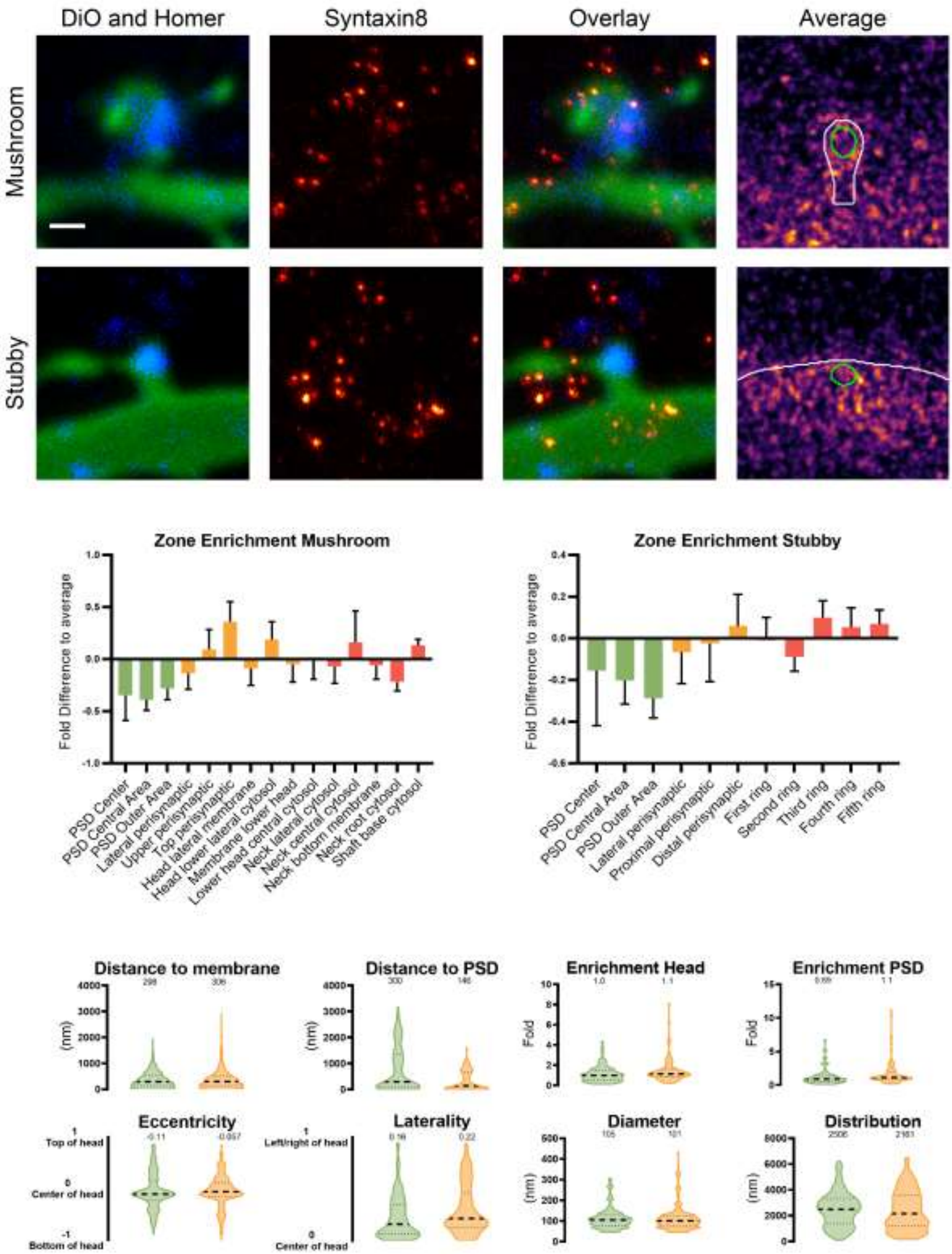

Figure 109: Syntaxin 8 nanoscale localization and abundance. Continued on next page. 


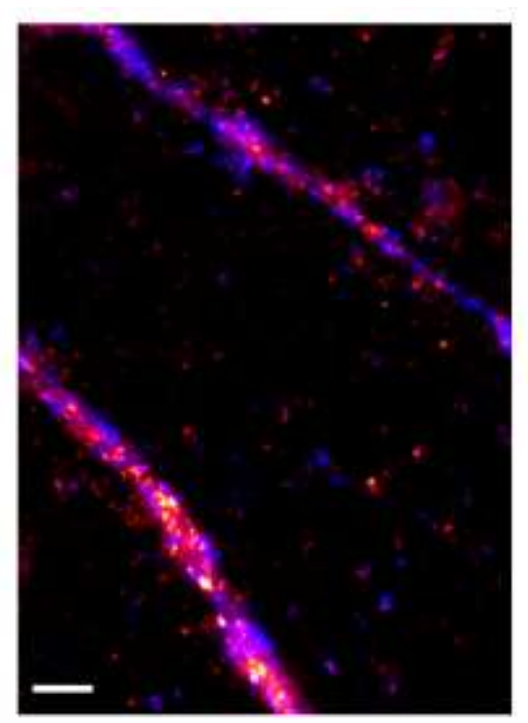

\begin{tabular}{|c|c|c|}
\hline $\begin{array}{c}\text { Whole cell copy } \\
\text { number }\end{array}$ & \multicolumn{2}{|c|}{$1742277.72 \pm 239717.18$} \\
\hline $\begin{array}{c}\text { Spine copy } \\
\text { number }\end{array}$ & \multicolumn{2}{|c|}{$303.62 \pm 69.23$} \\
\hline$\%$ in PSD & \multicolumn{2}{|c|}{13.71} \\
\hline PSD copy number & \multicolumn{2}{|c|}{$36.61 \pm 8.35$} \\
\hline \begin{tabular}{c|c|} 
Spine copy \\
number
\end{tabular} & Mushroom & Stubby \\
\hline PSD copy number & $224.34 \pm 51.15$ & $352.89 \pm 80.47$ \\
\hline$\%$ of protein & $0.01 \pm 0.00 \%$ & $0.01 \pm 0.00 \%$ \\
\hline Molarity $[\mu \mathrm{M}]$ & $1.88 \pm 0.43$ & $1.89 \pm 0.43$ \\
\hline
\end{tabular}

\section{References}

Antibody: Synaptic Systems 110083

PDB Identifier: modified Syntaxin1

\section{Literature:}

Antonin et al., 2002; Chen et al., 2014; He and Linder, 2009 


\section{Syntaxin13 (Syntaxin12, Gene: Stx12, Uniprot ID: G3V7P1)}

Known function: Qa SNARE, Homotypic endosome fusion, Delivery of AMPAR to PM

Known organization: Transmembrane protien, On early and sorting endosomes

Known Interactions: Syntaxin6, VAMP4, Vti1a
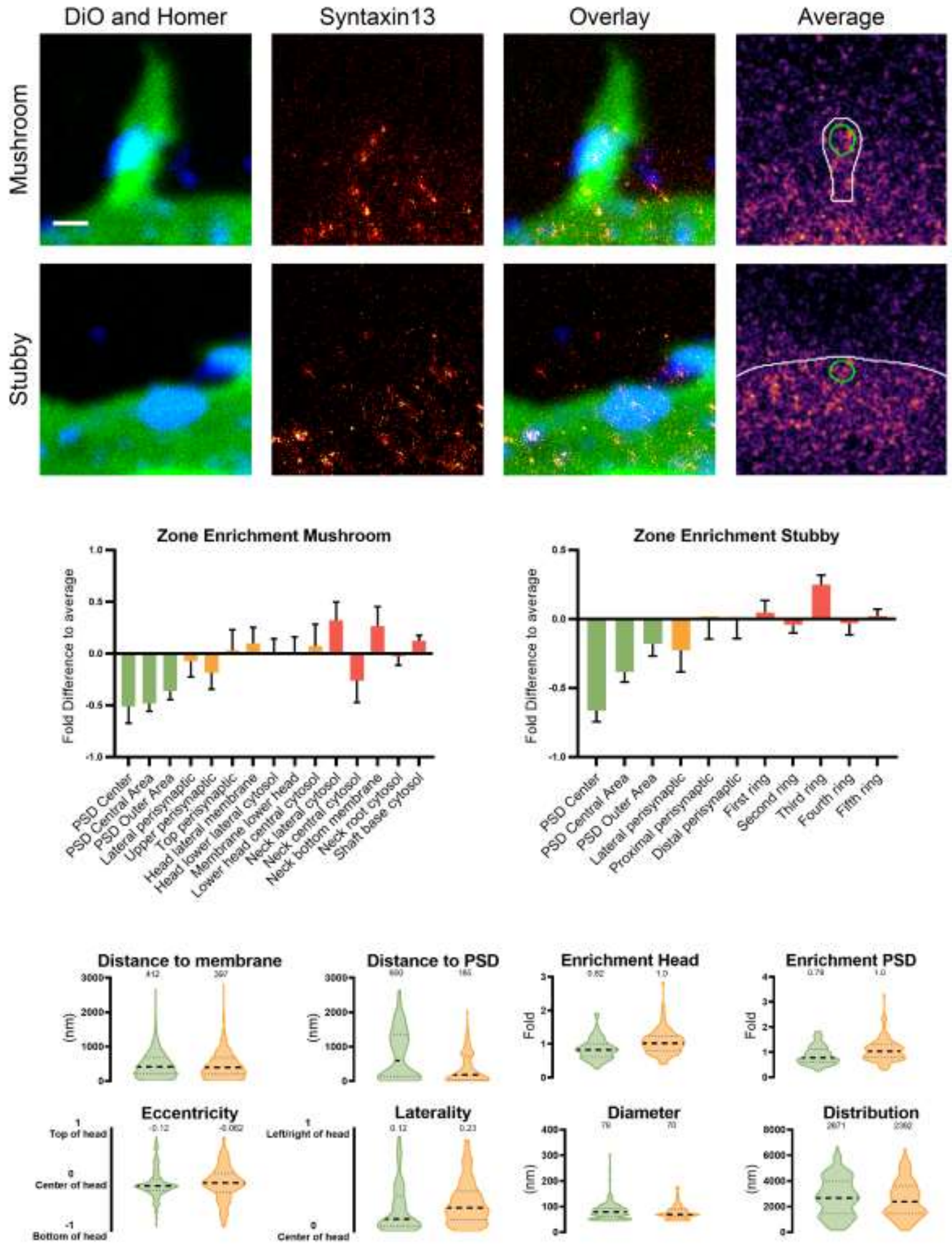

Figure 110: Syntaxin13 nanoscale localization and abundance. Continued on next page. 


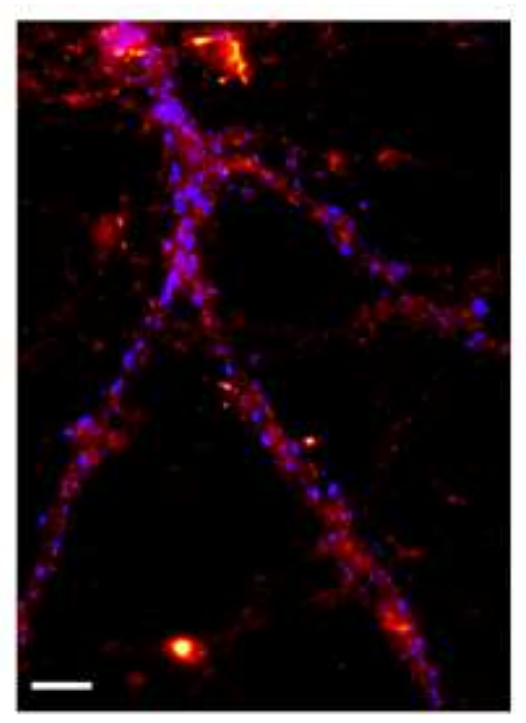

\begin{tabular}{|c|c|c|}
\hline $\begin{array}{c}\text { Whole cell copy } \\
\text { number }\end{array}$ & \multicolumn{2}{|c|}{$4084257.85 \pm 498526.15$} \\
\hline $\begin{array}{c}\text { spine copy } \\
\text { number }\end{array}$ & \multicolumn{2}{|c|}{$528.88 \pm 141.29$} \\
\hline$\%$ in PSD & \multicolumn{2}{|c|}{13.48} \\
\hline PSD copy number & \multicolumn{2}{|c|}{$62.81 \pm 16.78$} \\
\hline Mushroom & Stubby \\
\hline $\begin{array}{c}\text { Spine copy } \\
\text { number }\end{array}$ & $412.29 \pm 110.14$ & $626.38 \pm 167.33$ \\
\hline PSD copy number & $48.97 \pm 13.08$ & $74.39 \pm 19.87$ \\
\hline$\%$ of protein & $0.03 \pm 0.01 \%$ & $0.03 \pm 0.01 \%$ \\
\hline Molarity $[\mu \mathrm{M}]$ & $3.46 \pm 0.92$ & $3.36 \pm 0.90$ \\
\hline
\end{tabular}

\section{References}

Antibody: Reinhardt Jahn laboratory cl. 151.1 PDB Identifier: modified Syntaxin1

\section{Literature:}

Aikawa et al., 2006; Antonin et al., 2002; Brandhorst et al., 2006; Hirling et al., 2000; Hong, 2005; McBride et al., 1999; Park et al., 2004; Petrini et al., 2009; Zwilling et al., 2007 


\section{Syntaxin16 (Gene: Stx16, Uniprot ID: D3Z9R7)}

Known function: Qa SNARE, Homotypic endosome fusion, Endosome-TGN transport, Important for dendritic protein traffic

Known organization: Tail-anchored, On Endosomes, TGN

Known Interactions: Syntaxin6, VAMP4, Vti1a
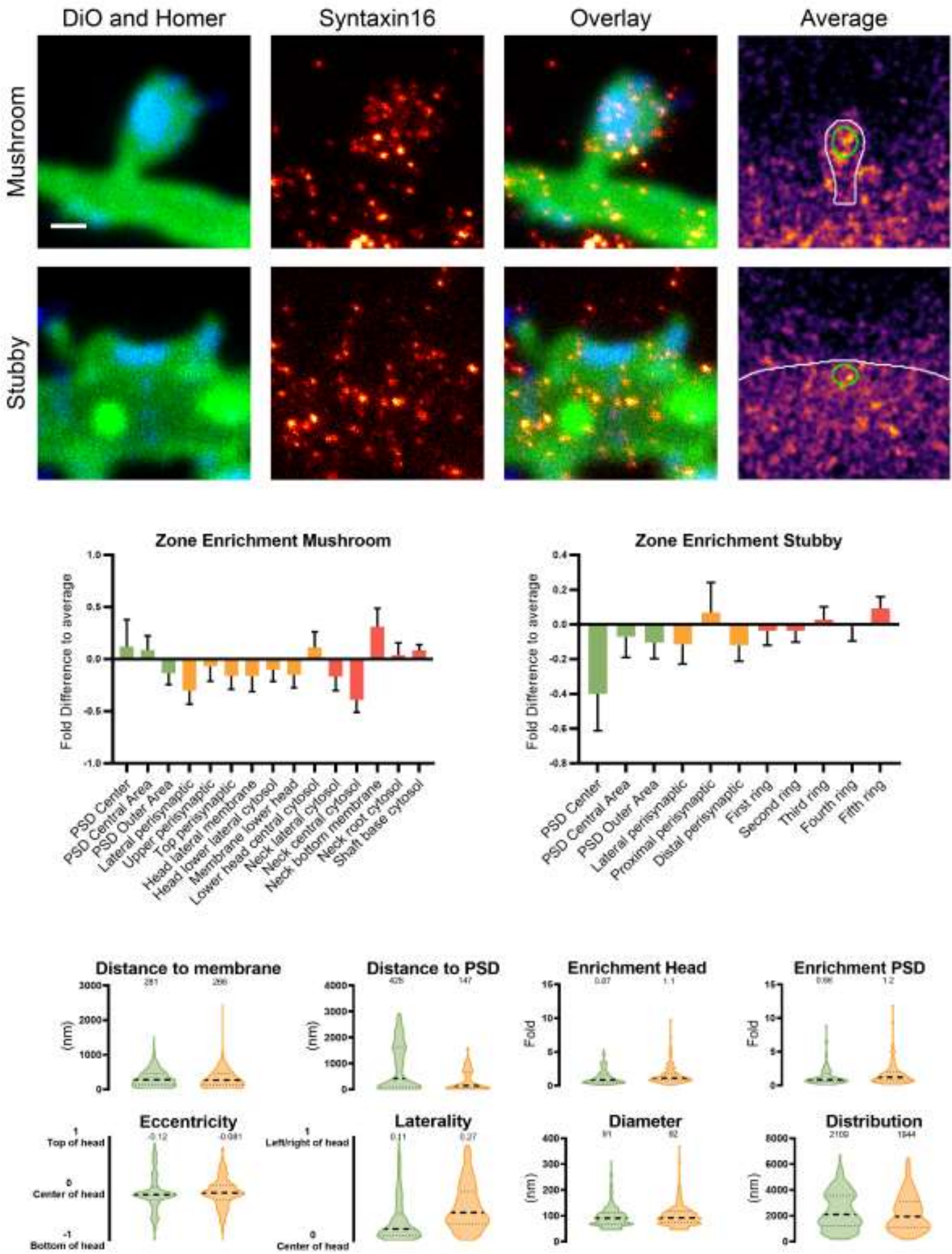

Figure 111: Syntaxin 16 nanoscale localization and abundance. Continued on next page. 


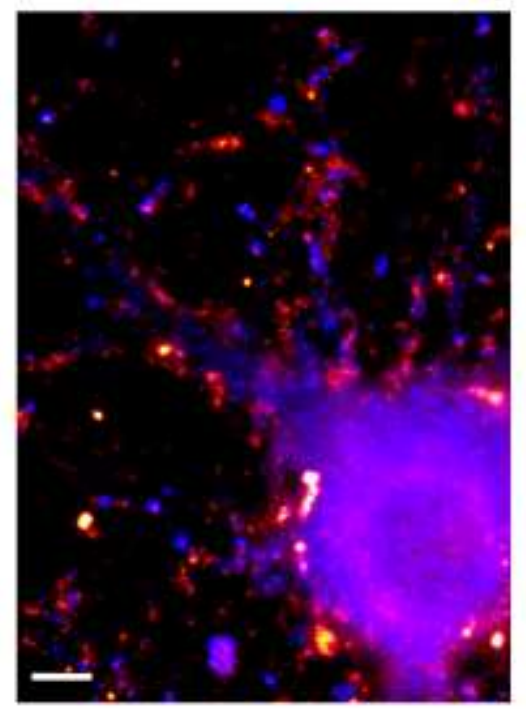

\begin{tabular}{|c|c|c|}
\hline $\begin{array}{c}\text { Whole cell copy } \\
\text { number }\end{array}$ & \multicolumn{2}{|c|}{$166101.09 \pm 18387.59$} \\
\hline $\begin{array}{c}\text { Spine copy } \\
\text { number }\end{array}$ & \multicolumn{2}{|c|}{$48.49 \pm 9.35$} \\
\hline$\%$ in PSD & \multicolumn{2}{|c|}{17.60} \\
\hline PSD copy number & \multicolumn{2}{|c|}{$7.25 \pm 1.40$} \\
\hline \begin{tabular}{c|c|} 
Spine copy \\
number
\end{tabular} & Mushroom & Stubby \\
\hline PSD copy number & $6.34 \pm 8.17$ & $55.77 \pm 10.75$ \\
\hline$\%$ of protein & $0.00 \pm 0.00 \%$ & $0.00 \pm 0.00 \%$ \\
\hline Molarity $[\mu \mathrm{M}]$ & $0.36 \pm 0.07$ & $0.30 \pm 0.06$ \\
\hline
\end{tabular}

\section{References}

Antibody: Synaptic Systems 110162

PDB Identifier: Modified Syntaxin1

\section{Literature:}

Amessou et al., 2007; Antonin et al., 2002; Brandhorst et al., 2006; Chua and Tang, 2008; Fischer von Mollard and Stevens, 1998; Ganley et al., 2004; Mallard et al., 2002; McBride et al., 1999; Shitara et al., 2013; Simonsen et al., 1998; Tang, 2008; Tang et al., 1998; Zwilling et al., 2007 
VAMP1 (Synaptobrevin1, Gene: Vamp1, Uniprot ID: Q63666)

Known function: R-SNARE, SV release, Delivery of NMDAR to PM

Known organization: Transmembrane protein, On SV and secretory vesciles

Known Interactions: Syntaxin1, Syntaxin4, SNAP25
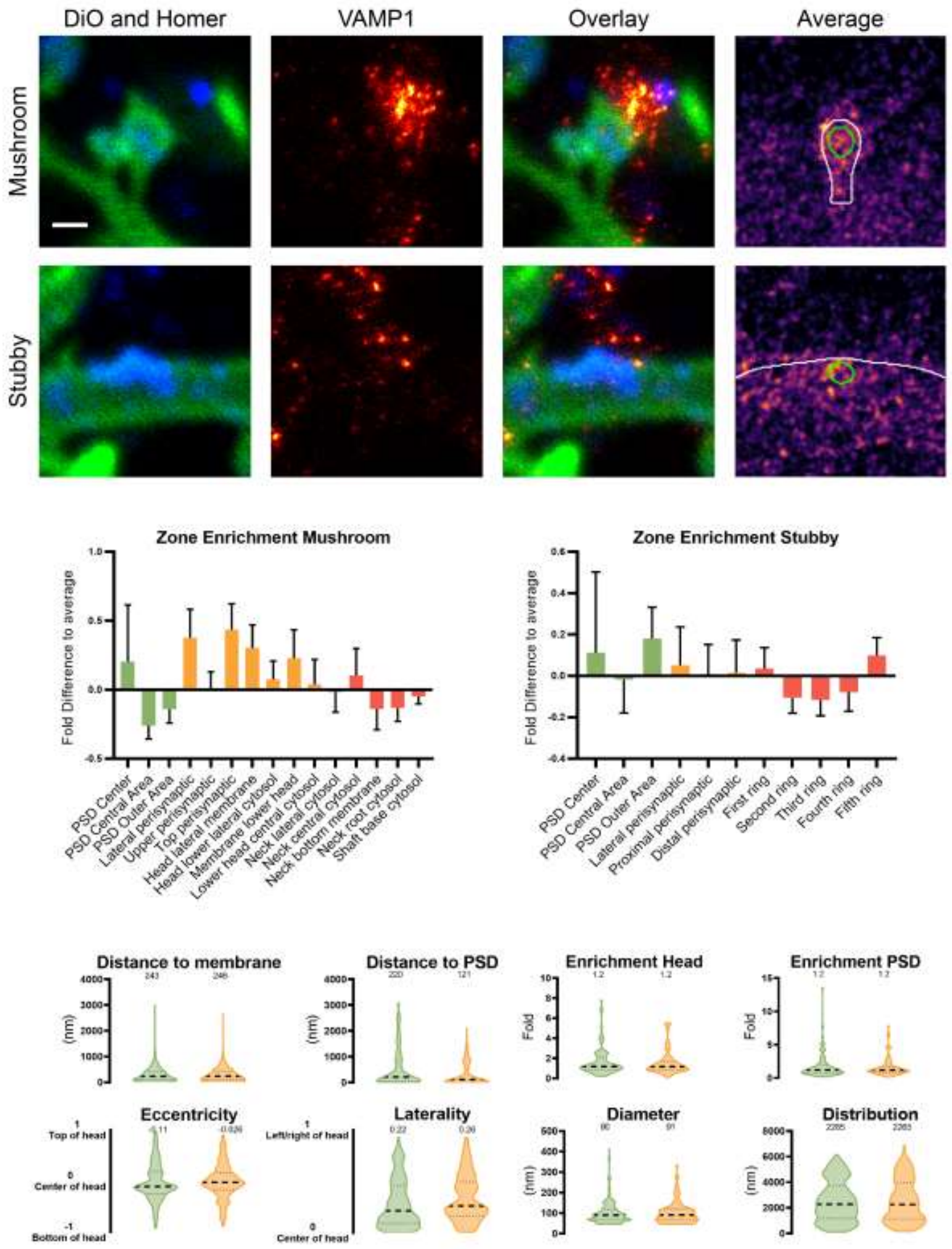

Figure 112: VAMP1 nanoscale localization and abundance. Continued on next page. 


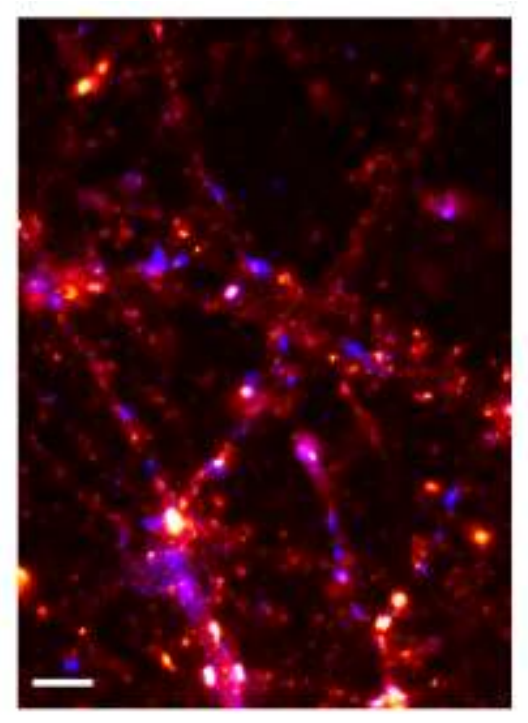

\begin{tabular}{|c|c|c|}
\hline $\begin{array}{c}\text { Whole cell copy } \\
\text { number }\end{array}$ & \multicolumn{2}{|c|}{$8616551.20 \pm 1397968.54$} \\
\hline $\begin{array}{c}\text { Spine copy } \\
\text { number }\end{array}$ & \multicolumn{2}{|c|}{$1258.97 \pm 352.98$} \\
\hline$\%$ in PSD & \multicolumn{2}{|c|}{23.27} \\
\hline PSD copy number & \multicolumn{2}{|c|}{$237.66 \pm 66.63$} \\
\hline & Mushroom & Stubby \\
\hline \begin{tabular}{c|c|} 
Spine copy \\
number
\end{tabular} & $\begin{array}{c}1106.27 \\
\pm 310.17\end{array}$ & 1435.83 \\
\hline PSD copy number & $208.84 \pm 58.55$ & $271.05 \pm 75.99$ \\
\hline$\%$ of protein & $0.03 \pm 0.01 \%$ & $0.03 \pm 0.01 \%$ \\
\hline Molarity $[\mu \mathrm{M}]$ & $9.27 \pm 2.60$ & $7.70 \pm 2.16$ \\
\hline
\end{tabular}

\section{References}

Antibody: Synaptic Systems 104002

PDB Identifier: modified VAMP2

\section{Literature:}

Calakos et al., 1994; Gu and Huganir, 2016; Trimble et al., 1988 
VAMP2 (Synaptobrevin2, Gene: Vamp2, Uniprot ID: P63045)

Known function: R SNARE, SV release, Delivery of AMPAR and GABAAR to PM

Known organization: Transmembrane protein, On SV and small postsynaptic vesicles close to PSD

Known Interactions: Syntaxin1, Syntaxin4, SNAP25, Synaptotagmin1, CDC42
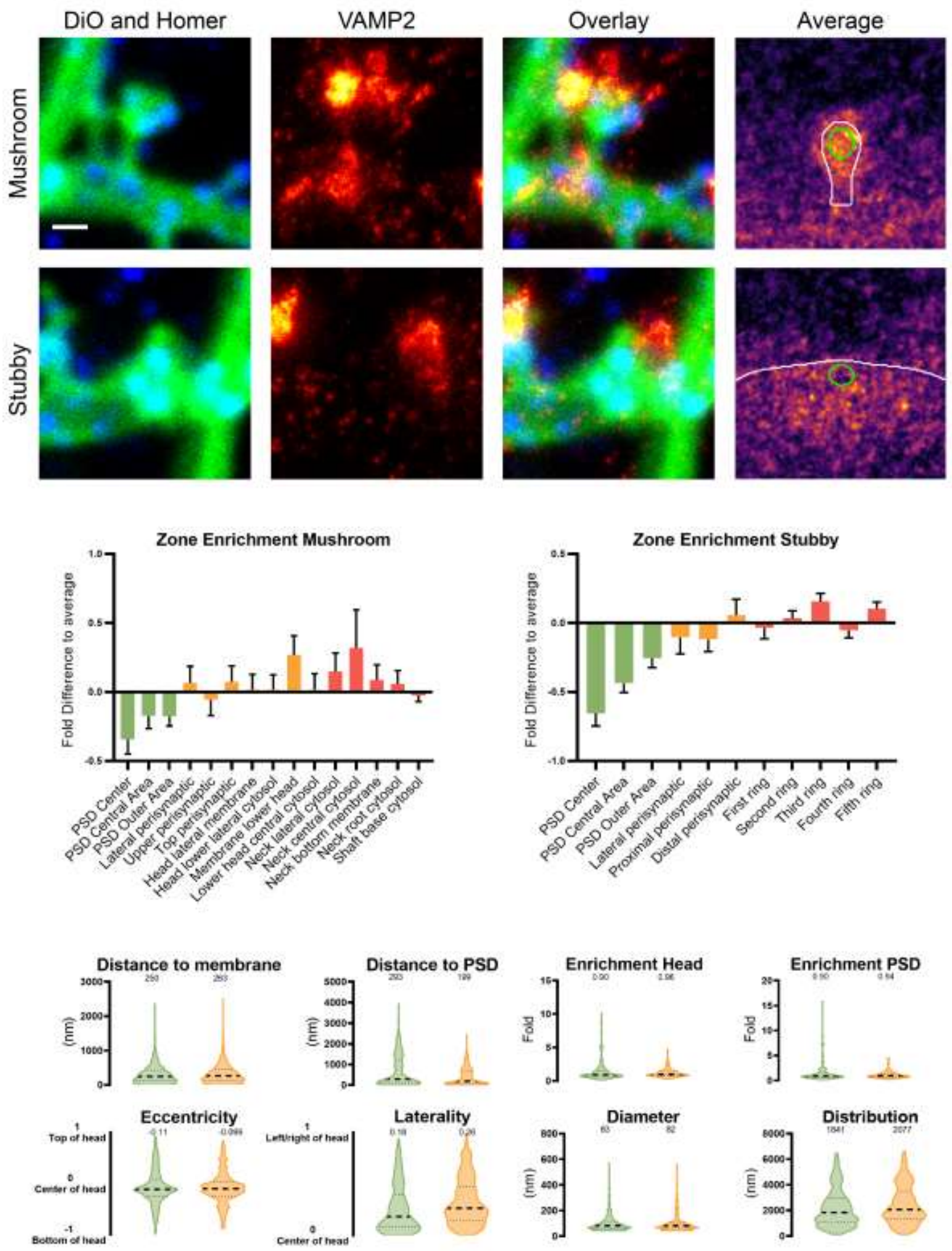

Figure 113: VAMP2 nanoscale localization and abundance. Continued on next page. 


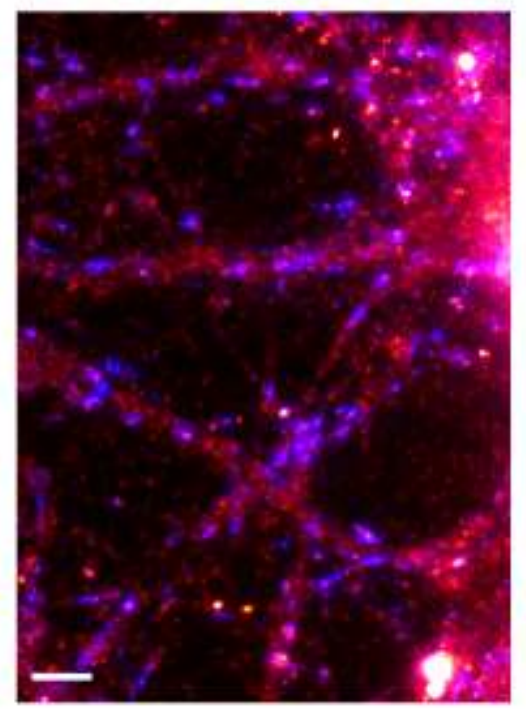

\begin{tabular}{|c|c|c|}
\hline $\begin{array}{c}\text { Whole cell copy } \\
\text { number }\end{array}$ & \multicolumn{2}{|c|}{$749558699.46 \pm 94440652.76$} \\
\hline $\begin{array}{c}\text { spine copy } \\
\text { number }\end{array}$ & \multicolumn{2}{|c|}{$82230.32 \pm 29643.82$} \\
\hline$\%$ in PSD & \multicolumn{2}{|c|}{16.58} \\
\hline PSD copy number & \multicolumn{2}{|c|}{$11696.81 \pm 4216.67$} \\
\hline & Mushroom & Stubby \\
\hline Spine copy & 80195.55 & 78146.54 \\
\hline number & \pm 28910.29 & \pm 28171.62 \\
\hline PSD copy number & 11407.37 & 11115.91 \\
\hline \% of protein & $2.35 \pm 0.85 \%$ & $1.47 \pm 0.53 \%$ \\
\hline Molarity $[\mu M]$ & $672.14 \pm 242.30$ & $418.94 \pm 151.03$ \\
\hline
\end{tabular}

\section{References}

Antibody: Synaptic Systems 104211

PDB Identifier: $2 \mathrm{kog}$

\section{Literature:}

Calakos et al., 1994; Gu et al., 2016; Hu et al., 2002; Hussain and Davanger, 2015; Jurado et al., 2013; Nevins and Thurmond, 2005 
VAMP7 (Sybl1, Ti-VAMP, Gene: Vamp7, Uniprot ID: Q9JHW5)

Known function: R SNARE, Lysosome secretion

Known organization: Transmembrane protein, On endosomes, TGN and lysosomes,

Extrasynaptic

Known Interactions: Syntaxin1, Syntaxin4, SNAP23, SNAP25
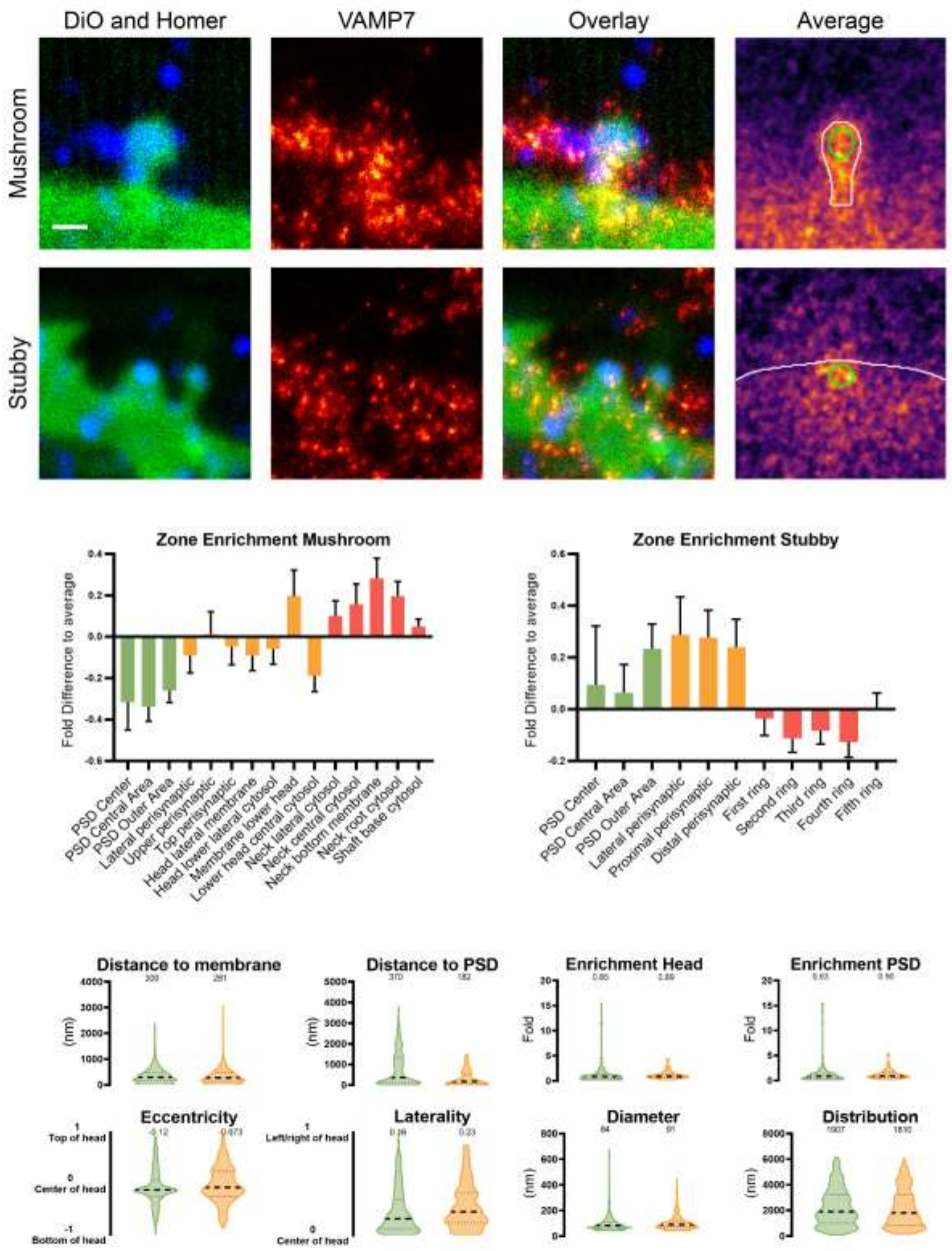

Figure 114: VAMP7 nanoscale localization and abundance. Continued on next page. 


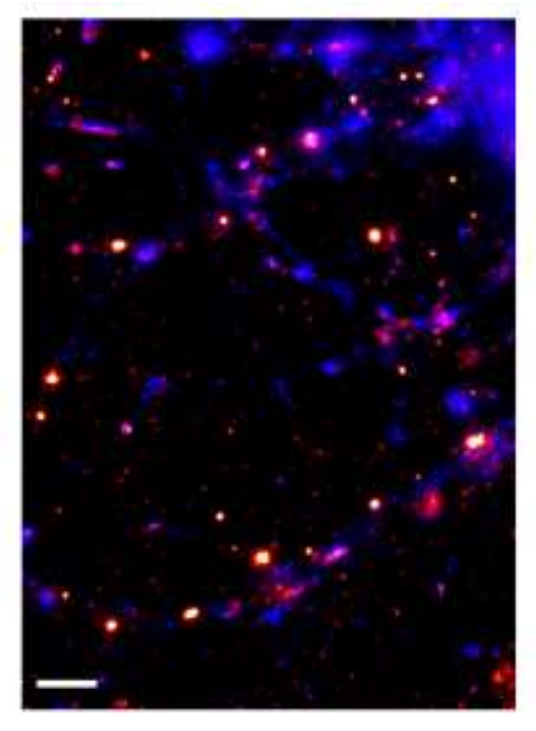

\begin{tabular}{|c|c|c|}
\hline $\begin{array}{c}\text { Whole cell copy } \\
\text { number }\end{array}$ & \multicolumn{2}{|c|}{$1232792.55 \pm 96535.35$} \\
\hline $\begin{array}{c}\text { spine copy } \\
\text { number }\end{array}$ & \multicolumn{2}{|c|}{$169.34 \pm 59.64$} \\
\hline$\%$ in PSD & \multicolumn{2}{|c|}{17.03} \\
\hline PSD copy number & \multicolumn{2}{|c|}{$24.64 \pm 8.68$} \\
\hline \begin{tabular}{c|c|} 
Spine copy \\
number
\end{tabular} & Mushroom & Stubby \\
\hline PSD copy number & $22.33 \pm 7.86$ & $26.21 \pm 9.23$ \\
\hline$\%$ of protein & $0.01 \pm 0.00 \%$ & $0.01 \pm 0.00 \%$ \\
\hline Molarity $[\mu \mathrm{M}]$ & $1.29 \pm 0.45$ & $0.97 \pm 0.34$ \\
\hline
\end{tabular}

\section{References}

Antibody: Abcam ab68776

PDB Identifier: 2 dmw

\section{Literature:}

Advani et al., 1999; Alberts et al., 2003; Martinez-Arca et al., 2003; Rao et al., 2004; Scheuber et al., 2006 


\section{Vti1a (Vti112, Gene: Vti1a, Uniprot ID: Q9JI51)}

Known function: Qb SNARE, Endosome-TGN transport, Homotypic endosome fusion Known organization: Transmembrane protein, On endosomes, TGN, SV, CCV

Known Interactions: Syntaxin6, Syntaxin16, VAMP4
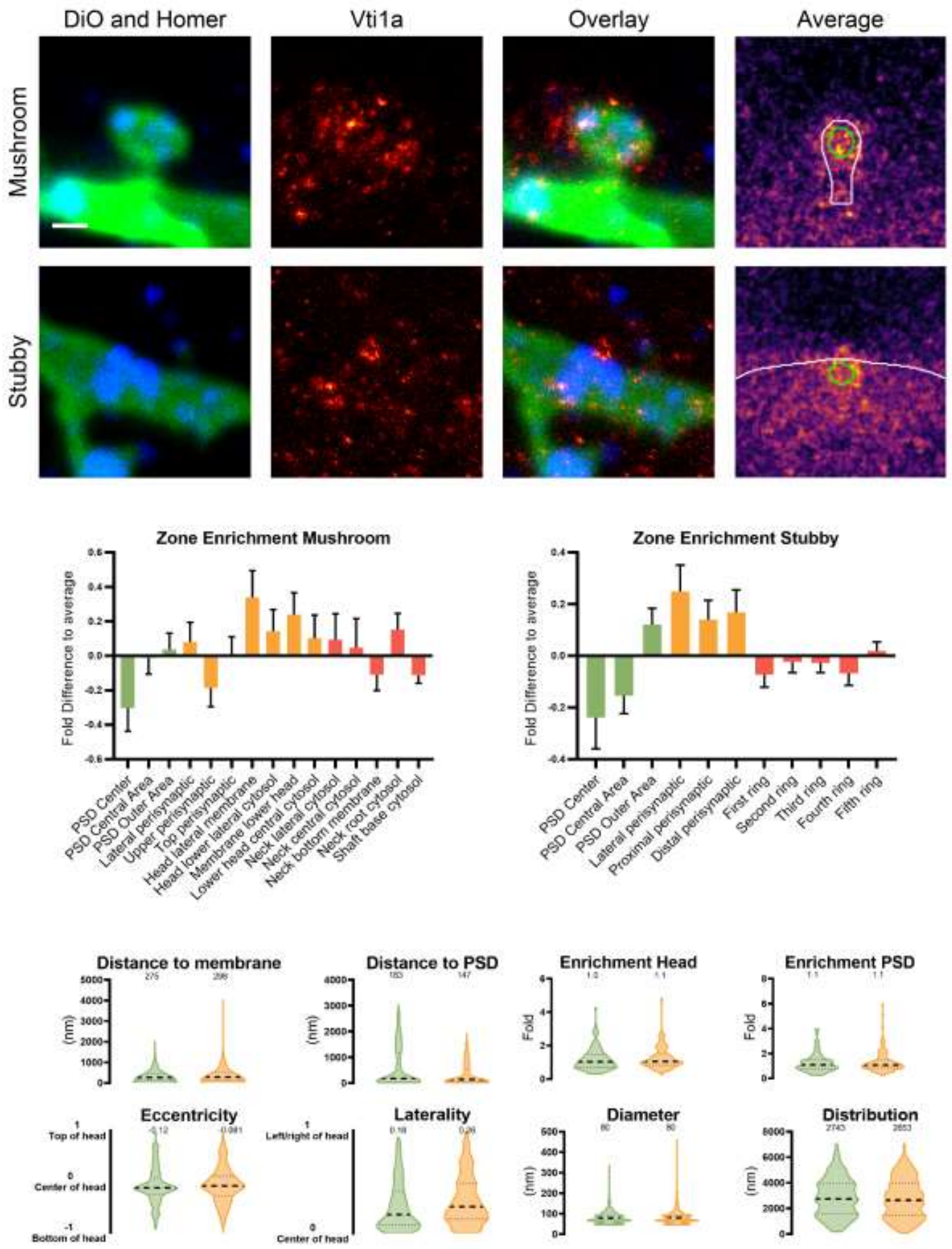

Figure 115: Vti1a nanoscale localization and abundance. Continued on next page. 


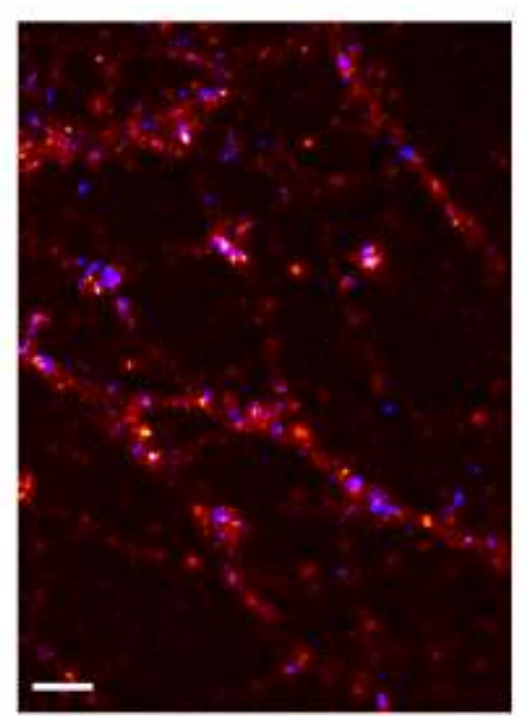

\begin{tabular}{|c|c|c|}
\hline $\begin{array}{c}\text { Whole cell copy } \\
\text { number }\end{array}$ & \multicolumn{2}{|c|}{$4833694.67 \pm 568662.42$} \\
\hline $\begin{array}{c}\text { Spine copy } \\
\text { number }\end{array}$ & \multicolumn{2}{|c|}{$599.16 \pm 160.26$} \\
\hline$\%$ in PSD & \multicolumn{2}{|c|}{19.26} \\
\hline PSD copy number & \multicolumn{2}{|c|}{$96.78 \pm 25.89$} \\
\hline & Mushroom & Stubby \\
\hline \begin{tabular}{c|c|} 
Spine copy \\
number
\end{tabular} & $\begin{array}{c}587.68 \\
\pm 157.19\end{array}$ & $\begin{array}{c}572.37 \\
\pm 153.09\end{array}$ \\
\hline PSD copy number & $94.92 \pm 25.39$ & $92.45 \pm 24.73$ \\
\hline$\%$ of protein & $0.03 \pm 0.01 \%$ & $0.02 \pm 0.01 \%$ \\
\hline Molarity $[\mu \mathrm{M}]$ & $4.93 \pm 1.32$ & $3.07 \pm 0.82$ \\
\hline
\end{tabular}

\section{References}

Antibody: BD Biosciences 611220

Structure: from Takamori et al. 2006

\section{Literature:}

Amessou et al., 2007; Antonin et al., 2002; Brandhorst et al., 2006; Fischer von Mollard and Stevens, 1998; Mallard et al., 2002; McBride et al., 1999; Shitara et al., 2017; Zwilling et al., 2007 


\section{Organelle proteins}

The dendrite contains representatives of many organelles, some unique to this compartment. I investigate their distribution and the quantity of key proteins within these compartments in the following section.

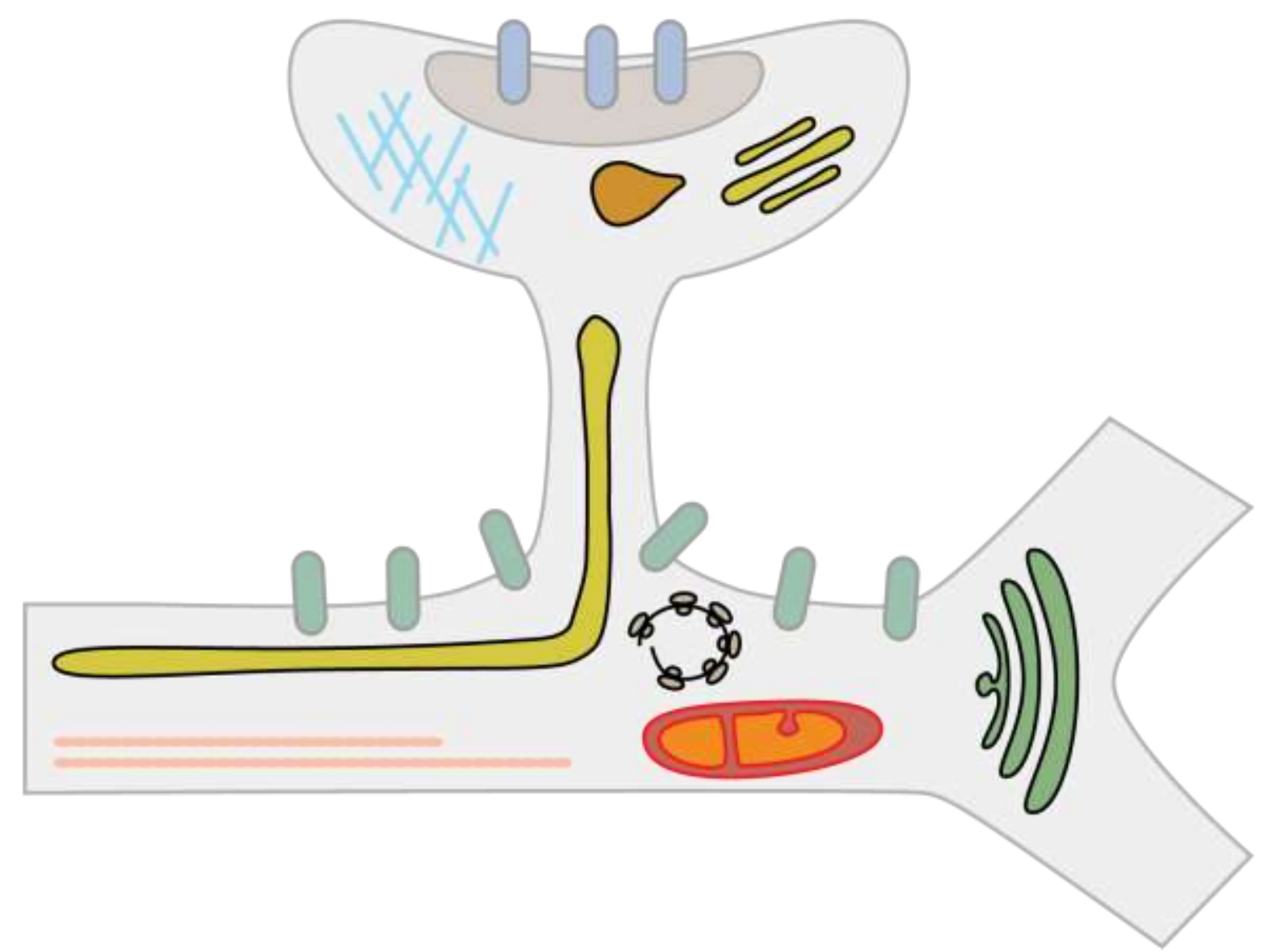

Figure 116: Depiction of organelles in dendritic spines. 


\section{Calreticulin (CALBP, Gene: Calr, Uniprot ID: P18418)}

Known function: ER resident chaperone, Calcium buffer

Known organization: ER lumen

Known Interactions: None
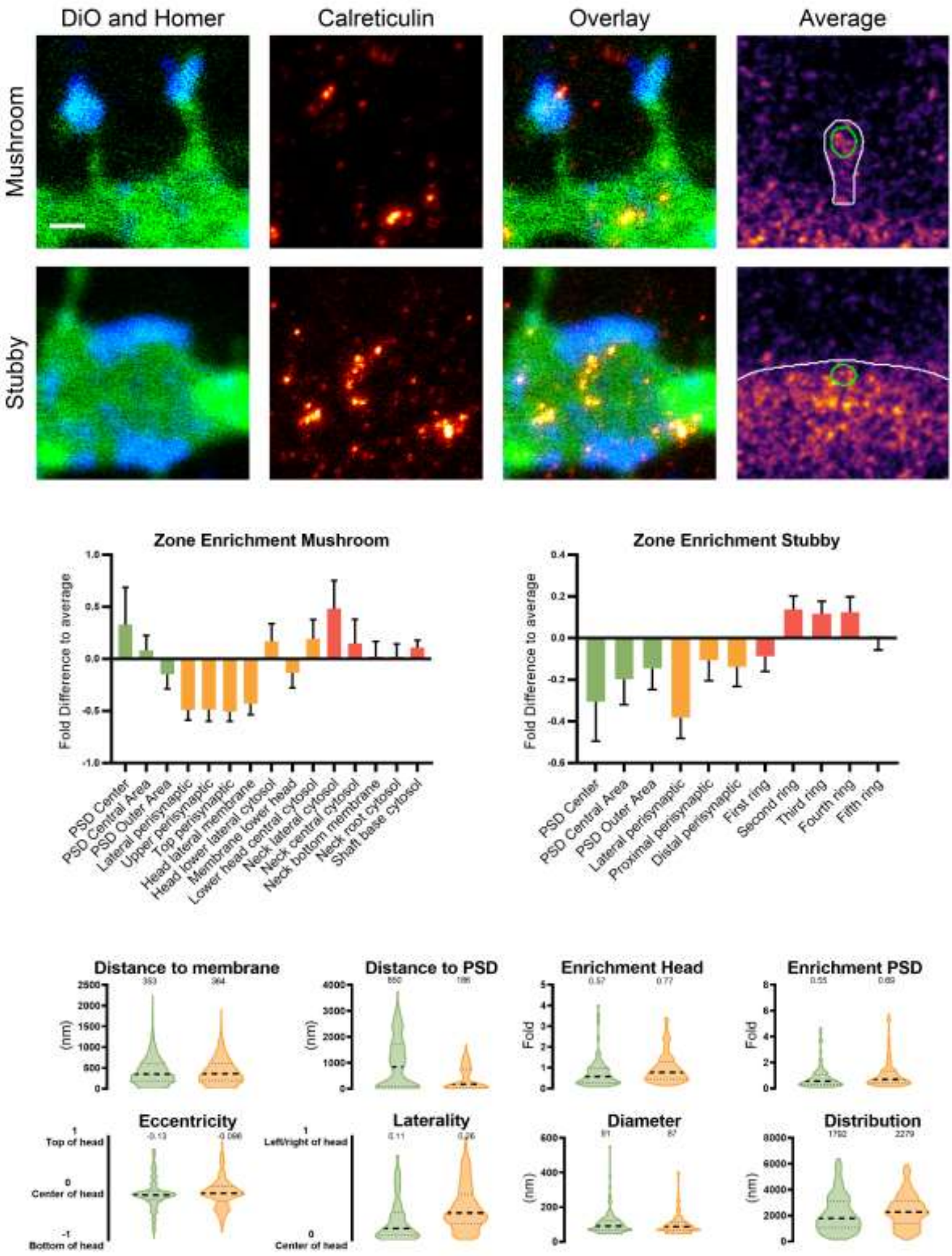

Figure 117: Calreticulin: nanoscale localization and abundance. Continued on next page. 


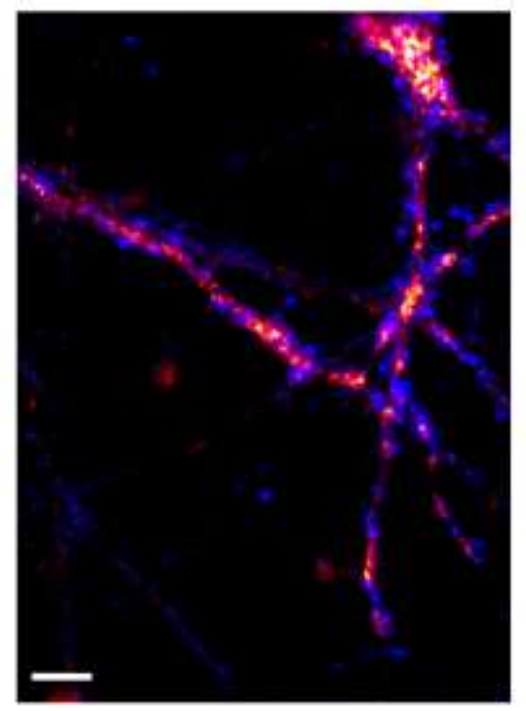

\begin{tabular}{|c|c|c|}
\hline $\begin{array}{c}\text { Whole cell copy } \\
\text { number }\end{array}$ & \multicolumn{2}{|c|}{$54765462.29 \pm 6744999.73$} \\
\hline $\begin{array}{c}\text { Spine copy } \\
\text { number }\end{array}$ & \multicolumn{2}{|c|}{$4906.70 \pm 1542.63$} \\
\hline$\%$ in PSD & \multicolumn{2}{|c|}{14.02} \\
\hline PSD copy number & \multicolumn{2}{|c|}{$603.42 \pm 189.71$} \\
\hline Spine copy & 3068.45 & 7062.81 \\
\hline number & \pm 964.70 & \pm 2220.49 \\
\hline PSD copy number & $377.35 \pm 118.64$ & $868.57 \pm 273.07$ \\
\hline$\%$ of protein & $0.34 \pm 0.11 \%$ & $0.50 \pm 0.16 \%$ \\
\hline Molarity $[\mu \mathrm{M}]$ & $25.72 \pm 8.09$ & $37.86 \pm 11.90$ \\
\hline
\end{tabular}

\section{References}

Antibody: Cell Signaling 12238

PDB Identifier: $1 \mathrm{hhn}, 300 \mathrm{v}$

\section{Literature:}

Bedard et al., 2005; Hebert and Molinari, 2007; Nakamura et al., 2001 


\section{ERp72 (ERp70, CaBP2, Gene: Pdia4, Uniprot ID: P38659)}

Known function: ER-resident chaperone, Isomerizes disulfide bonds

Known organization: ER lumen

Known Interactions: None
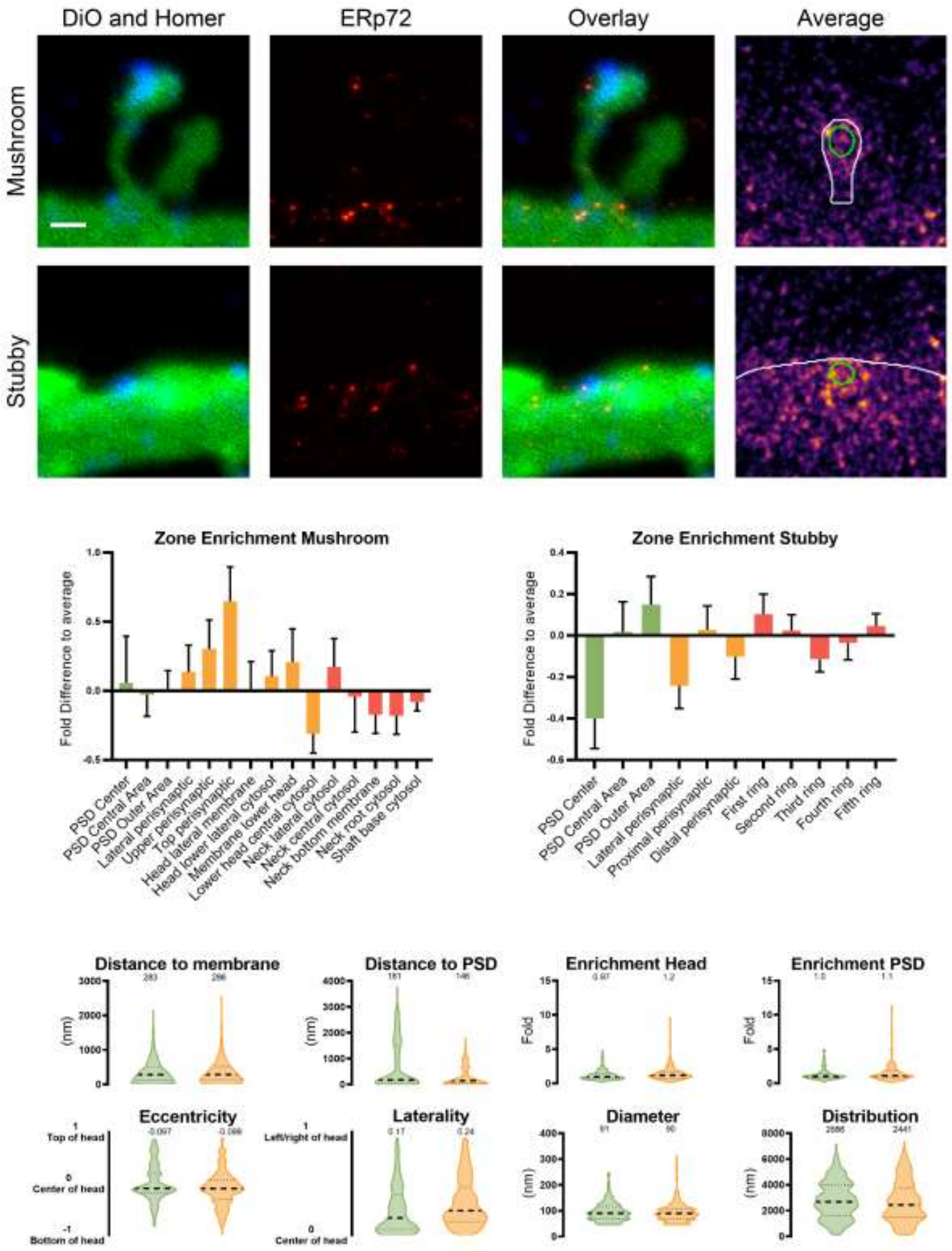

Figure 118: ERp72 nanoscale localization and abundance. Continued on next page. 


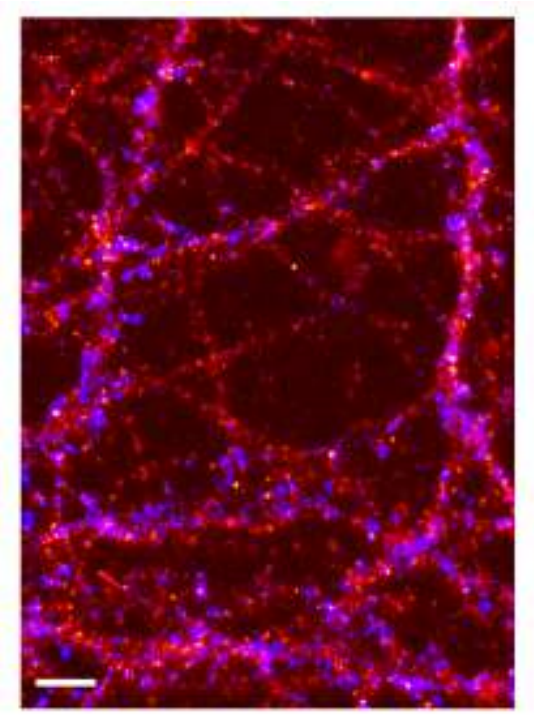

\begin{tabular}{|c|c|c|}
\hline $\begin{array}{c}\text { Whole cell copy } \\
\text { number }\end{array}$ & \multicolumn{2}{|c|}{$741978.91 \pm 208711.33$} \\
\hline $\begin{array}{l}\text { Spine copy } \\
\text { number }\end{array}$ & \multicolumn{2}{|c|}{$62.28 \pm 22.72$} \\
\hline$\%$ in PSD & \multicolumn{2}{|c|}{21.78} \\
\hline \multirow[t]{2}{*}{ PSD copy number } & \multicolumn{2}{|c|}{$11.14 \pm 4.06$} \\
\hline & Mushroom & Stubby \\
\hline $\begin{array}{l}\text { Spine copy } \\
\text { number }\end{array}$ & $45.71 \pm 16.67$ & $75.25 \pm 27.45$ \\
\hline PSD copy number & $8.17 \pm 2.98$ & $13.46 \pm 4.91$ \\
\hline$\%$ of protein & $0.01 \pm 0.00 \%$ & $0.01 \pm 0.00 \%$ \\
\hline Molarity $[\mu \mathrm{M}]$ & $0.38 \pm 0.14$ & $0.40 \pm 0.15$ \\
\hline
\end{tabular}

\section{References}

Antibody: Cell Signaling 5033

PDB Identifier: 2dj1, 3ec3

\section{Literature:}

Lièvremont et al., 1997; Mazzarella et al., 1990; Rupp et al., 1994 


\section{Ribosomal protein L7a (Gene: Rpl7a, Uniprot ID: P62425)}

Known function: Translation

Known organization: Cytosolic, on 605 subunit

Known Interactions: 40 S ribosomal subunit
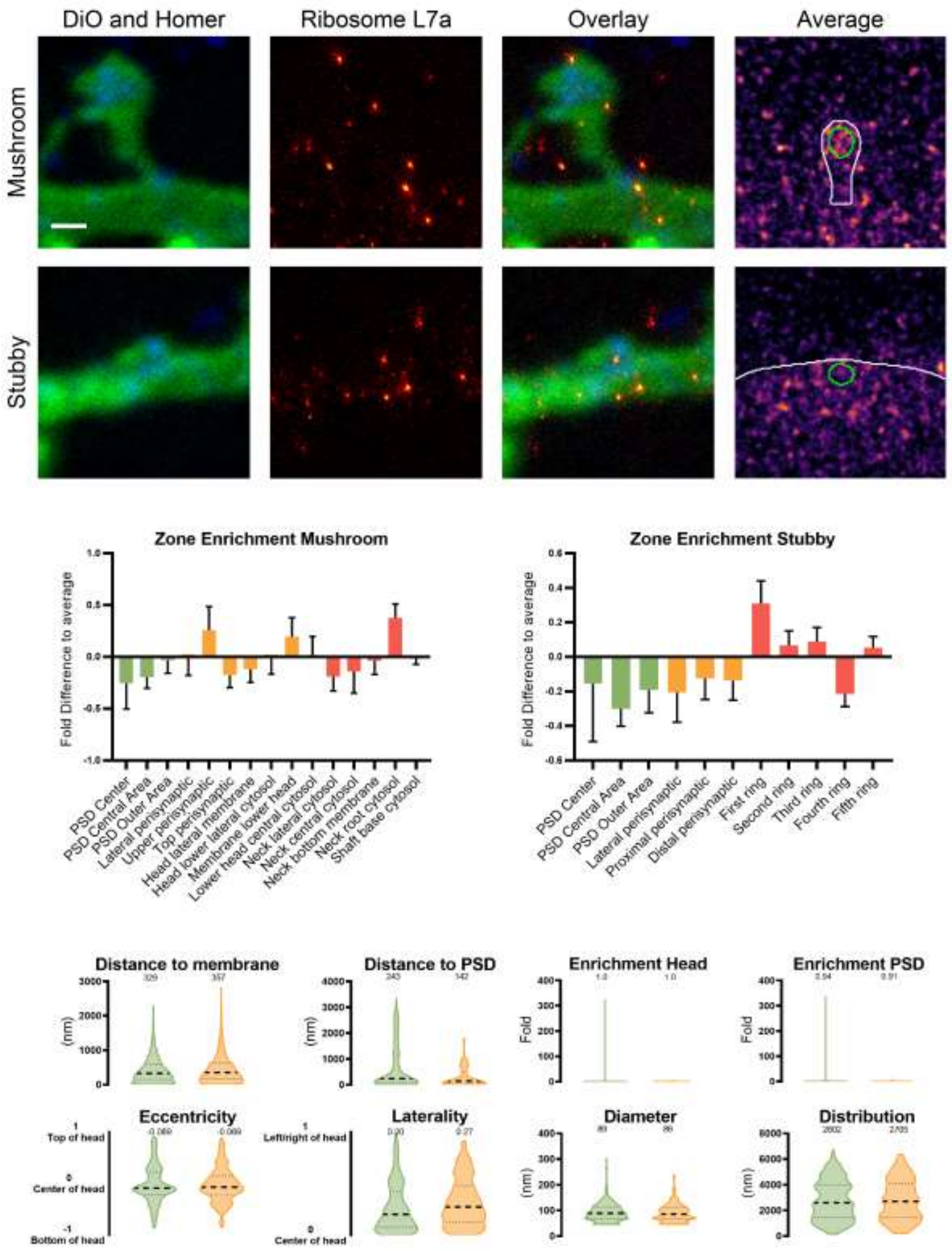

Figure 119: Ribosomal protein L7a nanoscale localization and abundance. Continued on next page. 


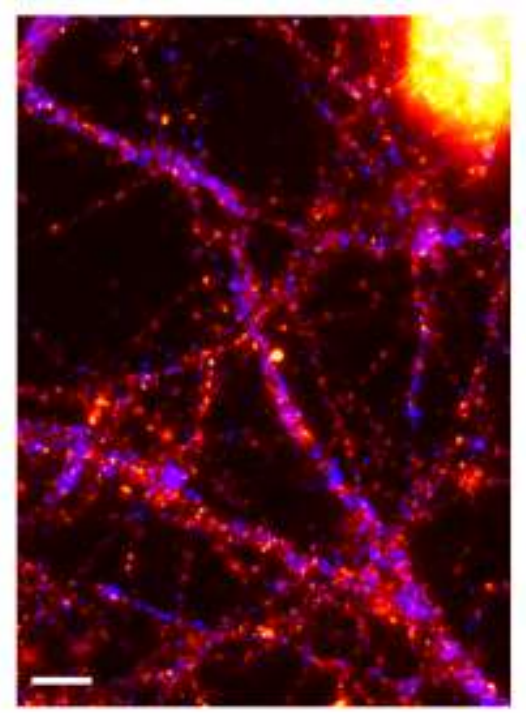

\begin{tabular}{|c|}
\hline $\begin{array}{c}\text { Whole cell copy } \\
\text { number }\end{array}$ \\
\hline $\begin{array}{c}\text { Spine copy } \\
\text { number }\end{array}$ \\
\hline$\%$ in PSD \\
\hline PSD copy number \\
\hline
\end{tabular}

$34562077.05 \pm 6388085.24$

\begin{tabular}{|c|c|c|}
\hline & Mushroom & Stubby \\
\hline $\begin{array}{c}\text { Spine copy } \\
\text { number }\end{array}$ & $\begin{array}{c}346.46 \\
\pm 419.77\end{array}$ & $\begin{array}{c}554.65 \\
\pm 672.02\end{array}$ \\
\hline PSD copy number & $52.22 \pm 63.27$ & $83.60 \pm 101.29$ \\
\hline$\%$ of protein & $0.02 \pm 0.03 \%$ & $0.02 \pm 0.03 \%$ \\
\hline Molarity $[\mu \mathrm{M}]$ & $2.90 \pm 3.52$ & $2.97 \pm 3.60$ \\
\hline
\end{tabular}

Mushroom molecular model

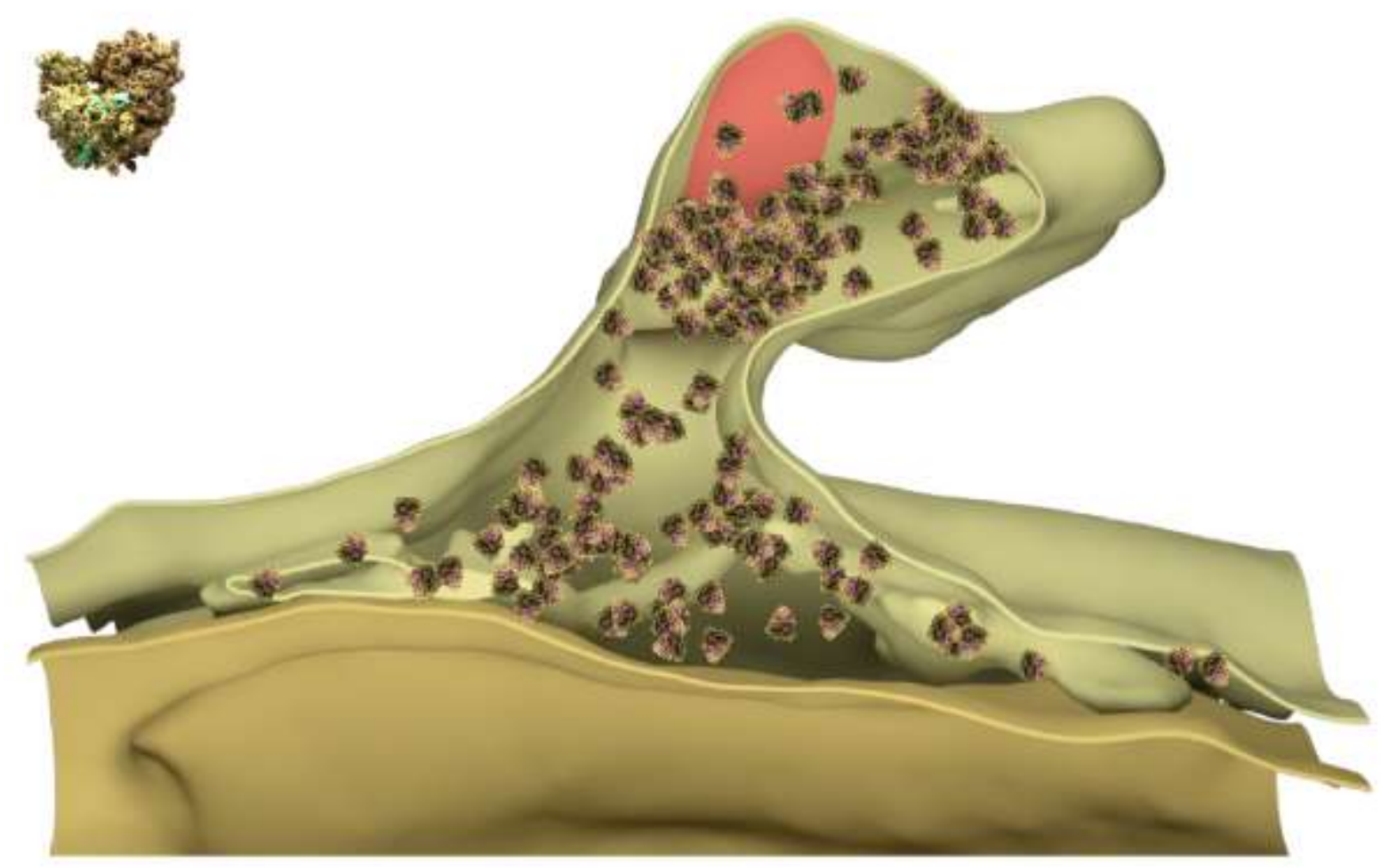

\section{References}

Antibody: Cell Signaling 2403

PDB Identifier: 4ugo

\section{Literature:}

Ban et al., 2014; de la Cruz et al., 2015 


\title{
Ribosomal protein S3 (Gene: Rps3, Uniprot ID: P62909)
}

\author{
Known function: Translation
}

Known organization: Cytosolic, on 405 subunit

Known Interactions: $60 \mathrm{~S}$ ribosomal subunit
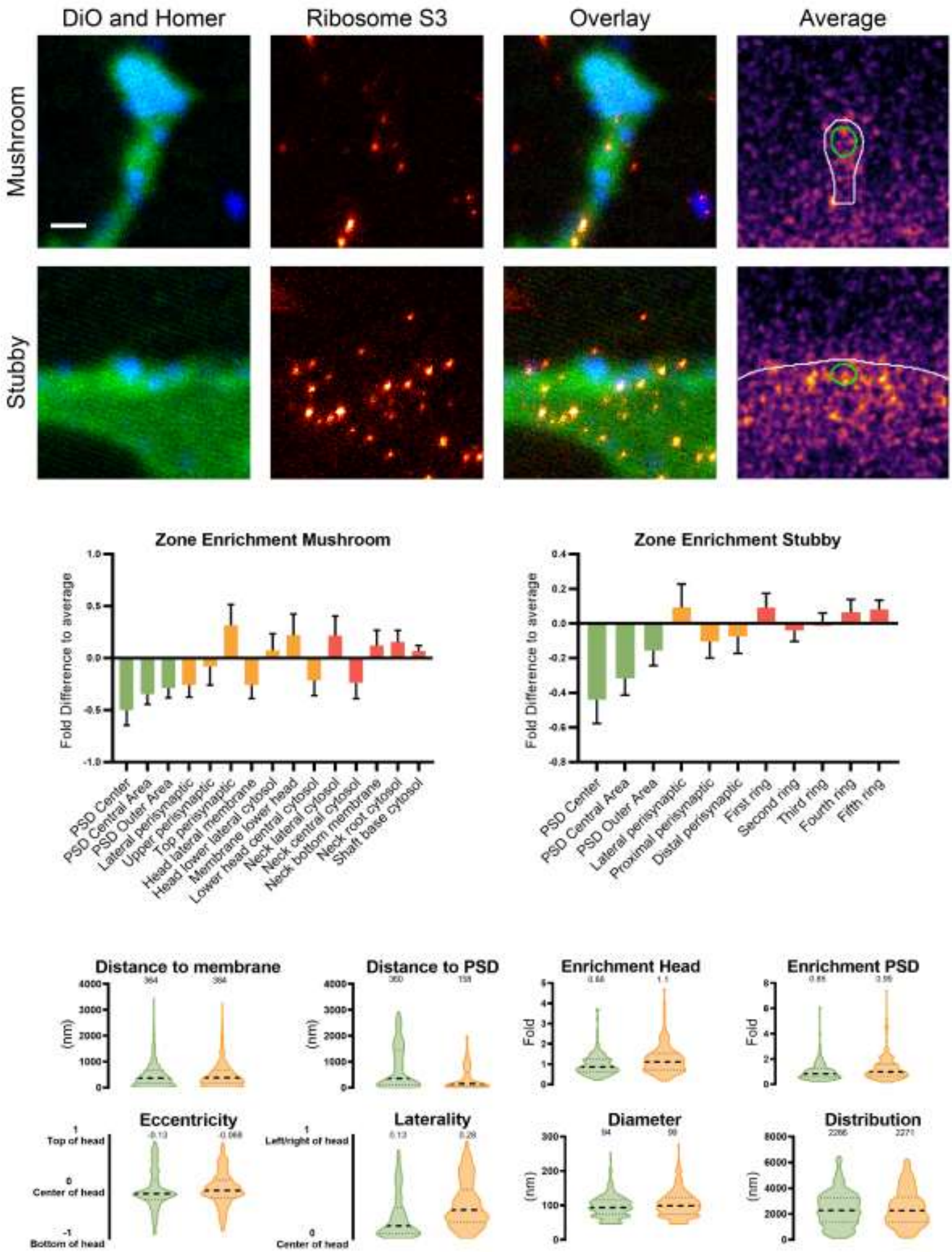

Figure 120: Ribosomal protein S3 nanoscale localization and abundance. Continued on next page. 


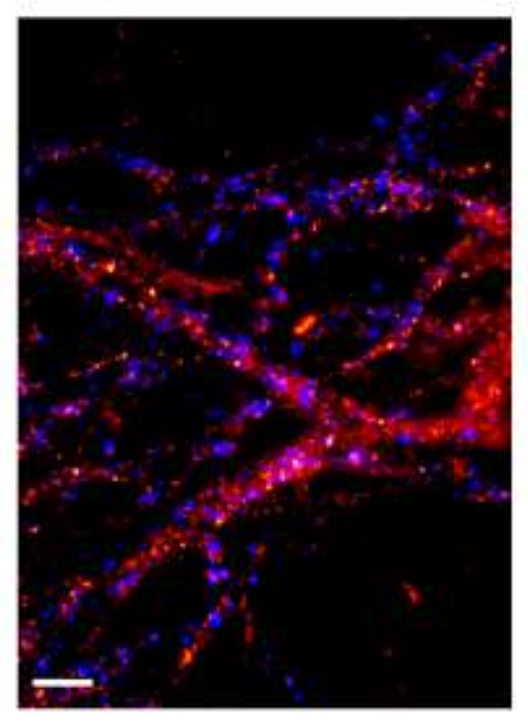

\begin{tabular}{|c|c|c|}
\hline $\begin{array}{l}\text { whole cell copy } \\
\text { number }\end{array}$ & \multicolumn{2}{|c|}{ not detected } \\
\hline $\begin{array}{l}\text { Spine copy } \\
\text { number }\end{array}$ & \multicolumn{2}{|c|}{$\begin{array}{c}8509.40 \pm 10000.94 \\
\text { (from comparative imaging) }\end{array}$} \\
\hline$\%$ in PSD & \multicolumn{2}{|c|}{15.04} \\
\hline \multirow[t]{2}{*}{ PSD copy number } & \multicolumn{2}{|c|}{$1112.61 \pm 1307.63$} \\
\hline & Mushroom & Stubby \\
\hline $\begin{array}{l}\text { Spine copy } \\
\text { number }\end{array}$ & $\begin{array}{l}6283.09 \\
\pm 7384.40\end{array}$ & $\begin{array}{c}11421.63 \\
\pm 13423.63\end{array}$ \\
\hline PSD copy number & $\begin{array}{c}821.52 \\
\pm 965.51\end{array}$ & $\begin{array}{c}1493.38 \\
\pm 1755.14\end{array}$ \\
\hline$\%$ of protein & $\pm \%$ & $\pm \%$ \\
\hline Molarity $[\mu \mathrm{M}]$ & $52.66 \pm 61.89$ & $61.23 \pm 71.96$ \\
\hline
\end{tabular}

\section{References}

Antibody: Cell Signaling 9538

PDB Identifier: 4ugo

\section{Literature:}

Ban et al., 2014; de la Cruz et al., 2015 


\section{Ribosomal protein S6 (Gene: Rps6, Uniprot ID: P62755)}

Known function: Translation

Known organization: Cytosolic, on 40 S subunit

Known Interactions: $60 \mathrm{~S}$ ribosomal subunit
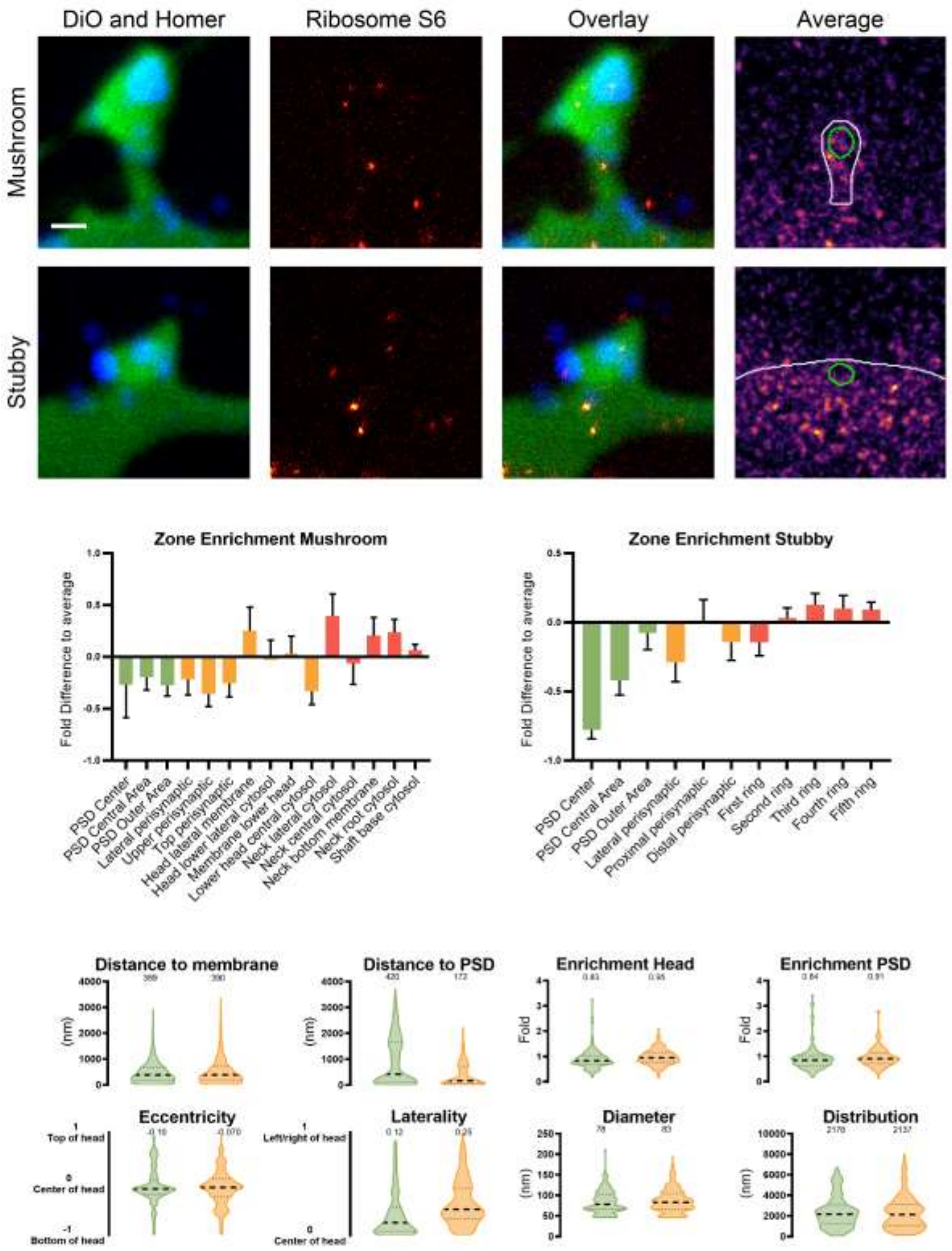

Figure 121: Ribosomal protein S6 nanoscale localization and abundance. Continued on next page. 


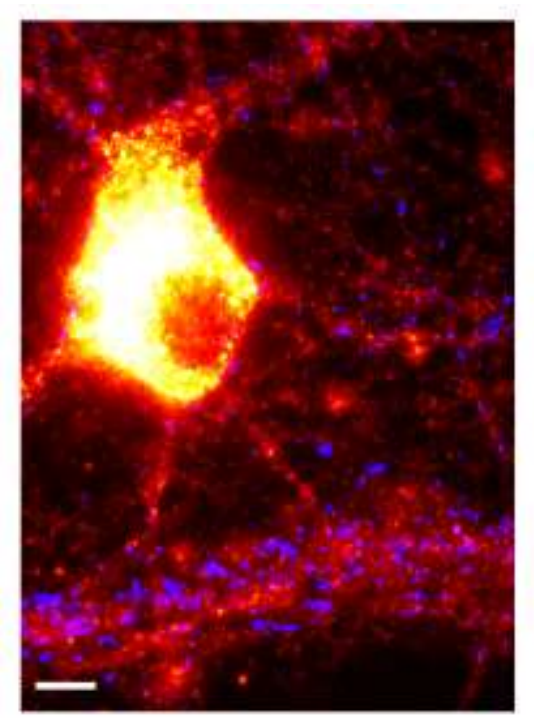

\begin{tabular}{|c|c|c|}
\hline $\begin{array}{c}\text { Whole cell copy } \\
\text { number }\end{array}$ & \multicolumn{2}{|c|}{$50580108.78 \pm 8484683.82$} \\
\hline $\begin{array}{c}\text { Spine copy } \\
\text { number }\end{array}$ & \multicolumn{2}{|c|}{ not detected } \\
\hline$\%$ in PSD & \multicolumn{2}{|c|}{ na } \\
\hline PSD copy number & \multicolumn{2}{|c|}{ na } \\
\hline \begin{tabular}{c|c|} 
Spine copy \\
number
\end{tabular} & na & na \\
\hline PSD copy number & na & na \\
\hline$\%$ of protein & na & na \\
\hline Molarity $[\mu M]$ & \pm & \pm \\
\hline
\end{tabular}

\section{References}

Antibody: Cell Signaling 2217

PDB Identifier: 4ugo

\section{Literature:}

Ban et al., 2014; de la Cruz et al., 2015 
TGN38 (Gene: Ttgn1, Uniprot ID: P19814)

Known function: TGN-PM cycling

Known organization: Transmembrane protein, TGN

Known Interactions: None
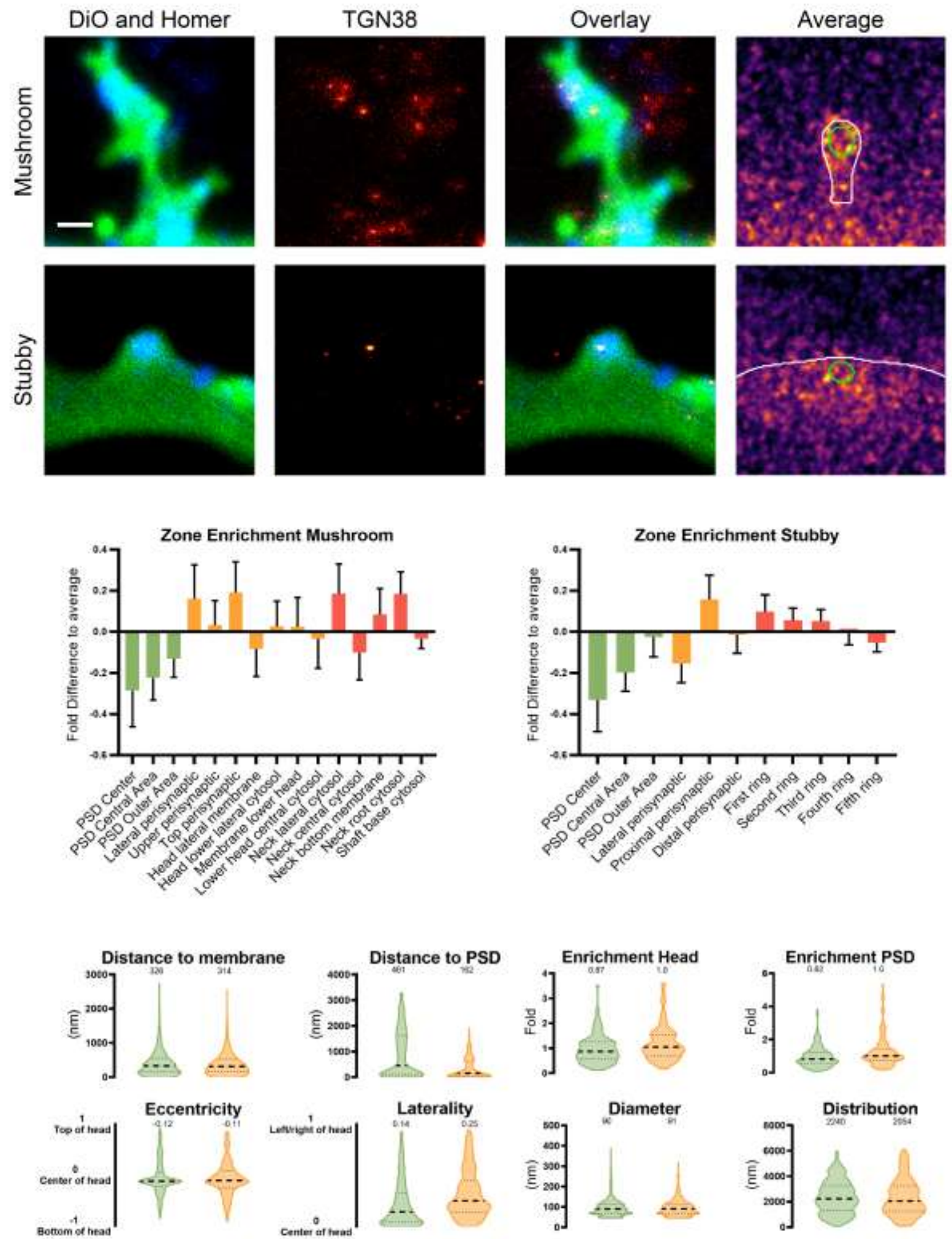

Figure 122: TGN38 nanoscale localization and abundance. Continued on next page. 


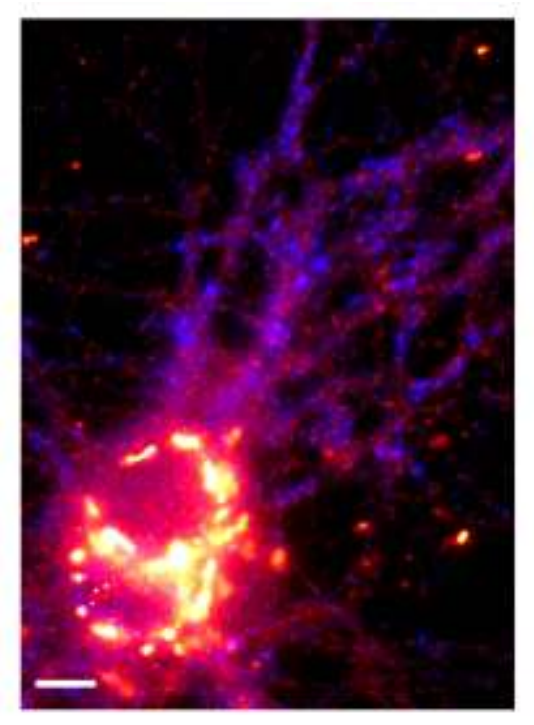

\begin{tabular}{|c|c|c|}
\hline $\begin{array}{c}\text { Whole cell copy } \\
\text { number }\end{array}$ & \multicolumn{2}{|c|}{$209201.42 \pm 32085.51$} \\
\hline $\begin{array}{c}\text { Spine copy } \\
\text { number }\end{array}$ & \multicolumn{2}{|c|}{$4.65 \pm 5.51$} \\
\hline$\%$ in PSD & \multicolumn{2}{|c|}{17.41} \\
\hline PSD copy number & \multicolumn{2}{|c|}{$0.69 \pm 0.82$} \\
\hline \begin{tabular}{c|c|} 
Spine copy \\
number
\end{tabular} & $3.83 \pm 4.54$ & $5.64 \pm 6.68$ \\
\hline PSD copy number & $0.57 \pm 0.67$ & $0.84 \pm 0.99$ \\
\hline$\%$ of protein & $0.00 \pm 0.00 \%$ & $0.00 \pm 0.00 \%$ \\
\hline Molarity $[\mu \mathrm{M}]$ & $0.03 \pm 0.04$ & $0.03 \pm 0.04$ \\
\hline
\end{tabular}

\section{References}

Antibody: Sigma Aldrich T9826

Structure: Modelled by Burkhard Rammner

\section{Literature:}

Gardiol et al., 1999; Luzio et al., 1990; Reaves et al., 1993 


\section{TOM20 (Gene: Tomm20, Uniprot ID: Q62760)}

Known function: Part of the translocase of the outer mitochondrial membrane, Recognizes presequence of preproteins

Known organization: Transmembrane protein, Mitochondrion, Forms clusters Known Interactions: None
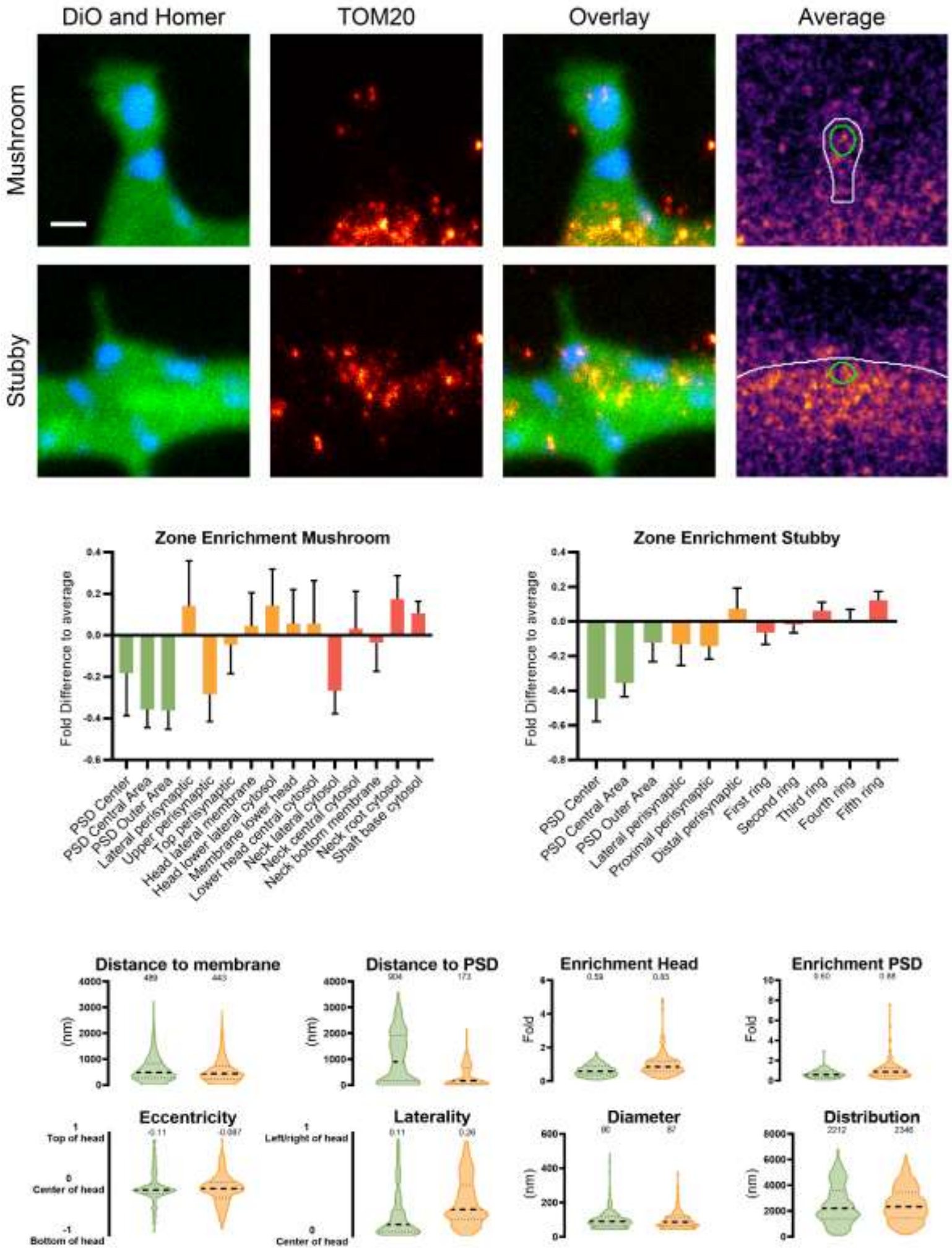

Figure 123: TOM20 nanoscale localization and abundance. Continued on next page. 


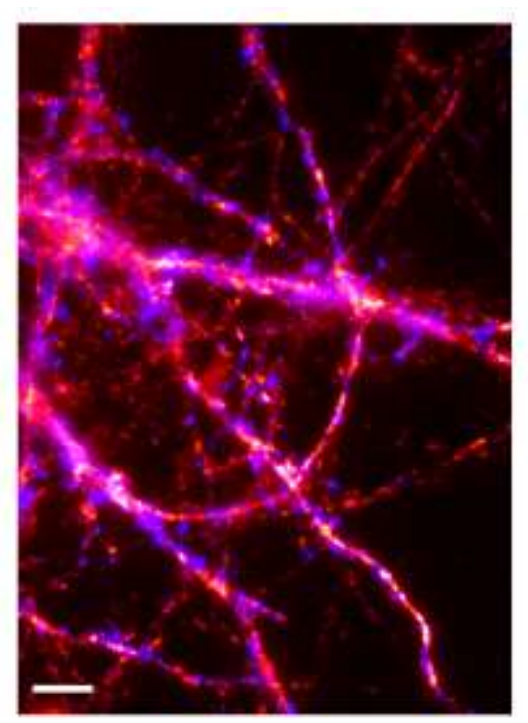

\begin{tabular}{|c|c|c|}
\hline $\begin{array}{c}\text { Whole cell copy } \\
\text { number }\end{array}$ & \multicolumn{2}{|c|}{$9032340.15 \pm 2045079.31$} \\
\hline $\begin{array}{c}\text { Spine copy } \\
\text { number }\end{array}$ & \multicolumn{2}{|c|}{$640.71 \pm 353.45$} \\
\hline$\%$ in PSD & \multicolumn{2}{|c|}{12.15} \\
\hline PSD copy number & \multicolumn{2}{|c|}{$69.40 \pm 38.29$} \\
\hline & Mushroom & Stubby \\
\hline \begin{tabular}{c|c|} 
Spine copy \\
number
\end{tabular} & $\begin{array}{c}484.07 \\
\pm 267.04\end{array}$ & $\begin{array}{c}705.69 \\
\pm 389.30\end{array}$ \\
\hline PSD copy number & $52.44 \pm 28.93$ & $76.44 \pm 42.17$ \\
\hline$\%$ of protein & $0.02 \pm 0.01 \%$ & $0.02 \pm 0.01 \%$ \\
\hline Molarity $[\mu \mathrm{M}]$ & $4.06 \pm 2.24$ & $3.78 \pm 2.09$ \\
\hline
\end{tabular}

\section{References}

Antibody: Sigma Aldrich WH0009804M1

PDB Identifier: 1om2

\section{Literature:}

Donnert et al., 2007; Saitoh et al., 2007; Söllner et al., 1989; Wurm et al., 2011 


\section{Synaptic scaling changes the nanoscale localization and abundance of key proteins}

The data presented so far demonstrates the molecular architecture of a dendritic spine at rest. But the postsynapse is known to undergo various plasticity regimens to adapt its strength. I set out to study one of them, synaptic scaling, for a subset of potential key proteins in this process. Synaptic scaling is a homeostatic process that neurons employ to keep their firing rate at a set constant. When the strength of the input to a neuron increases, it responds by reducing the efficiency of its spines globally or locally. Similarly, when the input strength decreases, the neuron responds by enhancing spine efficiency (Turrigiano, 2008). I used chronic treatment with Bicuculline, a GABA blocker, to increase the baseline network activity of the neuronal cultures, causing synaptic downscaling. To elicit synaptic upscaling, I used either TTX to block synchronous release (while spontaneous release is still occurring) or CNQX+AP5 to block AMPA and NMDA receptors, abolishing almost all glutamatergic signaling. The latter is therefore expected to have a more dramatic effect on synaptic upscaling than TTX. First, I determined whether the treatments changed the frequency of mushroom or stubby dendritic spines (Table 10). Interestingly, the percentage of the spine classes did not change for most treatments. Only when blocking glutamate mediated transmission completely with $\mathrm{CNQX}+\mathrm{AP} \%$, the percentage of mushroom spines decreased, which was compensated by an increase in 'other' class spines.

Table 10: Spine class frequency during homeostatic plasticity. Only during CNQX+AP5 treatment did the frequency of spine classes change, showing a decrease in mushroom spines and an increase in other spines. The percentage of stubby classes spines was constant throughout all treatments.

\begin{tabular}{|l|r|r|r|r|}
\hline Spine class & Untreated & Bicuculline & TTX & CNQX + AP5 \\
\hline Mushroom & $75.37 \%$ & $74.55 \%$ & $75.68 \%$ & $64.36 \%$ \\
\hline Stubby & $24.46 \%$ & $24.55 \%$ & $23.46 \%$ & $23.66 \%$ \\
\hline Other & $0.17 \%$ & $0.89 \%$ & $0.87 \%$ & $11.98 \%$ \\
\hline
\end{tabular}

As the spine class distribution did not show major changes the different treatments can compared to each other directly and I present the results in a standardized form, similar to the previous section. Again, the average distribution of the protein is shown, as well as the percentage change in staining intensity, compared to the untreated control. The lower panel shows the zone enrichment analysis, which was performed exactly as for the average distribution at rest. 


\section{Actin}

I found F-Actin to be decreased in Bicuculline, a trend that was interestingly very pronounced but not significant for mushroom synapses. The localization also moved toward the center of the PSD after bicuculline treatment for both synapse classes, and also after TTX treatment in stubby synapses.

\section{Mushroom}

C

A

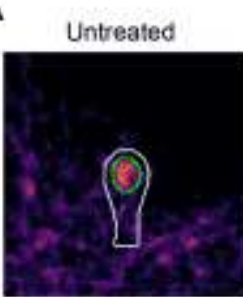

$\pi x$

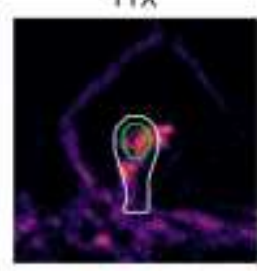

CNQX+AP5

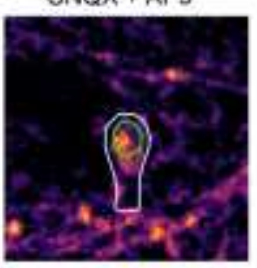

B

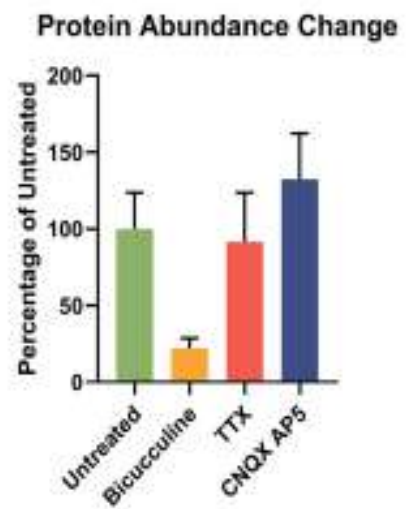

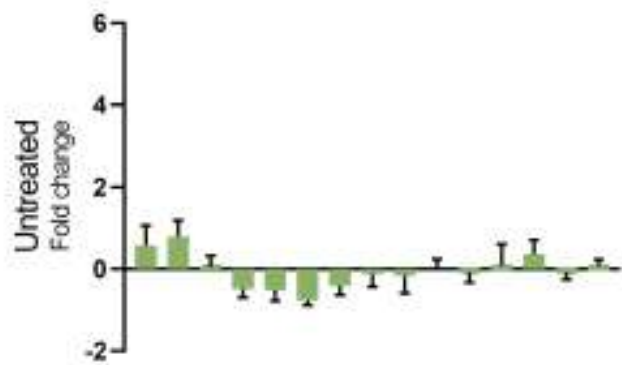
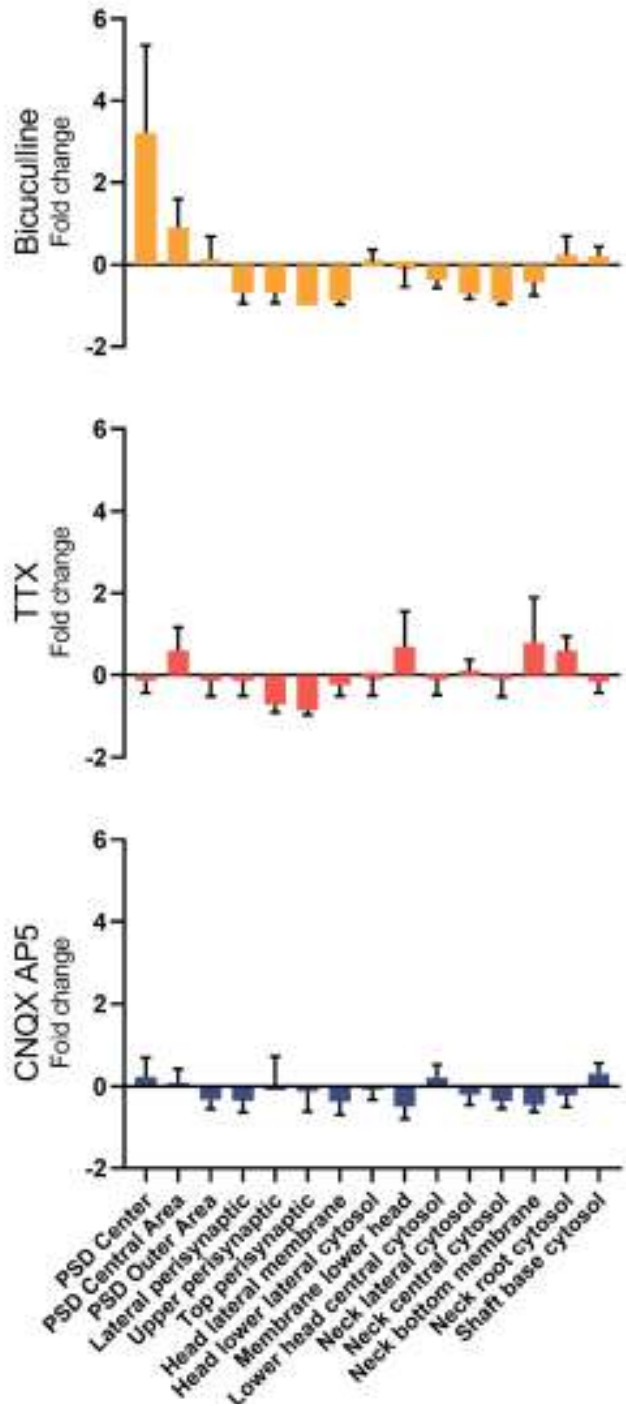

Figure 124: Changes in actin localization and abundance after synaptic scaling. A) The average distribution of actin in the treatments. Scale bar is $500 \mathrm{~nm}$. B) The change in total intensity compared to untreated was calculated. C) The Enrichment of actin in the different zones for each treatment. Values are Mean \pm SEM. Continued on next page. 


\section{Stubby}

C

A

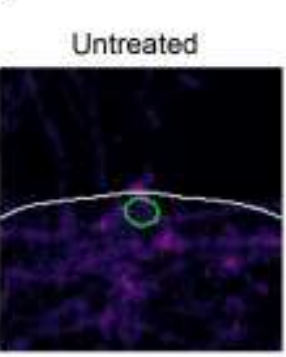

TTX

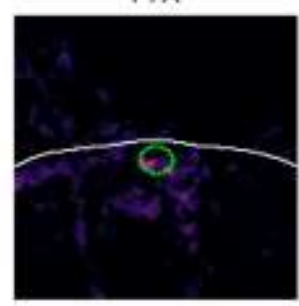

B

Protein Abundance Change

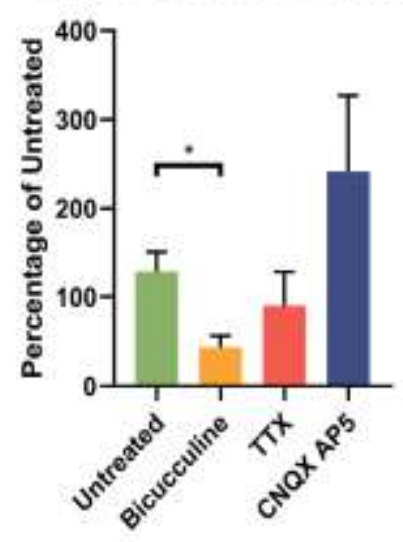

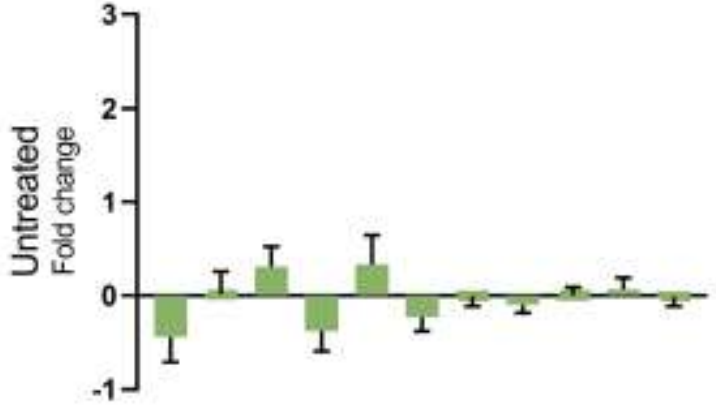
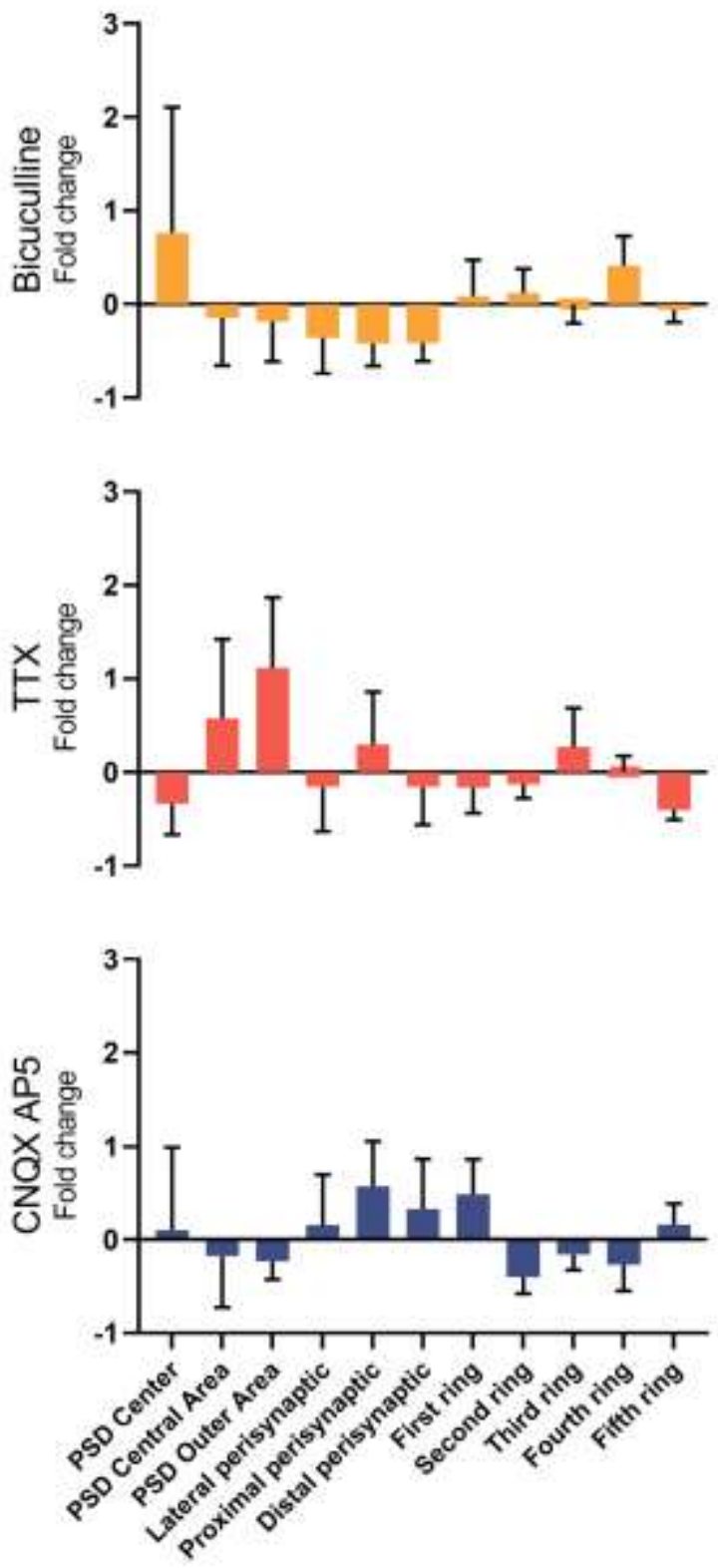


\section{Clathrin light chain}

Clathrin abundance was increased after Bicuculline treatment for both synapse classes, whereas it was significantly decreased after CNQX+AP5 treatment for mushroom spines. After Bicuculline treatment, Clathrin also showed a trend to be positioned at the center of the PSD, compared to Untreated.

\section{Mushroom}

C

A

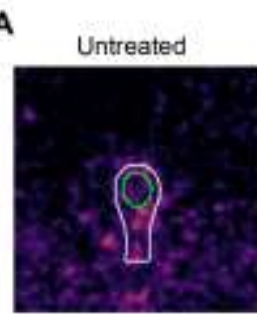

TTX

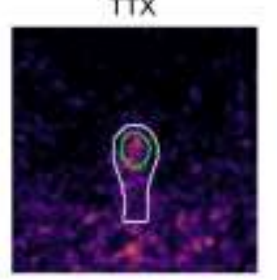

B

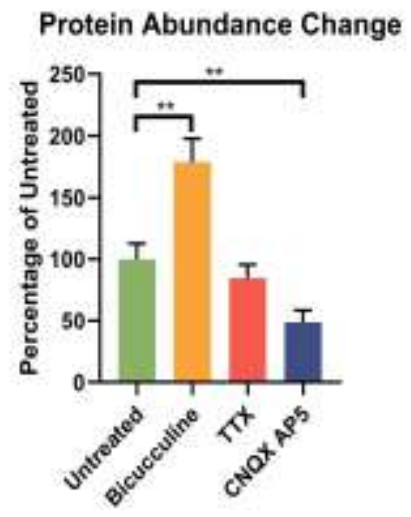

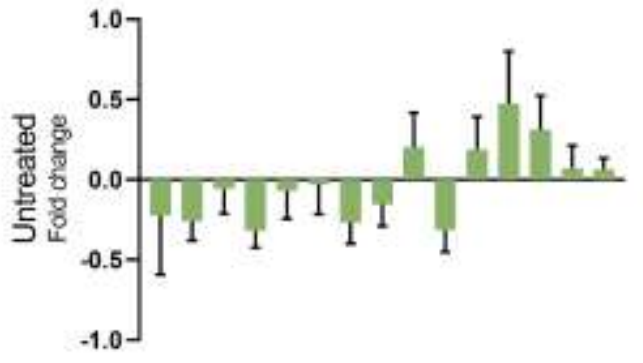
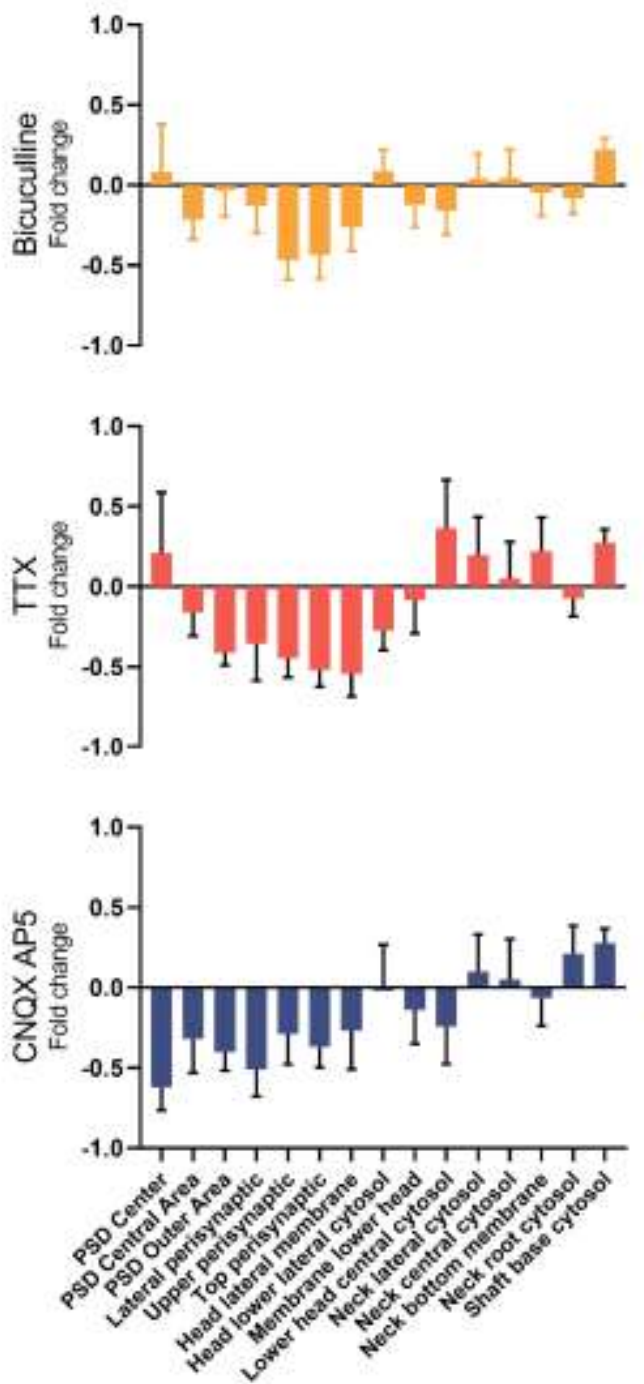

Figure 125: Changes in Clathrin LC localization and abundance after synaptic scaling. The figure is organized as in Figure 124. Continued on next page. 


\section{Stubby}

$c$
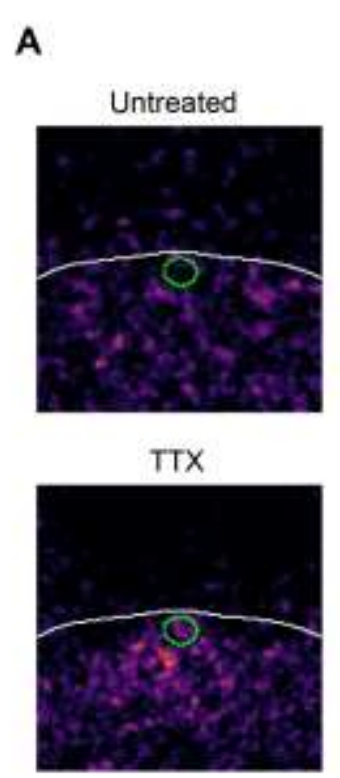

B

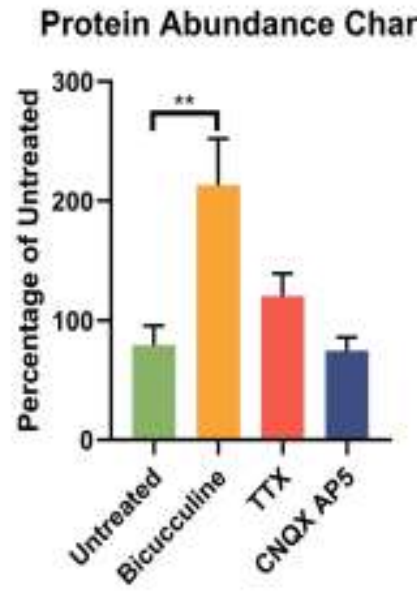

Bicuculline

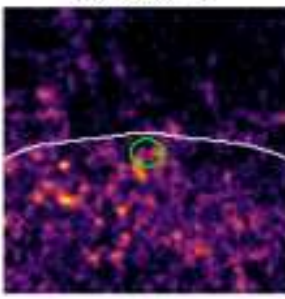

CNQX + AP5
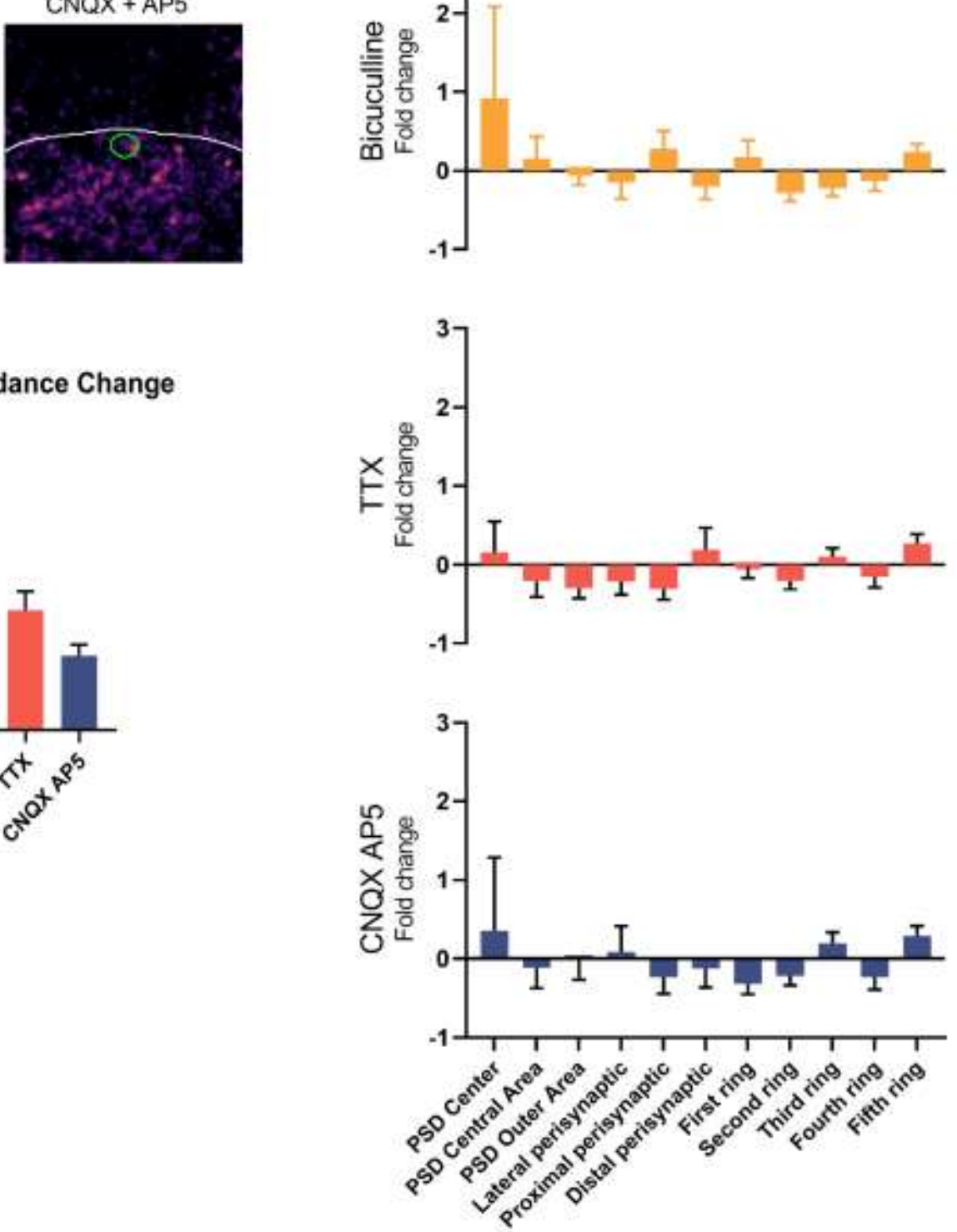


\section{GluN2B}

The NMDA receptor subunit was increased after blocking glutamatergic signaling using CNQX+AP5 in Mushroom spines and a showed a centralization of the receptor. Stubby synapses also showed an increase, but not to the same extent, and GluN2B was also recruited to more perisynaptic regions.

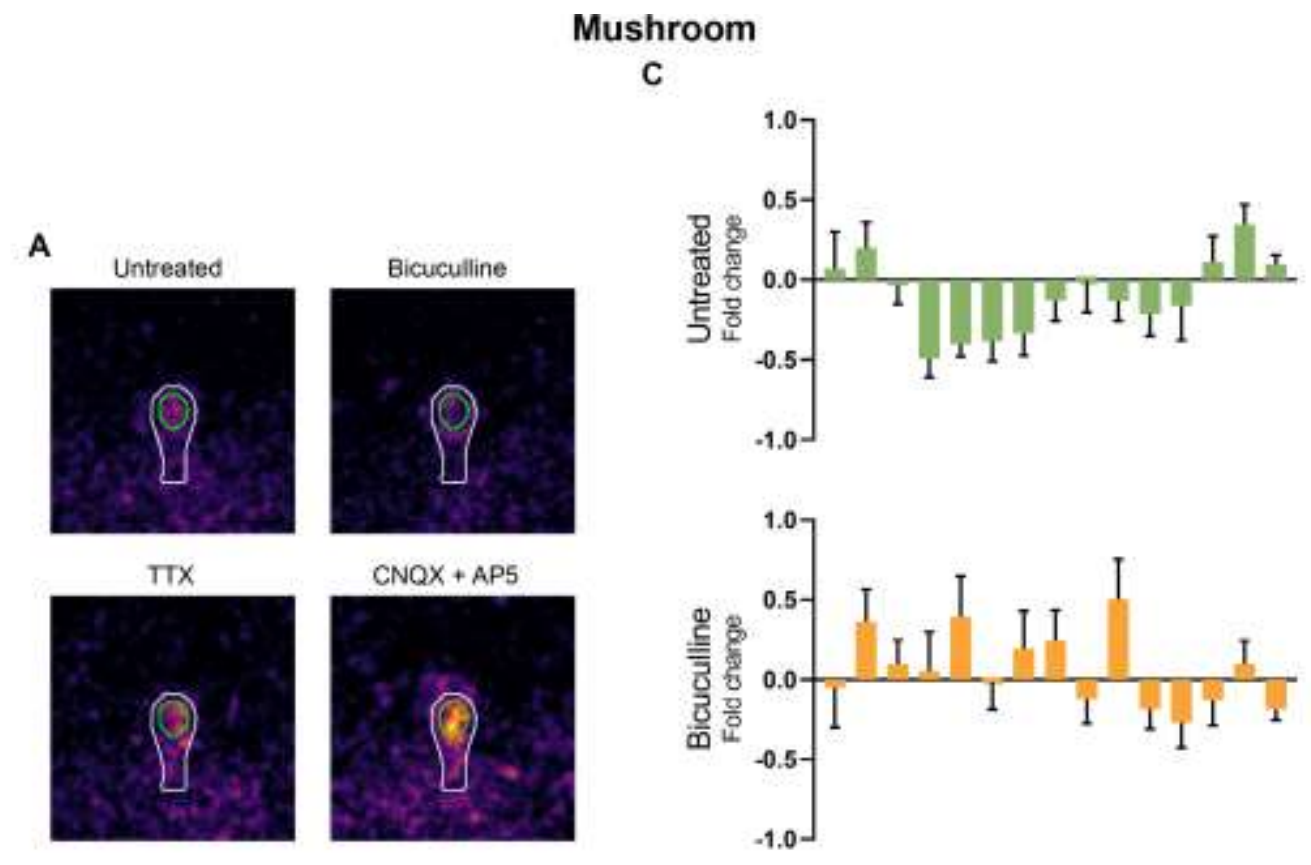

B
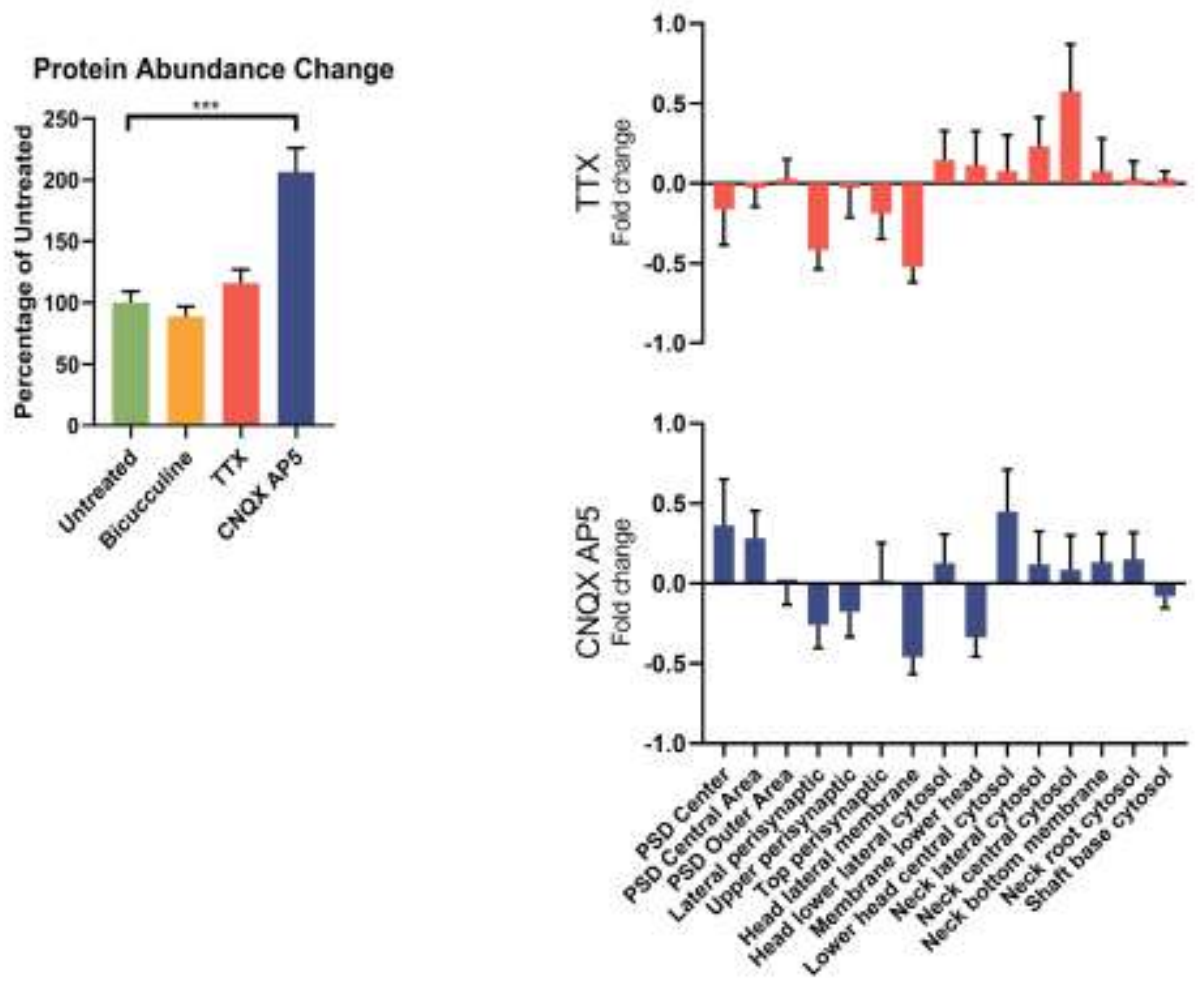

Figure 126: Changes in GluN2B localization and abundance after synaptic scaling. The figure is organized as in Figure 124. Continued on next page. 


\section{Stubby}

c
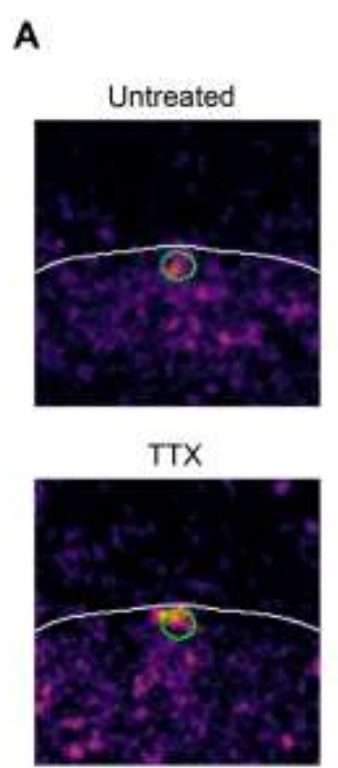

B

Protein Abundance Change

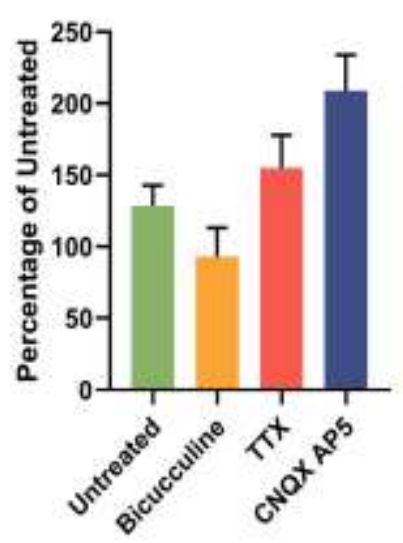

Bicuculline

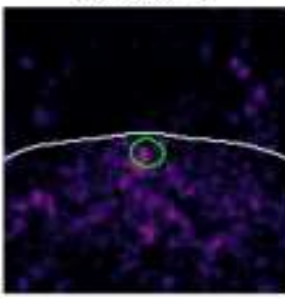

CNQX + AP5
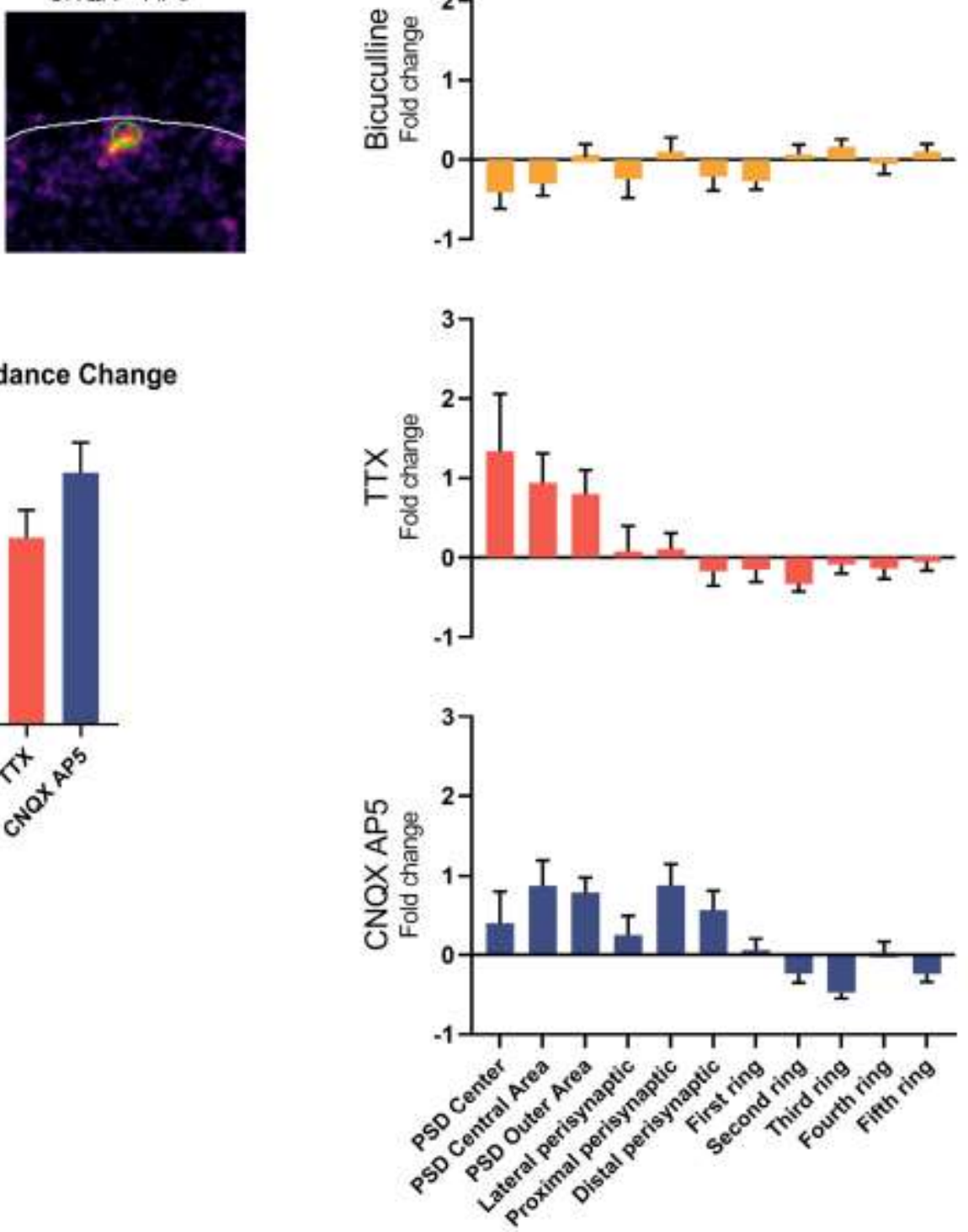


\section{GluR1}

This AMPAR subunit was differentially regulated between mushroom and stubby synapses. Mushroom spines decreased the abundance and PSD localization of GluR1 after all treatments. As expected, stubby synapses showed an increase in GluR1 after CNQX+AP5 treatment, which was also accompanied by a recruitment to the PSD.

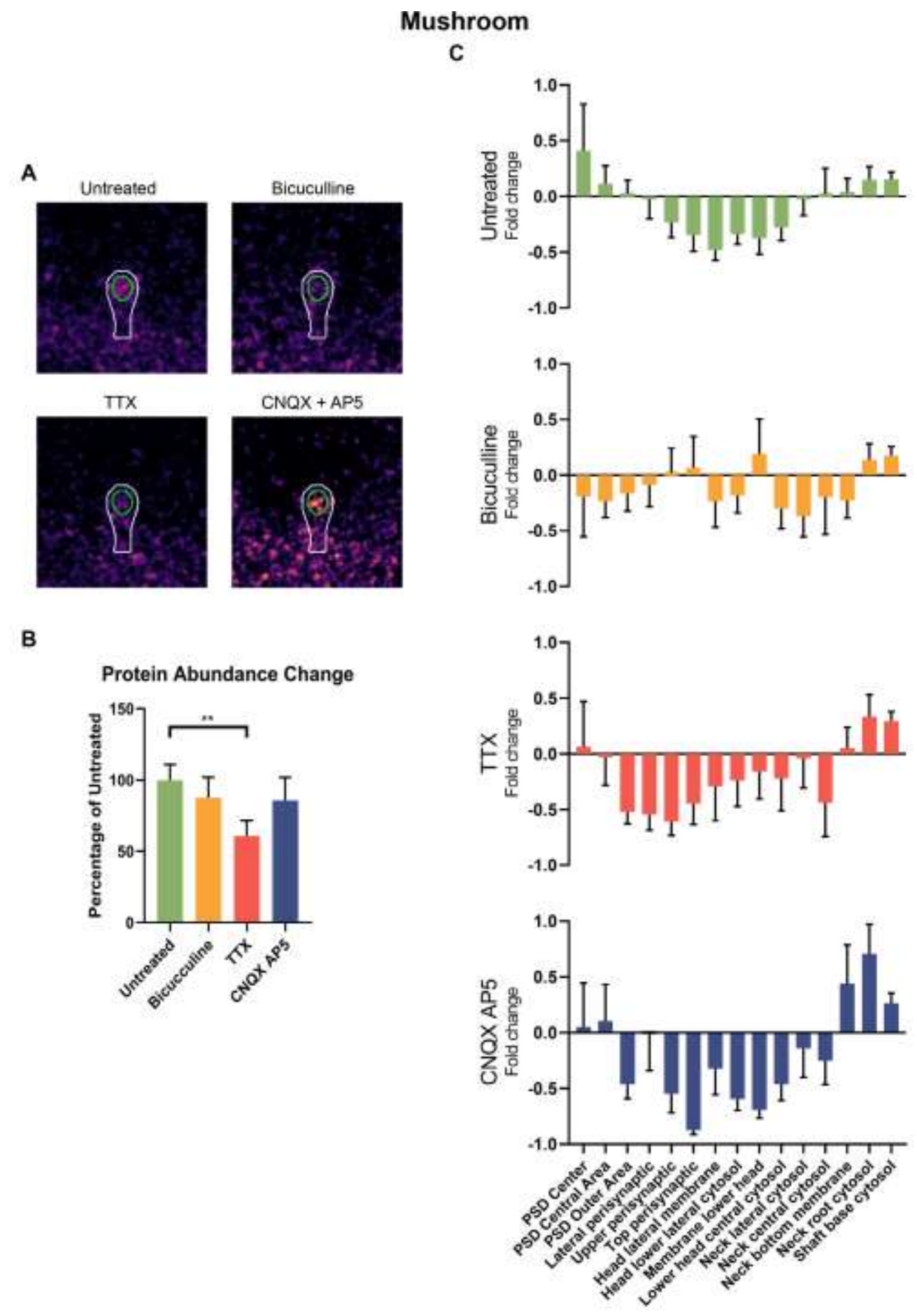

Figure 127: Changes in GluR1 localization and abundance after synaptic scaling. The figure is organized as in Figure 124. Continued on next page. 


\section{Stubby}

c
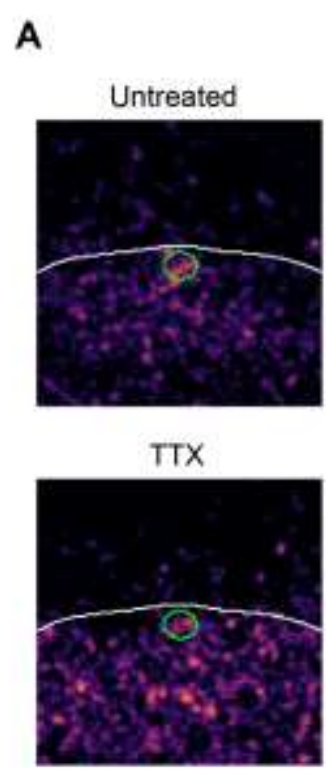

B

Protein Abundance Change

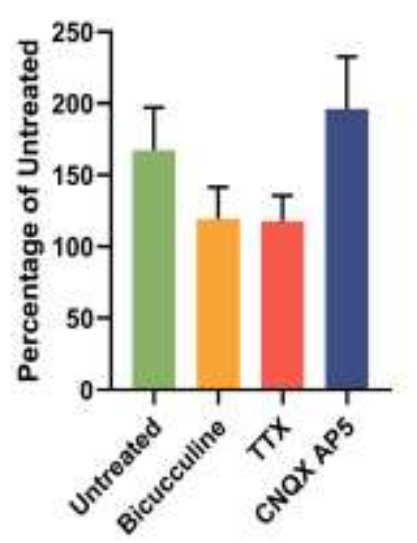

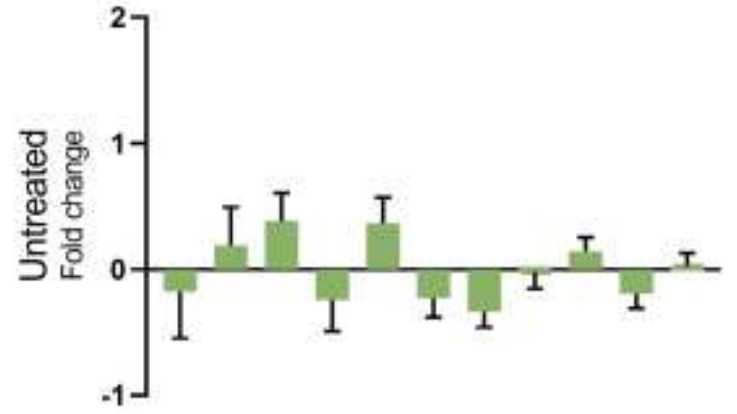
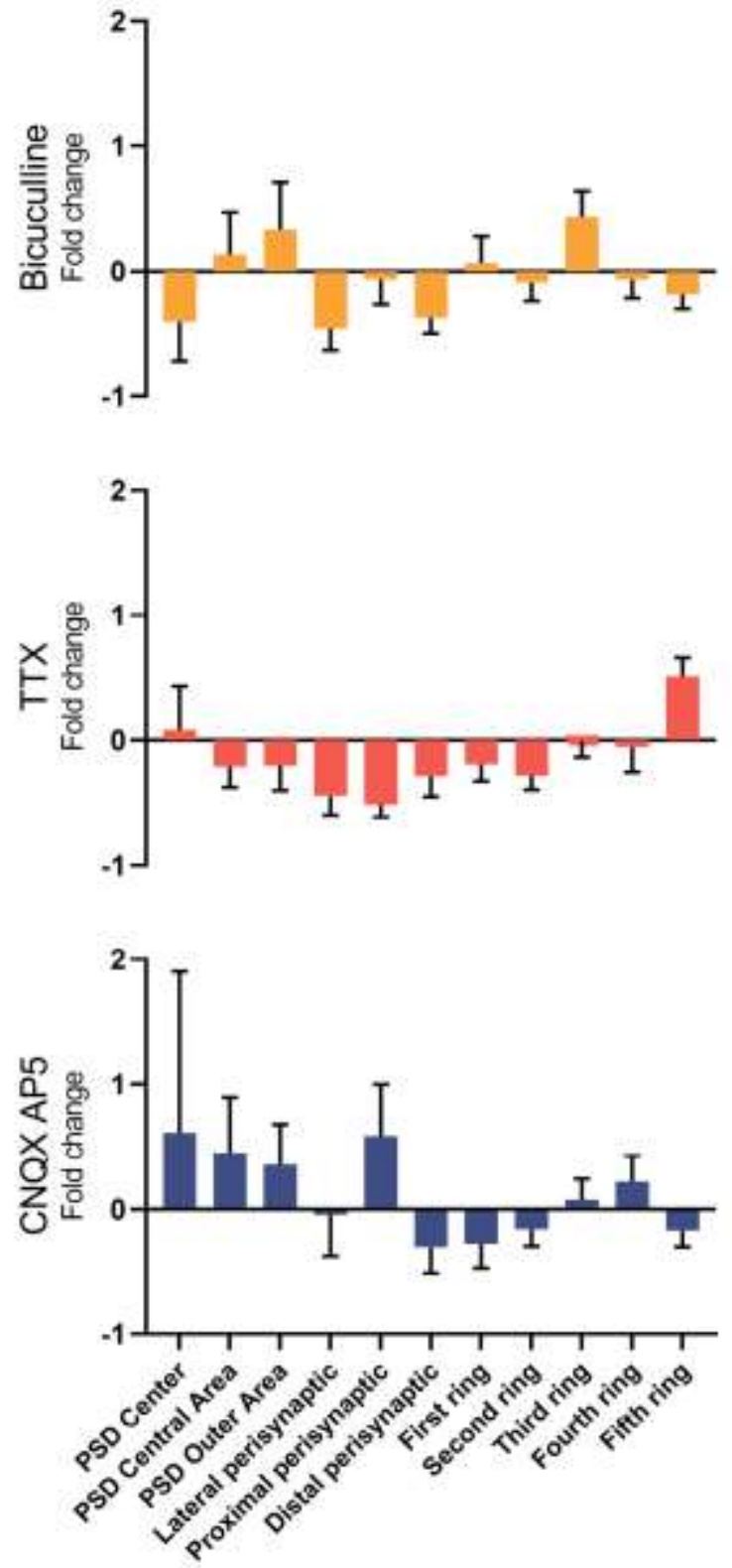


\section{GluR2}

This constitutively cycling AMPAR did not change its localization in any treatment. Surprisingly, it was significantly increased after bicuculline treatment in mushroom spines, but the increase itself was very minor.

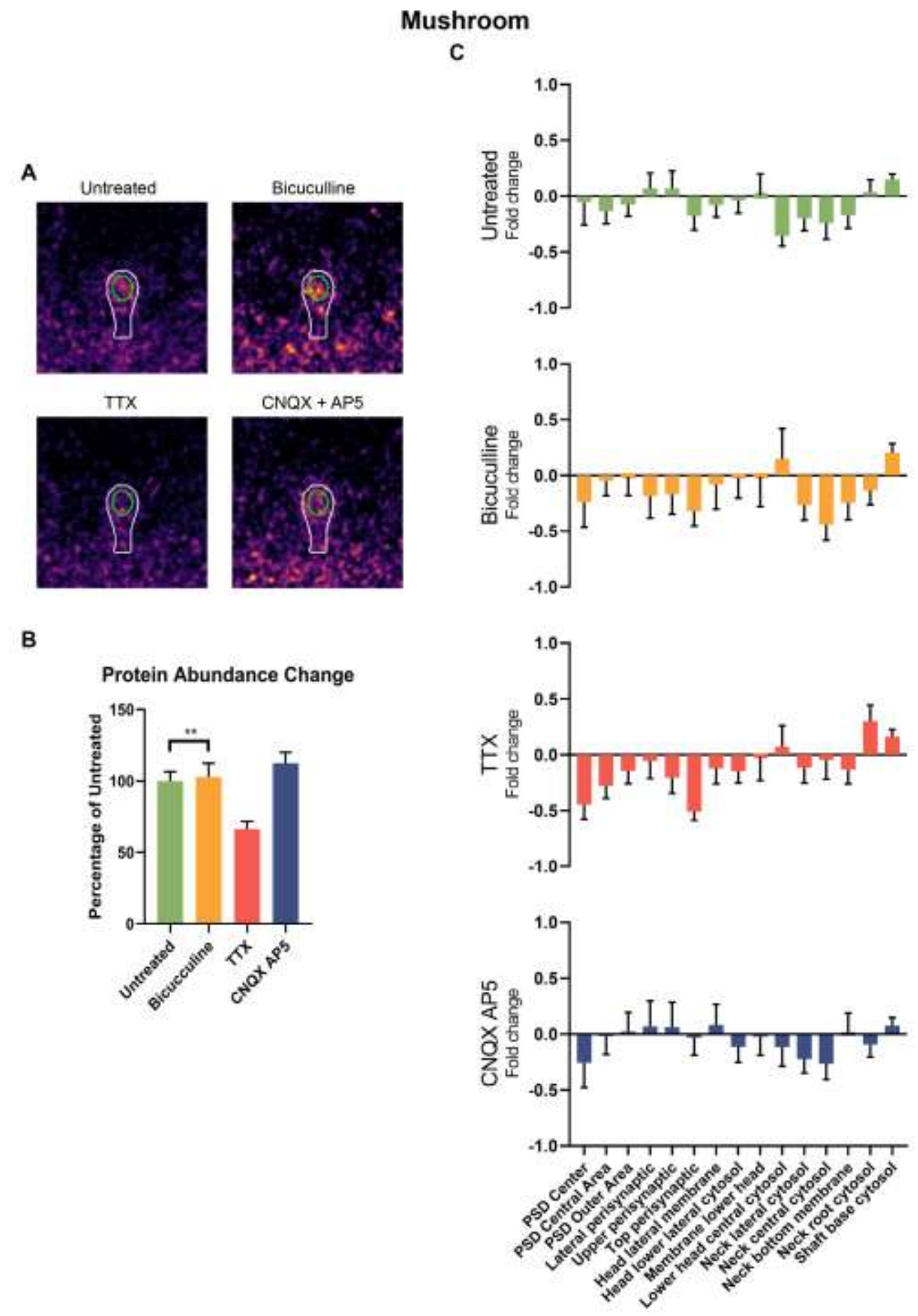

Figure 128: Changes in GluR2 localization and abundance after synaptic scaling. The figure is organized as in Figure 124. Continued on next page. 


\section{Stubby}

C
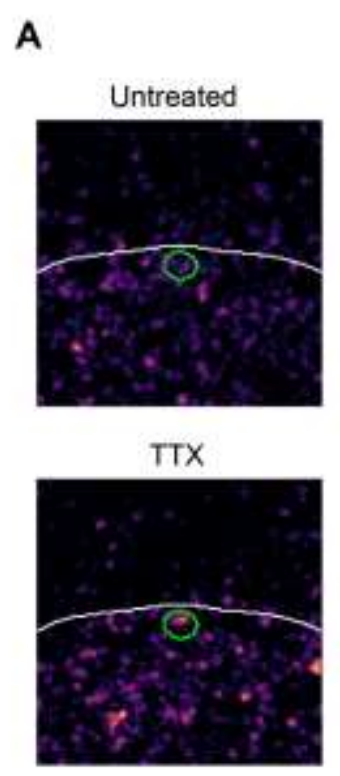

B

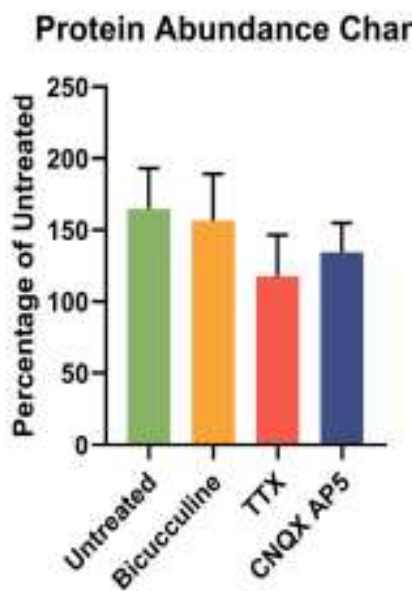

Bicuculline

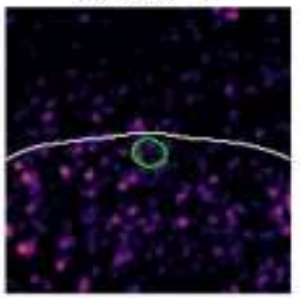

CNQX + AP5

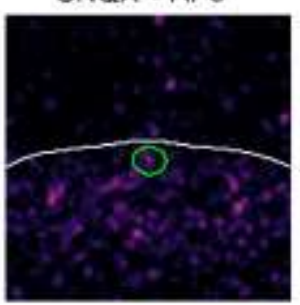

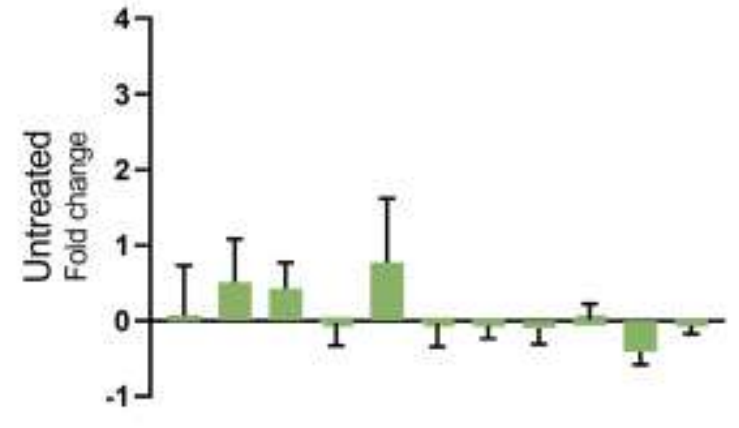
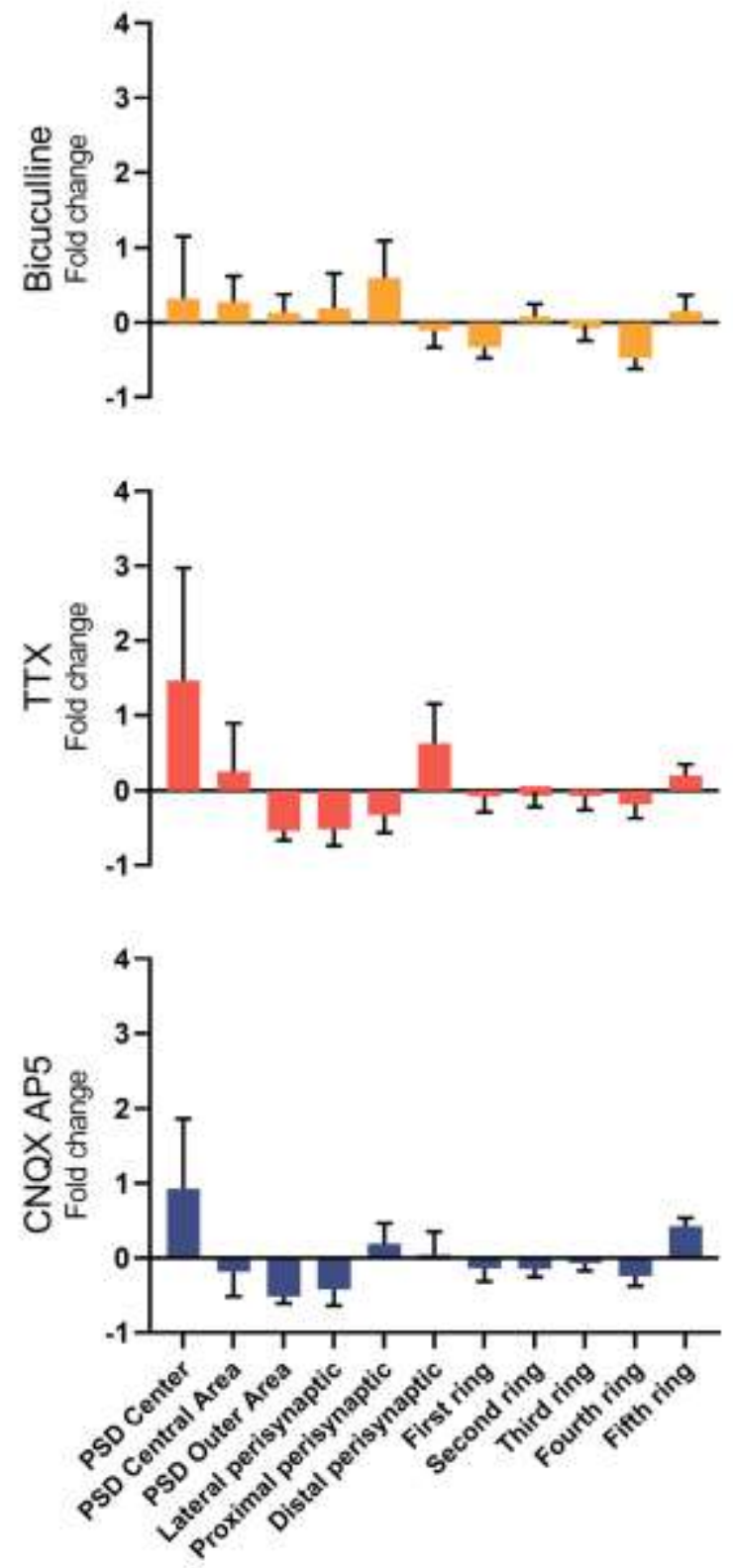


\section{mGluR5}

This metabotropic glutamate receptor showed a dramatic increase in abundance for TTX and CNQX+AP5 treated neurons, and also for bicuculline treated stubby synapses. Interestingly, its localization was not changed dramatically in both conditions. Synaptic downscaling via bicuculline also showed a trend toward increasing mGluR5.

\section{Mushroom}

C

A

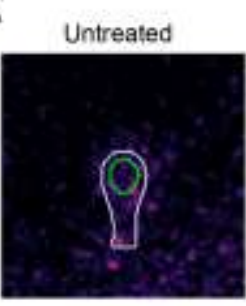

TTX

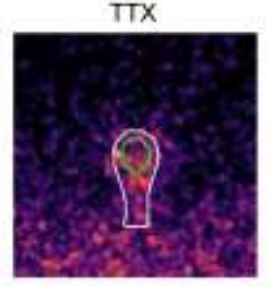

B

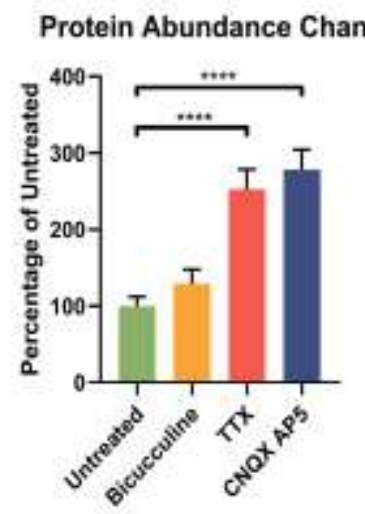

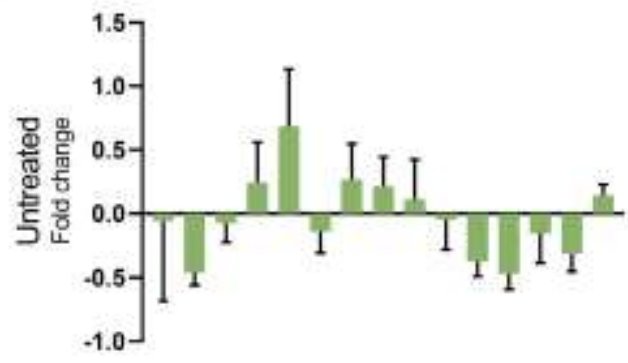
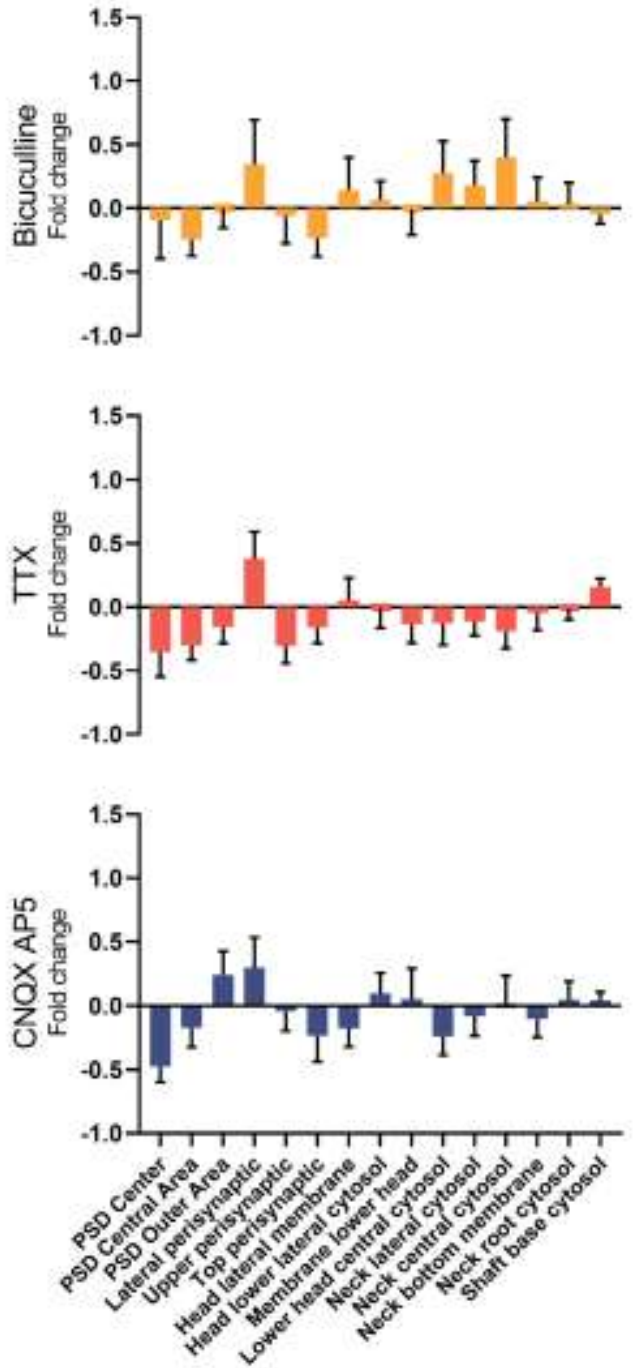

Figure 129: Changes in mGluR5 localization and abundance after synaptic scaling. The figure is organized as in Figure 124. Continued on next page. 


\section{Stubby}

$c$
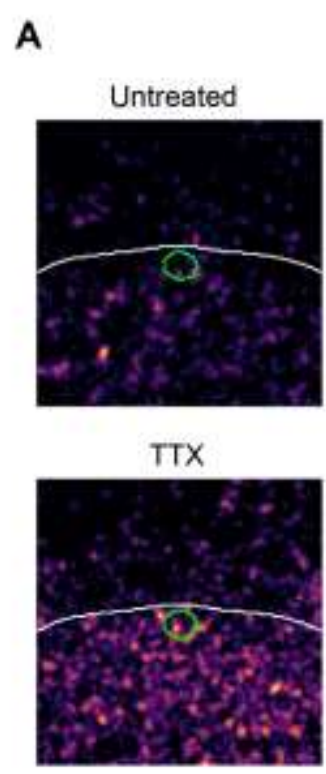

B
Bicuculline

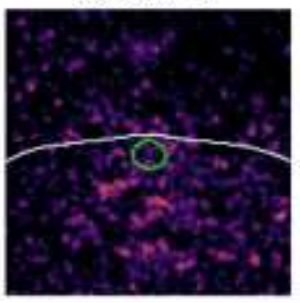

CNQX + AP5

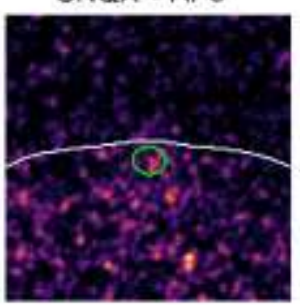

Protein Abundance Change

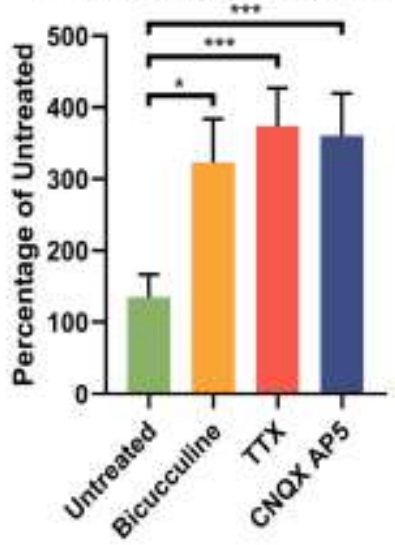

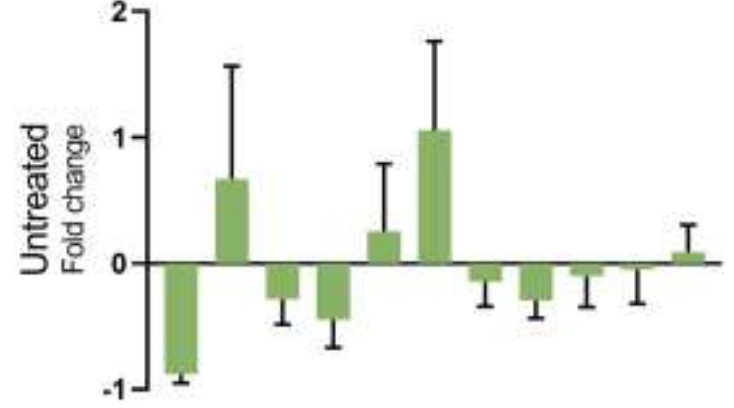
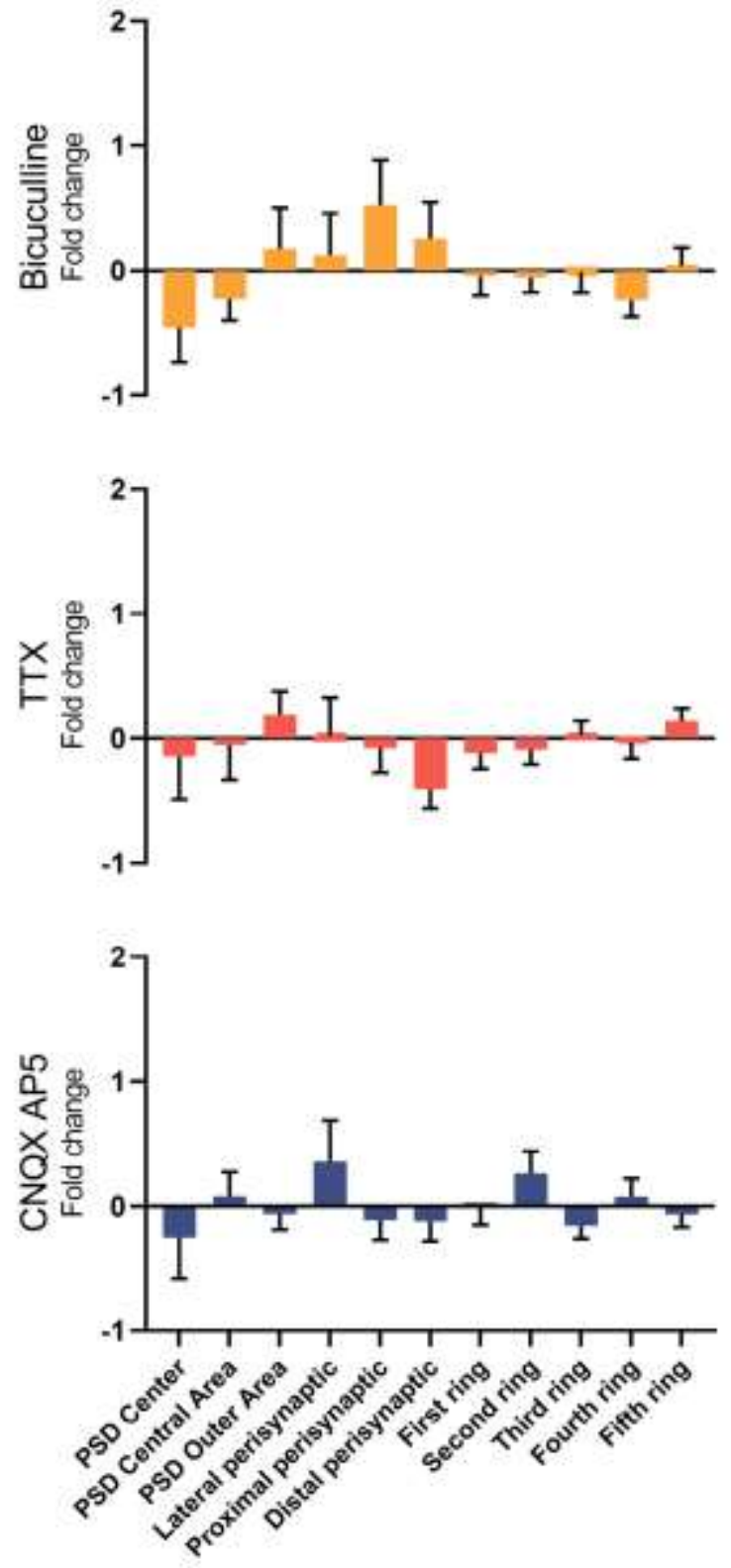


\section{SNAP47}

This SNARE protein exhibited an interesting phenotype in bicuculline treated mushroom spines, where its abundance was decreased, but it was highly localized to the PSD. A similar effect was observed in TTX treated stubby synapses. Although it was increased in CNQX+AP5 treated cells, this effect did not reach significance.

\section{Mushroom}

A

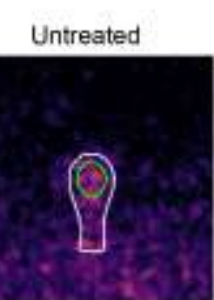

$\pi x$

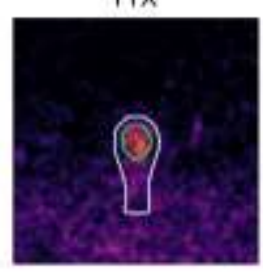

CNOX + APS

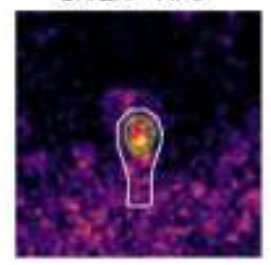

B

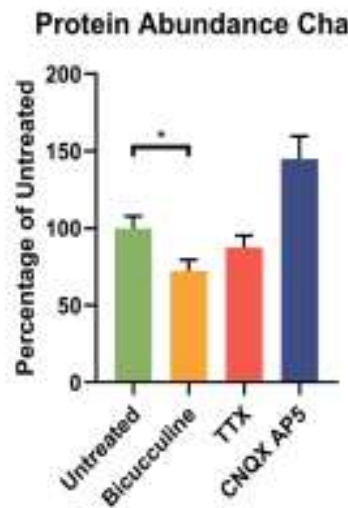

C
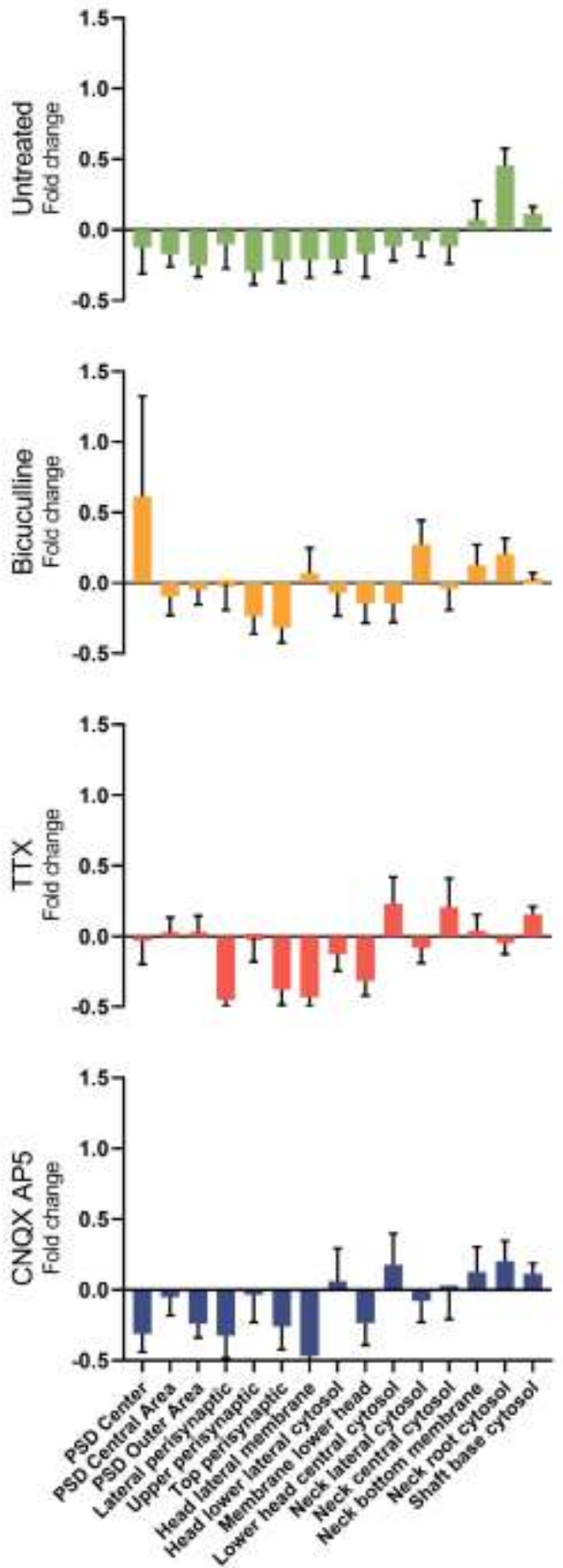

Figure 130: Changes in SNAP47 localization and abundance after synaptic scaling. The figure is organized as in Figure 124. Continued on next page. 


\section{Stubby}

$c$
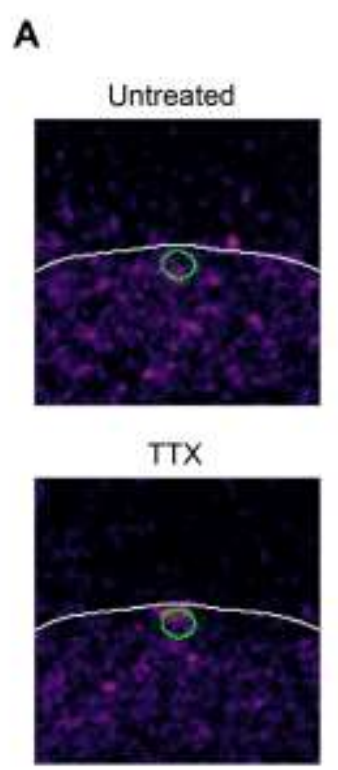

B

Protein Abundance Change

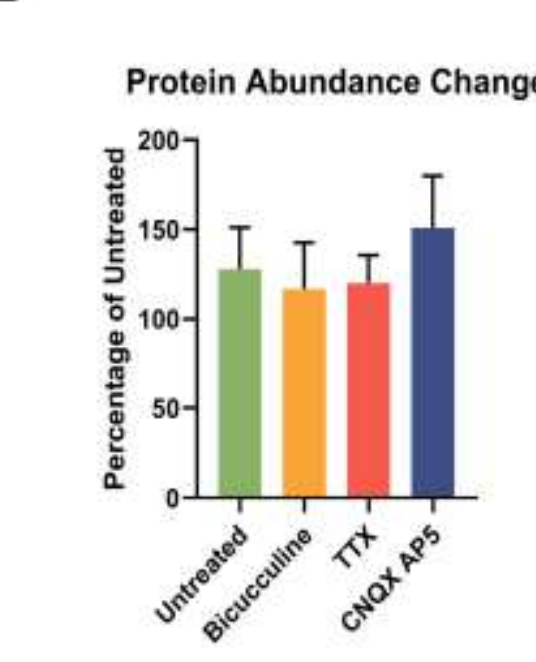

Bicuculline

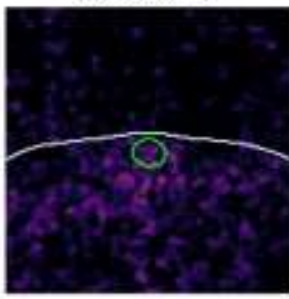

CNQX + AP5

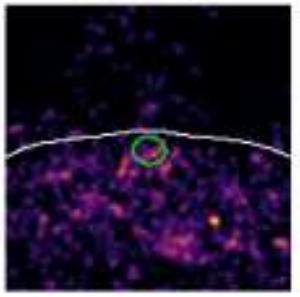

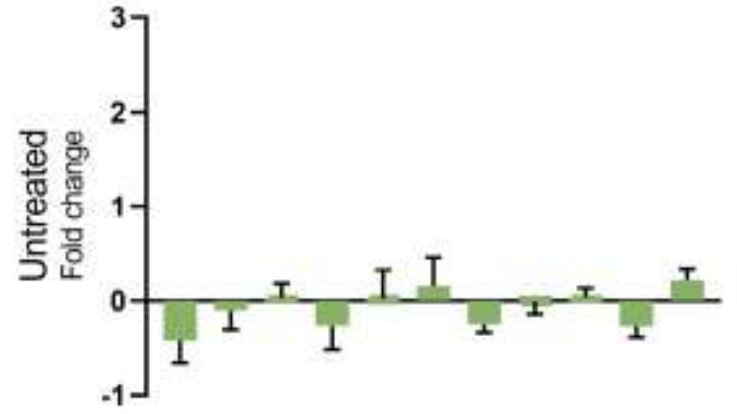
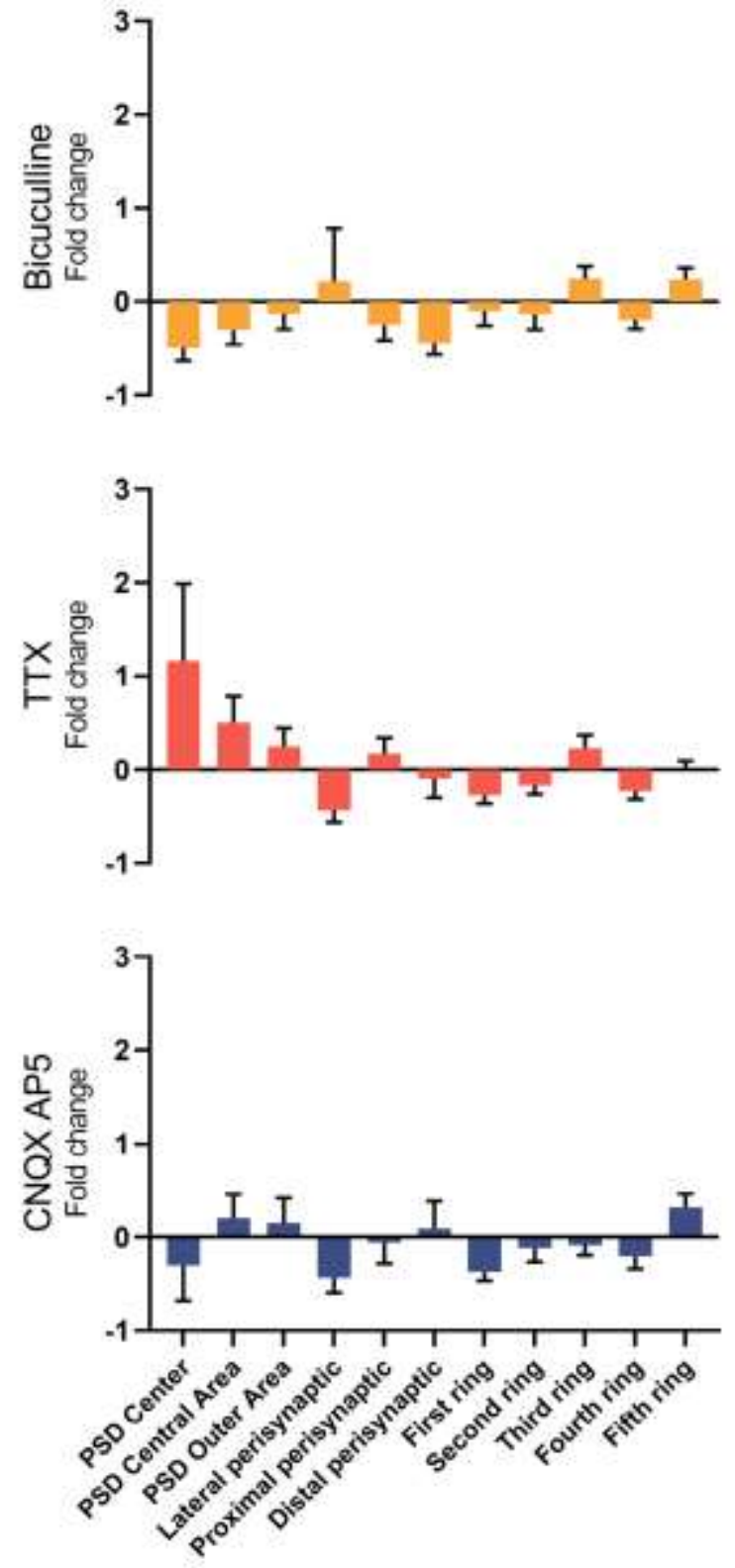


\section{Tubulin}

Tubulin showed a very consistent localization, that did not change during the treatments. Its abundance was increased after TTX or CNQX+AP5 treatment, although this effect was not significant in mushroom spines.

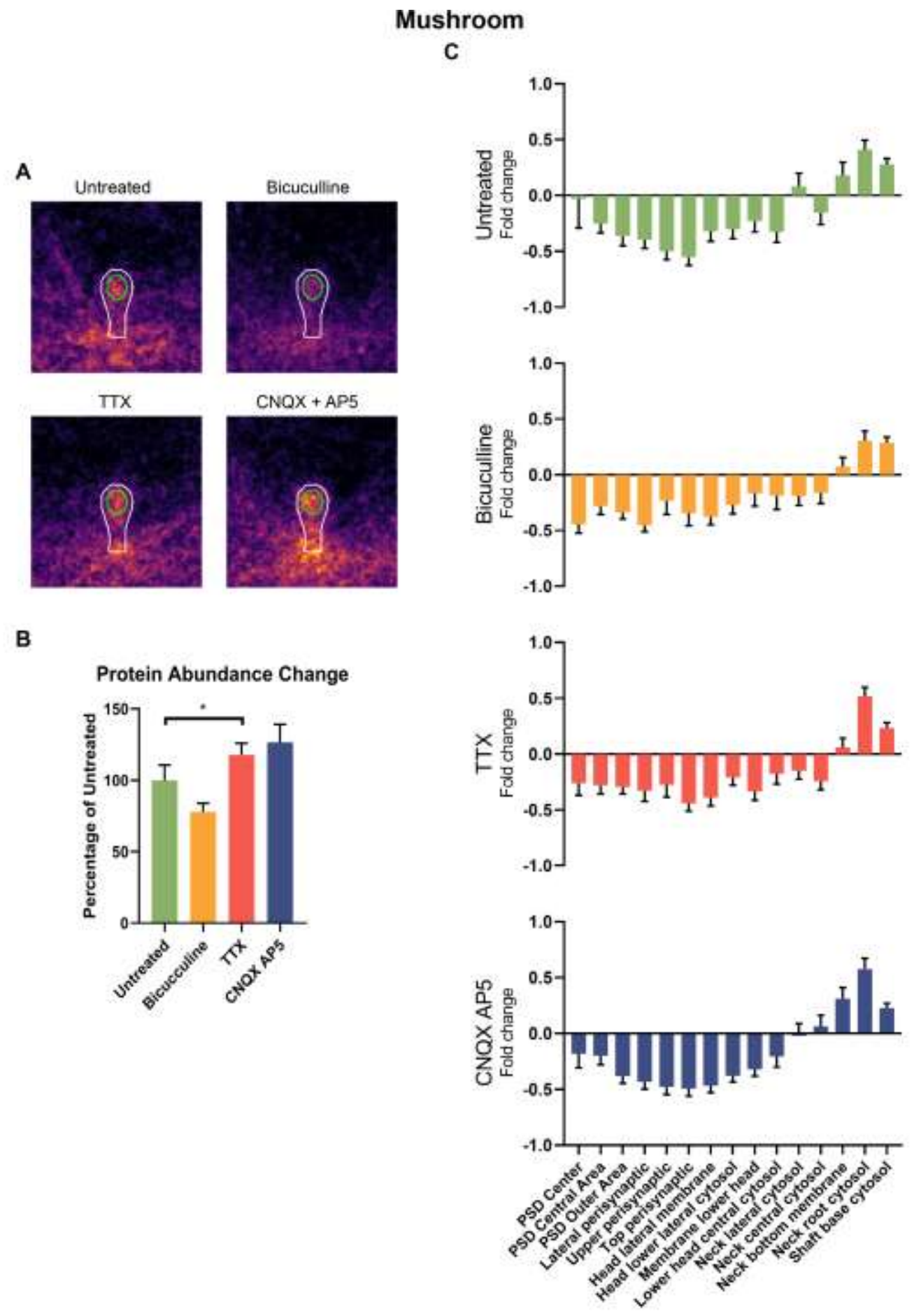

Figure 131: Changes in tubulin localization and abundance after synaptic scaling. The figure is organized as in Figure 124. Continued on next page. 


\section{Stubby}

C

A

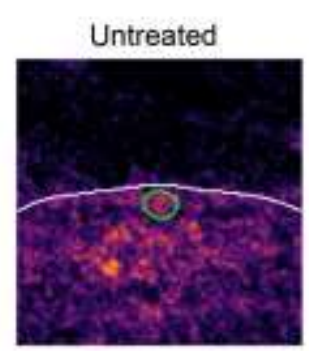

Bicuculline
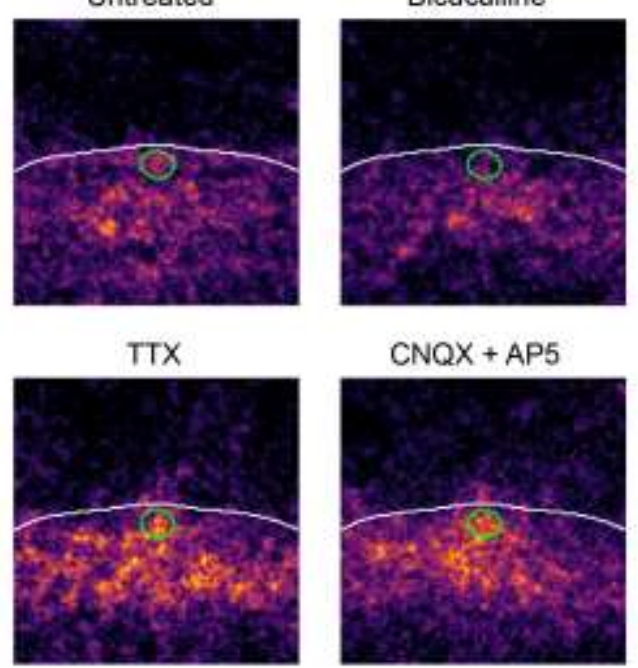

B

Protein Abundance Change

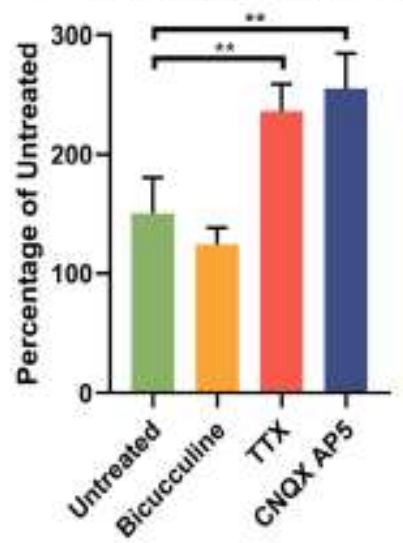

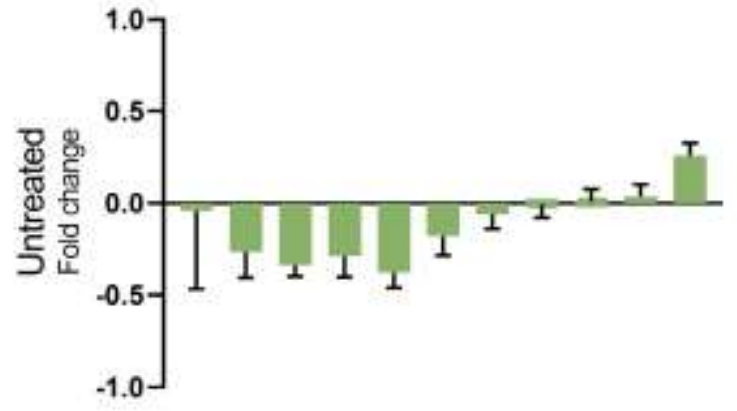
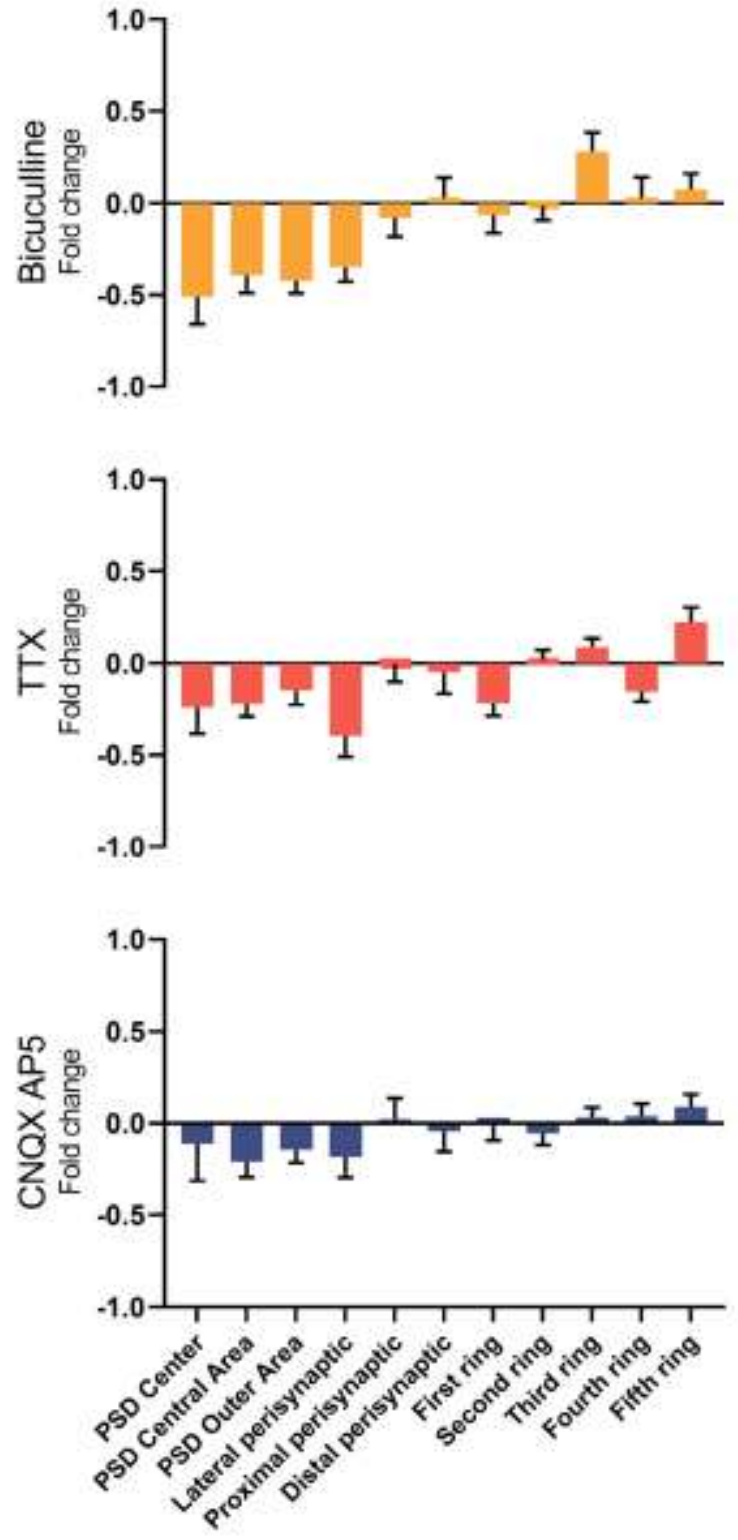


\section{Discussion}

In this thesis, I present a quantitative analysis of dendritic spines at an unprecedented level of detail. To my knowledge this is the most comprehensive database on this organelle to date. There are several readily observed results in this thesis:

1) Mushroom and stubby spines in vitro are almost identical in most of the measured parameters of their ultrastructure.

2) Most proteins showed similar copy numbers and localization in both spine types, probably to ensure efficient synaptic transmission and to avoid bottle necks. For example, interacting SNARE proteins of are present at similar amounts, whereas receptors show low abundance. This also argues for a conserved and probably modular architecture of the postsynapse.

3) I report two large quantitative databases, the dendritic proteome with nanoscale localization, which I termed the Dendrite Nanomap, as well as the neuronal proteome, providing absolute quantification of over 6000 proteins. These databases lay the foundation for future modeling studies, where it is essential to use the correct initial protein concentrations and locations for results, resembling the in vivo dendritic spine

\section{The ultrastructure of spines in culture}

To build an accurate model of the dendritic spine in culture, I needed to first study its morphology. Most ultrastructural research has been done on brain samples, with only few works focusing on neuronal cultures (Boyer et al., 1998; Schikorski and Stevens, 1997). Because these studies pooled shaft and spine synapses, I set out to analyze the postsynaptic ultrastructure for both classes separately.

\section{Mushroom and stubby spines have similar morphology}

Surprisingly, the two different spine classes analyzed did not show any significant differences in the parameters I calculated. The only difference was the correlation of stubby PSD Area to volume, which was not present in mushroom spines. This is at odds with previous studies observing this correlation, albeit in vivo (Boyer et al., 1998; Harris and Stevens, 1989). Because the brain is densely packed with synapses, they might compete for space. Since the cultures are much sparser, this restriction is not present and might explain the uncorrelated morphology of spines. Additionally, astrocytes enclosing the synapse might also regulate the size. The increased fraction of PSD area to surface of stubby synapses over mushroom spines is probably due to the neck of mushroom spines. These add surface, while not containing any PSD. 


\section{Dendritic spines in culture are similar to those in vivo}

The parameters I measured are like those derived from in vivo samples, albeit sometimes higher than previously reported cell culture data (Table 11). The difference in the cell culture data might be explained by different culturing protocols (although both data are from Banker type cultures), especially the density at which the neurons are plated influences spine number and probably also spine size (Cullen et al., 2010). Unfortunately, the number of neurons plated in the work from Boyer and colleagues is given, but not the area on which they are plated (Boyer et al., 1998). I can therefore not compare the densities. Also, the network activity will influence the structure of the formed synapses (Alvarez and Sabatini, 2007).

In the cultures I found an almost 1:1 ratio of mushroom to stubby spines, similar to what has been reported for hippocampal cultures before (Boyer et al., 1998). This high fraction of stubby synapses is reminiscent of the situation in the immature brain, where the majority of synapses is formed on stubby spines, while the adult brain only has a small minority of stubby postsynapses (Harris et al., 1992). Thus, the culture likely reflects an intermediate state. Because I report a separate model for each class, one can apply it to immature as well as adult brains.

Still, even in brain samples there is a high variability of spine morphology, from which my model does not differ too much. Therefore, the model I derive here for hippocampal cultures may also be applicable to in vivo dendritic spines. At any rate, more parameters of the situation in vivo could be derived by comparing Banker cultures to slices, using comparative imaging (Richter et al., 2018) 
Table 11: Comparison of measured spine morphology to previously published cell culture and in vivo data. Mean $\pm S D$; Data extracted from a(Schikorski and Stevens, 1997), b(Boyer et al., 1998), ' (Spacek and Harris, 1997), ${ }^{d}$ (Harris and Stevens, 1989) and ${ }^{\mathrm{e}}$ (Harris et al., 1992).

\begin{tabular}{|c|c|c|c|c|c|}
\hline & \multicolumn{2}{|c|}{$\begin{array}{c}\text { This study } \\
\end{array}$} & \multirow{2}{*}{$\begin{array}{l}\text { Cell culture } \\
\text { combined }\end{array}$} & \multicolumn{2}{|c|}{ Brain } \\
\hline & Mushroom & Stubby & & Mushroom & Stubby \\
\hline PSD Area $\left[\mu m^{2}\right]$ & $\begin{array}{l}0.106 \pm 0.06 \\
6\end{array}$ & $\begin{array}{l}0.142 \pm 0.09 \\
0\end{array}$ & $\begin{array}{l}0.028 \pm 0.020 \\
\text { a }\end{array}$ & $\begin{array}{r}0,043 \pm 0,031 \\
a \\
0.48^{\mathrm{c}} \\
0.069 \pm 0.08^{\mathrm{d}} \\
0,21 \pm 0,10^{\mathrm{e}}\end{array}$ & $\begin{array}{r}0.19^{c} \\
0,15 \pm 0,09^{e}\end{array}$ \\
\hline $\begin{array}{l}\text { Surface Area } \\
{\left[\mu \mathrm{m}^{2}\right]}\end{array}$ & $\begin{array}{l}1.513 \pm 0.74 \\
3\end{array}$ & $\begin{array}{l}1.387 \pm 0.50 \\
9\end{array}$ & & $\begin{array}{r}2.98^{\mathrm{c}} \\
0.83 \pm 0.63^{\mathrm{d}} \\
1.50 \pm 0.62^{\mathrm{e}}\end{array}$ & $\begin{array}{r}1.95^{\mathrm{c}} \\
0,95 \pm 0,48^{\mathrm{e}}\end{array}$ \\
\hline $\begin{array}{l}\text { Fraction PSD } \\
\text { of Surface Area }\end{array}$ & $6.6 \pm 1.4 \%$ & $9.2 \pm 3.2 \%$ & & $\begin{array}{r}16.11 \%^{\mathrm{c}} \\
8,31 \%^{\mathrm{d}} \\
14 \pm 3 \%\end{array}$ & $\begin{array}{r}9.74 \%^{c} \\
16 \pm 3 \%^{\mathrm{e}}\end{array}$ \\
\hline Volume $\left[\mu \mathrm{m}^{3}\right]$ & $\begin{array}{l}0.189 \pm 0.15 \\
8\end{array}$ & $\begin{array}{l}0.179 \pm 0.10 \\
9\end{array}$ & $\begin{array}{l}0.058 \pm 0.034 \\
\mathrm{~b}\end{array}$ & $\begin{array}{r}0,038 \pm 0,036 \\
a \\
0.43^{\mathrm{c}} \\
0.062 \pm 0.08^{\mathrm{d}} \\
0.18 \pm 0.09^{\mathrm{e}}\end{array}$ & $\begin{array}{r}0.24^{\mathrm{c}} \\
0,11 \pm 0,07^{\mathrm{e}}\end{array}$ \\
\hline $\begin{array}{l}\text { Vacuole } \\
\text { volume }\left[\mu \mathrm{m}^{3}\right]\end{array}$ & $\begin{array}{l}1.088 \pm 2.10 \\
2\end{array}$ & $\begin{array}{l}1.139 \pm 1.95 \\
9\end{array}$ & & $\begin{array}{r}0.0093^{c} \\
\text { (only spine } \\
\text { apparatus) }\end{array}$ & $\begin{array}{r}0.0083^{c} \\
\text { (only spine } \\
\text { apparatus) }\end{array}$ \\
\hline
\end{tabular}




\section{The quantitative neuronal proteome reveals general regulatory principles}

After investigating the ultrastructure of the spines in culture, I determined the quantitative proteome of neurons in culture. To do so, I used a label-free, quantitative mass spectrometry approach. This work is the most comprehensive description of postsynaptic copy numbers in absolute terms. A landmark study of the mouse brain proteome detected 7792 proteins, comprising neurons and glia (Wang et al., 2006). I detected 6194 protein groups, but from a pure hippocampal culture. To my knowledge, this is the most comprehensive mass spectrometry dataset of this model system to date. This is a significant advance in quantitative proteomics of rat neurons, as so far only few neuronal proteins have been counted in absolute numbers, most studies rather look at relative changes between treatments. But it is equally important to also know the absolute abundance of a protein. This allows us, for example, to estimate which concentrations of pharmacological interventions are reasonable, or identify which proteins might pose bottle necks for a specific process in the cell. Because the scope of this thesis was on the dendritic spine, no detailed analysis on the whole-cell copy numbers was performed.

During this study, I tried to estimate several key parameters, such as protein molarity for the neurons. This was difficult, because no accurate measurements of the volume of neurons in culture are available. I projected the neuron volume to be $5000 \mu^{3}$, which is on the upper end of mammalian cell cultures (Bohil et al., 2006; Cohen and Studzinski, 1967; Krombach et al., 1997; Luby-Phelps, 2000; PUCK et al., 1956; Zhao et al., 2008). Similarly, the protein concentration or molarity of neurons is not known. My calculations are above what one would estimate from bacteria or cell culture data (Molarity of $20.35 \mathrm{mM}$ vs $4.98 \mathrm{mM}$; $2.2 \mathrm{ng}$ per cell vs. 0.3 ng/cell; Milo, 2013; Wiśniewski et al., 2014). Again, the morphology of cell cultures or bacteria is much simpler than neurons, making this comparison difficult. It is surprising how little we still know about the basic morphology of this common model system, and we urgently need accurate quantifications for further studies.

\section{Subcellular copy numbers show a highly skewed distribution}

Using GO analysis, the proteome quickly revealed that the mean and median copy numbers differ between subcellular compartments. While nuclear proteins showed the highest mean copy number, this was not the case when the median was analyzed. There, mitochondrial proteins were significantly higher than any other compartment, which showed similar median copy numbers. This reveals several things:

First, all compartments showed a smaller median than mean copy number, indicative of a skewed distribution towards higher values (i.e. there are few proteins for each compartment 
that are extremely high abundant, compared to the rest). This was most pronounced in the nucleus. Second, several nuclear proteins are known to be present in extremely high numbers, such as histones. These were also very abundant in my dataset, and together with the cytoskeletal proteins in the nucleus caused this skewedness. Third, the high average copy number in the cytoplasm is mainly caused by cytoskeletal proteins, as well as several highly abundant membrane proteins, such as VAMP2 and SNAP25.

On the other hand, the higher median for mitochondrial proteins also suggests that fewer proteins with low copy numbers are present. I could reliably observe this when analyzing the distribution of the proteins, where the lower tail of the mitochondria was decreasing continuously. One of the main functions of mitochondria is to produce ATP, they provide roughly $93 \%$ of ATP in the nervous systems (Harris et al., 2012). To do so, it needs high numbers of the involved proteins. Most of the proteins that are within the mitochondria are produced in the cytoplasm of the cell first and are then inserted into the mitochondria, with only a handful of proteins translated in mitochondria themselves (Dudek et al., 2013). Also, many of the proteins within mitochondria interact with each other to form the supercomplexes of the respiratory chain. This probably explains why the copy numbers in mitochondria are closer together than in the other compartments. Conversely, many different mechanisms are running in parallel in the other compartments, which have very different requirements regarding their copy number. For example, cytoskeletal proteins need to be present in high amounts to regulate the morphology and enable efficient trafficking, whereas receptors can be present at low number and still fulfill their function. This causes a higher spread of the distribution, and the imbalance in the copy numbers required for the different processes increases the skewedness.

Finally, I observed an interesting copy number distribution for ER and Golgi proteins, which showed a distinct population of proteins at lower levels, roughly between $10^{3}$ and $10^{5}$ copies per cell. It would be interesting to study which proteins comprise this population and whether they have common functions or protein motifs.

\section{The copy number decreases with protein size}

I confirmed a weak negative correlation of protein copy number to its size (Mandad et al., 2018). As possible interpretation lies in the energy efficiency of the cell. As protein synthesis is an energy-intensive process, it is highly regulated. Still, proteins do not always fold correctly after translation and then need to be degraded again (Chen et al., 2011). The longer a protein gets, the higher the lost energy is. Therefore, it is favorable to only produce long proteins when they are really needed. Usually, translation is one of the highest energy consuming processes in cells, mammalian cells use about $20 \%$ of their energy for protein biosynthesis (Rolfe and 
Brown, 1997). This ratio is decreased in neurons, probably because they are postmitotic and therefore do not need to reproduce proteins they lost after division. It would therefore be interesting to study, whether the correlation of protein size to copy number is higher in dividing cells. Another explanation is, that larger proteins have higher chance to accumulate damage. Their larger surface exposes them to more potentially hazardous interactions, also leading to the observed phenotype.

\section{The quantitative neuronal proteome as a tool}

Since hippocampal neuronal cultures are extensively used to study neuronal function, this database itself is of great interest to the neuroscience community. There are several interesting questions that one could investigate, using this dataset:

\section{Are proteins in the same pathway correlated?}

For the presynapse it has been shown that proteins involved in the same pathway are often correlated in their abundance (Wilhelm et al., 2014). It would be interesting to study whether this is a common regulatory principle within the whole neuron as well. From the cellular copy numbers, one can also estimate which proteins might be potential bottlenecks of a given molecular function. Equally, I already set up unsupervised learning algorithms to cluster proteins that show similar localization. Following the hypothesis that proteins that show similar distributions are involved in the same pathway, these data could for example indicate which SNAREs are working together in the dendritic spine.

\section{What are neuronal copy numbers in other compartments?}

Using the whole-neuron dataset, one can easily extend the calculation of subcellular copy numbers to other organelles, as the epifluorescence imaging is fast and could also be automatized. Any other sub-compartment that has good markers available could in principle be studied. This included the membrane enclosed organelles, such as mitochondria, ER, Golgi apparatus or the nucleus. Also, finer structures, such as the axon initial segment could be investigated, because my method does not rely on fractionation.

\section{How strong is the molecular crowding effect in neurons?}

The cytoplasm of cells is very dense with proteins, which influences diffusion, translation, chromosome condensation and cell shape (Banks and Fradin, 2005; Miyoshi and Sugimoto, 2008). This effect was termed 'molecular crowding' and its influence on the regulation of neurons has not been studied fully. Using my dataset, a general density of the neuron could be calculated at high fidelity, enabling an unprecedented view inside cells. Apart from the physical barrier proteins can pose, their hydrophobicity can also cause them to form droplet like phases (Li et al., 2012; Shin et al., 2017; Zeng et al., 2016). Taking advantage of coarse- 
grained simulations, one could study the formation of these phases and how they influence the structuring of the cytosol (McGuffee and Elcock, 2010).

\section{The dendritic proteome}

Although dendritic spines have been studied for decades, we only have scant reports of postsynaptic copy numbers. These mostly encompassed receptors or ion channels studied by electrophysiological methods or imaging approaches, both of which are very labor intensive (Chen et al., 2005; Nusser et al., 1998; Sabatini and Svoboda, 2000; Sugiyama et al., 2005). Several semiquantitative mass spectrometry studies have been published, but they only describe relative amounts and often are restricted to PSD fractions (Cheng et al., 2006; Distler et al., 2014; Peng et al., 2004b; Schanzenbächer et al., 2016, 2018). As the name implies, this fraction is comprised of the PSD core and some postsynaptic membrane clinging to the scaffold. The dendritic cytosol or peri- to extrasynaptic membranes are completely missing, which is a major limitation of these studies, also revealed by the infrequent reports of membrane-associated proteins in the current literature (Collins et al., 2006). This is the reason why I used the combination of imaging and mass spectrometry. Still, there are some caveats of my analysis:

Because I use the $\mathrm{R}^{2}$ of the synaptic region, the copy numbers I report have the highest confidence for proteins that are present in the spine head at least to some extent. This limits my analysis for proteins that are usually found only in the dendritic shaft, as can be seen from the low copy number of TOM20. There are two ways one could address this: One could estimate the intensity distribution from the super-resolution images to calculate how much protein is in the synapse versus the shaft and extrapolate the shaft copy numbers. Alternatively, I already measured the enrichment of synapse to shaft for each protein by marking a shaft region during the synapse alignment. This value could also be used to estimate the abundance in the shaft.

Proteins that are only present in subpopulations of dendritic spines also show reduced copy numbers due to the averaging. For example, several of the calcium binding proteins are known to be present in only a fraction of neurons, which, in part, explains their low abundance. This is a difficult issue to address. Ideally, one would have an additional marker for these populations. As most imaging setups can easily use 4 different colors this would be feasible but searching for these subpopulations will increase the imaging time profoundly.

Another interesting phenomenon is the general increase of protein abundance in stubby to mushroom class spines (Figure 12C). How does this come about? There are two explanations: 
First, stubby synapses were larger, with the ratio of the median volumes being 1.34. This is already very close to the observed 1.44 -fold increased copy numbers in stubby synapses. Additionally, the epifluorescence imaging might analyze some additional dendritic shaft volume for stubby synapses, but not for mushroom synapses. Synapses on mushroom class spine are most of the times sufficiently apart from the dendritic shaft that only the synaptic region plus the thin neck is picked up during imaging. The remaining area around the mushroom is usually devoid of other cellular structures (except for the associated presynapse). Stubby synapses on the other hand are, by definition, very close to the shaft. Therefore, some signal from the shaft is picked up, because of the low lateral and axial resolution. Thus, the synaptic copy number reported for stubby synapses probably present a slight overestimation.

Intriguingly, postsynaptic copy numbers seem to correlate with their presynaptic counterpart (Figure 12B). The postsynaptic proteins showed a general trend to be less abundant than in the presynapse. Again, the observed volume differs, with the synaptosome volume being 2.43 fold larger than the postsynaptic (Wilhelm et al., 2014). Because the slope of the linear regression is only 0.72 , this suggests that the postsynaptic protein density is higher than the presynaptic. Also, I studied hippocampal neurons, whereas the presynaptic numbers were derived from cortical synaptosomes, which one should keep in mind for this comparison. Probably, the observed correlation is caused by common pathways operating in both compartments. For example, microtubules and their associated transport machinery are present in both compartments, also endosomal recycling takes place at both sides of the synapse. Still, several proteins are specifically enriched in one compartment. For example the SNARE proteins responsible for SV release are present in much higher copies in the presynapse than they are in the postsynapse, whereas SNAP23, that has been described to be mostly postsynaptic, also shows this behavior here (Suh et al., 2010). 


\section{The Nanomap is a window to dendritic spines at unprecedented detail}

\section{Cytoskeleton and associated proteins}

Actin and tubulin are the main cytoskeleton components. Many studies have shown that actin is primarily abundant in the head of mushroom spines, whereas tubulin is rarely invading into the spine head and is more prominent in the shaft (Chazeau and Giannone, 2016; Kaech et al., 2001). In line with these observations, I found actin to be highly enriched in the head and less abundant in the shaft, while tubulin was showing the opposite distribution. Mushroom spines showed a higher actin copy number than stubby class synapses, which is most likely due to their more elaborate and larger morphology. Because of the averaging, the periodic actin structure cannot be identified in the average images anymore. Interestingly, tubulin was even more abundant in the average dendritic spines than actin, but I found a much higher copy number in stubby class synapses than mushroom spines. This is probably due to the more shaft-like morphology of stubby synapses and in line with the observation, that tubulin is not often invading into mushroom spines (Jaworski et al., 2009). Surprisingly, I did not detect $\beta$-3tubulin to be present in the spines, due to its negative correlation with Homer1. This suggests that this specific tubulin isoform is excluded from the spines and not present on the dynamic microtubules invading the PSD.

The other important structural elements are intermediate filaments, consisting of the Neurofilament light, medium and heavy chain triplet, as well as $\alpha$-internexin. They have been largely thought to be presynaptic, but lately also postsynaptic functions have been identified (Jordan et al., 2004; Yuan et al., 2015a). In my work I did only detect low amounts of the neurofilaments, which were mostly present in non-PSD areas. These low amounts make the proposed lattice of $\alpha$-internexin with tubulin in the PSD unlikely (Suzuki et al., 2018). The high standard error comes from the fact, that the distribution correlation of $\alpha$-internexin with Homer was centered around 0 . This means that several synapses do not contain $\alpha$-internexin at all and also explains why neurofilaments have been often difficult to detect in the postsynapse. The lower abundance of Neurofilament $\mathrm{H}$, in comparison with Neurofilament $\mathrm{L}$, is in agreement with its late expression that mostly starts in postnatal stages (Benson et al., 1996; Shaw and Weber, 1982).

Drebrin, $\boldsymbol{\beta}$-2-spectrin and Cortactin are actin associated proteins. Drebrin mirrored the distribution of actin very well, showing a high enrichment in the PSD areas. It is present in high amounts in a 1:6 ratio to actin, which is very close to the reported 1:5 ratio reported from in vitro studies (Ishikawa et al., 1994). Therefore, most actin is probably bound by drebrin in dendritic spines, with the remaining actin molecules either being free actin, or interacting with 
other actin-binding proteins. The typical actin rings that shape the morphology of the dendritic shaft and neck use $\beta$-2-spectrin as spacers (Bär et al., 2016; Sidenstein et al., 2016; Xu et al., 2013). I found it to be mostly localized to the neck and shaft regions as well, but surprisingly it was present in very low numbers in the synapse only. This is most likely because the rings are largely absent from the spine head, which I use for the calculation of the copy numbers (Bär et al., 2016). Finally, Cortactin showed a very unspecific signal, with a de-enrichment in the PSD areas. This is in contrast to the studies by MacGillavry and colleagues, which found it to be highly enriched in the PSD (MacGillavry et al., 2016). Taking the low quality of my Cortactin images into account, my antibody, which differs from the one used by MacGillavry and colleagues, is probably less specific.

MAP2 showed a similar distribution as its interaction partner, the tubulins, being mostly present in the shaft areas. It also showed a complementary distribution across synapse classes, with more MAP2 being present in stubby class synapses than in mushroom spines. However, its low abundance for a cytoskeleton-associated protein makes it difficult to assess its role in the synapse. The stabilization of microtubules seems not to play a high role in dendritic spines, which is in line with the observation that only dynamic microtubules enter spines, whereas the stable bundles are mostly in the shaft (Jaworski et al., 2009; Kaech et al., 2001; Landis and Reese, 1983).

The motor protein Myosin5a, which acts along actin filaments, localized primarily to perisynaptic regions. This localization was similar to these of the ER-resident proteins ERp72, Calretinin and Calreticulin, which is in agreement with its role to position the ER in the synapse (Miyata et al., 2000; Rudolf et al., 2011). It was present in large amounts, with almost double the amount in stubby class synapses than in mushroom class synapses. This suggests a much higher volume of transport happening in stubby class synapses, probably because the synapse is still under development.

Finally, Septin7 has been reported to control the diffusion across the spine neck by forming arc- or ring-like domains on the base of the neck (Ewers et al., 2014; Kinoshita et al., 2002). According to this function, I observe it to be mostly localized to the neck regions. I also found it to be present in perisynaptic regions, which is probably because of its regulation of PSD95 (Yadav et al., 2017). Again, its low abundance makes it difficult to assess to what extent the proposed rings are really able to limit the diffusion across the neck membrane. Maybe it acts hand in hand with actin to fulfill its function. 


\section{Scaffold proteins}

As expected, all scaffold proteins were highly enriched in the PSD, with only DLGAP1 showing an actual decrease in enrichment in the PSD, compared to the average localization. This was true mostly for the mushroom class synapses, which also show an increased distance of DLGAP1 spots to the PSD compared with the other scaffold proteins.

Shank2 was not as specifically localized to the PSD as the other Shank isoforms. It also shows distinct populations with higher distance to the PSD, a low eccentricity and high laterality. This suggests that it has an additional hotspot at the bottom of the spine head. As it interacts with the cytoskeleton, it might be involved in the formation and stability of the head to neck junction, or it might position specific molecules there.

All of the scaffold proteins showed a spot diameter close to $80 \mathrm{~nm}$, corroborating the recently discovered nanomodule organization of postsynapses (Broadhead et al., 2016; Hruska et al., 2018; MacGillavry et al., 2013). In addition, I observed similar protein numbers between mushroom and stubby class spines, further supporting a modular organization of synapses.

In several works, it was proposed that the PSD is layered, with PSD95 being at the top, DLGAP1 beneath it, followed by Shank and Homer proteins (Dani et al., 2010; Valtschanoff and Weinberg, 2001). Comparing their average distribution, I could see that Shank is indeed lower than PSD95, but Homer1 was in between both proteins. This is more in line with other observations using immunogold EM, which find Homer1 to be very central and close to the PSD (Tao-Cheng et al., 2014). Because the localization of DLGAP1 was not as specific as for the other proteins, a comparison is difficult, although it seems that there is at least a pool of DLGAP1 proteins in between PSD95 and Shank. 


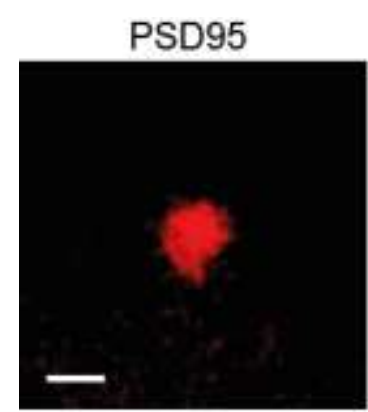

PSD95

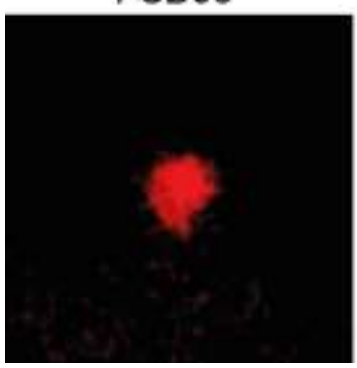

Shank1

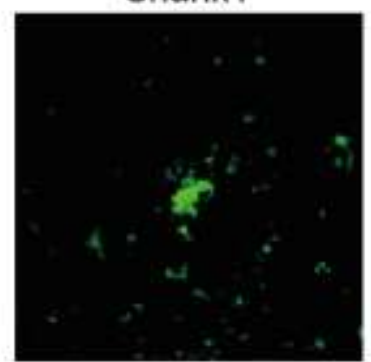

Shank1

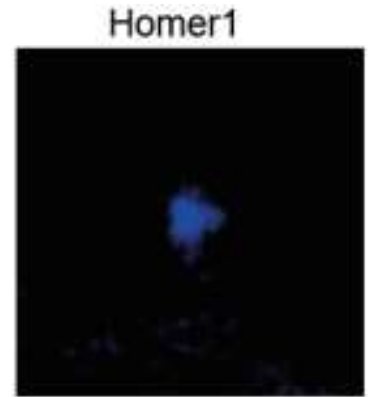

DLGAP1
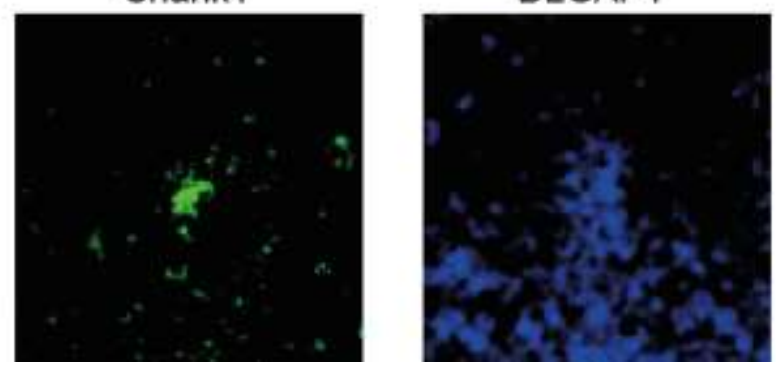

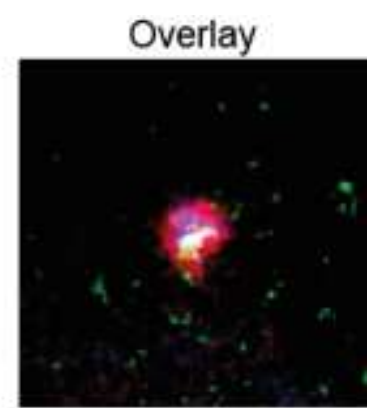

Overlay

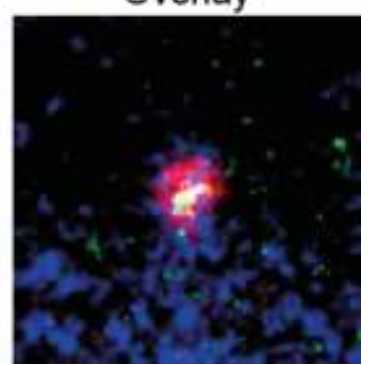

Figure 132: Observed layering of the PSD. Shank1 was found below PSD95, Homer1 was similarly distributed as PSD95, albeit being more central. The position of DLGAP1 was not as specific as for the other proteins. Scale bar $500 \mathrm{~nm}$.

For the formation of the mesh of Homer $1 b$ and Shank1, a ratio of $1: 1$ is optimal (Hayashi et al., 2009). In my data, I observe roughly 6x more Homer1 than Shank1 proteins (686.35 \pm 200.68 Homer1 vs. $112.21 \pm 5.14$ Shank1). Other works focusing on isolated PSDs did find lower numbers, probably an effect of the fractionation (Cheng et al., 2006; Lowenthal et al., 2015; Peng et al., 2004c), whereas the only other paper reporting copy numbers from intact spines is close to my data (Sugiyama et al., 2005). However, the mesh is formed only by the isoform Homer1b, whereas Homer1a is actually inhibiting the formation of the mesh (Hayashi et al., 2009). Since I do not discriminate between these isoforms, I cannot conclude whether the proteins do indeed exist in the optimal ratio.

Recently, a trans-synaptic nanocolumn was described, that aligns presynaptic release sites with postsynaptic receptor complexes, involving PSD95 and RIM1 (Tang et al., 2016). Despite the fact, that they are organized together, there is not direct correlation of their copy numbers (38.63 \pm 4.23 RIM1 proteins vs. $299.10 \pm 67.48$ PSD95 proteins; RIM1 number from Wilhelm et al., 2014). 


\section{Receptors}

Dopamine receptor types showed differing localization pattern. In accordance with published data, I found the Dopamine D1 receptor to be mostly extrasynaptic, whereas the D2 receptor was also found on the head (Garzón et al., 2013; Ladepeche et al., 2013a). There, it was present mostly on perisynaptic regions. Unfortunately, I could not detect any of the dopamine receptors in the mass spectrometry, either because they ionize poorly, as is often the case for membrane proteins, or they are only very sparsely present in the hippocampus.

The AMPA receptors GluR1 and GluR2 were both found mostly central on the PSD, with another population in extrasynaptic regions, whereas GluR3 was only found extrasynaptically. The second pool is most likely AMPAR not bound by PSD proteins or AMPAR on endosomes, since AMPAR are highly mobile in the dendritic membrane and they, especially GluR3, are also cycled (Borgdorff and Choquet, 2002; Passafaro et al., 2001). Using the synaptic copy numbers, I calculated that an average synapse contains $\sim 46$ assembled AMPAR tetramers, with GluR2 being the dominant subunit. This is in line with other studies using cell culture or tissue (Antal et al., 2008; Cheng et al., 2006; Masugi-Tokita et al., 2007; Nusser et al., 1998; Tanaka et al., 2005), and the observation that spines contain -4 postsynaptic nanodomains, which each contain 20-25 receptors (Broadhead et al., 2016; Compans et al., 2016; Hruska et al., 2018; Levet et al., 2015; Nair et al., 2013). Neuroproteomic data using isolated PSDs report lower numbers (Cheng et al., 2006; Peng et al., 2004a). The lower number in neuroproteomic data is caused by the subcellular fractionation for PSDs, which loses all diffusing or trafficking receptors (Carlin et al., 1980).

For NMDA receptors, GluN2B was very specifically present at the very center of the PSD, whereas GluN2A was much more dispersed. The current literature is not clear on whether GluN2A or GluN2B are more central in the PSD, with two studies reporting central GluNA2 in vivo (Shinohara et al., 2008; Zhang and Diamond, 2009), whereas two other in vitro studies report a central GluN2B localization (Dani et al., 2010; MacGillavry et al., 2013). I also observed that GluN2B is present in higher numbers than GluN2A on postsynapses. As GluN2B is the dominant prenatal isoform of the GluN2 family, this shows that the cell culture has not undergone the GluN2B/GluN2A switch, suggesting a network in a prenatal, or early postnatal stage (Sheng et al., 1994b). This also suggests that the nanoscale localization of GluN2A and GluN2B interchange during the switch. The subunit GluN1 showed a more homogenous localization on the head. As GluN1 is present in all assembled NMDA receptors, this is probably reflecting the combined localization of GluN2A and GluN2B. 
Kainate receptor and the metabotropic glutamate receptors were localized on the PSD for mushroom class synapses, whereas they were mostly perisynaptic on stubby synapses. Probably, metabotropic signaling is not as important for these classes of synapses, maybe because they are not fully mature yet. This is also reflected by the low PSD enrichment of GluR2 in stubby synapses.

The neurotrophin receptors LNGFR and TrkB showed a differential localization, with LNGFR being within the PSD, whereas TrkB was positioned mostly on peri- and extrasynaptic regions. This is an interesting phenomenon, because LNGFR has much lower affinity for all four neurotrophins, while LNGFR has high affinity for BDNF and neurotrophin 4, but not the others (Leal et al., 2015). The central localization of LNGFR might make up for some of its lack of binding specificity, as the concentration of the release neurotrophins is higher in the synaptic cleft than in the areas surrounding the spine. TrkB was detected at very low copy numbers and LNGFR was not detected at all in the mass spectrometric analysis. This suggests either a low abundance of these receptors in hippocampal cultures, or a bad ionization of the peptides, which is a common problem of transmembrane proteins. IGF1R showed a similar distribution as TrkB but could also not be quantified. I am currently performing quantitative WB experiments to calculate the copy numbers of both missing growth factor receptors in my cultures.

Finally, mAChR1 was present in very low copies, which localized to the center of mushroom class synapses, whereas this was less pronounced in stubby class synapses. Acetylcholine probably plays a minor role in cultures though, as I did not detect any vAChT, suggesting that there is actually very little to none cholinergic signaling happening in the cultures. This is probably different in vivo as there are cholinergic neurons enervating the hippocampus as well. These are probably cut away and their axons die during the culture preparation, which explains the missing $v A C h T$ signal, whereas the postsynaptic neurons are retained. 


\section{Ion Channels}

Without ion channels neurons would not be able to propagate their signals. Whereas presynaptic channels are well studied, postsynaptic ion channels are less well understood. Because there is a huge family of possible postsynaptic ion channels I focused on a few isoforms of each category.

Calcium channels are an important entry point for calcium in the postsynapse, besides NMDAR, calcium-permeable AMPAR and intracellular calcium stores. I did find the channel subunit $\mathrm{Ca}_{\mathrm{v}} 1.3$ to be enriched in the center of the PSD for both synapse classes. This is probably due to its interaction with Shank proteins. Unfortunately, it was not picked up in the mass spectrometric analysis, most likely because membrane proteins are inherently difficult to ionize and thus are not often detected. I am currently performing quantitative western blotting to as an alternative route to estimating its postsynaptic copy number. The subunit $\mathrm{Ca}_{\mathrm{v}} 2.1$ did not show a very specific enrichment, except for an absence in the PSD of mushroom class synapses. On the other hand, it was very lowly abundant, with a copy number of only 6.11 per PSD. It is currently estimated that a spine contains a total of 1-20 VGCCs, with the calcium signal being dominated by $R$ type channels (Sabatini and Svoboda, 2000). Therefore, a low number of $\mathrm{Ca}_{\mathrm{v}} 2.1$, a P-Q channel, is not very surprising, although it has been implicated to be involved in LTP. Maybe it exerts its function more on the shaft, which was not studied in detail here.

Potassium channels are the driving force for the negative resting potential of neurons, which is maintained by inwardly rectifying channels. The Kir2.1 and $K_{v} 1.1$ rectifiers were located at the center of the PSD, which is in line with their interaction with PSD95 (Fomina et al., 2011; Frank et al., 2016; Kim et al., 1995). The $\mathrm{K}_{\mathrm{v}} 2.1$ on the other hand was very sparse at the PSD. Its reported presence at the soma, and its low abundance in spines reported here match very well. Kv2.1 is present in two distinct subpopulations, large clusters of 1-2 $\mu \mathrm{m}$ diameter that are non-conduction, or single, conducting channels (Misonou et al., 2005; Murakoshi and Trimmer, 1999). I only observed small spots on the dendrites, showing that only the conducting single channels are present there. Surprisingly, only a few hundred $K_{v} 2.1$ channels are present on the whole neuron, which is at odds with its reported function as the dominant delayed rectifier in the hippocampus (Murakoshi and Trimmer, 1999). Probably its distinct and specific localization to the soma enables it to fulfill this function even at low numbers.

Sodium channels are the main depolarizing channels in the axon during action potentials. Their role in dendrites is controversial. For a long time, it has been thought that signal propagation in dendrites is only electrotonic, but the discovery of dendritic spikes changed this 
(Hausser et al., 2000). By now, several postsynaptic sodium channels have been reported, albeit at much lower density than in the axon (Lorincz and Nusser, 2010). I found the subunits $\mathrm{Na}_{v} 1.1$ and $\mathrm{Na}_{v} 1$.3. as well as $\mathrm{Na} \beta 1$ to be present mostly at the base of synapses. $\mathrm{Na}_{v} 1.1$ was much more abundant than $\mathrm{Na}_{v} 1.3$, congruent with its reported high expression (Goldin, 1999), while $\mathrm{Na}_{v} 1.3$ showed a very low copy number. Interestingly, $\mathrm{Na} \beta 1$ is even more abundant. Its localization to the same regions as the other sodium channel subunits suggests that it mainly acts as a modulator of sodium channel function, not as an adhesion molecule here, but the high copy number makes it likely that there are additional sodium channel subunits. One candidate is $\mathrm{Na}_{v} 1.6$, which has already been reported to be present on postsynapses (Lorincz and Nusser, 2010). 


\section{Signaling proteins}

Within dendritic spines a complex signaling machinery is essential to appropriately respond to the binding of glutamate to its receptors. Especially calcium signaling is essential for synaptic plasticity. To elucidate the function of these machineries I studied several key regulators, but these can only by the start of a complete understanding of the signaling happening in the postsynapse.

The Akt pathway is involved in many different functions throughout different tissues (Kennedy et al., 1997, 1999). In spines it is, among others, involved in the regulated expression of AMPAR (Gobert et al., 2008; Karpova et al., 2006; Man et al., 2003; Pen et al., 2016; Qin et al., 2005). I found it to be localized primarily to shaft regions, where also its target proteins of the translation machinery are found. This is also in line with another immunogold EM study, that found it primarily in the dendritic shaft (Znamensky et al., 2003). Interestingly, it was present in low copy numbers only, but this might be enough to exert its effect as a kinase.

APP is the precursor of $A \beta$, one critical protein of Alzheimer's disease. It was not enriched in the PSD but was more present in the mushroom head than in the shaft. For Stubby synapses, the distribution was more ubiquitous. Interestingly, it was present at much lower copies than in the presynapse $(47.28 \pm 11.33$ vs $6283.6 \pm 584.51$; Wilhelm et al., 2014), but other super resolution studies have shown that APP often colocalizes with SV, which might explain the predominant presynaptic localization (Groemer et al., 2011).

Many different calcium-binding proteins have been described in dendritic spines. The most important one is calmodulin, which has over 100 target proteins (Xia and Storm, 2005). It was enriched in perisynaptic regions. In the membrane areas, it is most likely in the calcium unbound form and interacts with Neurogranin (Baudier et al., 1991; Prichard et al., 1999). The interaction with PSD95 is only transient and keeps it at perisynaptic sites, which explains why I did not detect it at the center of the PSD in my images (Chowdhury et al., 2017). Calmodulin was also the most abundant calcium binding protein I detected, which is in agreement with its central role for calcium signaling in dendritic spines. Calbindin and Calretinin are present on only a subset of excitatory hippocampal neurons (Villa et al., 1994). They were mainly localized to the lower head or extrasynaptic regions, which is further away from the calcium entry points than other calcium binders, such as CaMKII or calmodulin. Also, their copy numbers are very low for calcium-binding proteins. Therefore, they most likely play a minor role in calcium signaling. 
CaMKII is the central kinase of dendritic spines with numerous targets and among the most abundant proteins in the brain and the synapse (Hell, 2014). As expected, it was positioned close to the PSD, an effect that was particularly dramatic in stubby synapses. Some studies have shown an interaction of CaMKII with microtubules, which are more abundant in stubby class synapses and might explain this phenomenon. Also, CaMKII is subject to dynamic relocalization when it is phosphorylated and some studies have shown binding of CaMKII to microtubules and MAP2 in its phosphorylated form (Baratier et al., 2006; Baudier and Cole, 1987; Lemieux et al., 2012; Schulman, 1984). Since the antibody I use is specific for the phosphorylated form this might be the reason why I see CaMKII also in perisynaptic regions in mushroom spines, as microtubules are rarely reaching close to the PSD (Kaech et al., 2001; Landis and Reese, 1983).

As expected, CaMKII is among the most abundant postsynaptic proteins. It makes up $4.43 \%$ of the mushroom protein content, and $4.69 \%$ of the flat class protein content, which is in agreement with other studies, showing that CaMKII constitutes $2-6 \%$ of the total spine protein (Chen et al., 2005). In both spine classes, it was present at a molarity of approximately $300 \mu \mathrm{M}$, which is higher than previously reported values using fluorescence microscopy $(138 \mu \mathrm{M}$, Otmakhov and Lisman, 2012). This discrepancy of might be explained by the two different methods used. Otmakhov and colleagues used two-photon microscopy and selected circular ROls over the spine head, which probably also include regions outside the spine head. This might lead to an underestimation of the copy number.

The neurotrophin BDNF is essential for the proper development of the brain (Leal et al., 2015). In my study it did not show a specific enrichment to a region within the dendritic spine and it was present only at low numbers. Although BDNF signaling is present in axons as well as dendrites of the hippocampus (Adachi et al., 2005; Kohara et al., 2001; Matsuda et al., 2009), BDNF containing vesicles are only rarely detected in dendrites (Cooney et al., 2002; Harward et al., 2016) and super-resolution microscopy found that most of these vesicles are presynaptic (Andreska et al., 2014). Therefore, a minority of dendritic spines probably contains BDNF granules, which then also will have higher copy numbers. Also, BDNF is a very potent signaling molecule that can even act in an autocrine fashion, so even the release of few molecules is probably enough to bind its target receptors (Harward et al., 2016).

The core of secretory granules consists of proteins of the Chromogranin/Secretogranin family and their exocytosis involves CAPS1. Chromogranin A and B show similar distribution, ubiquitous distribution and low abundance, in agreement with the low number of secretory granules in dendrites (Cooney et al., 2002). On the other hand, Secretogranin II is enriched at the PSD of mushroom spines and is present in much higher copy numbers. As they are cleaved 
into different hormones, this suggests that Secretogranin II mediated signaling is more pronounced in hippocampal neurons than Chromogranin mediated signaling. CAPS1 mimics the distribution of Secretogranin II, suggesting that it is preferentially associated with these vesicles as well.

CDC42 is one of the actin regulating GTPases in the dendritic spine. I found it mostly in the spine head and neck for mushroom synapses, whereas it showed a ubiquitous distribution outside the PSD of stubby synapses. It was present in fairly high copy numbers, but the actin cytoskeleton of spines is also very high, which might necessitate also high copy numbers of its regulating proteins.

The neuronal NO synthetase was positioned within the PSD of both synapse classes, through its interactions with PSD95 and NMDAR (Aarts et al., 2002; Aoki et al., 1997). This positioning is crucial for its function, as the produced NO diffuses in all directions and also across membrane barriers. In order to reach its targets, for example NMDAR, or to act as an efficient retrograde signal to the presynapse (Bon and Garthwaite, 2003), it needs to be close to these locations. 


\section{Trafficking proteins}

Within dendritic spines, many different compartments exist, which necessitates highly regulated trafficking. In this study I analyzed the essential parts of the classical clathrinmediated endocytosis pathway and determined the position of the different endosomal compartments.

The exact location of endocytosis on the postsynapse is currently under debate. Several groups have found endocytic zones adjacent to the PSD (Blanpied et al., 2002; Lu et al., 2007; Rácz et al., 2004; Rosendale et al., 2017), whereas others hypothesize that endocytosis takes place at the base of the spine (Cooney et al., 2002; Tao-Cheng et al., 2011). In my work, I found the endocytic machinery (AP180, Clathrin, Dynamin) localized mostly at the perisynaptic regions in mushroom spines, corroborating endocytic zones close to the PSD. This was less pronounced for the stubby class synapses.

The clathrin molecules are the key proteins for clathrin-mediated endocytosis. They form triskelia in a 1:1 ratio of heavy and light chain (Ungewickell and Branton, 1981), my postsynaptic copy numbers are not exactly matching this, showing $3 x$ more light chain molecules than heavy chain. This mismatch is not due to my analysis, as clathrin light chains are more abundant in the whole cell in general (61754332.85 \pm 7545013.76 vs $9863953.46 \pm$ 1883721.98). Because clathrin heavy chains alone can already form assemblies, it has been hypothesized that the light chains act as regulators of the coat assembly (Ybe et al., 1998). Maybe they need to be present in larger quantities to fulfill this function.

To investigate the maximally possible, simultaneous endocytosis events, I used the mean of the two copy numbers, which is 9122.65 for mushroom class spines and 12500.47 for stubby spines. Roughly 165 clathrin molecules are needed for the endocytosis of a vesicle (Cheng et al., 2007; McMahon and Boucrot, 2011), which yields a total of 55 simultaneous events in mushroom spines, and 76 events in stubby spines. As the postsynapse does not have as high recycling demands as the presynapse, this is most likely not a bottle neck for endocytosis.

Similarly, dynamin is the GTPase responsible for pinching off the endocytosed structures from the PM (Takei et al., 1996). To fulfill this function, at least 52 molecules are needed (Shnyrova et al., 2013), which results in a maximum of a maximum of 37 events in mushroom spines and 43 events in stubby spines. Although this is less than the maximum events possible from clathrin, it still does not pose a significant bottleneck to endocytosis, as we never observed above 10 endosomes or vesicles in one spine (Figure 9). 
Surprisingly, the clathrin uncoating ATPase HSC70 was not colocalized with clathrin on average, but rather showed an opposite distribution. It was preferentially located to the PSD, especially in stubby synapses. Because the postsynapse has less endocytic events than the presynapse, HSC70 might also perform other functions there, which is also exemplified by its lower copy number in the postsynapse (8210.1 \pm 404.50 vs $50.48 \pm 13.52)$. Given its localization it maybe acts more as a chaperone for receptors or scaffold proteins, to ensure that they are always properly folded.

The two GRIP proteins control AMPAR trafficking, GRIP1 promotes AMPAR cycling, whereas GRIP2 inhibits it (Hanley and Henley, 2010). Because the antibody does recognize both proteins, inferring function from the observed localization is difficult, but I observed an avoidance of the PSD. While initially GRIP was thought to stabilize AMPAR at the PSD (Dong et al., 1997, 1999), several studies now showed that GRIP proteins rather control the cycling of AMPAR containing vesicles (Braithwaite et al., 2002; Hanley and Henley, 2010). Therefore, the localization in more perisynaptic regions makes sense, as this is where GluR2, the AMPAR subunit bound by GRIP, vesicles are mostly found (Hussain et al., 2015).

Rab proteins control the trafficking of endosomal compartments and are used as markers for their function. Rab3 is found mostly on SVs and neuroendocrine vesicles. In mushroom class synapses, it was found mostly at lower regions of the head, or at the base of synapses, whereas it was more central in stubby synapses. Assuming that Rab3 is on neuroendocrine vesicles, this would suggest that stubby class synapses are undergoing more retrograde signaling than mushroom synapses. Because the localization of Rab3 does not match those of BDNF or the Chromogranins, it is probably not present on the same vesicles though.

Both Rab4 and Rab5 are involved in basal AMPAR trafficking. I found both to be present mostly the head regions, with Rab4 being closer to the PSD. This fits well with the normal AMPAR recycling pathway, which is Rab5 to Rab4 (and then sometimes Rab11) endosomes, followed by exocytosis (Hausser and Schlett, 2017).

Rab9 marked endosomes are responsible for endosome to TGN trafficking. In dendritic spines, the spine apparatus is probably the destination organelle for these endosomes, which is often found more distant to the PSD (Spacek and Harris, 1997). In agreement with this, Rab9 organelles were found more in the lower head regions of mushroom classes, or on the shaft.

Concomitantly, recycling endosomes marked by Rab11 were found both inside the head as well on the shaft. As endosomal recycling is not only taking place for receptor molecules, it is 
very reasonable that recycling endosomes are also present at both locations. In fact, Rab11 has been shown to be part of the AMPAR and TrkB recycling pathway, and its localization matches well with these function (Brown et al., 2007; Huang et al., 2013; Lazo et al., 2013). 


\section{SNAREs and associated proteins}

SNARE proteins are essential for intracellular transport and trafficking. In this study, I analyzed almost all SNAREs present in the rat, which is, to my knowledge, the most comprehensive, simultaneous description in dendritic spines to date. Because the full biosynthetic pathway, with all involved organelles, is present in the postsynapse, the trafficking from one to the other needs to be happening as well (Cooney et al., 2002; Horton et al., 2005; Spacek and Harris, 1997). It is therefore not unsurprising, that I found a high number of SNAREs also to be present postsynaptically. This postsynaptic function of SNARE proteins is now started to getting appreciated by several research groups, mainly studying the trafficking of receptors and retrograde signaling (Arendt et al., 2015; Gu and Huganir, 2016; Gu et al., 2016b; Jurado et al., 2013; Kennedy and Ehlers, 2011; Suh et al., 2010).

All SNAREs were mostly localized to the more caudal lateral regions of the head for mushroom spines, or peri- and extrasynaptic regions for both classes. Exceptions include SNAP47, VAMP1 and Syntaxin6 in mushroom class spines, as well as VAMP7 in stubby synapses. The mostly peri- or extrasynaptic localization of SNARE proteins is in line with their presence on various intracellular organelles, like endosomes, ER, Golgi, which I also found predominantly in the shaft (Hong, 2005; Koerbs, 2017; Salimi, 2017). Because the distribution of the SNARE proteins is so similar, it is difficult to draw conclusions on their endosomal compartment or function in the postsynapse. This is even exacerbated by the fact, that I analyze the average of several hundred spines for each SNARE, because trafficking is an inherently dynamic process. Therefore, detailed studies focusing on specific SNAREs are needed to determine their specific function.

Since SNAP47 is involved in the insertion of AMPAR into the PM during LTP (Jurado et al., 2013), its localization right to the PSD is probably to facilitate a fast insertion once LTP is triggered. This has also been observed using immunogold EM (Münster-Wandowski et al., 2017). For a few other SNARE proteins, their postsynaptic localization was also determined using immunogold EM. Interestingly, SNAP47 showed an almost 1:1:1 stoichiometry with Synaptotagmin7 and Syntaxin3, both of which are also involved in AMPAR exocytosis during LTP (Jurado et al., 2013; Wu et al., 2017). This strongly suggests that they are all present on the same vesicles. The low copy number of all three proteins also indicates that the pool of AMPAR containing vesicles is very small, which also might explain why they are so difficult to detect in EM (Hussain and Davanger, 2015). It is difficult to answer the question whether SNARE proteins working together are in general present in correlated copy numbers, because they often take part in multiple fusion steps. 
I did reproduce the lateral localization of Syntaxin4 and SNAP23 (Kennedy et al., 2010; Suh et al., 2010), whereas I did not detect VAMP2 to be enriched close to the PSD (Hussain and Davanger, 2015). The enrichment close to the PSD might be masked by the location of endosomes of constitutively trafficking AMPAR, because VAMP2 is involved in AMPAR delivery during basal activity and LTP (Jurado et al., 2013).

Looking at the copy numbers of postsynaptic SNAREs, VAMP2 sticks out as the most abundant postsynaptic SNARE. Its copy number is almost double than the next SNARE protein, SNAP23. A similar situation can be observed in the presynapse, although with SNAP25 as the second most abundant protein (Wilhelm et al., 2014). There, it is suggested that the high abundance of exocytotic SNAREs is to ensure fast SV release. Because AMPAR containing vesicles and endosomes are not as frequent as presynaptic SV (Hussain and Davanger, 2015), the high postsynaptic copy number cannot be explained just by the fact that the AMPAR release needs to be fast for LTP. Therefore, VAMP2 is either so abundant because it is involved in other, yet unknown trafficking steps, or because of the complicated control of its localization. It has been shown that VAMP2 is first trafficked to both axon and dendrite. In the dendrite, it is then selectively endocytosed again, whereas it is retained in the axon (Pennuto et al., 2003; Sampo et al., 2003). The fact that VAMP2 is among the top 10 most abundant proteins for the whole neuron make it likely that this initial unpolarized transport results in the high observed copy number in dendritic spines.

Synaptotagmins showed a differential distribution within spines. While Synaptotagmin 4 was not present directly at the PSD, Synaptotagmin5 and especially Synaptotagmin7 were enriched there in mushroom spines (whereas all isoforms where not present at PSDs in stubby synapses). Synaptotagmin 7 shows a similar distribution as SNAP47, both of which are involved in regulated AMPAR exocytosis during LTP (Jurado et al., 2013; Wu et al., 2017). As mentioned above, this, together with their highly similar copy numbers, suggests that they are located on the same vesicles. The other isoforms, Synaptotagmin 4 and 5, are both implicated in LDCV release, which has slow release kinetics (Kennedy and Ehlers, 2011; Ohnuma et al., 2001; Saegusa et al., 2002; Yoshihara et al., 2005). The localization to extrasynaptic areas might explain this delay in release, as the vesicles need to be transported to the PSD before they are released. The fact, that all three isoforms are not enriched in stubby class synapses also suggests that they do not employ LDCV or retrograde signaling.

Alpha/beta SNAP and NSF showed a strikingly similar distribution in mushroom class synapses, concurrent with their collective function. They were both enriched in the PSD, as well as the lower lateral cytosol of the head. As these are probably the two main release sites 
of dendritic spines (Kennedy and Ehlers, 2011), the disassembly machinery is also located at both sites to ensure a fast replenishment of functional SNAREs. This is underscored by the fact that they are also relatively high abundant, compared with most SNARE proteins. 


\section{Organelle proteins}

The Dendrite and dendritic spines possess many different organelles, some specific to this region, like Golgi outposts or the spine apparatus. To elucidate the position of these organelles in my model, I localized them using specific markers.

Calreticulin is an abundant ER resident protein. I found it to be localized mainly to the neck and shaft regions, which is in agreement with the localization of the SER in dendrites (Spacek and Harris, 1997). Interestingly, there was also a peak at the PSD, which might be indicative of spine apparati. These are found in the head regions, but only in a subset of mushroom spines. In agreement with this, this peak was absent in the Stubby class synapses. The copy number of Calreticulin was much higher in Stubby synapses, which again suggests a higher presence of ER tubules there. Using a total calcium concentration in the ER of $1 \mathrm{mM}$ and the volume of the ER at the spines (Michalak et al., 2009), the molar ratio of calcium to calreticulin is at 2.5:1 for mushroom class spines and 1:1 for stubby synapses. Since calreticulin is able to bind up to $25 \mathrm{~mol}$ of calcium per mol of protein, the concentration of calreticulin is sufficiently high to efficiently buffer the ER luminal calcium, in agreement with the reports that over $50 \%$ of ER luminal calcium is bound by calreticulin (Nakamura et al., 2001).

ERp72 is another ER luminal protein, that acts as a protein disulfide isomerase (Rupp et al., 1994). It showed a different localization than calreticulin, being enriched in the lower parts of the mushroom head. Maybe it is present in smooth ER, that sometimes invaginates into the neck, where it could be involved in protein quality control. The reported low copy number makes an important function unlikely though. Since there are many other PDI proteins present in mammals, other proteins of this class might be more important in dendritic spines.

Ribosomes are also present in the dendrite, where local translation is happening. They have often been reported to be mainly localized to the base of the dendritic neck, where they form rough $\mathrm{ER}$, or sometimes also in the head as free polyribosomes. Especially after LTP, they are thought to redistribute into the head (Bourne et al., 2007; Ostroff et al., 2002; Steward and Levy, 1982; Steward and Reeves, 1988). I used three different ribosomal proteins as markers for their localization, one of the $40 \mathrm{~S}$ subunit (Rpl7a) and two of the 60S subunit (Rps3 and Rps6). All three of them showed a similar distribution, which showed a slight trend to localize at the dendritic shaft. The number of the different ribosomal proteins were highly variable, as exemplified by the high SEM of both quantified proteins. Together with the finding that the composition of ribosomal proteins on the ribosome differs between functional states makes an assessment difficult. The high number of Rps6 make an efficient translation likely though. 
The trans-golgi marker TGN38 was mainly localized to the lower head regions of mushrooms, as well as the dendritic shaft, which is consistent with the localization of golgi outposts (Gardiol et al., 1999; Zhou et al., 2014). On the other hand, it's very low copy number indicate that it is not very important for the local trafficking in dendrites.

Mitochondria are an important source of ATP and usually found in the dendritic shaft, where they also localize in my data (Bourne and Harris, 2008). My calculated copy numbers for TOM20 are relatively low, which is most likely because of their localization further away from the synapse. 


\section{Synaptic scaling influences the molecular composition}

Having measured the molecular architecture of dendritic spines, I wanted to see how much it changes during activity. To this end, I used homeostatic plasticity to investigate the quantitative changes for key proteins during synaptic scaling. Surprisingly, the overall morphology of the dendritic spines, as measured by the spine class frequencies, did show few changes. Only CQNX+AP5 treatment induced a decrease in mushroom spines, with an increase in 'other' spines. These other spines are mostly composed of strong homer signal at the base of mushroom spines or filopodia. Contrary to TTX, where spontaneous release is still possible, and therefore synapses participate in limited signaling, CNQX+AP5 completely block glutamatergic transmission. This deprivation might cause mushroom spines to deteriorate and pull back into the dendritic shaft. At the same time, the neuron is looking for input and tries to form new synapses. An interesting hypothesis explaining the phenotype is that mushroom spines do indeed pull back into the dendritic shaft, bringing their PSD with them. They then start looking for new input, probably forming filopodia, with the PSD still at their base.

Like the morphology, I found the molecular composition of dendritic spines to be rather stable during homeostatic plasticity. In turn, this means that my previous quantification of the dendritic spines at resting conditions is applicable to many different culturing conditions. In the following, I will discuss the differences during the treatments, where I found them.

First, the actin cytoskeleton that shapes dendritic spines, was decreased when network activity was increased and vice versa. As the changes in network activity were long, chronic treatment, this is not due to an acute rearrangement of the cytoskeleton. Rather, it suggests that the size of the synapses per se changes, decreasing their volume when scaling down occurs, and vice versa. As receptors have been shown to follow the same behavior (Ibata et al., 2008; O'Brien et al., 1998; Turrigiano et al., 1998; Watt et al., 2000; Wierenga, 2005), the size of the PSD most likely shrinks as well, which in turn is correlated with the spine volume (Harris and Stevens, 1989; Nusser et al., 1998). Interestingly, only TTX showed a slight but significant increase in mushroom spine head width, measured from the DiO staining. Therefore, the volume of the spine head does not change during homeostatic plasticity. Whether its fine ultrastructure does change could not be assessed, as the membrane labeling was only observed in regular confocal imaging. 

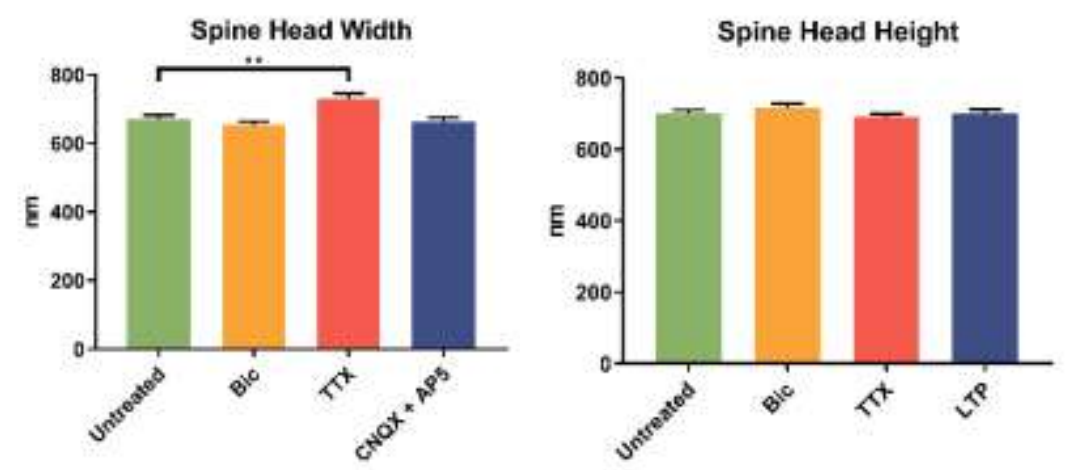

Figure 133: Synaptic scaling does not change the size of mushroom dendritic spines.

Alternatively, the density of the cytoskeleton could change, because fewer molecules need to be positioned within downscaled synapses (Schanzenbächer et al., 2016). Many receptors and signaling molecules are linked to actin (Du et al., 1998; Hell, 2014), directly or indirectly, therefore this is a viable hypothesis, but further studies are necessary to elucidate this effect.

Tubulin showed very constant localization, but its abundance changed in the same way as actin. As tubulin is the major cytoskeletal element in the dendritic shaft, the stable localization was not surprising (Kaech et al., 2001). The decrease in bicuculline treated neurons might suggest decreased trafficking along the dendrite, which is in line with the observed decrease in receptor proteins, as well as probably scaffolds, in dendritic spines. The opposite trafficking effect would also explain the increased microtubule network after synaptic upscaling.

Interestingly, Clathrin light chain was dramatically increased in cultures with elevated activity. This suggests a high amount of endocytosis occurring in these synapses. The increased firing rate of likely fewer receptors might cause them to be damaged faster, which in turn would require a higher rate of endocytosis. Very little is known about the quality control of glutamate receptors. From the presynapse we know that SV are under tight quality control (Truckenbrodt et al., 2018). The postsynapse could follow a similar stringent paradigm, whereby AMPAR subunits such as GluR2 are continuously recycled, and eventually removed after a predetermined number of cycling events. Such a mechanism may lead to the observed phenotype.

The other trafficking protein that was investigated, SNAP47, did not show the same phenotype as Clathrin LC. Its function is largely unknown, and it has only recently been shown to be involved in the delivery of AMPAR during LTP (Jurado et al., 2013). Its abundance during synaptic scaling did change however, showing a decrease in bicuculline treated neurons. I also observed that it relocalized to the PSD in these neurons. It is possible that this change in 
localization entails a repositioning of AMPAR containing vesicles, waiting to be released in response to an LTP-triggering stimulus. However, this is unlikely, since AMPA and NMDA receptors did not exhibit the same relocalization. Surprisingly, the relative abundance of SNAP47 was increased by almost 50\%, in CNQX+AP5 treated mushroom spines, but this effect was not significant. This suggests an increase in AMPAR endocytosis, but not in clathrinmediated endocytosis. It has recently been reported that homeostatic plasticity relies on clathrin-independent pathways for AMPAR recycling, which may resolve this conflicting finding (Glebov et al., 2015).

Glutamate receptors are among the best studied molecules in homeostatic plasticity (Turrigiano, 2008). Most work has focused on AMPAR, where the exact mechanisms are still controversial with some observing GluR1 to be the predominantly regulated subunit (Gainey et al., 2009; Hou et al., 2008; Sutton and Schuman, 2006; Thiagarajan et al., 2005), while others suggest that GluR2 is the main effector (Cingolani et al., 2008; Gainey et al., 2009; O'Brien et al., 1998; Wierenga, 2005). This disparity is further complicated by the fact, that there are global, as well as local synaptic scaling mechanisms. I did not find any drastic changes in GluR1 during homeostatic plasticity induction, except for TTX-treated mushroom samples. Here, its synaptic abundance was unexpectedly decreased, the same was true for GluR2. How can this supposed misregulation be explained? Many of the studies so far used mixed cultures of glia and neurons and it has been shown that glia secreted factors are also involved in synaptic scaling (Kaneko et al., 2008; Stellwagen and Malenka, 2006). Since I used pure neuronal cultures where the contact of neurons and glia is limited, this signaling might be missing, which could cause the aforementioned effects. To control for this, I will need to repeat these experiments in mixed cultures, but this is out of the scope of this thesis.

In my work I found NMDA receptors to be primarily regulated following a blockade of glutamatergic signaling with CNQX+AP5. This increased their abundance and localization to the PSD, as expected. Metabotropic glutamate receptors showed even higher increases in synaptic abundance, in line with previously described observations (Hu et al., 2010), but stubby spines actually showed an increase in bicuculline treated stubby neurons. Since mGluR5 has various roles, among them controlling intracellular calcium concentrations, it might be mediated in all three treatments to adequately adjust the changing environment of the spine and to be primed to react to any potential changes. However, more detailed studies would be needed to unravel this effect. 


\section{Limitations of this study}

\section{The detection of Homer1 and DiO was limited to confocal resolution}

When I started this project, I only had access to a 1 color STED system. Therefore, I needed to image Homer1 and DiO in confocal resolution, which limits the resolution for these two markers. I could circumvent the confocal resolution by using the FWHM of the Homer1 signal, which has been shown to be a good proxy of the Homer1 structure obtained by superresolution microscopy ( $\mathrm{Li}$ and Blanpied, 2016). There is no such approximation for the $\mathrm{DiO}$ signal though, which does limit my resolution, especially in the neck area. I therefore did not analyze the morphology of the neck in detail. Using deconvolution, I might be able to improve the DiO signal, which could enable me to look at the distribution of proteins in the neck in more detail. If one chooses more conservative deconvolution parameters, it could be run without supervision. Because of the highly annotated nature of my dataset, which also already includes a tracing of the neck, the following analysis of the neck could be fully automatized.

Unfortunately, I am missing a model for thin spines, the dominant dendritic form in the adult brain (Harris et al., 1992), but the differentiation between thin and mushroom spines is difficult in confocal images. Reinvestigating the imaging classification with improved analysis methods based on the large database I have now accumulated might enable me to extract also a separate thin class.

My data is also limited in axial resolution, because I only had confocal resolution in z-axis. With the advance of 3D STED, an increased axial resolution is possible, but this comes with highly increased imaging time, since one needs to sample the spines at least at a step size of half the axial resolution. Assuming a z-resolution of $80 \mathrm{~nm}$, the step size would be $40 \mathrm{~nm}$, which will require me to at least have $12+$ slices per spine. Because of the number of proteins I investigated, this increase in imaging time would make the project unfeasible. On the other hand, the membrane label DiO, is not usable in STED microscopy, because it is not very photostable. Alternatively, one could use 3D STORM imaging that ideally would also be able to use 3 colors. But like 3D STED z-stack imaging, STORM is inherently slower in its image acquisition time. During this study I tried to implement a 3-color 3D STORM imaging scheme at the laboratory of Bo Huang, using simultaneous detection of the fluorophores (similar to Bossi et al., 2008; Lampe et al., 2012), rather than sequential (Bates et al., 2007). The separation of the used fluorophores was difficult though and the resolution decreased dramatically. For both approaches, 3D STED and 3D STORM, an increase in z-resolution always comes with a decrease in lateral resolution. Because I average over many spines per protein in this study, the axial distribution differences of a protein cancel each other out. The 
model is then constructed assuming rotational symmetry along the head-neck axis. This is a good compromise to make this average model feasible. For single proteins, more detailed analysis using sequential multicolor 3D STORM would be possible. This would especially be interesting for scaffold proteins, to elucidate their structure in more detail.

\section{The in vitro culture misses some elements present in the brain}

All work in this study was done on cultures, which lack several elements present in the brain. The density of neurons and synapses is lower than in the brain, there is no fully mature extracellular matrix, low number of inhibitory neurons and low network activity. Still, hippocampal neuron cultures are a widely used model systems and DIV21 cultures are similar in function and structure to synapses in situ, making my work relevant for many applications (Kaech and Banker, 2006; Papa et al., 1995). Furthermore, it has been shown multiple times that dendritic spines are made up out of PSD 'building blocks' that are aligned with the presynaptic active zone (Broadhead et al., 2016; Hruska et al., 2018; MacGillavry et al., 2013; Nair et al., 2013; Tang et al., 2016). The structure of these blocks is most likely highly conserved even between neuronal cultures and in vivo, my model of the dendritic spine is therefore equally well applicable to dendritic spines in living animals.

Also, I specifically chose a Banker style culture, which limits the contact of glia and neurons. Although this might slightly change the structure of the synapses formed, it was essential for the analysis carried out here. Only this in vitro approach enabled us to perform quantitative, unlabeled mass spectrometry on a pure neuronal sample (Figure 6), something which cannot be done using mixed cultures, slices or whole-brain samples.

\section{The manual morphology assessment is limiting protein throughput}

To average the dendritic spines, they needed to be aligned according to their morphology. I tried different correlation-based approaches, but the complex morphology of especially mushroom spines impaired an easy, automated alignment algorithm. Therefore, I had to resort to manual annotation of spine landmarks, which always introduces a small subjective element to the analysis. After I had annotated about 2000 spines for both classes, I tried to train a convolutional neural network to automate this task. Unfortunately, I only achieved $\sim 70 \%$ accuracy with both training from scratch and retraining different network architectures. This required me to manually curate every spine detected, which was very labor intensive. On the other hand, the large curated database that I have now could serve as a basis to again try to train a neural network. This would greatly accelerate the further study of dendritic spines. 


\section{The Nanomap as a quantitative foundation for further studies}

The Dendrite Nanomap is an extensive collection of localization and abundance data of many proteins. Naturally, it can be used as the starting point for many more studies.

\section{Integrate known interactions}

First, the presented data does so far not include known interactions between the proteins included in this study. Although I have already done extensive literature research to find the data describing the known protein complexes, integrating these into the model was not achievable within the time frame. The interaction data also needs to be carefully assessed, because often it is only known that two proteins can interact, but not how many of the proteins in the spine actually do so. Nonetheless, this data is exciting, but challenging to build into the model. One could try to reduce the complexity by only implementing the interactions of key proteins, such as the scaffolds, to make this more feasible.

\section{Investigating spine subclasses}

Within the two defined spine classes, a large variability still exists. It would be interesting to investigate whether I can describe finer subclasses, for example differentiating between Mushroom spines with long neck and short neck. Using the large database, I could then further study whether a given protein shows a preferential enrichment in one of these subclasses, using the fluorescence signal intensity. In addition, I will investigate whether the nanoscale localization of the protein is different between the subclasses, by making subclass-specific average distributions. I already set up the analysis routines to do so, using fuzzy c-means clustering (Bezdek, 1981), but the interpretation of the results could not be finished during the time of this thesis.

\section{What is the function and composition of synapses at the base of mushroom spines?}

During this study, I regularly observed synapses on the base of mushroom spines. So far, I did not investigate these synapses, but the analysis can easily be extended to them. Another study has also found these synapses and proposes that initially these shaft synapses are formed at the contact site of axons and dendrites (Reilly et al., 2011). Subsequently, dendritic spines emerge from adjacent sites of this shaft synapse and form their own synapse with the close by axon. It would interesting to study, whether these initial shaft synapses have their own molecular composition, distinct from the other spine classes. Also, it would be exciting to explore whether the shaft and spine synapses show associated signaling or not, using for example calcium imaging. 


\section{Extending the model to the dendritic shaft}

So far, the nanomap mainly describes the dendritic spines, whereas the dendrite shaft has not been studied in detail. Since the acquired average images also contain this area, I plan to extend the model also the dendritic shaft. This will reveal the basic organization, mostly encompassing protein concentrations or molarity, which is important trafficking or translation in dendrites. Using the organelle markers, I could also estimate which volume is occupied by mitochondria and ER.

\section{Diffusion of proteins in and out of spines}

My model describes a static snapshot of an average dendritic spine. In vivo, the spine is a dynamic system where proteins are constantly cycled, produced and degraded. It is highly interesting to study these dynamic processes within spines as well. For a handful of proteins, the diffusion has been studied in detail, but for many other proteins, their transport in- and out of spines is not known (Harvey et al., 2008; Lee and Yasuda, 2009; Lee et al., 2009; Sabatini et al., 2001). The dendritic model I present would benefit these studies immensely, as one could use it as the equilibrium state to which the diffusion converges. We also know, that the diffusion is additionally regulated by activity, the effect of which I observed in the homeostatic plasticity experiments (Bloodgood and Sabatini, 2005).

\section{Hebbian plasticity}

Learning is based on the strengthening (LTP) or weakening (LTD) of synapses after stimuli. The hippocampus has long been the model organism for these two synaptic plasticity schemes. However, the induction of LTP in cultures is challenging, and many different treatments to do so have been proposed (Molnár, 2011). Therefore, I chose not to include LTP as a separate treatment in this study, but one can use my model as the baseline from which a synapse is either potentiated or depressed. One would then only need to quantify relative changes, which could be calculated to absolute numbers with the known baseline copy number provided in this thesis.

\section{Is a protein's half-life related to its location?}

The half-life of many proteins have been determined in culture (Cohen et al., 2013; Dörrbaum et al., 2018; Heo et al., 2018; Mathieson et al., 2018), as well as also in vivo (Fornasiero et al., 2018), and it has been shown that they differ between subcellular compartments in the brain. Taking this one step further, I can speculate that it is not only different between compartments, but also between the location on this compartment. Interestingly, the amino acid sequence itself accounts for a large part of the lifetime already (Mandad et al., 2018). Given the fact that proteins colocalizing on the nanoscale in dendritic spines share common sequences and domains, such as the PDZ domain, nanoscale differences in lifetime become even more likely. 
Combining these datasets with my localization information would reveal whether proteins indeed show differential turnover based on their nanoscale localization. For example, it is conceivable that proteins close to the glutamatergic signaling machinery, and therefore close to drastic changes in ion concentrations or secondary messengers such as NO, would have shorter lifetimes than proteins further away from these mechanisms. I could then construct a map of the protein's lifetime in 3D, investigating which other processes might underlie this regulation.

\section{A sound quantitative base for in silico approaches}

Dendritic spines are an exciting candidate for modelling and simulation studies. Its small volume and interesting morphology lend itself to many different analyses, mostly studying diffusion of proteins, often AMPAR, or the generation of the EPSP in the postsynapse. Usually these models are based on highly simplified assumptions (Gulledge et al., 2012; Maio et al., 2017):

1. The spine has a spherical head, with a linear tube connecting it to a shaft compartment. When complex processes are considered, the model sometimes is even represented as a cube, because the simulations are otherwise too computationally expensive (Gallimore et al., 2016)

2. Free diffusion within membrane and cytosol, except for the PSD. Alternatively, a well-mixed system is considered, which assumes the instantaneous equilibration of the protein concentrations across the whole volume.

3. Receptor numbers are unknown and often placed uniformly distributed with the PSD.

All these assumptions are disparate from the real situation in real spines and are often simply because there is no available data on the nanoscale distribution or number of the proteins in question. But it has been shown repeatedly that these factors have a strong influence on synaptic function. For example, AMPAR positioning in relation to the release site is crucial for synaptic strength, because of their low affinity for glutamate (Franks et al., 2002; MacGillavry et al., 2013; Nair et al., 2013; Raghavachari and Lisman, 2004; Savtchenko and Rusakov, 2014; Tarusawa et al., 2009). My model provides important parameters for several situations frequently investigated in silico:

AMPAR diffusion is highly important for the regulation of synaptic strength. It has long been known that AMPAR are dynamically moving in and out of synapses. This mobility, as well as their position and number is essential for postsynaptic function (reviewed in Choquet and Triller, 2013; Compans et al., 2016). To adequately model this, one needs a quantitative understanding of several key parameters: 
- Number of AMPAR

- Number and location of scaffold proteins that can bind them (i.e. 'open slots')

- The structure of the membrane, possible diffusion barriers such as septin or actin fences

- Intra- and extracellular crowding

I provide quantitative measurements for these, and many more proteins, often for the first time, with the exception of extracellular crowding. But because most of these studies are done in vitro, the effect of extracellular crowding is negligible, and the medium composition is known. Now, these studies can also incorporate the effect of diffusion barrier, such as septin or actin fences, which have been neglected so far.

Molecular crowding also controls the diffusion of proteins. Like the crowding one can model in the neuronal cytosol, this can also be extended to dendritic spines. It has already been reported that AMPAR diffusion is also influences by molecular crowding ( $\mathrm{Li}$ and Blanpied, 2016; Li et al., 2016; Santamaria et al., 2010). So far, this effect was only studied in the PSD, now I can also study it in the other regions of the dendritic spine.

Electrical signaling within dendritic spines is heavily influenced by its morphology. Especially the thin neck has a high resistance of several hundred $\mathrm{M} \Omega$ and electrically isolates the spine head from the dendrite (Beaulieu-Laroche and Harnett, 2018; Yuste, 2013). This poses the question how exactly EPSP traverses across the spine neck to reach the shaft. My presented data can extend the current modelling approaches by supplying the number and location of many of the relevant proteins for ions, namely ion channels, calcium buffers, calcium stores and the $\mathrm{Na}^{+} / \mathrm{K}^{+}$ATPase, which needed to be estimated so far (Bartol et al., 2015). I also provide detailed data on the morphology of the spine, which is important for electrodiffusion (Holcman and Yuste, 2015).

\section{Towards a quantitative understanding of the neuron}

Besides the information we can gain on postsynaptic function, with this study, we now have comprehensive quantitative information on the dendritic spine, as well as the associated presynapse (Wilhelm et al., 2014). We can therefore now combine both compartments, enabling a holistic view on the synapse, the main functional unit of the brain. At the next level, we need to understand how these synapses together influence the behavior of an individual neuron. To do so, we need additional information on the morphology of neurons, their ion regional ion buffering capacities and their molecular composition. Some of this information can be extracted from my work here, for example combining the quantitative neuron proteome copy 
with further imaging studies to calculate protein numbers in other compartments, whereas others, such as the overall morphology of neurons, need independent studies. We could then start to understand, model and predict neuronal function in yet unknown detail. 


\section{References}

Aarts, M., Liu, Y., Liu, L., Besshoh, S., Arundine, M., Gurd, J.W., Wang, Y.-T., Salter, M.W., and Tymianski, M. (2002). Treatment of ischemic brain damage by perturbing NMDA receptorPSD-95 protein interactions. Science 298, 846-850.

Adachi, N., Kohara, K., and Tsumoto, T. (2005). Difference in trafficking of brain-derived neurotrophic factor between axons and dendrites of cortical neurons, revealed by live-cell imaging. BMC Neurosci. 6, 42.

Advani, R.J., Yang, B., Prekeris, R., Lee, K.C., Klumperman, J., and Scheller, R.H. (1999). VAMP-7 mediates vesicular transport from endosomes to lysosomes. J. Cell Biol. 146, 765776.

Agnati, L.F., Guidolin, D., Cervetto, C., Borroto-Escuela, D.O., and Fuxe, K. (2016). Role of iso-receptors in receptor-receptor interactions with a focus on dopamine iso-receptor complexes. Rev. Neurosci. 27, 1-25.

Ahle, S., and Ungewickell, E. (1986). Purification and properties of a new clathrin assembly protein. EMBO J. 5, 3143-3149.

Aikawa, Y., Xia, X., and Martin, T.F.J. (2006). SNAP25, but not syntaxin 1A, recycles via an ARF6-regulated pathway in neuroendocrine cells. Mol. Biol. Cell 17, 711-722.

Al-Hallaq, R.A., Conrads, T.P., Veenstra, T.D., and Wenthold, R.J. (2007). NMDA diheteromeric receptor populations and associated proteins in rat hippocampus. J. Neurosci. 27, 8334-8343.

Alberts, P., Rudge, R., Hinners, I., Muzerelle, A., Martinez-Arca, S., Irinopoulou, T., Marthiens, V., Tooze, S., Rathjen, F., Gaspar, P., et al. (2003). Cross talk between tetanus neurotoxininsensitive vesicle-associated membrane protein-mediated transport and L1-mediated adhesion. Mol. Biol. Cell 14, 4207-4220.

Alvarez, V. a, and Sabatini, B.L. (2007). Anatomical and physiological plasticity of dendritic spines. Annu. Rev. Neurosci. 30, 79-97.

Ambrosini, A., Bresciani, L., Fracchia, S., Brunello, N., and Racagni, G. (1995). Metabotropic glutamate receptors negatively coupled to adenylate cyclase inhibit N-methyl-D-aspartate receptor activity and prevent neurotoxicity in mesencephalic neurons in vitro. Mol. Pharmacol. 47, 1057-1064.

Amessou, M., Fradagrada, A., Falguières, T., Lord, J.M., Smith, D.C., Roberts, L.M., Lamaze, C., and Johannes, L. (2007). Syntaxin 16 and syntaxin 5 are required for efficient retrograde 
transport of several exogenous and endogenous cargo proteins. J. Cell Sci. 120, 1457-1468.

Andersen, B.B., Gundersen, H.J.G., and Pakkenberg, B. (2003). Aging of the human cerebellum: A stereological study. J. Comp. Neurol. 466, 356-365.

Andreska, T., Aufmkolk, S., Sauer, M., and Blum, R. (2014). High abundance of BDNF with in glutamatergic presynapses of cultured hippocampal neurons. Front. Cell. Neurosci. 8, 107.

Angelov, B., and Angelova, A. (2017). Nanoscale clustering of the neurotrophin receptor TrkB revealed by super-resolution STED microscopy. Nanoscale 9, 9797-9804.

Antal, M., Fukazawa, Y., Eordogh, M., Muszil, D., Molnar, E., Itakura, M., Takahashi, M., Shigemoto, R., Eördögh, M., Muszil, D., et al. (2008). Numbers, densities, and colocalization of AMPA- and NMDA-type glutamate receptors at individual synapses in the superficial spinal dorsal horn of rats. J. Neurosci. 28, 9692-9701.

Antonin, W., Holroyd, C., Fasshauer, D., Pabst, S., Von Mollard, G.F., and Jahn, R. (2000). A SNARE complex mediating fusion of late endosomes defines conserved properties of SNARE structure and function. EMBO J. 19, 6453-6464.

Antonin, W., Dulubova, I., Araç, D., Pabst, S., Plitzner, J., Rizo, J., and Jahn, R. (2002). The N-terminal Domains of Syntaxin 7 and vti1b Form Three-helix Bundles That Differ in Their Ability to Regulate SNARE Complex Assembly. J. Biol. Chem. 277, 36449-36456.

Aoki, C., Rhee, J., Lubin, M., and Dawson, T.M. (1997). NMDA-R1 subunit of the cerebral cortex co-localizes with neuronal nitric oxide synthase at pre-and postsynaptic sites and in spines. Brain Res. 750, 25-40.

Arendt, K.L., Zhang, Y., Jurado, S., Malenka, R.C., Südhof, T.C., and Chen, L. (2015). Retinoic acid and LTP recruit postsynaptic AMPA receptors using distinct SNARE-dependent mechanisms. Neuron 86, 442-456.

Arnold, W.P., Mittal, C.K., Katsuki, S., and Murad, F. (1977). Nitric oxide activates guanylate cyclase and increases guanosine 3':5'-cyclic monophosphate levels in various tissue preparations. Proc. Natl. Acad. Sci. U. S. A. 74, 3203-3207.

Awasthi, A., Ramachandran, B., Ahmed, S., Benito, E., Shinoda, Y., Nitzan, N., Heukamp, A., Rannio, S., Martens, H., Barth, J., et al. (2018). Synaptotagmin-3 drives AMPA receptor endocytosis, depression of synapse strength, and forgetting. Science 1483, 300.

Bacaj, T., Wu, D., Yang, X., Morishita, W., Zhou, P., Xu, W., Malenka, R.C., and Südhof, T.C. (2013). Synaptotagmin-1 and Synaptotagmin-7 Trigger Synchronous and Asynchronous Phases of Neurotransmitter Release. Neuron 80, 947-959. 
Bacaj, T., Ahmad, M., Jurado, S., Malenka, R.C., and Sudhof, T.C. (2015). Synaptic Function of Rab11Fip5: Selective Requirement for Hippocampal Long-Term Depression. J. Neurosci. $35,7460-7474$.

Ban, N., Beckmann, R., Cate, J.H., Dinman, J.D., Dragon, F., Ellis, S.R., Lafontaine, D.L., Lindahl, L., Liljas, A., Lipton, J.M., et al. (2014). A new system for naming ribosomal proteins. Curr. Opin. Struct. Biol. 24, 165-169.

Banks, D.S., and Fradin, C. (2005). Anomalous diffusion of proteins due to molecular crowding. Biophys. J. 89, 2960-2971.

Bär, J., Kobler, O., van Bommel, B., Mikhaylova, M., Tonnesen, J., Katona, G., Rozsa, B., Nagerl, U. V., Adrian, M., Kusters, R., et al. (2016). Periodic F-actin structures shape the neck of dendritic spines. Sci. Rep. 6, 1-9.

Baratier, J., Peris, L., Brocard, J., Gory-Fauré, S., Dufour, F., Bosc, C., Fourest-Lieuvin, A., Blanchoin, L., Salin, P., Job, D., et al. (2006). Phosphorylation of microtubule-associated protein STOP by calmodulin kinase II. J. Biol. Chem. 281, 19561-19569.

Bard, L., Sainlos, M., Bouchet, D., Cousins, S., Mikasova, L., Breillat, C., Stephenson, F.A., Imperiali, B., Choquet, D., and Groc, L. (2010). Dynamic and specific interaction between synaptic NR2-NMDA receptor and PDZ proteins. Proc. Natl. Acad. Sci. 107, 19561-19566.

Barker, P.A. (1998). p75NTR: A study in contrasts. Cell Death Differ. 5, 346-356.

Barrera, N.P., and Robinson, C. V (2011). Advances in the mass spectrometry of membrane proteins: from individual proteins to intact complexes. Annu. Rev. Biochem. 80, 247-271.

Barria, A., and Malinow, R. (2005). NMDA Receptor Subunit Composition Controls Synaptic Plasticity by Regulating Binding to CaMKII. Neuron 48, 289-301.

Bartol, T.M., Keller, D.X., Kinney, J.P., Bajaj, C.L., Harris, K.M., Sejnowski, T.J., and Kennedy, M.B. (2015). Computational reconstitution of spine calcium transients from individual proteins. Front. Synaptic Neurosci. 7, 1-24.

Bartos, J.A., Ulrich, J.D., Li, H., Beazely, M.A., Chen, Y., MacDonald, J.F., and Hell, J.W. (2010). Postsynaptic Clustering and Activation of Pyk2 by PSD-95. J. Neurosci. 30, 449-463.

Bates, M., Huang, B., Dempsey, G.T., and Zhuang, X. (2007). Multicolor Super-Resolution Imaging with Photo-Switchable Fluorescent Probes. Science (80-. ). 317, 1749-1753.

Bats, C., Groc, L., and Choquet, D. (2007). The Interaction between Stargazin and PSD-95 Regulates AMPA Receptor Surface Trafficking. Neuron 53, 719-734. 
Baudier, J., and Cole, R.D. (1987). Phosphorylation of tau proteins to a state like that in Alzheimer's brain is catalyzed by a calcium/calmodulin-dependent kinase and modulated by phospholipids. J. Biol. Chem. 262, 17577-17583.

Baudier, J., Deloulme, J.C., Van Dorsselaer, A., Black, D., and Matthes, H.W. (1991). Purification and characterization of a brain-specific protein kinase $C$ substrate, neurogranin (p17). Identification of a consensus amino acid sequence between neurogranin and neuromodulin (GAP43) that corresponds to the protein kinase C phosphorylation si. J. Biol. Chem. 266, 229-237.

Beaulieu-Laroche, L., and Harnett, M.T. (2018). Dendritic Spines Prevent Synaptic Voltage Clamp. Neuron 97, 75-82.e3.

Beaulieu, J.M., Espinoza, S., and Gainetdinov, R.R. (2015). Dopamine receptors - IUPHAR review 13. Br. J. Pharmacol. 172, 1-23.

Bedard, K., Szabo, E., Michalak, M., and Opas, M. (2005). Cellular Functions of Endoplasmic Reticulum Chaperones Calreticulin, Calnexin, and ERp57. In International Review of Cytology, pp. 91-121.

Benke, T.A., Lüthi, A., Isaac, J.T.R., and Collingridge, G.L. (1998). Modulation of AMPA receptor unitary conductance by synaptic activity. Nature 393, 793-797.

Benson, D.L., Watkins, F.H., Steward, O., and Banker, G. (1994). Characterization of GABAergic neurons in hippocampal cell cultures. J. Neurocytol. 23, 279-295.

Benson, D.L., Mandell, J.W., Shaw, G., and Banker, G. (1996). Compartmentation of alphainternexin and neurofilament triplet proteins in cultured hippocampal neurons. J. Neurocytol. 25, 181-196.

Berggard, T., Miron, S., Onnerfjord, P., Thulin, E., Akerfeldt, K.S., Enghild, J.J., Akke, M., and Linse, S. (2002). Calbindin D28k Exhibits Properties Characteristic of a Ca2+ Sensor. J. Biol. Chem. 277, 16662-16672.

Berry, K.P., and Nedivi, E. (2017). Spine Dynamics: Are They All the Same? Neuron 96, 4355.

Bezdek, J.C. (1981). Pattern Recognition with Fuzzy Objective Function Algorithms (New York: Plenum Press).

Bhattacharyya, S., Biou, V., Xu, W., Schlüter, O., and Malenka, R.C. (2009). A critical role for PSD-95/AKAP interactions in endocytosis of synaptic AMPA receptors. Nat. Neurosci. 12, 172-181. 
Bibel, M., Hoppe, E., and Barde, Y.A. (1999). Biochemical and functional interactions between the neurotrophin receptors trk and p75NTR. EMBO J. 18, 616-622.

Bin, N.R., Ma, K., Harada, H., Tien, C.W., Bergin, F., Sugita, K., Luyben, T.T., Narimatsu, M., Jia, Z., Wrana, J.L., et al. (2018). Crucial Role of Postsynaptic Syntaxin 4 in Mediating Basal Neurotransmission and Synaptic Plasticity in Hippocampal CA1 Neurons. Cell Rep. 23, 29552966.

Blanpied, T.A., Scott, D.B., and Ehlers, M.D. (2002). Dynamics and regulation of clathrin coats at specialized endocytic zones of dendrites and spines. Neuron 36, 435-449.

Block, M.R., Glick, B.S., Wilcox, C.A., Wieland, F.T., and Rothman, J.E. (1988). Purification of an $\mathrm{N}$-ethylmaleimide-sensitive protein catalyzing vesicular transport. Proc. Natl. Acad. Sci. U. S. A. $85,7852-7856$.

Blom, H., Rönnlund, D., Scott, L., Spicarova, Z., Widengren, J., Bondar, A., Aperia, A., and Brismar, H. (2011). Spatial distribution of $\mathrm{Na}+-\mathrm{K}+-\mathrm{ATPase}$ in dendritic spines dissected by nanoscale superresolution STED microscopy. BMC Neurosci. 12, 16.

Blom, H., Rönnlund, D., Scott, L., Spicarova, Z., Rantanen, V., Widengren, J., Aperia, A., and Brismar, H. (2012). Nearest neighbor analysis of dopamine D1 receptors and $\mathrm{Na}(+)-\mathrm{K}(+)-$ ATPases in dendritic spines dissected by STED microscopy. Microsc. Res. Tech. 75, 220 228.

Blom, H., Bernhem, K., Brismar, H., Blom, H., Bernhem, K., and Brismar, H. (2016). Sodium pump organization in dendritic spines. Neurophotonics 3, 041803.

Bloodgood, B.L., and Sabatini, B.L. (2005). Neuronal activity regulates diffusion across the neck of dendritic spines. Science 310, 866-869.

Bock, D.D., Lee, W.-C.A., Kerlin, A.M., Andermann, M.L., Hood, G., Wetzel, A.W., Yurgenson, S., Soucy, E.R., Kim, H.S., and Reid, R.C. (2011). Network anatomy and in vivo physiology of visual cortical neurons. Nature 471, 177-182.

Boeckers, T.M., Kreutz, M.R., Winter, C., Zuschratter, W., Smalla, K.H., Sanmarti-Vila, L., Wex, H., Langnaese, K., Bockmann, J., Garner, C.C., et al. (1999a). Proline-rich synapseassociated protein-1/cortactin binding protein 1 (ProSAP1/CortBP1) is a PDZ-domain protein highly enriched in the postsynaptic density. J. Neurosci. 19, 6506-6518.

Boeckers, T.M., Winter, C., Smalla, K.H., Kreutz, M.R., Bockmann, J., Seidenbecher, C., Garner, C.C., and Gundelfinger, E.D. (1999b). Proline-rich synapse-associated proteins ProSAP1 and ProSAP2 interact with synaptic proteins of the SAPAP/GKAP family. Biochem. 
Biophys. Res. Commun. 264, 247-252.

Boehm, J., Kang, M.-G., Johnson, R.C., Esteban, J., Huganir, R.L., and Malinow, R. (2006). Synaptic incorporation of AMPA receptors during LTP is controlled by a PKC phosphorylation site on GluR1. Neuron 51, 213-225.

Bohil, A.B., Robertson, B.W., and Cheney, R.E. (2006). Myosin-X is a molecular motor that functions in filopodia formation. Proc. Natl. Acad. Sci. 103, 12411-12416.

Bon, C.L.M., and Garthwaite, J. (2003). On the role of nitric oxide in hippocampal long-term potentiation. J. Neurosci. 23, 1941-1948.

Borgdorff, A.J., and Choquet, D. (2002). Regulation of AMPA receptor lateral movements. Nature 417, 649-653.

Bosch, M., Castro, J., Saneyoshi, T., Matsuno, H., Sur, M., and Hayashi, Y. (2014). Structural and molecular remodeling of dendritic spine substructures during long-term potentiation. Neuron 82, 444-459.

Bossi, M., Fölling, J., Belov, V.N., Boyarskiy, V.P., Medda, R., Egner, A., Eggeling, C., Schönle, A., and Hell, S.W. (2008). Multicolor far-field fluorescence nanoscopy through isolated detection of distinct molecular species. Nano Lett. 8, 2463-2468.

Bourne, J.N., and Harris, K.M. (2008). Balancing structure and function at hippocampal dendritic spines. Annu. Rev. Neurosci. 31, 47-67.

Bourne, J.N., Sorra, K.E., Hurlburt, J., and Harris, K.M. (2007). Polyribosomes are increased in spines of CA1 dendrites $2 \mathrm{~h}$ after the induction of LTP in mature rat hippocampal slices. Hippocampus 17, 1-4.

Boyer, C., Schikorski, T., and Stevens, C.F. (1998). Comparison of hippocampal dendritic spines in culture and in brain. J. Neurosci. 18, 5294-5300.

Braithwaite, S.P., Xia, H., and Malenka, R.C. (2002). Differential roles for NSF and GRIP/ABP in AMPA receptor cycling. Proc. Natl. Acad. Sci. 99, 7096-7101.

Brand, F., Schumacher, S., Kant, S., Menon, M.B., Simon, R., Turgeon, B., Britsch, S., Meloche, S., Gaestel, M., and Kotlyarov, A. (2012). The Extracellular Signal-Regulated Kinase 3 (Mitogen-Activated Protein Kinase 6 [MAPK6])-MAPK-Activated Protein Kinase 5 Signaling Complex Regulates Septin Function and Dendrite Morphology. Mol. Cell. Biol. 32, 2467-2478.

Brandhorst, D., Zwilling, D., Rizzoli, S.O., Lippert, U., Lang, T., and Jahn, R. (2006). Homotypic fusion of early endosomes: SNAREs do not determine fusion specificity. Proc. Natl. Acad. Sci. 
103, 2701-2706.

Braun, A.P., and Schulman, H. (2003). The Multifunctional Calcium/Calmodulin-Dependent Protein Kinase: From Form to Function. Annu. Rev. Physiol. 57, 417-445.

Brenman, J.E., Christopherson, K.S., Craven, S.E., McGee, A.W., and Bredt, D.S. (1996a). Cloning and characterization of postsynaptic density 93 , a nitric oxide synthase interacting protein. J. Neurosci. 16, 7407-7415.

Brenman, J.E., Chao, D.S., Gee, S.H., McGee, A.W., Craven, S.E., Santillano, D.R., Wu, Z., Huang, F., Xia, H., Peters, M.F., et al. (1996b). Interaction of nitric oxide synthase with the postsynaptic density protein PSD-95 and alpha1-syntrophin mediated by PDZ domains. Cell 84, 757-767.

Broadhead, M.J., Horrocks, M.H., Zhu, F., Muresan, L., Benavides-Piccione, R., DeFelipe, J., Fricker, D., Kopanitsa, M. V., Duncan, R.R., Klenerman, D., et al. (2016). PSD95 nanoclusters are postsynaptic building blocks in hippocampus circuits. Sci. Rep. 6, 24626.

Brown, T.C., Tran, I.C., Backos, D.S., and Esteban, J.A. (2005). NMDA Receptor-Dependent Activation of the Small GTPase Rab5 Drives the Removal of Synaptic AMPA Receptors during Hippocampal LTD. Neuron 45, 81-94.

Brown, T.C., Correia, S.S., Petrok, C.N., and Esteban, J.A. (2007). Functional Compartmentalization of Endosomal Trafficking for the Synaptic Delivery of AMPA Receptors during Long-Term Potentiation. J. Neurosci. 27, 13311-13315.

Bucci, C., Parton, R.G., Mather, I.H., Stunnenberg, H., Simons, K., Hoflack, B., and Zerial, M. (1992). The small GTPase rab5 functions as a regulatory factor in the early endocytic pathway. Cell 70,715-728.

Burbea, M., Dreier, L., Dittman, J.S., Grunwald, M.E., and Kaplan, J.M. (2002). Ubiquitin and AP180 regulate the abundance of GLR-1 glutamate receptors at postsynaptic elements in C. elegans. Neuron 35, 107-120.

Burri, L., Varlamov, O., Doege, C.A., Hofmann, K., Beilharz, T., Rothman, J.E., Sollner, T.H., and Lithgow, T. (2003). A SNARE required for retrograde transport to the endoplasmic reticulum. Proc. Natl. Acad. Sci. 100, 9873-9877.

Cai, Z., Saugstad, J.A., Sorensen, S.D., Ciombor, K.J., Zhang, C., Schaffhauser, H., Hubalek, F., Pohl, J., Duvoisin, R.M., and Conn, P.J. (2001). Cyclic AMP-dependent protein kinase phosphorylates group III metabotropic glutamate receptors and inhibits their function as presynaptic receptors. J. Neurochem. 78, 756-766. 
Cajal, S.R.Y. (1894). The Croonian Lecture: La Fine Structure des Centres Nerveux. Proc. R. Soc. London.

Cajigas, I.J., Tushev, G., Will, T.J., tom Dieck, S., Fuerst, N., and Schuman, E.M. (2012). The local transcriptome in the synaptic neuropil revealed by deep sequencing and high-resolution imaging. Neuron 74, 453-466.

Calabrese, V., Mancuso, C., Calvani, M., Rizzarelli, E., Butterfield, D.A., and Stella, A.M.G. (2007). Nitric oxide in the central nervous system: neuroprotection versus neurotoxicity. Nat. Rev. Neurosci. 8, 766-775.

Calakos, N., Bennett, M.K., Peterson, K.E., and Scheller, R.H. (1994). Protein-protein interactions contributing to the specificity of intracellular vesicular trafficking. Science (80-. ). 263, 1146-1149.

Carlin, R.K., Grab, D.J., Cohen, R.S., and Siekevitz, P. (1980). Isolation and characterization of postsynaptic densities from various brain regions: enrichment of different types of postsynaptic densities. J. Cell Biol. 86, 831-845.

Carroll, R.C., Beattie, E.C., Xia, H., Luscher, C., Altschuler, Y., Nicoll, R.A., Malenka, R.C., and von Zastrow, M. (1999). Dynamin-dependent endocytosis of ionotropic glutamate receptors. Proc. Natl. Acad. Sci. 96, 14112-14117.

Catarino, T., Ribeiro, L., Santos, S.D., and Carvalho, A.L. (2013). Regulation of synapse composition by protein acetylation: the role of acetylated cortactin. J. Cell Sci. 126, 149-162.

Chao, L.H., Stratton, M.M., Lee, I.H., Rosenberg, O.S., Levitz, J., Mandell, D.J., Kortemme, T., Groves, J.T., Schulman, H., and Kuriyan, J. (2011). A mechanism for tunable autoinhibition in the structure of a human Ca 2+/calmodulin-dependent kinase II holoenzyme. Cell 146, $732-$ 745.

De Chaumont, F., Dallongeville, S., Chenouard, N., Hervé, N., Pop, S., Provoost, T., MeasYedid, V., Pankajakshan, P., Lecomte, T., Le Montagner, Y., et al. (2012). Icy: An open bioimage informatics platform for extended reproducible research. Nat. Methods 9, 690-696.

Chazeau, A., and Giannone, G. (2016). Organization and dynamics of the actin cytoskeleton during dendritic spine morphological remodeling. Cell. Mol. Life Sci. 73, 3053-3073.

Chen, B., Retzlaff, M., Roos, T., and Frydman, J. (2011). Cellular strategies of protein quality control. Cold Spring Harb. Perspect. Biol. 3, a004374.

Chen, B., Zhao, L., Li, X., Ji, Y.-S.S., Li, N., Xu, X.-F.F., and Chen, Z.-Y.Y. (2014). Syntaxin 8 modulates the post-synthetic trafficking of the TrkA receptor and inflammatory pain 
transmission. J. Biol. Chem. 289, 19556-19569.

Chen, D., Bernstein, A.M., Lemons, P.P., and Whiteheart, S.W. (2000). Molecular mechanisms of platelet exocytosis: role of SNAP-23 and syntaxin 2 in dense core granule release. Blood 95, 921-929.

Chen, W., Feng, Y., Chen, D., and Wandinger-Ness, A. (1998). Rab11 is required for transgolgi network-to-plasma membrane transport and a preferential target for GDP dissociation inhibitor. Mol. Biol. Cell 9, 3241-3257.

Chen, X., Vinade, L., Leapman, R.D., Petersen, J.D., Nakagawa, T., Phillips, T.M., Sheng, M., and Reese, T.S. (2005). Mass of the postsynaptic density and enumeration of three key molecules. Proc. Natl. Acad. Sci. U. S. A. 102, 11551-11556.

Cheng, D., Hoogenraad, C.C., Rush, J., Ramm, E., Schlager, M. a, Duong, D.M., Xu, P., Wijayawardana, S.R., Hanfelt, J., Nakagawa, T., et al. (2006). Relative and absolute quantification of postsynaptic density proteome isolated from rat forebrain and cerebellum. Mol. Cell. Proteomics 5, 1158-1170.

Cheng, Y., Boll, W., Kirchhausen, T., Harrison, S.C., and Walz, T. (2007). Cryo-electron Tomography of Clathrin-coated Vesicles: Structural Implications for Coat Assembly. J. Mol. Biol. 365, 892-899.

Chicurel, M.E., and Harris, K.M. (1992). Three-dimensional analysis of the structure and composition of CA3 branched dendritic spines and their synaptic relationships with mossy fiber boutons in the rat hippocampus. J. Comp. Neurol. 325, 169-182.

Choi, J., Ko, J., Racz, B., Burette, A., Lee, J.-R., Kim, S., Na, M., Lee, H.W., Kim, K., Weinberg, R.J., et al. (2005). Regulation of Dendritic Spine Morphogenesis by Insulin Receptor Substrate 53, a Downstream Effector of Rac1 and Cdc42 Small GTPases. J. Neurosci. 25, 869-879.

Choi, Y.-B., Tenneti, L., Le, D.A., Ortiz, J., Bai, G., Chen, H.-S.V., and Lipton, S.A. (2000). Molecular basis of NMDA receptor-coupled ion channel modulation by S-nitrosylation. Nat. Neurosci. 3, 15-21.

Choquet, D., and Triller, A. (2013). The dynamic synapse. Neuron 80, 691-703.

Chowdhury, D., Turner, M., Patriarchi, T., Hergarden, A.C., Anderson, D., Zhang, Y., Sun, J., Chen, C., Ames, J.B., and Hell, J.W. (2017). Ca ${ }^{2+} /$ calmodulin binding to PSD-95 mediates homeostatic synaptic scaling down. EMBO J. 37, e201695829.

Christel, C.J., Schaer, R., Wang, S., Henzi, T., Kreiner, L., Grabs, D., Schwaller, B., and Lee, A. (2012). Calretinin regulates $\mathrm{Ca} 2+-$ dependent inactivation and facilitation of $\mathrm{Ca}(\mathrm{v}) 2.1 \mathrm{Ca} 2+$ 
channels through a direct interaction with the a12.1 subunit. J. Biol. Chem. 287, 39766-39775. Chua, C.E.L., and Tang, B.L. (2008). Syntaxin 16 is enriched in neuronal dendrites and may have a role in neurite outgrowth. Mol. Membr. Biol. 25, 35-45.

Cingolani, L.A., Thalhammer, A., Yu, L.M.Y., Catalano, M., Ramos, T., Colicos, M.A., and Goda, Y. (2008). Activity-Dependent Regulation of Synaptic AMPA Receptor Composition and Abundance by $\beta 3$ Integrins. Neuron 58, 749-762.

Citri, A., and Malenka, R.C. (2008). Synaptic plasticity: multiple forms, functions, and mechanisms. Neuropsychopharmacology 33, 18-41.

Clary, D.O., Griff, I.C., and Rothman, J.E. (1990). SNAPs, a family of NSF attachment proteins involved in intracellular membrane fusion in animals and yeast. Cell 61, 709-721.

Cohen, L.S., and Studzinski, G.P. (1967). Correlation between cell enlargement and nucleic acid and protein content of hela cells in unbalanced growth produced by inhibitors of DNA synthesis. J. Cell. Physiol. 69, 331-339.

Cohen, L.D., Zuchman, R., Sorokina, O., Müller, A., Dieterich, D.C., Armstrong, J.D., Ziv, T., and Ziv, N.E. (2013). Metabolic Turnover of Synaptic Proteins: Kinetics, Interdependencies and Implications for Synaptic Maintenance. PLoS One 8, e63191.

Colbran, R.J., and Brown, A.M. (2004). Calcium/calmodulin-dependent protein kinase II and synaptic plasticity. Curr. Opin. Neurobiol. 14, 318-327.

Collins, M.O., Husi, H., Yu, L., Brandon, J.M., Anderson, C.N.G., Blackstock, W.P., Choudhary, J.S., and Grant, S.G.N. (2006). Molecular characterization and comparison of the components and multiprotein complexes in the postsynaptic proteome. Supplement. J. ... 97 Supp/ 1, 1623.

Compans, B., Choquet, D., and Hosy, E. (2016). Review on the role of AMPA receptor nanoorganization and dynamic in the properties of synaptic transmission. Neurophotonics 3 , 041811.

Constals, A., Penn, A.C., Compans, B., Toulmé, E., Phillipat, A., Marais, S., Retailleau, N., Hafner, A.S., Coussen, F., Hosy, E., et al. (2015). Glutamate-Induced AMPA Receptor Desensitization Increases Their Mobility and Modulates Short-Term Plasticity through Unbinding from Stargazin. Neuron 85, 787-803.

Contractor, A., Mulle, C., and Swanson, G.T. (2011). Kainate receptors coming of age: milestones of two decades of research. Trends Neurosci. 34, 154-163. 
Cooney, J.R., Hurlburt, J.L., Selig, D.K., Harris, K.M., and Fiala, J.C. (2002). Endosomal compartments serve multiple hippocampal dendritic spines from a widespread rather than a local store of recycling membrane. J. Neurosci. 22, 2215-2224.

Correia, S.S., Bassani, S., Brown, T.C., Lisé, M.-F., Backos, D.S., El-Husseini, A., Passafaro, M., and Esteban, J.A. (2008). Motor protein-dependent transport of AMPA receptors into spines during long-term potentiation. Nat. Neurosci. 11, 457-466.

Courel, M., Soler-Jover, A., Rodriguez-Flores, J.L., Mahata, S.K., Elias, S., Montero-Hadjadje, M., Anouar, Y., Giuly, R.J., O'Connor, D.T., and Taupenot, L. (2010). Pro-hormone Secretogranin II Regulates Dense Core Secretory Granule Biogenesis in Catecholaminergic Cells. J. Biol. Chem. 285, 10030-10043.

Cox, J., and Mann, M. (2008). MaxQuant enables high peptide identification rates, individualized p.p.b.-range mass accuracies and proteome-wide protein quantification. Nat. Biotechnol. 26, 1367-1372.

Cox, J., Neuhauser, N., Michalski, A., Scheltema, R.A., Olsen, J. V, and Mann, M. (2011). Andromeda: a peptide search engine integrated into the MaxQuant environment. J. Proteome Res. 10, 1794-1805.

Cullen, D.K., Gilroy, M.E., Irons, H.R., and Laplaca, M.C. (2010). Synapse-to-neuron ratio is inversely related to neuronal density in mature neuronal cultures. Brain Res. 1359, 44-55.

D’Abaco, G.M., Ng, K., Paradiso, L., Godde, N.J., Kaye, A., and Novak, U. (2006). ADAM22, Expressed in Normal Brain but not in High-Grade Gliomas, Inhibits Cellular Proliferation via the Disintegrin Domain. Neurosurgery 58, 179-186.

D'Ercole, A.J., Ye, P., Calikoglu, A.S., and Gutierrez-Ospina, G. (1996). The role of the insulinlike growth factors in the central nervous system. Mol. Neurobiol. 13, 227-255.

D’Este, E., Kamin, D., Göttfert, F., El-Hady, A., Hell, S.W.W., D’Este, E., Kamin, D., Göttfert, F., El-Hady, A., and Hell, S.W.W. (2015). STED Nanoscopy Reveals the Ubiquity of Subcortical Cytoskeleton Periodicity in Living Neurons. Cell Rep. 10, 1246-1251.

D’Este, E., Kamin, D., Velte, C., Göttfert, F., Simons, M., and Hell, S.W. (2016). Subcortical cytoskeleton periodicity throughout the nervous system. Sci. Rep. 6, 22741.

Dakoji, S., Tomita, S., Karimzadegan, S., Nicoll, R.A., and Bredt, D.S. (2003). Interaction of transmembrane AMPA receptor regulatory proteins with multiple membrane associated guanylate kinases. Neuropharmacology 45, 849-856.

Damke, H., Baba, T., Warnock, D.E., and Schmid, S.L. (1994). Induction of mutant dynamin 
specifically blocks endocytic coated vesicle formation. J. Cell Biol. 127, 915-934.

Dani, A., Huang, B., Bergan, J., Dulac, C., and Zhuang, X. (2010). Superresolution Imaging of Chemical Synapses in the Brain. Neuron 68, 843-856.

Dargan, S.L., Schwaller, B., and Parker, I. (2004). Spatiotemporal patterning of IP3-mediated Ca2+ signals in Xenopus oocytes by Ca2+-binding proteins. J. Physiol. 556, 447-461.

Deinhardt, K., Salinas, S., Verastegui, C., Watson, R., Worth, D., Hanrahan, S., Bucci, C., and Schiavo, G. (2006). Rab5 and Rab7 Control Endocytic Sorting along the Axonal Retrograde Transport Pathway. Neuron 52, 293-305.

Díaz, E., Schimmöller, F., and Pfeffer, S.R. (1997). A novel Rab9 effector required for endosome-to-TGN transport. J. Cell Biol. 138, 283-290.

Dimmeler, S., Fleming, I., Fisslthaler, B., Hermann, C., Busse, R., and Zeiher, A.M. (1999). Activation of nitric oxide synthase in endothelial cells by Akt-dependent phosphorylation. Nature 399, 601-605.

Distler, U., Schmeisser, M.J., Pelosi, A., Reim, D., Kuharev, J., Weiczner, R., Baumgart, J., Boeckers, T.M., Nitsch, R., Vogt, J., et al. (2014). In-depth protein profiling of the postsynaptic density from mouse hippocampus using data-independent acquisition proteomics. Proteomics 14, 2607-2613.

Dombeck, D.A., Khabbaz, A.N., Collman, F., Adelman, T.L., and Tank, D.W. (2007). Imaging large-scale neural activity with cellular resolution in awake, mobile mice. Neuron 56, 43-57.

Dong, H., O’Brien, R.J., Fung, E.T., Lanahan, A.A., Worley, P.F., and Huganir, R.L. (1997). GRIP: a synaptic PDZ domain-containing protein that interacts with AMPA receptors. Nature 386, 279-284.

Dong, H., Zhang, P., Liao, D., and Huganir, R.L. (1999). Characterization, expression, and distribution of GRIP protein. Ann. N. Y. Acad. Sci. 868, 535-540.

Donnert, G., Keller, J., Wurm, C.A., Rizzoli, S.O., Westphal, V., Schönle, A., Jahn, R., Jakobs, S., Eggeling, C., and Hell, S.W. (2007). Two-color far-field fluorescence nanoscopy. Biophys. J. 92, L67-L69.

Dörrbaum, A.R., Kochen, L., Langer, J.D., and Schuman, E.M. (2018). Local and global influences on protein turnover in neurons and glia. Elife 7.

Du, J., Haak, L.L., Phillips-Tansey, E., Russell, J.T., and McBain, C.J. (2000). Frequencydependent regulation of rat hippocampal somato-dendritic excitability by the $\mathrm{K}+$ channel 
subunit Kv2.1. J. Physiol. 522 Pt 1, 19-31.

Du, Y., Weed, S.A., Xiong, W.C., Marshall, T.D., and Parsons, J.T. (1998). Identification of a novel cortactin $\mathrm{SH} 3$ domain-binding protein and its localization to growth cones of cultured neurons. Mol. Cell. Biol. 18, 5838-5851.

Dudek, J., Rehling, P., and van der Laan, M. (2013). Mitochondrial protein import: common principles and physiological networks. Biochim. Biophys. Acta 1833, 274-285.

Dunaevsky, a, Tashiro, a, Majewska, a, Mason, C., and Yuste, R. (1999). Developmental regulation of spine motility in the mammalian central nervous system. Proc. Natl. Acad. Sci. U. S. A. $96,13438-13443$.

Eckenstaler, R., Lessmann, V., Brigadski, T., and Company, T. (2016). CAPS1 effects on intragranular $\mathrm{pH}$ and regulation of BDNF release from secretory granules in hippocampal neurons. J. Cell Sci. 129, 1378-1390.

Egorov, A. V., Hamam, B.N., Fransén, E., Hasselmo, M.E., and Alonso, A.A. (2002). Graded persistent activity in entorhinal cortex neurons. Nature 420,173-178.

Ehlen, M., lorns, E., and Perkel, J.M. (2014). The antibody challenge. Biotechniques 56, 111114.

Ehlers, M.D., Zhang, S., Bernhadt, J.P., and Huganir, R.L. (1996). Inactivation of NMDA receptors by direct interaction of calmodulin with the NR1 subunit. Cell $84,745-755$.

Eisenberg, E., and Greene, L.E. (2007). Multiple Roles of Auxilin and Hsc70 in ClathrinMediated Endocytosis. Traffic 8, 640-646.

Elias, G.M., and Nicoll, R.A. (2007). Synaptic trafficking of glutamate receptors by MAGUK scaffolding proteins. Trends Cell Biol. 17, 343-352.

Eng, L.F. (1985). Glial fibrillary acidic protein (GFAP): the major protein of glial intermediate filaments in differentiated astrocytes. J. Neuroimmunol. 8, 203-214.

Ernfors, P., Ibáñez, C.F., Ebendal, T., Olson, L., and Persson, H. (1990). Molecular cloning and neurotrophic activities of a protein with structural similarities to nerve growth factor: developmental and topographical expression in the brain. Proc. Natl. Acad. Sci. U. S. A. 87, 5454-5458.

Evers, D.M., Matta, J.A., Hoe, H.-S., Zarkowsky, D., Lee, S.H., Isaac, J.T., and Pak, D.T.S. (2010). Plk2 attachment to NSF induces homeostatic removal of GluA2 during chronic overexcitation. Nat. Neurosci. 13, 1199-1207. 
Ewers, H., Tada, T., Petersen, J.D., Racz, B., Sheng, M., and Choquet, D. (2014). A septindependent diffusion barrier at dendritic spine necks. PLoS One 9, 1-19.

Faelber, K., Posor, Y., Gao, S., Held, M., Roske, Y., Schulze, D., Haucke, V., Noé, F., and Daumke, O. (2011). Crystal structure of nucleotide-free dynamin. Nature 477, 556-560.

Farah, C.A., and Leclerc, N. (2008). HMWMAP2: New perspectives on a pathway to dendritic identity. Cell Motil. Cytoskeleton 65, 515-527.

Farina, M., van de Bospoort, R., He, E., Persoon, C.M., van Weering, J.R.R.T., Broeke, J.H., Verhage, M., and Toonen, R.F. (2015). CAPS-1 promotes fusion competence of stationary dense-core vesicles in presynaptic terminals of mammalian neurons. Elife 4, 1-22.

Feinshreiber, L., Singer-Lahat, D., Ashery, U., and Lotan, I. (2009). Voltage-gated potassium channel as a facilitator of exocytosis. In Annals of the New York Academy of Sciences, pp. 87-92.

Feng, W., Tu, J., Yang, T., Vernon, P.S., Allen, P.D., Worley, P.F., and Pessah, I.N. (2002). Homer regulates gain of ryanodine receptor type 1 channel complex. J. Biol. Chem. 277, $44722-44730$.

von Figura, K., and Hasilik, A. (1986). Lysosomal enzymes and their receptors. Annu. Rev. Biochem. 55, 167-193.

Fink, C.C., Bayer, K.-U., Myers, J.W., Ferrell, J.E., Schulman, H., and Meyer, T. (2003). Selective regulation of neurite extension and synapse formation by the beta but not the alpha isoform of CaMKII. Neuron 39, 283-297.

Fischer-Colbrie, R., Laslop, A., and Kirchmair, R. (1995). Secretogranin II: molecular properties, regulation of biosynthesis and processing to the neuropeptide secretoneurin. Prog. Neurobiol. 46, 49-70.

Fischer, M., Kaech, S., Wagner, U., Brinkhaus, H., and Matus, a (2000). Glutamate receptors regulate actin-based plasticity in dendritic spines. Nat. Neurosci. 3, 887-894.

Fischer von Mollard, G., and Stevens, T.H. (1998). A human homolog can functionally replace the yeast vesicle-associated SNARE Vti1p in two vesicle transport pathways. J. Biol. Chem. 273, 2624-2630.

Fischer von Mollard, G., Mignery, G.A., Baumert, M., Perin, M.S., Hanson, T.J., Burger, P.M., Jahn, R., and Südhof, T.C. (1990). rab3 is a small GTP-binding protein exclusively localized to synaptic vesicles. Proc. Natl. Acad. Sci. 87, 1988-1992. 
Fischer von Mollard, G., Stahl, B., Walch-Solimena, C., Takei, K., Daniels, L., Khoklatchev, A., De Camilli, P., Südhof, T.C., and Jahn, R. (1994). Localization of Rab5 to synaptic vesicles identifies endosomal intermediate in synaptic vesicle recycling pathway. Eur. J. Cell Biol. 65, 319-326.

Flannery, A.R., Czibener, C., and Andrews, N.W. (2010). Palmitoylation-dependent association with CD63 targets the Ca2+sensor synaptotagmin VII to lysosomes. J. Cell Biol. $191,599-613$.

Flaumenhaft, R., Croce, K., Chen, E., Furie, B., and Furie, B.C. (1999). Proteins of the exocytotic core complex mediate platelet alpha-granule secretion. Roles of vesicle-associated membrane protein, SNAP-23, and syntaxin 4. J. Biol. Chem. 274, 2492-2501.

Foa, L., and Gasperini, R. (2009). Developmental roles for Homer: More than just a pretty scaffold. J. Neurochem. 108, 1-10.

Fomina, S., Howard, T.D., Sleator, O.K., Golovanova, M., O’Ryan, L., Leyland, M.L., Grossmann, J.G., Collins, R.F., and Prince, S.M. (2011). Self-directed assembly and clustering of the cytoplasmic domains of inwardly rectifying Kir2.1 potassium channels on association with PSD-95. Biochim. Biophys. Acta 1808, 2374-2389.

Fornasiero, E.F., Mandad, S., Wildhagen, H., Alevra, M., Rammner, B., Keihani, S., Opazo, F., Urban, I., Ischebeck, T., Sakib, M.S., et al. (2018). Precisely measured protein lifetimes in the mouse brain reveal differences across tissues and subcellular fractions. Nat. Commun. 9 , 4230.

Fox, P.D., Loftus, R.J., and Tamkun, M.M. (2013). Regulation of Kv2.1 K(+) conductance by cell surface channel density. J. Neurosci. 33, 1259-1270.

Frank, R.A., and Grant, S.G. (2017). Supramolecular organization of NMDA receptors and the postsynaptic density. Curr. Opin. Neurobiol. 45, 139-147.

Frank, R.A.W., Komiyama, N.H., Ryan, T.J., Zhu, F., O'Dell, T.J., and Grant, S.G.N. (2016). NMDA receptors are selectively partitioned into complexes and supercomplexes during synapse maturation. Nat. Commun. 7, 11264.

Frank, R.A.W., Zhu, F., Komiyama, N.H., and Grant, S.G.N. (2017). Hierarchical organization and genetically separable subfamilies of PSD95 postsynaptic supercomplexes. J. Neurochem. $142,504-511$.

Franks, K.M., Bartol, T.M., and Sejnowski, T.J. (2002). A Monte Carlo model reveals independent signaling at central glutamatergic synapses. Biophys. J. 83, 2333-2348. 
Frappier, T., Stetzkowski-Marden, F., and Pradel, L.A. (1991). Interaction domains of neurofilament light chain and brain spectrin. Biochem. J. 275 ( Pt 2, 521-527.

Fukata, Y., Adesnik, H., Iwanaga, T., Bredt, D.S., Nicoll, R.A., and Fukata, M. (2006). Epilepsyrelated ligand/receptor complex LGI1 and ADAM22 regulate synaptic transmission. Science 313, 1792-1795.

Fukuda, M., and Mikoshiba, K. (2000). Calcium-dependent and -independent heterooligomerization in the synaptotagmin family. J. Biochem. 128, 637-645.

Fukunaga, Y., Matsubara, M., Nagai, R., and Miyazawa, A. (2005). The Interaction between PSD-95 and Ca2+/Calmodulin Is Enhanced by PDZ-Binding Proteins. J. Biochem. 138, 177182.

Fulton, D., Gratton, J.P., McCabe, T.J., Fontana, J., Fujio, Y., Walsh, K., Franke, T.F., Papapetropoulos, A., and Sessa, W.C. (1999). Regulation of endothelium-derived nitric oxide production by the protein kinase Akt. Nature 399, 597-601.

Gainey, M.A., Hurvitz-Wolff, J.R., Lambo, M.E., and Turrigiano, G.G. (2009). Synaptic scaling requires the GluR2 subunit of the AMPA receptor. J. Neurosci. 29, 6479-6489.

Gallimore, A.R., Aricescu, A.R., Yuzaki, M., and Calinescu, R. (2016). A Computational Model for the AMPA Receptor Phosphorylation Master Switch Regulating Cerebellar Long-Term Depression. PLOS Comput. Biol. 12, e1004664.

Gallimore, A.R., Kim, T., Tanaka-Yamamoto, K., and De Schutter, E. (2018). Switching On Depression and Potentiation in the Cerebellum. Cell Rep. 22, 722-733.

Ganley, I.G., Carroll, K., Bittova, L., and Pfeffer, S. (2004). Rab9 GTPase Regulates Late Endosome Size and Requires Effector Interaction for Its Stability. Mol. Biol. Cell 15, 54205430 .

Gardiol, a, Racca, C., and Triller, A. (1999). Dendritic and postsynaptic protein synthetic machinery. J Neurosci. 19, 168-179.

Gardoni, F., Polli, F., Cattabeni, F., and Di Luca, M. (2006). Calcium-calmodulin-dependent protein kinase II phosphorylation modulates PSD-95 binding to NMDA receptors. Eur. J. Neurosci. 24, 2694-2704.

Garner, C.C., Nash, J., and Huganir, R.L. (2000). PDZ domains in synapse assembly and signalling. Trends Cell Biol. 10, 274-280.

Garzón, M., Duffy, A.M., Chan, J., Lynch, M.-K., Mackie, K., and Pickel, V.M. (2013). Dopamine 
$D_{2}$ and acetylcholine $\alpha 7$ nicotinic receptors have subcellular distributions favoring mediation of convergent signaling in the mouse ventral tegmental area. Neuroscience 252, 126-143.

Gazit, N., Vertkin, I., Shapira, I., Helm, M., Slomowitz, E., Sheiba, M., Mor, Y., Rizzoli, S., and Slutsky, I. (2016). IGF-1 Receptor Differentially Regulates Spontaneous and Evoked Transmission via Mitochondria at Hippocampal Synapses. Neuron 89, 1-15.

Gerrard, S.R., Levi, B.P., and Stevens, T.H. (2000). Pep12p is a multifunctional yeast syntaxin that controls entry of biosynthetic, endocytic and retrograde traffic into the prevacuolar compartment. Traffic 1, 259-269.

Getting, S.J., Segieth, J., Ahmad, S., Biggs, C.S., and Whitton, P.S. (1996). Biphasic modulation of GABA release by nitric oxide in the hippocampus of freely moving rats in vivo. Brain Res. 717, 196-199.

Glasser, M.F., Smith, S.M., Marcus, D.S., Andersson, J.L.R., Auerbach, E.J., Behrens, T.E.J., Coalson, T.S., Harms, M.P., Jenkinson, M., Moeller, S., et al. (2016). The Human Connectome Project's neuroimaging approach. Nat. Neurosci. 19, 1175-1187.

Glebov, O.O., Tigaret, C.M., Mellor, J.R., and Henley, J.M. (2015). Clathrin-Independent Trafficking of AMPA Receptors. J. Neurosci. 35, 4830-4836.

Gobert, D., Topolnik, L., Azzi, M., Huang, L., Badeaux, F., Desgroseillers, L., Sossin, W.S., and Lacaille, J.-C. (2008). Forskolin induction of late-LTP and up-regulation of 5' TOP mRNAs translation via mTOR, ERK, and PI3K in hippocampal pyramidal cells. J. Neurochem. 106, 1160-1174.

Goldin, A.L. (1999). Diversity of mammalian voltage-gated sodium channels. Ann. N. Y. Acad. Sci. 868, 38-50.

Gomes, R.A., Hampton, C., El-Sabeawy, F., Sabo, S.L., and McAllister, A.K. (2006). The Dynamic Distribution of TrkB Receptors before, during, and after Synapse Formation between Cortical Neurons. J. Neurosci. 26, 11487-11500.

Gorski, J.A., Zeiler, S.R., Tamowski, S., and Jones, K.R. (2003). Brain-derived neurotrophic factor is required for the maintenance of cortical dendrites. J. Neurosci. 23, 6856-6865.

Gray, E.G. (1959). Axo-somatic and axo-dendritic synapses of the cerebral cortex. J. Anat. 93, 420-433.

Gray, N.W., Fourgeaud, L., Huang, B., Chen, J., Cao, H., Oswald, B.J., Hémar, A., and McNiven, M.A. (2003). Dynamin 3 is a component of the postsynapse, where it interacts with mGluR5 and Homer. Curr. Biol. 13, 510-515. 
Greer, P.L., and Greenberg, M.E. (2008). From Synapse to Nucleus: Calcium-Dependent Gene Transcription in the Control of Synapse Development and Function. Neuron 59, 846860.

Groemer, T.W., Thiel, C.S., Holt, M., Riedel, D., Hua, Y., Hüve, J., Wilhelm, B.G., and Klingauf, J. (2011). Amyloid Precursor Protein Is Trafficked and Secreted via Synaptic Vesicles. PLoS One 6, e18754.

Grove, D.E., Fan, C.-Y., Ren, H.Y., and Cyr, D.M. (2011). The endoplasmic reticulumassociated Hsp40 DNAJB12 and Hsc70 cooperate to facilitate RMA1 E3-dependent degradation of nascent CFTRDeltaF508. Mol. Biol. Cell 22, 301-314.

Grutzendler, J., Kasthuri, N., and Gan, W. (2002). Long-term dendritic spine stability in the adult cortex. Nature 420, 812-816.

Gu, Y., and Huganir, R.L. (2016). Identification of the SNARE complex mediating the exocytosis of NMDA receptors. Proc. Natl. Acad. Sci. 113, 12280-12285.

Gu, Y., Chiu, S.-L., Liu, B., Wu, P.-H., Delannoy, M., Lin, D.-T., Wirtz, D., and Huganir, R.L. (2016a). Differential vesicular sorting of AMPA and GABAA receptors. Proc. Natl. Acad. Sci. U. S. A. 113, E922-31.

Gu, Y., Chiu, S.-L., Liu, B., Wu, P.-H., Delannoy, M., Lin, D.-T., Wirtz, D., and Huganir, R.L. (2016b). Differential vesicular sorting of AMPA and GABA A receptors. Proc. Natl. Acad. Sci. 113, E922-E931.

Gulledge, A.T., Carnevale, N.T., and Stuart, G.J. (2012). Electrical advantages of dendritic spines. PLoS One 7.

Guthrie, P.B., Segal, M., and Kater, S.B. (1991). Independent regulation of calcium revealed by imaging dendritic spines. Nature $354,76-80$.

Gutierrez, M.G., Munafó, D.B., Berón, W., and Colombo, M.I. (2004). Rab7 is required for the normal progression of the autophagic pathway in mammalian cells. J. Cell Sci. 117, 26872697.

Halt, A.R., Dallapiazza, R.F., Zhou, Y., Stein, I.S., Qian, H., Juntti, S., Wojcik, S., Brose, N., Silva, A.J., and Hell, J.W. (2012). CaMKII binding to GluN2B is critical during memory consolidation. EMBO J. 31, 1203-1216.

Hamilton, A.M., and Zito, K. (2013). Breaking it down: the ubiquitin proteasome system in neuronal morphogenesis. Neural Plast. 2013, 196848. 
Hanley, L.J., and Henley, J.M. (2010). Differential roles of GRIP1a and GRIP1b in AMPA receptor trafficking. Neurosci. Lett. 485, 167-172.

Hanson, P.I., Otto, H., Barton, N., and Jahn, R. (1995). The N-ethylmaleimide-sensitive fusion protein and alpha-SNAP induce a conformational change in syntaxin. J. Biol. Chem. 270, 16955-16961.

Hanus, C., and Schuman, E.M. (2013). Proteostasis in complex dendrites. Nat. Rev. Neurosci. 14, 638-648.

Harris, K.M., and Stevens, J.K. (1989). Dendritic spines of CA 1 pyramidal cells in the rat hippocampus: serial electron microscopy with reference to their biophysical characteristics. J. Neurosci. 9, 2982-2997.

Harris, J.J., Jolivet, R., and Attwell, D. (2012). Synaptic energy use and supply. Neuron 75, 762-777.

Harris, K.M., Jensen, F.E., and Tsao, B. (1992). Three-dimensional structure of dendritic spines and synapses in rat hippocampus (CA1) at postnatal day 15 and adult ages: implications for the maturation of synaptic physiology and long-term potentiation. J. Neurosci. 12, 2685-2705.

Harris, K.P., Zhang, Y. V., Piccioli, Z.D., Perrimon, N., Littleton, J.T., and Troy Littleton, J. (2016). The postsynaptic t-SNARE syntaxin 4 controls traffic of neuroligin 1 and synaptotagmin 4 to regulate retrograde signaling. Elife 5, 1-26.

Hartmann, M., Heumann, R., and Lessmann, V. (2001). Synaptic secretion of BDNF after highfrequency stimulation of glutamatergic synapses. EMBO J. 20,5887-5897.

Harvey, C.D., Yasuda, R., Zhong, H., and Svoboda, K. (2008). The Spread of Ras Activity Triggered by Activation of a Single Dendritic Spine. Science (80-. ). 321, 136-140.

Harward, S.C., Hedrick, N.G., Hall, C.E., Parra-Bueno, P., Milner, T.A., Pan, E., Laviv, T., Hempstead, B.L., Yasuda, R., and McNamara, J.O. (2016). Autocrine BDNF-TrkB signalling within a single dendritic spine. Nature 538, 99-103.

Hausser, A., and Schlett, K. (2017). Coordination of AMPA receptor trafficking by Rab GTPases. Small GTPases 0, 1-14.

Hausser, M., Spruston, N., and Stuart, G.J. (2000). Diversity and dynamics of dendritic signaling. Science (80-. ). 290, 739-744.

Hawrylycz, M.J., Lein, E.S., Guillozet-Bongaarts, A.L., Shen, E.H., Ng, L., Miller, J.A., van de 
Lagemaat, L.N., Smith, K.A., Ebbert, A., Riley, Z.L., et al. (2012). An anatomically comprehensive atlas of the adult human brain transcriptome. Nature 489, 391-399.

Hay, J.C., Chao, D.S., Kuo, C.S., and Scheller, R.H. (1997). Protein interactions regulating vesicle transport between the endoplasmic reticulum and Golgi apparatus in mammalian cells. Cell 89, 149-158.

Hayashi, K., Ishikawa, R., Ye, L.H., He, X.L., Takata, K., Kohama, K., and Shirao, T. (1996). Modulatory role of drebrin on the cytoskeleton within dendritic spines in the rat cerebral cortex. J. Neurosci. 16, 7161-7170.

Hayashi, M.K., Ames, H.M., and Hayashi, Y. (2006). Tetrameric hub structure of postsynaptic scaffolding protein homer. J. Neurosci. 26, 8492-8501.

Hayashi, M.K., Tang, C., Verpelli, C., Narayanan, R., Stearns, M.H., Xu, R.M., Li, H., Sala, C., and Hayashi, Y. (2009). The Postsynaptic Density Proteins Homer and Shank Form a Polymeric Network Structure. Cell 137, 159-171.

He, Y., and Linder, M.E. (2009). Differential palmitoylation of the endosomal SNAREs syntaxin 7 and syntaxin 8. J. Lipid Res. 50, 398-404.

Hebert, D.N., and Molinari, M. (2007). In and Out of the ER: Protein Folding, Quality Control, Degradation, and Related Human Diseases. Physiol. Rev. 87, 1377-1408.

Heine, M., Groc, L., Frischknecht, R., Beique, J.-C., Lounis, B., Rumbaugh, G., Huganir, R.L., Cognet, L., and Choquet, D. (2008). Surface Mobility of Postsynaptic AMPARs Tunes Synaptic Transmission Supplement. Science (80-. ). 320, 201-205.

Hell, J.W. (2014). CaMKII: Claiming center stage in postsynaptic function and organization. Neuron 81, 249-265.

Heo, S., Diering, G.H., Na, C.H., Nirujogi, R.S., Bachman, J.L., Pandey, A., and Huganir, R.L. (2018). Identification of long-lived synaptic proteins by proteomic analysis of synaptosome protein turnover. Proc. Natl. Acad. Sci. U. S. A. 115, E3827-E3836.

Hering, H., Lin, C.-C., and Sheng, M. (2003). Lipid rafts in the maintenance of synapses, dendritic spines, and surface AMPA receptor stability. J. Neurosci. 23, 3262-3271.

Herring, B.E., and Nicoll, R.A. (2016a). Long-Term Potentiation: From CaMKII to AMPA Receptor Trafficking. Annu. Rev. Physiol. 78, 351-365.

Herring, B.E., and Nicoll, R.A. (2016b). Kalirin and Trio proteins serve critical roles in excitatory synaptic transmission and LTP. Proc. Natl. Acad. Sci. 113, 201600179. 
Hersch, S.M., Gutekunst, C.A., Rees, H.D., Heilman, C.J., and Levey, A.I. (1994). Distribution of $\mathrm{m} 1-\mathrm{m} 4$ muscarinic receptor proteins in the rat striatum: light and electron microscopic immunocytochemistry using subtype-specific antibodies. J. Neurosci. 14, 3351-3363.

Heuser, J.E., and Reese, T.S. (1973). Evidence for recycling of synaptic vesicle membrane during transmitter release at the frog neuromuscular junction. J. Cell Biol. 57, 315-344.

Hirao, K., Hata, Y., Deguchi, M., Yao, I., Ogura, M., Rokukawa, C., Kawabe, H., Mizoguchi, A., and Takai, Y. (2000). Association of synapse-associated protein 90/ postsynaptic density-95associated protein (SAPAP) with neurofilaments. Genes Cells 5, 203-210.

Hirbec, H., Perestenko, O., Nishimune, A., Meyer, G., Nakanishi, S., Henley, J.M., and Dev, K.K. (2002). The PDZ proteins PICK1, GRIP, and syntenin bind multiple glutamate receptor subtypes. Analysis of PDZ binding motifs. J. Biol. Chem. 277, 15221-15224.

Hirbec, H., Francis, J.C., Lauri, S.E., Braithwaite, S.P., Coussen, F., Mulle, C., Dev, K.K., Coutinho, V., Meyer, G., Isaac, J.T.R., et al. (2003). Rapid and differential regulation of AMPA and kainate receptors at hippocampal mossy fibre synapses by PICK1 and GRIP. Neuron 37, 625-638.

Hirokawa, N., Funakoshi, T., Sato-Harada, R., and Kanai, Y. (1996). Selective stabilization of tau in axons and microtubule-associated protein $2 \mathrm{C}$ in cell bodies and dendrites contributes to polarized localization of cytoskeletal proteins in mature neurons. J. Cell Biol. 132, 667-679.

Hohl, T.M., Parlati, F., Wimmer, C., Rothman, J.E., Söllner, T.H., and Engelhardt, H. (1998). Arrangement of subunits in $20 \mathrm{~S}$ particles consisting of NSF, SNAPs, and SNARE complexes. Mol. Cell 2, 539-548.

Holbro, N., Grunditz, A., and Oertner, T.G. (2009). Differential distribution of endoplasmic reticulum controls metabotropic signaling and plasticity at hippocampal synapses. Proc. Natl. Acad. Sci. U. S. A. 106, 15055-15060.

Holcman, D., and Yuste, R. (2015). The new nanophysiology: regulation of ionic flow in neuronal subcompartments. Nat. Rev. Neurosci. 16, 685-692.

Hollingsworth, E.B., McNeal, E.T., Burton, J.L., Williams, R.J., Daly, J.W., and Creveling, C.R. (1985). Biochemical characterization of a filtered synaptoneurosome preparation from guinea pig cerebral cortex: cyclic adenosine 3':5'-monophosphate-generating systems, receptors, and enzymes. J. Neurosci. 5, 2240-2253.

Holt, M., Varoqueaux, F., Wiederhold, K., Takamori, S., Urlaub, H., Fasshauer, D., and Jahn, R. (2006). Identification of SNAP-47, a novel Qbc-SNARE with ubiquitous expression. J. Biol. 
Chem. 281, 17076-17083.

Hong, W. (2005). SNAREs and traffic. Biochim. Biophys. Acta - Mol. Cell Res. 1744, 120-144.

Hoogenraad, C.C., Popa, I., Futai, K., Sanchez-Martinez, E., Wulf, P.S., van Vlijmen, T., Dortland, B.R., Oorschot, V., Govers, R., Monti, M., et al. (2010). Neuron Specific Rab4 Effector GRASP-1 Coordinates Membrane Specialization and Maturation of Recycling Endosomes. PLoS Biol. 8, e1000283.

Hopper, R., Lancaster, B., and Garthwaite, J. (2004). On the regulation of NMDA receptors by nitric oxide. Eur. J. Neurosci. 19, 1675-1682.

Horton, A.C., and Ehlers, M.D. (2003). Dual modes of endoplasmic reticulum-to-Golgi transport in dendrites revealed by live-cell imaging. J. Neurosci. 23, 6188-6199.

Horton, A.C., and Ehlers, M.D. (2004). Secretory trafficking in neuronal dendrites. Nat. Cell Biol. 6, 585-591.

Horton, A.C., Racz, B., Monson, E.E., Lin, A.L., Weinberg, R.J., and Ehlers, M.D. (2005). Polarized Secretory Trafficking Directs Cargo for Asymmetric Dendrite Growth and Morphogenesis. 48, 757-771.

Hou, Q., Zhang, D., Jarzylo, L., Huganir, R.L., and Man, H.-Y. (2008). Homeostatic regulation of AMPA receptor expression at single hippocampal synapses. Proc. Natl. Acad. Sci. U. S. A. 105, 775-780.

Hruska, M., Henderson, N., Le Marchand, S.J., Jafri, H., and Dalva, M.B. (2018). Synaptic nanomodules underlie the organization and plasticity of spine synapses. Nat. Neurosci. 21, 1. Hu, J.-H.H., Park, J.M., Park, S., Xiao, B., Dehoff, M.H., Kim, S., Hayashi, T., Schwarz, M.K., Huganir, R.L., Seeburg, P.H., et al. (2010). Homeostatic Scaling Requires Group I mGluR Activation Mediated by Homer1a. Neuron 68, 1128-1142.

Hu, K., Carroll, J., Fedorovich, S., Rickman, C., Sukhodub, A., and Davletov, B. (2002). Vesicular restriction of synaptobrevin suggests a role for calcium in membrane fusion. Nature $415,646-650$.

Huang, D.W., Sherman, B.T., Tan, Q., Collins, J.R., Alvord, W.G., Roayaei, J., Stephens, R., Baseler, M.W., Lane, H.C., and Lempicki, R.A. (2007). The DAVID Gene Functional Classification Tool: A novel biological module-centric algorithm to functionally analyze large gene lists. Genome Biol. 8.

Huang, S.-H., Wang, J., Sui, W.-H., Chen, B., Zhang, X.-Y., Yan, J., Geng, Z., and Chen, Z.- 
Y. (2013). BDNF-Dependent Recycling Facilitates TrkB Translocation to Postsynaptic Density during LTP via a Rab11-Dependent Pathway. J. Neurosci. 33, 9214-9230.

Huang, S.P., Tsai, M.Y., Tzou, Y.M., Wu, W.G., and Wang, C. (1993). Aspartyl residue 10 is essential for ATPase activity of rat hsc70. J. Biol. Chem. 268, 2063-2068.

Hudson, A.W., and Birnbaum, M.J. (1995). Identification of a nonneuronal isoform of synaptotagmin. Proc. Natl. Acad. Sci. U. S. A. 92, 5895-5899.

Hunter, J.D. (2007). Matplotlib: A 2D graphics environment. Comput. Sci. Eng.

Hussain, S., and Davanger, S. (2015). Postsynaptic VAMP/synaptobrevin facilitates differential vesicle trafficking of GluA1 and GluA2 AMPA receptor subunits. PLoS One 10, 1-24.

Hussain, N.K., Thomas, G.M., Luo, J., and Huganir, R.L. (2015). Regulation of AMPA receptor subunit GluA1 surface expression by PAK3 phosphorylation. Proc. Natl. Acad. Sci. 201518382.

Hussain, S., Ringsevjen, H., Egbenya, D.L., Skjervold, T.L., and Davanger, S. (2016). SNARE Protein Syntaxin-1 Colocalizes Closely with NMDA Receptor Subunit NR2B in Postsynaptic Spines in the Hippocampus. Front. Mol. Neurosci. 9, 1-13.

Ibata, K., Sun, Q., and Turrigiano, G.G. (2008). Rapid synaptic scaling induced by changes in postsynaptic firing. Neuron 57, 819-826.

lezzi, M., Kouri, G., Fukuda, M., and Wollheim, C.B. (2004). Synaptotagmin V and IX isoforms control Ca2+ -dependent insulin exocytosis. J. Cell Sci. 117, 3119-3127.

Inagaki, S., Ohoka, Y., Sugimoto, H., Fujioka, S., Amazaki, M., Kurinami, H., Miyazaki, N., Tohyama, M., and Furuyama, T. (2001). Sema4c, a transmembrane semaphorin, interacts with a post-synaptic density protein, PSD-95. J. Biol. Chem. 276, 9174-9181.

Ishikawa, R., Hayashi, K., Shirao, T., Xue, Y., Takagi, T., Sasaki, Y., and Kohama, K. (1994). Drebrin, a development-associated brain protein from rat embryo, causes the dissociation of tropomyosin from actin filaments. J. Biol. Chem. 269, 29928-29933.

Jaffrey, S.R., Snowman, A.M., Eliasson, M.J.L., Cohen, N.A., and Snyder, S.H. (1998). CAPON: A protein associated with neuronal nitric oxide synthase that regulates its interactions with PSD95. Neuron 20, 115-124.

Jahn, R., and Fasshauer, D. (2012). Molecular machines governing exocytosis of synaptic vesicles. Nature 490, 201-207.

Jan, Y.N., and Jan, L.Y. (2010). Branching out: Mechanisms of dendritic arborization. Nat. Rev. 
Neurosci. 11, 316-328.

Jarsky, T., Roxin, A., Kath, W.L., and Spruston, N. (2005). Conditional dendritic spike propagation following distal synaptic activation of hippocampal CA1 pyramidal neurons. Nat. Neurosci. 8, 1667-1676.

Jaudon, F., Raynaud, F., Wehrlé, R., Bellanger, J.-M., Doulazmi, M., Vodjdani, G., Gasman, S., Fagni, L., Dusart, I., Debant, A., et al. (2015). The RhoGEF DOCK10 is essential for dendritic spine morphogenesis. Mol. Biol. Cell 26, 2112-2127.

Jaworski, J., Kapitein, L.C., Gouveia, S.M., Dortland, B.R., Wulf, P.S., Grigoriev, I., Camera, P., Spangler, S.A., Di Stefano, P., Demmers, J., et al. (2009). Dynamic microtubules regulate dendritic spine morphology and synaptic plasticity. Neuron 61, 85-100.

Jedlicka, P., Vlachos, A., Schwarzacher, S.W., and Deller, T. (2008). A role for the spine apparatus in LTP and spatial learning. Behav. Brain Res. 192, 12-19.

Jenkins, M.A., Christel, C.J., Jiao, Y., Abiria, S., Kim, K.Y., Usachev, Y.M., Obermair, G.J., Colbran, R.J., and Lee, A. (2010). Ca2+-Dependent Facilitation of Cav1.3 Ca2+ Channels by Densin and Ca2+/Calmodulin-Dependent Protein Kinase II. J. Neurosci. 30, 5125-5135.

Jiang, X., Lautermilch, N.J., Watari, H., Westenbroek, R.E., Scheuer, T., and Catterall, W.A. (2008). Modulation of CaV2.1 channels by Ca2+/calmodulin-dependent protein kinase II bound to the C-terminal domain. Proc. Natl. Acad. Sci. 105, 341-346.

Jin, L.E., Wang, M., Yang, S.-T.T., Yang, Y., Galvin, V.C., Lightbourne, T.C., Ottenheimer, D., Zhong, Q., Stein, J., Raja, A., et al. (2017). MGluR2/3 mechanisms in primate dorsolateral prefrontal cortex: Evidence for both presynaptic and postsynaptic actions. Mol. Psychiatry 22, 1615-1625.

Jordan, B.A., Fernholz, B.D., Boussac, M., Xu, C., Grigorean, G., Ziff, E.B., and Neubert, T.A. (2004). Identification and Verification of Novel Rodent Postsynaptic Density Proteins. Mol. Cell. Proteomics 3, 857-871.

Ju, W., Morishita, W., Tsui, J., Gaietta, G., Deerinck, T.J., Adams, S.R., Garner, C.C., Tsien, R.Y., Ellisman, M.H., and Malenka, R.C. (2004). Activity-dependent regulation of dendritic synthesis and trafficking of AMPA receptors. Nat. Neurosci. 7, 244-253.

Jurado, S., Goswami, D., Zhang, Y., Molina, A.J.M., Südhof, T.C., and Malenka, R.C. (2013). LTP Requires a Unique Postsynaptic SNARE Fusion Machinery. Neuron 77, 542-558.

Kaech, S., and Banker, G. (2006). Culturing hippocampal neurons. Nat. Protoc. 1, 2406-2415. 
Kaech, S., Parmar, H., Roelandse, M., Bornmann, C., and Matus, A. (2001). Cytoskeletal microdifferentiation: a mechanism for organizing morphological plasticity in dendrites. Proc. Natl. Acad. Sci. U. S. A. 98, 7086-7092.

Kaneko, M., Stellwagen, D., Malenka, R.C., and Stryker, M.P. (2008). Tumor Necrosis Factora Mediates One Component of Competitive, Experience-Dependent Plasticity in Developing Visual Cortex. Neuron.

Kang, H., and Schuman, E.M. (1995). Long-lasting neurotrophin-induced enhancement of synaptic transmission in the adult hippocampus. Science $267,1658-1662$.

Kang, H.J., Kawasawa, Y.I., Cheng, F., Zhu, Y., Xu, X., Li, M., Sousa, A.M.M., Pletikos, M., Meyer, K.A., Sedmak, G., et al. (2011). Spatio-temporal transcriptome of the human brain. Nature 478, 483-489.

Karpova, A., Sanna, P.P., and Behnisch, T. (2006). Involvement of multiple phosphatidylinositol 3-kinase-dependent pathways in the persistence of late-phase long term potentiation expression. Neuroscience 137, 833-841.

Kato, A., Ozawa, F., Saitoh, Y., Fukazawa, Y., Sugiyama, H., and Inokuchi, K. (1998). Novel members of the Vesl/Homer family of PDZ proteins that bind metabotropic glutamate receptors. J. Biol. Chem. 273, 23969-23975.

Kato, A.S., Siuda, E.R., Nisenbaum, E.S., and Bredt, D.S. (2008). AMPA Receptor SubunitSpecific Regulation by a Distinct Family of Type II TARPs. Neuron 59, 986-996.

Kazarinova-Noyes, K., Malhotra, J.D., McEwen, D.P., Mattei, L.N., Berglund, E.O., Ranscht, B., Levinson, S.R., Schachner, M., Shrager, P., Isom, L.L., et al. (2001). Contactin associates with $\mathrm{Na}+$ channels and increases their functional expression. J. Neurosci. 21, 7517-7525.

Keen, J.H. (1987). Clathrin assembly proteins: affinity purification and a model for coat assembly. J. Cell Biol. 105, 1989-1998.

Kennedy, M.J., and Ehlers, M.D. (2011). Mechanisms and Function of Dendritic Exocytosis. Neuron 69, 856-875.

Kennedy, M.J., Davison, I.G., Robinson, C.G., and Ehlers, M.D. (2010). Syntaxin-4 defines a domain for activity-dependent exocytosis in dendritic spines. Cell 141, 524-535.

Kennedy, S.G., Wagner, A.J., Conzen, S.D., Jordán, J., Bellacosa, A., Tsichlis, P.N., and Hay, N. (1997). The PI 3-kinase/Akt signaling pathway delivers an anti-apoptotic signal. Genes Dev. $11,701-713$. 
Kennedy, S.G., Kandel, E.S., Cross, T.K., and Hay, N. (1999). Akt/Protein kinase B inhibits cell death by preventing the release of cytochrome c from mitochondria. Mol. Cell. Biol. 19, 5800-5810.

Kenyon, C. (2010). A pathway that links reproductive status to lifespan in Caenorhabditis elegans. In Annals of the New York Academy of Sciences, pp. 156-162.

Khvotchev, M. V, Ren, M., Takamori, S., Jahn, R., and Südhof, T.C. (2003). Divergent functions of neuronal Rab11b in Ca2+-regulated versus constitutive exocytosis. J. Neurosci. 23, 10531-10539.

Kim, E., Niethammer, M., Rothschild, A., Jan, Y.N., and Sheng, M. (1995). Clustering of Shaker-type $\mathrm{K}+$ channels by interaction with a family of membrane-associated guanylate kinases. Nature 378, 85-88.

Kim, E., Naisbitt, S., Hsueh, Y.P., Rao, A., Rothschild, A., Craig, A.M., and Sheng, M. (1997). GKAP, a novel synaptic protein that interacts with the guanylate kinase-like domain of the PSD-95/SAP90 family of channel clustering molecules. J. Cell Biol. 136, 669-678.

Kinoshita, M., Field, C.M., Coughlin, M.L., Straight, A.F., and Mitchison, T.J. (2002). Self- and actin-templated assembly of Mammalian septins. Dev. Cell 3, 791-802.

Kitagawa, N., Mazon, H., Heck, A.J.R., and Wilkens, S. (2008). Stoichiometry of the peripheral stalk subunits $E$ and $G$ of yeast V1-ATPase determined by mass spectrometry. J. Biol. Chem. 283, 3329-3337.

Kitano, J., Nishida, M., Itsukaichi, Y., Minami, I., Ogawa, M., Hirano, T., Mori, Y., and Nakanishi, S. (2003). Direct Interaction and Functional Coupling between Metabotropic Glutamate Receptor Subtype 1 and Voltage-sensitive Cav2.1 Ca2+ Channel. J. Biol. Chem. 278, 25101-25108.

Koerbs, C. (2017). Quantitative Anatomie der dendritischen Dornfortsätze Betreuungsausschuss : Prüfungskommission :

Kohara, K., Kitamura, A., Morishima, M., and Tsumoto, T. (2001). Activity-dependent transfer of brain-derived neurotrophic factor to postsynaptic neurons. Science (80-. ). 291, 2419-2423.

Kopec, C.D., Li, B., Wei, W., Boehm, J., and Malinow, R. (2006). Glutamate receptor exocytosis and spine enlargement during chemically induced long-term potentiation. J. Neurosci. 26, 2000-2009.

Korkotian, E., and Segal, M. (2001). Regulation of dendritic spine motility in cultured hippocampal neurons. J. Neurosci. 21, 6115-6124. 
Korkut, C., Li, Y., Koles, K., Brewer, C., Ashley, J., Yoshihara, M., and Budnik, V. (2013). Regulation of Postsynaptic Retrograde Signaling by Presynaptic Exosome Release. Neuron 77, 1039-1046.

Korte, M., Carroll, P., Wolf, E., Brem, G., Thoenen, H., and Bonhoeffer, T. (1995). Hippocampal long-term potentiation is impaired in mice lacking brain-derived neurotrophic factor. Proc. Natl. Acad. Sci. U. S. A. 92, 8856-8860.

Krapivinsky, G., Krapivinsky, L., Manasian, Y., Ivanov, A., Tyzio, R., Pellegrino, C., Ben-Ari, Y., Clapham, D.E., and Medina, I. (2003). The NMDA receptor is coupled to the ERK pathway by a direct interaction between NR2B and RasGRF1. Neuron 40, 775-784.

Krombach, F., Münzing, S., Allmeling, A.M., Gerlach, J.T., Behr, J., and Dörger, M. (1997). Cell size of alveolar macrophages: an interspecies comparison. Environ. Health Perspect. 105, $1261-1263$.

Krömer, A., Glombik, M.M., Huttner, W.B., and Gerdes, H.H. (1998). Essential role of the disulfide-bonded loop of chromogranin B for sorting to secretory granules is revealed by expression of a deletion mutant in the absence of endogenous granin synthesis. J. Cell Biol. 140, 1331-1346.

Krug, M., Lössner, B., and Ott, T. (1984). Anisomycin blocks the late phase of long-term potentiation in the dentate gyrus of freely moving rats. Brain Res. Bull. 13, 39-42.

de la Cruz, J., Karbstein, K., and Woolford, J.L. (2015). Functions of Ribosomal Proteins in Assembly of Eukaryotic Ribosomes In Vivo. Annu. Rev. Biochem. 84, 93-129.

Ladepeche, L., Yang, L., Bouchet, D., and Groc, L. (2013a). Regulation of Dopamine D1 Receptor Dynamics within the Postsynaptic Density of Hippocampal Glutamate Synapses. PLoS One 8, 1-8.

Ladepeche, L., Dupuis, J.P., Bouchet, D., Doudnikoff, E., Yang, L., Campagne, Y., Bezard, E., Hosy, E., and Groc, L. (2013b). Single-molecule imaging of the functional crosstalk between surface NMDA and dopamine D1 receptors. Proc. Natl. Acad. Sci. 110, 18005-18010.

Lai, H.C., and Jan, L.Y. (2006). The distribution and targeting of neuronal voltage-gated ion channels. Nat. Rev. Neurosci. 7, 548-562.

Lampe, A., Haucke, V., Sigrist, S.J., Heilemann, M., and Schmoranzer, J. (2012). Multi-colour direct STORM with red emitting carbocyanines. Biol. Cell 104, 229-237.

Landis, D.M.D., and Reese, T.S. (1983). Cytoplasmic organization in cerebellar dendritic spines. J. Cell Biol. 97, 1169-1178. 
Lang, T., Margittai, M., Hölzler, H., and Jahn, R. (2002). SNAREs in native plasma membranes are active and readily form core complexes with endogenous and exogenous SNAREs. J. Cell Biol. 158, 751-760.

Lau, C.G., Takayasu, Y., Rodenas-Ruano, A., Paternain, A. V., Lerma, J., Bennett, M.V.L., and Zukin, R.S. (2010). SNAP-25 is a target of protein kinase C phosphorylation critical to NMDA receptor trafficking. J. Neurosci. 30, 242-254.

Lazo, O.M., Gonzalez, A., Ascaño, M., Kuruvilla, R., Couve, A., and Bronfman, F.C. (2013). BDNF regulates Rab11-mediated recycling endosome dynamics to induce dendritic branching. J. Neurosci. 33, 6112-6122.

Leal, G., Afonso, P.M., Salazar, I.L., and Duarte, C.B. (2015). Regulation of hippocampal synaptic plasticity by BDNF. Brain Res. 1621, 82-101.

Lee, S.-J.R., and Yasuda, R. (2009). Spatiotemporal Regulation of Signaling In and Out of Dendritic Spines: CaMKII and Ras. Open Neurosci. J. 3, 117-127.

Lee, F.J.S., Xue, S., Pei, L., Vukusic, B., Chéry, N., Wang, Y., Wang, Y.T., Niznik, H.B., Yu, X., and Liu, F. (2002). Dual regulation of NMDA receptor functions by direct protein-protein interactions with the dopamine D1 receptor. Cell 111, 219-230.

Lee, H.-K., Barbarosie, M., Kameyama, K., Bear, M.F., and Huganir, R.L. (2000). Regulation of distinct AMPA receptor phosphorylation sites during bidirectional synaptic plasticity. Nature 405, 955-959.

Lee, M.-J., Jang, S., Nahm, M., Yoon, J.-H., and Lee, S. (2013). Tbc1d15-17 regulates synaptic development at the Drosophila neuromuscular junction. Mol. Cells 36, 163-168.

Lee, S.-J.R., Escobedo-Lozoya, Y., Szatmari, E.M., and Yasuda, R. (2009). Activation of CaMKII in single dendritic spines during long-term potentiation. Nature 458, 299-304.

Lein, E.S., Hawrylycz, M.J., Ao, N., Ayres, M., Bensinger, A., Bernard, A., Boe, A.F., Boguski, M.S., Brockway, K.S., Byrnes, E.J., et al. (2007). Genome-wide atlas of gene expression in the adult mouse brain. Nature 445, 168-176.

Lemieux, M., Labrecque, S., Tardif, C., Labrie-Dion, É., LeBel, É., and De Koninck, P. (2012). Translocation of CaMKII to dendritic microtubules supports the plasticity of local synapses. J. Cell Biol. 198, 1055-1073.

Lemmon, M.A., and Schlessinger, J. (2010). Cell signaling by receptor tyrosine kinases. Cell 141, 1117-1134. 
Lendvai, B., Stern, E. a, Chen, B., and Svoboda, K. (2000). Experience-dependent plasticity of dendritic spines in the developing rat barrel cortex in vivo. Nature 404, 876-881.

Leung, Y.M., Kwan, E.P., Ng, B., Kang, Y., and Gaisano, H.Y. (2007). SNAREing VoltageGated $\mathrm{K}+$ and ATP-Sensitive $\mathrm{K}+$ Channels: Tuning $\beta$-Cell Excitability with Syntaxin-1A and Other Exocytotic Proteins. Endocr. Rev. 28, 653-663.

Levet, F., Hosy, E., Kechkar, A., Butler, C., Beghin, A., Choquet, D., and Sibarita, J.-B. (2015). SR-Tesseler: a method to segment and quantify localization-based super-resolution microscopy data. Nat. Methods 12, 1065-1071.

Lewis, M.J., Rayner, J.C., and Pelham, H.R.B. (1997). A novel SNARE complex implicated in vesicle fusion with the endoplasmic reticulum typically with a C-terminal transmembrane domain pre. EMBO J. 16, 3017-3024.

Leyland, M.L., and Dart, C. (2004). An alternatively spliced isoform of PSD-93/Chapsyn 110 binds to the inwardly rectifying potassium channel, Kir2.1. J. Biol. Chem. 279, 43427-43436.

Li, T.P., and Blanpied, T.A. (2016). Control of transmembrane protein diffusion within the postsynaptic density assessed by simultaneous single-molecule tracking and localization microscopy. Front. Synaptic Neurosci. 8, 1-14.

Li, P., Banjade, S., Cheng, H.C., Kim, S., Chen, B., Guo, L., Llaguno, M., Hollingsworth, J. V., King, D.S., Banani, S.F., et al. (2012). Phase transitions in the assembly of multivalent signalling proteins. Nature.

Li, T.P., Song, Y., MacGillavry, H.D., Blanpied, T.A., and Raghavachari, S. (2016). Protein Crowding within the Postsynaptic Density Can Impede the Escape of Membrane Proteins. J. Neurosci. 36, 4276-4295.

Lièvremont, J.P., Rizzuto, R., Hendershot, L., and Meldolesi, J. (1997). BiP, a major chaperone protein of the endoplasmic reticulum lumen, plays a direct and important role in the storage of the rapidly exchanging pool of Ca2+. J. Biol. Chem. 272, 30873-30879.

Lin, D.-T., Makino, Y., Sharma, K., Hayashi, T., Neve, R., Takamiya, K., and Huganir, R.L. (2009). Regulation of AMPA receptor extrasynaptic insertion by $4.1 \mathrm{~N}$, phosphorylation and palmitoylation. Nat. Neurosci. 12, 879-887.

Lindner, R., and Ungewickell, E. (1992). Clathrin-associated proteins of bovine brain coated vesicles. An analysis of their number and assembly-promoting activity. J. Biol. Chem. 267, 16567-16573.

Lisé, M.-F., Wong, T.P., Trinh, A., Hines, R.M., Liu, L., Kang, R., Hines, D.J., Lu, J., Goldenring, 
J.R., Wang, Y.T., et al. (2006). Involvement of myosin Vb in glutamate receptor trafficking. J. Biol. Chem. 281, 3669-3678.

Lissin, D. V, Gomperts, S.N., Carroll, R.C., Christine, C.W., Kalman, D., Kitamura, M., Hardy, S., Nicoll, R.A., Malenka, R.C., and von Zastrow, M. (1998). Activity differentially regulates the surface expression of synaptic AMPA and NMDA glutamate receptors. Proc. Natl. Acad. Sci. U. S. A. 95, 7097-7102.

Liu, X.-Y., Chu, X.-P., Mao, L.-M., Wang, M., Lan, H.-X., Li, M.-H., Zhang, G.-C., Parelkar, N.K., Fibuch, E.E., Haines, M., et al. (2006). Modulation of D2R-NR2B interactions in response to cocaine. Neuron 52, 897-909.

Lledo, P.M., Zhang, X., Südhof, T.C., Malenka, R.C., and Nicoll, R.A. (1998). Postsynaptic membrane fusion and long-term potentiation. Science 279, 399-403.

Loebrich, S., Djukic, B., Tong, Z.J., Cottrell, J.R., Turrigiano, G.G., and Nedivi, E. (2013). Regulation of glutamate receptor internalization by the spine cytoskeleton is mediated by its PKA-dependent association with CPG2. Proc. Natl. Acad. Sci. 110, E4548-E4556.

Lombardi, D., Soldati, T., Riederer, M.A., Goda, Y., Zerial, M., and Pfeffer, S.R. (1993). Rab9 functions in transport between late endosomes and the trans Golgi network. EMBO J. 12, 677682.

Lonart, G., Wang, J., and Johnson, K.M. (1992). Nitric oxide induces neurotransmitter release from hippocampal slices. Eur. J. Pharmacol. 220, 271-272.

Lorincz, A., and Nusser, Z. (2010). Molecular identity of dendritic voltage-gated sodium channels. Science 328, 906-909.

Lovero, K.L., Fukata, Y., Granger, A.J., Fukata, M., and Nicoll, R.A. (2015). The LGl1ADAM22 protein complex directs synapse maturation through regulation of PSD-95 function. Proc. Natl. Acad. Sci. 112, E4129-E4137.

Lowenthal, M.S., Markey, S.P., and Dosemeci, A. (2015). Quantitative Mass Spectrometry Measurements Reveal Stoichiometry of Principal Postsynaptic Density Proteins. J. Proteome Res. 150415143859000.

Lu, W., and Ziff, E.B. (2005). PICK1 interacts with ABP/GRIP to regulate AMPA receptor trafficking. Neuron $47,407-421$.

Lu, J., Helton, T.D., Blanpied, T.A., Rácz, B., Newpher, T.M., Weinberg, R.J., and Ehlers, M.D. (2007). Postsynaptic positioning of endocytic zones and AMPA receptor cycling by physical coupling of dynamin-3 to Homer. Neuron 55, 874-889. 
Luby-Phelps, K. (2000). Cytoarchitecture and physical properties of cytoplasm: volume, viscosity, diffusion, intracellular surface area. Int. Rev. Cytol. 192, 189-221.

Lugli, G., Larson, J., Martone, M.E., Jones, Y., and Smalheiser, N.R. (2005). Dicer and elF2c are enriched at postsynaptic densities in adult mouse brain and are modified by neuronal activity in a calpain-dependent manner. J. Neurochem. 94, 896-905.

Luo, F., Bacaj, T., and Sudhof, T.C. (2015). Synaptotagmin-7 Is Essential for Ca2+-Triggered Delayed Asynchronous Release But Not for Ca2+-Dependent Vesicle Priming in Retinal Ribbon Synapses. J. Neurosci. 35, 11024-11033.

Luzio, J.P., Brake, B., Banting, G., Howell, K.E., Braghetta, P., and Stanley, K.K. (1990). Identification, sequencing and expression of an integral membrane protein of the trans-Golgi network (TGN38). Biochem. J. 270, 97-102.

MacGillavry, H.D., Song, Y., Raghavachari, S., and Blanpied, T.A. (2013). Nanoscale scaffolding domains within the postsynaptic density concentrate synaptic ampa receptors. Neuron 78, 615-622.

MacGillavry, H.D., Kerr, J.M., Kassner, J., Frost, N.A., and Blanpied, T.A. (2016). Shankcortactin interactions control actin dynamics to maintain flexibility of neuronal spines and synapses. Eur. J. Neurosci. 43, 179-193.

Maejima, T., Hashimoto, K., Yoshida, T., Aiba, A., and Kano, M. (2001). Presynaptic inhibition caused by retrograde signal from metabotropic glutamate to cannabinoid receptors. Neuron $31,463-475$.

Magupalli, V.G., Mochida, S., Yan, J., Jiang, X., Westenbroek, R.E., Nairn, A.C., Scheuer, T., and Catterall, W.A. (2013). Ca2+-independent activation of Ca2+/Calmodulin- dependent protein kinase II bound to the C-terminal domain of CaV2.1 calcium channels. J. Biol. Chem. 288, 4637-4648.

Maidorn, M. (2017). Development of Nanobodies to Image Synaptic Proteins in SuperResolution Microscopy Dissertation.

Maio, V.D., Ventriglia, F., and Santillo, S. (2017). Stochastic, structural and functional factors influencing AMPA and NMDA synaptic response variability: a review. Neuronal Signal. 1, NS20160051.

Malenka, R.C., and Bear, M.F. (2004). LTP and LTD: An embarrassment of riches. Neuron 44, 5-21.

Malhotra, J.D., Kazen-Gillespie, K., Hortsch, M., and Isom, L.L. (2000). Sodium channel beta 
subunits mediate homophilic cell adhesion and recruit ankyrin to points of cell-cell contact. J. Biol. Chem. 275, 11383-11388.

Mallard, F., Tang, B.L., Galli, T., Tenza, D., Saint-Pol, A., Yue, X., Antony, C., Hong, W., Goud, B., and Johannes, L. (2002). Early/recycling endosomes-to-TGN transport involves two SNARE complexes and a Rab6 isoform. J. Cell Biol. 156, 653-664.

Man, H.-Y.Y., Wang, Q., Lu, W.-Y.Y., Ju, W., Ahmadian, G., Liu, L., D’Souza, S., Wong, T.. P., Taghibiglou, C., Lu, J., et al. (2003). Activation of PI3-kinase is required for AMPA receptor insertion during LTP of mEPSCs in cultured hippocampal neurons. Neuron 38, 611-624.

Mandad, S., Rahman, R.-U., Centeno, T.P., Vidal, R.O., Wildhagen, H., Rammner, B., Keihani, S., Opazo, F., Urban, I., Ischebeck, T., et al. (2018). The codon sequences predict protein lifetimes and other parameters of the protein life cycle in the mouse brain. Sci. Rep. 8, 16913.

Mansouri, M., Kasugai, Y., Fukazawa, Y., Bertaso, F., Raynaud, F., Perroy, J., Fagni, L., Kaufmann, W.A., Watanabe, M., Shigemoto, R., et al. (2015). Distinct subsynaptic localization of type 1 metabotropic glutamate receptors at glutamatergic and GABAergic synapses in the rodent cerebellar cortex. Eur. J. Neurosci. 41, 157-167.

Martinez-Arca, S., Rudge, R., Vacca, M., Raposo, G., Camonis, J., Proux-Gillardeaux, V., Daviet, L., Formstecher, E., Hamburger, A., Filippini, F., et al. (2003). A dual mechanism controlling the localization and function of exocytic v-SNAREs. Proc. Natl. Acad. Sci. 100, 9011-9016.

Masugi-Tokita, M., Tarusawa, E., Watanabe, M., Molnár, E., Fujimoto, K., and Shigemoto, R. (2007). Number and density of AMPA receptors in individual synapses in the rat cerebellum as revealed by SDS-digested freeze-fracture replica labeling. J. Neurosci. 27, 2135-2144.

Mathieson, T., Franken, H., Kosinski, J., Kurzawa, N., Zinn, N., Sweetman, G., Poeckel, D., Ratnu, V.S., Schramm, M., Becher, I., et al. (2018). Systematic analysis of protein turnover in primary cells. Nat. Commun. 9, 689 .

Matsuda, N., Lu, H., Fukata, Y., Noritake, J., Gao, H., Mukherjee, S., Nemoto, T., Fukata, M., and Poo, M.-M. (2009). Differential activity-dependent secretion of brain-derived neurotrophic factor from axon and dendrite. J. Neurosci. 29, 14185-14198.

Matsuzaki, M., Ellis-Davies, G.C., Nemoto, T., Miyashita, Y., lino, M., and Kasai, H. (2001). Dendritic spine geometry is critical for AMPA receptor expression in hippocampal CA1 pyramidal neurons. Nat. Neurosci. 4, 1086-1092.

Matsuzaki, M., Honkura, N., Ellis-Davies, G.C.R., and Kasai, H. (2004a). Structural basis of 
long-term potentiation in single dendritic spines. Nature 429, 761-766.

Matsuzaki, M., Honkura, N., Ellis-Davies, G.C.R., and Kasai, H. (2004b). Structural basis of long-term potentiation in single dendritic spines. Nature 429, 761-766.

Mayer, M.L., Westbrook, G.L., and Guthrie, P.B. (1984). Voltage-dependent block by Mg 2+ of NMDA responses in spinal cord neurones. Nature 309, 261-263.

Mazzarella, R.A., Srinivasan, M., Haugejorden, S.M., and Green, M. (1990). ERp72, an abundant luminal endoplasmic reticulum protein, contains three copies of the active site sequences of protein disulfide isomerase. J. Biol. Chem. 265, 1094-1101.

McBride, H.M., Rybin, V., Murphy, C., Giner, A., Teasdale, R., and Zerial, M. (1999). Oligomeric complexes link Rab5 effectors with NSF and drive membrane fusion via interactions between EEA1 and syntaxin 13. Cell 98, 377-386.

McGlade-McCulloh, E., Yamamoto, H., Tan, S.-E., Brickey, D.A., and Soderling, T.R. (1993). Phosphorylation and regulation of glutamate receptors by calcium/calmodulin-dependent protein kinase II. Nature 362, 640-642.

McGuffee, S.R., and Elcock, A.H. (2010). Diffusion, crowding \& protein stability in a dynamic molecular model of the bacterial cytoplasm. PLoS Comput. Biol. 6.

McMahon, H.T., and Boucrot, E. (2011). Molecular mechanism and physiological functions of clathrin-mediated endocytosis. Nat. Rev. Mol. Cell Biol. 12, 517-533.

McMahon, H.T., and Südhof, T.C. (1995). Synaptic core complex of synaptobrevin, syntaxin, and SNAP25 forms high affinity alpha-SNAP binding site. J. Biol. Chem. 270, 2213-2217.

McMahon, H.T., Ushkaryov, Y.A., Edelmann, L., Link, E., Binz, T., Niemann, H., Jahn, R., and Südhof, T.C. (1993). Cellubrevin is a ubiquitous tetanus-toxin substrate homologous to a putative synaptic vesicle fusion protein. Nature 364, 346-349.

McNew, J.A., Parlati, F., Fukuda, R., Johnston, R.J., Paz, K., Paumet, F., Söllner, T.H., and Rothman, J.E. (2000). Compartmental specificity of cellular membrane fusion encoded in SNARE proteins. Nature 407, 153-159.

Merriam, E.B., Millette, M., Lumbard, D.C., Saengsawang, W., Fothergill, T., Hu, X., Ferhat, L., and Dent, E.W. (2013). Synaptic Regulation of Microtubule Dynamics in Dendritic Spines by Calcium, F-Actin, and Drebrin. J. Neurosci. 33, 16471-16482.

Michalak, M., Groenendyk, J., Szabo, E., Gold, L.I., and Opas, M. (2009). Calreticulin, a multiprocess calcium-buffering chaperone of the endoplasmic reticulum. Biochem. J. 417, 651-666. 
Mikati, M.A., Grintsevich, E.E., and Reisler, E. (2013). Drebrin-induced stabilization of actin filaments. J. Biol. Chem. 288, 19926-19938.

Milo, R. (2013). What is the total number of protein molecules per cell volume? A call to rethink some published values. BioEssays 35, 1050-1055.

Minichiello, L., Calella, A.M., Medina, D.L., Bonhoeffer, T., Klein, R., and Korte, M. (2002). Mechanism of TrkB-mediated hippocampal long-term potentiation. Neuron 36, 121-137.

Misonou, H., Mohapatra, D.P., and Trimmer, J.S. (2005). Kv2.1: A voltage-gated K+channel critical to dynamic control of neuronal excitability. In NeuroToxicology, pp. 743-752.

Miyata, M., Finch, E.A., Khiroug, L., Hashimoto, K., Hayasaka, S., Oda, S.I., Inouye, M., Takagishi, Y., Augustine, G.J., and Kano, M. (2000). Local calcium release in dendritic spines required for long-term synaptic depression. Neuron 28, 233-244.

Miyoshi, D., and Sugimoto, N. (2008). Molecular crowding effects on structure and stability of DNA. Biochimie 90, 1040-1051.

Mizutani, A., Kuroda, Y., Futatsugi, A., Furuichi, T., and Mikoshiba, K. (2008). Phosphorylation of Homer3 by Calcium/Calmodulin-Dependent Kinase II Regulates a Coupling State of Its Target Molecules in Purkinje Cells. J. Neurosci. 28, 5369-5382.

Mohrmann, K., Gerez, L., Oorschot, V., Klumperman, J., and van der Sluijs, P. (2002). rab4 Function in Membrane Recycling from Early Endosomes Depends on a Membrane to Cytoplasm Cycle. J. Biol. Chem. 277, 32029-32035.

Molgaard, S., Ulrichsen, M., Boggild, S., Holm, M.-L., Vaegter, C., Nyengaard, J., and Glerup, S. (2014). Immunofluorescent visualization of mouse interneuron subtypes. F1000Research $3,242$.

von Mollard, G.F., Südhof, T.C., and Jahn, R. (1991). A small GTP-binding protein dissociates from synaptic vesicles during exocytosis. Nature 349, 79-81.

Molnár, E. (2011). Long-term potentiation in cultured hippocampal neurons. Semin. Cell Dev. Biol. 22, 506-513.

Monteiro, P., and Feng, G. (2017). SHANK proteins: Roles at the synapse and in autism spectrum disorder. Nat. Rev. Neurosci. 18, 147-157.

Montero-Hadjadje, M., Elias, S., Chevalier, L., Benard, M., Tanguy, Y., Turquier, V., Galas, L., Yon, L., Malagon, M.M., Driouich, A., et al. (2009). Chromogranin A Promotes Peptide Hormone Sorting to Mobile Granules in Constitutively and Regulated Secreting Cells. J. Biol. 
Chem. 284, 12420-12431.

Moosmang, S., Haider, N., Klugbauer, N., Adelsberger, H., Langwieser, N., Müller, J., Stiess, M., Marais, E., Schulla, V., Lacinova, L., et al. (2005). Role of Hippocampal Cav1.2 Ca2+ Channels in NMDA Receptor-Independent Synaptic Plasticity and Spatial Memory. J. Neurosci. 25, 9883-9892.

Müller, U.C., and Zheng, H. (2012). Physiological functions of APP family proteins. Cold Spring Harb. Perspect. Med. 2, a006288.

Müller, W., and Connor, J.A. (1991). Dendritic spines as individual neuronal compartments for synaptic Ca2+ responses. Nature $354,73-76$.

Münster-Wandowski, A., Heilmann, H., Bolduan, F., Trimbuch, T., Yanagawa, Y., and Vida, I. (2017). Distinct Localization of SNAP47 Protein in GABAergic and Glutamatergic Neurons in the Mouse and the Rat Hippocampus. Front. Neuroanat. 11, 1-18.

Murakoshi, H., and Trimmer, J.S. (1999). Identification of the Kv2.1 K+ channel as a major component of the delayed rectifier $\mathrm{K}+$ current in rat hippocampal neurons. J. Neurosci. 19, 1728-1735.

Murakoshi, H., Wang, H., and Yasuda, R. (2011). Local, persistent activation of Rho GTPases during plasticity of single dendritic spines. Nature 472, 100-104.

Murphy, J.E., Pleasure, I.T., Puszkin, S., Prasad, K., and Keen, J.H. (1991). Clathrin assembly protein AP-3. The identity of the $155 \mathrm{~K}$ protein, AP 180, and NP185 and demonstration of a clathrin binding domain. J. Biol. Chem. 266, 4401-4408.

Musacchio, A., Smith, C.J., Roseman, A.M., Harrison, S.C., Kirchhausen, T., and Pearse, B.M.F. (1999). Functional organization of clathrin in coats: combining electron cryomicroscopy and X-ray crystallography. Mol. Cell 3, 761-770.

Nair, D., Hosy, E., Petersen, J.D., Constals, A., Giannone, G., Choquet, D., and Sibarita, J.B.J.-B. (2013). Super-resolution imaging reveals that AMPA receptors inside synapses are dynamically organized in nanodomains regulated by PSD95. J. Neurosci. 33, 13204-13224.

Naisbitt, S., Eunjoon, K., Tu, J.C., Xiao, B., Sala, C., Valtschanoff, J., Weinberg, R.J., Worley, P.F., and Sheng, M. (1999). Shank, a novel family of postsynaptic density proteins that binds to the NMDA receptor/PSD-95/GKAP complex and cortactin. Neuron 23, 569-582.

Nakamura, K., Zuppini, A., Arnaudeau, S., Lynch, J., Ahsan, I., Krause, R., Papp, S., De Smedt, H., Parys, J.B., Müller-Esterl, W., et al. (2001). Functional specialization of calreticulin domains. J. Cell Biol. 154, 961-972. 
Nakayama, A.Y., Harms, M.B., and Luo, L. (2000). Small GTPases Rac and Rho in the maintenance of dendritic spines and branches in hippocampal pyramidal neurons. J. Neurosci. 20, 5329-5338.

Nanou, E., Scheuer, T., and Catterall, W.A. (2016). Calcium sensor regulation of the Ca V 2.1 Ca 2+ channel contributes to long-term potentiation and spatial learning. Proc. Natl. Acad. Sci. 113, 13209-13214.

Natori, S., and Huttner, W.B. (1996). Chromogranin B (secretogranin I) promotes sorting to the regulated secretory pathway of processing intermediates derived from a peptide hormone precursor. Proc. Natl. Acad. Sci. U. S. A. 93, 4431-4436.

Nevins, A.K., and Thurmond, D.C. (2005). A Direct Interaction between Cdc42 and Vesicleassociated Membrane Protein 2 Regulates SNARE-dependent Insulin Exocytosis. J. Biol. Chem. 280, 1944-1952.

Newpher, T.M., and Ehlers, M.D. (2008). Glutamate receptor dynamics in dendritic microdomains. Neuron 58, 472-497.

Niethammer, M., Kim, E., and Sheng, M. (1996). Interaction between the C terminus of NMDA receptor subunits and multiple members of the PSD-95 family of membrane-associated guanylate kinases. J. Neurosci. 16, 2157-2163.

Noguchi, J., Nagaoka, A., Watanabe, S., Ellis-Davies, G.C.R., Kitamura, K., Kano, M., Matsuzaki, M., and Kasai, H. (2011). In vivo two-photon uncaging of glutamate revealing the structure-function relationships of dendritic spines in the neocortex of adult mice. J. Physiol. 589, 2447-2457.

Norris, F.A., Ungewickell, E., and Majerus, P.W. (1995). Inositol hexakisphosphate binds to clathrin assembly protein 3 (AP-3/AP180) and inhibits clathrin cage assembly in vitro. J. Biol. Chem. 270, 214-217.

Nusser, Z., Lujan, R., Laube, G., Roberts, J.D.B., Molnar, E., and Somogyi, P. (1998). Cell type and pathway dependence of synaptic AMPA receptor number and variability in the hippocampus. Neuron 21, 545-559.

O’Brien, R.J., Kamboj, S., Ehlers, M.D., Rosen, K.R., Fischbach, G.D., and Huganir, R.L. (1998). Activity-dependent modulation of synaptic AMPA receptor accumulation. Neuron 21, 1067-1078.

O'Connell, K.M.S., Loftus, R., and Tamkun, M.M. (2010). Localization-dependent activity of the Kv2.1 delayed-rectifier K+ channel. Proc. Natl. Acad. Sci. U. S. A. 107, 12351-12356. 
Ogawa, Y., Oses-Prieto, J., Kim, M.Y., Horresh, I., Peles, E., Burlingame, A.L., Trimmer, J.S., Meijer, D., and Rasband, M.N. (2010). ADAM22, a Kv1 channel-interacting protein, recruits membrane-associated guanylate kinases to juxtaparanodes of myelinated axons. J. Neurosci. 30, 1038-1048.

Oh, S.W., Harris, J.A., Ng, L., Winslow, B., Cain, N., Mihalas, S., Wang, Q., Lau, C., Kuan, L., Henry, A.M., et al. (2014). A mesoscale connectome of the mouse brain. Nature 508, 207214.

Ohkuma, S., Katsura, M., Chen, D.Z., Narihara, H., and Kuriyama, K. (1996). Nitric oxideevoked [3H] gamma-aminobutyric acid release is mediated by two distinct release mechanisms. Brain Res. Mol. Brain Res. 36, 137-144.

Ohnuma, K., Whim, M.D., Fetter, R.D., Kaczmarek, L.K., and Zucker, R.S. (2001). Presynaptic target of $\mathrm{Ca} 2+$ action on neuropeptide and acetylcholine release in Aplysia californica. J. Physiol.

Okamoto, K.I., Nagai, T., Miyawaki, A., and Hayashi, Y. (2004). Rapid and persistent modulation of actin dynamics regulates postsynaptic reorganization underlying bidirectional plasticity. Nat. Neurosci. 7, 1104-1112.

Olivo-Marin, J.C. (2002). Extraction of spots in biological images using multiscale products. Pattern Recognit. 35, 1989-1996.

Olsen, J. V., de Godoy, L.M.F., Li, G., Macek, B., Mortensen, P., Pesch, R., Makarov, A., Lange, O., Horning, S., and Mann, M. (2005). Parts per Million Mass Accuracy on an Orbitrap Mass Spectrometer via Lock Mass Injection into a C-trap. Mol. Cell. Proteomics 4, 2010-2021.

Olwin, B.B., Keller, C.H., and Storm, D.R. (1984). Calcium-dependent and calciumindependent affinities of calmodulin for calmodulin-binding proteins determined by fluorescence techniques. Adv. Cyclic Nucleotide Protein Phosphorylation Res. 16, 227-243.

Opazo, P., Labrecque, S., Tigaret, C.M., Frouin, A., Wiseman, P.W., De Koninck, P., and Choquet, D. (2010). CaMKII triggers the diffusional trapping of surface AMPARs through phosphorylation of stargazin. Neuron 67, 239-252.

Osten, P., Srivastava, S., Inman, G.., Vilim, F.., Khatri, L., Lee, L.., States, B.., Einheber, S., Milner, T.., Hanson, P.., et al. (1998). The AMPA Receptor GluR2 C Terminus Can Mediate a Reversible, ATP-Dependent Interaction with NSF and $\alpha$ - and $\beta$-SNAPs. Neuron 21, 99-110.

Ostroff, L.E., Fiala, J.C., Allwardt, B., and Harris, K.M. (2002). Polyribosomes redistribute from dendritic shafts into spines with enlarged synapses during LTP in developing rat hippocampal 
slices. Neuron 35, 535-545.

Otmakhov, N., and Lisman, J. (2012). Measuring CaMKII concentration in dendritic spines. J. Neurosci. Methods 203, 106-114.

Oyler, G.A., Higgins, G.A., Hart, R.A., Battenberg, E., Billingsley, M., Bloom, F.E., and Wilson, M.C. (1989). The identification of a novel synaptosomal-associated protein, SNAP-25, differentially expressed by neuronal subpopulations. J. Cell Biol. 109, 3039-3052.

Pan, P.-Y., Cai, Q., Lin, L., Lu, P.-H., Duan, S., and Sheng, Z.-H. (2005). SNAP-29-mediated Modulation of Synaptic Transmission in Cultured Hippocampal Neurons. J. Biol. Chem. 280, 25769-25779.

Pandya, N.J., Klaassen, R. V., van der Schors, R.C., Slotman, J.A., Houtsmuller, A., Smit, A.B., and Li, K.W. (2016). Group 1 metabotropic glutamate receptors 1 and 5 form a protein complex in mouse hippocampus and cortex. Proteomics 16, 2698-2705.

Papa, M., Bundman, M.C., Greenberger, V., and Segal, M. (1995). Morphological analysis of dendritic spine development in primary cultures of hippocampal neurons. J. Neurosci. 15, 111.

Park, M., Penick, E.C., Edwards, J.G., Kauer, J.A., and Ehlers, M.D. (2004). Recycling endosomes supply AMPA receptors for LTP. Science 305, 1972-1975.

Park, M., Salgado, J.M., Ostroff, L., Helton, T.D., Robinson, C.G., Harris, K.M., and Ehlers, M.D. (2006). Plasticity-induced growth of dendritic spines by exocytic trafficking from recycling endosomes. Neuron 52, 817-830.

Park, S., Park, J.M., Kim, S., Kim, J.-A., Shepherd, J.D., Smith-Hicks, C.L., Chowdhury, S., Kaufmann, W., Kuhl, D., Ryazanov, A.G., et al. (2008). Elongation Factor 2 and Fragile X Mental Retardation Protein Control the Dynamic Translation of Arc/Arg3.1 Essential for mGluR-LTD. Neuron 59, 70-83.

Parker, M.J., Zhao, S., Bredt, D.S., Sanes, J.R., and Feng, G. (2004). PSD93 regulates synaptic stability at neuronal cholinergic synapses. J. Neurosci. 24, 378-388.

Parnass, Z., Tashiro, A., and Yuste, R. (2000). Analysis of spine morphological plasticity in developing hippocampal pyramidal neurons. Hippocampus 10, 561-568.

Passafaro, M., Piëch, V., and Sheng, M. (2001). Subunit-specific temporal and spatial patterns of AMPA receptor exocytosis in hippocampal neurons. Nat. Neurosci. 4, 917-926.

Patterson, S.L., Abel, T., Deuel, T.A., Martin, K.C., Rose, J.C., and Kandel, E.R. (1996). 
Recombinant BDNF rescues deficits in basal synaptic transmission and hippocampal LTP in BDNF knockout mice. Neuron 16, 1137-1145.

Pei, L., Lee, F.J.S., Moszczynska, A., Vukusic, B., and Liu, F. (2004). Regulation of dopamine D1 receptor function by physical interaction with the NMDA receptors. J. Neurosci. 24, 11491158.

Pen, Y., Borovok, N., Reichenstein, M., Sheinin, A., and Michaelevski, I. (2016). Membranetethered AKT kinase regulates basal synaptic transmission and early phase LTP expression by modulation of post-synaptic AMPA receptor level. Hippocampus 26, 1149-1167.

Peng, J., Kim, M.J., Cheng, D., Duong, D.M., Gygi, S.P., and Sheng, M. (2004a). Semiquantitative proteomic analysis of rat forebrain postsynaptic density fractions by mass spectrometry. J. Biol. Chem. 279, 21003-21011.

Peng, J., Kim, M.J., Cheng, D., Duong, D.M., Gygi, S.P., and Sheng, M. (2004b). Semiquantitative proteomic analysis of rat forebrain postsynaptic density fractions by mass spectrometry- Supplement. J. Biol. Chem.

Peng, J., Kim, M.J., Duong, D.M., Gygi, S.P., Sheng, M., Jong, M., and Cheng, D. (2004c). Semiquantitative Proteomic Analysis of Rat Forebrain Postsynaptic Density Fractions by Mass Spectrometry Supplemental material : Density Fractions by Mass Spectrometry.

Pennuto, M., Bonanomi, D., Benfenati, F., and Valtorta, F. (2003). Synaptophysin I controls the targeting of VAMP2/synaptobrevin II to synaptic vesicles. Mol. Biol. Cell 14, 4909-4919.

Pérez-Otaño, I., Luján, R., Tavalin, S.J., Plomann, M., Modregger, J., Liu, X.-B., Jones, E.G., Heinemann, S.F., Lo, D.C., and Ehlers, M.D. (2006). Endocytosis and synaptic removal of NR3A-containing NMDA receptors by PACSIN1/syndapin1. Nat. Neurosci. 9, 611-621.

Perez, R.G., Zheng, H., Van der Ploeg, L.H., and Koo, E.H. (1997). The beta-amyloid precursor protein of Alzheimer's disease enhances neuron viability and modulates neuronal polarity. J. Neurosci. 17, 9407-9414.

Perroy, J., Raynaud, F., Homburger, V., Rousset, M.-C., Telley, L., Bockaert, J., and Fagni, L. (2008). Direct interaction enables cross-talk between ionotropic and group I metabotropic glutamate receptors. J. Biol. Chem. 283, 6799-6805.

Pierce, J.P., van Leyen, K., and McCarthy, J.B. (2000). Translocation machinery for synthesis of integral membrane and secretory proteins in dendritic spines. Nat. Neurosci. 3, 311-313.

Poindexter, K., Nelson, N., DuBose, R.F., Black, R.A., and Cerretti, D.P. (1999). The identification of seven metalloproteinase-disintegrin (ADAM) genes from genomic libraries. 
Gene 237, 61-70.

Predescu, S.A., Predescu, D.N., Shimizu, K., Klein, I.K., and Malik, A.B. (2005). Cholesteroldependent Syntaxin-4 and SNAP-23 Clustering Regulates Caveolar Fusion with the Endothelial Plasma Membrane. J. Biol. Chem. 280, 37130-37138.

Prichard, L., Deloulme, J.C., and Storm, D.R. (1999). Interactions between neurogranin and calmodulin in vivo. J. Biol. Chem. 274, 7689-7694.

PUCK, T.T., MARCUS, P.I., and CIECIURA, S.J. (1956). Clonal growth of mammalian cells in vitro; growth characteristics of colonies from single HeLa cells with and without a feeder layer. J. Exp. Med. 103, 273-283.

Qin, Y., Zhu, Y., Baumgart, J.P., Stornetta, R.L., Seidenman, K., Mack, V., van Aelst, L., and Zhu, J.J. (2005). State-dependent Ras signaling and AMPA receptor trafficking. Genes Dev. 19, 2000-2015.

Racca, C., Stephenson, F.A., Streit, P., Roberts, J.D., and Somogyi, P. (2000). NMDA receptor content of synapses in stratum radiatum of the hippocampal CA1 area. J. Neurosci. 20, 25122522.

Rácz, B., Blanpied, T.A., Ehlers, M.D., and Weinberg, R.J. (2004). Lateral organization of endocytic machinery in dendritic spines. Nat. Neurosci. 7, 917-918.

Raghavachari, S., and Lisman, J.E. (2004). Properties of Quantal Transmission at CA1 Synapses. J. Neurophysiol. 92, 2456-2467.

Raimondi, A., Ferguson, S.M., Lou, X., Armbruster, M., Paradise, S., Giovedi, S., Messa, M., Kono, N., Takasaki, J., Cappello, V., et al. (2011). Overlapping Role of Dynamin Isoforms in Synaptic Vesicle Endocytosis. Neuron 70, 1100-1114.

Ramón y Cajal, S. (1888). Estructura de los centros nerviosos de las aves. Rev. Trim. Histol. Norm. Pat. 1, 1-10.

Ramsey, M.M., Adams, M.M., Ariwodola, O.J., Sonntag, W.E., and Weiner, J.L. (2005). Functional Characterization of Des-IGF-1 Action at Excitatory Synapses in the CA1 Region of Rat Hippocampus. J. Neurophysiol. 94, 247-254.

Rao, M. V., Mohan, P.S., Kumar, A., Yuan, A., Montagna, L., Campbell, J., Veeranna, Espreafico, E.M., Julien, J.P., and Nixon, R.A. (2011). The Myosin Va Head Domain Binds to the Neurofilament-L Rod and Modulates Endoplasmic Reticulum (ER) Content and Distribution within Axons. PLoS One 6, e17087. 
Rao, S.K., Huynh, C., Proux-Gillardeaux, V., Galli, T., and Andrews, N.W. (2004). Identification of SNAREs Involved in Synaptotagmin VII-regulated Lysosomal Exocytosis. J. Biol. Chem. 279, 20471-20479.

Reaves, B., Horn, M., and Banting, G. (1993). TGN38/41 recycles between the cell surface and the TGN: brefeldin A affects its rate of return to the TGN. Mol. Biol. Cell 4, 93-105.

Reilly, J.E., Hanson, H.H., and Phillips, G.R. (2011). Persistence of excitatory shaft synapses adjacent to newly emerged dendritic protrusions. Mol. Cell. Neurosci. 48, 129-136.

Ren, G., Helwani, F.M., Verma, S., McLachlan, R.W., Weed, S.A., and Yap, A.S. (2009). Cortactin Is a Functional Target of E-cadherin-activated Src Family Kinases in MCF7 Epithelial Monolayers. J. Biol. Chem. 284, 18913-18922.

Renna, M., Schaffner, C., Winslow, A.R., Menzies, F.M., Peden, A.A., Floto, R.A., and Rubinsztein, D.C. (2011). Autophagic substrate clearance requires activity of the syntaxin-5 SNARE complex. J. Cell Sci. 124, 469-482.

Ribrault, C., Sekimoto, K., and Triller, A. (2011). From the stochasticity of molecular processes to the variability of synaptic transmission. Nat. Rev. Neurosci. 12, 375-387.

Riccio, A., Alvania, R.S., Lonze, B.E., Ramanan, N., Kim, T., Huang, Y., Dawson, T.M., Snyder, S.H., and Ginty, D.D. (2006). A Nitric Oxide Signaling Pathway Controls CREB-Mediated Gene Expression in Neurons. Mol. Cell 21, 283-294.

Richter, K.N., Revelo, N.H., Seitz, K.J., Helm, M.S., Sarkar, D., Saleeb, R.S., D’Este, E., Eberle, J., Wagner, E., Vogl, C., et al. (2017). Glyoxal as an alternative fixative to formaldehyde in immunostaining and super-resolution microscopy. EMBO J. e201695709.

Richter, K.N., Wildhagen, H., Helm, M.S., Ußling, J.-E., Schikorski, T., and Rizzoli, S.O. (2018). Comparative synaptosome imaging: a semi-quantitative method to obtain copy numbers for synaptic and neuronal proteins. Sci. Rep. 8, 14838.

Roche, K.W., Tu, J.C., Petralia, R.S., Xiao, B., Wenthold, R.J., and Worley, P.F. (1999). Homer $1 \mathrm{~b}$ regulates the trafficking of group I metabotropic glutamate receptors. J. Biol. Chem. 274, 25953-25957.

Rodriguez-Tébar, A., Dechant, G., and Barde, Y.A. (1990). Binding of brain-derived neurotrophic factor to the nerve growth factor receptor. Neuron 4, 487-492.

Rolfe, D.F., and Brown, G.C. (1997). Cellular energy utilization and molecular origin of standard metabolic rate in mammals. Physiol. Rev. 77, 731-758. 
Rosendale, M., Jullié, D., Choquet, D., Perrais, D., Julli??, D., Choquet, D., and Perrais, D. (2017). Spatial and Temporal Regulation of Receptor Endocytosis in Neuronal Dendrites Revealed by Imaging of Single Vesicle Formation. Cell Rep. 18, 1840-1847.

Rostaing, P., Real, E., Siksou, L., Lechaire, J.-P.P., Boudier, T., Boeckers, T.M., Gertler, F., Gundelfinger, E.D., Triller, A., and Marty, S. (2006). Analysis of synaptic ultrastructure without fixative using high-pressure freezing and tomography. Eur. J. Neurosci. 24, 3463-3474.

Rothnie, A., Clarke, A.R., Kuzmic, P., Cameron, A., and Smith, C.J. (2011). A sequential mechanism for clathrin cage disassembly by $70-\mathrm{kDa}$ heat-shock cognate protein (Hsc70) and auxilin. Proc. Natl. Acad. Sci. 108, 6927-6932.

Roy, A., Kucukural, A., and Zhang, Y. (2010). I-TASSER: A unified platform for automated protein structure and function prediction. Nat. Protoc.

Rudolf, R., Bittins, C.M., and Gerdes, H.-H.H. (2011). The role of myosin v in exocytosis and synaptic plasticity. J. Neurochem. 116, 177-191.

Rupp, K., Birnbach, U., Lundström, J., Van, P.N., and Söling, H.D. (1994). Effects of CaBP2, the rat analog of ERp72, and of CaBP1 on the refolding of denatured reduced proteins. Comparison with protein disulfide isomerase. J. Biol. Chem. 269, 2501-2507.

Sabatini, B.L., and Svoboda, K. (2000). Analysis of calcium channels in single spines using optical fluctuation analysis. Nature 408, 589-593.

Sabatini, B.L., Maravall, M., and Svoboda, K. (2001). Ca2+ signaling in dendritic spines. Curr. Opin. Neurobiol. 11, 349-356.

Sadakata, T., Kakegawa, W., Shinoda, Y., Hosono, M., Katoh-Semba, R., Sekine, Y., Sato, Y., Tanaka, M., Iwasato, T., Itohara, S., et al. (2013). CAPS1 Deficiency Perturbs Dense-Core Vesicle Trafficking and Golgi Structure and Reduces Presynaptic Release Probability in the Mouse Brain. J. Neurosci. 33, 17326-17334.

Saegusa, C., Fukuda, M., and Mikoshiba, K. (2002). Synaptotagmin V is targeted to densecore vesicles that undergo calcium-dependent exocytosis in PC12 cells. J. Biol. Chem. 277, 24499-24505.

Sagane, K., Ohya, Y., Hasegawa, Y., and Tanaka, I. (1998). Metalloproteinase-like, disintegrin-like, cysteine-rich proteins MDC2 and MDC3: novel human cellular disintegrins highly expressed in the brain. Biochem. J. 334 ( Pt 1, 93-98.

Saitoh, T., Igura, M., Obita, T., Ose, T., Kojima, R., Maenaka, K., Endo, T., and Kohda, D. (2007). Tom20 recognizes mitochondrial presequences through dynamic equilibrium among 
multiple bound states. EMBO J. 26, 4777-4787.

Salimi, V. (2017). Die präzise Ultrastruktur der Organellen der dendritischen Spines.

Sambandan, S., Akbalik, G., Kochen, L., Rinne, J., Kahlstatt, J., Glock, C., Tushev, G., Alvarez-Castelao, B., Heckel, A., and Schuman, E.M. (2017). Activity-dependent spatially localized miRNA maturation in neuronal dendrites. Science (80-. ). 355, 634-637.

Sampo, B., Kaech, S., Kunz, S., and Banker, G. (2003). Two distinct mechanisms target membrane proteins to the axonal surface. Neuron 37, 611-624.

Sans, N., Petralia, R.S., Wang, Y.X., Blahos, J., Hell, J.W., and Wenthold, R.J. (2000). A developmental change in NMDA receptor-associated proteins at hippocampal synapses. J. Neurosci. 20, 1260-1271.

Santamaria, F., Gonzalez, J., Augustine, G.J., and Raghavachari, S. (2010). Quantifying the Effects of Elastic Collisions and Non-Covalent Binding on Glutamate Receptor Trafficking in the Post-Synaptic Density. PLoS Comput. Biol. 6, e1000780.

Satoh, K., Yanai, H., Senda, T., Kohu, K., Nakamura, T., Okumura, N., Matsumine, A., Kobayashi, S., Toyoshima, K., and Akiyama, T. (1997). DAP-1, a novel protein that interacts with the guanylate kinase-like domains of hDLG and PSD-95. Genes Cells 2, 415-424.

Savtchenko, L.P., and Rusakov, D. a (2014). Moderate AMPA receptor clustering on the nanoscale can efficiently potentiate synaptic current. Philos. Trans. R. Soc. Lond. B. Biol. Sci.

Scannevin, R.H., Murakoshi, H., Rhodes, K.J., and Trimmer, J.S. (1996). Identification of a cytoplasmic domain important in the polarized expression and clustering of the Kv2.1 K+channel. J. Cell Biol. 135, 1619-1632.

Schaffhauser, H., Cai, Z., Hubalek, F., Macek, T.A., Pohl, J., Murphy, T.J., and Conn, P.J. (2000). cAMP-dependent protein kinase inhibits mGluR2 coupling to G-proteins by direct receptor phosphorylation. J. Neurosci. 20, 5663-5670.

Schanzenbächer, C.T., Sambandan, S., Langer, J.D., and Schuman, E.M. (2016). Nascent Proteome Remodeling following Homeostatic Scaling at Hippocampal Synapses. Neuron 92, 358-371.

Schanzenbächer, C.T., Langer, J.D., and Schuman, E.M. (2018). Time- and polaritydependent proteomic changes associated with homeostatic scaling at central synapses. Elife 7, 1-20.

Scheuber, A., Rudge, R., Danglot, L., Raposo, G., Binz, T., Poncer, J.-C., and Galli, T. (2006). 
Loss of AP-3 function affects spontaneous and evoked release at hippocampal mossy fiber synapses. Proc. Natl. Acad. Sci. 103, 16562-16567.

Schiffelholz, T., and Aldenhoff, J.B. (2002). Novel object presentation affects sleep-wake behavior in rats. Neurosci. Lett. 328, 41-44.

Schikorski, T., and Stevens, C.F. (1997). Quantitative ultrastructural analysis of hippocampal excitatory synapses. J. Neurosci. 17, 5858-5867.

Schlossman, D.M., Schmid, S.L., Braell, W.A., and Rothman, J.E. (1984). An enzyme that removes clathrin coats: Purification of an uncoating ATPase. J. Cell Biol. 99, 723-733.

Schmitz, D., Mellor, J., and Nicoll, R.A. (2001a). Presynaptic kainate receptor mediation of frequency facilitation at hippocampal mossy fiber synapses. Science (80-. ).

Schmitz, D., Mellor, J., Frerking, M., and Nicoll, R.A. (2001b). Presynaptic kainate receptors at hippocampal mossy fiber synapses. Proc. Natl. Acad. Sci. U. S. A. 98, 11003-11008.

Schneider, C.A., Rasband, W.S., and Eliceiri, K.W. (2012). NIH Image to ImageJ: 25 years of image analysis. Nat. Methods 9, 671-675.

Schnell, E., Sizemore, M., Karimzadegan, S., Chen, L.L., Bredt, D.S., and Nicoll, R.A. (2002). Direct interactions between PSD-95 and stargazin control synaptic AMPA receptor number. Proc. Natl. Acad. Sci. 99, 13902-13907.

Schratt, G.M., Nigh, E.A., Chen, W.G., Hu, L., and Greenberg, M.E. (2004). BDNF regulates the translation of a select group of mRNAs by a mammalian target of rapamycinphosphatidylinositol 3-kinase-dependent pathway during neuronal development. J. Neurosci. 24, 7366-7377.

Schulman, H. (1984). Phosphorylation of microtubule-associated proteins by a Ca2+/calmodulin-dependent protein kinase. J. Cell Biol. 99, 11-19.

Schultze, W., Eulenburg, V., Lessmann, V., Herrmann, L., Dittmar, T., Gundelfinger, E.D., Heumann, R., and Erdmann, K.S. (2001). Semaphorin4F interacts with the synapseassociated protein SAP90/PSD-95. J. Neurochem. 78, 482-489.

Schwanhäusser, B., Busse, D., Li, N., Dittmar, G., Schuchhardt, J., Wolf, J., Chen, W., and Selbach, M. (2011). Global quantification of mammalian gene expression control. Nature 473, 337-342.

Schwenk, J., Harmel, N., Brechet, A., Zolles, G., Berkefeld, H., Müller, C.S., Bildl, W., Baehrens, D., Hüber, B., Kulik, A., et al. (2012). High-Resolution Proteomics Unravel 
Architecture and Molecular Diversity of Native AMPA Receptor Complexes. Neuron 74, 621633.

Shaikh, S.A., Dolino, D.M., Lee, G., Chatterjee, S., MacLean, D.M., Flatebo, C., Landes, C.F., Jayaraman, V., Amico-Ruvio, S.A., Popescu, G.K., et al. (2016). Stargazin Modulation of AMPA Receptors. Cell Rep. 17, 328-335.

Shaw, G., and Weber, K. (1982). Differential expression of neurofilament triplet proteins in brain development. Nature 298, 277-279.

Shen, K., and Meyer, T. (1999). Dynamic control of caMKII translocation and localization in hippocampal neurons by NMDA receptor stimulation. Science (80-. ). 284, 162-166.

Sheng, M., and Kim, E. (2011). The postsynaptic organization of synapses. Cold Spring Harb. Perspect. Biol. 3.

Sheng, M., Cummings, J., Roldan, L.A., Jan, Y.N., and Jan, L.Y. (1994a). Changing subunit composition of heteromeric NMDA receptors during development of rat cortex. Nature 368, 144-147.

Sheng, M., Cummings, J., Roldan, L.A., Jan, Y.N., and Jan, L.Y. (1994b). Changing subunit composition of heteromeric NMDA receptors during development of rat cortex. Nature 368, 144-147.

Shi, S., Hayashi, Y., Esteban, J.A., and Malinow, R. (2001). Subunit-specific rules governing AMPA receptor trafficking to synapses in hippocampal pyramidal neurons. Cell 105, 331-343.

Shimojo, M., Courchet, J., Pieraut, S., Torabi-Rander, N., Sando, R., Polleux, F., and Maximov, A. (2015). SNAREs Controlling Vesicular Release of BDNF and Development of Callosal Axons. Cell Rep. 11, 1054-1066.

Shin, Y., Berry, J., Pannucci, N., Haataja, M.P., Toettcher, J.E., and Brangwynne, C.P. (2017). Spatiotemporal Control of Intracellular Phase Transitions Using Light-Activated optoDroplets. Cell 168, 159-171.e14.

Shinohara, Y., Hirase, H., Watanabe, M., Itakura, M., Takahashi, M., and Shigemoto, R. (2008). Left-right asymmetry of the hippocampal synapses with differential subunit allocation of glutamate receptors. Proc. Natl. Acad. Sci. U. S. A. 105, 19498-19503.

Shiraishi-Yamaguchi, Y., Sato, Y., Sakai, R., Mizutani, A., Knöpfel, T., Mori, N., Mikoshiba, K., and Furuichi, T. (2009). Interaction of Cupidin/Homer2 with two actin cytoskeletal regulators, Cdc42 small GTPase and Drebrin, in dendritic spines. BMC Neurosci. 10, 1-14. 
Shirane, M., and Nakayama, K.I. (2006). Protrudin induces neurite formation by directional membrane trafficking. Science $314,818-821$.

Shitara, A., Shibui, T., Okayama, M., Arakawa, T., Mizoguchi, I., Shakakura, Y., Takuma, T., and Takuma, T. (2013). VAMP4 is required to maintain the ribbon structure of the Golgi apparatus. Mol. Cell. Biochem. 380, 11-21.

Shitara, A., Shibui, T., Okayama, M., Arakawa, T., Mizoguchi, I., Sakakura, Y., and Takuma, T. (2017). VAMP4 and its cognate SNAREs are required for maintaining the ribbon structure of the Golgi apparatus. J. Oral Biosci. 59, 192-196.

Shnyrova, A. V, Bashkirov, P. V, Akimov, S.A., Pucadyil, T.J., Zimmerberg, J., Schmid, S.L., and Frolov, V.A. (2013). Geometric catalysis of membrane fission driven by flexible dynamin rings. Science 339, 1433-1436.

Shuford, C.M., Sederoff, R.R., Chiang, V.L., and Muddiman, D.C. (2012). Peptide production and decay rates affect the quantitative accuracy of protein cleavage isotope dilution mass spectrometry (PC-IDMS). Mol. Cell. Proteomics 11, 814-823.

Sidenstein, S.C., D’Este, E., Böhm, M.J., Danzl, J.G., Belov, V.N., Hell, S.W., D’Este, E., Böhm, M.J., Danzl, J.G., Belov, V.N., et al. (2016). Multicolour Multilevel STED nanoscopy of Actin/Spectrin Organization at Synapses. Sci. Rep. 6, 26725.

Sieber, J.J., Willig, K.I., Heintzmann, R., Hell, S.W., and Lang, T. (2006). The SNARE motif is essential for the formation of syntaxin clusters in the plasma membrane. Biophys. J. 90, 28432851.

Sieber, J.J., Willig, K.I., Kutzner, C., Gerding-Reimers, C., Harke, B., Donnert, G., Rammner, B., Eggeling, C., Hell, S.W., Grubmuller, H., et al. (2007). Anatomy and Dynamics of a Supramolecular Membrane Protein Cluster. Science (80-. ). 317, 1072-1076.

Simons, M., de Strooper, B., Multhaup, G., Tienari, P.J., Dotti, C.G., and Beyreuther, K. (1996). Amyloidogenic processing of the human amyloid precursor protein in primary cultures of rat hippocampal neurons. J. Neurosci. 16, 899-908.

Simonsen, A., Bremnes, B., Rønning, E., Aasland, R., and Stenmark, H. (1998). Syntaxin-16, a putative Golgi t-SNARE. Eur. J. Cell Biol. 75, 223-231.

Skeberdis, V.A., Lan, J., Opitz, T., Zheng, X., Bennett, M. V, and Zukin, R.S. (2001). mGluR1 mediated potentiation of NMDA receptors involves a rise in intracellular calcium and activation of protein kinase C. Neuropharmacology 40, 856-865.

Skou, J.C. (1957). The influence of some cations on an adenosine triphosphatase from 
peripheral nerves. Biochim. Biophys. Acta 23, 394-401.

Slunt, H.H., Thinakaran, G., Von Koch, C., Lo, A.C., Tanzi, R.E., and Sisodia, S.S. (1994). Expression of a ubiquitous, cross-reactive homologue of the mouse beta-amyloid precursor protein (APP). J. Biol. Chem. 269, 2637-2644.

Smith, R.S., and Araneda, R.C. (2010). Cholinergic Modulation of Neuronal Excitability in the Accessory Olfactory Bulb. J. Neurophysiol. 104, 2963-2974.

Söllner, T., Griffiths, G., Pfaller, R., Pfanner, N., and Neupert, W. (1989). MOM19, an import receptor for mitochondrial precursor proteins. Cell 59, 1061-1070.

Söllner, T., Bennett, M.K., Whiteheart, S.W., Scheller, R.H., and Rothman, J.E. (1993a). A protein assembly-disassembly pathway in vitro that may correspond to sequential steps of synaptic vesicle docking, activation, and fusion. Cell 75, 409-418.

Söllner, T., Whiteheart, S.W., Brunner, M., Erdjument-Bromage, H., Geromanos, S., Tempst, P., and Rothman, J.E. (1993b). SNAP receptors implicated in vesicle targeting and fusion. Nature 362, 318-324.

Song, I., and Huganir, R.L. (2002). Regulation of AMPA receptors during synaptic plasticity. Trends Neurosci. 25, 578-588.

Soppet, D., Maragos, J., Raid, S.W., Blair, J., Stanton, B.R., Parade, L.F., Escandon, E., Burton, L.E., Nikolics, K., Middlemas, D.S., et al. (1991). The neurotrophic factors brainderived neurotrophic factor and neurotrophin-3 are ligands for the trkB tyrosine kinase receptor. Cell 65, 895-903.

Sorra, K.E., and Harris, K.M. (2000). Overview on the structure, composition, function, development, and plasticity of hippocampal dendritic spines. Hippocampus 10, 501-511.

Sousa, R., Tannery, N.H., Zhou, S., and Lafer, E.M. (1992). Characterization of a novel synapse-specific protein. I. Developmental expression and cellular localization of the F1-20 protein and mRNA. J. Neurosci. 12, 2130-2143.

Spacek, J., and Harris, K.M. (1997). Three-dimensional organization of smooth endoplasmic reticulum in hippocampal CA1 dendrites and dendritic spines of the immature and mature rat. J. Neurosci. 17, 190-203.

Stanika, R., Campiglio, M., Pinggera, A., Lee, A., Striessnig, J., Flucher, B.E., and Obermair, G.J. (2016). Splice variants of the CaV1.3 L-type calcium channel regulate dendritic spine morphology. Sci. Rep. 6, 34528. 
Steegmaier, M., Yang, B., Yoo, J.S., Huang, B., Shen, M., Yu, S., Luo, Y., and Scheller, R.H. (1998). Three novel proteins of the syntaxin/SNAP-25 family. J. Biol. Chem. 273, 3417134179.

Stellwagen, D., and Malenka, R.C. (2006). Synaptic scaling mediated by glial TNF-alpha. Nature 440, 1054-1059.

Steward, O., and Levy, W.B. (1982). Preferential localization of polyribosomes under the base of dendritic spines in granule cells of the dentate gyrus. J. Neurosci. 2, 284-291.

Steward, O., and Reeves, T.M. (1988). Protein-synthetic machinery beneath postsynaptic sites on CNS neurons: association between polyribosomes and other organelles at the synaptic site. J. Neurosci. 8, 176-184.

Su, Q., Mochida, S., Tian, J.-H.H., Mehta, R., and Sheng, Z.-H.H. (2001). SNAP-29: A general SNARE protein that inhibits SNARE disassembly and is implicated in synaptic transmission. Proc. Natl. Acad. Sci. 98, 14038-14043.

Sugiyama, Y., Kawabata, I., Sobue, K., and Okabe, S. (2005). Determination of absolute protein numbers in single synapses by a GFP-based calibration technique. Nat. Methods 2 , $677-684$.

Suh, Y., Atzmon, G., Cho, M.-O., Hwang, D., Liu, B., Leahy, D.J., Barzilai, N., and Cohen, P. (2008). Functionally significant insulin-like growth factor I receptor mutations in centenarians. Proc. Natl. Acad. Sci. 105, 3438-3442.

Suh, Y.H., Terashima, A., Petralia, R.S., Wenthold, R.J., Isaac, J.T.R.R., Roche, K.W., and Roche, P.A. (2010). A neuronal role for SNAP-23 in postsynaptic glutamate receptor trafficking. Nat. Neurosci. 13, 338-343.

Sullivan, K.F., and Cleveland, D.W. (1986). Identification of conserved isotype-defining variable region sequences for four vertebrate beta tubulin polypeptide classes. Proc. Natl. Acad. Sci. U. S. A. 83, 4327-4331.

Sun, M., Watanabe, T., Bochimoto, H., Sakai, Y., Torii, S., Takeuchi, T., and Hosaka, M. (2013). Multiple Sorting Systems for Secretory Granules Ensure the Regulated Secretion of Peptide Hormones. Traffic 14, 205-218.

Sun, X., Zhao, Y., and Wolf, M.E. (2005). Dopamine receptor stimulation modulates AMPA receptor synaptic insertion in prefrontal cortex neurons. J. Neurosci. 25, 7342-7351.

Sutton, M. a., and Schuman, E.M. (2006). Dendritic Protein Synthesis, Synaptic Plasticity, and Memory. Cell 127, 49-58. 
Sutton, M.A., Ito, H.T., Cressy, P., Kempf, C., Woo, J.C., and Schuman, E.M. (2006). Miniature Neurotransmission Stabilizes Synaptic Function via Tonic Suppression of Local Dendritic Protein Synthesis. Cell 125, 785-799.

Suzuki, T., Mitake, S., Okumura-Noji, K., Shimizu, H., Tada, T., and Fujii, T. (1997). Excitable membranes and synaptic transmission: Postsynaptic mechanisms. Localization of $\alpha$-internexin in the postsynaptic density of the rat brain. Brain Res. 765, 74-80.

Suzuki, T., Kametani, K., Guo, W., and Li, W. (2018). Protein components of post-synaptic density lattice, a backbone structure for type I excitatory synapses. J. Neurochem. 144, 390407.

Szíber, Z., Liliom, H., Morales, C.O.O., Ignácz, A., Rátkai, A.E., Ellwanger, K., Link, G., Szűcs, A., Hausser, A., and Schlett, K. (2017). Ras and Rab interactor 1 controls neuronal plasticity by coordinating dendritic filopodial motility and AMPA receptor turnover. Mol. Biol. Cell 28, 285-295.

Takamori, S., Holt, M., Stenius, K., Lemke, E.A., Grønborg, M., Riedel, D., Urlaub, H., Schenck, S., Brügger, B., Ringler, P., et al. (2006). Molecular anatomy of a trafficking organelle. Cell 127, 831-846.

Takei, K., Mundigl, O., Daniell, L., and De Camilli, P. (1996). The synaptic vesicle cycle: a single vesicle budding step involving clathrin and dynamin. J. Cell Biol. 133, 1237-1250.

Takeuchi, M., Hata, Y., Hirao, K., Toyoda, A., Irie, M., and Takai, Y. (1997). SAPAPs. A family of PSD-95/SAP90-associated proteins localized at postsynaptic density. J. Biol. Chem. 272, 11943-11951.

Tanaka, J., Matsuzaki, M., Tarusawa, E., Momiyama, A., Molnar, E., Kasai, H., and Shigemoto, R. (2005). Number and density of AMPA receptors in single synapses in immature cerebellum. J. Neurosci. 25, 799-807.

Tanaka, T., Saito, H., and Matsuki, N. (1997). Inhibition of GABAA Synaptic Responses by Brain-Derived Neurotrophic Factor (BDNF) in Rat Hippocampus. J. Neurosci. 17, 2959-2966.

Tang, B.L. (2008). Emerging aspects of membrane traffic in neuronal dendrite growth. Biochim. Biophys. Acta - Mol. Cell Res. 1783, 169-176.

Tang, A.-H.H., Chen, H., Li, T.P., Metzbower, S.R., MacGillavry, H.D., and Blanpied, T.A. (2016). A trans-synaptic nanocolumn aligns neurotransmitter release to receptors. Nature 536, 210-214.

Tang, B.L., Low, D.Y.H., Lee, S.S., Tan, A.E.H., and Hong, W. (1998). Molecular Cloning and 
Localization of Human Syntaxin 16, a Member of the Syntaxin Family of SNARE Proteins. Biochem. Biophys. Res. Commun. 242, 673-679.

Tao-Cheng, J.-H., Crocker, V.T., Winters, C.A., Azzam, R., Chludzinski, J., and Reese, T.S. (2011). Trafficking of AMPA receptors at plasma membranes of hippocampal neurons. J. Neurosci. 31, 4834-4843.

Tao-Cheng, J.-H., Yang, Y., Reese, T.S., and Dosemeci, A. (2015). Differential Distribution of Shank and GKAP at the Postsynaptic Density. PLoS One 10, e0118750.

Tao-Cheng, J.H., Thein, S., Yang, Y., Reese, T.S., and Gallant, P.E. (2014). Homer is concentrated at the postsynaptic density and does not redistribute after acute synaptic stimulation. Neuroscience 266, 80-90.

Tarusawa, E., Matsui, K., Budisantoso, T., Molnár, E., Watanabe, M., Matsui, M., Fukazawa, Y., and Shigemoto, R. (2009). Input-specific intrasynaptic arrangements of ionotropic glutamate receptors and their impact on postsynaptic responses. J. Neurosci. 29, 1289612908.

Taylor, C.J., Ireland, D.R., Ballagh, I., Bourne, K., Marechal, N.M., Turner, P.R., Bilkey, D.K., Tate, W.P., and Abraham, W.C. (2008). Endogenous secreted amyloid precursor protein- $\alpha$ regulates hippocampal NMDA receptor function, long-term potentiation and spatial memory. Neurobiol. Dis. 31, 250-260.

Techlovská, Š., Chambers, J.N., Dvořáková, M., Petralia, R.S., Wang, Y.-X., Hájková, A., Nová, A., Franková, D., Prezeau, L., and Blahos, J. (2014). Metabotropic glutamate receptor 1 splice variants mGluR1a and mGluR1b combine in mGluR1a/b dimers in vivo. Neuropharmacology 86, 329-336.

Thiagarajan, T.C., Lindskog, M., and Tsien, R.W. (2005). Adaptation to synaptic inactivity in hippocampal neurons. Neuron 47, 725-737.

Tiffany, A.M., Manganas, L.N., Kim, E., Hsueh, Y.P., Sheng, M., and Trimmer, J.S. (2000). PSD-95 and SAP97 exhibit distinct mechanisms for regulating $\mathrm{K}(+)$ channel surface expression and clustering. J. Cell Biol. 148, 147-158.

Tomita, S., Adesnik, H., Sekiguchi, M., Zhang, W., Wada, K., Howe, J.R., Nicoll, R.A., and Bredt, D.S. (2005). Stargazin modulates AMPA receptor gating and trafficking by distinct domains. Nature 435, 1052-1058.

Tønnesen, J., and Nägerl, U.V. (2016). Dendritic Spines as Tunable Regulators of Synaptic Signals. Front. Psychiatry 7, 101. 
Tønnesen, J., Katona, G., Rózsa, B., and Nägerl, U.V. (2014). Spine neck plasticity regulates compartmentalization of synapses. Nat. Neurosci. 17, 678-685.

Trimble, W.S., Cowan, D.M., and Scheller, R.H. (1988). VAMP-1: a synaptic vesicleassociated integral membrane protein. Proc. Natl. Acad. Sci. U. S. A. 85, 4538-4542.

Trimmer, J.S. (1991). Immunological identification and characterization of a delayed rectifier K+ channel polypeptide in rat brain. Proc. Natl. Acad. Sci. U. S. A. 88, 10764-10768.

Truckenbrodt, S., Viplav, A., Jähne, S., Vogts, A., Denker, A., Wildhagen, H., Fornasiero, E.F., and Rizzoli, S.O. (2018). Newly produced synaptic vesicle proteins are preferentially used in synaptic transmission. EMBO J. e98044.

Tu, J.C., Xiao, B., Yuan, J.P., Lanahan, A.A., Leoffert, K., Li, M., Linden, D.J., and Worley, P.F. (1998). Homer binds a novel proline-rich motif and links group 1 metabotropic glutamate receptors with IP3 receptors. Neuron 21, 717-726.

Tu, J.C., Xiao, B., Naisbitt, S., Yuan, J.P., Petralia, R.S., Brakeman, P., Doan, A., Aakalu, V.K., Lanahan, A. a., Sheng, M., et al. (1999). Coupling of mGluR/Homer and PSD-95 complexes by the Shank family of postsynaptic density proteins. Neuron $23,583-592$.

Turrigiano, G.G. (2008). The Self-Tuning Neuron: Synaptic Scaling of Excitatory Synapses. Cell 135, 422-435.

Turrigiano, G.G., and Nelson, S.B. (2004). Homeostatic plasticity in the developing nervous system. Nat. Rev. Neurosci. 5, 97-107.

Turrigiano, G.G., Leslie, K.R., Desai, N.S., Rutherford, L.C., and Nelson, S.B. (1998). Activitydependent scaling of quantal amplitude in neocortical neurons. Nature 391, 892-896.

Uchimura, N., and North, R.A. (1990). Muscarine reduces inwardly rectifying potassium conductance in rat nucleus accumbens neurones. J. Physiol. 422, 369-380.

Uchino, S., Wada, H., Honda, S., Nakamura, Y., Ondo, Y., Uchiyama, T., Tsutsumi, M., Suzuki, E., Hirasawa, T., and Kohsaka, S. (2006). Direct interaction of post-synaptic density95/Dlg/ZO-1 domain-containing synaptic molecule Shank3 with GluR1 alpha-amino-3hydroxy-5-methyl-4-isoxazole propionic acid receptor. J. Neurochem. 97, 1203-1214.

Ungewickell, E., and Branton, D. (1981). Assembly units of clathrin coats. Nature 289, 420 422.

Ungewickell, E., Ungewickell, H., and Holstein, S.E. (1997). Functional interaction of the auxilin $\mathrm{J}$ domain with the nucleotide- and substrate-binding modules of Hsc70. J. Biol. Chem. 272, 
19594-19600.

Valtschanoff, J.G., and Weinberg, R.J. (2001). Laminar organization of the NMDA receptor complex within the postsynaptic density. J. Neurosci. 21, 1211-1217.

Veenstra, T.D., Johnson, K.L., Tomlinson, A.J., Naylor, S., and Kumar, R. (1997). Determination of calcium-binding sites in rat brain calbindin $\mathrm{D}(28 \mathrm{~K})$ by electrospray ionization mass spectrometry. Biochemistry 36, 3535-3542.

Veit, M., Söllner, T.H., and Rothman, J.E. (1996). Multiple palmitoylation of synaptotagmin and the t-SNARE SNAP-25. FEBS Lett. 385, 119-123.

Vikstrom, K.L., Vaidyanathan, R., Levinsohn, S., O’Connell, R.P., Qian, Y., Crye, M., Mills, J.H., and Anumonwo, J.M.B. (2009). SAP97 regulates Kir2.3 channels by multiple mechanisms. Am. J. Physiol. Circ. Physiol. 297, H1387-H1397.

Villa, A., Podini, P., Panzeri, M.C., Racchetti, G., and Meldolesi, J. (1994). Cytosolic Ca2+ Binding Proteins during Rat Brain Ageing: Loss of Calbindin and Calretinin in the Hippocampus, with no Change in the Cerebellum. Eur. J. Neurosci. 6, 1491-1499.

Wade, N., Bryant, N.J., Connolly, L.M., Simpson, R.J., Luzio, J.P., Piper, R.C., and James, D.E. (2001). Syntaxin 7 Complexes with Mouse Vps10p Tail Interactor 1b, Syntaxin 6, Vesicleassociated Membrane Protein (VAMP)8, and VAMP7 in B16 Melanoma Cells. J. Biol. Chem. 276, 19820-19827.

Wang, H., Qian, W.-J., Chin, M.H., Petyuk, V.A., Barry, R.C., Liu, T., Gritsenko, M.A., Mottaz, H.M., Moore, R.J., Camp, D.G., et al. (2006). Characterization of the Mouse Brain Proteome Using Global Proteomic Analysis Complemented with Cysteinyl-Peptide Enrichment. J. Proteome Res. 5, 361-369.

Wang, S., Stanika, R.I., Wang, X., Hagen, J., Kennedy, M.B., Obermair, G.J., Colbran, R.J., and Lee, A. (2017). Densin-180 Controls the Trafficking and Signaling of L-Type VoltageGated $\mathrm{Ca}_{\vee} 1.2 \mathrm{Ca}^{2+}$ Channels at Excitatory Synapses. J. Neurosci. 37, 4679-4691.

Wang, W., Wang, F., Yang, Y.-J., Hu, Z.-L., Long, L.-H., Fu, H., Xie, N., and Chen, J.-G. (2011). The flavonoid baicalein promotes NMDA receptor-dependent long-term potentiation and enhances memory. Br. J. Pharmacol. 162, 1364-1379.

Wang, Z., Edwards, J.G., Riley, N., Provance, D.W., Karcher, R., Li, X.-D. dong, Davison, I.G., Ikebe, M., Mercer, J.A., Kauer, J.A., et al. (2008). Myosin Vb Mobilizes Recycling Endosomes and AMPA Receptors for Postsynaptic Plasticity. Cell 135, 535-548.

Wang, Z., Miao, G., Xue, X., Guo, X., Yuan, C., Wang, Z., Zhang, G., Chen, Y., Feng, D., Hu, 
J., et al. (2016). The Vici Syndrome Protein EPG5 Is a Rab7 Effector that Determines the Fusion Specificity of Autophagosomes with Late Endosomes/Lysosomes. Mol. Cell 63, 781795.

Washbourne, P. (2004). Cycling of NMDA Receptors during Trafficking in Neurons before Synapse Formation. J. Neurosci. 24, 8253-8264.

Watson, R.T., and Pessin, J.E. (2000). Functional cooperation of two independent targeting domains in syntaxin 6 is required for its efficient localization in the trans-golgi network of 3T3L1 adipocytes. J. Biol. Chem. 275, 1261-1268.

Watt, a J., van Rossum, M.C., MacLeod, K.M., Nelson, S.B., and Turrigiano, G.G. (2000). Activity coregulates quantal AMPA and NMDA currents at neocortical synapses. Neuron 26, 659-670.

Weber, J.P., Toft-Bertelsen, T.L., Mohrmann, R., Delgado-Martinez, I., and Sørensen, J.B. (2014). Synaptotagmin-7 is an asynchronous calcium sensor for synaptic transmission in neurons expressing SNAP-23. PLoS One 9, 1-22.

Weidman, P.J., Melançon, P., Block, M.R., and Rothman, J.E. (1989). Binding of an Nethylmaleimide-sensitive fusion protein to Golgi membranes requires both a soluble protein(s) and an integral membrane receptor. J. Cell Biol. 108, 1589-1596.

Wen, H., Linhoff, M.W., McGinley, M.J., Li, G.-L., Corson, G.M., Mandel, G., and Brehm, P. (2010). Distinct roles for two synaptotagmin isoforms in synchronous and asynchronous transmitter release at zebrafish neuromuscular junction. Proc. Natl. Acad. Sci. U. S. A. 107, 13906-13911.

Wendler, F., and Tooze, S. (2001). Syntaxin 6: the promiscuous behaviour of a SNARE protein. Traffic 2, 606-611.

Westenbroek, R.E., Merrick, D.K., and Catterall, W.A. (1989). Differential subcellular localization of the RI and RII Na+ channel subtypes in central neurons. Neuron 3, 695-704.

Westenbroek, R.E., Sakurai, T., Elliott, E.M., Hell, J.W., Starr, T. V, Snutch, T.P., and Catterall, W.A. (1995). Immunochemical identification and subcellular distribution of the alpha $1 \mathrm{~A}$ subunits of brain calcium channels. J. Neurosci. 15, 6403-6418.

Whitaker, W.R., Faull, R.L., Waldvogel, H.J., Plumpton, C.J., Emson, P.C., and Clare, J.J. (2001). Comparative distribution of voltage-gated sodium channel proteins in human brain. Brain Res. Mol. Brain Res. 88, 37-53.

Whiteheart, S.W., Griff, I.C., Brunner, M., Clary, D.O., Mayer, T., Buhrow, S.A., and Rothman, 
J.E. (1993). SNAP family of NSF attachment proteins includes a brain-specific isoform. Nature 362, 353-355.

Wierenga, C.J. (2005). Postsynaptic Expression of Homeostatic Plasticity at Neocortical Synapses. J. Neurosci. 25, 2895-2905.

Wilhelm, B.G., Mandad, S., Truckenbrodt, S., Kröhnert, K., Schäfer, C., Rammner, B., Koo, S.J., Claßen, G. a, Krauss, M., Haucke, V., et al. (2014). Composition of isolated synaptic boutons reveals the amounts of vesicle trafficking proteins. Science 344, 1023-1028.

Wilson, D.W., Wilcox, C.A., Flynn, G.C., Chen, E., Kuang, W.-J., Henzel, W.J., Block, M.R., Ullrich, A., and Rothman, J.E. (1989). A fusion protein required for vesicle-mediated transport in both mammalian cells and yeast. Nature 339, 355-359.

Wirth, M.J., Brun, A., Grabert, J., Patz, S., and Wahle, P. (2003). Accelerated dendritic development of rat cortical pyramidal cells and interneurons after biolistic transfection with BDNF and NT4/5. Development 130, 5827-5838.

Wiśniewski, J.R., Hein, M.Y., Cox, J., and Mann, M. (2014). A "proteomic ruler" for protein copy number and concentration estimation without spike-in standards. Mol. Cell. Proteomics 13, 3497-3506.

de Wit, H., Lichtenstein, Y., Kelly, R.B., Geuze, H.J., Klumperman, J., and van der Sluijs, P. (2001). Rab4 regulates formation of synaptic-like microvesicles from early endosomes in PC12 cells. Mol. Biol. Cell 12, 3703-3715.

Wong, S.H., Xu, Y., Zhang, T., Griffiths, G., Lowe, S.L., Subramaniam, V.N., Seow, K.T., and Hong, W. (1999). GS32, a novel Golgi SNARE of $32 \mathrm{kDa}$, interacts preferentially with syntaxin 6. Mol. Biol. Cell 10,119-134.

Wu, H. (2000). Intramolecular interactions regulate SAP97 binding to GKAP. EMBO J.

Wu, H., and Parsons, J.T. (1993). Cortactin, an 80/85-kilodalton pp60src substrate, is a filamentous actin-binding protein enriched in the cell cortex. J. Cell Biol. 120, 1417-1426.

Wu, D., Bacaj, T., Morishita, W., Goswami, D., Arendt, K.L., Xu, W., Chen, L., Malenka, R.C., and Südhof, T.C. (2017). Postsynaptic synaptotagmins mediate AMPA receptor exocytosis during LTP. Nature 544, 316-321.

Wu, K., Aoki, C., Elste, A., Rogalski-Wilk, A.A., and Siekevitz, P. (1997). The synthesis of ATP by glycolytic enzymes in the postsynaptic density and the effect of endogenously generated nitric oxide. Proc. Natl. Acad. Sci. U. S. A. 94, 13273-13278. 
Wu, L.G., Westenbroek, R.E., Borst, J.G., Catterall, W.A., and Sakmann, B. (1999). Calcium channel types with distinct presynaptic localization couple differentially to transmitter release in single calyx-type synapses. J. Neurosci. 19, 726-736.

Wurm, C.A., Neumann, D., Lauterbach, M.A., Harke, B., Egner, A., Hell, S.W., and Jakobs, S. (2011). Nanoscale distribution of mitochondrial import receptor Tom20 is adjusted to cellular conditions and exhibits an inner-cellular gradient. Proc. Natl. Acad. Sci. 108, 13546-13551.

Wyszynski, M., Lin, J., Rao, A., Nigh, E., Beggs, A.H., Craig, A.M., and Sheng, M. (1997). Competitive binding of alpha-actinin and calmodulin to the NMDA receptor. Nature 385,439 442.

Xia, Z., and Storm, D.R. (2005). The role of calmodulin as a signal integrator for synaptic plasticity. Nat. Rev. Neurosci. 6, 267-276.

Xia, J., Zhang, X., Staudinger, J., and Huganir, R.L. (1999). Clustering of AMPA receptors by the synaptic PDZ domain-containing protein PICK1. Neuron 22, 179-187.

Xiao, B., Tu, J.C., Petralia, R.S., Yuan, J.P., Doan, A., Breder, C.D., Ruggiero, A., Lanahan, A.A., Wenthold, R.J., and Worley, P.F. (1998). Homer regulates the association of group 1 metabotropic glutamate receptors with multivalent complexes of homer-related, synaptic proteins. Neuron 21, 707-716.

Xu, B., Gottschalk, W., Chow, A., Wilson, R.I., Schnell, E., Zang, K., Wang, D., Nicoll, R.A., Lu, B., and Reichardt, L.F. (2000). The role of brain-derived neurotrophic factor receptors in the mature hippocampus: modulation of long-term potentiation through a presynaptic mechanism involving TrkB. J. Neurosci. 20, 6888-6897.

Xu, K., Zhong, G., and Zhuang, X. (2013). Actin, spectrin, and associated proteins form a periodic cytoskeletal structure in axons. Science 339, 452-456.

Yadav, S., Oses-Prieto, J.A., Peters, C.J., Zhou, J., Pleasure, S.J., Burlingame, A.L., Jan, L.Y., and Jan, Y.-N.N. (2017). TAOK2 Kinase Mediates PSD95 Stability and Dendritic Spine Maturation through Septin7 Phosphorylation. Neuron 93, 379-393.

Yamamoto, Y., Kimura, T., Momohara, S., Takeuchi, M., Tani, T., Kimata, Y., Kadokura, H., and Kohno, K. (2010). A Novel ER J-protein DNAJB12 Accelerates ER-associated Degradation of Membrane Proteins Including CFTR. Cell Struct. Funct. 35, 107-116.

Yang, J., Siao, C.-J., Nagappan, G., Marinic, T., Jing, D., McGrath, K., Chen, Z.-Y., Mark, W., Tessarollo, L., Lee, F.S., et al. (2009). Neuronal release of proBDNF. Nat. Neurosci. 12, 113115. 
Yang, J., Harte-Hargrove, L.C., Siao, C.-J., Marinic, T., Clarke, R., Ma, Q., Jing, D., LaFrancois, J.J., Bath, K.G., Mark, W., et al. (2014). proBDNF Negatively Regulates Neuronal Remodeling, Synaptic Transmission, and Synaptic Plasticity in Hippocampus. Cell Rep. 7, 796-806.

Yao, I. (1999). Synamon, a Novel Neuronal Protein Interacting with Synapse- associated Protein 90 / Postsynaptic Density-95-associated Protein *. J. Biol. Chem. 274, 27463-27466.

Ybe, J.A., Greene, B., Liu, S.H., Pley, U., Parham, P., and Brodsky, F.M. (1998). Clathrin selfassembly is regulated by three light-chain residues controlling the formation of critical salt bridges. EMBO J. 17, 1297-1303.

Ye, W., and Lafer, E.M. (1995). Clathrin Binding and Assembly Activities of Expressed Domains of the Synapse-specific Clathrin Assembly Protein AP-3. J. Biol. Chem. 270, 1093310939.

Yoshihara, M., Adolfsen, B., Galle, K.T., and Littleton, J.T. (2005). Retrograde signaling by Syt 4 induces presynaptic release and synapse-specific growth. Science (80-. ). 310, 858-863.

Young-Pearse, T.L., Chen, A.C., Chang, R., Marquez, C., and Selkoe, D.J. (2008). Secreted APP regulates the function of full-length APP in neurite outgrowth through interaction with integrin beta1. Neural Dev. 3, 15.

Yuan, A., Rao, M. V., Sasaki, T., Chen, Y., Kumar, A., Veeranna, Liem, R.K.H., Eyer, J., Peterson, A.C., Julien, J.-P., et al. (2006). Alpha-Internexin Is Structurally and Functionally Associated with the Neurofilament Triplet Proteins in the Mature CNS. J. Neurosci. 26, 1000610019.

Yuan, A., Rao, M. V, Veeranna, and Nixon, R.A. (2012). Neurofilaments at a glance. J. Cell Sci. 125, 3257-3263.

Yuan, A., Sershen, H., Veeranna, Basavarajappa, B.S., Kumar, A., Hashim, A., Berg, M., Lee, J.-H.H., Sato, Y., Rao, M. V., et al. (2015a). Neurofilament subunits are integral components of synapses and modulate neurotransmission and behavior in vivo. Mol. Psychiatry 20, 986994.

Yuan, A., Sershen, H., Veeranna, Basavarajappa, B.S., Kumar, A., Hashim, A., Berg, M., Lee, J.-H., Sato, Y., Rao, M. V, et al. (2015b). Functions of neurofilaments in synapses. Mol. Psychiatry 20, 915-915.

Yuan, J.P., Kiselyov, K., Shin, D.M., Chen, J., Shcheynikov, N., Kang, S.H., Dehoff, M.H., Schwarz, M.K., Seeburg, P.H., Muallem, S., et al. (2003). Homer binds TRPC family channels 
and is required for gating of TRPC1 by IP3 receptors. Cell 114, 777-789.

Yudowski, G.A., Puthenveedu, M.A., Leonoudakis, D., Panicker, S., Thorn, K.S., Beattie, E.C., and von Zastrow, M. (2007). Real-Time Imaging of Discrete Exocytic Events Mediating Surface Delivery of AMPA Receptors. J. Neurosci. 27, 11112-11121.

Yuste, R. (2013). Electrical Compartmentalization in Dendritic Spines. Annu. Rev. Neurosci. $36,429-449$.

Zeng, M., Shang, Y., Araki, Y., Guo, T., Huganir, R.L., and Zhang, M. (2016). Phase Transition in Postsynaptic Densities Underlies Formation of Synaptic Complexes and Synaptic Plasticity. Cell 166, 1163-1175.e12.

Zhang, Y. (2008). I-TASSER server for protein 3D structure prediction. BMC Bioinformatics.

Zhang, J., and Diamond, J.S. (2009). Subunit- and pathway-specific localization of NMDA receptors and scaffolding proteins at ganglion cell synapses in rat retina. J. Neurosci. 29, 4274-4286.

Zhang, H., Maximov, A., Fu, Y., Xu, F., Tang, T.-S., Tkatch, T., Surmeier, D.J., and Bezprozvanny, I. (2005). Association of CaV1.3 L-Type Calcium Channels with Shank. J. Neurosci. 25, 1037-1049.

Zhang, T., Wong, S.H., Tang, B.L., Xu, Y., Peter, F., Subramaniam, V.N., and Hong, W. (1997). The mammalian protein (rbet1) homologous to yeast Bet1p is primarily associated with the pre-Golgi intermediate compartment and is involved in vesicular transport from the endoplasmic reticulum to the Golgi apparatus. J. Cell Biol. 139, 1157-1168.

Zhang, T., Wong, S.H., Tang, B.L., Xu, Y., and Hong, W. (1999). Morphological and functional association of Sec22b/ERS-24 with the pre-Golgi intermediate compartment. Mol. Biol. Cell 10, 435-453.

Zhang, Y., Matt, L., Patriarchi, T., Malik, Z.A., Chowdhury, D., Park, D.K., Renieri, A., Ames, J.B., and Hell, J.W. (2014). Capping of the N-terminus of PSD-95 by calmodulin triggers its postsynaptic release. EMBO J. 33, 1341-1353.

Zhao, L., Kroenke, C.D., Song, J., Piwnica-Worms, D., Ackerman, J.J.H., and Neil, J.J. (2008). Intracellular water-specific MR of microbead-adherent cells: the HeLa cell intracellular water exchange lifetime. NMR Biomed. 21, 159-164.

Zheng, Z., Lauritzen, J.S., Perlman, E., Robinson, C.G., Nichols, M., Milkie, D., Torrens, O., Price, J., Fisher, C.B., Sharifi, N., et al. (2018). A Complete Electron Microscopy Volume of the Brain of Adult Drosophila melanogaster. Cell 174, 730-743.e22. 
Zhou, S., Sousa, R., Tannery, N.H., and Lafer, E.M. (1992). Characterization of a novel synapse-specific protein. II. cDNA cloning and sequence analysis of the F1-20 protein. J. Neurosci. 12, 2144-2155.

Zhou, W., Chang, J., Wang, X., Savelieff, M.G., Zhao, Y., Ke, S., and Ye, B. (2014). GM130 Is Required for Compartmental Organization of Dendritic Golgi Outposts. Curr. Biol. 24, 12271233.

Zhou, Y., Takahashi, E., Li, W., Halt, A., Wiltgen, B., Ehninger, D., Li, G.-D., Hell, J.W., Kennedy, M.B., and Silva, A.J. (2007). Interactions between the NR2B receptor and CaMKII modulate synaptic plasticity and spatial learning. J. Neurosci. 27, 13843-13853.

Zhu, H., Domingues, F.S., Sommer, I., and Lengauer, T. (2006). NOXclass: prediction of protein-protein interaction types. BMC Bioinformatics 7, 27.

Znamensky, V., Akama, K.T., McEwen, B.S., and Milner, T.A. (2003). Estrogen levels regulate the subcellular distribution of phosphorylated Akt in hippocampal CA1 dendrites. J. Neurosci. 23, 2340-2347.

Zuo, Y., Lin, A., Chang, P., and Gan, W.B. (2005). Development of long-term dendritic spine stability in diverse regions of cerebral cortex. Neuron 46, 181-189.

Zwilling, D., Cypionka, A., Pohl, W.H., Fasshauer, D., Walla, P.J., Wahl, M.C., and Jahn, R. (2007). Early endosomal SNAREs form a structurally conserved SNARE complex and fuse liposomes with multiple topologies. EMBO J. 26, 9-18. 


\section{List of Figures}

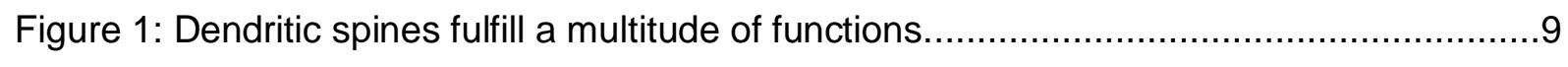

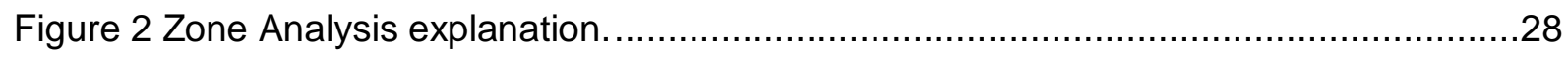

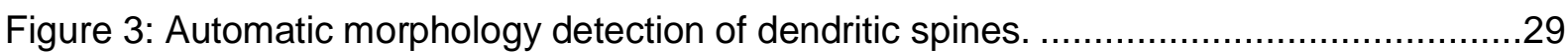

Figure 4: Assignment of organelle and zone identities for the construction of the 3D model. 36

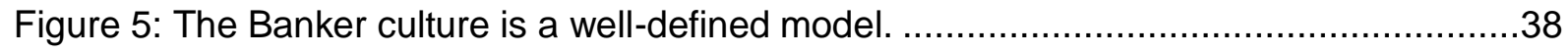

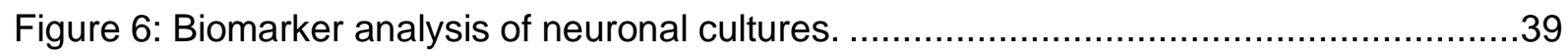

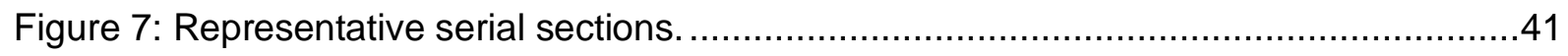

Figure 8: Representative 3D Reconstructions of dendritic spines. .................................42

Figure 9: The quantitative morphological parameters of mushroom and stubby spines in culture

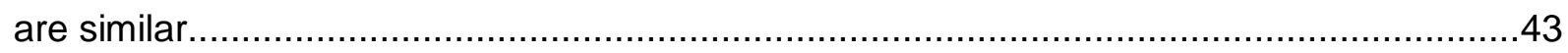

Figure 10: A general description of the quantitative neuronal proteome. ...........................45

Figure 11: Quantitative mass spectrometry of neuronal cultures........................................46

Figure 12: Dendritic spine copy numbers......................................................................... 49

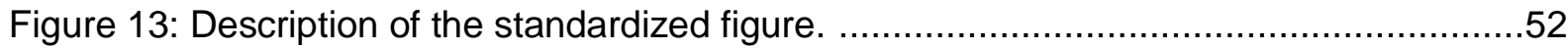

Figure 14: Depiction of the cytoskeleton in dendritic spines..........................................5

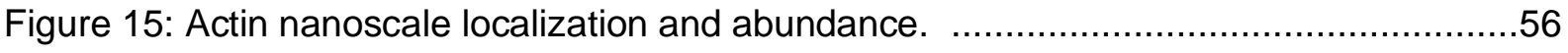

Figure 16: $\alpha$-Internexin nanoscale localization and abundance........................................58

Figure 17: $\beta$-2-spectrin nanoscale localization and abundance........................................60

Figure 18: $\beta 3$-tubulin nanoscale localization and abundance...........................................62

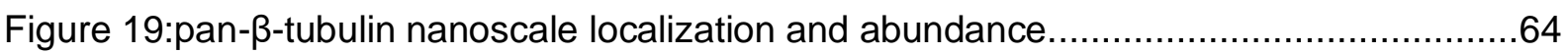

Figure 20: Cortactin nanoscale localization and abundance. .........................................66

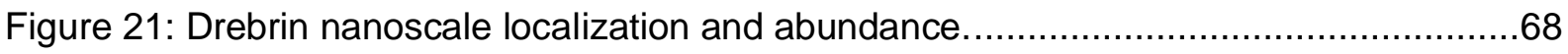

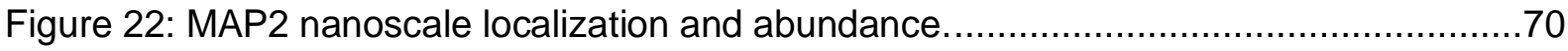

Figure 23: Myosin5a nanoscale localization and abundance. .........................................

Figure 24: Neurofilament heavy nanoscale localization and abundance. ..............................74

Figure 25: Neurofilament light nanoscale localization and abundance...............................76

Figure 26: Septin7 nanoscale localization and abundance. ............................................78

Figure 27: Depiction of scaffold proteins in dendritic spines......................................... 81

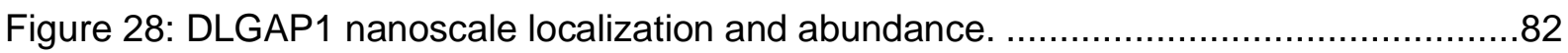

Figure 29: Homer1 nanoscale localization and abundance............................................84

Figure 30: Homer2 nanoscale localization and abundance............................................ 86

Figure 31: Homer3 nanoscale localization and abundance ........................................... 88

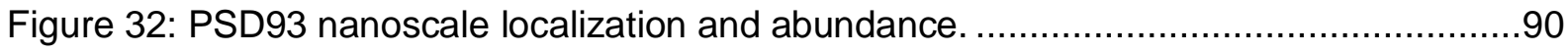

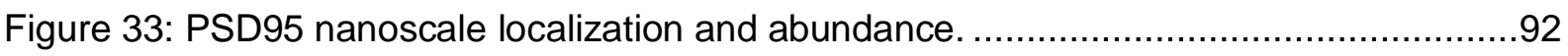

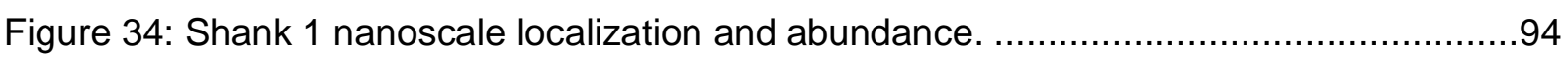

Figure 35: Shank2 nanoscale localization and abundance. .........................................96 
Figure 36: Shank3 nanoscale localization and abundance. ..........................................

Figure 37: Depiction of receptors in dendritic spines................................................... 101

Figure 38: Dopamine Receptor D1 nanoscale localization and abundance. ......................102

Figure 39: Dopamine Receptor D2 nanoscale localization and abundance. .......................104

Figure 40: GluK1 nanoscale localization and abundance. ..........................................106

Figure 41: GluN1 nanoscale localization and abundance. ............................................ 108

Figure 42: GluN2A nanoscale localization and abundance..........................................110

Figure 43: GluN2B nanoscale localization and abundance.........................................112

Figure 44: GluR1 nanoscale localization and abundance. ..............................................114

Figure 45: GluR2 nanoscale localization and abundance. .........................................116

Figure 46: GluR3 nanoscale localization and abundance. ..........................................118

Figure 47: IGF1R nanoscale localization and abundance.......................................... 120

Figure 48: LNGFR nanoscale localization and abundance. .........................................122

Figure 49: mAChR1 nanoscale localization and abundance. .......................................124

Figure 50: mGluR1 nanoscale localization and abundance. ........................................126

Figure 51: mGluR2 nanoscale localization and abundance. .......................................128

Figure 52: mGluR5 nanoscale localization and abundance. .......................................130

Figure 53: TrkB nanoscale localization and abundance...............................................132

Figure 54: Depiction of ion channels in dendritic spines. ................................................ 135

Figure 55: $\mathrm{Ca}_{\mathrm{v}} 1.3$ nanoscale localization and abundance...........................................136

Figure 56: Cav2.1 nanoscale localization and abundance........................................... 138

Figure 57: Kir2.1 nanoscale localization and abundance............................................140

Figure 58: $\mathrm{K}_{\mathrm{v}} 1.1$ nanoscale localization and abundance. ............................................ 142

Figure 59: $\mathrm{K}_{\mathrm{v}} 2.1$ nanoscale localization and abundance. .............................................. 144

Figure 60: $\mathrm{Na} \beta 1$ nanoscale localization and abundance..........................................146

Figure 61: $\mathrm{Na}_{\mathrm{v}} 1.1$ nanoscale localization and abundance............................................148

Figure 62: $\mathrm{Na}_{\mathrm{v}} 1.3$ nanoscale localization and abundance.............................................150

Figure 63: $\mathrm{Na}^{+} / \mathrm{K}^{+}$ATPase nanoscale localization and abundance. .................................152

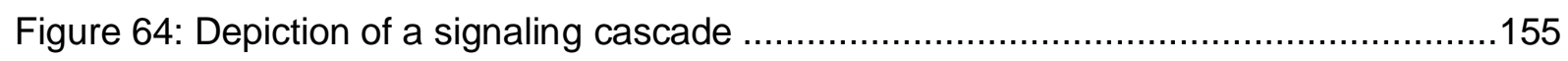

Figure 65: ADAM22 nanoscale localization and abundance. ......................................... 156

Figure 66: Akt nanoscale localization and abundance. ..............................................158

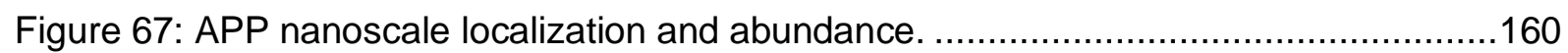

Figure 68: BDNF nanoscale localization and abundance............................................162

Figure 69: Calbindin nanoscale localization and abundance.......................................164

Figure 70: Calmodulin nanoscale localization and abundance..........................................166

Figure 71: Calretinin nanoscale localization and abundance. ......................................168

Figure 72: CaMKII nanoscale localization and abundance.........................................170 
Figure 73: CAPS1 nanoscale localization and abundance............................................. 172

Figure 74: CDC42 nanoscale localization and abundance............................................ 174

Figure 75: Chromogranin A nanoscale localization and abundance................................176

Figure 76: Chromogranin B nanoscale localization and abundance................................178

Figure 77: nNOS nanoscale localization and abundance ...........................................180

Figure 78: Secretogranin II nanoscale localization and abundance. ..................................182

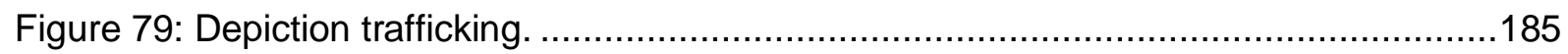

Figure 80: AP180 nanoscale localization and abundance..........................................186

Figure 81: Clathrin heavy chain nanoscale localization and abundance. ............................188

Figure 82: Clathrin light chain nanoscale localization and abundance. ..............................190

Figure 83: Dynamin1-3 nanoscale localization and abundance. ......................................192

Figure 84: GRIP1/2 nanoscale localization and abundance......................................... 194

Figure 85: HSC70 nanoscale localization and abundance. ...........................................196

Figure 86: Rab3 nanoscale localization and abundance............................................198

Figure 87: Rab4 nanoscale localization and abundance............................................200

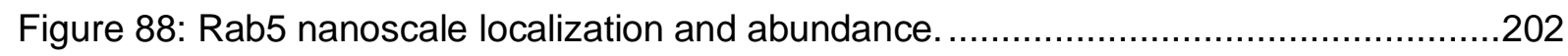

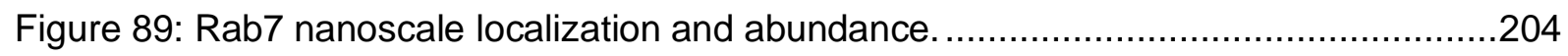

Figure 90: Rab9 nanoscale localization and abundance.............................................206

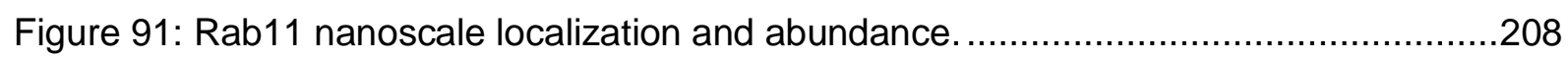

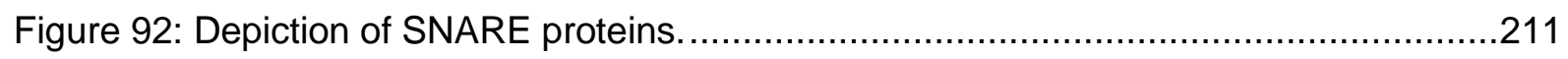

Figure 93: $\alpha / \beta$ SNAP nanoscale localization and abundance.........................................212

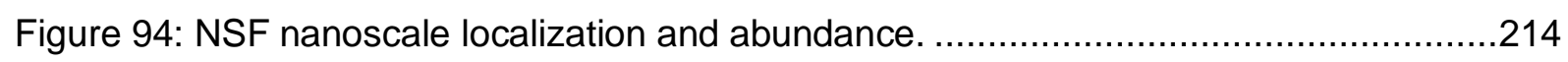

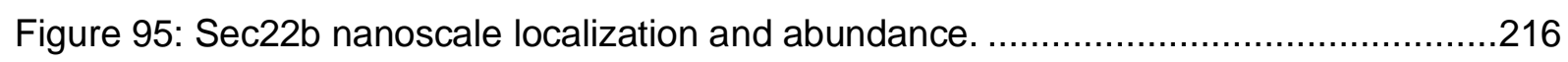

Figure 96: SNAP23 nanoscale localization and abundance...........................................218

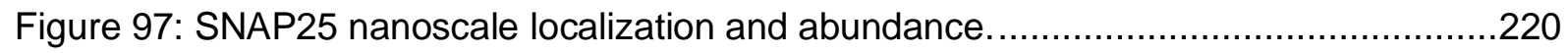

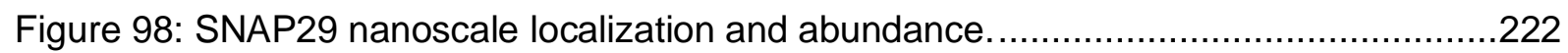

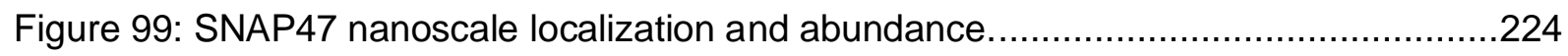

Figure 100: Synaptotagmin 4 nanoscale localization and abundance................................226

Figure 101: Synaptotagmin 5 nanoscale localization and abundance...............................228

Figure 102: Synaptotagmin 7 nanoscale localization and abundance..............................230

Figure 103: Syntaxin1a nanoscale localization and abundance.......................................232

Figure 104: Syntaxin2 nanoscale localization and abundance.......................................234

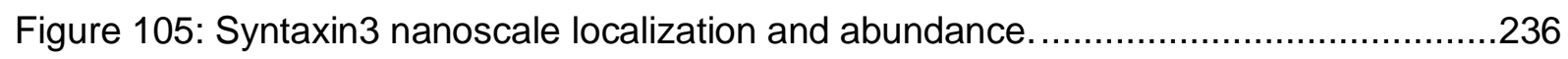

Figure 106: Syntaxin4 nanoscale localization and abundance......................................238

Figure 107: Syntaxin 5 nanoscale localization and abundance........................................240

Figure 108: Syntaxin 6 nanoscale localization and abundance.....................................242

Figure 109: Syntaxin 8 nanoscale localization and abundance.....................................244 
Figure 110: Syntaxin13 nanoscale localization and abundance.....................................246

Figure 111: Syntaxin 16 nanoscale localization and abundance.....................................248

Figure 112: VAMP1 nanoscale localization and abundance. .......................................250

Figure 113: VAMP2 nanoscale localization and abundance. .........................................252

Figure 114: VAMP7 nanoscale localization and abundance. .........................................254

Figure 115: Vti1a nanoscale localization and abundance. ...........................................256

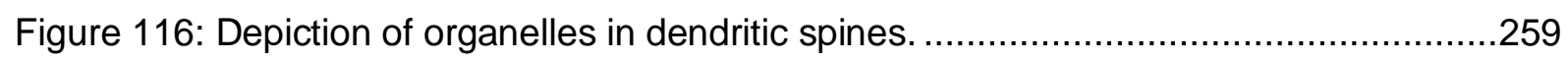

Figure 117: Calreticulin: nanoscale localization and abundance ....................................260

Figure 118: ERp72 nanoscale localization and abundance. ..........................................262

Figure 119: Ribosomal protein L7a nanoscale localization and abundance. ......................264

Figure 120: Ribosomal protein S3 nanoscale localization and abundance.........................266

Figure 121: Ribosomal protein S6 nanoscale localization and abundance........................268

Figure 122: TGN38 nanoscale localization and abundance. ..........................................270

Figure 123: TOM20 nanoscale localization and abundance...........................................272

Figure 124: Changes in actin localization and abundance after synaptic scaling. A) The average distribution of actin in the treatments. Scale bar is $500 \mathrm{~nm}$. B) The change in total intensity compared to untreated was calculated. C) The Enrichment of actin in the different zones for each treatment. Values are Mean \pm SEM......................................................276

Figure 125: Changes in Clathrin LC localization and abundance after synaptic scaling. The

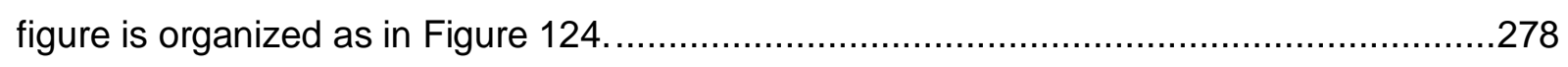

Figure 126: Changes in GluN2B localization and abundance after synaptic scaling. The figure

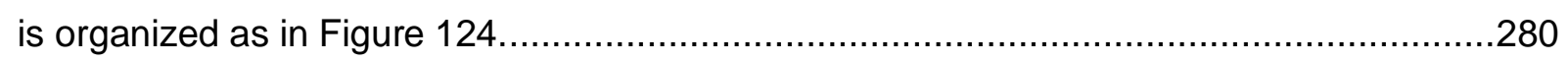

Figure 127: Changes in GluR1 localization and abundance after synaptic scaling. . The figure

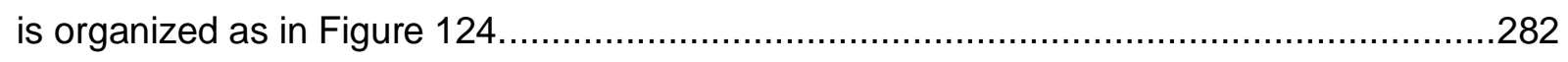

Figure 128: Changes in GluR2 localization and abundance after synaptic scaling. . The figure

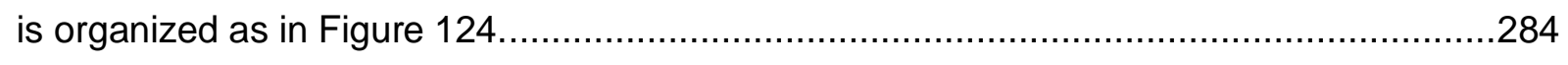

Figure 129: Changes in mGluR5 localization and abundance after synaptic scaling. . The figure

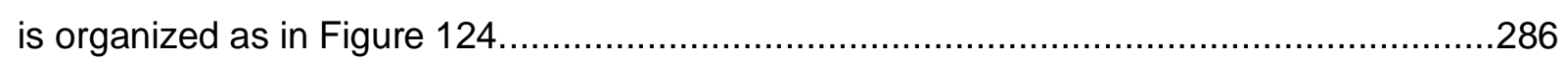

Figure 130: Changes in SNAP47 localization and abundance after synaptic scaling. . The

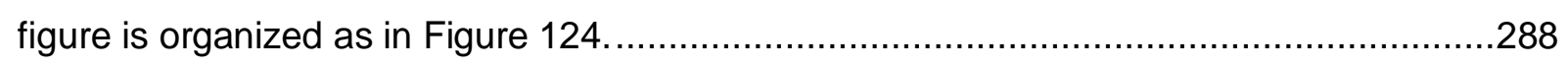

Figure 131: Changes in tubulin localization and abundance after synaptic scaling. The figure

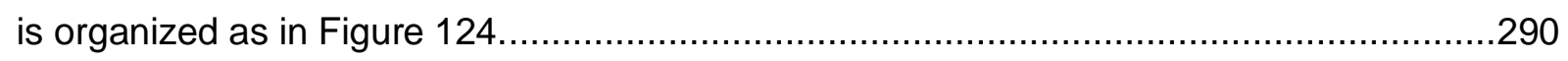

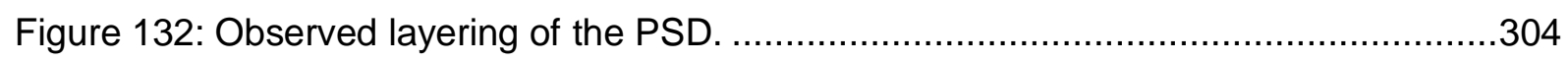

Figure 133: Synaptic scaling does not change the size of mushroom dendritic spines. .......321 


\section{List of Tables}

Table 1: Primary antibodies, Nanobodies and small molecules used to detect proteins........17

Table 2: Antibodies used as cell-type markers..........................................................21

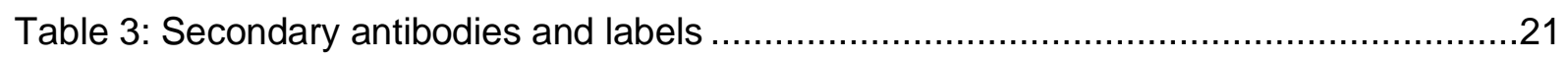

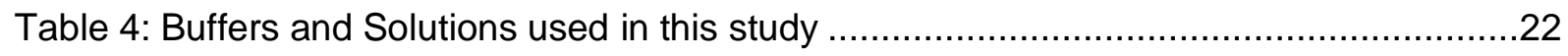

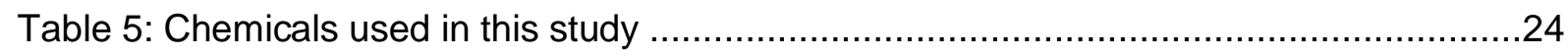

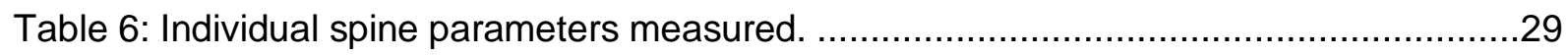

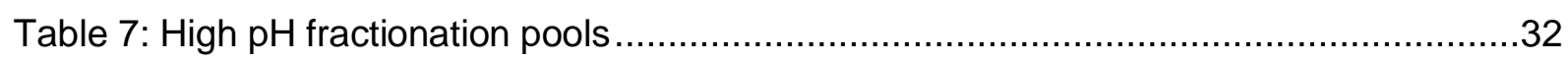

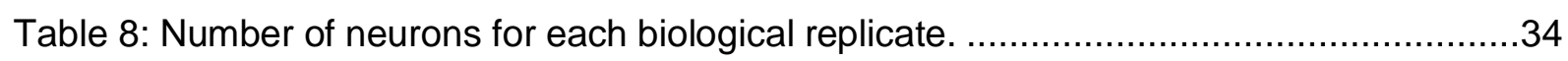

Table 9: Distribution of the spine classes over the whole dataset. ......................................51

Table 10: Spine class frequency during homeostatic plasticity. ......................................275

Table 11: Comparison of measured spine morphology to previously published cell culture and in vivo data. 


\section{List of Equations}

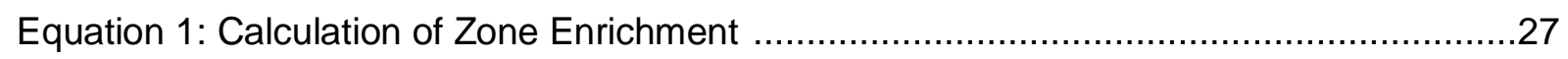

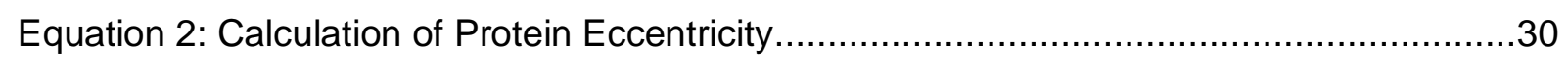

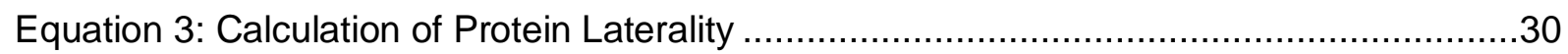

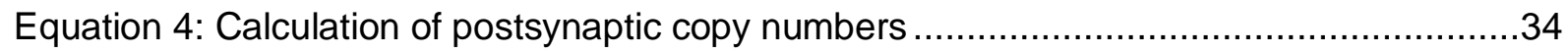




\section{List of Abbreviations}

SV

PSD

NMDA

NMDAR

AMPA

AMPAR

EPSP

TARP

LTP

LTD

BDNF

ER

CaMKII

GEF

SNARE

STED

CNQX

AP5

TTX

PFA

PM

CCV

TGN

EM
Synaptic vesicle

Postsynaptic density

N-Methyl-D-aspartic acid

NMDA receptor

a-amino-3-hydroxy-5-methyl-4-isoxazolepropionic acid

AMPA receptor

Excitatory postsynaptic potential

Transmembrane AMPA receptor regulating protein

Long-term potentiation

Long-term depression

Brain derived neurotrophic factor

Endoplasmic reticulum

$\mathrm{Ca}^{2+} /$ calmodulin-dependent protein kinase II

Guanine nucleotide exchange factor

soluble N-ethylmaleimide-sensitive-factor attachment receptor

Stimulated Emission Depletion

6-cyano-7-nitroquinoxaline-2,3-dione

(2R)-amino-5-phosphonovaleric acid; $(2 R)$-amino-5-

phosphonopentanoate

Tetrodotoxin

Paraformaldehyde

Plasma membrane

Clathrin-coated vesicle

Trans-golgi network

electron microscopy 


\section{List of Publications}

Richter, K.N., Wildhagen, H., Helm, M.S., Ußling, J.-E., Schikorski, T., and Rizzoli, S.O. (2018). Comparative synaptosome imaging: a semi-quantitative method to obtain copy numbers for synaptic and neuronal proteins. Sci. Rep. 8, 14838.

Mentel, M., Ionescu, A.E., Puscalau-Girtu, I., Helm, M.S., Badea, R.A., Rizzoli, S.O., and Szedlacsek, S.E. (2018). WDR1 is a novel EYA3 substrate and its dephosphorylation induces modifications of the cellular actin cytoskeleton. Sci. Rep. 8, 2910.

Richter, K.N., Revelo, N.H., Seitz, K.J., Helm, M.S., Sarkar, D., Saleeb, R.S., D’Este, E., Eberle, J., Wagner, E., Vogl, C., et al. (2017). Glyoxal as an alternative fixative to formaldehyde in immunostaining and super-resolution microscopy. EMBO J. e201695709.

Gazit, N., Vertkin, I., Shapira, I., Helm, M., Slomowitz, E., Sheiba, M., Mor, Y., Rizzoli, S., and Slutsky, I. (2016). IGF-1 Receptor Differentially Regulates Spontaneous and Evoked Transmission via Mitochondria at Hippocampal Synapses. Neuron 89, 1-15.

Jähne, S., Rizzoli, S.O., and Helm, M.S. (2015). The structure and function of presynaptic endosomes. Exp. Cell Res. 335, 172-179. 